NUREG/CR-XXXX

ORNL/TM-2006/530

\title{
A Physically Based Correlation of Irradiation-Induced Transition Temperature Shifts for RPV Steels
}

Prepared by

E. D. Eason, Modeling and Computing Services, LLC

G. R. Odette, University of California, Santa Barbara

R. K. Nanstad, Oak Ridge National Laboratory

T. Yamamoto, University of California, Santa Barbara

Prepared at

Oak Ridge National Laboratory, Oak Ridge, Tennessee, Managed by UT-Battelle, LLC, under Contract No. DE-AC05-000R22725

M. T. EricksonKirk, NRC Project Manager

Prepared for

The Division of Fuel, Engineering, and Radiological Research

U.S. Nuclear Regulatory Commission

Washington, DC 20555-0001

NRC Job Code W6953 



\section{Abstract}

Irradiation embrittlement of reactor pressure vessel beltline materials is currently evaluated using U.S. Nuclear Regulatory Commission Regulatory Guide 1.99 Revision 2 (RG1.99/2), which presents methods for estimating the shift in Charpy transition temperature at $30 \mathrm{ft}-\mathrm{lb}$ (TTS) and the drop in Charpy upper shelf energy ( $\triangle \mathrm{USE}$ ). The purpose of the work reported here is to improve on the TTS correlation in RG1.99/2 using the broader database now available and current understanding of embrittlement mechanisms.

The key areas expanded in the current database are low-flux, low-copper, and long-time, high-fluence exposures, previously sparse areas. The model incorporates both physically motivated features and empirical calibration to the U.S. power reactor surveillance data. It contains two terms, corresponding to the best-understood radiation damage features, matrix damage and copper-rich precipitates. The new model includes the variables copper, nickel, and fluence that are in RG1.99/2, but also includes irradiation temperature, neutron flux, phosphorus, and manganese.

The report includes a chapter summarizing embrittlement mechanisms and one comparing the Irradiation Variables (IVAR) database with the TTS model predictions. The key new insight in the TTS modeling effort, that flux effects are evident in both low (or no) copper and higher copper materials, is supported by the IVAR data. 

Foreword

[To be supplied by M. T. EricksonKirk] 



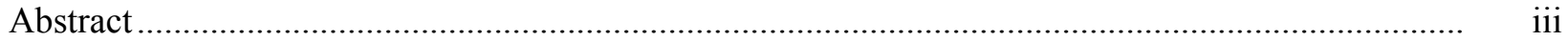

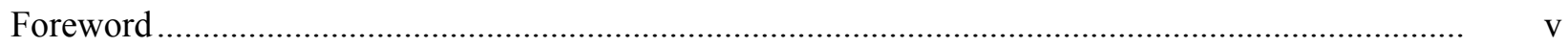

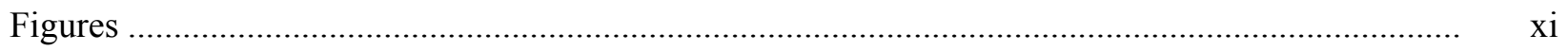

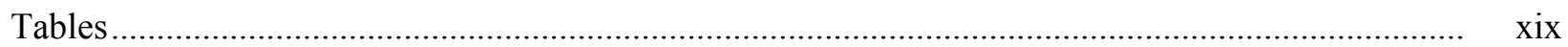

Abbreviated Terms, Nomenclature, and Unit Conversions ......................................................... xxi

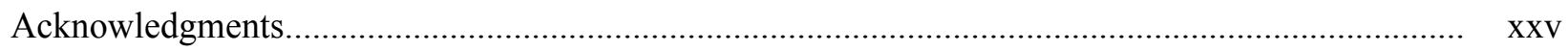

1. Introduction

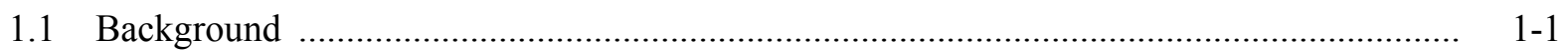

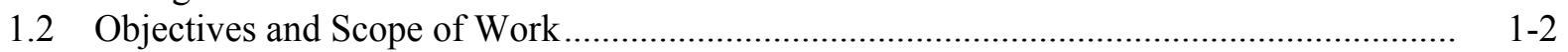

1.3 Overview and Organization of Report …................................................................ $1-3$

1.4 Radiation Damage Mechanisms Background …............................................................... $1-3$

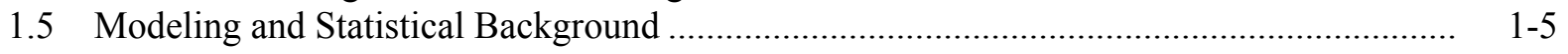

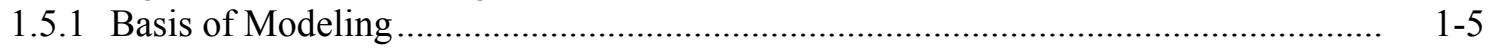

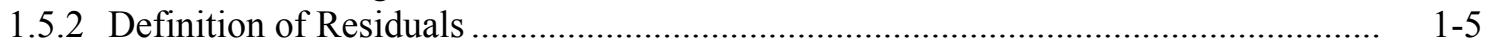

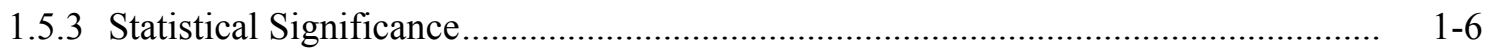

1.5.4 Treatment of Outliers ..............................................................................

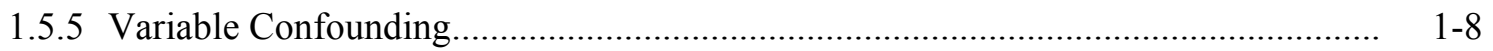

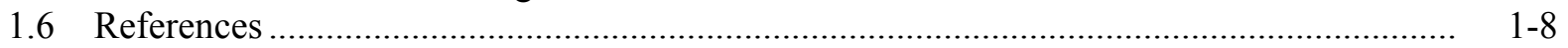

2. Embrittlement Mechanisms and Physical Models Underpinning the TTS Model ...................... 2-1

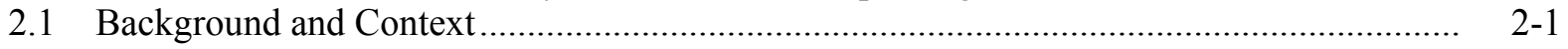

2.2 Key Embrittlement Mechanisms ................................................................................. $2-5$

2.2.1 The Basic Hardening Mechanism of Embrittlement and Primary

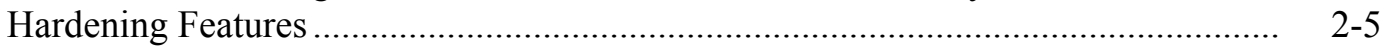

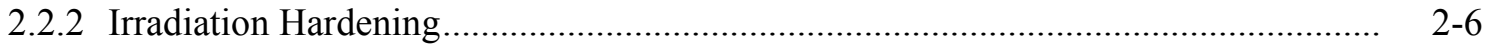

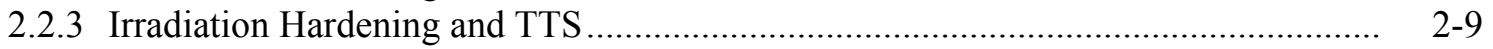

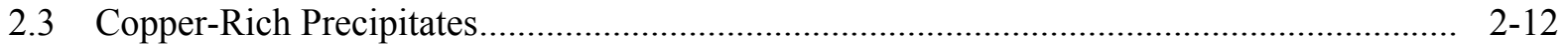

2.3.1 Radiation-Enhanced Diffusion and Flux Effects on CRP Hardening ....................... 2-12

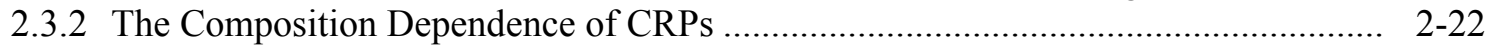

2.3.3 The Fluence Dependence of CRP Hardening .................................................... 2-27

2.3.4 Effects of Irradiation Temperature, $\mathrm{T}_{\mathrm{i}}$, on CRP Hardening .................................... 2-31

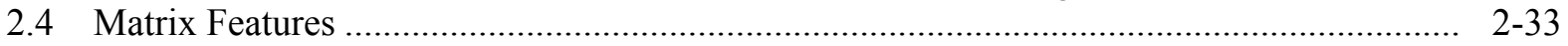

2.4.1 Phosphide Phases (PPs) ............................................................................................ 2-33

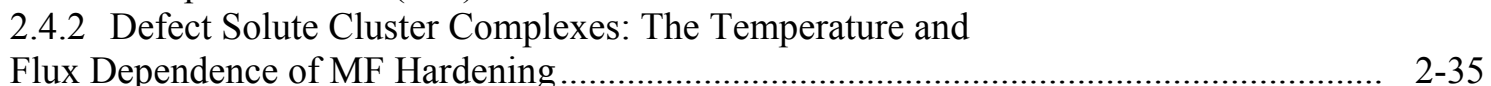

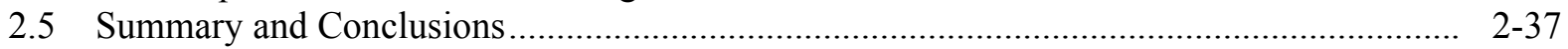

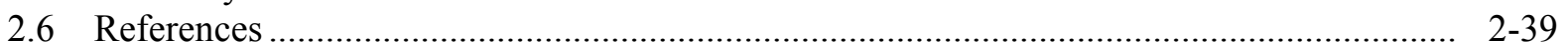


3. Transition Temperature Shift (TTS) Database …………………………………………........... 3-1

3.1 Power Reactor Embrittlement Database (PR-EDB) ........................................................... 3-1

3.2 Guidance from Research Programs (IVAR Database) ………………………………........ 3-1

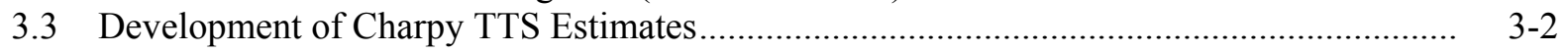

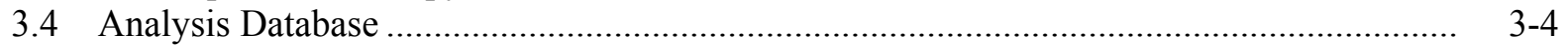

3.4.1 Database for Developing the Matrix Feature (MF) Term ........................................ 3-4

3.4.2 Database for Developing the Copper-Rich Precipitate (CRP) Term .......................... 3- 3-5

3.4.3 Calibration and Validation Datasets ………………………………………….... 3-. $3-7$

3.4.4 Range of Data by Independent Variable ………………......................................... 3-7

3.4.5 Comparison with Prior Databases........................................................................... 3-. 3-9

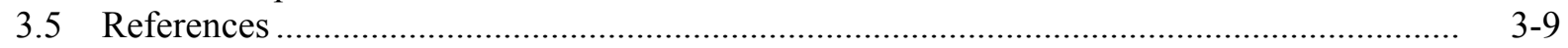

4. Transition Temperature Shift (TTS) Model ………………………………………………..... 4-1

4.1 TTS Analysis Methodology ……………………………………………………...... 4-1

4.1.1 Summary of Steps Taken to Develop a Draft Model............................................... 4. 4-2

4.1.2 Summary of Steps Taken to Calibrate the Baseline TTS Model ............................... 4. 4-3

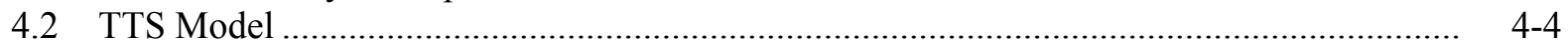

4.2.1 Threshold for High-Cu Behavior ......................................................................... 4. 4-4

4.2.2 Matrix Feature (MF) Term........................................................................... 4.. 4

4.2.3 Copper-Rich Precipitate (CRP) Term .................................................................. 4-12

4.3 Quality of Fit of Revised TTS Model ……………………………………………..... 4-16

4.3.1 Model TTS vs Measured TTS Plots....................................................................... 4-17

4.3.2 Statistical Measures ………………………………......................................... 4-17

4.3.3 Residual Analysis ........................................................................................ 4- 4

4.3.4 Calibration vs Validation Datasets and Predictive Capability ..................................... 4- 48

4.3.5 Comparison with SRM Data ................................................................................... 4-20

4.3.6 Numerical Convergence Checks ............................................................................. 4-21

4.3.7 Comparison with Prior Models ………………………………………………... 4-21

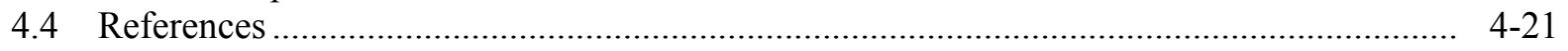

5. Effects of Individual Variables on TTS................................................................................... 5-1

$5.1 \quad$ Material Variables ................................................................................................... $5-1$

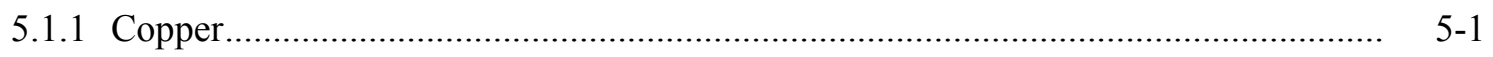

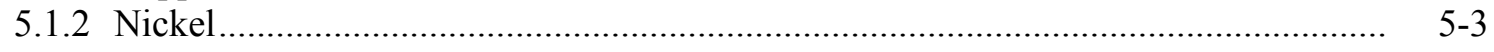

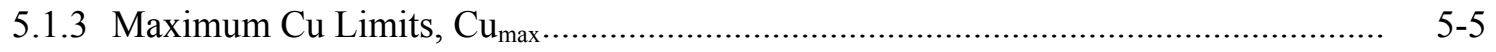

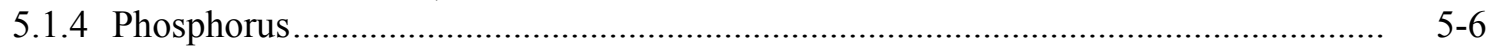

5.1.5 Manganese, Product Form ……………………………………………………. 5 $\quad$ 5-8

5.1.6 Additional Comments on Composition and Product Form........................................ 5-9

$5.2 \quad$ Exposure Variables …………………………………............................................ 5 5-11

5.2.1 Fluence, Effective Fluence, and Flux …………………………………………..... 5-11

5.2.2 Irradiation Temperature ................................................................................... 5 5-15

5.3 References .................................................................................................... 5 5-16

6. Comparison of the IVAR Database with the Calibrated TTS Model Predictions ........................... 6-1

6.1 The IVAR Program and Database ................................................................................ 6-1

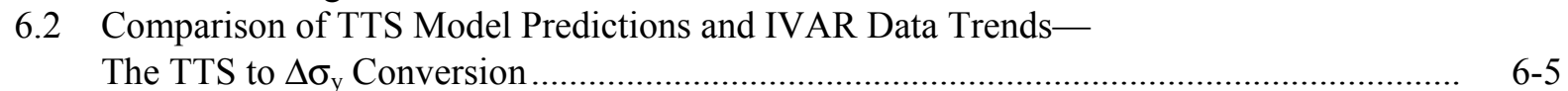

6.3 The Fluence and Irradiation Temperature Dependence of $\Delta \sigma_{\mathrm{y}}$ in Welds and Plates ........... 6 6-6

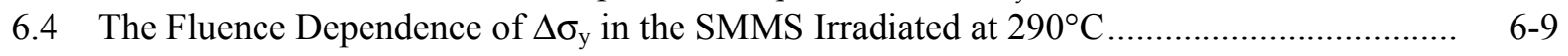

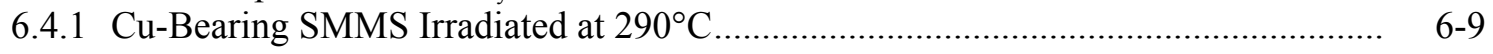

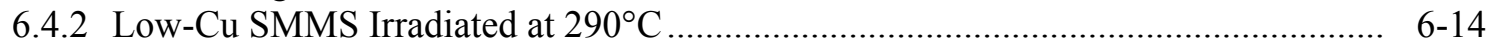


6.5 The Irradiation Temperature Dependence of $\Delta \sigma_{\mathrm{y}}$ in the SMMS ....................................... 6-18

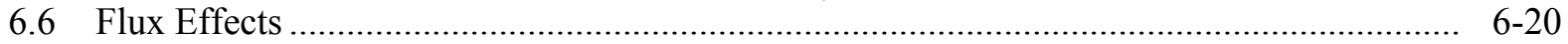

6.6.1 Flux Effects on Hardening in $\mathrm{Cu}-$ Bearing Steels .................................................... 6-20

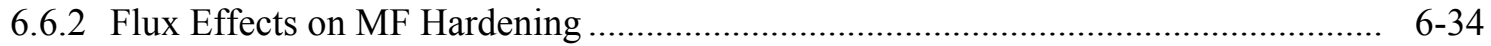

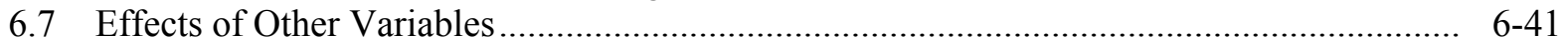

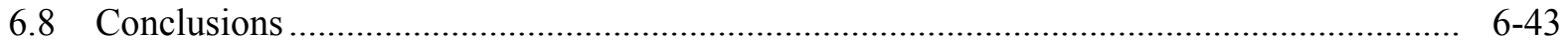

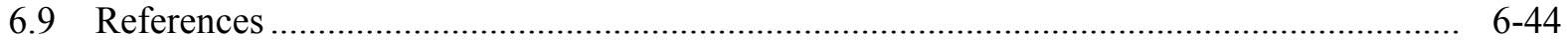

7 Discussion and TTS Model Simplification ..............................................................................

7.1 Discussion of the Irradiation Temperature Term in the CRP Part
of the Baseline Model ................................................................................................

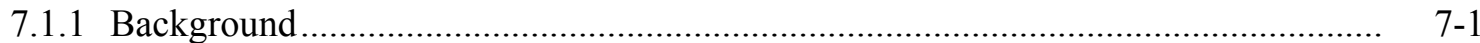

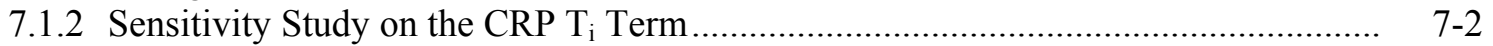

7.1.3 Detailed Review of Low- $\mathrm{T}_{i}$ and High- $\mathrm{T}_{\mathrm{i}}$ Surveillance Data .................................... 7-4

7.1.4 Conclusions and Recommendations from the CRP $\mathrm{T}_{\mathrm{i}}$ Term Sensitivity Study .......... 7-5

7.2 Discussion of the Maximum $\mathrm{Cu}_{\mathrm{e}}$ Values for Material Groups............................................. 7-6

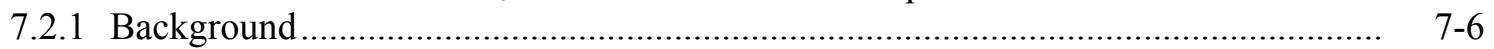

7.2.2 Analysis of Surveillance Data Usable for Calibrating the $\mathrm{Cu}_{\max }$ Values .................... 7-7

7.2.3 Specific Issues Regarding the $\mathrm{Cu}_{\max }$ Limits..........................................................

7.2.4 Sensitivity Study on Combining the Low-Ni Group in a Larger

"All Other" Grouping............................................................................................ 7-11

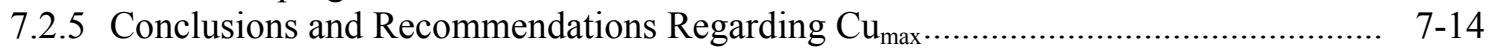

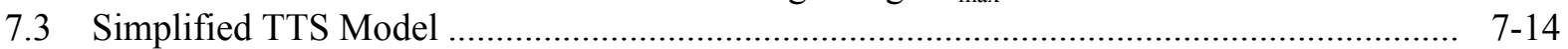

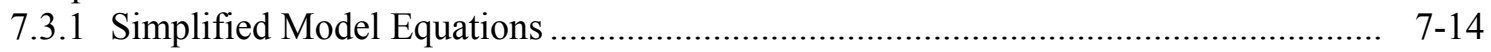

7.3.2 Variables, Units, Definitions, and Ranges of Applicability .................................. 7-15

7.3.3 Goodness of Fit of the Simplified Model ........................................................ 7-17

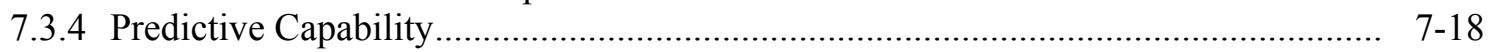

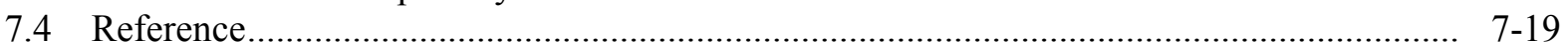

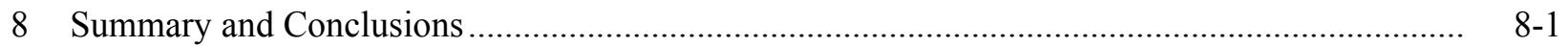

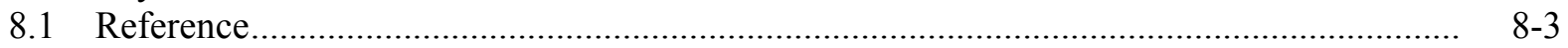

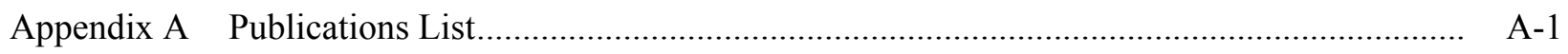

Appendix B Irradiation Variable (IVAR) Program Data Base ................................................... B-1

Appendix C Analysis Data Base …................................................................................... C-1

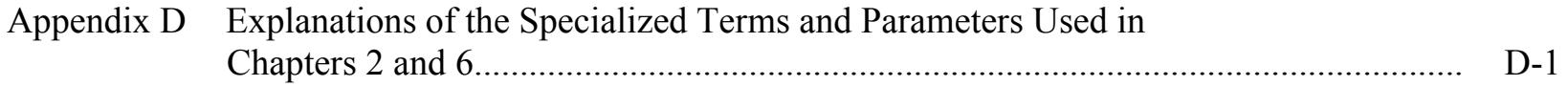

Appendix E Points Excluded from Fitting Sets ................................................................. E-1

Appendix F Residual Plots for Low-Cu and High-Cu Datasets, Baseline Model ........................ F-1

Appendix G Means, Standard Deviations, and Range of Chemistry Variables by Materials Group, Surveillance Data................................................................. G-1

Appendix H $\quad$ Residual Plots for the Simplified Model ........................................................... H-1 



\section{Figures}

1.1 Plot of Charpy impact results showing the effects of irradiation at $88^{\circ} \mathrm{C}$ to a fluence of $1.5 \times 10^{23} \mathrm{n} \cdot \mathrm{m}^{-2} \mathrm{~s}^{-1}(>1 \mathrm{MeV})$ on the CVN impact toughness...

2-1 A simplified description of the sequence of events leading to irradiationinduced TTS.

2.2 A molecular dynamics simulation of displacement cascade in Fe produced by primary recoil atoms (PRAs) created by high-energy neutrons.....

2.3 Primary vacancy and SIA defects and defect clusters created in cascades by PRAs

2.4 Kinetic lattice Monte Carlo simulations of cascade aging to form vacancy solute $(\mathrm{Cu})$ cluster complexes and their remnants that are believed to be the primary source of MF hardening

2.5 (a) Schematic illustration of the vacancy exchange mechanism that resuts in diffusional motion of a substitutional solute

2.6 Schematic illustration of the primary types of nanometer-scale features that cause the irradiation hardening and embrittlement of RPV steels

2.7 (a) Molecular dynamics simulation of the critical dislocation bowing angle for penetrating coherent $\mathrm{Cu}$ precipitates with various $\mathrm{r}_{\mathrm{p}}$ by Bacon and Osetskiy (BO)

2.8 Schematic illustration of the effect of the critical dislocation bowing angle on strength superposition

2.9 Superposition of various levels of strengthening from 1-nm pure $\mathrm{Cu}$ precipitates and CRPs with $180 \mathrm{MPa}$ of pre-existing strong obstacle strengthening and $\alpha_{\mathrm{o}}=0.9$

2.10 Measured versus predicted $\Delta \sigma_{y}$ from CRPs based on SANS measurements of $f_{p}$ and $r_{p}$ used in a modified Russell-Brown precipitate hardening and computer simulation derived superposition model [Eqs. (1) to (3)] ....

2.11 Two feature model hardening analyses for a $0.4 \% \mathrm{Cu} \mathrm{LC}$ alloy irradiated at $290{ }^{\circ} \mathrm{C}$

2.12 Illustration of the basis for determining TTS from $\Delta \sigma_{y}$

2.13 The $\mathrm{C}_{\mathrm{c}}=\mathrm{TTS} / \Delta \sigma_{\mathrm{y}}$ derived from the model described in Fig. 2.12 and the text

2.14 The solubility of $\mathrm{Cu}$ in $\alpha$-Fe in equilibrium with the fcc (solid line) and bcc

$\mathrm{Cu}$ phases (dashed line) as a function of temperature.

2.15 Schematic illustration of solute vacancy trap enhanced recombination 
2.16 The diffusion scaling parameter, $\mathrm{D}^{*} \mathrm{t} / \phi \mathrm{t}=\mathrm{K}+\mathrm{D}_{\mathrm{Cu}} / \phi$, as a function of flux for

the solute trap enhanced recombination- $\mathrm{D}_{\mathrm{Cu}}$ model.

2.17 The dose rate effect scaling parameter $\mathrm{p}$ for $\phi_{\mathrm{r}}=4.45 \times 10^{14} \mathrm{n} / \mathrm{m}^{2}$ versus $\phi$

for the $K(\phi)$ curves shown in Fig. 2.16 (a).

2.18 SANS data on $\mathrm{f}_{\mathrm{p}}$ and $\mathrm{r}_{\mathrm{p}}$ for a $0.4 \mathrm{wt} \% \mathrm{Cu}, 1.25 \mathrm{wt} \% \mathrm{Ni}$ split melt model steel alloys (LD) irradiated at three flux levels between 0.6 to $10 \times 10^{15} \mathrm{n} / \mathrm{m}^{2}-\mathrm{s}$ in IVAR at $290^{\circ} \mathrm{C}$, plotted on both fluence, $\phi \mathrm{t}$, and effective fluence, $\phi \mathrm{t}_{\mathrm{e}}$, scales using a value of $\mathrm{p}=0.5$

2.19 Illustration of the two feature hardening model and the effect of flux, $\phi$, on the fluence, $\phi \mathrm{t}$, dependence of the CRP hardening contribution

2.20 Illustration of the thermodynamics leading to CRP enrichment on $\mathrm{Mn}$ and Ni......

2.21 SANS data on $\mathrm{r}_{\mathrm{p}}, \mathrm{N}_{\mathrm{p}}$, and $\mathrm{f}_{\mathrm{p}}$ for a $0.4 \mathrm{wt} \% \mathrm{Cu}$ split melt model steels irradiated at high IVAR flux at $290^{\circ} \mathrm{C}$

2.22 The total $\mathrm{Cu}\left(\mathrm{X}_{\mathrm{Cu}}\right)$ required for a balance between that contained in the precipitates

$\left(f_{p}\right)$ plus that dissolved in the Fe matrix in local equilibrium with the bcc phase of

$\mathrm{Cu}$, for an interface energy $\gamma_{\mathrm{pm}}=0.4 \mathrm{~J} / \mathrm{m}^{2}$ and lattice coherency mismatch parameter $\delta=0.03$, plotted vs $\mathrm{r}_{\mathrm{p}}$ for various $\mathrm{N}_{\mathrm{p}}$ (open symbols) and the corresponding estimated hardening $\Delta \sigma_{\mathrm{y}}$ (filled symbols)

2.23 Example of a cluster dynamics model of the nucleation-growth and coarsening $\mathrm{Cu}$ precipitation kinetics for an $\mathrm{Fe} 0.3 \mathrm{wt} \% \mathrm{Cu}$ alloy irradiated at $300^{\circ} \mathrm{C}$.

2.24 The Avrami equation for different values of $\beta$

2.25 Illustration of the effects of alloy composition on the shape and position of the CRP/MNP effective fluence function

2.26 SANS data on $r_{p}, N_{p}$, and $f_{p}$ for a 0.4 wt $\% \mathrm{Cu}, 0.8$ wt $\% \mathrm{Ni}, 1.4$ wt $\%$ Mn SMMS irradiated at three temperatures in IVAR.

2.27 Three-dimensional tomographic atom probe data on for model alloys irradiated in IVAR to $1.77 \times 10^{19} \mathrm{n} / \mathrm{cm}^{2}$ at $290^{\circ} \mathrm{C}$ and high flux

2.28 Illustration of vacancy-solute ( $\mathrm{Cu}$ surrogate) cluster complexes that form in cascades from lattice Monte Carlo simulations and the further evolution of these features by a solute flux from long range RED

2.29 Electrical resistivity and Seebeck coefficient changes in a $\mathrm{Cu}$-free alloy with $0.8 \mathrm{wt} \% \mathrm{Ni}, 1.4 \mathrm{wt} \% \mathrm{Mn}$, and $0.005 \mathrm{wt} \% \mathrm{P}$ irradiated at various fluxes and temperatures over a range of fluence plotted against $\Delta \sigma_{\mathrm{y}}$. 
3.1 Examples of symmetric and asymmetric tanh curve fits (from Fig. 2.1 in Eason, E. D., J. E. Wright, and G. R. Odette, Improved Embrittlement Correlations for Reactor Pressure Vessel Steels, NUREG/CR-6551, U.S. Nuclear Regulatory

Commission, Washington, D.C., 1998)

4.1 Apparent location of $\mathrm{Cu}$ threshold based on coefficient in Eq. (4-2)

fitted to each $\mathrm{Cu}$ category

4.2 Apparent location of $\mathrm{Cu}$ threshold based on standard deviation of residuals relative to Eq. (4-2) for each $\mathrm{Cu}$ category

4.3 Apparent location of $\mathrm{Cu}$ threshold based on average residual relative to Eq. (4-2) for each $\mathrm{Cu}$ category.

4.4 Residuals in low-Cu PWR data relative to single coefficient model, plotted against P

4.5 Residuals in low-Cu PWR data with $\mathrm{Mn}>1.35 \mathrm{wt} \%$ relative to single

coefficient model, plotted against $\mathrm{P}$.

4.6 Residuals in low-Cu PWR data with $\mathrm{Mn} \leq 1.35 \mathrm{wt} \%$ relative to single coefficient model, plotted against $\mathrm{P}$

4.7 Effect of effective fluence on MF term at various flux values

4.8 Model shift vs measured shift, Eq. (4-3), all data with $\mathrm{Cu} \leq 0.072 \mathrm{wt} \%$.

4.9 Schematic of CRP term showing effect of key variables on changes

in plateau height and location

4.10 Model shift vs measured shift, all PWR calibration and validation data

with $\mathrm{Cu}>0.072$ wt \%

4.11 Model shift vs measured shift, all BWR calibration and validation data

with $\mathrm{Cu}>0.072 \mathrm{wt} \%$

4.12 Model shift vs measured shift for all SRM data

5.1 Effect of $\mathrm{Cu}$ on CRP term amplitude and transition fluence

5.2 Effect of $\mathrm{Cu}$ and fluence on TTS Model (MF term + CRP term)

5.3 Effect of $\mathrm{Cu}$ on TTS for average Ni Linde 80 welds at three fluence values

5.4 Effect Effect of $\mathrm{Cu}$ on TTS for three weld groups and $2 \times 10^{18} \mathrm{n} / \mathrm{cm}^{2}$ fluence

5.5 Effect of Ni on CRP term amplitude and transition fluence

5.6 Effect of Ni and fluence on TTS (MF term + CRP term)

5.7 Effect of $\mathrm{Ni}$ on TTS at typical $\mathrm{Cu}$ and three fluence values 
5.8 Effect of Ni on TTS at various $\mathrm{Cu}$ levels and $2 \times 10^{18} \mathrm{n} / \mathrm{cm}^{2}$ fluence .............................. 5-5

5.9 Effect of $\mathrm{P}$ on TTS at high- and low- $\mathrm{Cu}$ and $6.5 \times 10^{18} \mathrm{n} / \mathrm{cm}^{2}$ fluence............................ 5-7

5.10 Effect of Mn on TTS at high- and low-Cu and $6.5 \times 10^{18} \mathrm{n} / \mathrm{cm}^{2}$ fluence,

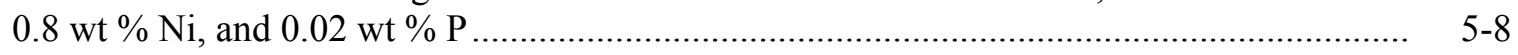

5.11 Effect of flux on TTS in CRP term ........................................................................

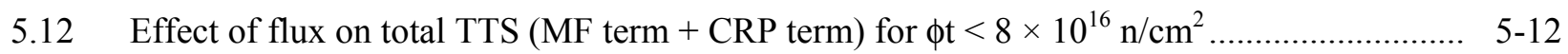

5.13 Schematic of effective fluence in the TTS model (solid curve) and

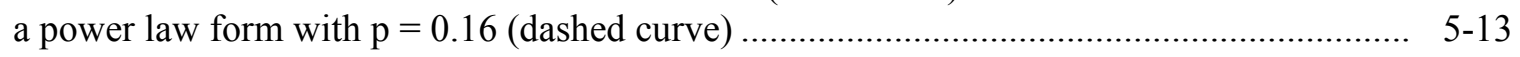

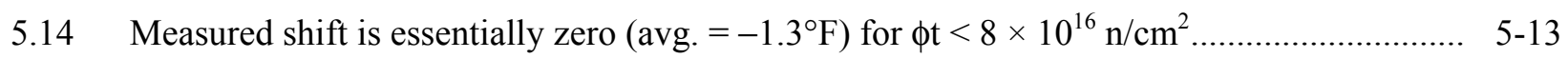

5.15 Significant residual trend using fluence instead of effective fluence, $\phi<4.39 \times 10^{10} \mathrm{n} / \mathrm{cm}^{2} / \mathrm{s}$ and $\phi \mathrm{t}>8 \times 10^{16} \mathrm{n} / \mathrm{cm}^{2}$

5.16 Effect of estimated irradiation temperature …........................................................... $5-15$

6.1 The IVAR irradiation facility and specimen capsules ............................................... $6-2$

6.2 The relations used to convert TTS predictions to $\Delta \sigma_{\mathrm{y}}$ data for welds and plates................... $6-6$

$6.3(\mathrm{a}-\mathrm{d})$. The TTS model predictions and the IVAR $\Delta \sigma_{\mathrm{y}}$ data plotted against the square root of fluence for four commercial $\mathrm{Cu}$-bearing welds

$6.3(\mathrm{e}-\mathrm{h})$. The TTS model predictions and the IVAR $\Delta \sigma_{\mathrm{y}}$ data plotted against the square root of fluence for a $\mathrm{Cu}$ bearing, and low-Cu weld and two commercial plates

6.4 The TTS model predictions and the IVAR $\Delta \sigma_{\mathrm{y}}$ data at two fluences for intermediate flux irradiations of five commercial welds and a plate at 270,290 , and $310^{\circ} \mathrm{C}$

6.5 (a-c). The TTS model predictions and the IVAR $\Delta \sigma_{\mathrm{y}}$ data plotted against the square root of fluence for three $0.8 \mathrm{wt} \% \mathrm{Ni}$ SMMS with 0.1 (a), 0.2 (b), and 0.4 (c) wt \% Cu

6.6 (a-c). The TTS nominal (solid lines) and adjusted (dashed lines) model predictions and the IVAR $\Delta \sigma_{\mathrm{y}}$ data plotted against the square root of fluence for three $0.4 \mathrm{wt} \%$ $\mathrm{Cu}$ SMMS with (0.2 (a) 0.4 (b) and 1.25 (c) wt \% Ni

$6.7(\mathrm{a}-\mathrm{c})$. The TTS model predictions and the IVAR $\Delta \sigma_{\mathrm{y}}$ data plotted against the square root of fluence for three $0.4 \mathrm{wt} \% \mathrm{Cu}$ SMMS with 0.0 (a), 0.8 (b), and $1.6(\mathrm{c}) \mathrm{wt} \% \mathrm{Mn}$

$6.8(\mathrm{a}-\mathrm{d})$. The solid lines in a-c are TTS model predictions for a nominal CE plate product form coefficients $(\mathrm{B}=135)$ and the IVAR $\Delta \sigma_{\mathrm{y}}$ data at two fluences plotted against $\mathrm{Cu}(\mathrm{a}), \mathrm{Ni}(\mathrm{b})$, and $\mathrm{Mn}$ (c). 
6.9 (a-d). The TTS model predictions (solid lines) and best fit chemistry factor (dashed lines) for the IVAR $\Delta \sigma_{y}$ data plotted against the square root of fluence for $\mathrm{Cu}$-free SMMS

6.10 (a-d). The TTS model predictions (solid lines) and best fit chemistry factor (dashed lines) for the IVAR $\Delta \sigma_{\mathrm{y}}$ data plotted against the square root of fluence for (a) and (b) 0.0 and $1.6 \mathrm{wt} \% \mathrm{Ni} \mathrm{Cu}$-free SMMS and (c) and (d) 0.0 .and $1.6 \mathrm{wt} \% \mathrm{Mn}$ 6-15

6.11 (a-c). The TTS model predictions (solid lines) and best fit chemistry factor (dashed lines) for the IVAR $\Delta \sigma_{\mathrm{y}}$ data plotted against the square root of fluence:

(a) and (b) for Cu-free SMMS with 0.031 (a) and $0.35 \mathrm{wt} \% \mathrm{P}$ (b); and a igh $\mathrm{Ni}(1.6 \mathrm{wt} \% \mathrm{Ni})$ low $\mathrm{Cu}$ weld (c).

6.12 (a-d). The solid lines are TTS model predictions at $290^{\circ} \mathrm{C}$ for IVAR SMMS compositions, including one low-Cu weld, several $\mathrm{Cu}$-free SMMS and a $0.1 \mathrm{wt} \% \mathrm{Cu}$ SMMS

6.13 (a-b). TTS model predictions and the $\Delta \sigma_{\mathrm{y}}$ data for four $\mathrm{Cu}$-free SMMS irradiated in IVAR at 270,390 , and $310^{\circ} \mathrm{C}$ at intermediate flux to two fluences

6.14 (a-d). TTS model predictions and the $\Delta \sigma_{\mathrm{y}}$ data for intermediate flux IVAR irradiations to two fluences at 270,290 , and $310^{\circ} \mathrm{C}$ : (a) and (b) SMMS with 0.8 wt $\% \mathrm{Ni}, 1.4 \mathrm{wt} \% \mathrm{Mn}$ and $0.1,0.2$, and $0.4 \mathrm{wt} \% \mathrm{Cu}$; (c) and (d) SMMS with 0.4 wt $\% \mathrm{Cu}, 1.4 \mathrm{wt} \% \mathrm{Mn}$ and $0.2,0.8$ and $1.25 \mathrm{wt} \% \mathrm{Ni}$

6.14 (e-f). The TTS model predictions and the $\Delta \sigma_{\mathrm{y}}$ data for intermediate flux IVAR irradiations to two fluences at 270,290 , and $310^{\circ} \mathrm{C}$ for SMMS with $0.4 \mathrm{wt} \%$ $\mathrm{Cu} ; 0.8 \mathrm{wt} \% \mathrm{Ni}$; and $0.0,0.8$, and $1.6 \mathrm{wt} \% \mathrm{Mn}$

6.15 (a-f) (a, d). The fitted recombination model (FRM) predictions and the $290^{\circ} \mathrm{C}$ IVAR $\Delta \sigma_{\mathrm{y}}$ data for two Cu-bearing commercial welds on an effective fluence, $\phi \mathrm{t}_{\mathrm{e}}$, scale; $(b, e)$ the corresponding measured minus predicted residuals for the FRM that are well centered and approximately independent of flux; (c, f) the corresponding residuals for the TTS model, showing a systematic effect of flux that is not accounted for in the TTS model developed in Chap. 3

$6.15(\mathrm{~g}-1)(\mathrm{g}, \mathrm{j})$. The fitted recombination model (FRM) predictions and the $290^{\circ} \mathrm{C}$ IVAR $\Delta \sigma_{\mathrm{y}}$ data for two $\mathrm{Cu}$-bearing commercial welds on an effective fluence, $\phi \mathrm{t}_{\mathrm{e}}$, scale; $(\mathrm{h}, \mathrm{k})$ the corresponding measured minus predicted residuals for the FRM that are well centered and approximately independent of flux; (i, l) the corresponding residuals for the TTS model, showing a systematic effect of flux that is not accounted for in the TTS model developed in Chap. 3

$6.15(\mathrm{~m}-\mathrm{o})(\mathrm{m})$. The fitted recombination model (FRM) predictions and the $290^{\circ} \mathrm{C}$ IVAR $\Delta \sigma_{\mathrm{y}}$ data for a $\mathrm{Cu}$-bearing commercial welds on an effective fluence, $\phi \mathrm{t}_{\mathrm{e}}$, scale; (n) the corresponding measured minus predicted residuals for the FRM that are well centered and approximately independent of flux; (o) the corresponding residuals for the TTS model, showing a systematic effect of flux that is not accounted for in the TTS model developed in Chap. 3 
$6.15(\mathrm{p}-\mathrm{u})(\mathrm{p}, \mathrm{s})$. The fitted recombination model (FRM) predictions and the $290^{\circ} \mathrm{C}$ IVAR $\Delta \sigma_{\mathrm{y}}$ data for two $\mathrm{Cu}$-bearing commercial plates on an effective fluence, $\phi \mathrm{t}_{\mathrm{e}}$, scale; $(\mathrm{q}, \mathrm{t})$ the corresponding measured minus predicted residuals for the FRM that are well centered and approximately independent of flux; $(r, u)$ the corresponding residuals for the TTS model, showing a systematic effect of flux that is not accounted for in the TTS model developed in Chap. 3.

$6.16(\mathrm{a}-\mathrm{f})(\mathrm{a}, \mathrm{d})$. The fitted recombination model (FRM) predictions and the $290^{\circ} \mathrm{C}$ IVAR $\Delta \sigma_{\mathrm{y}}$ data for two SMMS with $0.8 \mathrm{wt} \% \mathrm{Ni}, 1.4 \mathrm{wt} \% \mathrm{Mn}$ and 0.1 and $0.2 \mathrm{wt} \% \mathrm{Cu}$ on an effective fluence, $\phi \mathrm{t}_{\mathrm{e}}$, scale; $(\mathrm{b}, \mathrm{e})$ the corresponding measured minus predicted residuals for the FRM that are well centered and approximately independent of flux; (c, f) the corresponding residuals for the TTS model, showing a systematic effect of flux that is not accounted for in the TTS model developed in Chap. 3 .....

$6.16(\mathrm{~g}-\mathrm{i})(\mathrm{g})$. The fitted recombination model (FRM) predictions and the $290^{\circ} \mathrm{C}$ IVAR $\Delta \sigma_{\mathrm{y}}$ data for a SMMS with $0.8 \mathrm{wt} \% \mathrm{Ni}, 1.4 \mathrm{wt} \% \mathrm{Mn}$ and $0.4 \mathrm{wt} \% \mathrm{Cu}$ on an effective fluence, $\phi \mathrm{t}_{\mathrm{e}}$, scale; (h) the corresponding measured minus predicted residuals for the FRM that are well centered and approximately independent of flux; (i) the corresponding residuals for the TTS model, showing a systematic effect of flux that is not accounted for in the TTS model developed in Chap. 3 ....

6.17 (a-f). (a,d). The fitted recombination model (FRM) predictions and the $290^{\circ} \mathrm{C}$ IVAR $\Delta \sigma_{\mathrm{y}}$ data for two SMMS with $0.4 \mathrm{wt} \% \mathrm{Cu}, 1.4 \mathrm{wt} \%$ $\mathrm{Mn}$, and 0.2 and $1.25 \mathrm{wt} \% \mathrm{Ni}$ on an effective fluence, $\phi \mathrm{t}_{\mathrm{e}}$, scale; $(\mathrm{b}, \mathrm{e})$ the corresponding measured minus predicted residuals for the FRM that are well centered and approximately independent of flux; (c, f) the corresponding residuals for the TTS model, showing a systematic effect of flux that is not accounted for in the TTS model developed in Chap. 3

6.18 (a-f.) (a, d). The fitted recombination model (FRM) predictions and the $290^{\circ} \mathrm{C}$ IVAR $\Delta \sigma_{\mathrm{y}}$ data for two SMMS with $0.4 \mathrm{wt} \% \mathrm{Cu}, 0.8 \mathrm{wt} \% \mathrm{Ni}$, and 0.0 and $0.8 \mathrm{wt} \% \mathrm{Mn}$ on an effective fluence, $\phi \mathrm{t}_{\mathrm{e}}$, scale; $(\mathrm{b}, \mathrm{e})$ the corresponding measured minus predicted residuals for the FRM that are well centered and approximately independent of flux; (c, f) the corresponding residuals for the TTS model, showing a systematic effect of flux that is not accounted for in the TTS model developed in Chap. 3

$6.18(\mathrm{~g}-\mathrm{i}) .(\mathrm{g})$. The fitted recombination model (FRM) predictions and the $290^{\circ} \mathrm{C}$ IVAR $\Delta \sigma_{\mathrm{y}}$ data for two SMMS with $0.4 \mathrm{wt} \% \mathrm{Cu}, 0.8 \mathrm{wt} \% \mathrm{Ni}$, and $1.6 \mathrm{wt} \% \mathrm{Mn}$ on an effective fluence, $\phi \mathrm{t}_{\mathrm{e}}$, scale; (h) the corresponding measured minus predicted residuals for the FRM that are well centered and approximately independent of flux; (i) the corresponding residuals for the TTS model, showing a systematic effect of flux that is not accounted for in the TTS model developed in Chap. 3.

6.19 The flux scaling $\mathrm{p}$ values for a range of $\mathrm{X}_{\mathrm{t}}$ and low and high nominal $\mathrm{D}_{\mathrm{C}}$ 
6.20 The $\mathrm{M} \phi=\phi \mathrm{t}_{\mathrm{e}} / \phi \mathrm{t}$ effective fluence multiplying factor as a function of flux for the TTS model, the FRM for various subsets of IVAR data and the corresponding $\mathrm{M} \phi$ reported by Williams

6.21 The fluence at 50\% of the maximum CRP hardening, $\phi t 0.5$, for the TTS and FRM models and various subsets of IVAR data ignoring the effect of the TTS to $\Delta \sigma_{\mathrm{y}}$ conversion

6.22 Schematic illustration of the ability of the TTS model to fit a flux effect in scattered data over a wide range of dose rates compared to the more precise data from the controlled IVAR experiment

6.23 (a-f). (a, d). The fitted MF model for the $290^{\circ} \mathrm{C}$ IVAR $\Delta \sigma_{\mathrm{y}}$ data on two $\mathrm{Cu}$-free SMMS with $0.8 \mathrm{wt} \% \mathrm{Ni}, 1.6 \mathrm{wt} \% \mathrm{Mn}$, and $0.005 \mathrm{wt} \% \mathrm{P}$ on an effective fluence, $\phi \mathrm{t}_{\mathrm{e}}$, scale

6.23 (g-1). $(g, j)$. The fitted MF model for the $290^{\circ} \mathrm{C}$ IVAR $\Delta \sigma_{\mathrm{y}}$ data on two $\mathrm{Cu}$-free SMMS with 0.8 wt \% Ni, 1.4 to 1.6 wt $\% \mathrm{Mn}$, and $0.005 \mathrm{wt} \% \mathrm{P}$ on an effective fluence, $\phi \mathrm{t}_{\mathrm{e}}$, scale

6.24 (a-f.) (a, d). The fitted MF model for the $290^{\circ} \mathrm{C}$ IVAR $\Delta \sigma_{\mathrm{y}}$ data on two $\mathrm{Cu}$-free SMMS with 0.8 and $1.6 \mathrm{wt} \% \mathrm{Ni}, 1.6 \mathrm{wt} \% \mathrm{Mn}$, and $0.005 \mathrm{wt} \% \mathrm{P}$ on an effective fluence, $\phi \mathrm{t}_{\mathrm{e}}$, scale

$6.24(\mathrm{~g}-1) .(\mathrm{g}, \mathrm{j})$. The fitted MF model for the $290^{\circ} \mathrm{C}$ IVAR $\Delta \sigma_{\mathrm{y}}$ data

on two $\mathrm{Cu}$-free SMMS with $0.8 \mathrm{wt} \% \mathrm{Ni}, 0.005 \mathrm{wt} \% \mathrm{P}$, and 0.0 and $0.8 \mathrm{wt} \% \mathrm{Mn}$ on an effective fluence, $\phi \mathrm{t}_{\mathrm{e}}$, scale .

6.25 (a-f.) (a, d). The fitted MF model for the $290^{\circ} \mathrm{C}$ IVAR $\Delta \sigma_{\mathrm{y}}$ data for two

$\mathrm{Cu}$-free SMMS with $0.8 \mathrm{wt} \% \mathrm{Ni}, 1.6 \mathrm{wt} \% \mathrm{Mn}$, and 0.031 and $0.35 \mathrm{wt} \% \mathrm{P}$ on an effective fluence, $\phi \mathrm{t}_{\mathrm{e}}$, scale.

$6.25(\mathrm{~g}-1)(\mathrm{g}, \mathrm{j})$. The fitted MF model for the $290^{\circ} \mathrm{C}$ IVAR $\Delta \sigma_{\mathrm{y}}$ data fortwo low-Cu welds with $0.8-1.6 \mathrm{wt} \% \mathrm{Ni}$ on an effective fluence, $\phi \mathrm{t}_{\mathrm{e}}$, scale

6.26 The $\Delta \sigma_{\mathrm{y}}$ for three simple model alloys irradiated in IVAR at high flux and $290^{\circ} \mathrm{C}$ to $1.77 \times 10^{19} \mathrm{n} / \mathrm{cm}^{2}$, showing a Mn-P interaction leading to higher hardening.

6.27 Averaged high fluence $\Delta \sigma_{\mathrm{y}}$ data for IVAR irradiations at intermediate and high flux at $290^{\circ} \mathrm{C}$ plotted as a function of $\mathrm{P}$ for SMMS with $0.8 \mathrm{wt} \% \mathrm{Ni}, 1.6 \mathrm{wt} \% \mathrm{Mn}$ and 0.0 and $0.1 \mathrm{wt} \% \mathrm{Cu}$.

7.1 Residual trend with temperature for high-Cu data, modified model without CRP $T_{i}$ term, showing apparent residual effect (slope is not significant).

7.2 Nonsignificant linear residual trend when a single calibrated $\mathrm{Cu}_{\max }$ value is used, all available data with $\mathrm{Cu}>0.243 \mathrm{wt} \%$

7.3 Effect of $\mathrm{Cu}$ on TTS for various Ni levels, "all other" materials (similar to Fig. 5-4 but revised for two material group categories) 
7.4 Effect of Ni on TTS for various $\mathrm{Cu}$ levels, "All Other" group, and $2 \times 10^{18} \mathrm{n} / \mathrm{cm}^{2}$ fluence.

7.5 Effect of using $\mathrm{Cu}_{\max }=0.301$ on the low-Ni welds that determined the $\mathrm{Cu}_{\max }=0.37$ value for the "other weld" category in the baseline model

7.6 Simplified Model shift (Eq. 7-2 through 7-5) vs measured shift, all PWR calibration and validation data with $\mathrm{Cu}>0.072 \mathrm{wt} \%$...

7.7 Simplified Model shift (Eq. 7-2 through 7-5) vs measured shift, all BWR calibration and validation data with $\mathrm{Cu}>0.072 \mathrm{wt} \%$ 


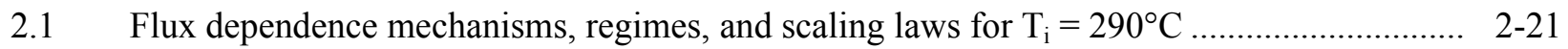

3.1 Sampling of standard reference material data .............................................................. $3-6$

3.2 Distribution of data by dataset (including SRM samples) ................................................ $3-7$

3.3 Independent variables in the embrittlement shift model and their ranges and mean values over all calibration and validation data (855 datapoints)

4.1 Standard deviation $\left(\mathrm{S}_{\mathrm{d}}\right)$ of residuals about the embrittlement shift model in various subsets, all PWR and BWR calibration and validation data except SRM.

4.2 Comparison of calibration and validation subsets by mean and standard deviation $\left(\mathrm{S}_{\mathrm{d}}\right)$ of residuals

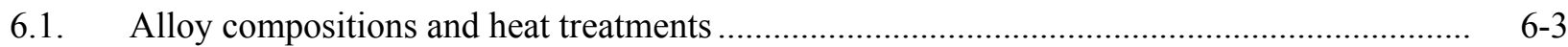

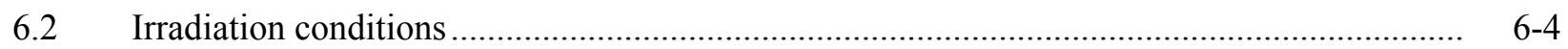

7.1 Residual analysis of the points that are at least $15^{\circ} \mathrm{F}$ above and below average temperature, modified model without $\mathrm{CRP} \mathrm{T}_{\mathrm{i}}$ term

7.2 Distribution of data for calibrating $\mathrm{Cu}$ saturation limits (calibration and validation data)

7.3. Independent variables in the embrittlement shift model and their ranges and mean values over all calibration and validation data (855 datapoints)

7.4. Standard deviation $\left(S_{d}\right)$ of residuals about the simplified embrittlement shift model in various subsets, all PWR and BWR calibration and validation data except SRM

7.5. Comparison of calibration and validation subsets by mean and standard deviation $\left(\mathrm{S}_{\mathrm{d}}\right)$ of residuals for the simplified TTS model 



\section{Acronyms and Abbreviations}

\begin{tabular}{|c|c|}
\hline ASTM & American Society for Testing and Materials \\
\hline B\&W & Babcock and Wilcox \\
\hline BWR & boiling water reactor \\
\hline CDM & cluster dynamics model \\
\hline $\mathrm{CE}$ & Combustion Engineering \\
\hline $\mathrm{CF}$ & chemistry factor \\
\hline CRP & copper-rich precipitate \\
\hline DBT & ductile-brittle transition \\
\hline dpa & displacements per atom \\
\hline EPRI & Electric Power Research Institute \\
\hline FRM & fitted recombination model \\
\hline IAEA & International Atomic Energy Agency \\
\hline IGF & intergranular fracture \\
\hline IVAR & Irradiation Variable (program) \\
\hline JRQ & Japanese designation for a specific heat of RPV steel \\
\hline LBP & late-blooming phase \\
\hline LSE & lower shelf energy \\
\hline LWR & light water reactor \\
\hline $\mathrm{M} \& \mathrm{CS}$ & Modeling and Computing Services \\
\hline $\mathrm{MC}$ & Monte-Carlo \\
\hline MD & molecular dynamic \\
\hline MF & matrix feature \\
\hline MNP & Mn-Ni-Si-rich precipitate \\
\hline NRC & U.S. Nuclear Regulatory Commission \\
\hline ORNL & Oak Ridge National Laboratory \\
\hline PP & phosphide phases \\
\hline PR-EDB & Power Reactor Embrittlement Database \\
\hline PWHT & postweld heat treatment \\
\hline PRA & primary recoiling atom \\
\hline PWR & pressurized water reactor \\
\hline RED & radiation-enhanced diffusion \\
\hline RPV & reactor pressure vessel \\
\hline RSC & resistivity-Seebeck coefficient \\
\hline RSS & square root of the sum of the squares \\
\hline SANS & small-angle neutron scattering \\
\hline SAW & submerged arc weld \\
\hline SIA & self-interstitial atom \\
\hline SMF & stable matrix feature \\
\hline SMMS & split metal model steel \\
\hline SRM & standard reference material \\
\hline SSP & Supplemental Surveillance Program \\
\hline TTS & transition temperature shift \\
\hline UCSB & University of California, Santa Barbara \\
\hline USE & upper shelf energy \\
\hline VSR & variable stress relief \\
\hline
\end{tabular}




\section{Nomenclature}

\begin{tabular}{|c|c|}
\hline$\alpha_{j}$ & obstacle strength of hardening feature $\mathrm{j}$ \\
\hline$\alpha(n)$ & the rate at which the cluster of the size, $n$, emits a mobile monomer \\
\hline$\beta$ & precipitation kinetics parameter depending on the controlling mechanism \\
\hline$\beta(v)$ & the rate at which the cluster of the size, $n$, absorbs a mobile monomer \\
\hline$\delta$ & lattice parameter mismatch between precipitate and matrix \\
\hline$\Delta \rho$ & change in resistivity \\
\hline$\Delta \mathrm{S}$ & change in Seebeck coefficient \\
\hline$\Delta \sigma_{\mathrm{y}}$ & change in yield strength \\
\hline$\Delta \sigma_{\mathrm{yj}}$ & change in yield strength due to hardening feature $\mathrm{j}$ \\
\hline$\Delta \mathrm{USE}$ & change in Charpy upper shelf energy due to irradiation $\left(=\mathrm{USE}_{\mathrm{i}}-\mathrm{USE}_{\mathrm{u}}\right)$ \\
\hline$\gamma_{\mathrm{pm}}$ & precipitate-matrix interface energy \\
\hline$\mu$ & shear modulus \\
\hline$\phi$ & flux, $\mathrm{n} / \mathrm{cm}^{2} / \mathrm{s}, \mathrm{E}>1 \mathrm{MeV}$ \\
\hline$\phi_{\mathrm{r}}$ & reference flux \\
\hline$\phi(\mathrm{E})$ & spectrum \\
\hline$\phi t$ & fluence, $\mathrm{n} / \mathrm{cm}^{2}, \mathrm{E}>1 \mathrm{MeV}$ \\
\hline$\phi \mathrm{t}_{\mathrm{e}}$ & effective fluence \\
\hline$\Gamma_{\mathrm{ij}}$ & thermodynamic activity coefficient of solute $\mathrm{i}$ in phase $\mathrm{j}\left(=\mathrm{a}_{\mathrm{ij}} / \mathrm{X}_{\mathrm{ij}}\right)$ \\
\hline$\mu$ & shear modulus \\
\hline$\sigma^{*}$ & microcleavage fracture stress \\
\hline$\sigma_{\mathrm{v}}$ & neutron spectrum averaged vacancy production cross section \\
\hline$\sigma_{\mathrm{yj}}$ & intrinsic contribution of hardening feature $\mathrm{j}$ to yield strength before superposition \\
\hline$\sigma_{\text {ypm }}$ & maximum CRP hardening \\
\hline$\rho$ & dislocation density \\
\hline A & product form parameter for $\mathrm{TTS}_{\mathrm{mf}}$ \\
\hline$a_{i j}$ & thermodynamic activity of solute $\mathrm{i}$ in phase $\mathrm{j}$ \\
\hline $\mathrm{b}$ & Burger's vector \\
\hline B & product form parameter for $\mathrm{TTS}_{\mathrm{crp}}$ \\
\hline $\mathrm{C}_{\mathrm{c}}$ & ratio of TTS to $\Delta \sigma_{\mathrm{y}}\left({ }^{\circ} \mathrm{C} / \mathrm{MPa}\right)$ \\
\hline $\mathrm{C}_{\text {Cue }}$ & effective $\mathrm{Cu}$ concentration \\
\hline $\mathrm{C}_{\mathrm{p}}$ & $\mathrm{P}$ coefficient for TTS $\left({ }^{\circ} \mathrm{C} / \mathrm{wt} \% \mathrm{P}\right)$ \\
\hline $\mathrm{C}_{\mathrm{T}}$ & irradiation temperature coefficient for irradiation hardening \\
\hline $\mathrm{CF}$ & Chemistry factor quantifying the effect of the composition of MF hardening \\
\hline $\mathrm{CF}_{\mathrm{P}}$ & Phosphorus CF \\
\hline $\mathrm{Cu}_{\min }$ & minimum $\mathrm{Cu}$ needed for $\mathrm{CRP}$ formation \\
\hline $\mathrm{Cu}_{\max }$ & maximum effective dissolved $\mathrm{Cu}$ prior to irradiation \\
\hline $\mathrm{Cu}_{\mathrm{e}}$ & effective $\mathrm{Cu}$ \\
\hline $\mathrm{D}^{*}$ & radiation-enhanced diffusion coefficient of $\mathrm{Cu}$ \\
\hline $\mathrm{D}_{\mathrm{Cu}}$ & thermal diffusion coefficient of $\mathrm{Cu}$ \\
\hline $\mathrm{D}_{\mathrm{i}}$ & diffusion coefficient of SIAs \\
\hline $\mathrm{D}_{\text {sd }}$ & diffusion coefficient for Fe self-diffusion \\
\hline $\mathrm{D}_{\mathrm{S}}$ & diffusion coefficient of a species $\mathrm{S}$ \\
\hline$D_{v}$ & vacancy diffusion coefficient $\left(=1.1 \times 10^{-16} \mathrm{~m}^{2} / \mathrm{s} @ 290^{\circ} \mathrm{C}\right)$ \\
\hline$f_{j}$ & volume fraction of hardening feature $\mathrm{j}$ \\
\hline$f_{\mathrm{pm}}^{1}$ & maximum precipitate volume fraction \\
\hline $\begin{array}{l}\text { Ipm } \\
\text { fluence }\end{array}$ & $\mathrm{n} / \mathrm{cm}^{2}, \mathrm{E}>1 \mathrm{MeV}$ \\
\hline flux & $\mathrm{n} / \mathrm{cm}^{2} / \mathrm{s}, \mathrm{E}>1 \mathrm{MeV}$ \\
\hline $\mathrm{G}_{\mathrm{i}}$ & generation rate of SIAs \\
\hline
\end{tabular}


$\mathrm{T}$

$\mathrm{T}_{30}$

$\mathrm{T}_{\mathrm{ann}}$

$\mathrm{T}_{\mathrm{c}}$

$\mathrm{T}_{\mathrm{i}}$

$t_{\text {sr }}$

$t_{\mathrm{t}}$

$\mathrm{T}_{\mathrm{sr}}$ tanh

$\mathrm{X}_{\mathrm{Cu}}$

$\mathrm{X}_{\text {Cue }}$ fraction of vacancies that recombine with SIAs before reaching sinks fraction of vacancies that reach sinks

generation rate of a species $\mathrm{S}$

generation rate of vacancies

heat of solution of solute $i$ in phase $j$

solute index for precipitation thermodynamics

hardening feature index; by type ( $\mathrm{mf}, \mathrm{p}$, crp, mnp, pp for MF, precipitate, CRP, MNP, PP) or by strength (w, m, o for weak, medium, high strength)

thermodynamics index for the $\mathrm{j}$ phase containing element $\mathrm{i}$

Boltzmann's constant

radiation-enhanced diffusion factor

constraint factor $>1$

effective fluence multiplier reflecting flux effect $\left(=\phi t_{\mathrm{e}} / \phi \mathrm{t}\right)$

number of atoms in a cluster

number density of hardening feature $\mathrm{j}$

number density of the cluster of size, $n$

dose rate effect exponent, which varies from 0 to 1 depending on the flux regime

gas constant

recombination factor

radius of hardening feature $\mathrm{j}$

radius of a cluster of $\mathrm{n} \mathrm{Cu}$ atoms

recombination radius

superposition factor to control the balance between LS and RSS laws

standard deviation

standard error

total defect sink strength

total defect sink strength for a species $\mathrm{S}$

$\mathrm{Cu}$ supersaturation

temperature

temperature at $30 \mathrm{ft}-\mathrm{lb}(41 \mathrm{~J})$ in Charpy tests

annealing temperature

elastic cleavage temperature

irradiation temperature

stress relief time

overall time constant for precipitate formation (time for $f_{p}$ to reach $0.63 f_{p m}$ )

stress relief temperature

hyperbolic tangent

Taylor Factor $(\approx 3)$

transition temperature measured at $\approx 10 \mathrm{~J}$ for irradiatetd material

transition temperature measured at $\approx 10 \mathrm{~J}$ for unirradiated material

TTS measured at $10 \mathrm{~J}=\mathrm{TT}_{10 \mathrm{i}}-\mathrm{TT}_{10 \mathrm{u}}$

TTS due to hardening feature $\mathrm{p}$

TTS measured at $41 \mathrm{~J}$ due to the reduction in USE

TTS due to MF

TTS due to CRP

Charpy upper shelf energy for irradiated material

Charpy upper shelf energy for unirradiated material

the volume of a $\mathrm{Cu}$ or $\mathrm{Fe}$ atom

the volume of a mole of $\mathrm{Cu}$ atoms

initial $\mathrm{Cu}$ in solution

effective atomic fraction of $\mathrm{Cu}$ initially in solution 
$\mathrm{X}_{\text {Cup }} \quad$ precipitate $\mathrm{Cu}$ content

$\mathrm{X}_{\mathrm{Cur}} \quad$ residual $\mathrm{Cu}$ in solution in equilibrium with precipitates

$\mathrm{X}_{\mathrm{ij}}$ atomic fraction of solute $\mathrm{i}$ in phase $\mathrm{j}$

$\mathrm{X}_{\mathrm{S}} \quad$ atomic fraction of a species $\mathrm{S}$

$\mathrm{X}_{\mathrm{v}} \quad$ atomic fraction of vacancies under irradiation

$\mathrm{X}_{\mathrm{ve}} \quad$ atomic fraction of vacancies at thermal equilibrium

\section{Unit Conversions}

Fahrenheit to Celsius: $T_{\mathrm{C}}=\left(T_{\mathrm{F}}-32\right) / 1.8$

Foot-pounds to Joules: $1 \mathrm{ft}-\mathrm{lb}=1.356 \mathrm{~J}$

Note: The U.S. RPV surveillance database is maintained in English units. Thus, the modeling task was performed and is reported in English units. Other parts of this report, namely Chap. 2 on embrittlement mechanisms and Chap. 6 on comparisons of the IVAR data with the TTS model, are reported in SI units. 


\section{Acknowledgments}

The authors extend their appreciation to the members of ASTM E10.02.02 for extensive reviews of the surveillance database, to J. A. Wang for assistance and advice regarding the PR-EDB database, to Thomas M. Rosseel, Program Manager of the Heavy-Section Steel Irradiation Program, Mikhail Sokolov and Roger Stoller for helpful discussions and technical reviews of the report, to Walter Koncinski for technical editing, and to Brenda Phillips for preparation of the manuscript. We also gratefully acknowledge the very significant contributions of UCSB student Mr. Jonathan Smith to the analysis of the IVAR database and comparison of the IVAR database with the TTS model predictions. We also thank the members of the UCSB Materials Reliability and Performance Group, especially Mr. Doug Klingensmith and Mr. David Gragg, whose tireless efforts made developing the IVAR database possible. We also wish to express our appreciation for the contributions of our many collaborators around the world, who helped to develop an improved fundamental understanding of embrittlement mechanisms that forms the foundation of Chapter 2, with special thanks going to Professor Brian Wirth (UC Berkeley), Mike Miller (ORNL) and the staff of the National Institute of Standards and Technology Center for Neutron Research. The research at UCSB was supported by the US NRC Contract Number 04-01-064. Finally, appreciation is also extended to Carolyn Fairbanks, Mark EricksonKirk, Bob Hardies, Allen Hiser, Cayetano Santos, and Simon Sheng of the U.S. Nuclear Regulatory Commission for their advice, encouragement, and financial support. 



\section{Introduction}

\subsection{Background}

Pressure vessels for light water reactors (LWRs) are designed and fabricated in accordance with the requirements in consensus codes that are based on mechanical and physical properties of the steels used to construct the vessels. In the absence of radiation damage to the reactor pressure vessel (RPV), fracture of the vessel is difficult to postulate because the fracture toughness of the RPV in the unirradiated condition is generally very high at and above room temperature. However, exposure to high-energy neutrons can result in embrittlement of radiation-sensitive RPV materials. The degrading effects of neutron irradiation on carbon and low-alloy pressure vessel steels have been recognized and investigated since the early 1950s. In those steels at LWR operating temperatures $\left[\sim 520-570^{\circ} \mathrm{F}\left(\sim 270-300^{\circ} \mathrm{C}\right)\right]$, radiation damage is produced when neutrons of sufficient energy displace atoms; the displacements result in displacement cascades, which produce large numbers of vacancy and interstitial-type defects. Although the inside surface of the RPV is exposed to neutrons of varying energies, the higher-energy neutrons produce the bulk of the damage. In a typical LWR, the flux of such high-energy neutrons $(>1 \mathrm{MeV})$ is from about $10^{13}$ to $10^{16} \mathrm{n} \cdot \mathrm{m}^{-2} \mathrm{~s}^{-1}$.

Irradiation embrittlement of RPV beltline materials is currently evaluated according to U.S. Nuclear Regulatory Commission (NRC) Regulatory Guide 1.99, Revision 2 [1], which presents methods (based on data correlations) for estimating the Charpy transition temperature shift (TTS) at $30 \mathrm{ft}-\mathrm{lb}$ (41 J) as well as the drop in upper shelf energy (USE). Figure 1.1 shows an example for one particular RPV weld with high copper content [2].

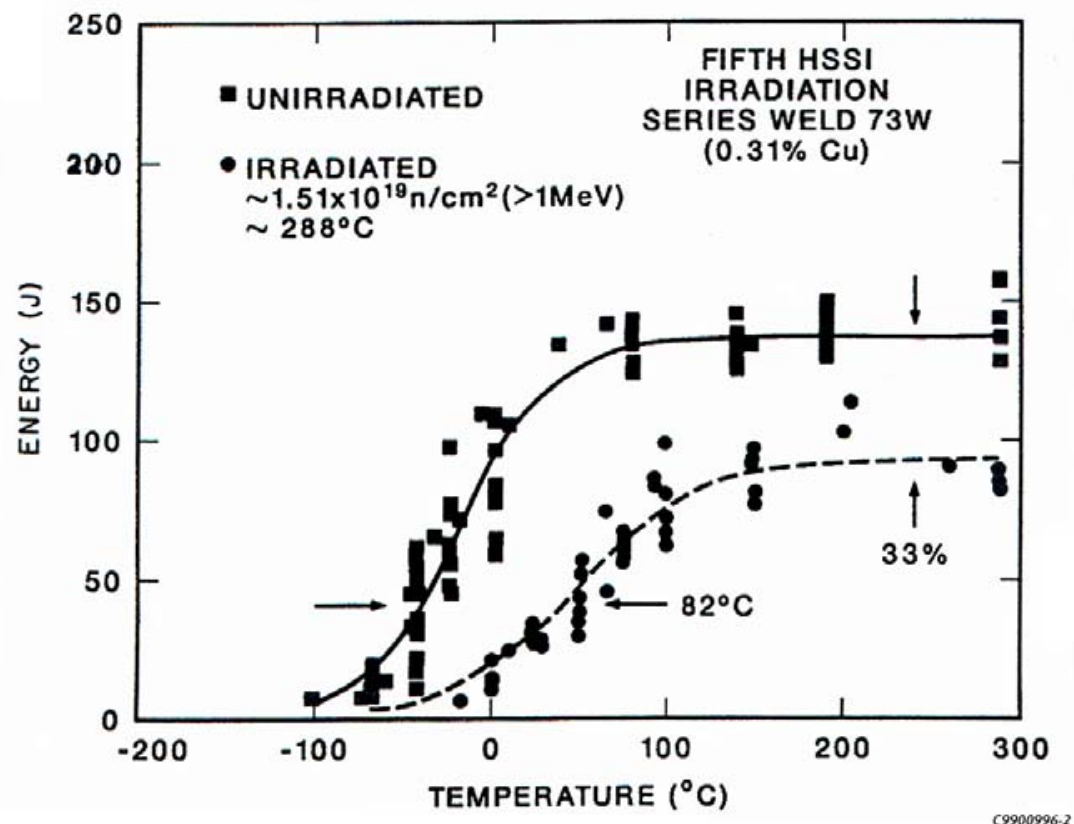

Fig. 1.1. Plot of Charpy impact results showing the effects of irradiation at $288^{\circ} \mathrm{C}$ to a fluence of $1.5 \times 10^{23} \mathrm{n} \cdot \mathrm{m}^{-2} \mathrm{~s}^{-1}(>1 \mathrm{MeV})$ on the CVN impact toughness. 
Improved correlation models, based on a broader database and a better understanding of embrittlement mechanisms, are presented in NUREG/CR-6551, published in November 1998 [3]. The models incorporate material chemical composition and various exposure variables to enable predictions of TTS and USE changes. The embrittlement shift model in NUREG/CR-6551 was updated in July 2000 with additional surveillance data collected since the earlier work; this is referred to in this report as the Draft 2000 model [4]. Another embrittlement shift model was developed at about the same time on the same mid-2000 database under the auspices of the Electric Power Research Institute (EPRI) and the American Society for Testing and Materials (ASTM) E10.02 subcommittee (the E900 model [5]), published as E900-02 [6] in 2002. Similar correlation models were developed on different databases in the United Kingdom [7,8]. A large database of test reactor data from single-variable experiments is being analyzed in terms of a physically motivated model with some features similar to the correlation models $[9,10]$.

Motivation for a new modeling effort came from the fact that 62 additional low-flux boiling water reactor (BWR) shifts became available in 2003. These data were significantly underpredicted by the previous shift models [4,5], so it was necessary to investigate the cause of the underprediction. Additional pressurized water reactor (PWR) data from surveillance reports (about 140 shifts) were also added to the database in 2003 and 2004. Finally, the reliability of the database was improved when all old and new surveillance data were reviewed for completeness, duplicates, and discrepancies during summer and fall 2004, in cooperation with the ASTM Subcommittee E10.02 on Radiation Effects in Structural Materials. Thus a larger, better balanced and, therefore, more reliable database was made available for analysis.

This report is a record of work performed in part by the Heavy Section Steel Irradiation Program (see Appendix A).

\subsection{Objectives and Scope of Work}

The present work is an update to the earlier embrittlement shift correlation models [3,4], based on the expanded surveillance database and on the continuing advances in mechanistic understanding. The objective is to produce an improved embrittlement shift correlation model with demonstrated predictive capability.

The work reported here includes several steps:

- collection of new data that have become available since the last database revision in 2000;

- review and updating of both new and previously collected data, including identifying duplicates and discrepancies;

- model development to address the low-flux data that were not well fitted by previous models and to improve the agreement with recent physical insights and the expanded database;

- calibration of the revised model;

- validation of the model on surveillance data not used for fitting, to the extent possible; and

- comparison with test reactor results not used for fitting.

The model revision presented here is calibrated to the surveillance data from U.S. power reactors; so data from test reactor irradiations were used only for insight during the calibration process. After the model was calibrated to surveillance data, an extensive comparison was made between the model and the Irradiation Variable (IVAR) program test reactor data, as described in Chap. 6. (See Appendix B, "Irradiation Variable [IVAR] Program Data Base.”) Subsequent sensitivity studies on surveillance data led to minor simplifications of the model as discussed in Chap. 7.

The revised model is able to fit both PWR and BWR data, within a format similar to earlier models, using a modification of the "effective fluence" $\left(\phi \mathrm{t}_{\mathrm{e}}\right)$ approach to flux effects that has been previously suggested by Odette and others [9-11]. The revised model contains, sometimes in different form, all 
effects that were in earlier models by the same authors [3,4], except the "long-time" effect in [4], which became clearly nonsignificant with the latest expansion of the database and the use of a separate standard reference material (SRM) coefficient. The revised model (discussed in detail in Chap. 4) combines two terms, the first of which depends on irradiation temperature $\left(\mathrm{T}_{\mathrm{i}}\right)$, material chemical composition, and effective fluence ( $\phi \mathrm{t}_{\mathrm{e}}$ ), while the second depends only on those latter two factors. The general form of the model is shown in Eq. (1-1); the complete model includes various coefficients to account for material specific product forms, chemical composition ranges, and weld flux types:

$$
T T S=f\left(T_{i}, P, M n, \phi t_{e}\right)+g\left(N i, C u, P, \phi t_{e}\right) .
$$

The Mn effect in the revised model is an example of a change in model form - the effect of Mn was implicitly included in the prior models via the product form coefficients, while in the revised model it is also incorporated explicitly. The revised model also incorporates the variation in fluence at which copperrich precipitate (CRP) damage becomes important as a function of $\mathrm{Cu}$ and $\mathrm{Ni}$, a known effect (see p. 87 in [3] that could not be calibrated reliably before the recent database expansions; see also [7],[9],[10]).

\subsection{Overview and Organization of Report}

This report presents the revised embrittlement shift model and discusses its quality of fit, both of data used for model calibration and of other data that were not used for calibration. The focus of the report is on the revised model and its quality of fit. The model development process has been an iterative effort over several years as the surveillance database has increased in size and the understanding of radiation damage has matured. Two of the previous iterations are described in $[3,4]$ and more recent iterations are summarized in this report.

The report is organized to provide some background on radiation damage and statistical terminology in the remainder of Chap. 1, followed by a more detailed description in Chap. 2 of the radiation damage mechanisms relevant to the revised model. Chapter 3 describes the surveillance database and the subsets used for model calibration and analysis. Chapter 4 summarizes the model development process, presents the model, and demonstrates quality of fit and validation on surveillance data not used for fitting. The individual variable effects are described and plotted and their statistical significance is discussed in Chap. 5. Then, the model of surveillance data is compared to an independent database of test reactor results, the IVAR database, in Chap. 6. The agreement with the independent IVAR database provides additional validation of predictive capability and illuminates some limitations of the surveillance database and the effects of those limitations on the model. Chapter 7 discusses minor simplifications and supporting sensitivity analysis, and presents the revised model in the form recommended for application. Chapter 8 , the final chapter, provides a brief summary and conclusions.

\subsection{Radiation Damage Mechanisms Background}

The mechanisms of irradiation embrittlement are discussed in Chap. 2 and are only briefly described in this section. Reactor spectrum neutrons generate high-energy primary recoil atoms. The primary recoil atoms slow down in a branching series of collisions with atoms that are ejected from their lattice sites, in what is known as a displacement cascade. This produces a large concentration of vacancies and selfinterstitial atoms in the cascade region. Many self-interstitial atoms quickly recombine with vacancies, thus healing the damage, or form clusters of like defects, typically in the form of complexes with various solute atoms. At RPV operating temperatures, the residual vacancies and interstitials subsequently diffuse long distances relative to the size of the cascade. The migrating single vacancies, interstitials, and small interstitial clusters, as well as vacancies emitted by the dissolution of vacancy clusters, recombine during long-range diffusion, or are absorbed at sinks. So-called "matrix features" (MFs), which mainly form in 
the cascades, produce hardening in both low- and high-Cu steels. They are believed to be vacancy-solute cluster complexes or their solute remnants; MFs may also grow by the long-range diffusion of solutes and vacancies. The hardening from MF increases with decreasing irradiation temperature and roughly with the square root of fluence. The excess concentration of vacancies under irradiation also accelerates precipitation of $\mathrm{Cu}$, along with $\mathrm{Ni}, \mathrm{Mn}$, and $\mathrm{Si}$, from the supersatured solution (the $\mathrm{Cu}$ solubility limit is less than $100 \mathrm{appm}$ at $290^{\circ} \mathrm{C}$ ) above threshold levels of about $0.07 \mathrm{wt} \% \mathrm{Cu}$. This precipitation results in the formation of CRPs, or at high levels of these elements, manganese-nickel rich precipitates (MNPs) $[12,13]$. The causes, character, and consequences of the MFs are not as well understood as are those of CRPs and MNPs, and improved treatments of their contributions to TTS are the subject of continuing study by the research community.

Evolution of CRPs or MNPs is the most important mechanism of embrittlement in irradiation sensitive western LWR steels [12]. Radiation-enhanced diffusion is a result of the much higher concentration of vacancies in the steel under irradiation, compared with that under thermal conditions. Thus, a given solute atom (e.g., $\mathrm{Cu}, \mathrm{Ni}, \mathrm{Mn}$, and $\mathrm{Si}$ ) has a much higher probability of having a neighboring vacancy during irradiation. With or without irradiation, diffusion of solutes, such as copper, takes place by thermal jumps into adjacent vacancies, but the number of these jumps in a given time interval is much higher during irradiation, corresponding to the increased concentration of vacancies. Subsequent repetition of this vacancy-solute exchange process results in random diffusion of copper. When a diffusing $\mathrm{Cu}$ atom encounters another $\mathrm{Cu}$ atom (or cluster of $\mathrm{Cu}$ atoms), they bind with one another. Small $\mathrm{Cu}$ clusters can redissolve, but at a sufficient size the $\mathrm{Cu}$ clusters form coherent precipitates that continue to grow by radiation-enhanced diffusion up to the point when the $\mathrm{Cu}$ is depleted from the matrix. The effective energy of $\mathrm{Ni}, \mathrm{Mn}$, and $\mathrm{Si}$ atoms is lower in the precipitates than in the matrix, so these elements also flow into CRPs along with $\mathrm{Cu}$. The resulting high number density of CRPs $\left(>10^{23} / \mathrm{m}^{3}\right.$ ) with typical diameters on the order of 1 to $3 \mathrm{~nm}$ efficiently pin dislocations, resulting in significant hardening in RPV steels, which increases with $\mathrm{Cu}$ contents above about $0.10 \mathrm{wt} \%$. The amount of solute diffusion under irradiation that occurs at a specified fluence increases with increasing dose rate (neutron flux). This results in a strong dose rate effect in the CRP hardening and TTS regime (due to matrix solute depletion).

At high levels the actual copper remaining in solid solution that is available for irradiation-induced precipitation may be less than the measured bulk copper content, due to pre-precipitation during postweld heat treatment (PWHT) performed during the fabrication of an RPV. As a result the maximum amount of copper that remains in solution following typical PWHT is about 0.25 to $0.3 \mathrm{wt} \%$ [12].

The synergistic interaction among $\mathrm{Cu}, \mathrm{Mn}$, and $\mathrm{Ni}$ is the reason why $\mathrm{Ni}$ enhances the effects of $\mathrm{Cu}$ on TTS, as reflected in the predictive embrittlement formulas based on the U.S. LWR surveillance database $[1,3,6]$, that of the Japanese LWR surveillance database [14], and in other commercial reactor vessel steels [15,16]. Moreover, atom probe tomography has observed the enrichment of CRPs with Ni, Mn, P, and $\mathrm{Si}$ as well as segregation of solutes such as $\mathrm{P}, \mathrm{Ni}$, and $\mathrm{Mn}$ to grain and lath boundaries in the microstructure [17]. In Effects of Nickel on Irradiation Embrittlement of Light Water Reactor Pressure Vessel Steels, a report by the International Atomic Energy Agency (IAEA) Cooperative Research Project, it was noted that, "for a given high level of nickel in the material and all other factors being equal, high manganese content leads to much greater radiation-induced embrittlement than low manganese content for both VVER-1000 and PWR materials"[18]. Notably, these interactions were long ago predicted based on theoretical models that were subsequently verified by careful single-variable experiments and very detailed nano-analytical characterization studies [19]. Some further references that discuss the effects of nickel with copper and manganese are [20-27].

While the existence of MNPs in Cu-bearing steels has long been well established, the models also predicted the possible formation of $\mathrm{Mn}-\mathrm{Ni}-\mathrm{Si}$ precipitates even in very low $\mathrm{Cu}$ steels. Since these precipitates were expected to be slow to nucleate relative to CRPs, thus requiring high fluence to cause hardening and TTS, they were called "late-blooming phases (LBPs)." Recently, careful experimental 
studies have verified the existence of LBPs, even in nominally Cu-free alloys [28]. The significance of LBP to the steels and irradiation conditions pertinent to the fleet of western RPVs is a topic of ongoing research.

Irradiation hardening results in TTS, which can be understood from the basic micromechanics of fracture. Body-centered cubic alloys, such as RPV steels, undergo a transition from cleavage to ductile fracture over a range of temperature known as the "ductile-brittle transition" (DBT), shown in Fig. 1.1. In the higher-temperature region, the yield stress of the alloy is insufficient to produce a stress concentration near the tip of a notch or crack that reaches the cleavage fracture stress. In this case, ductile fracture occurs by the nucleation, growth, and coalescence of internal voids that form on inclusion particles. The position and range of the DBT is controlled by the magnitude and rate of increase in the yield stress with decreasing temperature and the local critical stress-volume conditions leading to cleavage. As a result of irradiation-induced increases in yield stress, higher temperatures are required to keep the internal stress concentrations below the cleavage fracture stress, resulting in TTS.

For some steels, nonhardening embrittlement can be caused by radiation-enhanced solute segregation of elements such as phosphorus to grain boundaries that are effectively weakened to the point where they become the primary path for the propagation of brittle cracks. Thus this type of embrittlement, typically defined as irradiation-assisted temper embrittlement [29], is manifested as an intergranular (grain boundary) fracture rather than the usual transgranular cleavage fracture. In this case, TTS can occur even if the yield strength does not increase; in principle, combinations of irradiation hardening and grain boundary embrittlement can interact synergistically to produce very large TTS. In general, however, the amount of radiation-induced intergranular fracture in U.S. RPV steels (Mn-Mo-Ni steels) is low, probably due to their generally low sensitivity to temper embrittlement [30,31]. Consequently, intergranular fracture is not discussed in this report.

\subsection{Modeling and Statistical Background}

\subsubsection{Basis of Modeling}

The model presented in Chap. 4 is a hybrid incorporating both physically motivated features and empirical calibration. In this type of model, features of the model may be based on statistical significance in the modeling database, physical understanding, observations from independent sources (including data from controlled experiments), or all of these types of evidence. Most of the effects incorporated in the model presented are supported by more than one form of evidence. However, as a key ground rule for the modeling effort, the only data used in this report for calibration and statistical analysis are the U.S. reactor surveillance data, described in more detail in Chap. 3.

The calibration and much of the analysis of the model are inherently statistical, so the following paragraphs highlight a few statistical definitions and issues intended to help nonspecialists with the statistical details given in the report.

\subsubsection{Definition of Residuals}

Much of the discussion of models in this report refers to residuals. A residual is the difference between the model estimate and the measured TTS value:

$$
\text { Residual }=\text { Model TTS }- \text { Measured TTS } .
$$

In this calculation, the values of the independent variables that were recorded for the measured TTS (e.g., fluence, $\mathrm{Cu}, \mathrm{Ni}$ ) are used to make the model estimate. The residuals are just as often defined with the opposite sign (residual = measured TTS - model TTS), so it is important to be sure which definition is 
used when interpreting the direction of trends in residuals. Both definitions are used in this report, in sections prepared by different authors, and the corresponding definition is shown. As defined in Eq. (1-2), residuals are negative if the model underestimates the measured shift.

The least squares method finds the best fit by minimizing the sum of squared residuals, so by definition, a good fit will have relatively small residuals overall. Moreover, a good model will show no obvious trends if the residuals are plotted against model variables and against variables not included in the model.

\subsubsection{Statistical Significance}

Statistical significance, although not the only basis of the model, is frequently mentioned throughout this report. Statistical significance implies that the variable effect or difference in shift or residual slope that is being analyzed is large enough relative to the uncertainty from data scatter and limited sample size that it is unlikely to be caused by random variations. Any reference to "significant" in this report refers to statistical significance unless otherwise noted. Differences in shift and variable effects that are practically important (i.e., having cost, operational, or other consequences) may or may not be statistically significant, Two types of questions are frequently asked about statistical significance: (1) is A significantly different from B? or (2) is A significantly larger (or smaller) than B?

The first question gives rise to what is called a two-tail or two-sided significance test, in which a result is significant if the probability is small that the difference, in either direction, is due to random variation. The second question gives rise to what is called a one-tail or one-sided test, in which a result is significant if the probability is small that the difference, in a preselected direction, is due to random variation. The usual practice in statistical analysis is that a two-sided test is appropriate if the direction of an effect or comparison is not known in advance and if neither direction is substantially more important than the other. A one-sided test is appropriate if the direction of an effect or comparison is known in advance, or if differences in one direction are clearly more critical than in the other [32,33]. As a practical matter, a difference must be larger to show a specified level of significance on a two-sided basis than on a one-sided basis.

Both two-sided and one-sided tests of significance are used in this report, following the usual practice noted in the last paragraph, and the type of test used is stated. It could be argued that enough research and prior modeling has been done that by now most questions about the significance of embrittlement variable effects should be one-sided questions, as the direction of most variable effects is well known in advance of the statistical analysis. For instance, $\mathrm{Cu}$ is known to enhance embrittlement, so it makes little sense to ask if the data show that high-Cu shifts are significantly different from the low-Cu shifts; instead, if the question were raised at all, it would be asked whether the high-Cu shifts are significantly greater than the low-Cu shifts, hence a one-sided test. But some questions are more appropriate for two-sided tests. For example, throughout this report, tests on the significance of the slope of residual trends are two-sided unless otherwise noted, asking the question whether the slope of the residual trend is significantly different from zero. The reason is that the modeling objective is to make the model accurately fit the data trends (i.e., zero slopes to the residuals when plotted against modeling variables), so in most cases a significant residual trend for a variable in the model represents a modeling issue whether its slope is positive or negative. Moreover, although the direction of the physical variable effect is often known in advance, the direction of residual modeling error depends on the model and the specific data that are available and may not be known in advance.

An exception to the use of two-sided tests for residuals is appropriate if the residual offset or slope is caused by intentionally leaving out or disabling the effect of a term in the model to see whether it is significant. In this case, the expected direction of the residual slope or offset is known in advance because the analyst intentionally took out a previously calibrated effect, thus causing the residual slope or offset to occur in a predictable direction. The question in this case is whether the slope or offset is large enough in 
the predictable direction to imply a significant variable effect relative to the uncertainty, so a one-sided test is appropriate.

The level of probability (p) used in this report for concluding that an effect is significant is $\mathrm{p}<0.05$ unless otherwise stated. This is a typical significance level for engineering, where it is sometimes referred to as the $95 \%$ significance level. That level of significance means that the chance the observed effect or difference could arise from random variation is estimated to be less than $5 \%$. In the case of a two-sided test, such as the test for significance of residual slope, a slope that is significantly different from zero at $\mathrm{p}$ $<0.05$ implies it is far enough from zero in one direction that there is less than $2.5 \%$ chance that it could occur by random variation. The same would be true of a slope of the same magnitude in the opposite direction, and hence the chance of observing that magnitude of slope in either direction due to random variation is less than $5 \%$.

\subsubsection{Treatment of Outliers}

Despite best efforts, anomalous data points occur in engineering databases, in many cases because of measurement or documentation errors that cannot be identified or resolved by the available information. The question from an analyst's point of view is whether an apparent outlier is different enough from the bulk of the data to suggest that the point is in error. The risk in using such a point for model calibration can be substantial because extreme outliers produce large residuals, which can have a disproportionate effect on the sum of squared residuals that is minimized by least squares, thus biasing the fit. The risk in not using such points is the possibility that they are valid; hence, by omitting them the fitted model or the estimates of data scatter could be somewhat unrealistic. A biased fit from leaving outliers in a calibration set is the greater risk, particularly in large datasets as analyzed here. Since the purpose of the modeling is usually to characterize the average trend in the data, that can be accomplished using about $99 \%$ of the data nearest the average trend while ignoring the few points furthest away.

The procedure followed in this analysis was to calibrate a preliminary model, then apply an objective statistical criterion (Chauvenet's) to identify potential outliers among the residuals. These outlier points were investigated to the extent possible with the available documentation and with help in many cases from the ASTM E10.02 subcommittee (on Behavior and Use of Nuclear Structural Materials), in an attempt to determine whether there were recording errors, physical causes for the anomalous behavior, or extremes in test or irradiation conditions or composition. If a correctable error or other cause of the anomaly could be found, appropriate corrections were made and documented; otherwise, the points were set aside based on Chauvenet's criterion and the models were recalibrated without the identified outliers, producing the results presented in this report.

Chauvenet's criterion provides a quantitative and objective means of deciding whether or not an outlier point may be discarded [34,35]. Chauvenet's criterion calls for rejection of an outlier if the estimated probability of observing it is less than $1 /(2 N)$, where $N$ is the total number of points being analyzed. In other words, an outlier is rejected if the expected number of observations in the database as far from the mean as the outlier is less than $1 / 2$. In the modeling application, the mean of the distribution is typically set to be equal to the mean residual (or to zero), and the residuals relative to the model are the observations. If the standard deviation of the residuals is estimated by the model standard error $\left(\mathrm{S}_{\mathrm{e}}\right)$, the critical multiple of $S_{e}$ from the model, beyond which the data are suspect, can be calculated from the normal distribution. For example, if 100 points are used for calibration, an outlier might be rejected if further than $2.8 \mathrm{~S}_{\mathrm{e}}$ from the model, whereas if 1000 points are used, an outlier must be further than about $3.5 \mathrm{~S}_{\mathrm{e}}$ from the model to be rejected on that basis.

Chauvenet's criterion was used to justify removal of several surveillance data points in previous modeling efforts and in the present analysis. The outliers removed in previous modeling efforts were reconsidered in the present analysis and all remained Chauvenet outliers. A few points were also removed because they were irradiated under unusual conditions, including irradiation in two different reactors at 
substantially different flux and/or temperature and irradiation at flux values higher than the usual range for surveillance materials. One point could be removed on either basis. The points not used in the fit amounted to about $1 \%$ of the available data.

\subsubsection{Variable Confounding}

In data from statistically designed experiments, variables may be confounded by the experimental design such that their effects cannot be separately estimated, as discussed in many statistics texts (e.g., Chap. 14 of. [32]). Partial confounding may occur, in which estimation of the effects of two or more variables is feasible, but the estimates are affected to some degree by the other variable(s) [36]. In databases that are not from a single statistically designed experiment, such as the surveillance database, partial confounding often occurs from patterns in the data. For example, the only product form in the surveillance database with relatively low Mn values (below about $1 \mathrm{wt} \%$ ) is forging, so the analysis reported in later chapters found that the Mn variable is partially confounded with the product form variable.

The problem with variable confounding is that effects that are nominally attributed to one physical variable may be caused in part by one or more other variables, and the effect of some variables may not be readily calibrated because of relationships with other variables. This situation is noted where detected in the following chapters.

\subsection{References}

1. U.S. Nuclear Regulatory Commission, Radiation Embrittlement of Reactor Vessel Materials, Regulatory Guide 1.99 Revision 2, 1988.

2. Nanstad, R. K., McCabe, D. E., Menke, B. H., Iskander, S. K., Haggag, F. M., "Effects of Radiation on $\mathrm{K}_{\text {Ic }}$ Curves for High Copper Welds," pp. 214-33 in Effects of Radiation on Materials: 14th International Symposium (Volume II), ASTM STP 1046, N. H. Packan, R. E. Stoller, and A. S. Kumar, Eds., American Society for Testing and Materials, Philadelphia, 1990.

3. Eason, E. D., J. E. Wright, and G. R. Odette, Improved Embrittlement Correlations for Reactor Pressure Vessel Steels, NUREG/CR-6551, U.S. Nuclear Regulatory Commission, Washington D.C., 1998.

4. Kirk, M., C. Santos, E. Eason, J. Wright, and G. R. Odette, "Updated Embrittlement Trend Curve for Reactor Pressure Vessel Steels," in Transactions of the $17^{\text {th }}$ International Conference on Structural Mechanics in Reactor Technology (SMiRT 17), Prague, Czech Republic, August 17-22, 2003, 2003.

5. Server, W., C. English, D. Naiman, and S. Rosinski, "Charpy Embrittlement Correlations-Status of Combined Mechanistic and Statistical Bases for U.S. RPV Steels (MRP-45),” EPRI Report 1000705, 2001.

6. ASTM International, "Standard Guide of Predicting Neutron Radiation Damage to Reactor Vessel Materials,” E706 (IIF), E900-02, Annual Book of ASTM Standards, ASTM International, 2002.

7. Williams, T. J., and D. Ellis, "A Mechanistically-Based Model of Irradiation Damage in Low Alloy Steel Submerged Arc Welds," pp. 8-27 in Effects of Radiation on Materials: $20^{\text {th }}$ International Symposium, ASTM STP 1405, Ed. S.T. Rosinski, et al., ASTM, 2001.

8. Williams, T. J., D. Ellis, C. A. English, and J. Hyde, "A Model of Irradiation Damage in High Nickel Submerged Arc Welds,” Int. Jour. of Pressure Vessels and Piping 79, 649-60, 2002.

9. Odette, G. R., T. Yamamoto, and D. Klingensmith, The Effect of Dose Rate on Irradiation Hardening of RPV Steels: A Comprehensive Single Variable Database and Model Based Analysis, Letter Report UCSB-NRC-03/1, 2003. 
10. Odette, G. R., T. Yamamoto, D. Klingensmith, and H. Kishimoto, The Effect of Flux and Irradiation Temperature on Hardening in RPV Steels, Letter Report UCSB-NRC-03/2, 2003.

11. Electric Power Research Institute, Workshop on Dose Rate Effects in Reactor Pressure Vessel Materials Held November 12-14, 2001, Olympic Valley Calif., Conference Proceedings (CD), Ed. N. Soneda and R. G. Carter, EPRI 1006981, CRIEPI T02904, 2002.

12. Odette, G. R., and G. E. Lucas, "Embrittlement of Nuclear Reactor Pressure Vessels," J. of Metals 53 (7), 18-22, 2001.

13. Odette, G. R., and G. E. Lucas, "Recent Progress in Understanding Reactor Pressure Vessel Embrittlement,” Rad. Effects and Defects in Solids 144, 189-231, 1998.

14. Japanese Electric Association (JEA), Method of Surveillance Tests for Structural Materials of Nuclear Reactors, Japanese Electric Association Code (JEAC) 4201-2000, 2000.

15. Kryukov, A. M., Y. A. Nikolaev, and A. V. Nikolaeva, "Behavior of Mechanical Properties of Nickel-Alloyed Reactor Pressure Vessel Steel under Neutron Irradiation and Post-Irradiation Annealing,” Nuclear Engineering and Design 186 (3), 353-59, 1998.

16. Williams, T. J., P. R. Burch, C. A. English, and P. H. N. de la Cour Ray, ”The Effect of Irradiation Dose Rate and Temperature, and Copper and Nickel Content, on the Irradiation Shift of Low Alloy Steel Submerged Arc Welds,” pp. 121-131 in Third International Symposium on Environmental Degradation of Materials in Nuclear Power Systems_-Water Reactors, 1987, Ed. G. J. Theus and J. R. Weeks, 1988.

17. Miller, M. K., P. Pareige, and M. G. Burke, "Understanding Pressure Vessel Steels: An Atom Probe Perspective,” Materials Characterization 44, 235-54, 2000.

18. International Atomic Energy Agency, Effects of Nickel on Irradiation Embrittlement of Light Water Reactor Pressure Vessel Steels, IAEA-TECDOC-1441, Vienna, June 2005.

19. Odette, G.R. and Lucas, G.E. "The Effect of Nickel on Radiation Hardening of Pressure Vessel Steels", Effects of Irradiation on Materials-14th Symposium, ASTM-STP-1046, American Society for Testing and Materials, Philadelphia, 323-347, 1989.

20. Nanstad, R. K., M. A. Sokolov, and M. K. Miller, "Comparison of Nickel Effects on Embrittlement Mechanisms in a Radiation-Sensitive Weld and in Prototypic WWER-1000 and A533B Steels," Proceedings of IAEA Specialists' Meeting on Effects of Radiation on Reactor Pressure Vessels and Internals, Gus-Khrustalny, Russia, May, 2004.

21. Odette, G. R., and G. E. Lucas, "The Effect of Nickel on Irradiation Hardening of Pressure Vessel Steels," pp. 323-47 in Effects of Radiation on Materials: $14^{\text {th }}$ International Symposium (Volume II), ASTM STP 1046, Ed. N. H. Packan, R. E. Stoller, and A. S. Kumar, American Society for Testing and Materials, Philadelphia, 1990.

22. Odette, G. R., "Radiation Induced Microstructural Evolution in Reactor Pressure Vessel Steels," pp. 137-48 in Microstructure of Irradiated Materials, MRS Symposium Proceedings, Volume 373, Pittsburgh, 1995.

23. Gerard, R., A. Fabry, J. Van de Velde, J. L. Puzzolante, A. Verstrepen, T. Van Ransheeck, and E. van Walle, "In-Service Embrittlement of the Pressure Vessel Welds at the Doel I and II Nuclear Power Plants," pp. 294-319 in Effects of Radiation on Materials: $17^{\text {th }}$ International Symposium (Volume II), ASTM STP 1046, Ed. David S. Gelles, R. K. Nanstad, A. S. Kumar, and E. A. Little, American Society for Testing and Materials, 1996. 
24. Odette, G. R., C. L. Liu, and B. D. Wirth, On the Composition and Structure of Nanoprecipitates in Irradiated Pressure Vessel Steels,” pp. 457-69 in Microstructure Evolution During Irradiation, MRS Symposium Proceedings, Volume 439, Pittsburgh, 1997.

25. S. M. Rekhson, M. J. Leitman, Y. A. Nikolaev, A. M. Kryukov, V. I. Levit, Y. N., Korolyov, and A. V. Nikolaeva, "Radiation Embrittlement and Thermal Annealing Behavior of Cr-Ni-Mo Reactor Pressure Vessel Materials,” J. Nuc. Matls. 226, 144-55, 1995.

26. Burke, M. G., R. J. Stofanak, J. M. Hyde, C. A. English, and W. L. Server, "Irradiation Damage Investigation of A508 Grade 4N Steels: Ni and Flux Effects," pp. 13-1 through 13-30 in Workshop on Dose Rate Effects in Reactor Pressure Vessel Materials Held November 12-14, 2001, Olympic Valley Calif., Conference Proceedings (CD), Ed. N. Soneda and R. G. Carter, EPRI 1006981, CRIEPI T02904, 2002.

27. Burke, M. G., R. J. Stofanak, J. M. Hyde, C. A. English, and W. L. Server, "Microstructural Aspects of Irradiation Damage in A508 Gr 4N Forging Steel: Composition and Flux Effects," pp. 194-207 in The Effects of Radiation on Materials: $21^{\text {st }}$ International Symposium, ASTM STP 1447, Ed. M. L. Grossbeck, ASTM, 2003.

28. G. R. Odette, T. Yamamoto, and B. D. Wirth, "Late Blooming Phases and Dose Rate Effects in RPV Steels: Integrated Experiments and Models," Proceedings of the Second International Conference on Multiscale Materials Modeling, edited by N. M. Ghoniem, University of California (2004) 355.

29. McMahon, Jr., C. J., V. Vitek, and J. Kameda, "Mechanics and Mechanisms of Intergranular Fracture," Chap. 4 in Developments in Fracture Mechanics-2, Ed. G. G. Chell, Applied Science Publishers, Englewood, N.J., 1981.

30. McElroy. R. J., C. A. English, A. J. E. Foreman, G. Gage, J. M. Hyde, P. H. N. Ray, and I. A. Vatter, "Temper Embrittlement, Irradiation Induced Phosphorus Segregation and Implications for PostIrradiation Annealing of Reactor Pressure Vessels," pp. 296-316 in Effects of Radiation on Materials: $18^{\text {th }}$ International Symposium, ASTM STP 1325, Ed. R. K. Nanstad, M. L. Hamilton, F. A. Garner, and A. S. Kumar, American Society for Testing and Materials, West Conshohocken, Pennsylvania, 1999.

31. Nanstad, R. K., D. E. McCabe, M. A. Sokolov, C. A. English, and S. R. Ortner, "Investigation of Temper Embrittlement in Reactor Pressure Vessel Steels Following Thermal Aging, Irradiation, and Thermal Annealing," pp. 356-82 in Effects of Radiation on Materials: $20^{\text {th }}$ International Symposium, ASTM STP 1405, Ed. S. T. Rosinski, M. L. Grossbeck, T. R. Allen, and A. S. Kumar, American Society for Testing and Materials, West Conshohocken, Pennsylvania, 2001.

32. Miller, I. and J. E. Freund, pp. 162-65 in Probability and Statistics for Engineers, Englewood Cliffs, N.J.: Prentice-Hall, 1965.

33. Milton, J. S. and J. C. Arnold, pp. 234-37 in Probability and Statistics in the Engineering and Computing Sciences, New York: McGraw-Hill, 1986.

34. Taylor, J. R., An Introduction to Error Analysis, University Science Books, Mill Valley, California, 1982.

35. Young, H. D., Statistical Treatment of Experimental Data, McGraw-Hill Book Company, Inc., New York, 1962.

36. Haaland, P. D., pp. 85-92 in Experimental Design in Biotechnology, New York, Marcel Dekker, 1989. 


\section{Embrittlement Mechanisms and Physical Models Underpinning the TTS Model}

\subsection{Background and Context}

Irradiation hardening and embrittlement of RPV steels depend on the combination of a large number of metallurgical and irradiation variables [1-23]. Physical models and experimental insight on mechanisms are helpful in identifying potentially important embrittlement variables and their interactions, as well as in developing simplified embrittlement model equations that can be fitted to the TTS data base. (See Appendix C, “Analysis Data Base.”)

To begin we note that this chapter and Chap. 6 were both written after work on the TTS model (presented in Chaps. 3 to 5) had been basically completed. The foundation for what follows is a large literature on embrittlement that has developed over the recent years, including the results of a number of controlled single variable and variable combination experiments and mechanism studies, as well as extensive modeling efforts. The experimental studies include the Irradiation Variable (IVAR) program that is extensively discussed in Chap. 6. However, it must be emphasized that the current state of understanding of embrittlement is not complete, and that the overview that follows represents the author's own experience and viewpoints, that will be extended and refined in the future. It is also important to note that, while basic understanding of embrittlement mechanisms was used as a guide, the quantitative TTS model was derived by non-linear least square fitting of necessarily simplified analytical expressions to the scattered and non-ideally distributed surveillance database. Thus some differences between the TTS model and the description of the physics that follows are almost inevitable. (See Appendix D for an explanation of the specialized terms used in Chaps. 2 and 6.)

The basic mechanisms of irradiation embrittlement are illustrated in the block diagram shown in Fig. 2.1 [8]. High-energy neutrons interactions with atomic nuclei create energetic primary recoil atoms (PRAs) with energies up to several tens of $\mathrm{keV}$. The PRAs produce displacement cascades by a branching series of atomic collisions until the energies of the final generation of secondary recoiling atoms fall below that needed to displace atoms from their crystal lattice sites. The defects created in the cascade are in the form of single and small clusters of vacancies and self-interstitial atoms (SIAs). SIA defects are two atoms sharing one crystal lattice site. A molecular dynamics simulation of a cascade is shown in Fig. 2.2 [24]. The green atoms are displaced from the vacant lattice sites marked as the red symbols. The primary vacancy and SIA defects that remain after tens of ps, illustrated in Fig. 2.3, are the basic sources of radiation damage $[12,24,25]$. These point defects and small defect clusters are mobile and diffuse through the ferrite matrix at RPV operating temperatures. A fraction of the vacancies and SIA recombine and like defects quickly cluster in the cascade region, but most eventually migrate to sinks or undergo SIAvacancy recombination (self-healing) reactions during long-range diffusion [3,7-9,12,13,24-27]. Sinks are sites where the individual vacancies and SIA are destroyed, like at dislocation jogs, free surfaces and grain boundaries. Larger vacancy clusters dissolve over varying time scales [26,27]. Larger SIA clusters: (a) migrate one dimensionally to sinks; (b) shrink by absorbing excess vacancies; or (c) grow by absorbing an excess flux of SIAs to form dislocation loops [8,24,26,27]. The primary vacancies also locally interact with various solutes during the long-term aging of the cascades, as illustrated in the kinetic lattice Monte Carlo simulation shown in Fig. 2.4, to form defect-solute cluster complexes and their solute remnants, which are known as matrix features (MFs) [3,8,26,27].

Substitutional solutes diffuse by a vacancy exchange mechanism, as schematically illustrated in Fig. 2.5 (a). Thus the excess concentration of vacancies created by irradiation greatly accelerates solute diffusion rates. As illustrated by the kinetic lattice Monte Carlo simulation in Fig. 2.5 (b) for a Fe-0.3 at.\% $\mathrm{Cu}$ alloy [27], at highly supersaturated dissolved $\mathrm{Cu}$ concentrations (more than about 0.05 to $0.1 \mathrm{wt}$

$\% \mathrm{Cu}$ ), radiation-enhanced diffusion (RED) leads to the accelerated precipitation of a high number density of nanometer-scale coherent bcc copper-rich precipitate (CRP) phases [3,6-9,12,22,28-34]. The 


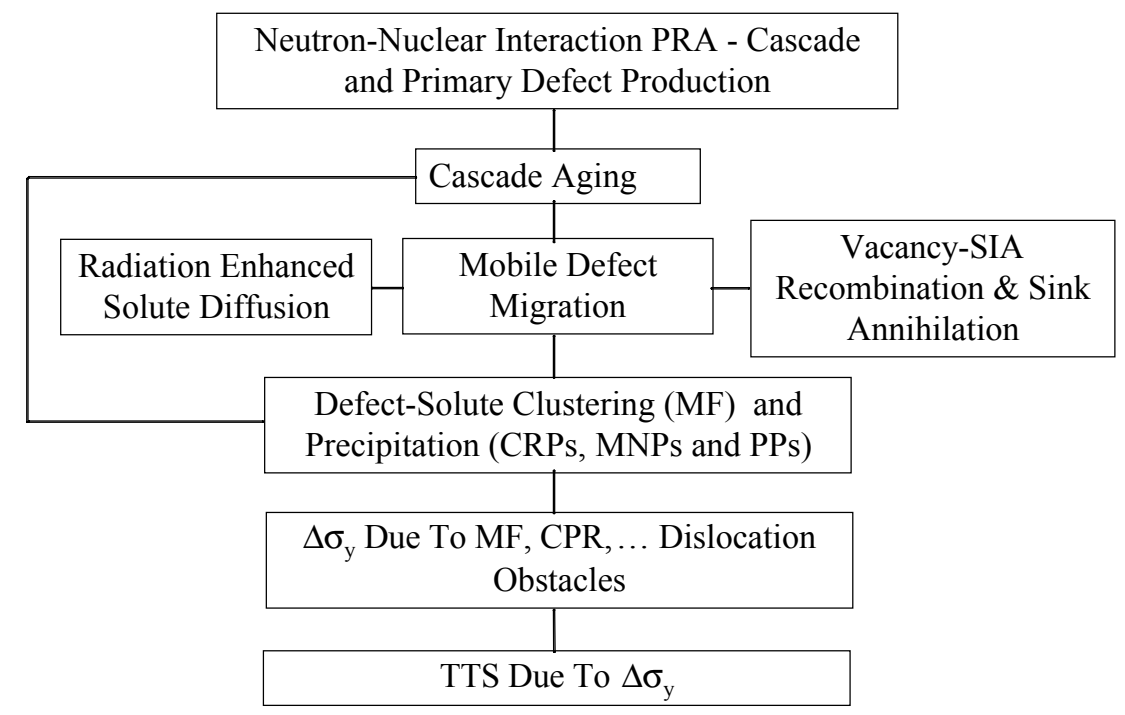

Fig. 2.1. A simplified description of the sequence of events leading to irradiation-induced TTS.
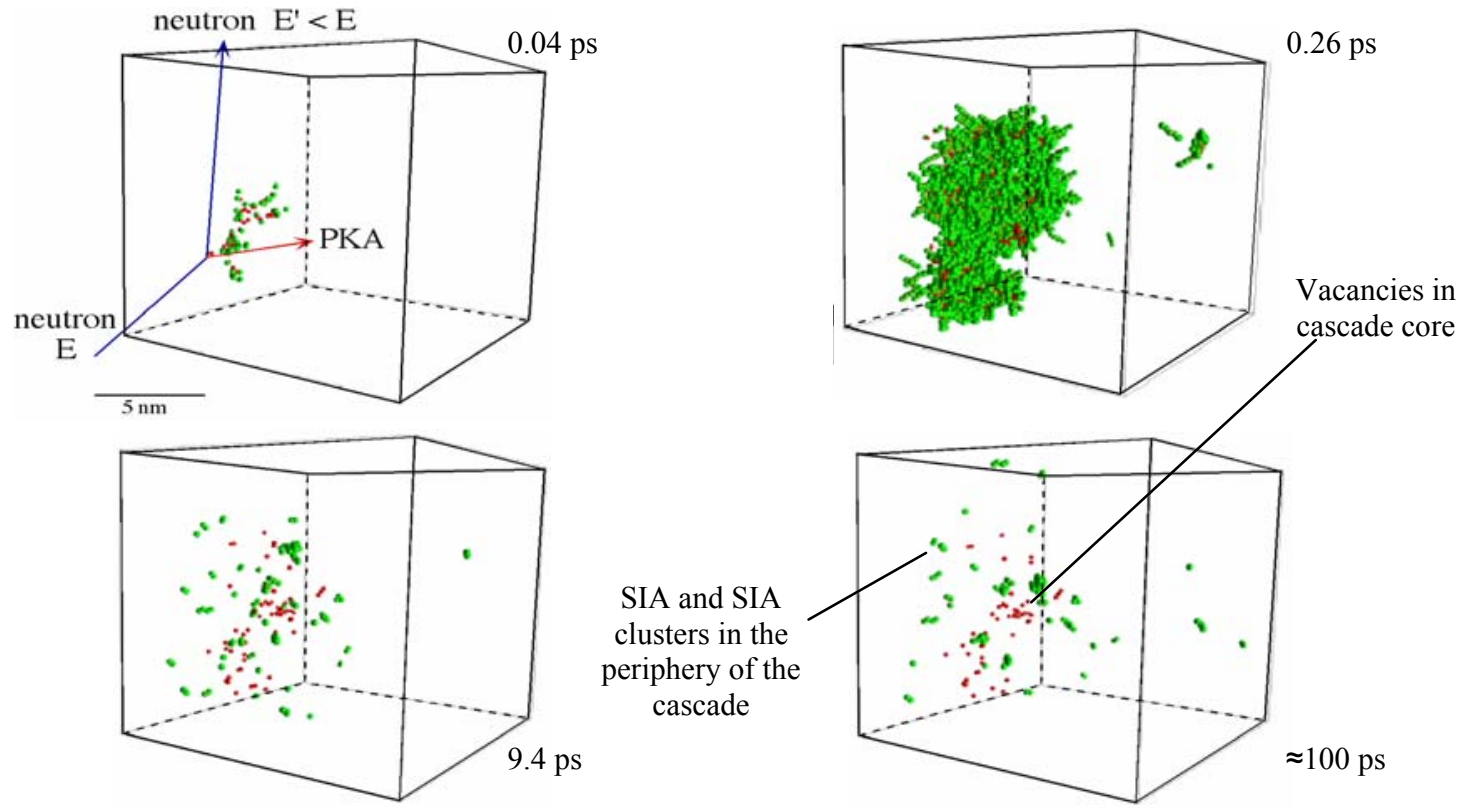

Fig. 2.2. A molecular dynamics simulation of displacement cascade in Fe produced by primary recoil atoms (PRAs) created by high-energy neutrons. The figures represent a time sequence starting from the initial PRA collisions (upper left) to the defects remaining after the thermal energy has dissipated from the cascade (lower right) after $\approx 100 \mathrm{ps}$. The green dots are SIA and the red dots are vacancies. Note the positions of the lattice Fe atoms are not shown. 


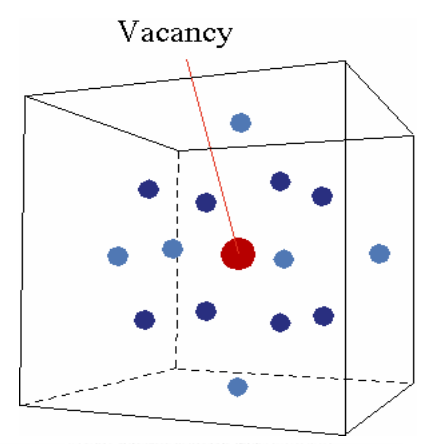

12 Vacancy cluster

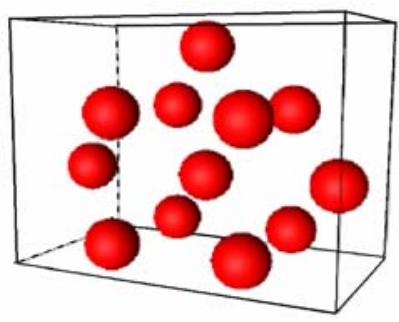

$<110>$ Split-Interstitial (SIA)

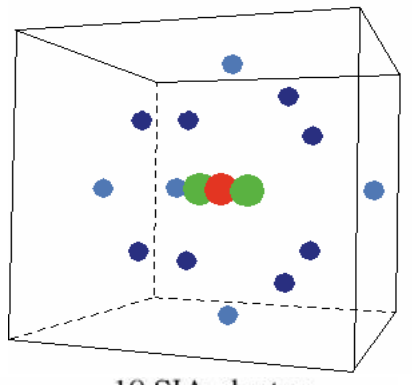

10 SIA cluster

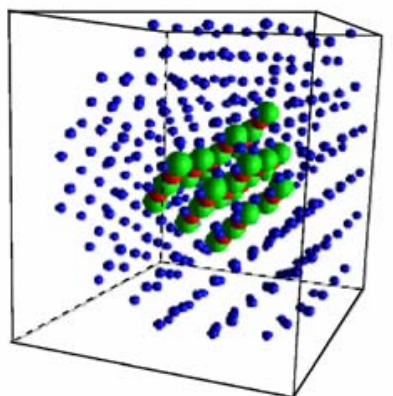

Fig. 2.3. Primary vacancy and SIA defects and defect clusters created in cascades by PRAs.

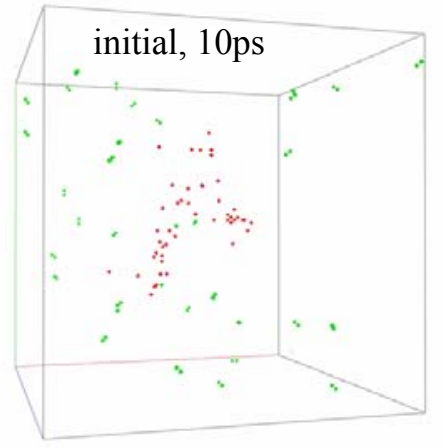

Cascade vacancy-solute clusters
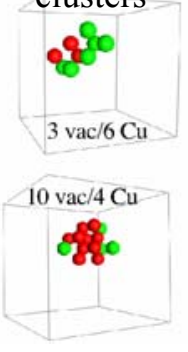
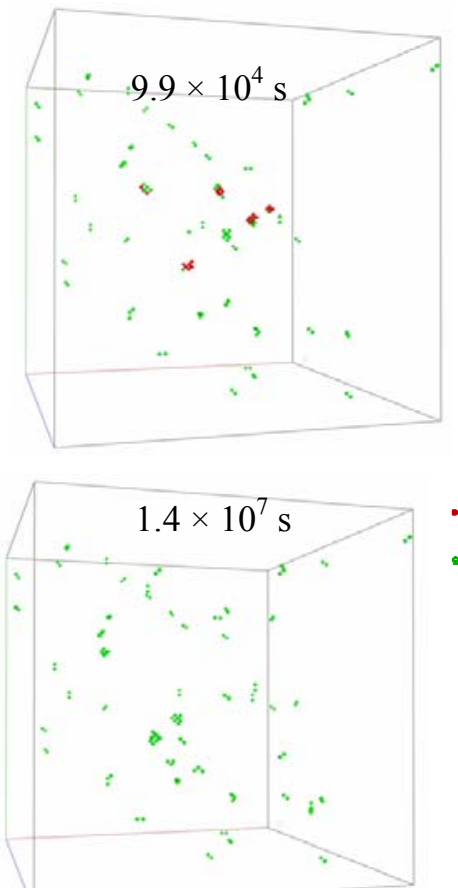

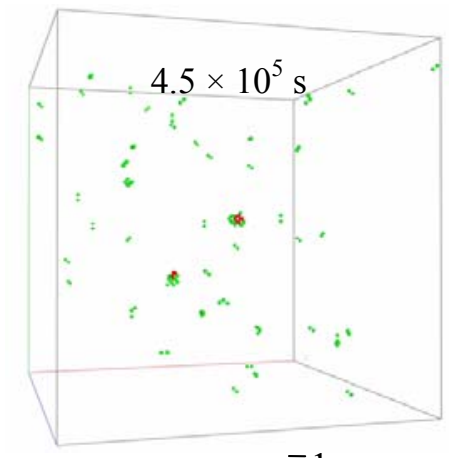

$-1 \mathrm{~nm}$

- clustered $\mathrm{Cu}$

Fig. 2.4. Kinetic lattice Monte Carlo simulations of cascade aging to form vacancy solute $(\mathrm{Cu})$ cluster complexes and their remnants that are believed to be the primary source of MF hardening. Note the positions of the Fe atoms are not shown. 

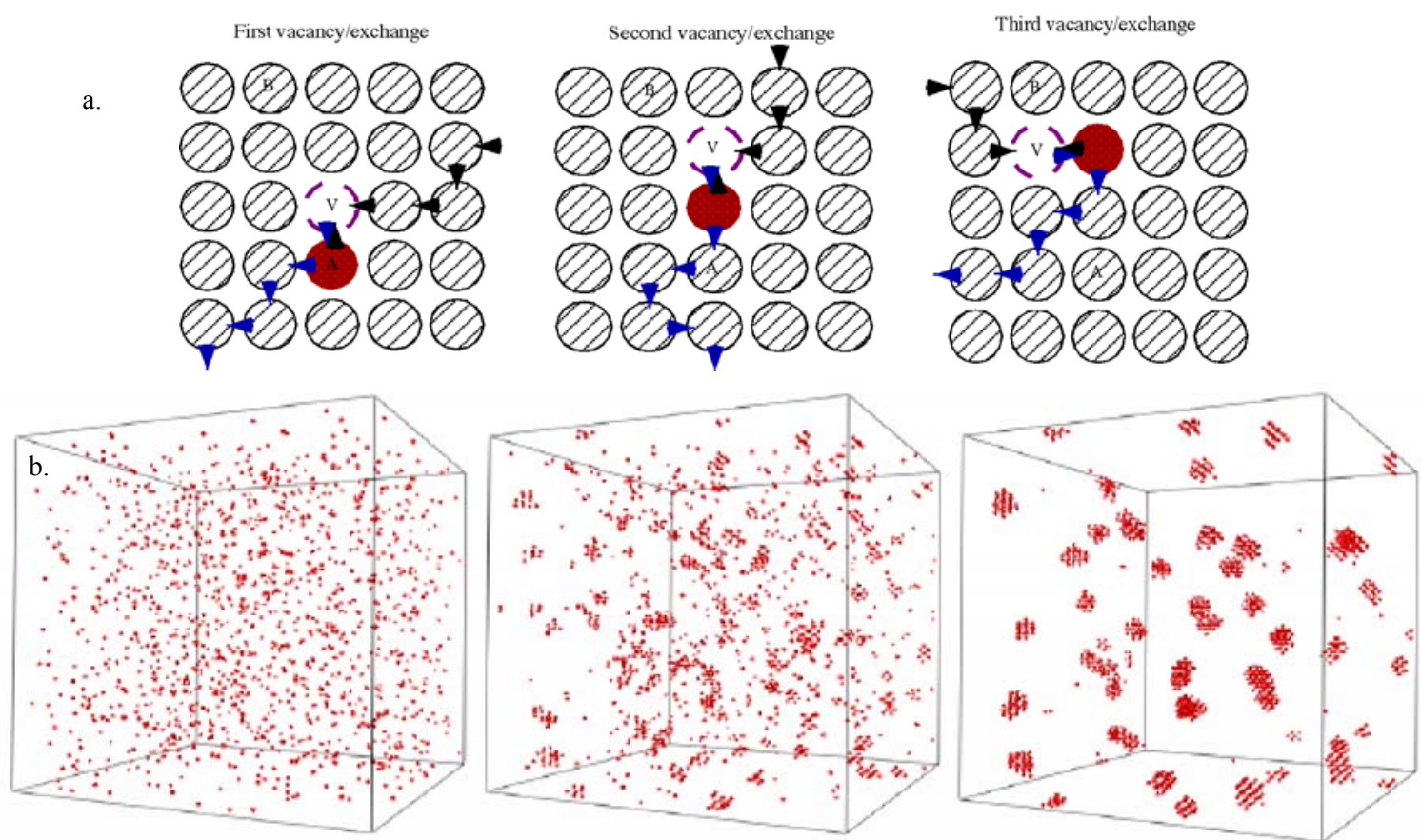

Fig. 2.5. (a) Schematic illustration of the vacancy exchange mechanism that resuts in diffusional motion of a substitutional solute. Note the path of the vacancies are shown by the arrows and, in this illustration, a new vacancy participates in each solute exchange; (b) a kinetic lattice Monte Carlo simulation of Cu clustering and precipitation in an $\mathrm{Fe}-0.3 \mathrm{Cu}$ alloy at $300^{\circ} \mathrm{C}$ based on the effects for a sequence of a large number of jumps of a single vacancy (not shown), corresponding to increasing time. The time scales roughly with the inverse of the number of vacancies per lattice site, hence is reduced under irradiation compared to thermal aging conditions. The Cu-clusters would eventually coarsen into a single precipitate. Note the positions of the $\mathrm{Fe}$ atoms are not shown.

CRPs are also enriched with varying amounts of $\mathrm{Mn}, \mathrm{Ni}, \mathrm{Si}$, and $\mathrm{P}$ in RPV steels containing these elements. In addition to CRPs, nanometer-scale Mn-Ni-Si rich precipitates (MNPs) also form under some conditions [3,6-9,12,22,28-33]. The nanometer-scale features, that are primarily responsible for irradiation hardening, are illustrated in Fig. 2.6. In addition to CRPs, MNPs, MFs, and dislocation loops, other potential hardening features include alloy phosphide precipitates (PPs) and dislocation solute atmospheres [34,35].

The defect cluster complex MF and fine-scale precipitates (or solute clusters) act as obstacles to dislocation glide, resulting in an increase in the yield $\left(\Delta \sigma_{\mathrm{y}}\right)$ and flow stress of the steel. The $\Delta \sigma_{\mathrm{y}}$ results in the elevation of the Charpy impact test transition temperature, typically defined as the $41 \mathrm{~J}$ (30 ft-lb) transition temperature shift (TTS). Thus, the sequence of embrittlement mechanisms is

Irradiation and metallurgical variables $\rightarrow$ Evolution of hardening features $\rightarrow \Delta \sigma_{\mathrm{y}} \rightarrow$ TTS

Hierarchical multiscale-multiphysics models and experiments have been used to link these various mechanisms and ultimately to relate the TTS to the combination of metallurgical and irradiation variables $[8,36]$. However, accurate TTS predictions require that simplified analytical representations of these models be fitted to the TTS surveillance database (or other embrittlement databases 

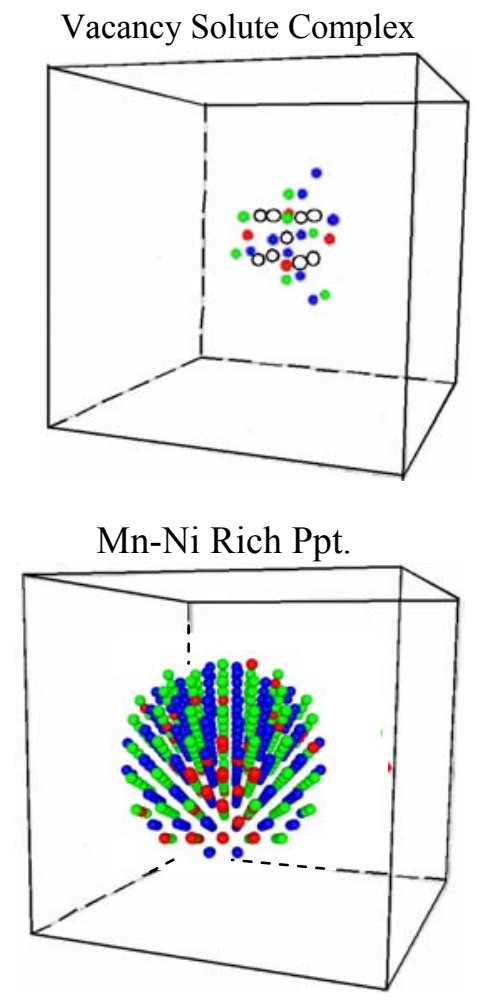

Solute Atmosphere
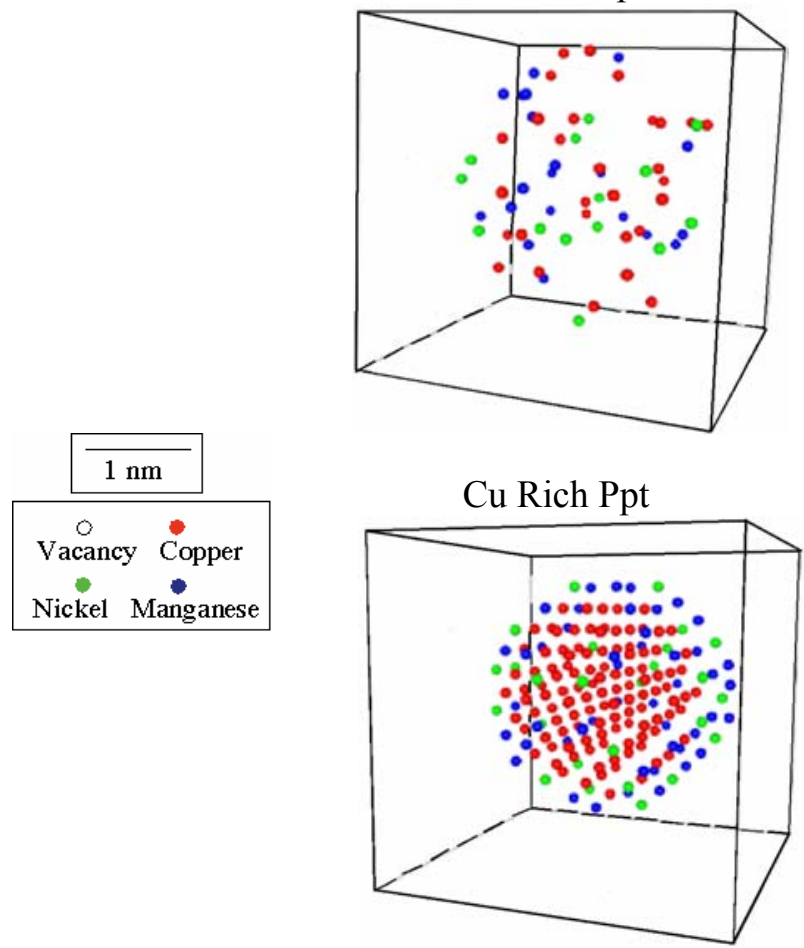

Fig. 2.6. Schematic illustration of the primary types of nanometer-scale features that cause the irradiation hardening and embrittlement of RPV steels. Note the positions of the Fe atoms are not shown.

In the following sections, the various mechanisms leading to embrittlement are briefly discussed in a sequence aimed both to frame an understanding of embrittlement and to provide a mechanistic foundation for the formulation of the physically motivated TTS models. The discussion starts with the basic

hardening mechanism of embrittlement and its underlying microstructural basis. Next, the effects of thecombination of metallurgical and irradiation variables on the hardening microstructures are discussed, first for CRPs. Representative examples of the effect of some metallurgical and irradiation variables on the CRP microstructure from the Irradiation Variables (IVAR) program are then presented. This is followed by a discussion of MFs, which form in both low-Cu and higher-Cu steels.

\subsection{Key Embrittlement Mechanisms}

\subsubsection{The Basic Hardening Mechanism of Embrittlement and Primary Hardening Features}

The primary mechanism of irradiation embrittlement in Mn-Mo-Ni RPV steels is an increase in the yield stress $\left(\Delta \sigma_{y}\right)$, produced by a high number density of nanometer-scale hardening features that develop as a consequence of irradiation. The nanofeatures include coherent $\mathrm{Cu}-\mathrm{Mn}-\mathrm{Ni}$-rich CRPs, although in some cases they contain more $\mathrm{Mn}, \mathrm{Ni}$, and $\mathrm{Si}$ than $\mathrm{Cu}$, and so are referred to as MNPs. In low-Cu alloys ( $\mathrm{Cu}$ less than about $\approx 0.07 \mathrm{wt} \%$ ), the primary hardening features are believed to be defect cluster-solute complex MFs. Dislocation loops, phosphide (PPs) and other fine-scale precipitates, as well as solute dislocation atmospheres may also contribute to the hardening in some cases. Irradiation hardening, as quantified by $\Delta \sigma_{y}$, depends on the combination of metallurgical and irradiation variables (alloy 
composition, heat treatment, product form, irradiation temperature, and neutron flux, fluence and spectra) and may exceed $350 \mathrm{MPa}$ in very irradiation-sensitive steels.

The following sections aim to provide a more quantitative, model-based descriptions of key embrittlement mechanisms. It must be emphasized that even these more detailed models are simplified and do not represent a fully comprehensive description of all the complexities involved in the embrittlement process. However, they do provide a good physical framework for assessing the strengths and limitations of necessarily even more simplified TTS models used to fit the surveillance database, as described in Chaps. 3 and 4.

\subsubsection{Irradiation Hardening}

The hardening features act as obstacles to dislocation glide, increasing the critical resolved shear stress for plastic deformation [37]. As expressed in Eq. 2-1, the strengthening produced by a specific type of feature depends on its radius $\left(\mathrm{r}_{\mathrm{j}}\right)$, number density $\left(\mathrm{N}_{\mathrm{j}}\right)$, volume fraction $\left(\mathrm{f}_{\mathrm{j}}\right)$, structure and composition ( $w h e r e ~ j=p$ for CRPs and $=m f$ for MFs) and can be characterized by an obstacle strength parameter, $\alpha_{j}$, as:

$$
\Delta \sigma_{\mathrm{yj}} \approx\left[0.55 \mathrm{TF} \alpha_{\mathrm{j}} \mu \mathrm{b} \vee \mathrm{f}_{\mathrm{j}}\right] / \mathrm{r}_{\mathrm{j}}
$$

Here TF is the Taylor factor $\approx 3, \mu$ is the shear modulus of $\mathrm{Fe} \approx 80 \mathrm{GPa}$, and $\mathrm{b}$ is the Burger's vector $\approx$ $0.248 \mathrm{~nm}$. The MFs are relatively weak obstacles $\left(\alpha_{\mathrm{mf}} \approx 0.05-0.1\right)$, since they are small and may be loose aggregates of solutes, while the well-formed CRPs have a range of medium strengths $\left(\alpha_{\mathrm{crp}} \approx 0.1\right.$ to 0.3$)$, which increases with the precipitate radius, $r_{p}$ [3]. This means that dislocations cut through the CRPs and MFs at a maximum local pinning force, corresponding to a critical bowing angle, characterized by the $\alpha_{j}$, which is controlled by the detailed obstacle-dislocation interaction mechanism. Obstacle-dislocation interaction mechanisms have been characterized experimentally, and modeled, most recently, by computer simulations. Figure 2.7 (a) shows the molecular dynamics simulations of the critical bowing angle, reported by Bacon and Osetskiy [38], when the dislocation cuts though a small coherent $\mathrm{Cu}$ precipitate with a radius $r_{p}$. Figure 2.8 (b) shows that the corresponding prediction of $\sigma_{y p} / ل_{p}$ vs $r_{p}$ is reasonably consistent with experimental data (various symbols) from the IVAR irradiations, where $f_{p}$ and $r_{p}$ have been measured by small-angle neutron scattering (SANS). Figure 2.7 also shows $\sigma_{y p} / ل_{p}$ for a fitted Russell-Brown model, which is in slightly better agreement with the data $[3,39]$. These pinning mechanisms involve dislocations cutting through the obstacles, in contrast to hardening by high strength, Orowan type obstacles, which require bypass by dislocation looping, with a corresponding maximum $\alpha_{0} \approx$ 1 [3,37]. Strong obstacles, particularly $\mathrm{Mo}_{2} \mathrm{C}$ precipitates, are responsible for producing a significant fraction of the pre-existing strength of unirradiated steels [3].

In developing embrittlement models, the individual hardening contributions from various irradiationinduced obstacles must be combined with one another, as well as with the various sources of the unirradiated alloy strength $[3,40]$. The limiting rules for such superposition are a linear sum (LS) law and a square root of the sum of the squares (RSS) law [40]. The RSS law accounts for the spacing of obstacles with similar strength on a slip plane. However, if mixtures of high- and medium-strength obstacles are present, the high-strength obstacles result in larger dislocation bowing angles $[3,40]$. The bowed and extended dislocation segments thus encounter more of the medium-strength obstacles than would be the case if only the latter were present. Thus the net $\sigma_{\mathrm{y}}$ for mixtures of medium- and high-strength obstacles is larger than is predicted by the simple RSS law. In the other limit, numerous very low strength obstacles do not significantly change the shape of gliding dislocations; hence, they act more like a lattice friction stress that simply adds to higher-strength obstacle contributions by an LS law. These concepts are schematically illustrated in Fig. 2.8. 


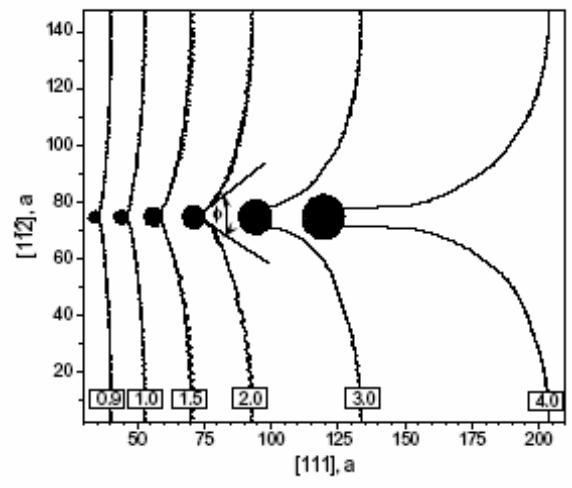

(a)

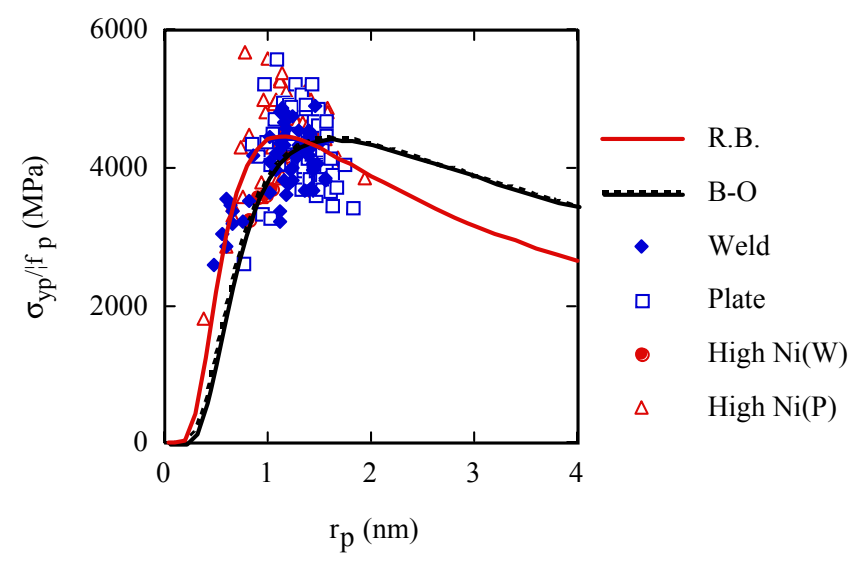

(b)

Fig. 2.7. (a) Molecular dynamics simulation of the critical dislocation bowing angle for penetrating coherent $\mathrm{Cu}$ precipitates with various $r_{p}$ by Bacon and Osetskiy (BO). (b) The precipitate hardening efficiency $\left(\sigma_{y p} / / f_{p}\right) v s r_{p}$ showing both a fitted Russell-Brown model (RB) along with the BO model compared to pairs of $\sigma_{y p} / \sqrt{ } f_{p}$ and $r_{p}$ data from a combination of SANS measurements $\left(f_{p}\right.$ and $\left.r_{p}\right)$ and tensile tests $\left(\sigma_{y p}\right)$.

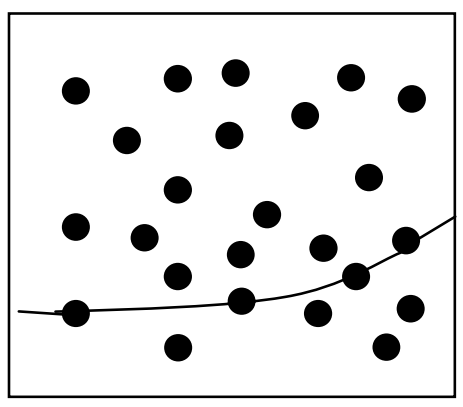

a.

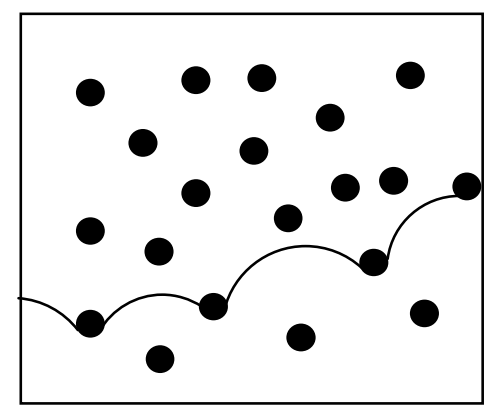

medium

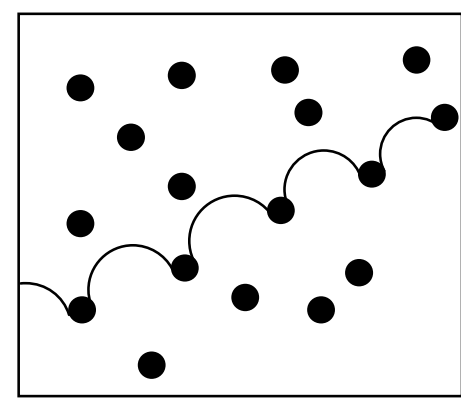

high

b.

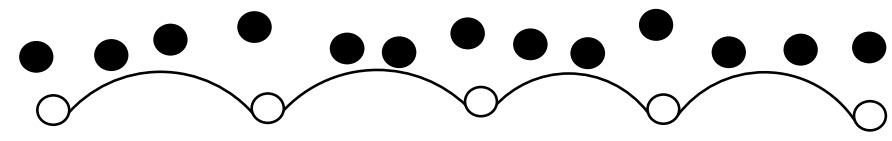

c.

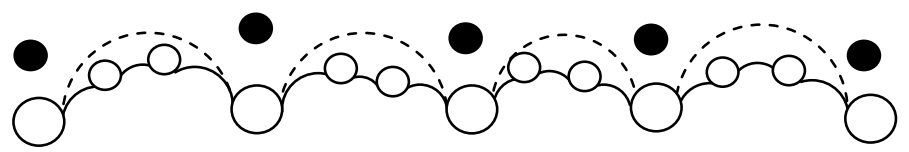

Fig. 2.8. Schematic illustration of the effect of the critical dislocation bowing angle on strength superposition. (a) Critical dislocation angle shapes for low-, medium- and highstrength obstacles. (b) The bowing for dislocation pinned by five medium-strength obstacles. The dislocation has not yet encountered obstacles shaded black at the critical bowing angle. (c) The dislocation bowing when high-strength obstacles have replaced the medium-strength obstacles. The dislocation is now also pinned by eight medium-strength obstacles instead of five as in Fig. 2.8b. The dashed line shows the bowing for strong obstacles alone at the critical bowing angle. 
Computer simulations have been developed to evaluate the net $\Delta \sigma_{y}[36,41]$ for various populations of obstacles with different $\alpha_{j}[3,36,41]$. In one study, the resulting computational database was fitted by an analytical model that can be used to calculate the net $\Delta \sigma_{\mathrm{y}}$ based on the individual yield stress contributions and $\alpha_{i}$ of weak $\left(\alpha_{\mathrm{w}}<0.1, \sigma_{\mathrm{yw}}\right)$, medium $\left(0.1<\alpha_{\mathrm{m}}<0.6, \sigma_{\mathrm{ym}}\right)$ and high $\left(\alpha_{\mathrm{o}}>0.6, \sigma_{\mathrm{yo}}\right)$ strength obstacles. The net $\sigma_{\mathrm{y}}$ is given by

$$
\sigma_{\mathrm{y}} \approx \sigma_{\mathrm{yw}}+(1-\mathrm{S})\left({\sigma_{\mathrm{ym}}}^{2}+\sigma_{\mathrm{yo}}{ }^{2}\right)^{1 / 2}+\mathrm{S}\left(\sigma_{\mathrm{ym}}+\sigma_{\mathrm{yo}}\right)
$$

Here the superposition factor $\mathrm{S}$ is given by [3]

$$
\mathrm{S} \approx \alpha_{\mathrm{o}}-\alpha_{\mathrm{m}}\left(5.0-3.3 \alpha_{\mathrm{o}}\right)
$$

Thus, features with similar $\alpha_{j}$ (all fairly low, medium, or high strength) obey an RSS superposition law, while those with very different $\alpha_{\mathrm{j}}$ (low and high strength) obey an LS law. The superposition of the strengthening contributions of medium strength and strong obstacles falls in between.

The LS law is most appropriate for adding the contribution of medium-strength irradiation-induced precipitates to simple model alloys, which initially have low unirradiated $\sigma_{\text {yu }}$ due to the absence of preexisting medium- and high-strength obstacles. The resulting $\Delta \sigma_{\mathrm{y}} \approx \sigma_{\mathrm{yp}}$ in this case is greater than if the same irradiation induced features were added to a complex steel alloy, with a large contribution from preexisting high-strength obstacles, where the net superposition falls between the LS and RSS limits. Figure 2.9 plots the net yield stress increase, $\Delta \sigma_{\mathrm{y}}$, vs the individual strengthening contribution of 1-nm CRPs, $\sigma_{\text {yp }}$, assuming the pre-existing strengthening is due to strong obstacles with $\alpha_{o}=0.9$ is $\sigma_{\text {уо }}=$ $180 \mathrm{MPa}$. A curve for pure $\mathrm{Cu}$ precipitates, which are somewhat weaker than CRPs in RPV steels, is also shown for comparison, along with the bounding LS and RSS laws. For example, if the $\sigma_{\mathrm{yp}}=100 \mathrm{MPa}$, the $\Delta \sigma_{\mathrm{y}}=48 \mathrm{MPa}$ for $1 \mathrm{~nm}$ CRPs, falling between the limiting cases of $100 \mathrm{MPa}$ (LS) and $23 \mathrm{MPa}$ (RSS).

Thus, if the details of a material's microstructure are known, the strengthening and superposition models described above can be applied rigorously, and have been shown to work well [3,8,27,42]. This is illustrated in Fig. 2.10 which shows, predicted vs measured CRP hardening for two medium-strength $\approx 0.4 \mathrm{wt} \% \mathrm{Cu}$-bearing RPV split melt model alloys with $\approx 0.8 \mathrm{wt} \%$ (LC) and $\approx 1.3 \mathrm{wt} \%$ (LD) Ni as well as a high-Cu $\approx 0.55 \mathrm{wt} \%$ and high-Ni $\approx 1.66 \mathrm{wt} \%$ weld $(\mathrm{WV})$ for a variety of irradiation conditions $[3,27,42]$. Here, the values of $f_{p}$ and $r_{p}$ were again determined from SANS measurements, and the $\Delta \sigma_{y p}$ was predicted based on the Russell Brown model (see Fig. 2.7) and Eqs. (2-2) and (2-3), again assuming a pre-irradiation $\sigma_{\text {уо }}=180 \mathrm{MPa}$ and $\alpha_{\mathrm{o}}=0.9$. The added MF contribution was based on the measured $\Delta \sigma_{\mathrm{y}}$ in alloys with the same nominal composition as the three alloys cited above, except that they do not contain $\mathrm{Cu}$.

Such detailed information is generally not available. Thus obstacle interaction and superposition effects must be effectively incorporated into the chemistry, product form and fluence function terms in the TTS model and fitting parameters. Since the MFs are fairly weak obstacles, a linear sum (LS) of $\Delta \sigma_{\mathrm{mf}}$ from MFs plus the net $\Delta \sigma_{\mathrm{p}}$ from CRPs roughly account for superposition effects and is a common approximation, leading to the so-called two-feature model adopted in the following chapters. In addition to these theoretical considerations, as illustrated in Fig. 2.11, the two-feature model appears to work well in practice. Figure 2.11a plots $\Delta \sigma_{\mathrm{y}}$ vs the square root of fluence for intermediate flux, $290^{\circ} \mathrm{C}$ irradiations (see Chap. 6) of the LC alloy, cited above, along with a $\mathrm{Cu}$-free alloy (LG), which otherwise has the same nominal composition as LC. Subtracting the $\Delta \sigma_{\mathrm{y}}$ for LG from that for LC gives an estimate of the net CRP hardening, shown as the long dashed line. As expected the CRP contribution approaches saturation. Figure $2.11 \mathrm{~b}$ plots corresponding SANS measurements of the CRP $f_{p}$ and $r_{p}$ for LC. The dotted line in 


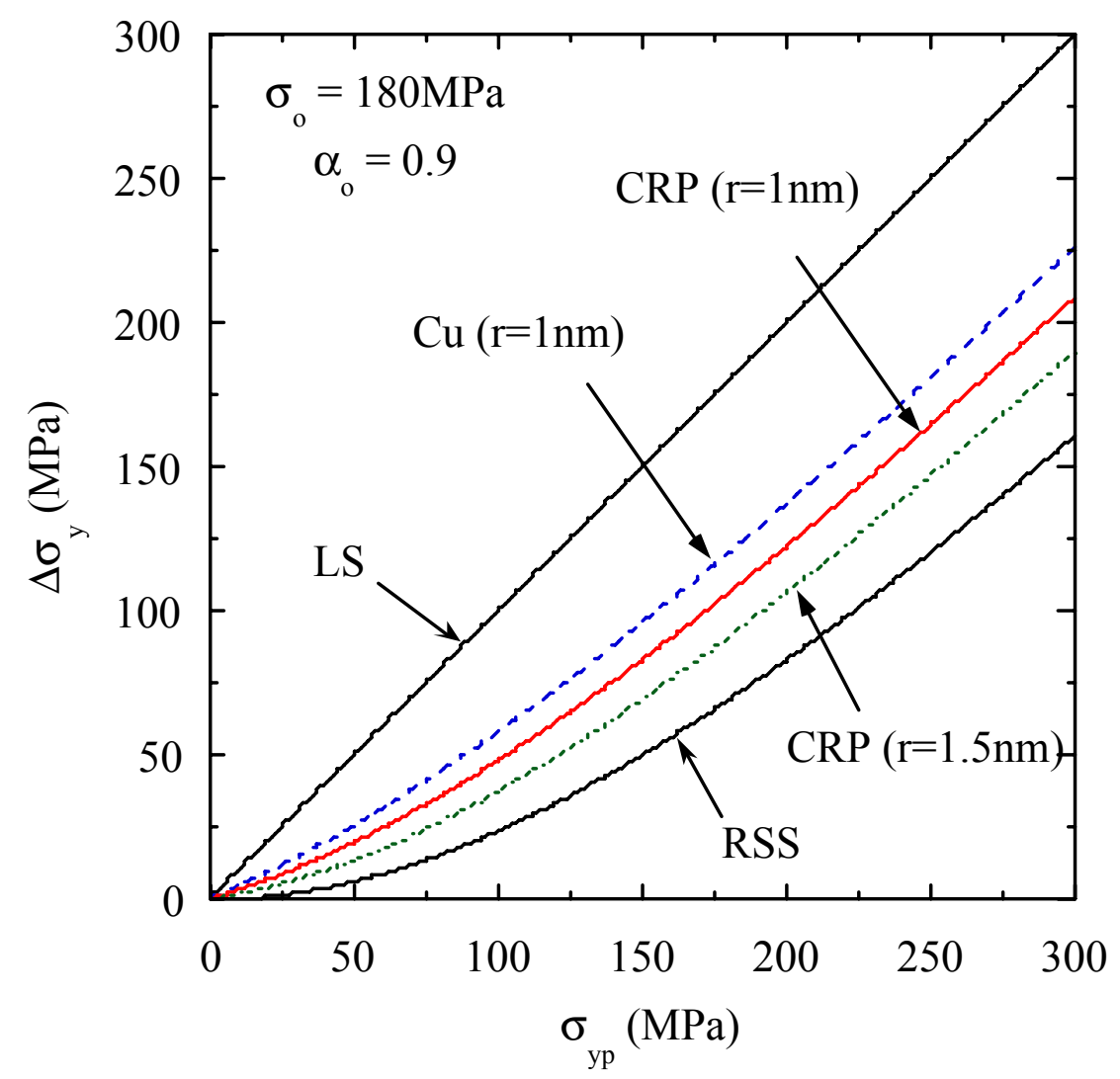

Fig. 2.9. Superposition of various levels of strengthening from 1-nm pure Cu precipitates and CRPs with $180 \mathrm{MPa}$ of pre-existing strong obstacle strengthening and $\alpha_{0}=0.9$.

Fig. 2.11a shows the predictions of $\sigma_{\mathrm{yp}}$ based on the Russell Brown hardening and superposition models described above. The general consistency of these results clearly supports the use of a two-feature model.

\subsubsection{Irradiation Hardening and TTS}

The transition temperature shift (TTS) in the Charpy temperature at $41 \mathrm{~J}(30 \mathrm{ft}-\mathrm{lb})$ can be related to $\Delta \sigma_{\mathrm{y}}$ based on well-established micromechanical models. There are two hardening contributions to the TTS [23]. The largest contribution is the shift in the maximum elastic cleavage fracture temperature typically occurring at about $10 \mathrm{~J}, \Delta \mathrm{TTS}_{10}$, which correlates directly with $\Delta \sigma_{\mathrm{y}}$. Figure 2.12 schematically illustrates this mechanism in terms of the so-called Davidenkov diagram. Here, $M(>1)$ is a constraint factor that elevates the internal tensile stresses near the notch tip to values greater than $\sigma_{\mathrm{y}}$, and $\sigma^{*}$ is the microcleavage fracture stress. Based on the conventional assumption that $\sigma^{*}$ does not vary with temperature and, for purposes of illustration, assuming a simple linear relation between the yield stress $\sigma_{\mathrm{y}}$ and $\mathrm{T},\left[\mathrm{d} \sigma_{\mathrm{y}} / \mathrm{dT}\right]$, the $\mathrm{TTS}_{10}$ is simply [23]

$$
\mathrm{TTS}_{10}=\mathrm{TT}_{10 \mathrm{i}}(\text { irradiated })-\mathrm{TT}_{10 \mathrm{u}}(\text { unirradiated })=\Delta \sigma_{\mathrm{y}} /\left[\mathrm{d} \sigma_{\mathrm{y}} / \mathrm{dT}\right]
$$

The actual non-linear relation between $\sigma_{\mathrm{y}}$ and $\mathrm{T}$ can be accounted for by using the average $<\left[\mathrm{d} \sigma_{\mathrm{y}} / \mathrm{dT}\right]>$ over the unirradiated, $\mathrm{TT}_{10 \mathrm{u}}$, to irradiated, $\mathrm{TT}_{10 \mathrm{i}}$, temperature interval. More generally, the elastic cleavage 


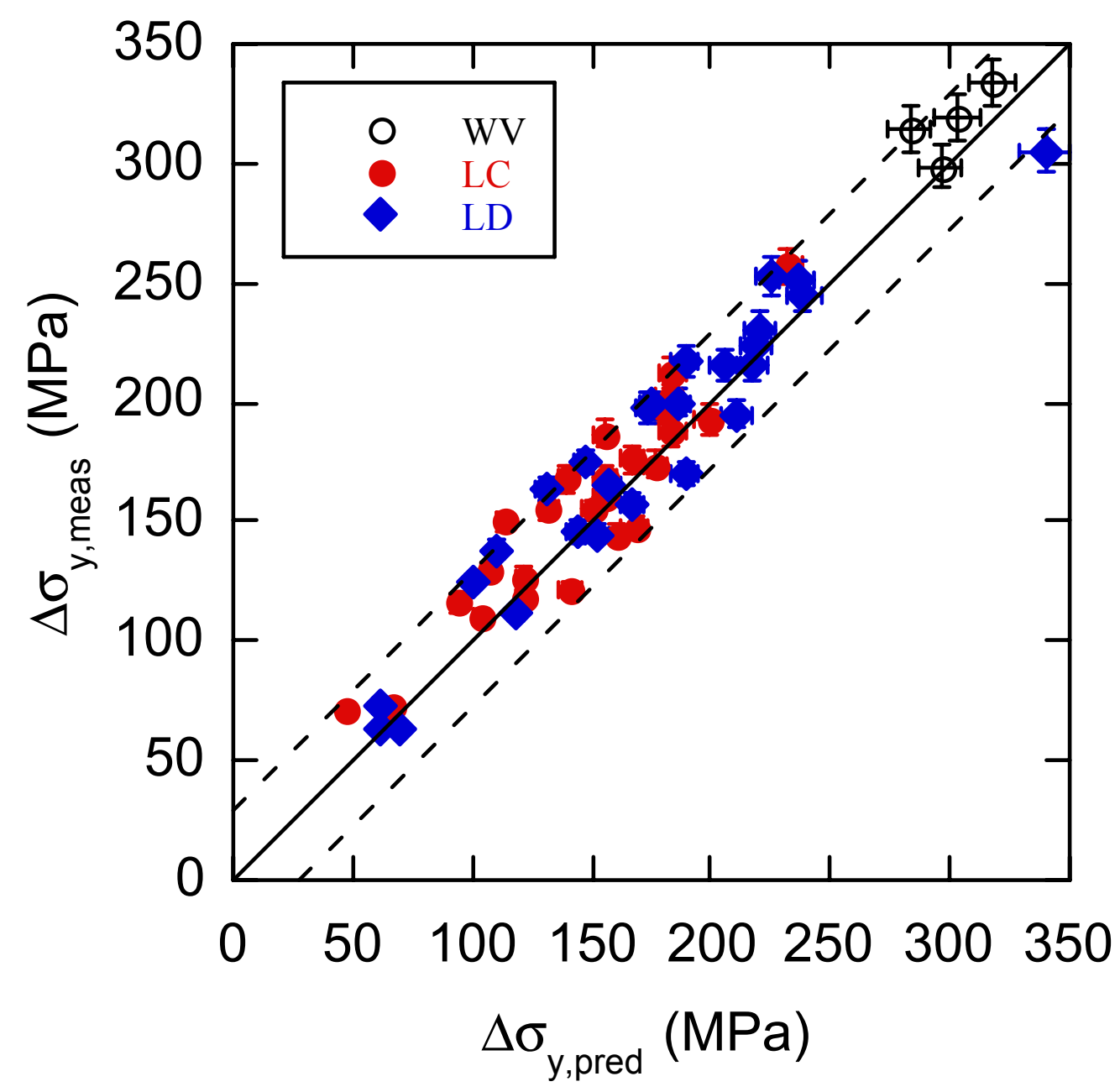

Fig. 2.10 Measured versus predicted $\Delta \sigma_{y}$ from CRPs based on SANS measurements of $f_{p}$ and $r_{p}$ used in a modified Russell-Brown precipitate hardening and computer simulation derived superposition model [Eqs. (1) to (3)]. The MF hardening is based on the addition of a much smaller $\Delta \sigma_{y}$ for alloys with similar compositions, except that they contained little or no $\mathrm{Cu}[27,42]$.

temperature shift can be specified by the difference in the temperatures at which the unirradiated and irradiated $\sigma_{\mathrm{y}}$ are equal [23],

$$
\sigma_{\mathrm{yu}}\left(\mathrm{T}_{\mathrm{cu}}\right)=\sigma_{\mathrm{yu}}\left(\mathrm{T}_{\mathrm{ci}}\right)+\Delta \sigma_{\mathrm{y}}
$$

Equation (2-5) accounts for the actual nonlinear relation between $\sigma_{\mathrm{y}}$ and $\mathrm{T}$, and predicts a corresponding nonlinear relation between the TTS that depends on $\mathrm{TT}_{10 \mathrm{u}}$ and $\Delta \sigma_{\mathrm{y}}$.

There is also a generally smaller contribution, TTS $_{\text {use, }}$, to the overall TTS at $41 \mathrm{~J}$, due to the reduction in the Charpy upper shelf energy, $\left(\Delta \mathrm{USE}=\mathrm{USE}_{\mathrm{i}}-\mathrm{USE}_{\mathrm{u}}<0\right)$ and the associated layover of the Charpy energy temperature curve in the ductile-brittle transition region. A previous study showed that the lower- 

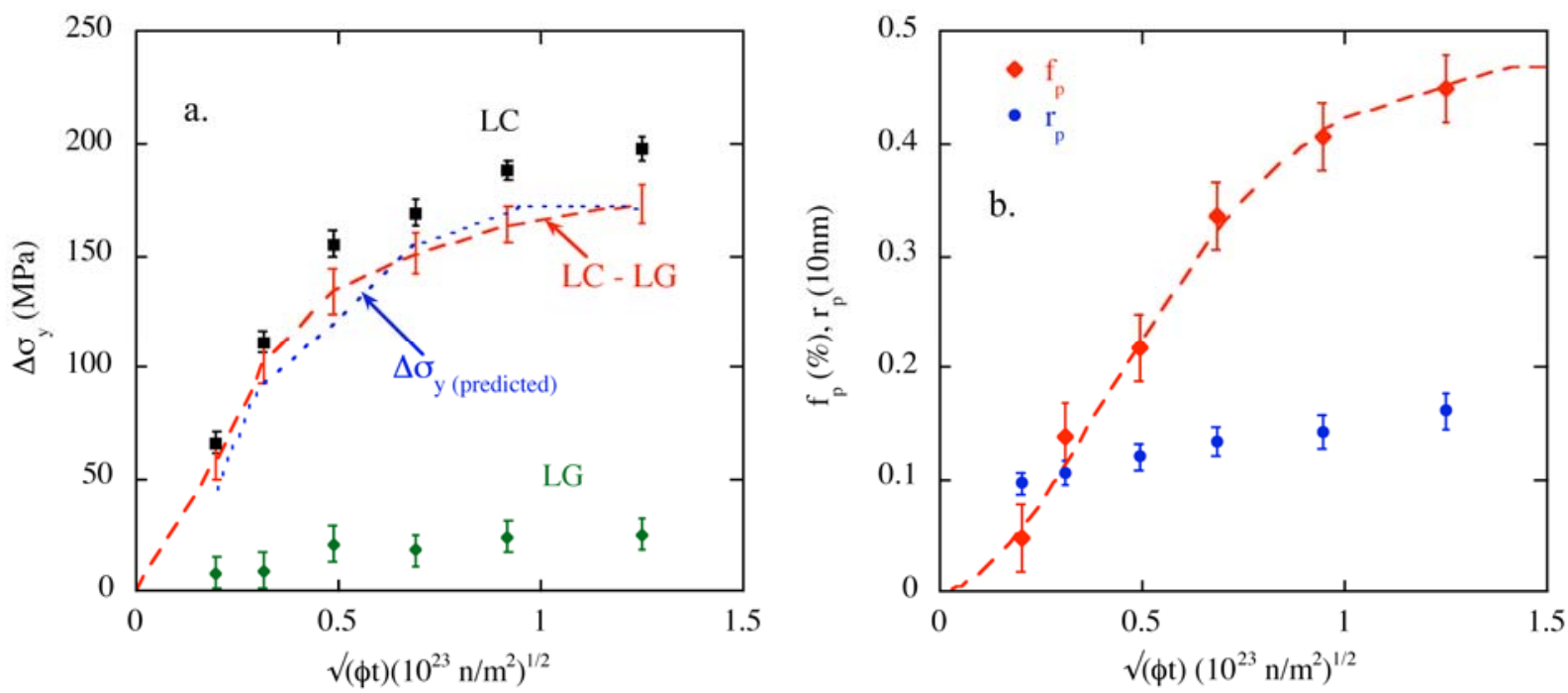

Fig. 2.11 Two feature model hardening analyses for a $0.4 \% \mathrm{Cu}$ LC alloy irradiated at $290^{\circ} \mathrm{C}$ (a) $\Delta \sigma_{y}$ vs the square root of fluence for intermediate flux irradiations (see Chap. 6) of the LC alloy, along with a Cu-free alloy (LG), which otherwise has the same nominal composition as LC, and the net CRP hardening (LC-LG), as shown as the long dashed line. (b) The corresponding SANS measurements of the CRP $f_{p}$ and $r_{p}$ for LC. The dotted line in Fig. 2.11a shows the predictions of $\sigma_{\mathrm{yp}}$ based on the Russell Brown hardening and superposition models. The general consistency of these results clearly supports the use of a two-feature model.

to-upper shelf transition occurs over an approximately constant temperature interval of $120 \pm 25^{\circ} \mathrm{C}$ [23] as illustrated in Fig. 2.12 (b). Note, that a recent unpublished study suggests that this transition temperature interval is not constant, but rather increases with radiation damage; the generality of this behavior willbe assessed in the future. However, even if confirmed, this detail would have little effect on the current discussion. The $\Delta$ USE can also be related empirically to $\Delta \sigma_{\mathrm{y}}$ based on a simple fitting model [23]. Thus the assumption of a constant transition interval combined with the empirical $\Delta \operatorname{USE}\left(\Delta \sigma_{\mathrm{y}}\right)$ model is used to estimate TTS $_{\text {use }}$ and TTS with an expression of the general form

$$
\mathrm{TTS}=\mathrm{TTS}_{10}+\mathrm{TTS}_{\mathrm{use}}=\mathrm{C}_{\mathrm{c}}\left(\mathrm{TT}_{10 \mathrm{u}}, \mathrm{USE}_{\mathrm{u}}, \Delta \sigma_{\mathrm{y}}\right) \Delta \sigma_{\mathrm{y}}
$$

Fig. 2.13 shows the predicted $\mathrm{C}_{\mathrm{c}}\left(\Delta \sigma_{\mathrm{y}}\right)$ for $\mathrm{TT}_{\mathrm{cu}}=-75^{\circ} \mathrm{C}$ and $\mathrm{USE}_{\mathrm{u}}=100 \mathrm{~J}$. Notably, $\mathrm{C}_{\mathrm{c}}$ increases with $\Delta \sigma_{\mathrm{y}} . \mathrm{C}_{\mathrm{c}}$ also increases at higher $\mathrm{TT}_{\mathrm{cu}}$ and lower initial $\mathrm{USE}_{\mathrm{u}}$. Observed $\mathrm{C}_{\mathrm{c}}$ values of $\approx 0.6 \pm 0.2^{\circ} \mathrm{C} / \mathrm{MPa}$, are consistent with these predictions [23].

The mechanisms and models described in this and the previous section provide the underpinning for the so-called two-feature TTS embrittlement model given by Eq. (2-7), which was calibrated to the surveillance data and presented in Chap. 4 of this report:

$$
\mathrm{TTS}=\mathrm{TTS}_{\mathrm{mf}}+\mathrm{TTS}_{\mathrm{crp}}
$$

As in the case of superposition of strengthening contributions, the nonlinear relation between $\mathrm{C}_{\mathrm{c}}$ and $\Delta \sigma_{\mathrm{y}}$ is approximately incorporated in the TTS model equations and effective fitting parameters.

In summary, the relations between TTS and $\Delta \sigma_{\mathrm{y}}$ are reasonably well understood and can be modeled. Standard micromechanical models predict weakly nonlinear relations between TTS and $\Delta \sigma_{\mathrm{y}}$ that depend 

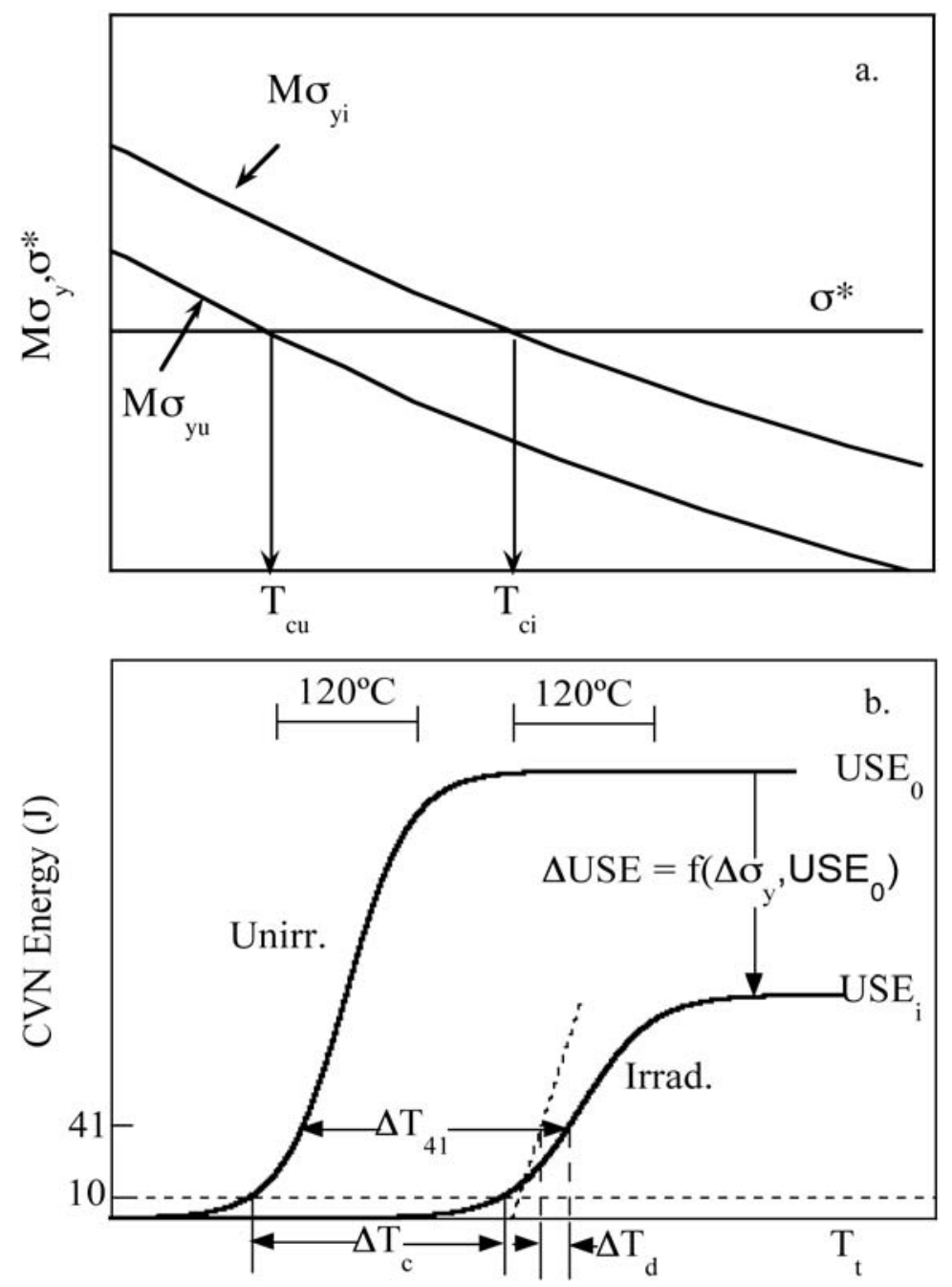

Fig. 2.12. Illustration of the basis for determining TTS from $\Delta \sigma_{\mathrm{y}}$. The model assumes that the critical cleavage stress and $\Delta \sigma_{y}$ are both independent of temperature. The $\Delta \sigma_{y}$ is used to estimate the shift in the elastic cleavage temperature $\left(T_{c}\right)$ defined at $10 \mathrm{~J}$. The extra TTS increment at $41 \mathrm{~J}$, due to the layover of the energy-temperature curve, is based on the observation that the temperature interval of the transition is $\approx 120^{\circ} \mathrm{C}$ and the use of an empirical relation between the reduction in the upper shelf energy $(\Delta \mathrm{USE})$ and $\Delta \sigma_{\mathrm{y}}$.

on the unirradiated properties of a particular steel. Such physical complexities are approximately incorporated in the two-feature TTS model presented in Chap. 4 of this report, in terms of averaged behavior, since the TTS equations are fit to the surveillance database.

\subsection{Copper-Rich Precipitates}

\subsubsection{Radiation-Enhanced Diffusion and Flux Effects on CRP Hardening}

Trace quantities of $\mathrm{Cu}(\mathrm{Cu} \approx 0.3 \mathrm{wt} . \%$ or less $)$ are left in solution after stress-relief treatments that are typically performed at around $600^{\circ} \mathrm{C}[3,4,6,7,12]$. However, as illustrated in Fig. 2.14, even at these low levels, $\mathrm{Cu}$ is highly supersaturated at the much lower vessel operating temperatures around $290^{\circ} \mathrm{C}[4,43]$ where the solubility of $\mathrm{Cu}$ is $\approx 74 \mathrm{appm}$, or $\approx 8.4 \times 10^{-3} \mathrm{wt} \%$. Thus $\mathrm{Cu}$ precipitates, first forming nmscale coherent bec transition phases, akin to so-called GP zones in aluminum alloys. Because of low 


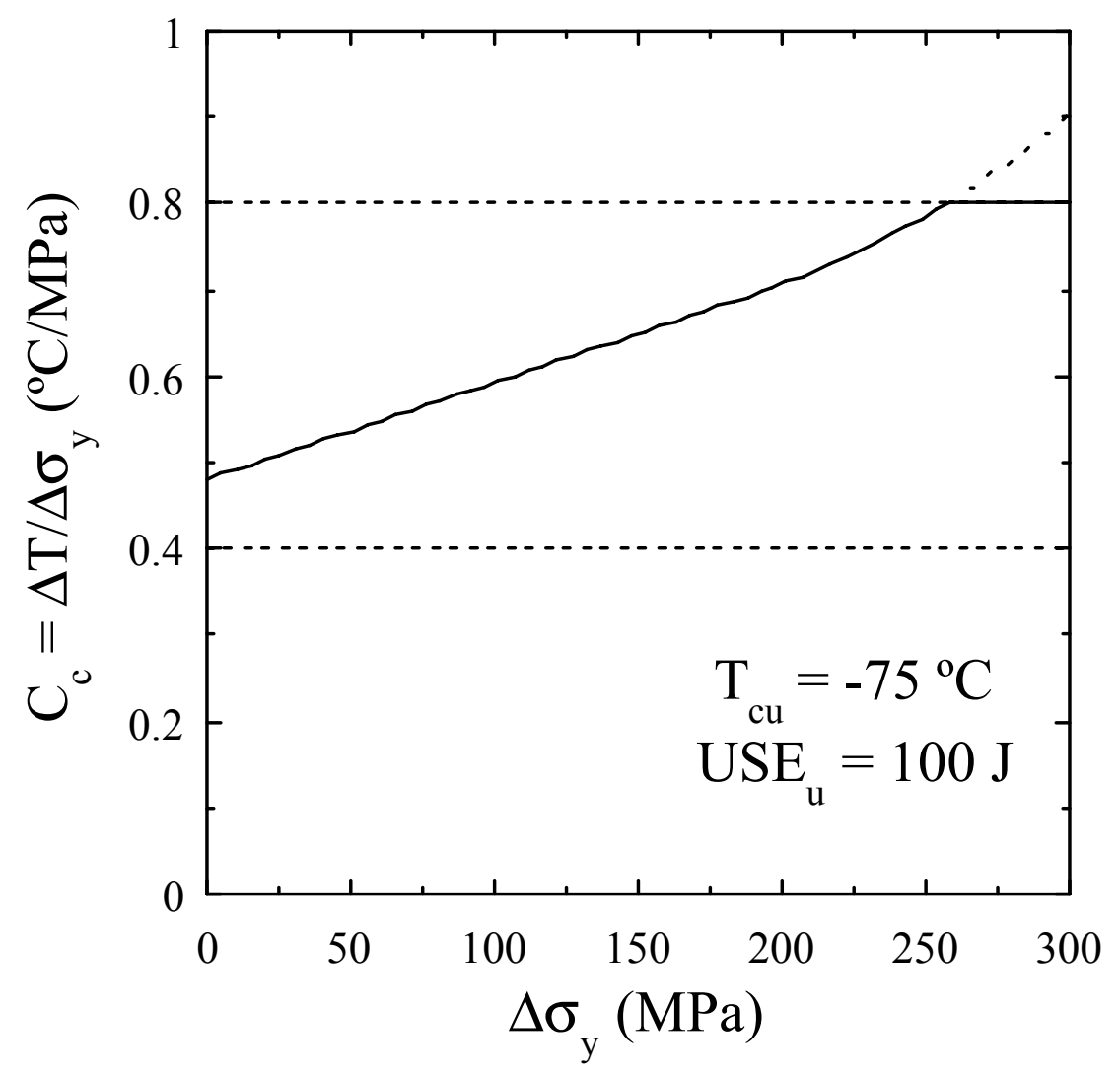

Fig. 2.13. The $\mathrm{C}_{\mathrm{c}}=\mathrm{TTS} / \Delta \sigma_{\mathrm{y}}$ derived from the model described in Fig. 2.12 and the text. The $\Delta \sigma_{y}$ dependence is primarily due to the variation in the slope of the $\sigma_{y}-T$ curve, with smaller contributions from the reduction in the USE. The value of $\mathrm{C}_{\mathrm{c}}$ depends on the unirradiated Charpy properties of the steel and $\Delta \sigma_{\mathrm{y}}$. The dashed lines mark the estimated bounds of the applicability of the model consistent with the range of observed $\mathrm{C}_{\mathrm{c}}$ values.

rates of thermal diffusion, $\mathrm{Cu}$ precipitation is relatively sluggish in the absence of irradiation. However, CRP nucleation and growth rates are greatly accelerated by radiation-enhanced diffusion (RED).

In the following section we estimate the magnitude of the radiation enhanced diffusion coefficient, $\mathrm{D}^{*}$, and model how it changes with variables such as the alloy composition and microstructure, irradiation temperature and, especially, the neutron flux. Substitutional solutes, like $\mathrm{Cu}$, diffuse by a vacancy exchange mechanism. Thus, the solute diffusion coefficient is proportional to the fractional concentration of vacancies, $X_{\mathrm{v}}$. In the absence of irradiation, thermodynamics dictates an equilibrium concentration of vacancies, $X_{v e}$, which controls the rate of both solute and self-diffusion. Under irradiation, there is an excess concentration of vacancies, $X_{v}>X_{v e}$, leading to a radiation enhanced diffusion coefficient, $\mathrm{D}^{*}>\mathrm{D}_{\mathrm{cu}}$, where $\mathrm{D}_{\mathrm{cu}}$ is the thermal diffusion coefficient of $\mathrm{Cu}$. Assuming all other variables are specified, the variation of $\mathrm{D}^{*}$ with flux is non-linear and exhibits four general regimes of behavior.

- At very low flux $\mathrm{D}^{*} \approx \mathrm{D}_{\mathrm{cu}}$ since $\mathrm{X}_{\mathrm{v}} \approx \mathrm{X}_{\mathrm{ve}}$

- At somewhat higher flux - when recombination is minimal - $D^{*}>D_{c u}$ is independent of flux

- At still higher flux - when recombination is dominant - $\mathrm{D}^{*}>>\mathrm{D}_{\mathrm{cu}}$ varies with the square root of flux 


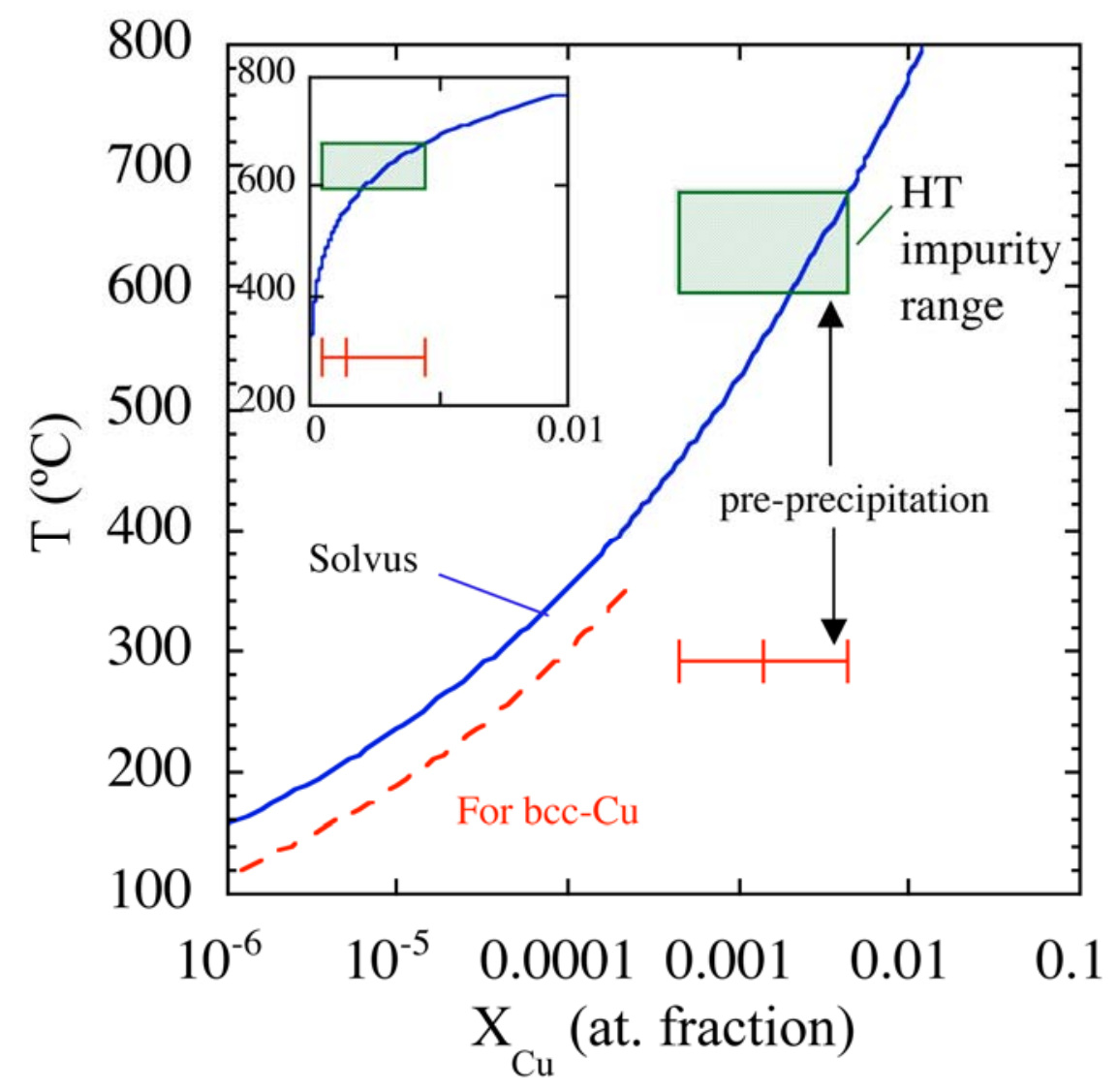

Fig. 2.14. The solubility of $\mathrm{Cu}$ in $\alpha$-Fe in equilibrium with the fcc (solid line) and bcc $\mathrm{Cu}$ phases (dashed line) as a function of temperature. The typical range of $\mathrm{Cu}$ impurity content is shown by the shaded box. At high-Cu levels beyond the solubility limit at the stress-relief temperature pre-precipitation limits the amount in solution prior to irradiation to a lower effective value, $\mathrm{Cu}_{\max }$. The $\mathrm{Cu}_{\max }$ depends on the heat treatment time and temperature history and the alloy composition, as well as the $\mathrm{Ni}$ content. The Cu remaining dissolved in the $\alpha$-Fe matrix is highly supersaturated around $290^{\circ} \mathrm{C}$ with respect to the coherent bcc phase. The supersaturated $\mathrm{Cu}$ undergoes accelerated precipitation under irradiation due to radiation enhanced diffusion (RED).

- At very high flux - when SIA-vacancy recombination occurs a high density of transient vacancy clusters is produced in displacement cascades $-D^{*}>>D_{c u}$ is once again constant and independent of flux [11]

The first three regimes are pertinent to surveillance and most test reactor irradiation conditions, hence, they will be the focus of the subsequent discussion. Rate theory can be used to model RED [12,13, 25 ] and $D^{*}$ by calculating the excess concentration of vacancies under irradiation. More details on the model described in this section are given in Ref. [13]. In the most basic sense, however, rate theory models determine the concentrations of reacting species by accounting for their generation, transport and fates that establish the kinetic balances between production, cluster accumulation (storage) and loss processes. The balances are expressed in terms of conservation equations containing the products of 
concentrations and rate coefficients, similar to those used to model chemical reaction kinetics. The fates of vacancies include recombination with SIA, self-clustering, cluster complex formation with solutes and annihilation at sinks, especially dislocations.

As a simple illustrative example, consider a species $S$ that is generated at a rate $G_{s}(S / a t o m-s)$ that undergoes diffusion to sinks where it is annihilated at a rate $X_{s} D_{s} S_{s}(S / a t o m-s)$. Here $X_{s}(S / a t o m), D_{s}$ $\left(\mathrm{m}^{2} / \mathrm{s}\right)$ and $\mathrm{S}_{\mathrm{s}}\left(\mathrm{m}^{-2}\right)$ are the fractional concentration, diffusion coefficient and total sink strength for $\mathrm{S}$, respectively. At steady-state, the production and loss of $S$ must balance, thus $G_{s}=X_{s} D_{s} S_{s}$, hence,

$$
\mathrm{X}_{\mathrm{s}}=\mathrm{G}_{\mathrm{s}} / \mathrm{D}_{\mathrm{s}} \mathrm{S}_{\mathrm{s}}
$$

The sink strength, $\mathrm{S}_{\mathrm{s}}$, is determined by solving the diffusion equation for the particular geometry. For example, for a specified dilute concentration of spherical sinks, with a number density, $\mathrm{N}_{\mathrm{s}}$, and a radius $\mathrm{r}_{\mathrm{s}}$, the sink strength $S_{s} \approx 4 \pi r_{s} N_{s}[25]$. Thus the concentration of $S, X_{s}$, is determined by the rate of generating $\mathrm{S}$, the sink microstructure, $\mathrm{N}_{\mathrm{s}}$ and $\mathrm{r}_{\mathrm{s}}$, and the diffusion coefficient of $\mathrm{S}, \mathrm{D}_{\mathrm{s}}$. In the case of radiation damage the $\mathrm{S}$ species are vacancies and SIA.

Displacement damage creates an equal number of vacancies and SIAs, sometimes called Frenkel pairs, at a rate

$$
\mathrm{G}_{\mathrm{v}}=\mathrm{G}_{\mathrm{i}}=\phi \sigma_{\mathrm{v}}
$$

Here $\phi$ is the neutron flux $\left(\mathrm{n} / \mathrm{m}^{2}\right.$-s, typically reported in terms of neutrons with energies $\left.>1 \mathrm{MeV}\right)$ and $\sigma_{\mathrm{v}}$ (vacancies- $\mathrm{m}^{2} / \mathrm{n}$-atom) is the neutron spectrum averaged vacancy production cross section [12,24]. A cross section can be thought of as an effective area per atomic nucleus for producing a nuclear reaction, in this case, generation of vacancies. Computer simulations of the formation and subsequent short-term rearrangement of displacement cascades leading to recombination, combined with neutron cross-section and reaction kinematics models, have been used to derive $\sigma_{\mathrm{v}}$ as a function of the neutron energy [24]. At higher fluxes, RED is primarily due to the excess vacancy concentration, $X_{\mathrm{v}}$. The RED coefficient (D*) can be expressed in terms of the ratio of atomic fraction of vacancies under irradiation $\left(\mathrm{X}_{\mathrm{v}}\right)$ to that at thermal equilibrium $\left(\mathrm{X}_{\mathrm{ve}}\right)$ and the thermal diffusion coefficient of $\mathrm{Cu}, \mathrm{D}_{\mathrm{cu}}$, as

$$
\mathrm{D}^{*} \approx \mathrm{D}_{\mathrm{cu}}\left[\mathrm{X}_{\mathrm{v}} / \mathrm{X}_{\mathrm{ve}}\right]
$$

At steady state, $\mathrm{X}_{\mathrm{v}}$ is constant; thus, as noted previously, vacancies (and SIAs) must be destroyed at the same rate as they are created. Vacancies are destroyed when they are absorbed at sinks, like dislocation jogs, or when they recombine with SIAs, which are both self-healing processes. In the simplest case, the vacancy and SIA destruction rates at sinks are $D_{v} X_{v} S_{t}$ and $D_{i} X_{i} S_{t}$, where $D_{v}$ and $D_{i}(>>$ $D_{v}$ ) are the vacancy and SIA diffusion coefficients, respectively, and $S_{t}$ is the total defect sink strength, taken here to be the same for vacancies and SIA. Thus the creation-destruction balance equation for vacancies is

$$
\mathrm{D}_{\mathrm{v}} \mathrm{X}_{\mathrm{v}} \mathrm{S}_{\mathrm{t}}+\text { recombination rate }=\phi \sigma_{\mathrm{v}}
$$

The recombination rate is $R X_{v} X_{i}$, where $R\left(s^{-1}\right)$ is a recombination factor $\approx 4 \pi r_{r}\left(D_{i}+D_{v}\right)$, where $r_{r}$ is the recombination radius. Without recombination, 


$$
X_{v}=\phi \sigma_{v} / S_{t} D_{v}+X_{v e}
$$

Thus, $X_{v}$ increases linearly with flux, and in the very low flux limit of no irradiation, $X_{v}=X_{v e}$.

The fraction of vacancies $\left(g_{r} \leq 1\right)$ that recombine with SIAs before reaching sinks is proportional to $\mathrm{X}_{\mathrm{i}} \mathrm{X}_{\mathrm{v}}$, or $\mathrm{X}_{\mathrm{v}}{ }^{2}$, since $\mathrm{X}_{\mathrm{i}}$ is proportional to $\mathrm{X}_{\mathrm{v}}$. Hence, recombination rates increase with increasing flux $(\phi)$, since $X_{v}$ also increases, but not in direct proportion. In the limit where recombination dominates, ignoring defect annihilation at sinks,

$$
\mathrm{G}_{\mathrm{v}}=\phi \sigma_{\mathrm{v}}=\mathrm{RX}_{\mathrm{v}} \mathrm{X}_{\mathrm{i}}=\left[\mathrm{D}_{\mathrm{v}} / \mathrm{D}_{\mathrm{i}}\right] \mathrm{RX_{ \textrm {v } } ^ { 2 }}
$$

Thus, $X_{v}$ varies with the square root of flux as

$$
X_{v}=\sqrt{ }\left(\phi \sigma_{v} /\left[\left(D_{v} / D_{i}\right) R\right]\right.
$$

Trapping of vacancies bound to solute atoms also increases recombination and $\mathrm{g}_{\mathrm{r}}$, since this lowers the effective diffusion coefficient $\left(\mathrm{D}_{\mathrm{v}}\right)$ for vacancies compared to alloys that do not contain such traps. Recombination rates also increase with decreasing irradiation temperature $\left(T_{i}\right)$ due to the lower $D_{v}$ and de-trapping rates. Combining Eqs. (2-11) and (2-12),

$$
\mathrm{X}_{\mathrm{v}}=\left(1-\mathrm{g}_{\mathrm{r}}\right) \phi \sigma_{\mathrm{v}} / \mathrm{S}_{\mathrm{t}} \mathrm{D}_{\mathrm{v}}+\mathrm{X}_{\mathrm{ve}}=\left[\mathrm{g}_{\mathrm{s}}\left(\phi, \mathrm{T}_{\mathrm{i}}, \mathrm{S}_{\mathrm{t}}, \mathrm{X}_{\mathrm{t}}, \mathrm{H}_{\mathrm{t}}\right) \phi \sigma_{\mathrm{v}}\right] /\left[\mathrm{S}_{\mathrm{t}} \mathrm{D}_{\mathrm{v}}\right]+\mathrm{X}_{\mathrm{ve}}
$$

Here $g_{s}$ is the fraction of vacancies that reach sinks, $\left(g_{s}=1-g_{r} \leq 1\right), X_{t}$ is the concentration of solute traps and $\mathrm{H}_{\mathrm{t}}$ is the corresponding trapping energy. Noting that the self-diffusion coefficient is $\mathrm{D}_{\mathrm{sd}} \approx \mathrm{D}_{\mathrm{v}} \mathrm{X}_{\mathrm{ve}}$, and using Eq. (2-10):

$$
\mathrm{D}^{*} \approx \mathrm{D}_{\mathrm{cu}}\left\{\phi\left[\mathrm{g}_{\mathrm{s}} \sigma_{\mathrm{v}}\right] /\left[\mathrm{S}_{\mathrm{t}} \mathrm{D}_{\mathrm{sd}}\right]+1\right\}=\mathrm{K} \phi+\mathrm{D}_{\mathrm{cu}}
$$

Here $\mathrm{K}\left(\mathrm{m}^{4}\right)$ is the RED factor, defined as

$$
\mathrm{K}=\left[\mathrm{g}_{\mathrm{s}} \sigma_{\mathrm{v}} / \mathrm{S}_{\mathrm{t}}\right]\left[\mathrm{D}_{\mathrm{cu}} / \mathrm{D}_{\mathrm{sd}}\right]
$$

Thus, both $\mathrm{g}_{\mathrm{s}}$ and $\mathrm{K}$ are functions of flux, irradiation temperature, and the total sink strength, $\mathrm{S}_{\mathrm{t}}$, as well as the parameters describing solute vacancy trapping enhanced recombination. In the absence of recombination, $\mathrm{g}_{\mathrm{s}}=1$ and $\mathrm{K}$ is inversely proportional to $\mathrm{S}_{\mathrm{t}}$, which is primarily determined by the dislocation density, and $\mathrm{K}$ is independent of flux if $\phi \mathrm{K}>\mathrm{D}_{\mathrm{cu}}$. The ratio of the $\mathrm{Cu}$ to self-diffusion coefficients term $\left[\mathrm{D}_{\mathrm{cu}} / \mathrm{D}_{\mathrm{sd}}\right]$ is a temperature and composition-dependent property of an alloy, and can be both modeled and measured [13, 43-46]. Both theory and experiment show that $\left[\mathrm{D}_{\mathrm{cu}} / \mathrm{D}_{\mathrm{sd}}\right]$ is greater than 1 , and has relatively large values at low temperatures, due to a high copper-vacancy binding energy [47]. As a result, both $D_{c u}$ and $D^{*}$ may be much larger at low temperatures than estimates based on extrapolations of $\mathrm{D}_{\mathrm{cu}}$ data from high temperature data [13,43-45]. However, the low temperature values of $\left[\mathrm{D}_{\mathrm{cu}} / \mathrm{D}_{\mathrm{sd}}\right]$, and hence, $\mathrm{K}$, are uncertain, even in simple $\mathrm{Fe}-\mathrm{Cu}$ alloys.

Experimental estimates of the maximum $\mathrm{K}_{\mathrm{m}}$ at $290^{\circ} \mathrm{C}$ for $\mathrm{g}_{\mathrm{s}}=1$ (no recombination) range from $\mathrm{K}_{\mathrm{m}} \approx$ $10^{-37}$ to $10^{-38} \mathrm{~m}^{4}$ [13]. An average $\mathrm{K}_{\mathrm{m}}=5 \times 10^{-38} \mathrm{~m}^{4}$ and nominal values of $\mathrm{S}_{\mathrm{t}}=2 \times 10^{14} / \mathrm{m}^{2}$ and $\sigma_{\mathrm{v}}=6 \times$ $10^{-26} \mathrm{~m}^{2} /$ neutron-atom, corresponds to $\left[\mathrm{D}_{\mathrm{cu}} / \mathrm{D}_{\mathrm{sd}}\right] \approx 33$ at $290^{\circ} \mathrm{C}$. Thus, for a typical PWR surveillance capsule flux of $5 \times 10^{14} \mathrm{n} / \mathrm{m}^{2}-\mathrm{s}, \mathrm{D}^{*} \approx 2.5 \times 10^{-23} \mathrm{~m}^{2} / \mathrm{s}$. This value compares to a high estimate (see below) 
of $\mathrm{D}_{\mathrm{cu}}$ at $290^{\circ} \mathrm{C}$ of $\approx 3 \times 10^{-25} \mathrm{~m}^{2} / \mathrm{s}$, or an RED acceleration factor of $\mathrm{D}^{*} \approx 84 \mathrm{D}_{\mathrm{cu}}$. At a typical BWR capsule flux of $2 \times 10^{13} \mathrm{n} / \mathrm{m}^{2}, \mathrm{D}^{*} \approx 4.3 \mathrm{D}_{\mathrm{cu}}$ Thus, at low flux, thermal diffusion may make a significant contribution to precipitation under irradiation.

Before further quantification of $\mathrm{D}^{*}$, we turn to the question of how RED affects $\mathrm{Cu}$ precipitation. For a specified alloy, fluence and irradiation temperature, the precipitate volume fraction, $\mathrm{f}_{\mathrm{p}}$, scales with $\mathrm{D}^{*} \mathrm{t}$ given by

$$
\mathrm{D}^{*} \mathrm{t}=\phi \mathrm{tK}\left(\phi, \mathrm{T}_{\mathrm{i}}\right)+\mathrm{D}_{\mathrm{cu}} \mathrm{t}
$$

Note, that $\left(D^{*} t\right)^{1 / 2}$ is a measure of the average distance that $\mathrm{Cu}$ diffuses under RED and that the initial rate of diffusion controlled growth of the $\mathrm{Cu}$ precipitate volume fraction, $\mathrm{df}_{\mathrm{p}} / \mathrm{dt}$, approximately scales with $(\mathrm{D} * \mathrm{t})^{3 / 2}[13]$.

The $\mathrm{D}^{*} \approx \phi \mathrm{K}$ is much greater than the thermal $\mathrm{D}_{\mathrm{cu}}$ at flux levels characteristic of PWR surveillance and test reactor irradiations. In this case, the effect of flux is controlled by $g_{s}(\phi) \leq 1$. At higher flux, in the recombination-dominated regime, $g_{s}$ is much less than 1 , and $K$ scales as $1 / \sqrt{ } \phi$, since recombination reduces $\mathrm{X}_{\mathrm{v}}$ and hence $\mathrm{K}$. Thus the $\mathrm{D}^{*} \mathrm{t}$, or amount of precipitation, at a specified fluence, also varies as $1 / ل_{\phi}$. At lower dose rates, when recombination is not important, $\mathrm{g}_{\mathrm{s}} \approx 1$, and both $\mathrm{K}$ and $\mathrm{D} * \mathrm{t}$ at a specified fluence are independent of flux. However, at very low flux, $\mathrm{D}_{\mathrm{cu}}$ is similar in magnitude to $\phi \mathrm{K}$, and in the limit, $\mathrm{D}^{*} \approx \mathrm{D}_{\mathrm{cu}}$ is independent of flux. In this case $\mathrm{D}^{*} \mathrm{t} \approx \mathrm{D}_{\mathrm{cu}} \mathrm{t}$ depends only on time, $\mathrm{t}$; since time at a specified fluence depends on $1 / \phi$, the corresponding $D * t$ also varies as $1 / \phi$.

Solutes like Mn and Ni have a positive binding energy with vacancies (that is, the vacancy energy decreases near a solute) $[13,47]$. The resulting trapping of vacancies at solutes increases the recombination rate, hence, decreases $\mathrm{g}_{\mathrm{s}}$. Figure 2.15 schematically illustrates this mechanism, which figuratively makes deeply trapped vacancies "sitting ducks" for recombination with SIA. Analytical expressions for $\mathrm{g}_{\mathrm{s}}$ are available, including treatment of solute trapping [13].

The solid lines in Fig. 2.16 (a) plot $D^{*} t / \phi t=D^{*} / \phi=K$ vs $\phi$, neglecting $D_{c u}$, for a typical set of recombination model parameters at $290^{\circ} \mathrm{C}: \mathrm{S}_{\mathrm{t}}=2 \times 10^{14} / \mathrm{m}^{2} ; \mathrm{H}_{\mathrm{t}}=30 \mathrm{~kJ} / \mathrm{mole} ; \mathrm{X}_{\mathrm{t}}=0.01$ and $0.03 ; \mathrm{D}_{\mathrm{v}}=$ $1.13 \times 10^{-16} \mathrm{~m}^{2} / \mathrm{s}$ and $\left[\mathrm{D}_{\mathrm{cu}} / \mathrm{D}_{\mathrm{sd}}\right]=33$ [13]. These curves flatten out at low flux, so it is important to properly represent the effects of thermal diffusion $\left(\mathrm{D}_{\mathrm{cu}}\right)$ on $\mathrm{D}^{*}$. Unfortunately, estimates of $\mathrm{D}_{\mathrm{cu}}$ are highly uncertain at low temperature. Values of $\mathrm{D}_{\mathrm{cu}}$ based on extrapolation of tracer diffusion data from high temperatures range from $\approx 2 \times 10^{-28}$ and $6 \times 10^{-27} \mathrm{~m}^{2} / \mathrm{s}$ at $290^{\circ} \mathrm{C}$ [43]. Atomistic models predict similar $\mathrm{D}_{\text {cu values between }} \approx 3 \times 10^{-28}$ and $9 \times 10^{-27} \mathrm{~m}^{2} / \mathrm{s}$. Extrapolating the lowest values of measured tracer $\mathrm{D}_{\mathrm{cu}}$ with activation energies from the atomistic models that are also thought to be appropriate for lower temperatures $\left(\approx 222\right.$ to $242 \mathrm{~kJ} / \mathrm{mole}$ ), yields tracer $D_{\text {cu }}$ values between $\approx 8 \times 10^{-27}$ and $5 \times 10^{-26} \mathrm{~m}^{2} / \mathrm{s}$.

However, these tracer diffusion coefficients do not account for either the effects of interstitial and substitutional solutes, or the thermodynamic factor that multiplies the tracer diffusion coefficient to account for chemical diffusion. The thermodynamic factor is approximately given by $\approx-2 \ln \mathrm{X}_{\mathrm{Cue}}$; for $\mathrm{Cu}$ at $290^{\circ} \mathrm{C}$ the factor is estimated to be about 20 . The effect of a solute $\mathrm{j}$ on $\mathrm{D}_{\mathrm{j}}$ is approximately given by the expression $D_{j}\left(X_{j}\right) \approx D_{j}\left(X_{j}=0\right)\left(1+b_{j} X_{j}\right)$. For $C u$ the atomistic model predicts that $b$ increases with decreasing temperature and that $\mathrm{b} \approx 43$ at $290^{\circ} \mathrm{C}$ [44]. However, experimental estimates at high temperature give a much higher $\mathrm{b} \approx 1760$ at $778^{\circ} \mathrm{C}$ [43]. Assuming $\mathrm{b}$ is the geometric mean of these values $\approx 275$ and that a typical total alloy concentration of solutes of $\mathrm{X} \approx 0.04$ behaves like $\mathrm{Cu},(1+\mathrm{bX}) \approx$ 12. The geometric mean of the limiting experimental values of the tracer diffusion coefficient is $\approx 1.1 \times$ $10^{-27} \mathrm{~m}^{2} / \mathrm{s}$; multiplying this value by the product of the thermodynamic and solute $(1+\mathrm{bX})$ factors $\approx 240$ yields a chemical $\mathrm{D}_{\mathrm{cu}} \approx 2.6 \times 10^{-25} \mathrm{~m}^{2} / \mathrm{s}$. 


\section{$q_{0}+0=0$ recombination}
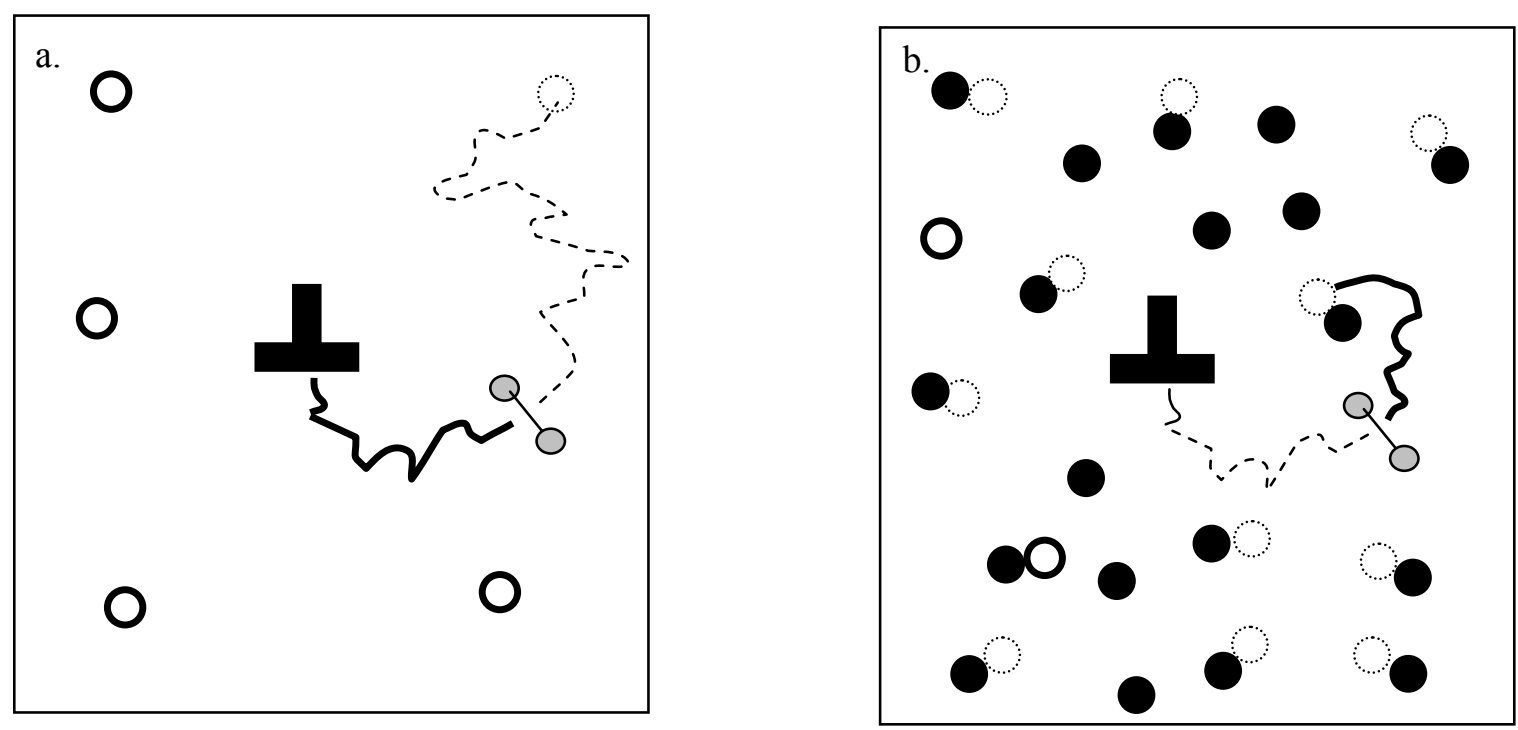

\section{- solute 0 vacancy

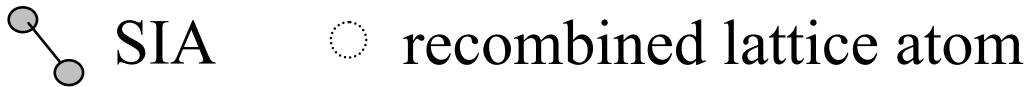

Fig. 2.15. Schematic illustration of solute vacancy trap enhanced recombination. (a) The SIA is more likely to annihilate at sinks like dislocations (the inverted T) when there are no vacancy traps; thus the concentration of mobile vacancies is higher. (b) The SIA is more likely to annihilate at solute (filled circles)-trapped vacancies, shown by the dashed open circles, than sinks, and the concentration of mobile vacancies, shown by the solid line circles, escaping recombination is lower.

Low temperature values of chemical $\mathrm{D}_{\mathrm{cu}}$ can also be indirectly estimated by fitting a diffusion controlled growth model to thermal precipitation data based on measured values of $N_{p}$ and $f_{p}[13,48]$. Analysis of a Fe- $0.9 \mathrm{wt} \% \mathrm{Cu}$ binary alloy thermally aged at $290^{\circ} \mathrm{C}$ for $7200 \mathrm{~h}$ yields a very high estimate of the chemical $D_{\text {cu }}$ of $\approx 5 \times 10^{-24} \mathrm{~m}^{2} / \mathrm{s}$. However, excess quench vacancies may produce a transient enhancement of $\mathrm{D}_{\mathrm{cu}}$ in this case. Nevertheless, $\mathrm{Cu}$ precipitation, and corresponding hardening, have also been observed in slowly cooled complex steels with high $\mathrm{Ni}$ and $\mathrm{Cu}$ contents including alloys aged at $\approx 290^{\circ} \mathrm{C}$ for $\approx$ only $7200 \mathrm{~h}[48,49]$. Thus the thermal aging data for complex alloys also qualitatively indicates a very high value of $D_{c u}$ of order $10^{-24} \mathrm{~m}^{2} / \mathrm{s}$, or possibly greater.

The dashed lines in Fig. 2.16 (a), for $\mathrm{X}_{\mathrm{t}}=0.03$ and 0.01, are based on nominally low and high values of the chemical $D_{c u}$ of $10^{-26}$ and $3 \times 10^{-25} \mathrm{~m}^{2} / \mathrm{s}$, respectively, although possibly lower and, even more likely, higher $\mathrm{D}_{\mathrm{cu}}$ values are certainly possible. However, these nominal low and high values of $\mathrm{D}_{\mathrm{cu}}$ give some sense of the possible role of thermal diffusion in extrapolating embrittlement models to very low flux.

It should be added that, in addition to RED, other radiation damage mechanisms may interact with thermal aging processes leading to accelerated embrittlement rates at very low flux. For example, formation of solute-defect cluster complexes directly in displacement cascades may combine synergistically with long-range thermal diffusion of solutes. The potential for, what might be called, radiation assisted thermal precipitation hardening, under long time-very low flux irradiation conditions, is not understood for the range of alloys and compositions in the TTS database. Thus, these various 

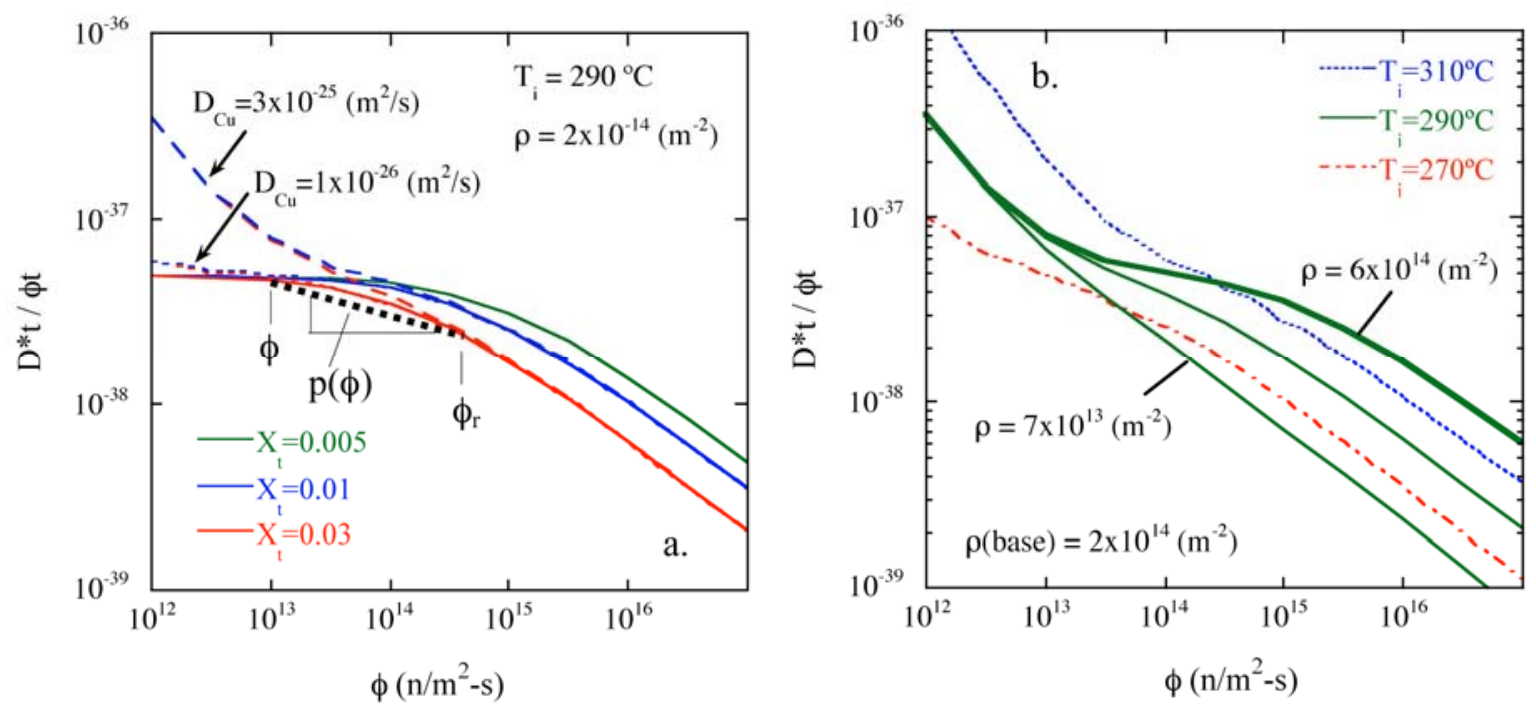

Fig. 2.16. The diffusion scaling parameter, $D^{*} t / \phi t=K+D_{c u} / \phi$, as a function of flux for the solute trap enhanced recombination- $D_{c u}$ model. Larger values of this parameter mean that more precipitation occurs at a given fluence. (a) The base model at $290^{\circ} \mathrm{C}$ with estimated low and high-Cu thermal diffusion coefficients, $D_{\mathrm{cu}}$ (b) The effect of variations in the recombination model parameters for the high $D_{\text {cu. }}$.

considerations suggest that the nominally high $\mathrm{D}_{\mathrm{cu}}$ curves in Fig. 2.16 (a) provides the most reasonable basis to extrapolate experimentally estimated values of $\mathrm{D}^{*}$ to lower flux.

Figure 2.16 (b) shows the effect of variations in the irradiation temperature and sink strength on $D * t / \phi t$ curves assuming the high value of $D_{c u}$. Lower irradiation temperatures $\left(T_{i}\right)$ shift the $D * t / \phi t$ curves down and to the left due to enhanced recombination; higher solute vacancy trap binding energies have the same effect. Higher sink densities $\left(\mathrm{S}_{\mathrm{t}}\right)$ reduce recombination, but also shift the curves down and to the left, since $X_{v}$ is reduced [13]. However, the general shapes of the curves remain similar and the magnitude of $\mathrm{K}$ is still in the range of $\approx 10^{-38}$ to $10^{-37} \mathrm{~m}^{4}$, in the pertinent flux range or slightly higher at $310^{\circ} \mathrm{C}$.

The effect of flux shown in the curves in Fig. 2.16 (a) can also be represented in terms of a flux dependent effective fluence, $\phi \mathrm{t}_{\mathrm{e}}$, as

$$
\phi \mathrm{t}_{\mathrm{e}}=\phi \mathrm{t}\left(\phi_{\mathrm{r}} / \phi\right)^{\mathrm{p}(\phi)}
$$

Here $\phi_{\mathrm{r}}$ is an arbitrary reference flux. Note, $\phi \mathrm{t}_{\mathrm{e}}=\phi \mathrm{t}$ at $\phi_{\mathrm{r}}$. As illustrated in Fig. 2.16 (a), the flux-scaling exponent, $\mathrm{p}(\phi)$, is the average slope of a line connecting $\ln [\mathrm{K}(\phi)]$ with $\ln \left[\mathrm{K}\left(\phi_{\mathrm{r}}\right)\right]$. Hence, $\mathrm{p}$ depends on both $\phi$ and $\phi_{\mathrm{r}}$. Figure 2.17 (a) plots $\mathrm{p}(\phi)$ for $\phi_{\mathrm{r}}=4.4 \times 10^{14} \mathrm{n} / \mathrm{m}^{2}$, which is the reference flux in the TTS model, versus $\phi$ for the $\mathrm{K}(\phi)$ curves shown in Fig. 2.16 (a). The p-curves decrease in going from high to intermediate flux, but go through a minimum whose position depends on $\mathrm{D}_{\mathrm{cu}}$.

Assuming a constant average $\mathrm{p}$ as in Eq. (2-20) provides a simplified formulation for the flux effect that can be readily incorporated into analytical expressions calibrated by fits to the TTS database.

$$
\phi \mathrm{t}_{\mathrm{e}}=\phi \mathrm{t}\left(\phi_{\mathrm{r}} / \phi\right)^{\mathrm{p}}
$$

While the value of $\mathrm{p}$ in Fig. 2.17 (b) explicitly depends on flux (as well as the alloy composition, microstructure and irradiation temperature), the variation between dose rates of $10^{13}$ and $10^{15} \mathrm{n} / \mathrm{m}^{2}-\mathrm{s}$ is not 

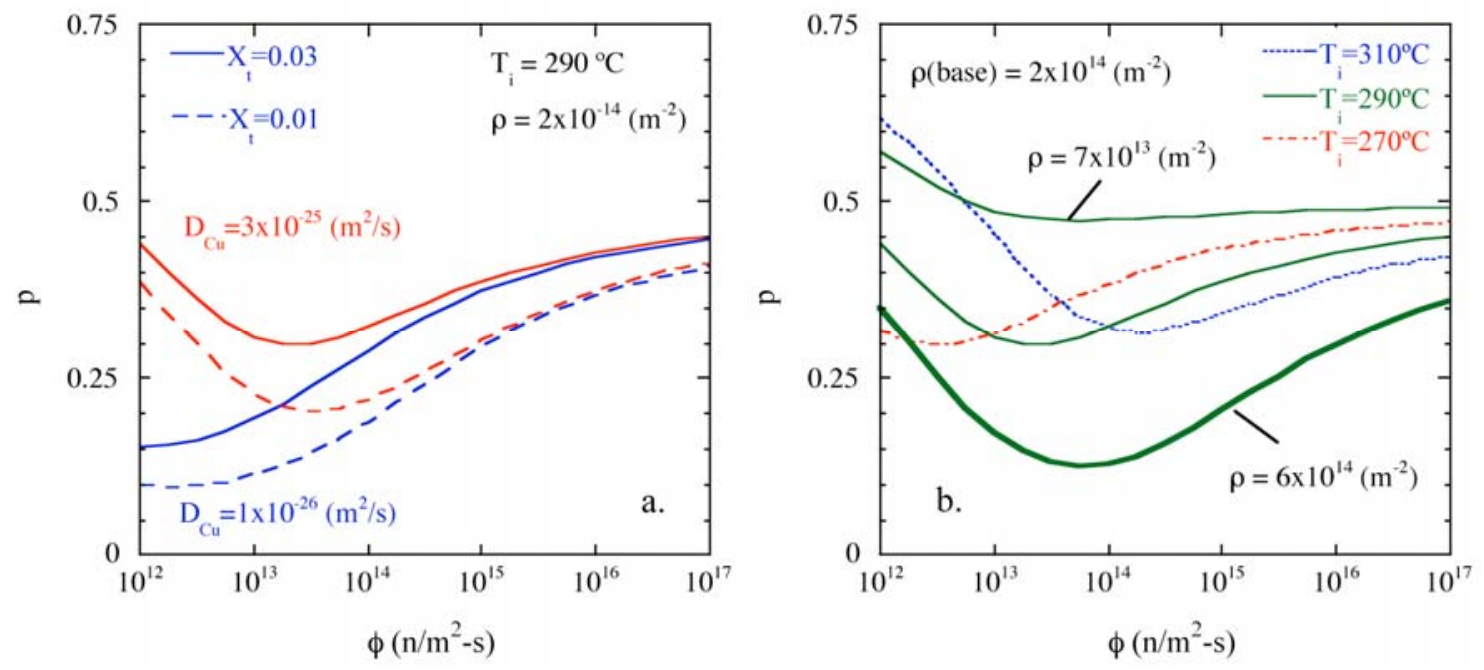

Fig. 2.17. The dose rate effect scaling parameter $p$ for $\phi_{r}=4.45 \times 10^{14} \mathrm{n} / \mathrm{m}^{2}$ versus $\phi$ for the $K(\phi)$ curves shown in Fig. 2.16 (a). (a) The base recombination model parameters and $X_{t}=0.01$ to 0.03 for low and high $D_{C u}$ (b) The effect of variations in the model parameters for $X_{t}=0.03$.

extremely large. For the high $D_{\text {cu }}$ case the average value and range of $p$ are $0.33 \pm 0.05$ and $0.24 \pm 0.07$ for $\mathrm{X}_{\mathrm{t}}=0.03$ and 0.01 , respectively. For the low $\mathrm{D}_{\mathrm{cu}}$ model, the corresponding averages are $0.29 \pm 0.09$ and $0.20 \pm 0.10$. Thus, using an effective average fitted $\mathrm{p}$ to fit and interpolate between embrittlement data at low and high flux, as is done in the TTS model, is a reasonable engineering approximation. Again note that, in the TTS model, the fitted effective flux scaling exponent $\mathrm{p}$ is also the average for a range of alloy compositions and microstructures, as well as irradiation temperatures.

Figure 2.18 shows SANS measurements of the effect of flux on the CRP $f_{p}$, and $r_{p}$ for a split melt model alloy containing $0.4 \mathrm{wt} \% \mathrm{Cu}, 1.4 \mathrm{wt} \% \mathrm{Mn}$ and $1.25 \mathrm{wt} \% \mathrm{Ni}$ irradiated at $290^{\circ} \mathrm{C}$. The diamonds, circles and squares are for fluxes of $\approx 8,3$ and $0.8 \times 10^{15} \mathrm{n} / \mathrm{m}^{2}$-s respectively. The $\mathrm{f}_{\mathrm{p}}$ and $\mathrm{r}_{\mathrm{p}}$ are plotted vs both the actual fluence (Fig. 2.18a and c), $\phi \mathrm{t}$, and the effective fluence (Fig. 2.18b and d), $\phi \mathrm{t}_{\mathrm{e}}$, assuming $\mathrm{p}$ $=0.5$ and $\phi_{\mathrm{r}}=3 \times 10^{15} \mathrm{n} / \mathrm{m}^{2}$-s. The shifts in the precipitation curves to higher fluence with higher flux is clear, while these data collapse onto one curve on the effective fluence, $\phi t_{e}$, scale. This flux effect is also clearly reflected in the corresponding $\Delta \sigma_{\mathrm{y}}$ data shown in Chap. 6. Table 2.1 summarizes the various flux regimes described in this section.

In summary, within the framework of the solute trap enhanced recombination model, the effect of flux on CRP precipitation and hardening is manifested in the pre-saturation regime. Higher flux shifts the CRP hardening curves to higher fluence, as schematically illustrated in Fig. 2.19. The plateau hardening does not depend on the flux. The observed effects of flux can be modeled based on a solute-enhanced recombination mechanism. Recombination models show that dose rate effects depend on flux, irradiation temperature, alloy composition, and microstructure. These conclusions are consistent with analysis of the IVAR database discussed in Chap. 6 and in Ref. [13]. However, flux effects can be approximately incorporated in TTS models using an effective fluence, $\phi \mathrm{t}_{\mathrm{e}}=\phi \mathrm{t}\left(\phi_{\mathrm{r}} / \phi\right)^{\mathrm{p}}$, by fitting a constant average effective flux scaling exponent, $\mathrm{p}$. This provides a reasonable basis to fit low flux BWR and high PWR flux surveillance data, and, more approximately, for interpolating between these extremes in dose rate. Extrapolation to even lower fluxes is uncertain, due to corresponding uncertainties in the thermal diffusion coefficient, $\mathrm{D}_{\mathrm{cu}}$, of $\mathrm{Cu}$. Nevertheless, using a nominally low value of $\mathrm{D}_{\mathrm{cu}}$ to extrapolate to very low fluxes is likely to under predict hardening and embrittlement, which may be further enhanced by synergisms between irradiation and thermal precipitation processes. 

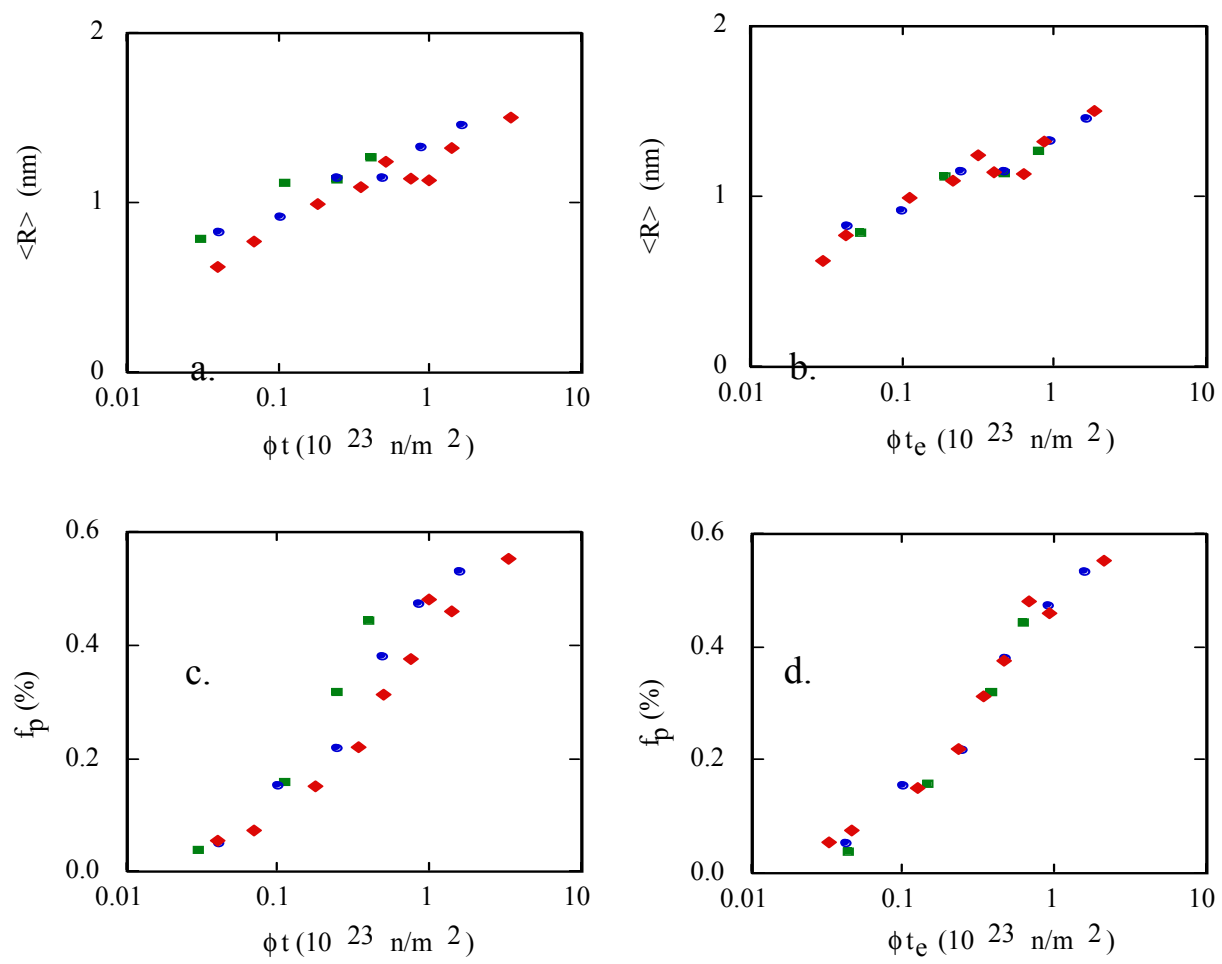

Fig. 2.18 SANS data on $f_{p}$ and $r_{p}$ for a $0.4 \mathrm{wt} \% \mathrm{Cu}, 1.25 \mathrm{wt} \%$ Ni split melt model steel alloys (LD) irradiated at three flux levels between 0.6 to $10 \times 10^{15} \mathrm{n} / \mathrm{m}^{2}-\mathrm{s}$ in IVAR at $290^{\circ} \mathrm{C}$, plotted on both fluence, $\phi t$, and effective fluence, $\phi t_{e}$, scales using a value of $p=0.5$.

Table 2.1. Flux dependence mechanisms, regimes, and scaling laws for $T_{i}=290^{\circ} \mathrm{C}$

\begin{tabular}{|c|c|c|c|}
\hline Dominant Mechanism & $\phi$-regime $\left(\mathrm{n} / \mathrm{m}^{2}-\mathrm{s}\right)$ & $\phi t_{\mathrm{pm} / 2}-\phi$ Scaling $^{\mathrm{a}}$ & Comments ${ }^{\mathrm{b}}$ \\
\hline & & & Depends on low temperature $\mathrm{D}_{\mathrm{cu}}$ \\
\hline $\begin{array}{l}\text { Thermal diffusion } \\
\quad \text { assisted }\end{array}$ & $<\approx 10^{3}$ & $\mathrm{D} * \mathrm{t} @ \phi \mathrm{t} \alpha 1 / \phi$ & $\begin{array}{l}\text { Thermal precipitation is observed in } \\
\text { sensitive model alloys and sensitive steels at } \\
290 \text { to } 350^{\circ} \mathrm{C} \text {. }\end{array}$ \\
\hline & & & $\begin{array}{l}\text { This flux range is pertinent to BWR vessels } \\
\text { and some low flux surveillance capsules. }\end{array}$ \\
\hline Fixed sink & $<10^{14}$ & $\mathrm{D}^{* \mathrm{t}} @ \phi \mathrm{t} \neq \mathrm{f}(\phi)$ & $\begin{array}{l}\text { There may be no purely sink dominated } \\
\text { regime - depends on alloy Ni \& Mn contents, } \\
\text { microstructure and low temperature } D_{c u} \text {. }\end{array}$ \\
\hline & & & $\begin{array}{l}\text { May be pertinent to PWR vessels at lower } \\
\text { flux levels. }\end{array}$ \\
\hline \multirow{2}{*}{$\begin{array}{l}\text { Solute trap } \\
\text { recombination }\end{array}$} & \multirow[b]{2}{*}{$>10^{14}$} & \multirow[b]{2}{*}{$\mathrm{D}^{* \mathrm{t}} @ \phi \mathrm{t} \alpha 1 / \sqrt{ } \phi$} & $\begin{array}{l}\text { Depends on alloy Ni \& Mn contents, } \\
\text { microstructure }\end{array}$ \\
\hline & & & $\begin{array}{l}\text { Pertinent to PWR surveillance capsules and } \\
\text { test reactor irradiations as well as higher flux } \\
\text { regions in PWR vessels. }\end{array}$ \\
\hline
\end{tabular}

${ }^{a}$ In regime dominated by the specified mechanism. Note the flux-scaling varies smoothly in transitions between regimes that are dominated by a specific mechanism. Also note that more than one mechanism may be important in the overlap between some regimes.

${ }^{\mathrm{b}}$ The flux levels at actual surveillance and vessel locations vary greatly, depending on details of the design and fuel assembly arrangements. For perspective, the peak flux in U.S. PWR and BWR RPVs are roughly $10^{15}$ and $10^{13}$ $\mathrm{n} / \mathrm{m}^{2}$-s, respectively. The flux levels are about two to four times higher in corresponding surveillance locations. 


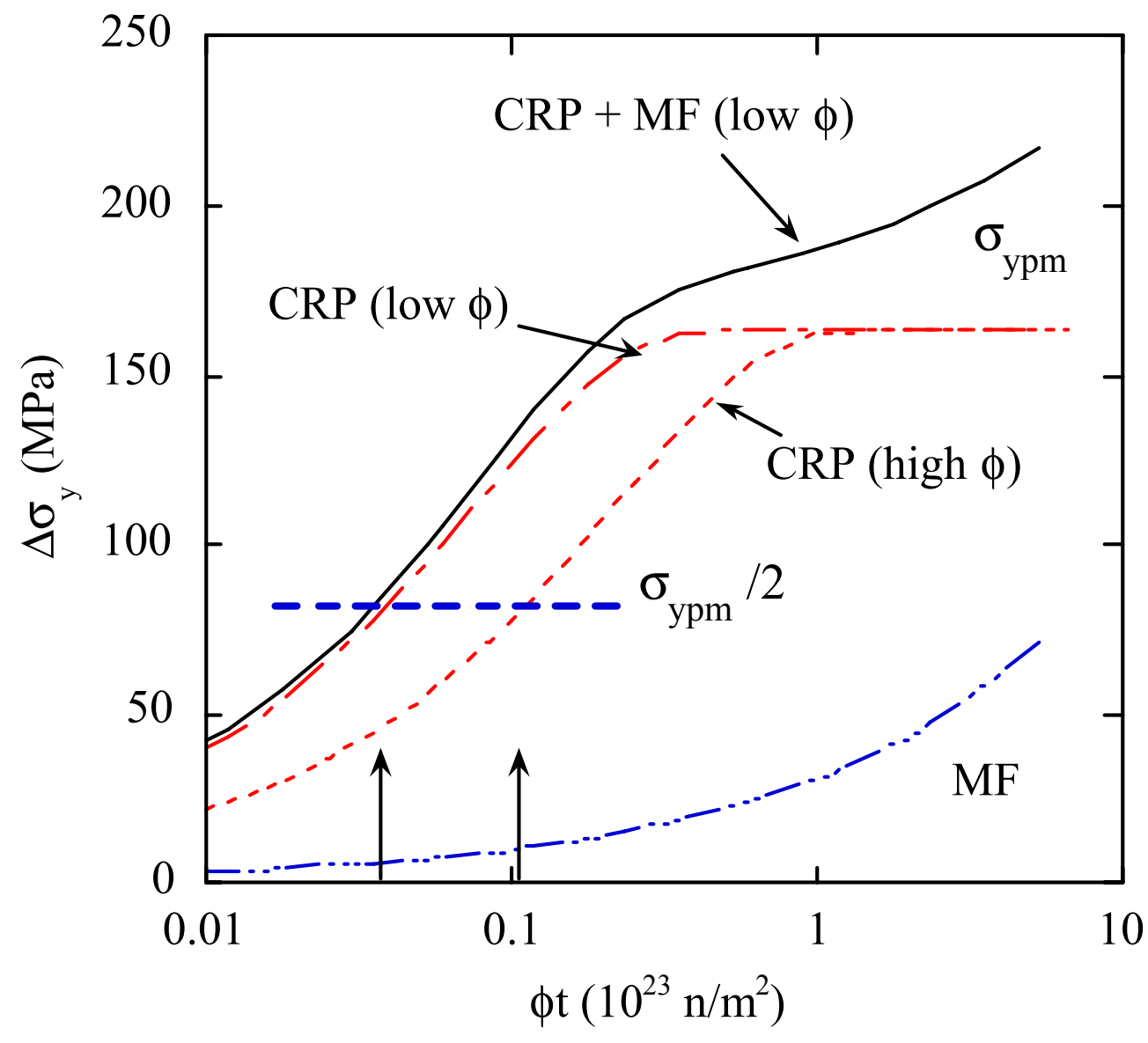

Fig. 2.19. Illustration of the two feature hardening model and the effect of flux, $\phi$, on the fluence, $\phi \mathbf{t}$, dependence of the CRP hardening contribution. The value of $\sigma_{\mathrm{ypm}} / 2$, which marks the fluence at $50 \%$ of the maximum CRP hardening, increases with increasing flux due to recombination.

\subsubsection{The Composition Dependence of CRPs}

We now turn our attention to the composition of the CRPs. At levels more than $\mathrm{Cu} \approx 0.1 \mathrm{wt} \%$, the nucleation of CRPs is primarily driven by the high supersaturations of dissolved $\mathrm{Cu}$. Once nucleated, CRPs grow by RED of solutes. The CRPs are also enriched in $\mathrm{Mn}, \mathrm{Ni}, \mathrm{Si}$, and $\mathrm{P}$, depending on the alloy content of these elements [3,8,9,12,27-33]. ${ }^{*}$ The CRP enrichment in solutes, such as Mn, can be understood and modeled within the framework of both classical thermodynamics [12] and atomistic

\footnotetext{
${ }^{*}$ A number of atom probe (AP) studies suggest that the precipitates contain up to $50 \%$ or more Fe. However, this interpretation is inconsistent with thermodynamic principles and data, as well as the results of other characterization methods such as SANS. Recent local area atom probe (LEAP) studies on Cu bearing IVAR alloys indicate a highly enriched $\mathrm{Cu}$ core (>80 - 90\% solute at sub $\mathrm{nm}$ dimensions) surrounded by shells enriched in $\mathrm{Ni}$, Mn and $\mathrm{Si}$. However, the outer shells may be somewhat ragged or diffuse and may also contain some thermodynamically dictated Fe. For nm scale precipitates almost all the atoms are at or near the interface, thus even small uncertainties in the positions of atoms ( 0.3 to $0.5 \mathrm{~nm}$ ) lead to an artifact of higher than actual precipitate Fe contents. Hence, some of the differences are due to the definition of what constitutes a precipitate. Note, in general, there is much better agreement on the total number of solute atoms in the precipitates which is the most important observation since it is the solutes that control the obstacle strength of the precipitate and provide an overall mass balance for various elements.
} 
simulations [31]. As an example, consider a simple A-B-C system (e.g., Fe-Cu-Mn). The activity coefficient $\Gamma_{\mathrm{ij}}$ is the ratio of the thermodynamic activity of solute $\mathrm{i}$ in phase $\mathrm{j}, \mathrm{a}_{\mathrm{ij}}$, to the corresponding atomic fraction, $\mathrm{X}_{\mathrm{ij}}, \Gamma_{\mathrm{ij}}=\mathrm{a}_{\mathrm{ij}} / \mathrm{X}_{\mathrm{ij}}$. Assume the primary matrix and precipitate phases are $\mathrm{A}$ (matrix, $\mathrm{m}$ ) and $\mathrm{B}$ (precipitate, p) rich (e.g., Fe and $\mathrm{Cu}$ ), respectively, and that $\mathrm{C}$ (e.g., $\mathrm{Mn}$ ) partitions between them at different $X_{\mathrm{Cm}}$ and $\mathrm{X}_{\mathrm{Cp}}$.

If the solute $\mathrm{C}$ has a limited solubility (forms a dilute solution) in the $\mathrm{A}$ matrix phase, $\Gamma_{\mathrm{Cm}}$ is approximately constant (Henry's law) and is given by

$$
\Gamma_{\mathrm{Cm}} \approx \exp \left(\mathrm{H}_{\mathrm{Cm}} / \mathrm{RT}\right)
$$

where $\mathrm{H}_{\mathrm{Cm}}$ is the heat of solution for dissolving $\mathrm{C}$ in the matrix phase (note, small thermal entropy effects have been neglected). For simplicity, assume that the precipitate-phase is an ideal B-C solution, with $\Gamma_{\mathrm{Cp}}=$ 1 (Rault's Law). Equilibrium requires that $\mathrm{a}_{\mathrm{Cp}}=\mathrm{a}_{\mathrm{Cm}}$, thus

$$
\mathrm{X}_{\mathrm{Cp}} / \mathrm{X}_{\mathrm{Cm}}=\Gamma_{\mathrm{Cm}} / \Gamma_{\mathrm{Cp}}=\exp \left(\mathrm{H}_{\mathrm{Cm}} / \mathrm{RT}\right)
$$

For example, $\mathrm{X}_{\mathrm{Cp}} / \mathrm{X}_{\mathrm{Cm}}=10$ at $290^{\circ} \mathrm{C}$ corresponds to $\mathrm{H}_{\mathrm{Cm}}=10.8 \mathrm{~kJ} / \mathrm{mole}$. Equation (2-22) also shows that solute enrichment in the precipitate phase decreases with increasing temperature.

The thermodynamics of real RPV alloys are more complex than in this simple example, and evaluation of CRP compositions requires the use of empirical thermodynamic parameters that can be found in the literature [27,31]. For example, in the Fe-Cu-Mn case, $\Gamma_{\mathrm{Mnp}}$ is less than $\Gamma_{\mathrm{Mnm}}$; thus $\mathrm{X}_{\mathrm{Mnp}} / \mathrm{X}_{\mathrm{Mnm}}$ $>1$. At $290^{\circ} \mathrm{C}$ and $\mathrm{X}_{\mathrm{MnFe}}=0.016, \mathrm{X}_{\mathrm{Mnp}} / \mathrm{X}_{\mathrm{Mnm}} \approx 12$; thus, $\mathrm{X}_{\mathrm{Mnp}} \approx 0.2$ and $\mathrm{X}_{\mathrm{Cup}} \approx 0.8$. In other words, if initially pure $\mathrm{Cu}$ precipitates in a Fe-Mn matrix are to approach equilibrium, Mn must flow out of the Ferich matrix phase and into the Cu-rich precipitate phase until $\mathrm{a}_{\mathrm{Mnm}}=\mathrm{a}_{\mathrm{Mnp}}$.

As schematically illustrated in Fig. 2.20, these basic thermodynamic and atomistic bonding concepts can be extended to treating additional solutes. Strong Mn-Ni, Cu-P, and Ni-Si interactions lead to the coenrichment of these elements in the CRPs along with Mn. If the Mn plus Ni content of the precipitates exceeds that of $\mathrm{Cu}$ they are called manganese-nickel rich precipitates (MNPs). These models also treat the effects of the CRP-Fe matrix interface excess free energy, which scales as $1 / r_{p}$, which is important at nanometer size scales. Higher $\mathrm{Ni}, \mathrm{Mn}, \mathrm{Si}$, and $\mathrm{P}$ decrease the precipitate-matrix interface energy, resulting in additional non-equilibrium solute enrichment and increasing the nucleation rates and number densities of the CRPs.

However, the major effect of solutes is to increase the volume fraction of precipitates, $f_{p}$. Thus, $f_{p}=$ $\mathrm{f}_{\text {Cup }} / \mathrm{X}_{\text {Cup }}$, where $\mathrm{f}_{\text {Cup }}$ is the volume fraction of precipitated $\mathrm{Cu}$ and $\mathrm{X}_{\text {Cup }}$ is the fraction of $\mathrm{Cu}$ in the precipitates. Since almost all the copper eventually precipitates, the maximum $\mathrm{f}_{\mathrm{pm}}$ can be estimated from the fraction of $\mathrm{Cu}$ initially in solution in the matrix $\mathrm{X}_{\mathrm{Cu}}$, as $\mathrm{f}_{\mathrm{p}} \approx\left(\mathrm{X}_{\mathrm{Cu}}-\mathrm{X}_{\mathrm{Cur}}\right) / \mathrm{X}_{\mathrm{Cup}}$, where $\mathrm{X}_{\mathrm{Cur}}$ is the residual $\mathrm{Cu}$ in solution in local equilibrium at the precipitate of radius $\mathrm{r}_{\mathrm{p}}$. The residual $\mathrm{Cu}, \mathrm{X}_{\mathrm{Cur}}$, is greater than $\mathrm{X}_{\mathrm{Cue}}$ due to three sources of excess free energy in precipitates relative to bulk $\mathrm{Cu}$ : 1) the excess free energy due to the precipitate-matrix interface energy $\left(\gamma_{\mathrm{pm}}\right)$, which is important for nanometer-size scales (the Gibbs Thompson effect); 2) the higher energy of the bcc coherent $\mathrm{Cu}$ precipitates versus the equilibrium fcc phase (see Fig. 2.14); and 3) the coherency strain energy associated with a lattice parameter mismatch between bcc $\mathrm{Cu}$ and $\mathrm{Fe}$. The $\mathrm{X}_{\mathrm{Cur}}$ can be estimated as

$$
\mathrm{X}_{\text {Cur }}=\mathrm{X}_{\text {Cue }}(\mathrm{bcc}) \exp \left(\left[\left(2 \gamma_{\mathrm{pm}} / \mathrm{r}_{\mathrm{p}}+4 \mu \delta^{2}\right) \mathrm{V}_{\mathrm{Cum}}\right] /[\mathrm{RT}]\right)
$$




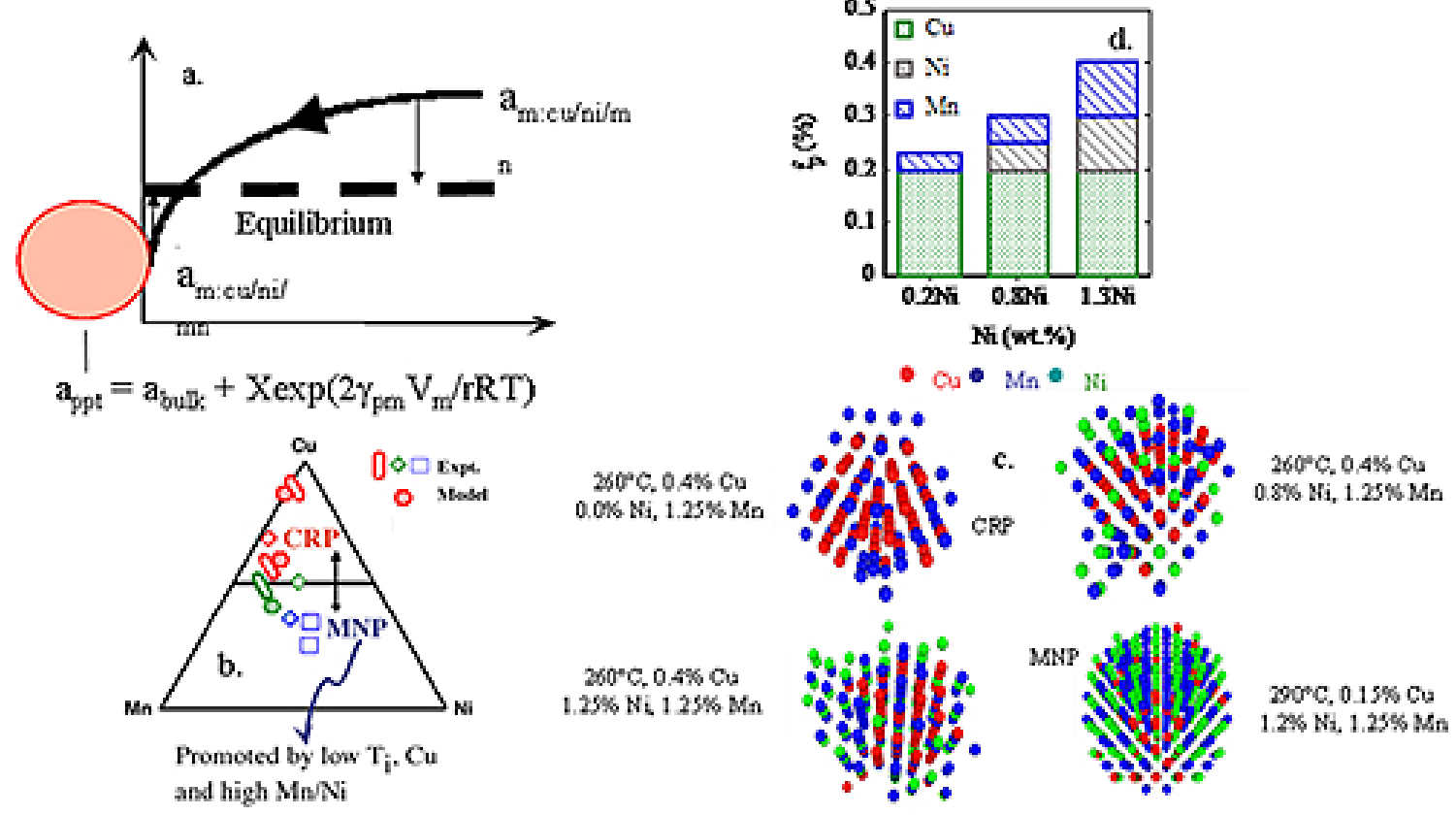

Fig. 2.20. Illustration of the thermodynamics leading to CRP enrichment on Mn and $\mathrm{Ni}$. (a) The $\mathrm{Cu}, \mathrm{Ni}$ and $\mathrm{Mn}$ activities are initially higher in the matrix than in the precipitate. As these elements flow into the CRP their activities decrease in the matrix and increase in the precipitate until equilibrium is established when they are equal in both phases. At small sizes the solute activity in the precipitate must include the contribution of the interface, which also depends on composition (b) A Gibb's triangle indicating the CRP and MNP composition ranges in Cu-bearing alloys. (c) Atomistic Monte Carlo simulations of CRPs and MNPs. (d) Illustration of the $\mathrm{Cu}, \mathrm{Ni}$ and Mn contributions to the CRP volume fraction as a function of alloy composition.

For pure copper precipitates at $290^{\circ} \mathrm{C}$, assuming an interface energy, $\gamma_{\mathrm{pm}} \approx 0.4 \mathrm{~J} / \mathrm{m}^{2}$, a molar volume of $\mathrm{Cu}, \mathrm{V}_{\mathrm{Cum}} \approx 7.1 \times 10^{-6} \mathrm{~m}^{3} /$ mole, a solubility of copper in equilibrium with coherent bcc precipitates, $\mathrm{X}_{\mathrm{Cue}} \approx$ $7.4 \times 10^{-5}, \delta=0.03$ and $\mu=70 \mathrm{GPa}$ (the weighted mean of $\mathrm{Cu}$ and $\mathrm{Fe}$ ), $\mathrm{X}_{\mathrm{Cur}}=\approx 0.042 \mathrm{wt} \%$ for a precipitate with $r_{p}=1 \mathrm{~nm}$ at $290^{\circ} \mathrm{C}$. While $X_{\text {Cur }}$ is fairly small, it is significant for steels with lower copper levels and, as discussed below, results in a threshold copper content, $\mathrm{Cu}_{\min }$, for forming CRPs.

The Mn and Ni enriched CRPs and MNPs can be very large, resulting in large $f_{p}$ and high levels of hardening and embrittlement as illustrated in Fig. 2.20 (d). For alloys with 1.3 to $1.6 \mathrm{wt} \% \mathrm{Mn}$, typical precipitate copper contents are $\mathrm{X}_{\mathrm{Cup}} \approx 0.7$ to 0.9 for low $\mathrm{Ni}(<0.5 \mathrm{wt} \%), \mathrm{X}_{\mathrm{Cup}} \approx 0.5$ to 0.7 for medium Ni ( 0.5 to $0.9 \mathrm{wt} \%$ ), and $\mathrm{X}_{\mathrm{Cup}} \approx 0.25$ to 0.5 for high $\mathrm{Ni}(>0.9 \mathrm{wt} \%)$. Hence, CRPs give way to Mn-Ni(-Si,..) rich precipitates (MNPs) in alloys with high $\mathrm{Ni}$ and $\mathrm{Mn}$ contents. The $\mathrm{Mn}$ and $\mathrm{Ni}$ enrichment in precipitates also increases with decreasing irradiation temperature.

Figure 2.21 shows SANS measurements of the mean radius, number density, and volume fraction of CRPs and MNPs as a function the alloy $\mathrm{Ni}$ and Mn contents, for alloys with $\approx 0.4 \mathrm{wt} \% \mathrm{Cu}$ irradiated at high flux at $290^{\circ} \mathrm{C}$ to $3.4 \times 10^{23} \mathrm{n} / \mathrm{m}^{2}$. The alloys with variations in Ni contain nominal concentrations of about 1.4 to $1.6 \mathrm{wt} \% \mathrm{Mn}$. The alloys with variations in Mn contain nominal concentrations of $0.8 \mathrm{wt} \%$ $\mathrm{Ni}$. The pie charts in the volume fraction plots are estimates of the precipitate composition based on the measured magnetic to nuclear scattering ratio [27,32]. The radius increases and decreases slightly with increasing $\mathrm{Ni}$ and $\mathrm{Mn}$, respectively. However, there are much larger increases in the corresponding number densities and volume fractions in both cases. At high alloy $\mathrm{Ni}$ and $\mathrm{Mn}$ the MNPs replace CRPs. Since the $\mathrm{Mn}$ and $\mathrm{Ni}$ activities decrease in the precipitates with decreasing $\mathrm{Cu}$, highly enriched MNPs are also favored over CRPs at lower Cu levels. Indeed, it has been recently shown that MNPs can even form 

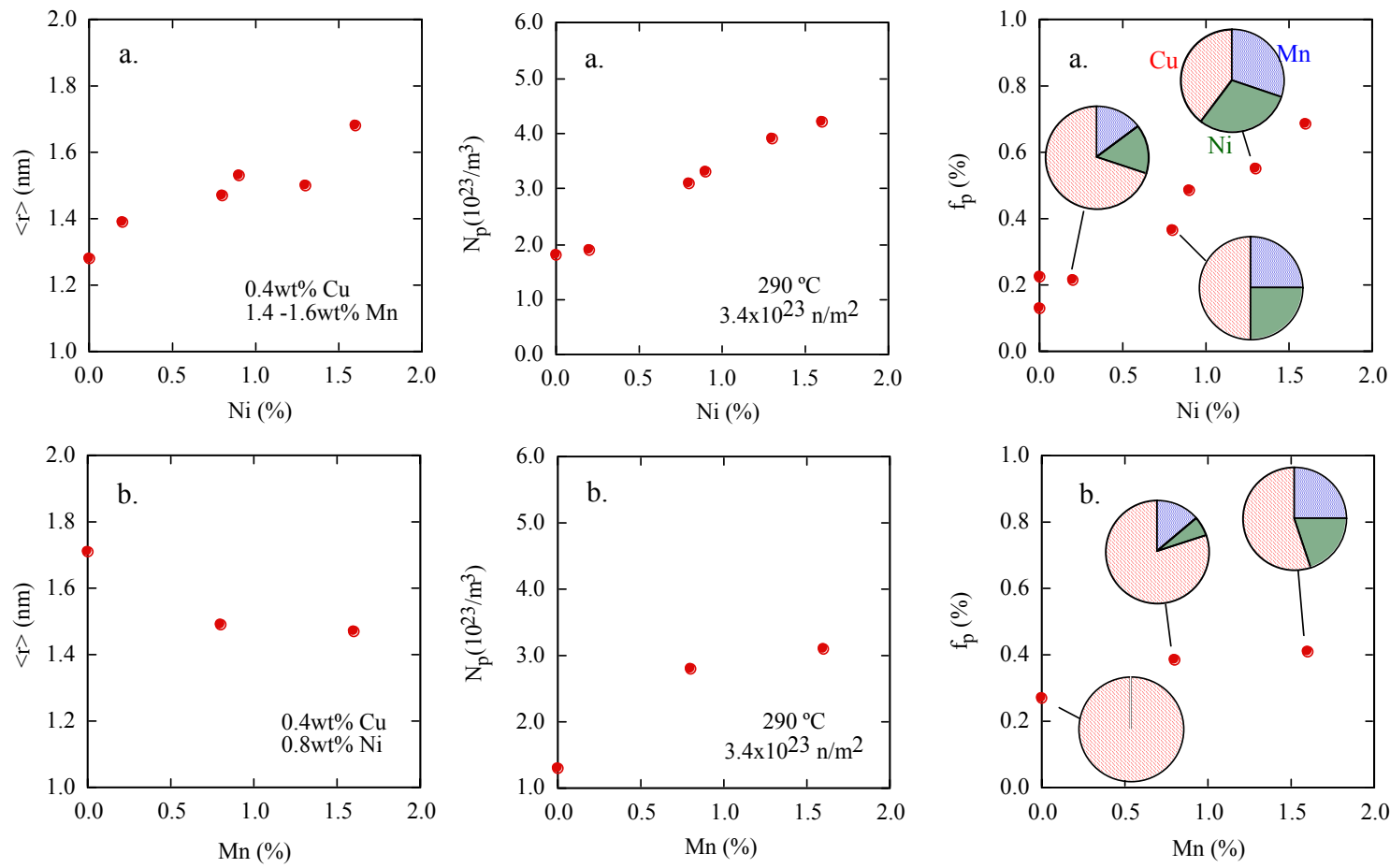

Fig. 2.21. SANS data on $r_{p}, N_{p}$, and $f_{p}$ for a 0.4 wt $\%$ Cu split melt model steels irradiated at high IVAR flux at $290^{\circ} \mathrm{C}$. (a) Effects on Ni variations in alloys with 1.4 to $1.6 \mathrm{wt} \% \mathrm{Mn}$; (b) Effects on Mn variations in alloys with $\approx 0.8 \mathrm{wt} . \% \mathrm{Ni}$. The pie charts in the $\mathrm{f}_{\mathrm{p}}$ plots show the estimated precipitate compositions.

in $\mathrm{Cu}$-free steels [50]. In this case $\mathrm{X}_{\text {Cup }}<$ to $<<0.1$. Since these nearly pure MNPs are slow to nucleate, they have been called "late-blooming" phases. These (and other) experimental observations are consistent with the thermodynamic concepts outlined above.

Thus, large volume fractions of CRPs and MNPs can form under irradiation. Fortunately, preprecipitation during stress relief heat treatments at around $600^{\circ} \mathrm{C}$ limits the maximum dissolved copper prior to irradiation at start-of-life, to an effective $\mathrm{Cu}_{\max }$, that may be lower than the bulk alloy copper content if the latter exceeds the solubility limit. An extensive study of pre-precipitation kinetics [4] yielded estimates of $\mathrm{Cu}_{\max } \approx 0.25 \pm 0.05$ wt $\%$ in medium $\mathrm{Ni}(\mathrm{Ni} \approx 0.8$ wt. $\%$ ) steels. This start-of-life copper increases to $\mathrm{Cu}_{\max } \approx 0.3 \mathrm{wt} \%$, or slightly more, at high $\mathrm{Ni}$ levels $\approx 1.6 \mathrm{wt} \%$. The apparent influence of $\mathrm{Ni}$ on the start-of-life $\mathrm{Cu}_{\max }$ may have both thermodynamic and kinetic origins. Thermodynamically, the bond strength between low solubility $\mathrm{Cu}$ and $\mathrm{Fe}$ is smaller than between high solubility $\mathrm{Ni}$ and both $\mathrm{Fe}$ and $\mathrm{Cu}$. Thus, as Ni replaces $\mathrm{Fe}$ atoms as neighbors of $\mathrm{Cu}$, the latter's solubility increases. The decrease in the matrix activity of $\mathrm{Cu}$ with increasing $\mathrm{Ni}$ also reduces the rate of $\mathrm{Cu}$ preprecipitation. The actual $\mathrm{Cu}_{\max }$ depends on the heat-treatment time-temperature history, as well as the alloy composition. In general $\mathrm{Cu}_{\max }$ may approach, but does not reach the equilibrium solubility limit. These estimates of $\mathrm{Cu}_{\max }$ are consistent with the parameters obtained by fitting the TTS model to the welds with high $\mathrm{Cu}$ and medium and higher $\mathrm{Ni}$ in the surveillance database. 
Test reactor data also show there is a minimum $\mathrm{Cu}$ threshold for forming CRPs, $\mathrm{Cu}_{\min }$, between $\approx$ 0.06 and 0.09 wt.\% $\mathrm{Cu}[3,5,8]$. This can be simply understood based on Eq. 2-23 and conservation of $\mathrm{Cu}$ requiring that

$$
\mathrm{f}_{\mathrm{p}}+\mathrm{X}_{\mathrm{cur}}=\mathrm{X}_{\mathrm{cu}}
$$

A given $\mathrm{N}_{\mathrm{p}}$ and $\mathrm{r}_{\mathrm{p}}$ specify the precipitate $\mathrm{f}_{\mathrm{p}}$ and $\mathrm{X}_{\mathrm{Cur}}$, hence, the initial dissolved $\mathrm{Cu}, \mathrm{X}_{\mathrm{Cu}}$, that is required to establish the balance in Eq. 2.24. As shown by the unfilled symbols in Fig. 2.22, assuming pure $\mathrm{Cu}$ precipitates and using Eq. 2-23 to determine $X_{\text {Cur }}, X_{C u}$ goes through a minimum as a function of $r_{p}$ that depends on $\mathrm{N}_{\mathrm{p}}$. Figure 2.22 shows that for $\mathrm{N}_{\mathrm{p}}=10^{23} / \mathrm{m}^{3}$ the minimum is greater than the threshold for forming CRPs in the TTS model, $\mathrm{X}_{\mathrm{Cu}} \approx 0.063$ at $\%$, which is equivalent to $0.072 \mathrm{wt} \% \mathrm{Cu}$. The minimum $\mathrm{X}_{\mathrm{Cu}}$ is slightly less than 0.063 at $\%$ for $\mathrm{N}_{\mathrm{p}}=10^{22}$ and $5 \times 10^{22} / \mathrm{m}^{3}$; however, as shown by the filled symbols for the corresponding $\Delta \sigma_{\mathrm{y}}$ curves, the hardening is small in these cases. Further, the homogeneous nucleation rates of $\mathrm{Cu}$ precipitates, as well as heterogeneous nucleation rates on small subcritical $\mathrm{Cu}$ clusters that may form in cascades, also very rapid drop-off very rapidly below $\approx 0.05$ to 0.09 wt. \% $\mathrm{Cu}$. These theoretical considerations are consistent with the minimum $\mathrm{Cu}$ for CRP formation that is in the TTS model of $\mathrm{Cu}=0.072 \mathrm{wt} \%$.

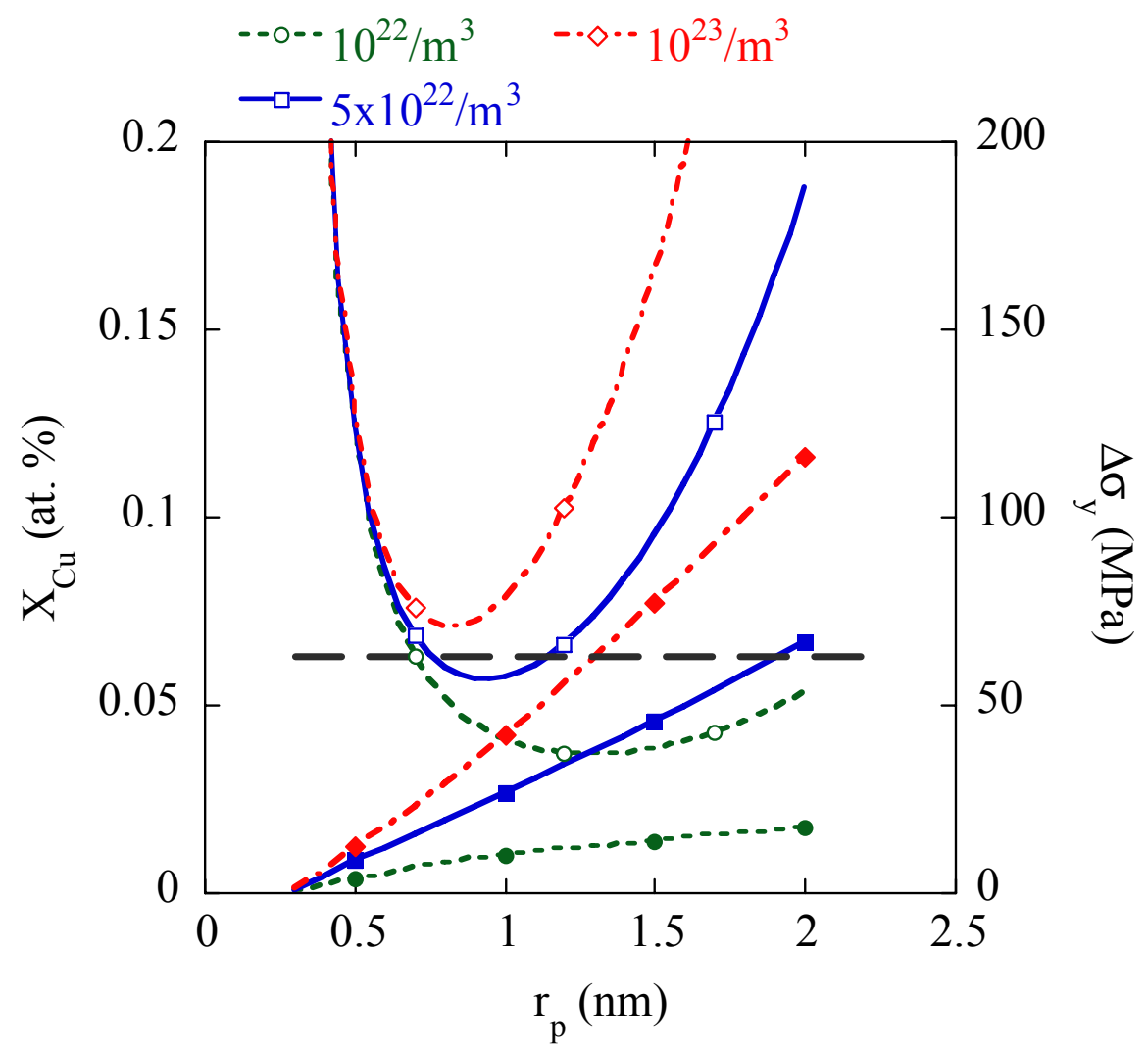

Fig. 2.22. The total $\mathrm{Cu}\left(\mathrm{X}_{\mathrm{Cu}}\right)$ required for a balance between that contained in the precipitates $\left(f_{p}\right)$ plus that dissolved in the Fe matrix in local equilibrium with the bcc phase of $\mathrm{Cu}$, for an interface energy $\gamma_{\mathrm{pm}}=0.4 \mathrm{~J} / \mathrm{m}^{2}$ and lattice coherency mismatch parameter $\delta=0.03$, plotted vs $r_{p}$ for various $N_{p}$ (open symbols) and the corresponding estimated hardening $\Delta \sigma_{y}$ (filled symbols). The horizontal dashed line represents the threshold for forming CRPs in the TTS model. 
In summary, the synergistic interactions between $\mathrm{Cu}-\mathrm{Mn}-\mathrm{Ni}$ (and by extension $\mathrm{P}$ and $\mathrm{Si}$ ) are experimentally fairly well characterized and can be thermodynamically modeled. Most notably, higheralloy $\mathrm{Mn}$ and $\mathrm{Ni}$ increase the precipitate volume fraction, $\mathrm{f}_{\mathrm{p}}$. Pre-precipitation limits the maximum effective dissolved $\mathrm{Cu}$ prior to irradiation, $\mathrm{Cu}_{\max }$, depending on both the stress-relief time and temperature and the alloy composition. Experimental estimates suggest that, following typical stress relief times around $600^{\circ}, \mathrm{Cu}_{\max } \approx 0.25 \pm 0.05 \%$ for medium $\mathrm{Ni}$ steels and $\approx 0.30 \mathrm{wt} \%$, or slightly more, in very high$\mathrm{Ni}$ steels, Both independent experimental observations and theory indicate that a threshold $\mathrm{Cu}$ content in the range of $\mathrm{Cu}_{\min } \approx 0.05$ to $0.09 \mathrm{wt} \%$ is required for CRP formation. These conclusions are broadly consistent with the treatment of alloy composition in the CRP term in the TTS model.

\subsubsection{The Fluence Dependence of CRP Hardening}

Precipitates evolve by long-range diffusion and clustering of $\mathrm{Cu}$ and other solute atoms. Rate theory can also be used to model the evolution of $\mathrm{Cu}$ precipitates as a function of fluence. The most detailed treatment is based on so-called cluster dynamics models (CDMs). CDMs represent the number clusters in each size category, $\mathrm{N}(\mathrm{n})$, from the mobile monomer species $\mathrm{n}=1$, up to a maximum number of atoms $\mathrm{n}=$ $\mathrm{n}_{\max }$, with a set of ordinary differential equations. In the simplest form,

$$
\mathrm{dN}(\mathrm{n}) / \mathrm{dt}=\beta(\mathrm{n}-1) \mathrm{N}(\mathrm{n}-1)+\alpha(\mathrm{n}+1) \mathrm{N}(\mathrm{n}+1)-[\alpha(\mathrm{n})+\beta(\mathrm{n})] \mathrm{N}(\mathrm{n})
$$

In this formulation, clusters with sizes $>1$ are assumed to be immobile. The $\alpha$ and $\beta$ coefficients are the rates at which the cluster of the indicated sizes, $n-1, n$ and $n+1$, emit or absorb a mobile monomer species, respectively. Note slightly different equations are used for the monomer and largest cluster. For diffusion controlled growth of spherical $\mathrm{Cu}$ clusters and using the capillary approximation for the GibbsThompson effect,

$$
\begin{gathered}
\beta(\mathrm{n})=4 \pi \mathrm{r}_{\mathrm{n}} \mathrm{D} * \mathrm{X}_{\mathrm{cum}} / \mathrm{V}_{\mathrm{Cu}} \\
\alpha=\left[4 \pi \mathrm{r}_{\mathrm{n}} \mathrm{D} * \mathrm{X}_{\mathrm{cue}} / \mathrm{V}_{\mathrm{Cu}}\right] \exp \left(2 \gamma_{\mathrm{pm}} \mathrm{V}_{\mathrm{Cu}} / \mathrm{r}_{\mathrm{n}} \mathrm{kT}\right)
\end{gathered}
$$

Here $r_{n}$ is the radius of a cluster of $n C u$ atoms. The set of $n=1$ to $n_{\max }$ coupled equations are integrated over time from an initial condition of supersaturated $\mathrm{Cu}$ in solution, typically with no clusters with $\mathrm{n}>1$. Note, these simplifying assumptions are not necessary, and there are many elaborations of CDMs to treat effects such as heterogeneous nucleation, mobile clusters and interface controlled kinetics.

Figure 2.23 shows a CDM prediction of a $300^{\circ} \mathrm{C}$ irradiation induced evolution of $\mathrm{Cu}$ precipitates in a Fe-0.3 at. \% Cu alloy with overlapping regimes of nucleation $(\mathrm{N})$, growth $(\mathrm{G})$ and coarsening (C) [3]. At low fluence, nucleation dominates, as manifested by a rapid increase in $\mathrm{N}_{\mathrm{p}}$ and $\mathrm{f}_{\mathrm{p}}$, At intermediate fluence, nucleation, growth and coarsening overlap, as manifested by a more rapid increase in $r_{p}$, a peak in $\mathrm{N}_{\mathrm{p}}$ and a continued increase in $\mathrm{f}_{\mathrm{p}}$ until it approaches an approximate plateau, when the matrix $\mathrm{Cu}$ is almost depleted. At still higher fluence, the precipitate evolution is dominated by coarsening, with a slowly decreasing $\mathrm{N}_{\mathrm{p}}$ and increasing $\mathrm{r}_{\mathrm{p}}$. The only adjustable parameter in these calculations is $\mathrm{K}$ factor in the RED diffusion coefficient $\mathrm{D}^{*}$, which sets the absolute fluence scale. The thermodynamic models described in the previous section can be used to properly enrich the precipitates with other solutes, assuming that $\mathrm{Cu}$ clustering controls the basic kinetics. The overall predictions of CDM are in good qualitative agreement with experimentally observed trends and provide a foundation for much simpler models used to fit the TTS database. 


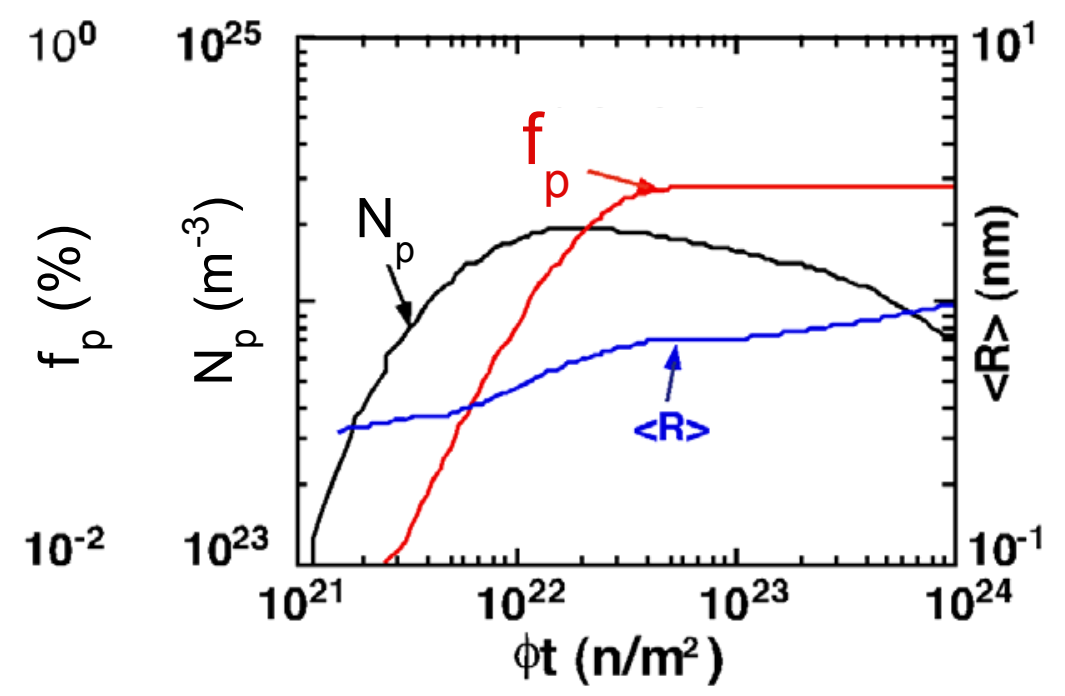

Fig. 2.23. Example of a cluster dynamics model of the nucleation-growth and coarsening $\mathrm{Cu}$ precipitation kinetics for an Fe $0.3 \mathrm{wt} \% \mathrm{Cu}$ alloy irradiated at $300^{\circ} \mathrm{C}$. Nominal thermodynamic parameters for the Fe-Cu system were used. The only adjustable parameter in this model is the radiation enhanced diffusion factor $\mathrm{K}$, which sets the fluence scale.

The factors that control the precipitation processes can be understood on a conceptual basis. There is a free energy activation barrier to CRP nucleation that is very sensitive to the $\mathrm{Cu}$ supersaturation. The CRP nucleation barrier has two apparent effects. First, the incubation fluence, marking the initiation of hardening by $\mathrm{CRPs}$, increases with decreasing $\mathrm{Cu}$, due to correspondingly lower nucleation and growth rates and number densities $\left(\mathrm{N}_{\mathrm{p}}\right)$ [1,13]. Second, below a threshold $\mathrm{Cu}_{\min }$, well-developed CRPs do not form; the threshold is $\mathrm{Cu}_{\min } \approx 0.05$ to $0.09 \mathrm{wt} \% \mathrm{Cu}$ at $\approx 290^{\circ} \mathrm{C}[3,5,13,18]$. The CRP number densities also decrease with increasing irradiation temperature, due to the lower $\mathrm{Cu}$ supersaturation. However, the precipitate number densities increase with higher alloy $\mathrm{Ni}, \mathrm{Mn}, \mathrm{Si}$ and $\mathrm{P}$ contents, due to the reduction of the CRP-Fe matrix interface energy by these solutes [12].

Once nucleated, the CRPs grow by RED of solutes. A standard expression to analytically represent precipitation (and other transformations) kinetics is the so-called Avrami (or Johnson-Mehl) equation, which has a general form $[13,51]$

$$
\mathrm{f}_{\mathrm{p}}(\mathrm{t})=\mathrm{f}_{\mathrm{pm}}\left\{1-\exp \left[-\left(\mathrm{t} / \mathrm{t}_{\mathrm{t}}\right)^{\beta}\right]\right\}
$$

where $f_{p m}$ is the maximum precipitate volume fraction, $t_{t}$ is the time for $f_{p}$ to reach $0.63 f_{p m}$ and $\beta$ is a parameter that depends on the rate controlling precipitation kinetics mechanism. For example, in the case of diffusion-controlled growth of a fixed number density of precipitates from very small size to saturation due to solute depletion, $\beta$ is $3 / 2$. In contrast, if the precipitate interface mobility is rate controlling, $\beta=3$. The maximum precipitation rate at short times is $f_{p}(t)=f_{p m}\left(t / t_{t}\right)^{\beta}$, and $d f_{p} / d t$ decreases at larger times due to depletion of solute from the matrix.

As illustrated by the CDMs, actual kinetics of CRP evolution is much more complex than can be described by any simple analytical expression such as Eq. (2-27). However, by considering $\beta$ to be an effective fit parameter, some of the missing physics can be captured [13]. As shown in Fig. 2.24, $\beta$ sets 


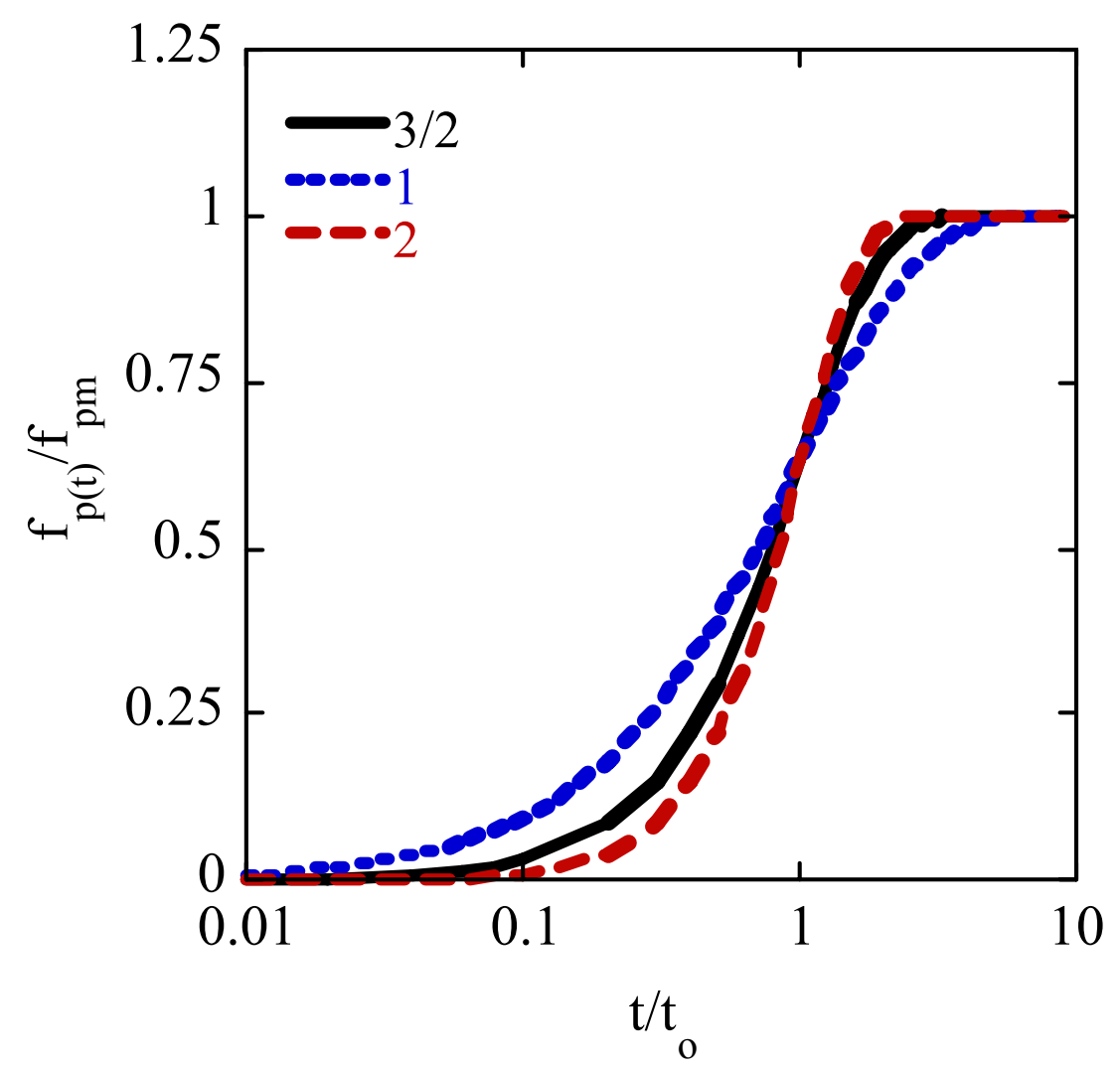

Fig. 2.24 The Avrami equation for different values of $\beta$.

the effective shape of the $f_{p}(t)$ curve between 0 and $f_{p m}$; decreasing $\beta$ increases the time interval for the precipitation.

The corresponding $\phi t_{\mathrm{e}}$ dependence of $\mathrm{f}_{\mathrm{p}}$ under irradiation can be approximately represented by [13]

$$
\mathrm{f}_{\mathrm{p}}\left(\phi \mathrm{t}_{\mathrm{e}}\right) \approx \mathrm{f}_{\mathrm{pm}}\left\{1-\exp \left[\left(-\phi \mathrm{t}_{\mathrm{e}} / \phi \mathrm{t}_{\mathrm{et}}\right)^{\beta}\right]\right\}
$$

In the case of pure diffusion-controlled growth, $\beta=3 / 2$ and $\phi t_{\mathrm{et}}$ can be related to $\mathrm{X}_{\mathrm{Cu}}, \mathrm{X}_{\mathrm{Cup}}, \mathrm{N}_{\mathrm{p}}$, and $\mathrm{D}^{*}$ [13]. Fitted values of $\beta(\approx 1)$ are typically less than $3 / 2$, suggesting that other mechanisms influence CRP growth [13]. For example, this could reflect a slow decrease in the RED coefficient, $\mathrm{D}^{*}$, due to the buildup of additional defect sinks and recombination centers. Assuming that almost all the $\mathrm{Cu}$ initially in solution, $\mathrm{X}_{\mathrm{Cu}}$, precipitates, and accounting for enrichment by other elements, such as $\mathrm{Ni}$ and $\mathrm{Mn}$,

$$
\mathrm{f}_{\mathrm{pm}} \approx \mathrm{X}_{\mathrm{Cu}} / \mathrm{X}_{\mathrm{Cup}}
$$

The effective $\phi t_{\text {et }}$ and $\beta$ can be determined by fitting data for $\mathrm{f}_{\mathrm{p}}$ as a function of the effective fluence at the reference flux, $\mathrm{f}_{\mathrm{p}}\left(\phi \mathrm{t}_{\mathrm{e}}\right)[13]$. For a specified alloy $\phi \mathrm{t}_{\mathrm{e}}$ can be approximated by

$$
\phi \mathrm{t}_{\mathrm{e}}=\phi \mathrm{t}\left(\phi_{\mathrm{r}} / \phi\right)^{\mathrm{p}}
$$


Recall, however, that this simple constant $\mathrm{p}$ scaling is not fully rigorous over a wide range of flux and that $\mathrm{p}$ also depends on the alloy composition and irradiation temperature as well as flux (as discussed in Sect. 2.2.3). Thus, $\mathrm{p}$ must also be considered an effective fitting parameter. Ignoring the detailed effects of the precipitate size in the peak hardening regime and superposition effects, the corresponding CRP hardening, $\Delta \sigma_{\mathrm{yp}}$, is simply given by [13]

$$
\Delta \sigma_{\mathrm{yp}}\left(\phi \mathrm{t}_{\mathrm{e}}\right) \approx \Delta \sigma_{\mathrm{ypm}}\left[\mathrm{f}_{\mathrm{p}}\left(\phi \mathrm{t}_{\mathrm{e}}\right) / \mathrm{f}_{\mathrm{pm}}\right]^{1 / 2}
$$

Thus,

$$
\Delta \sigma_{\mathrm{yp}}\left(\phi \mathrm{t}_{\mathrm{e}}\right)=\Delta \sigma_{\mathrm{ypm}}\left[1-\exp \left(-\phi \mathrm{t}_{\mathrm{e}} / \phi \mathrm{t}_{\mathrm{et}}\right)^{\beta}\right]^{1 / 2}
$$

Here $\Delta \sigma_{\mathrm{ypm}}$ is the maximum, or saturation, CRP hardening. The CRP contribution to the TTS, or TTS , is given by

$$
\operatorname{TTS}_{\mathrm{p}}=\mathrm{C}_{\mathrm{c}} \Delta \sigma_{\mathrm{yp}}
$$

Equations (2-30) to (2-33) represent physically based $\Delta \sigma_{\mathrm{y}}$ and TTS models that can be fit to experimental data. The $\mathrm{p}, \beta, \phi \mathrm{t}_{\mathrm{et}}$, and $\Delta \sigma_{\mathrm{ypm}}$, or $\mathrm{TTS}_{\mathrm{pm}}$, are effective fitting parameters that depend on metallurgical and irradiation variables in a way that can be physically understood and modeled. While these analytical expressions are greatly simplified relative to the complexities of the underlying embrittlement mechanisms, they can empirically reflect at large part of the physics that is not explicitly included when fitted to the TTS database. Hyperbolic tangent and error function (erf) equations can also be used to approximate $\Delta \sigma_{\mathrm{yp}}\left(\phi \mathrm{t}_{\mathrm{e}}\right)$ and $\operatorname{TTS}_{\mathrm{p}}\left(\phi \mathrm{t}_{\mathrm{e}}\right)$.

Based on both the physics of the CRP nucleation and growth mechanisms and a large body of data, any $\Delta \sigma_{\mathrm{yp}}$ or TTS model should reflect the following systematic variable effects:

- The $\Delta \sigma_{\mathrm{yp}}(\phi \mathrm{t})$ curve should manifest both low dose incubation and high dose saturation fluence. A $\ln \left(\Delta \sigma_{\mathrm{yp}}\right)$ versus $\ln (\phi \mathrm{t})$ plot should be sigmoidal, with an approximately linear shape in the transition. If simple diffusion controlled growth is rate controlling, the log-log transition slope is $\approx 3 / 4$.

- Threshold and maximum effective $\mathrm{Cu}$ contents for CRP formation are $\approx 0.06$ to 0.09 and $\approx 0.25$ to $0.30 \mathrm{wt} \%$, respectively.

- The saturation CRP hardening, $\Delta \sigma_{\mathrm{ypm}}$, increases rapidly with higher Ni and, to a lesser extent, Mn.

- The CRP hardening curve is shifted to higher fluence with increasing flux in the PWR and test reactor regime in a way that can be represented by an effective fluence, $\phi \mathrm{t}_{\mathrm{e}}$, that accounts for solute enhanced recombination. The recombination rate decreases with increasing irradiation temperature and decreasing alloy solute content flux. However, thermal $\mathrm{Cu}$ diffusion coefficients may be important at low flux, in and below the BWR regime.

- The CRP hardening curve is shifted to higher fluence with decreasing $\mathrm{Cu}$.

- The width of the CRP hardening curve transition increases with higher Ni.

The effects of $\mathrm{Ni}$ and $\mathrm{Cu}$ on the shape of TTS curves and their and position on the fluence scale are schematically illustrated in Fig. 2.25. 


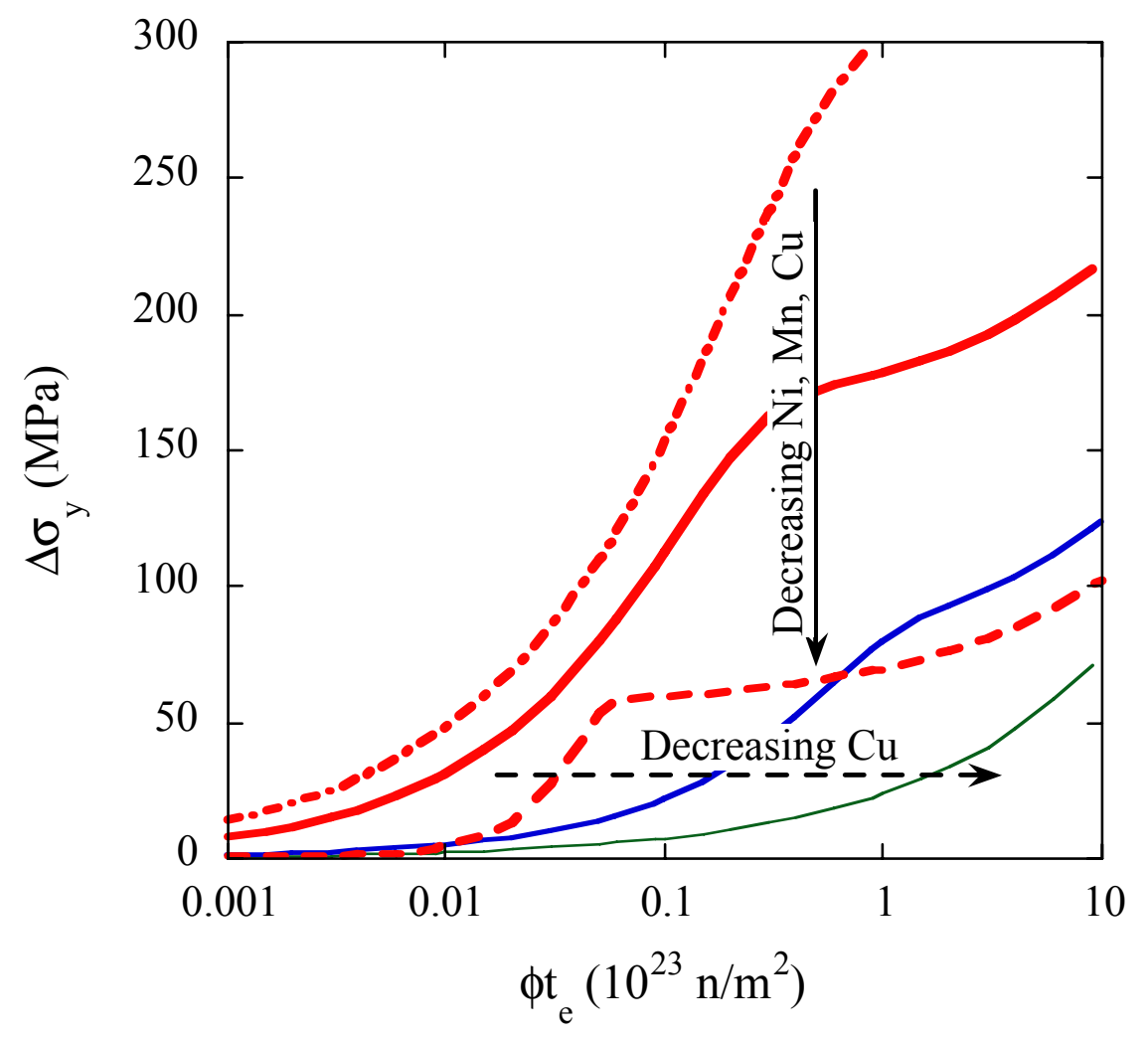

Fig. 2.25 Illustration of the effects of alloy composition on the shape and position of the CRPIMNP effective fluence function.

In summary, the fluence dependence of $\Delta \sigma_{\mathrm{y}}\left(\phi \mathrm{t}_{\mathrm{e}}\right)$ and $\operatorname{TTS}\left(\phi \mathrm{t}_{\mathrm{e}}\right)$ can be modeled by the Avrami-type equation, as well as with roughly equivalent tanh or erf representations. The effective fluence, $\phi \mathrm{t}_{\mathrm{e}}$, depends on the flux and other irradiation and material variables. The simplest flux scaling is given by $\phi t_{\mathrm{e}}$ $=\phi t\left(\phi_{\mathrm{r}} / \phi\right)^{\mathrm{p}}$, where $\phi_{\mathrm{r}}$ is a reference flux, and the fitting parameter $\mathrm{p}$ is assumed to be constant. The model fit parameters include $p$, which controls the flux scaling; $\beta$, which sets the width and shape of the precipitation hardening function; $\phi \mathrm{t}_{\mathrm{et}}$, which provides an effective fluence scale; and $\Delta \sigma_{\mathrm{ypm}}$ or $\mathrm{TTS}_{\mathrm{pm}}$, which are the saturation CRP hardening and transition temperature shift due to precipitation, respectively. The $\mathrm{p}$ and $\beta$ parameters generally depend on the alloy composition, flux and irradiation temperature, but are fitted averages in the TTS model.

\subsubsection{Effects of Irradiation Temperature, $T_{i}$, on CRP Hardening}

It has often been assumed that hardening and embrittlement due to irradiation-induced CRPs do not depend on the irradiation temperature, $T_{i}[2,7,9,16,22]$. However, this is not actually the case $[5,18]$. The irradiation temperature dependence of $\Delta \sigma_{\mathrm{yp}}$ and $\mathrm{TTS}_{\mathrm{p}}$ is due to a number of mechanisms.

- The CRP nucleation rates, hence $\mathrm{N}_{\mathrm{p}}$ and, to a lesser extent $\mathrm{f}_{\mathrm{p}}$, decrease with increasing irradiation temperature due to higher $\mathrm{Cu}$ solubility. The CRPs are larger at higher irradiation temperature and, if 
they grow beyond a radius of about $1.2 \mathrm{~nm}$, their hardening efficiency $\left(\Delta \sigma_{\mathrm{yp}} / \sqrt{\mathrm{p}}\right)$ gradually decreases with increasing $r_{p}$.

- The $\mathrm{f}_{\mathrm{p}}$ also decreases with increasing irradiation temperature, $\mathrm{T}_{\mathrm{i}}$, partly due to the reduction of $\mathrm{Ni}, \mathrm{Mn}$ and $\mathrm{Si}$ in the CRPs.

- At higher fluxes, the previous effects are somewhat offset by decreased recombination at higher irradiation temperature.

The effect of irradiation temperature on the CRP $r_{p}, N_{p}, f_{p}$, and estimated compositions is illustrated by the SANS data in Fig. 2.26 for an $0.4 \mathrm{wt} \% \mathrm{Cu}, 0.8 \mathrm{wt} \% \mathrm{Ni}, 1.4 \mathrm{wt} \% \mathrm{Mn}$ split melt model steel irradiated at medium flux to $1.6 \times 10^{23} \mathrm{n} / \mathrm{m}^{2}$ at 270,290 , and $310^{\circ} \mathrm{C}$. The corresponding effects of irradiation temperature on $\Delta \sigma_{\mathrm{y}}$ are also shown along with a plot of the predicted value based on the SANS data are shown in Figure 2.26d.
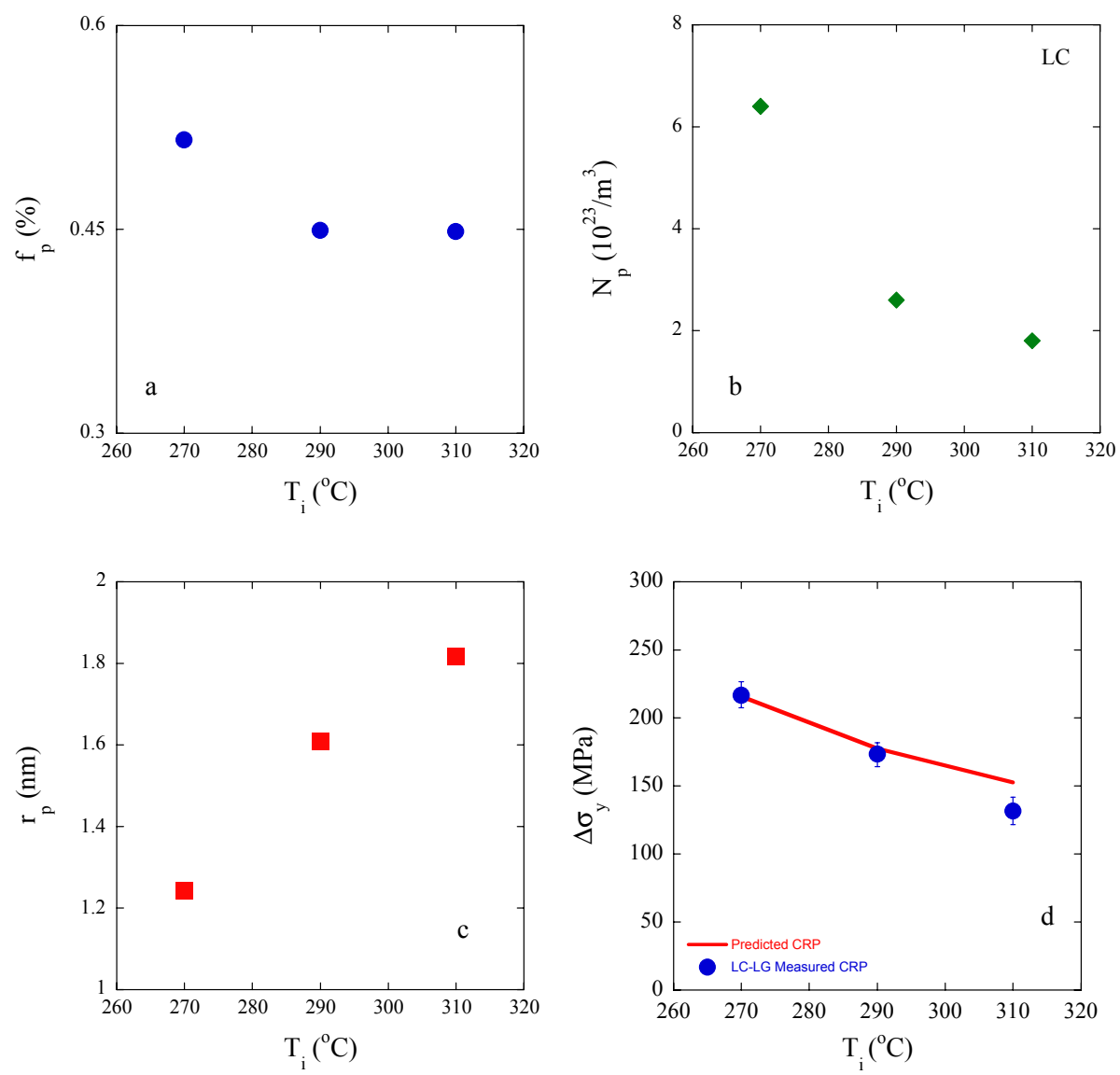

Fig. 2.26. SANS data on $r_{p}, N_{p}$, and $f_{p}$ for a 0.4 wt $\% \mathrm{Cu}, 0.8 \mathrm{wt} \% \mathrm{Ni}, 1.4$ wt \% Mn SMMS irradiated at three temperatures in IVAR. Figure $2.26 \mathrm{~d}$ shows the measured $\Delta \sigma_{y}$ along with predictions of the model and procedures described previously. 
The irradiation temperature dependence for CRP hardening can be modeled by a $f_{T}\left(T_{i}\right)$ function as

$$
\Delta \sigma_{\mathrm{y}}\left(\mathrm{T}_{\mathrm{i}}\right)=\Delta \sigma_{\mathrm{y}}\left(290^{\circ} \mathrm{C}\right)\left[1+\mathrm{C}_{\mathrm{T}}\left(290-\mathrm{T}_{\mathrm{i}}\right)\right]
$$

For higher fluence and intermediate flux irradiations, typical values of $\mathrm{C}_{\mathrm{T}}$ is $\approx 0.015 \pm 0.05 /{ }^{\circ} \mathrm{Cin} \mathrm{Cu}$ bearing alloys, while in lower-Cu steels the corresponding $\mathrm{C}_{\mathrm{T}}$ is $\approx 0.020 \pm 0.05 /{ }^{\circ} \mathrm{C}$. The highest irradiation temperature sensitivity is observed in steels that have $\mathrm{Cu}$ contents that are slightly greater than $\mathrm{Cu}_{\min }$. Equation (2-34) is equivalent to the linear form used in the TTS model for the temperature dependence of the MF term.

In summary, CRP hardening is not athermal, as is often assumed. The irradiation temperature dependence of the hardening can be treated using a linear $\mathrm{C}_{\mathrm{T}}\left(\mathrm{T}_{\mathrm{i}}\right)$ function.

\subsection{Matrix Features}

Matrix features (MFs) are generally defined as the dislocation obstacles that produce hardening in low $\mathrm{Cu}$ $(\leq 0.072 \mathrm{wt} \%)$ alloys. As noted previously, the general category of MFs includes dislocation loops, vacancy-solute clusters, dislocation atmospheres and fine-scale precipitates enriched in $\mathrm{P}$ or $\mathrm{Mn}, \mathrm{Ni}$ and $\mathrm{Si}$. At low P levels and typical low to intermediate flux irradiation conditions the dominant MFs are believed to be Mn-Ni-Si-P-Cu vacancy solute complexes or their remnants. However, at higher P levels alloy phosphide precipitates are observed. The effects of $\mathrm{P}$ and role of phosphide phases are discussed in the next section followed by a discussion of solute vacancy complex MFs.

\subsubsection{Phosphide Phases (PPs)}

It is well established that $\mathrm{P}$ contributes to the hardening and embrittlement of RPV steels $[1-4,9,22,52,53]$. While the amount of dissolved P in RPV alloys is generally very small, $(<0.05 \mathrm{wt} \%)$, it is very insoluble, and remains supersaturated following typical stress-relief heat treatments [12]. Thus, $P$ can undergo accelerated precipitation due to RED to form phosphide phases $[3,4,12,34,54]$. This is illustrated in Fig. 2.27 (a) where the tomographic atom probe map shows phoshide precipitates in a Fe$0.025 \mathrm{wt} \% \mathrm{P}$ binary model alloy irradiated in IVAR at $\approx 290^{\circ} \mathrm{C}$ and high flux to $\phi \mathrm{t} \approx 1.8 \times 10^{23} \mathrm{n} / \mathrm{m}^{2}$ [54].

The contribution of $\mathrm{P}$ to embrittlement is greatly enhanced by $\mathrm{Mn}$ in the TTS model. Jones proposed that the hardening associated with $\mathrm{P}$ is due to $\mathrm{Mn}_{3} \mathrm{P}$ precipitates [52]. Figure 2.27 (b) shows the corresponding APT P and Mn maps for a Fe-0.025\%-1.6 wt \% Mn ternary model alloy. In this case, the P clusters appear to be more diffuse, and significant segregation of both $\mathrm{P}$ and $\mathrm{Mn}$ to dislocations is observed. Notably, Mn doers not appear to be associated with the P clusters in this case, which is a puzzling observation in need of further examination. However, irradiation hardening in the same set of simple model alloys also reflects both a P precipitation and P-Mn interaction: for the $1.6 \mathrm{wt} \% \mathrm{Mn}$ and $0.025 \mathrm{wt} \% \mathrm{P}$ alloy, $\Delta \sigma_{\mathrm{y}}=151 \mathrm{MPa}$; for the Fe- $0.025 \% \mathrm{P}$ alloy, $\Delta \sigma_{\mathrm{y}}=76 \mathrm{MPa}$; for an $\mathrm{Fe} \approx .0125 \mathrm{wt} \% \mathrm{~N}$ alloy, $\Delta \sigma_{\mathrm{y}}=-31 \mathrm{MPa}$; and for a Fe-1.6\% wt \% Mn alloy, $\Delta \sigma_{\mathrm{y}}=46 \mathrm{MPa}$. Note the hardening from $\mathrm{P}$ in these cases may be partly due to formation of dislocation atmospheres, as well as precipitation. The softening in the Fe alloy containing $\mathrm{N}$ is probably the result of the dissolution of fine scale quench defects during long-term irradiation. We have also recently observed unusually low magnetic to nuclear scattering ratios $(<0.5)$ in a SANS study of a $\mathrm{Cu}$ free, $\approx 0.8 \mathrm{wt} \% \mathrm{Ni}, \approx 1.6 \mathrm{wt} \% \mathrm{Mn}, \approx 0.040 \mathrm{wt} \% \mathrm{P}$ split melt model steel that are also consistent with the presence of the $\mathrm{Mn}_{2-3} \mathrm{P}$ precipitates for medium flux irradiations to $\approx 1.6 \times 10^{23} \mathrm{n} / \mathrm{m}^{2}$ at $270^{\circ} \mathrm{C}$ and $290^{\circ} \mathrm{C}$.

The effect of Mn can be theoretically understood based on the strong bonding between $\mathrm{P}$ and various alloying elements, such as $\mathrm{Mo}, \mathrm{Cu}$, and especially $\mathrm{Mn}$. The alloy phosphides reduce $\mathrm{P}$ solubility in the ferrite matrix compared with that in pure Fe $[12,55]$. The stability and bonding strength of a phase is 

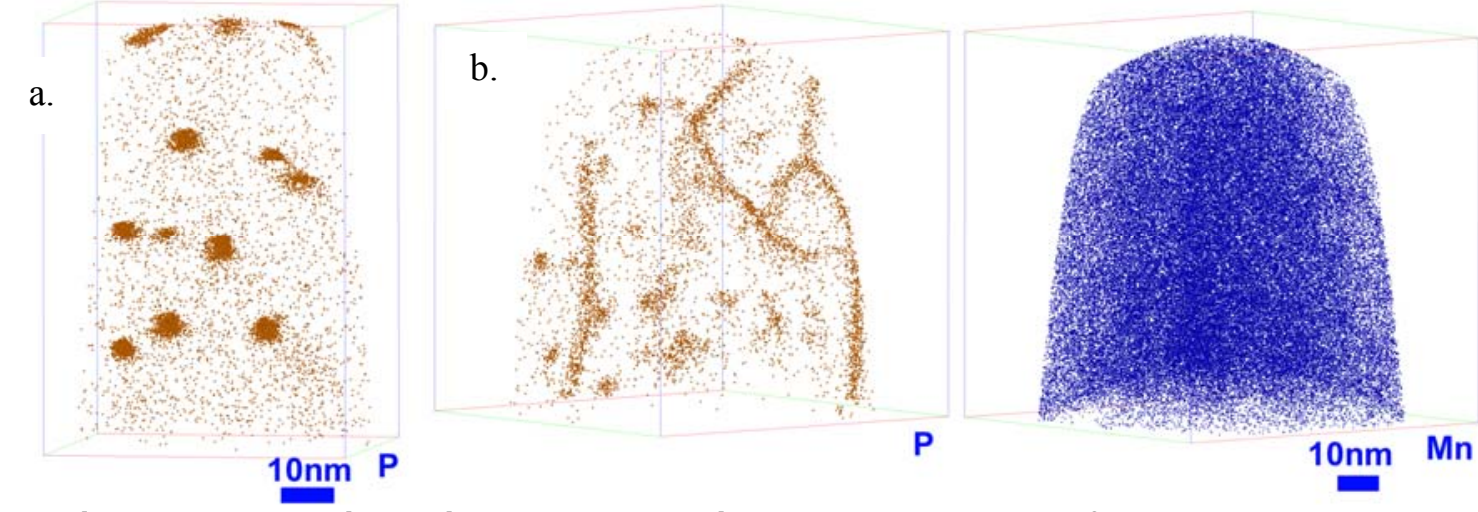

Fig. 2.27. Three-dimensional tomographic atom probe data on for model alloys irradiated in IVAR to $1.77 \times 10^{19} \mathrm{n} / \mathrm{cm}^{2}$ at $290^{\circ} \mathrm{C}$ and high flux. (a) An Fe-0.025 wt \% P alloy; (b) an Fe-0.025 wt \% P-1.6 wt \% Mn alloy. Note the extensive P segregation to dislocations in Fig. 2.27 (b).

reflected in its melting temperature. Thus it is notable that the $\mathrm{Mn}_{2} \mathrm{P}$ phase has the highest melting point in the Mn-P binary system. Assuming a mix of $\mathrm{Mn}_{2} \mathrm{P}$ and $\mathrm{Mn}_{3} \mathrm{P}$ phases, the maximum volume fraction of phosphide precipitates, is roughly three to four times the supersaturated atomic concentration of $\mathrm{P}$. Phosphorous precipitation kinetics are expected to be roughly similar to those for CRPs. Phosphorous also bonds with vacancies, hence it is expected to increase the thermal stability and hardening efficiency of MF vacancy solute (Cu-Ni-Mn-P) cluster complexes, discussed in the next section. Finally, segregation of $\mathrm{P}$ to form dislocation atmospheres by RED would also result in additional hardening.

Thus, the general form of the P-Mn contribution to the TTS MF term for low $\mathrm{Cu}$ steels is consistent with hardening by some combination of fine scale alloy phosphides, vacancy-solute complexes and dislocation atmospheres. Phosphorous may also enhance the formation of dislocation loops. However, the TTS MF P term is not consistent with expectation that phosphide hardening ultimately saturates due to depletion of $\mathrm{P}$ from the matrix.

There are several other complications to treating $\mathrm{P}$ effects on hardening and embrittlement. First, $\mathrm{P}$ also segregates to grain boundaries [34,54], as well as dislocations. Grain boundary P segregation reduces the amount of $\mathrm{P}$ available for precipitation under irradiation. However, accelerated grain boundary $\mathrm{P}$ segregation due to RED can also cause irradiation enhanced temper embrittlement, typically associated with brittle intergranular fracture [54,56]. Fortunately, irradiation enhanced temper embrittlement and intergranular fracture do not appear to be a very significant embrittlement mechanisms in irradiated LWR RPV steels [56,57].

The TTS model also includes an additional contribution of $\mathrm{P}$ to embrittlement in the CRP term. This would seem to be at odds with several early studies showing that the effect of $\mathrm{P}$ decreases with increasing $\mathrm{Cu}[52,53]$, as well as results of the IVAR irradiations, discussed in Chap. 6. The Cu-P synergism may be due to the fact that $\mathrm{P}$ is also enriched in CRPs due to strong P-Mn and P-Cu bonding. At higher $\mathrm{Cu}$ levels the effect of a relatively small amount of $P$ adding to the larger CRP volume fraction $\left(\mathrm{f}_{\mathrm{p}}\right)$ has little effect on net hardening. So the net consequence, in this case, may be a reduced $\Delta \sigma_{\mathrm{y}}$ since less $\mathrm{P}$ is available to form phosphides. Further, the net effect of strength contributions of phosphide precipitates decreases when superposed with a larger CRP contribution.

However, there are other mechanisms that could result in more CRP hardening at higher P. For example, at lower $\mathrm{Cu}$ levels, not too much above the $\mathrm{Cu}$ threshold for $\mathrm{CRP}$ hardening, the proportionately larger $f_{p}$, and especially $N_{p}$, due to the added $P$ would result in a larger increase of the CRP $\Delta \sigma_{y}$, perhaps offsetting any corresponding decrease in phosphide hardening. Further, since P decreases the size and 
increases the number density of CRPs, the CRPs may remain near their peak hardening efficiency radius of $\approx 1 \mathrm{~nm}$, especially at low flux levels typical of surveillance conditions.

In summary, a variety of mechanisms may lead to increases in hardening by $\mathrm{P}$ from both enhanced MF (phosphide precipitates, vacancy-solute complexes and dislocation atmospheres) and CRP (larger volume fractions and higher number densities) contributions. Significant P-Mn interactions are supported by both thermodynamic considerations and experimental observations. These conclusions are generally consistent with the treatment of P in the TTS model. However, phosphorous depletion leading to hardening saturation and a reduced effect of $\mathrm{P}$ due to $\mathrm{Cu}-\mathrm{P}$ interactions are expected in some cases, but are not reflected in the TTS model.

\subsubsection{Defect Solute Cluster Complexes: The Temperature and Flux Dependence of MF Hardening}

Nanometer-scale SIA dislocation loops develop in RPV steels at sufficiently high flux and fluence [35]. However, the significance of SIA loops for the lower flux and fluence irradiation conditions pertinent to RPV embrittlement has not been established (for example, by conclusive electron microscopy observations). In addition to loops and phosphide precipitates, MFs are believed to be primarily vacancysolute $(\mathrm{Mn}, \mathrm{Ni}, \mathrm{Cu}, \mathrm{P}, .$.$) cluster complexes, or their remnants left after the vacancy clusters have fully$ dissolved, forming solute aggregates in their wake [3,12,26-28,58,59]. Hence, MF hardening can be expected to depend on the alloy composition. This has been confirmed by studies showing that hardening in low $\mathrm{Cu}$ steels increases with $\mathrm{Cu}, \mathrm{Ni}, \mathrm{P}$ and $\mathrm{Mn}$, as discussed in Chap. 6. The MFs initially form by local spatially correlated vacancy and solute diffusion during long-term aging of displacement cascades. The MFs, and their remnants, may continue to grow by both the overlap of additional cascades [59] and by absorbing additional solutes and vacancies arriving by long-range RED. These concepts are schematically illustrated in Fig. 2.28. Simple dispersed barrier dislocation theory predicts that the MF are relatively weak obstacles, thus they would produce hardening in an additive term that increases with the square root of fluence.

Hardening in low $\mathrm{Cu}$ RPV steels caused by MF generally decreases with increasing irradiation temperature. The conventional view, reflected in all previous two-feature embrittlement models, has been that MF hardening is independent of flux. A combination of irradiation temperature dependence and flux independence is physically puzzling, since time-temperature kinetics usually go hand in hand. This behavior might be rationalized if an irradiation temperature dependent fraction of primary cascade features transform into MFs that are thermally stable at intermediate to high flux levels. However, to the extent that MFs recover in situ during irradiation, or grow by long range RED, some sensitivity to flux is to be expected. Both in-situ recovery and growth by long-range RED increase with decreasing flux. However, only the latter may be important at very low flux surveillance irradiation conditions. That is, at very low flux almost all the vacancies dissolve anyway; hence, the net hardening increases due to the rearrangement and growth of MF into stronger dislocation obstacles.

Observation of flux effects on MF is also complicated by the fact that the hardening levels are small; hence, it is difficult to establish reliable trends in data that are scattered. Further, it has proven to be difficult to characterize the precise character of MFs in RPV steels. Low-temperature post-irradiation annealing $\left(T_{\text {ann }}=T_{i}\right.$ to $\left.T_{i}+50^{\circ} \mathrm{C}\right)$ recovery measurements have been used to characterize the effects of various irradiation variables on MF hardening $[60,61]$. These studies clearly showed that MF hardening increases with decreasing irradiation temperature and increasing alloy Ni content. More recently, the presence of Mn and $\mathrm{Ni}$ in MF has been shown by combined electrical resistivity-Seebeck coefficient measurements that can be used to track solute (e.g., $\mathrm{Mn}$ and $\mathrm{Ni}$ ) clustering and precipitation [58]. This is illustrated in Fig. 2.29, where $\Delta \sigma_{\mathrm{y}}$ for a $\mathrm{Cu}$-free, $\approx 0.8 \mathrm{wt} \% \mathrm{Ni}, \approx 1.4 \mathrm{wt} \% \mathrm{Mn}, \approx 0.005 \mathrm{wt} \% \mathrm{P}$ alloy (LG) is plotted against measured changes in both the electrical resistivity $(\Delta \rho)$ and the Seebeck coefficient $(\Delta \mathrm{S})$ for a range of flux, fluence, and irradiation temperatures. 
- solutes (Cu)

- vacancies

)

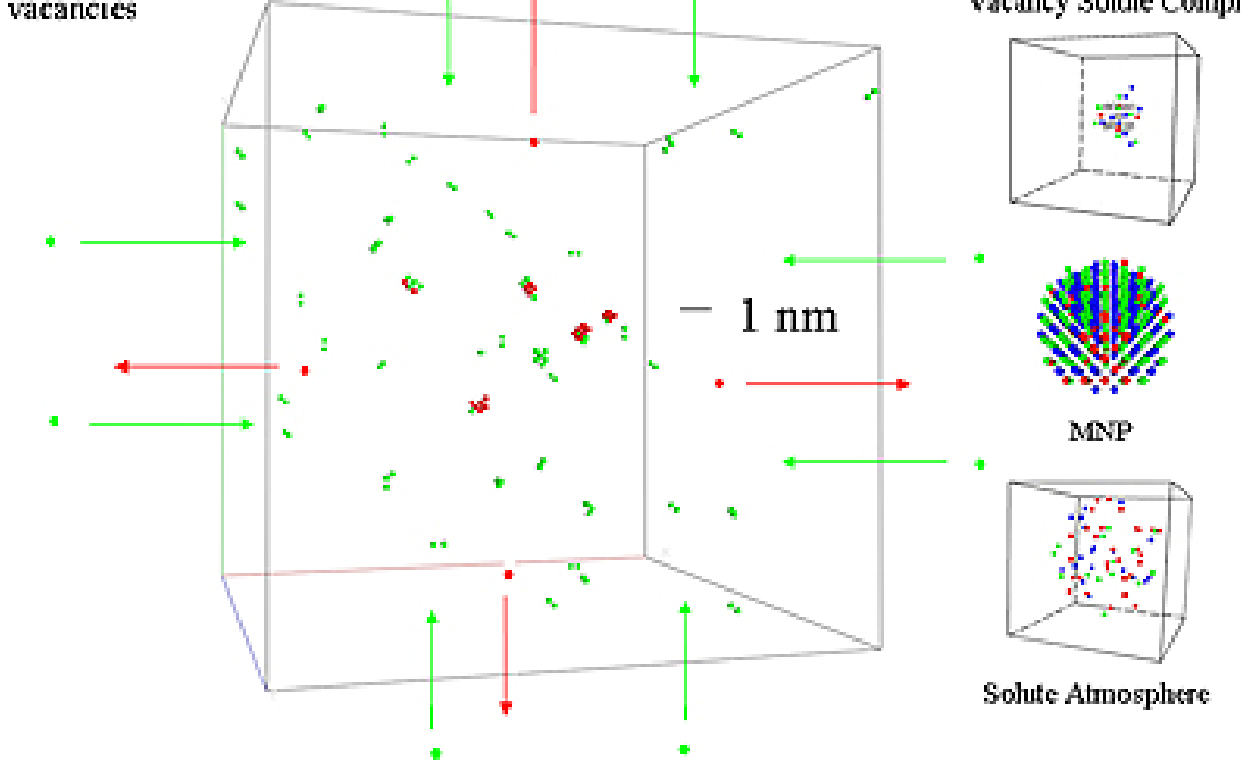

Fig. 2.28. Illustration of vacancy-solute (Cu surrogate) cluster complexes that form in cascades from lattice Monte Carlo simulations and the further evolution of these features by a solute flux from long range RED. The cascade vacancy clusters eventually dissolve and the emitted vacancies migrate to sinks or recombine with SIA. Cascade overlap also plays a role in the MF evolution. The residual features that form in both low-Cu and Cu-bearing steels represent a continuum that includes solute vacancy complexes, solute atmospheres, and MNP - depending on the alloy composition and irradiation conditions.
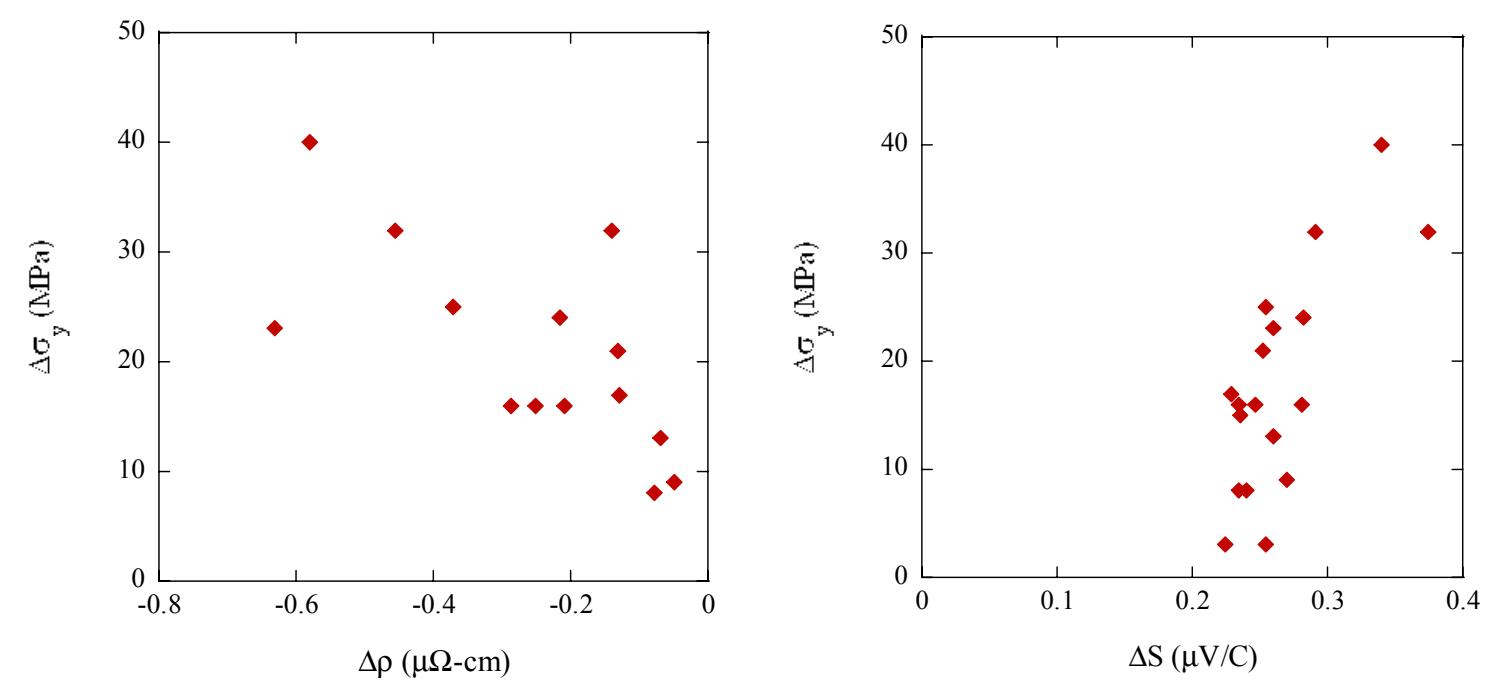

Fig. 2.29. Electrical resistivity and Seebeck coefficient changes in a Cu-free alloy with $0.8 \mathrm{wt} \% \mathrm{Ni}, 1.4 \mathrm{wt} \% \mathrm{Mn}$, and $0.005 \mathrm{wt} \% \mathrm{P}$ irradiated at various fluxes and temperatures over a range of fluence plotted against $\Delta \sigma_{\mathrm{y}}$. These changes can be related to clustering of $\mathrm{Mn}$ and Ni. 
Additional support for the hypothesis that MF hardening may depend on flux is provided by recent experimental studies that confirmed early model-based predictions that, under some conditions, welldefined $\mathrm{Mn}-\mathrm{Ni}(-\mathrm{Si})$ late blooming precipitate phases (LBPs) form in both complex pressure vessel type steels and simple model alloys that have very low $\mathrm{Cu}$ contents or that are $\mathrm{Cu}$ free [50]. For example, LBPs were observed in two high-Ni split melt $\mathrm{Cu}$ free model steels after intermediate flux irradiations at $270^{\circ} \mathrm{C}$ resulting in substantial hardening ranging from 160 to $190 \mathrm{MPa}$. Smaller volume fractions of similar features have been found in recent SANS studies for a variety of irradiation conditions. The LBPs are precipitates and are distinguished from MNPs by the definition that they do not require significant $\mathrm{Cu}$ to form. Taken together, studies of both low $\mathrm{Cu}$ and $\mathrm{Cu}$ bearing steels suggest that CRPs, MNPs, MFs and LBPs represent a continuum of radiation-induced nanoscale features, involving both cascade defect accumulation processes and long-range, dose-rate-dependent RED of solutes leading to cluster growth and precipitation. The effects of flux on RED contributions to MF hardening can also be represented in terms of an effective fluence, $\phi \mathrm{t}_{\mathrm{e}} \approx \phi \mathrm{t}\left(\phi_{\mathrm{r}} / \phi\right)^{\mathrm{p}}$. The IVAR data supporting this conclusion are discussed in Chap. 6.

In summary, MF hardening increases with the square root of fluence and with decreasing irradiation temperature. Higher, $\mathrm{Cu}, \mathrm{P}, \mathrm{Mn}$ and $\mathrm{Ni}$ concentrations increase MF hardening. While the conventional view has been that the MF hardening does not depend on flux, both theoretical consideration and new empirical evidence suggest that this may not be true in all situations, especially at low flux.

\subsection{Summary and Conclusions}

The implications of the overview of embrittlement mechanisms in this chapter can be summarized as follows. Transition temperature shifts are due to irradiation hardening $\left(\Delta \sigma_{\mathrm{y}}\right)$, primarily produced by nanometer-scale precipitates (CRPs), vacancy-solute complexes (MFs) and alloy phosphide precipitates (PPs). A MF plus CRP two-feature model provides an approximate, but reasonable, formulation for simple, parameterized, analytical equations for fitting the TTS surveillance database. It should be noted, however, that these simplified models can not practically be expected to treat all detailed mechanisms of embrittlement. For example, it would be very difficult to formulate simple analytical models that treat the complexities such as: strengthening superposition from multiple hardening features; the effects of the magnitude of hardening and unirradiated properties on the $\Delta \sigma_{y}$-TTS relation; the multiple potential roles of $\mathrm{P}$ and its synergisms with $\mathrm{Cu}$ and $\mathrm{Mn}$; and the detailed kinetics of CRP evolution. In part, the effects of simplifications are mitigated by fitting the TTS models to the surveillance database, which enforces adherence to important data trends, as represented by averaged effective fit parameters. Based on the information presented in this chapter, the following physical trends are expected to appear respectively in the MF and CRP terms of such a simplified TTS model. However, it should be emphasized that all these trends may not be observed in practice, in part due to limitations of both the surveillance database and fitting models.

For the MF term:

- The MF TTS term is expected to increase approximately with the square root of fluence and with decreasing irradiation temperature.

- MF embrittlement in low-Cu and Cu-free steels ( $<0.072 \mathrm{wt} \% \mathrm{Cu})$ increases with the alloy $\mathrm{Mn}, \mathrm{Ni}, \mathrm{P}$ and $\mathrm{Cu}$ contents.

- Large volume fractions of Mn-Ni rich MNPs can form under some conditions in low-Cu and $\mathrm{Cu}$-free steels, and these late-blooming phases produce high levels of hardening.

- The MF contribution in low-Cu steels also appears to depend on flux in a way that can be represented by an effective fluence, $\phi \mathrm{t}_{\mathrm{e}} \approx \phi \mathrm{t}\left(\phi_{\mathrm{r}} / \phi\right)^{\mathrm{p}}$, where $\phi_{\mathrm{r}}$ is a reference flux and $\mathrm{p}$ is a fitting constant.

- The flux dependences of MF and CRP hardening are similar. 
For the CRP term:

- The CRP hardening depends on flux due to a solute vacancy-trap-enhanced recombination mechanism.

- The effect of flux depends on the irradiation temperature and flux itself, as well as the alloy composition and microstructure.

- The flux effect can be treated in terms of an effective fluence, $\phi \mathrm{t}_{\mathrm{e}} \approx \phi \mathrm{t}\left(\phi_{\mathrm{r}} / \phi\right)^{\mathrm{p}}$, where $\phi_{\mathrm{r}}$ is a reference flux and where, in principle, the scaling exponent $\mathrm{p}$ depends on flux, irradiation temperature, and alloy composition and microstructure.

- In fitting the TTS database it is a reasonable approximation to treat flux scaling with an effective average $\mathrm{p}$, where $\mathrm{p}$ is assumed to be a constant and independent of the irradiation and metallurgical variables.

- Higher flux shifts the CRP TTS curves to higher fluence.

- The maximum, plateau CRP TTS increases with the alloy $\mathrm{Cu}, \mathrm{Ni}$, and $\mathrm{Mn}$ contents in a way that can be understood and modeled based on fundamental thermodynamic principles.

- Most notably, higher-alloy Mn and Ni increase the precipitate volume fraction, $\mathrm{f}_{\mathrm{p}}$.

- Pre-precipitation limits the maximum effective dissolved $\mathrm{Cu}$ prior to irradiation, $\mathrm{Cu}_{\max }$, depending on the stress-relief time and temperature and on the alloy composition.

- Best estimates suggest $\mathrm{Cu}_{\max } \approx 0.25 \pm 0.05 \%$ for medium $\mathrm{Ni}$ steels and $\mathrm{Cu}_{\max } \approx 0.30 \mathrm{wt} \%$ (or higher) in high-Ni steels following typical stress relief times around $600^{\circ} \mathrm{C}$.

- A threshold $\mathrm{Cu}$ content for forming CRPs that contribute to hardening is $\approx 0.05$ to $0.09 \mathrm{wt} \%$.

- CRP embrittlement is not athermal and decreases with increasing irradiation temperature.

For $\mathrm{P}$ contributions to the TTS:

- Phosphorous also increases embrittlement in low $\mathrm{Cu}$ steels, by a variety of mechanisms, including forming phosphide precipitates and enhancing MF contributions.

- The hardening kinetics of phosphide precipitates are expected to be qualitatively similar to that for CRPs.

- The phosphorous contribution to hardening would be expected to saturate at high fluence.

- Strong P-Mn interactions are consistent with the formation of stable $\mathrm{Mn}_{2} \mathrm{P}$ and $\mathrm{Mn}_{3} \mathrm{P}$ alloy phosphides;

- Phosphorous also affects CRP hardening by increasing the CRP volume fractions (slightly) and number densities (significantly).

- However, several other studies suggest that the effect of $\mathrm{P}$ decreases with increasing $\mathrm{Cu}$ at and above about $0.1 \mathrm{wt} \%$.

- This negative P-Cu synergism may be due to a combination of strength superposition reductions in the net phosphide contribution to TTS in steels with CRPs, as well as larger reductions in the phosphide TTS itself, compared to the corresponding smaller increases in the CRP contribution.

- Complex P-Cu synergisms are not well understood and may enhance or retard TTS, depending on the combination of other variables.

- It may be necessary to include phosphorous in both the MF and CRP terms or to use a separate phosphorous term in TTS fitting models. 


\subsection{References}

1. Odette, G. R., and G. E. Lucas, Irradiation Embrittlement of Reactor Pressure Vessel Steels, EPRI NP 6114, 1989.

2. Eason, E. D., J. E. Wright and G. R. Odette, Improved Embrittlement Correlations for Reactor Pressure Vessel Steels, NUREG/CR-6551, U.S. Nuclear Regulatory Commission, Washington, DC, 1998.

3. Odette, G. R., and G. E. Lucas, "Recent Progress in Understanding Reactor Pressure Vessel Embrittlement," Radiation Effects and Defects in Solids 144, 189, 1998.

4. Odette, G. R., G. E. Lucas, R. D. Klingensmith, et al., The Effects of Composition and Heat Treatment on Hardening and Embrittlement of Reactor Pressure Vessel Steels, NUREG/ CR-6778, U.S. Nuclear Regulatory Commission, Washington, DC, 2003 .

5. Odette, G. R., T. Yamamoto, R. D. Klingensmith, et al., The Effect of Flux and Irradiation, Temperature on Hardening in RPV Steels, UCSB-NRC-LR-03/2, 2003.

6. Odette, G. R., "On the Dominant Mechanism of Irradiation Embrittlement of Reactor Pressure Vessel Steels," Scripta Met. 17, 1183, 1983.

7. Fisher, S. B., and J. T. Buswell, "A Model For PWR Pressure-Vessel Embrittlement", Int. J. of Pressure Vessels and Piping 27-2, 91(1988.

8. Odette, G. R., B. D. Wirth, D. J. Bacon and N. M. Ghoneim, "Multiscale-Multiphysics Modeling of Radiation-Damaged Materials: Embrittlement of Pressure Vessel Steels," MRS Bulletin 26, 176, 2001 .

9. Williams, T. J., and D. Ellis, “A Mechanistically-Based Model of Irradiation Damage in Low Alloy Submerged Arc Welds," Effects of Radiation on Materials: 20th International Symposium, ASTM STP 1405, ed. S. T. Rosinski et al., American Society for Testing and Materials, West Conshohocken, PA, 8, 2001 .

10. Carter, R.G., and N. Soneda, Workshop on Dose Rate Effects in Reactor Pressure Vessel Materials, Electric Power Research Institute Conference Proceedings, EPRI 1006981, CD, 2002 .

11. Odette, G., R. E. V. Mader, G. E. Lucas, et al., "The Effect of Flux on the Irradiation Hardening and Embrittlement of Pressure Vessel Steels", Effects of Radiation on Materials: 16th International Symposium, ASTM-STP 1175, ed. A.S. Kumar et al., American Society for Testing and Materials, West Conshohocken, PA, 373, 1994.

12. Odette, G. R., "Modeling of Irradiation Embrittlement in Pressure Vessel Steels", Irradiation Effects on Pressure Vessel Steels, IAEA IRRWG-LMNPP98-3, International Atomic Energy Agency, Vienna, Austria, 438, 1998 .

13. Odette,G. R., T. Yamamoto, and R. D. Klingensmith, "On the Effect of Dose Rate on Irradiation Hardening of RPV Steels," Phil. Mag. 85, 2005779.

14. English, C.A., W. L. Server, and S. T. Rosinski, "Critical Review of Through-Wall Attenuation of Mechanical Properties in RPV Steels," Effects of Radiation on Materials: $21^{\text {st }}$ International Symposium, ASTM STP 1447, ed. M. L. Grossbeck et al., American Society for Testing and Materials, West Conshocken, PA, 221, 2004.

15. Langer, R., R. Bartsch and G. Nagel, "Irradiation Behavior of Submerged Arc Welding Materials with Different Copper Content," Effects of Radiation on Materials: 19th International Symposium, ASTM STP 1366, ed. M. L. Hamilton et al., American Society for Testing and Materials, West Conshocken, PA, 2000235. 
16. R. B. Jones and T. J. Williams, "The Temperature Dependence of Radiation Hardening and Embrittlement on Irradiation Temperature," Effects of Radiation on Materials: 17th International Symposium, ASTM STP 1270, ed. D. S. Gelles et al., American Society for Testing and Materials, West Conshocken, PA, 569,1996.

17. Odette, G. R., and G. E. Lucas, "Embrittlement of Nuclear Reactor Pressure Vessels," J. of Metals, 18, July 2001.

18. G. R. Odette, G. E. Lucas and R. D. Klingensmith, "The Influence of Metallurgical Variables on the Temperature Dependence of Irradiation Hardening in Pressure Vessel Steels," Effects of Radiation on Materials: 17th International Symposium, ASTM STP 1270, ed. D. S. Gelles et al., American Society for Testing and Materials, West Conshocken, PA, 606, 1996.

19. McElroy, R. J., and A. L. Lowe, Jr., "Irradiation Embrittlement Modeling of Linde 80 Weld Metals," Effects of Radiation on Materials: 17th International Symposium, ASTM STP 1270, ed. D. S. Gelles et al., American Society for Testing and Materials, West Conshocken, PA, 68, 1996.

20. Lucas, G. E., G. R. Odette, P. M. Lombrozo and J. W. Sheckherd, "Effects of Composition, Microstructure, and Temperature on Irradiation Hardening of Pressure Vessel Steels," Effects of Radiation on Materials: 12th International Symposium, ASTM STP 870, ed. F. A. Garner et al., American Society for Testing and Materials, West Conshocken, PA, 900, 1985.

21. Bolton, C. J., J. T. Buswell, R. B. Jones, R. Mskovic, and R. H. Priest, "The Modeling of Irradiation Embrittlement in Submerged Arc Welds, Effects of Radiation on Materials: 17th International Symposium, ASTM STP 1405, ed. D. S. Gelles et. al. American Society for Testing and Materials, West Conshohocken, PA, 103, 2001.

22. Williams, T. J., D. Ellis, C. A. English, and J. Hyde, "A Model of Irradiation Damage in High Nickel Submerged Arc Welds,” Int. J. of Pressure Vessels and Piping 79, 964, 2002.

23. Odette, G. R., P. M. Lombrozo and R. A. Wullaert, "Relationship Between Irradiation Hardening and Embrittlement of Pressure Vessel Steels," Effects of Radiation on Materials: 12th International Symposium, ASTM STP 870, ed. F. A. Garner et al., American Society for Testing and Materials, West Conshocken, PA, 840, 1985.

24. Stoller, R. E., G. R. Odette and B. D. Wirth, "Primary Damage Formation in bec Iron," J. of Nuc. Mater., 251, 49, 1997.

25. Mansur, L. K., A. D. Brailsford and W. G. Wolfer, "On The Meaning Of Sink Capture Efficiency and Sink Strength for Point-Defects," J. Nuc. Mater., 105, 1982.

26. Wirth, B. D., and G. R. Odette, "Kinetic Lattice Monte Carlo Simulations of Cascade Aging in Iron and Dilute Iron-Copper Alloys," Microstructural Processes in Irradiated Materials, MRS Soc. Symp. Proc. 540, ed. S. J. Zinkle et. al., Materials Research Society, Warrendale, PA, 637, 1999.

27. Wirth, B. D., "On the Character of Nano-Scale Features in Reactor Pressure Vessel Steels Under Neutron Irradiation," PhD. Thesis UC Santa Barbara, 1998.

28. Odette, G. R., and B. D. Wirth, "A Computational Microscopy Study of Nanostructural Evolution in Irradiated Pressure Vessel Steels," J. Nuc. Mat. 251, 157, 1997.

29. Odette, G. R., C. L. Liu, and B. D. Wirth, "On the Composition and Structure of Naonoprecipitates in Irradiated Pressure Vessel Steels," Microstructural Evolution During Irradiation, MRS Symp. Proc. 439, ed. I. M. Robertsonet. al. Materials Research Society, Warrendale, PA, 457, 1997.

30. Odette, G. R., "Radiation Induced Microstructural Evolution in Reactor Pressure Vessel Steels," Microstructural Evolution During Irradiation, Mat Res. Soc. Symp. Proc. 373, ed. I. M. Robertson et. al., Materials Research Society, Warrendale, PA, 137, 1995. 
31. Liu, C. L., G. R. Odette, B. D. Wirth, and G. E. Lucas, "A Lattice Monte Carlo Simulation of Nanophase Compositions and Structures in Irradiated Pressure Vessel Fe-Cu-Ni-Mn-Si Steels," Mat. Science \& Engin. A-Struct. 238-1, 202, 1997.

32. Wirth, B. D., G. R. Odette, W. A. Pavinich, G. E. Lucas, and S. Spooner, "Small Angle Neutron Scattering Study of Linde80 RVSP Welds," Effects of Radiation on Materials: 18th Symposium, ASTM STP 1325, ed. R. K. Nanstad et. al., American Society for Testing and Materials, West Conshohocken, PA, 102, 1999.

33. Odette, G. R., and G. E. Lucas, "The Effect of Nickel in Irradiation Hardening of Pressure Vessel Steels," Effects of Radiation on Materials: 14th International Symposium, ASTM STP 1046, ed. N. H. Packan et al., American Society for Testing and Materials, West Conshocken, PA,, 323, 1990.

34. Miller, M. K., and M. G. Burke, "An APFIM Survey of Grain Boundary Segregation and Precipitation in Irradiated Pressure Vessel Steels," Effects of Radiation on Materials: 16th International Symposium, ASTM STP 1175, ed. A. S. Kumar et al., American Society for Testing and Materials, West Conshocken, PA, 492, 1994.

35. Nicol, A. C., M. L. Jenkins, and M. A. Kirk, "Matrix Damage in Iron," Microstructural Processes in Irradiated Materials-2000, MRS Symp. Proc. 650, ed. G. E. Lucas et. al., Materials Research Society, Warrendale, PA, R1. 3, 2001.

36. Jumel, S., C. Domain, J. Ruste et al., "Simulation of Irradiation Effects in Reactor Pressure Vessel Steels: The Reactor for Virtual Experiments, REVE Project,” J. Test. Eval., 30-1, 37, 2002.

37. Martin, J. W., Micromechanisms in Particle-Hardened Alloys, Cambridge University Press, 1980

38. Bacon, D. J., and Y. N. Osetsky, "Hardening Due to Copper Precipitates in $\alpha$-iron Studied by Atomic-Scale Modeling," J. Nuc. Mat. 329-333, 1233, 2004.

39. Russell, K. C., and L. M. Brown, "A Dispersion Strengthening Model Based on Differing Elastic Moduli Applied to the Iron-Copper System," Acta Met., 20, 969, 1972.

40. Foreman, A. J. E., and M. J. Malkin, "Dislocation Movement Through Random Arrays of Obstacles," Phil. Mag. 14, 911, 1966.

41. Jumel, S., J. C. Van Duysen, J. Ruste and C. Domain, "Interactions Between Dislocations and Irradiation-Induced Defects in Light Water Reactor Pressure Vessel Steels," J. Nuc. Mat., 346, 79, 2005.

42. Odette, G. R., B. D. Wirth, and T. Yamamoto, unpublished analysis of a large body of recent SANS data.

43. Salje, G., and M. Feller-Kneipmeier, "The Diffusion and Solubility of Copper in Iron," J. Appl. Phys., 48-5, 1833, 1977.

44. Marian, J., B. D. Wirth G,R, Odette et al, "Cu Diffusion in Alpha-Fe: Determination of Solute Diffusivities using Atomic-Scale Simulations," Computational Materials Science 31:3-4, 347, 2005.

45. le Claire, A. D., "Solvent Self-Diffusion in Dilute B. C. C. Solid Solutions," Phil. Mag. 172, 819, 1970.

46. Lê, T. N., Barbu, A., Liu, D., and Maury, F., "Precipitation Kinetics of Dilute Fe-Cu and Fe-Cu-Mn Alloys Subjected To Electron-Irradiation,” Scripta Metall. et Mater. 26, 771, 1992.

47. Moslang, A., E. Albert, E. Recknagel and A. Weidinger, "Interaction of Vacancies With Impurities in Iron," Hyperfine Interactions, 15/16, 409, 1983.

48. Miller, M. K., K. F. Russell, G. R. Odette and D. Klingensmith, Precipitation in Thermally Aged and Neutron Irradiated Fe-Cu and Fe-Cu-Mn Model Alloys, ORNL/NRC/LTR-03/01, 2003. 
49. Odette, G. R., G. E. Lucas and D. Klingensmith, "Anomalous Hardening in Model Alloys and Steels Thermally Aged at $290^{\circ} \mathrm{C}$ and $350^{\circ} \mathrm{C}$ : Implications to Low Flux Irradiation Embrittlement," Effects of Radiation on Materials: 18th International Symposium, ASTM STP 1325, ed. R. K. Nanstad et al., American Society for Testing and Materials, West Conshocken, PA, 1089, 1999.

50. Odette, G. R., T. Yamamoto, and B. D. Wirth, "Late Blooming Phases and Dose Rate Effects in RPV Steels: Integrated Experiments and Models," Proceedings of the Second International Conference on Multiscale Materials Modeling, ed. N. M. Ghoniem, Univeristy of California, 355, 2004.

51. Machlin, E. S., An Introduction to Thermodynamics and Kinetics Relevant to Materials Science, $2^{\text {nd }}$ ed., Giro Press, Croton-on Hudson, NY, 1999.

52. Jones, R. B., and J. T. Buswell, "The Interactive Roles of Phosphorus, Tin and Copper in the Irradiation Embrittlement of PWR Pressure Vessel Steels," Environmental Degradation of Materials in Nuclear Power Systems - Water Reactors, Proceedings of the $3^{\text {rd }}$ International Symposium, ed. G. T. Theus et al., Metallurgical Society, Warrendale, PA, 111, 1988.

53. Hawthorne, J. R., Exploratory Studies of Element Interactions and Composition Dependences in Radiation Sensitivity Development, NUREG/CR-4437,U. S. Nuclear Regulatory Commission, Washington, DC, 1985.

54. Smithells Metals Reference Book $8^{\text {th }}$ Edition, ed. E. W. F. Gale and T. C. Totemeir, ASM/Elsevier, 2004.

55. Miller, M. K., K. Russell, and G. R, Odette, "Clustering and Precipitation in Low Copper and Copper Free Steels and Model Alloys," International Field Emission Symposium, 2006.

56. English, C. A., S. R. Ortner, G. Gage, W. L. Server and S. T. Rosinkski, "Review of Phosphorus Segregation and Intergranular Embrittlement in Reactor Pressure Vessel Steels," Effects of Radiation on Materials: 20th International Symposium, ASTM STP 1405, ed. S. T. Rosinski et al., American Society for Testing and Materials, West Conshohocken, PA, 151, 2001.

57. Nanstad, R. K., D. E. McCabe, M. A. Sokolov, C. A. English, and S. R. Ortner, "Investigation of Temper Embrittlement in Reactor Pressure Vessel Steels Following Thermal Aging, Irradiation, and Thermal Annealing," Effects of Radiation on Materials: $20^{\text {th }}$ International Symposium, ASTM STP 1405, ed. S. T. Rosinskiet. al., American Society for Testing and Materials, West Conshohocken, Pennsylvania, 2001.

58. Odette, G. R., and C. Cowan, "Use of Combined Electrical Resistivity and Seebeck Coefficient Measurements to Characterize Solute Redistribution Under Irradiation and Thermal Aging," Proceedings of the $10^{\text {th }}$ International Symposium on Environmental Degradation of Materials in Light Water Reactors, Ed. G. S. Was et. al., NACE, CD, 2001.

59. Wirth, B. D., G. R. Odette and R. E. Stoller, "Recent Progress Toward an Integrated MultiscaleMultiphysics Model of Reactor Pressure Vessel Embrittlement," Multiscale Modeling of Materials, MRS Soc. Symp. Proc. 677, ed. V. Bulatov et. al., Materials Research Society, Warrendale, PA, AA5. 2, 2001.

60. Mader, E., G. E. Lucas, and G. R. Odette, "The Effects of Metallurgical and Irradiation Variables on the Postirradiation Annealing Kinetics of Pressure Vessel Steels," Effects of Radiation on Materials: 15th International Symposium, ASTM STP 1125, ed. R. E. Stoller et al., American Society for Testing and Materials, West Conshocken, PA, 151, 1992.

61. Mader, E. V., "Kinetics of Irradiation Embrittlement and the Post-Irradiation Annealing of Nuclear Reactor Pressure Vessel Steels," PhD. Thesis, UC Santa Barbara, 1995. 


\section{Transition Temperature Shift (TTS) Database}

This chapter identifies the main sources of data utilized in this report and describes how the datasets used for model calibration and validation were prepared. The key databases include the Power Reactor Embrittlement Database (PR-EDB), which is an archive of information from U.S. reactor surveillance programs, the Irradiation Variable (IVAR) data, which are results from a major research program on irradiation effects, and the TTS database used for the analysis and modeling reported in Chaps. 4, 5, and 7. The TTS analysis database was developed from the PR-EDB and later surveillance reports as described in Sects. 3.3 and 3.4. (See Appendix B, "Irradiation Variable [IVAR] Program Data Base," and Appendix C, "Analysis Data Base.”)

\subsection{Power Reactor Embrittlement Database (PR-EDB)}

The PPR-EDB as reported in NUREG/CR-4816 [1] is an archival database that includes considerable detail on surveillance capsules that have been irradiated in U.S. power reactors. It is a relational database containing many linked attributes associated with surveillance data. In many cases, the PR-EDB includes multiple entries when data are presented in multiple sources, and generally more complete and detailed information than is needed specifically for embrittlement correlation development. Version 2 of the PREDB through Update $12^{1}$ was a primary source of data for the analysis and for cross-checking, identifying, and resolving discrepancies. Input data for the PR-EDB come from various surveillance reports, data logs, and official memorandums from nuclear power plants. Raw data files are constructed, and the input data are subjected to a rigorous quality assurance review prior to inclusion in the database. The data are categorized into (1) material history, (2) radiation environments, and (3) mechanical test results, along with the associated detailed references. The ORNL Radiation Safety Information Computational Center (865-574-6176, pdc@ornl.gov) is responsible for NRC software distribution, including the distribution of PR-EDB Version 2.

Newer results not yet in Version 2 (Update 12) to the PR-EDB were extracted in 2003 and 2004 from industry reports $[2,3]$ and individual surveillance capsule reports. The latter reports were identified and provided by C. Santos, who searched the ADAMS document-indexing facilities at the NRC through about March 2004.

\subsection{Guidance from Research Programs (IVAR Database)}

The Irradiation Variable (IVAR) database [4] is mentioned at various points in this report, particularly in Chap. 6, where it is directly compared with the transition temperature shift (TTS) model calibrated to the surveillance database. The IVAR materials, database, irradiations and testing program are described in some detail in Sect. 6.1; the IVAR database results from an extensive program, producing a large set of data from controlled, replicated experiments, using many different materials, including some that are typical of commercial RPV materials and others that have been remelted and heat-treated in order to explore individual and combined material variable trends. The key irradiation exposure variables were varied to clarify effects of fluence, flux, and irradiation temperature.

The significance of the IVAR database is that it provides independent evidence of variable trends, in many cases with less uncertainty, without confounding effects from other variables and with better signal to noise ratios than can the surveillance data. Some preliminary IVAR results were available and were used for modeling insight as the TTS model was developed, and additional details have since emerged from the comparison of the TTS model with the IVAR data presented in Chap. 6. Although some of the theory in Chap. 2 and some trends from IVAR data and other sources were used for insight, the TTS

\footnotetext{
${ }^{1}$ Update 12 to Version 2 of the PR-EDB was provided to the USNRC in 2004.
} 
model described in Chap. 4 was calibrated solely on the surveillance database, so the comparison with IVAR data in Chap. 6 provides an independent check on predictive capability for data not used in fitting.

\subsection{Development of Charpy TTS Estimates}

The PR-EDB includes reported transition temperature shifts (TTSs) and upper shelf energy (USE) drops taken directly from surveillance reports. However, multiple entries appear for some heats, making it unclear which values to use. Additionally, the reported values were determined using various techniques over the years, including visual inspection of raw Charpy plots and various fitting techniques. To ensure that mean estimates of shift and drop determined on a consistent, repeatable basis were used, a computer

program, FITCV, was written to fit the raw Charpy datasets from PR-EDB or other sources and to compute TTS and USE based on those fits. FITCV was first used and described in Sect. 2 of NUREG/CR-6551 [5] and is only summarized here. The same program has been used to estimate TTS for the additions to the database in 2000 and 2003-2004, so all shifts used in the present analysis are selfconsistent and consistent with those used in the Draft 2000, ASTM E900-02 and NUREG/CR-6551 models.

The general approach in FITCV is to fit modified hyperbolic tangent (tanh) curves to each set of raw Charpy data from the same heat of material tested in the same orientation and with the same neutron exposure. The program evaluates the two tanh fitting forms given by Eqs. (3-1) and (3-2) (and an exponential form if no upper shelf data are available), each calibrated by two independent least squares algorithms as an automatic check on convergence. The unirradiated and irradiated curves for the same heat and orientation are matched, and shifts and drops are computed from the difference of the fitted curves. The program chooses the best fit (minimum sum of squared residuals) automatically, but plots of the data and fits are also printed, showing the choice and the alternatives, and the choices are confirmed by individual review of the plots.

The general symmetric tanh model form often used for modeling Charpy curves [6] is one of the options fitted by FITCV:

$$
C_{v}=a_{3} \tanh \left(\frac{T-a_{1}}{a_{2}}\right)+\left(a_{3}+L S E\right)
$$

where

$$
\begin{array}{ll}
C_{v}= & \text { Charpy impact energy } \\
a_{3}= & \text { fitting parameter equal to }(U S E-L S E) / 2 \\
T= & \text { test temperature } \\
a_{1}= & \text { fitting parameter equal to temperature at the inflection point of the fitted curve } \\
a_{2}= & \text { fitting parameter related to the slope of the transition region } \\
L S E= & \text { lower shelf energy; } L S E=1.28 \mathrm{ft}-\mathrm{lbs} \text { (see Appendix B in [5]). }
\end{array}
$$

The symmetric tanh form in Eq. (3-1) does not do a good job of fitting the Charpy data for sets of data that exhibit an abrupt transition from the lower shelf and a more gradual transition to the upper shelf, which frequently occur. In such sets, the symmetric tanh curve does not agree well with the data in the lower transition region, near the $30 \mathrm{ft}-\mathrm{lb}(41 \mathrm{~J})$ temperature $\left(\mathrm{T}_{30}\right)$, which is the part of the curve needing the best possible fit because it is where the irradiation-induced shift is calculated. An asymmetric form of the tanh model fits the data better than the usual tanh model in those cases. 
The form of the asymmetric tanh is:

$$
C_{v}=c_{3} \tanh \left(\frac{T-c_{1}}{c_{4} T+c_{2}}\right)+\left(c_{3}+L S E\right)
$$

where $c_{1}, c_{2}$, and $c_{3}$ are analogous to $a_{1}, a_{2}, a_{3}$ in Eq. (3-1), and $c_{4}$ is a positive fitting parameter causing the hyperbolic tangent fit to have a more sharply curved transition in the lower region than in the upper region. The examples in Fig. 3.1 show that the asymmetric tanh is often able to come closer to the data than the symmetric tanh fit in the lower transition region. However, the symmetric tanh usually provides a better fit to sets with limited upper shelf data.

Note that Eq. (3-1) is a special case of Eq. (3-2), where $c_{4}=0$; hence, given sufficient data, Eq. (3-2) always fits the lower transition region at least as well as Eq. (3-1). Thus most of the shifts in the analysis database described in this report use Eq. (3-2).
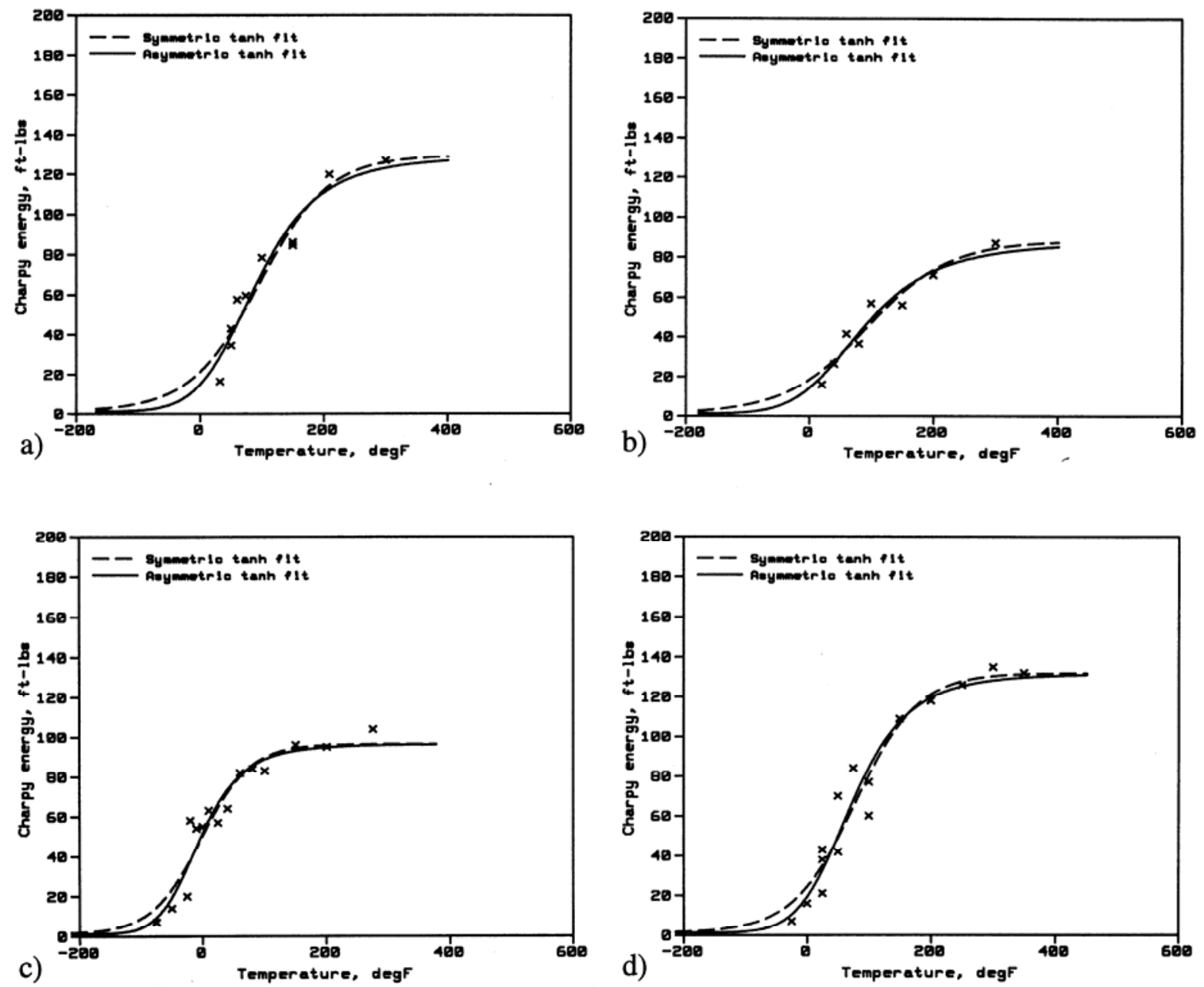

Fig. 3.1. Examples of symmetric and asymmetric tanh curve fits (from Fig. 2.1 in Eason, E. D., J. E. Wright, and G. R. Odette, Improved Embrittlement Correlations for Reactor Pressure Vessel Steels, NUREG/CR-6551, U.S. Nuclear Regulatory Commission, Washington, D.C., 1998). 
Most sets of raw Charpy data include only specimens from a single plant that are either unirradiated or irradiated in a single capsule. However, in the case of unirradiated standard reference materials, raw Charpy data associated with different plants were pooled together, so that one unirradiated $\mathrm{C}_{\mathrm{v}}$ vs. T curve was fitted for each standard reference material (SRM). The basis for this approach is that the unirradiated SRMs are the same three heats, regardless of which plant they were sent to, so combining the data from all plants gives the best average characterization of the heat in the unirradiated condition. This heat-based approach may have inadvertently increased the scatter for one of the SRMs, as discussed in Sect. 4.3.5 of this report, although it did not affect the correlation for plant materials.

Plate and forging materials were often tested in two orientations, TL and LT [7]. The shift for each orientation is about the same, although the unirradiated and irradiated values of $\mathrm{T}_{30}$ are not. The two orientations contribute independent measurements of unirradiated and irradiated $\mathrm{T}_{30}$, so they were treated as separate observations in the statistical analysis. The same approach was used in calibrating earlier surveillance models $[5,8-10]$.

\subsection{Analysis Database}

The term "analysis database" is used to denote the information used for analysis, model development, and calibration. It is a subset of the more complete details available in PR-EDB and surveillance reports. The analysis database is in the form of an Excel spreadsheet where each row is an individual shift observation for one heat and the columns contain the details that are used for identifying that shift and all the independent variables used for developing the embrittlement shift correlation. The analysis database includes data on surveillance welds, plates, and forgings, but heat-affected-zone data (which are in PR-EDB) are not included. This exclusion was deliberate and was made in conjunction with the ASTM E10.02 committee because of concerns regarding the heat-affected zone results. The same heat-affected zone exclusion was invoked for the same reasons in the earlier modeling efforts back to NUREG/CR-6551.

The analysis database used in the current modeling effort is contained in Appendix $\mathrm{C}$ of this report and is the result of the most recent cycle of updating, and was largely derived from the PR-EDB [1] as supplemented by recent capsule reports and corrections by members of the ASTM E10.02 committee. The steps in the updating process were (1) use the latest version of the PR-EDB (Version 2, Update 12), (2) search the available NRC documents for more recent surveillance reports, (3) tabulate and submit the extracted data for detailed review by the ASTM E10.02 committee in meetings held in January and June 2004, and (4) collaborate with Jy-An Wang of ORNL regarding data verification.

\subsubsection{Database for Developing the Matrix Feature (MF) Term}

The July 16, 2004 analysis database file, DupsDiscreps7-04.xls (which contains Version 7-04 of the data), was the starting point for the initial matrix feature (MF) modeling, although it was revised before that round of modeling was complete. The only result presented in this report that came from Version 704 is the analysis of the threshold separating low-Cu and high-Cu subsets. All other results presented in this report are from the database in file TTSDatabase8-04.xls (Version 8-04 of the data), completed August 23, 2004. Extensive comments are provided in TTSDatabase8-04.xls documenting the detailed changes to the data, including those made between Version 7-04 and Version 8-04. The main difference between the two databases is that some additional low- $\mathrm{Cu}$ points are included in Version 8-04. This difference arose because there were unknown values of irradiation temperature $\left(T_{i}\right)$ that initially eliminated nine points from Vogtle unit 2, all of which are at low $\mathrm{Cu}$. A value of $\mathrm{T}_{\mathrm{i}}$ was provided for those points before completing Version 8-04 of the database, and they were included for all subsequent modeling work. The omitted points do not affect the threshold $\mathrm{Cu}$ level determined in the analysis below because the Vogtle $2 \mathrm{Cu}$ values are not near the threshold. 
Preliminary analysis and modeling using the Version 7-04 dataset also identified anomalies in composition and apparent low-Cu outliers (see Appendix E). Requests were made to members of the ASTM E10.02 committee to check the unusual and questionable points. The responses from committee members included revised values of $S$ for the plate and welds from San Onofre Units 2 and 3, which were incorporated into the Version 8-04 database. Two of the Chauvenet outlier points identified in low-Cu materials are from weld WFA201, for which the unusually low Mo values (lowest in the database) were reviewed and confirmed. Another outlier had the largest negative shift $\left(-35^{\circ} \mathrm{F}\right)$ in the database; possible reasons for negative shifts include incorrect values of unirradiated $\mathrm{T}_{30}$ and fitting anomalies in the irradiated Charpy data. The four low-Cu outliers relative to the preliminary model remained Chauvenet outliers relative to the final model presented below. The revised PWR low-Cu fitting set contains 219 points after the nine VO2 data points were added and the four outliers were removed.

\subsubsection{Database for Developing the Copper-Rich Precipitate (CRP) Term}

The Version 8-04 database (August 2004) was used for the preliminary copper-rich precipitate (CRP) term modeling and for all of the final models presented here (both MF term and CRP term). The database incorporates the additional data, corrections, and low-Cu outlier deletions made during the preliminary low-Cu analysis discussed in Sect. 3.4.1. Additionally, the high-Cu Chauvenet outliers identified in the NUREG/CR-6551 and the July 2000 modeling efforts [5,8] were still Chauvenet outliers and were removed, along with three points with unusual irradiation conditions and one point that was identified by Chauvenet analysis but also reflects unusual irradiation conditions. The Version 8-04 database is given in Appendix C, which is a CD containing the electronic file TTSDatabase8-04R1.xls. The details of all points removed as outliers or anomalies are in Appendix E.

Previous modeling efforts had identified issues related to the SRM or correlation monitor data. Three SRM plates, referred to as ASTM, HSST-01, and HSST-02, are included in U.S. commercial reactor surveillance programs, each of which has been irradiated in many reactors. All three are in the high-Cu category $(\mathrm{Cu}>0.072 \mathrm{wt} \%)$. The first modeling issue with the SRMs is that they unbalance the database for modeling heat chemistry effects because a large fraction of all the available surveillance data comes from these three heats, which are similar in composition except for $\mathrm{Ni}$. A second issue is that including them in one or the other group of plate data can affect the results for those groups [note that plates in Combustion Engineering (CE)-manufactured vessels and non-CE-manufactured vessels are separated because they have significantly different shifts]. Furthermore, grouping the SRMs with either the CE or non-CE plate groups cannot be justified because in fact the three plates are not part of actual plant pressure vessels. A third issue is that the heat treatment of the plates may differ somewhat from plates that were welded into vessels and then postweld heat treated. Finally, concerns have been expressed for Plate HSST-02 because the unirradiated $\mathrm{T}_{30}$ value used in the analysis database (which is an average of values from several sources) may not be representative of the samples sent to some specific plants due to inhomogeneity of that plate. An error in unirradiated $\mathrm{T}_{30}$ would show up as a consistent offset between the model and measured shift TTS for that plate over a range in fluences. Such errors are of less concern for plates with an average amount of available data, but they could bias the correlation if the plate in question contributes much more data to the fit than most plates, as would be the case with HSST-02.

To address the SRM issues, the data on standard reference materials were initially removed from the high- $\mathrm{Cu}$ dataset, and a randomly selected subset of SRM data was added back into the calibration and validation datasets as described in the following paragraphs. The objective was to sample the SRMs to obtain a similar number of shifts as in the typical surveillance heats from plant vessels (usually two to five shifts in each orientation or four to ten shifts total for each plate or forging heat chemistry). Without such sampling, the SRMs would get undue weight in the fit, and the effects of the SRM chemistry or of uncertainties in unirradiated $\mathrm{T}_{30}$ would be magnified. The sampling plan shown in Table 3.1 was used. 
Table 3.1. Sampling of standard reference material data

\begin{tabular}{rccccc}
\hline \multirow{2}{*}{ Heat ID } & \multirow{2}{*}{$\begin{array}{c}\text { TL or } \\
\text { LT }\end{array}$} & Plant type & $\begin{array}{c}\text { Total shifts } \\
\text { available }\end{array}$ & $\begin{array}{c}\text { Salibration } \\
\text { set }\end{array}$ & $\begin{array}{c}\text { Validation } \\
\text { set }\end{array}$ \\
\hline SASTM & LT & PWR & 21 & 5 & 1 \\
SASTM & LT & BWR & 1 & 1 & 0 \\
SASTM & TL & PWR & 4 & 4 & 0 \\
SHSS01 & LT & PWR & 17 & 5 & 1 \\
SHSS02 & LT & PWR & 61 & 5 & 1 \\
SHSS02 & TL & PWR & 1 & 1 & 0 \\
SHHS02 & TL & BWR & 2 & 2 & 0 \\
Total & & & 107 & 23 & 3 \\
\hline
\end{tabular}

The random sampling of each of the three heats in the LT orientation was performed by first listing the data by increasing exposure time, assigning a uniformly distributed random number between 0 and 1 to each shift, then dividing the data on each heat into five subsets. For example, the SASTM LT PWR data were divided into five subsets, with the five lowest-time shifts in the first subset, the five next lowest-time shifts in the next subset, and so on up to the four highest time shifts in the last subset. Then one shift was picked from each of the five subsets by taking the shift in that subset with the smallest random number. Because the numbers were assigned randomly, it would also be random which shift would have the smallest random number. The choice of the smallest random number is arbitrary; any other unbiased method of picking one number (e.g., the largest random number, the random number nearest 0.5 ) should work as well. This procedure ensured that there would be five shifts, including at least one with high, medium, and low exposure time, from each SRM heat. All shifts from the heat/orientation combinations with fewer than five shifts (e.g., SASTM TL) were included in the calibration set without sampling.

The total number of SRM shifts after sampling is 23 in the calibration set, so a $10 \%$ validation sample should have no more than 3 shifts. The three validation shifts were selected by picking the shift in each heat in the LT orientation with a random number nearest to an arbitrary number, in particular, 0.55 . Without the sampling, the three SRM heats would have contributed a total of 104 shifts to the PWR high$\mathrm{Cu}$ dataset and 3 shifts to the BWR high-Cu dataset, amounting to over $18 \%$ of all the available high-Cu PWR data. With the sampling, the 3 SRM heats contribute a more reasonable 23 out of 485 shifts (less than 5\%) in the PWR high $\mathrm{Cu}$ dataset.

It should be noted that a different random sample of SRMs could produce slightly different results, but any such differences would be expected to affect mainly the SRM coefficient. Any difference in the SRM coefficient would also be small, based on the fact (shown in Sect. 4.3.5 and Fig. 4.12) that all SRM data, including points that are not included in the calibration and validation samples, are reasonably consistent with the model and generally within the $5 \%$ and $95 \%$ bounds estimated for plate materials. The effect of a different SRM sample on the other parts of the embrittlement shift model would be negligible for several reasons. First, the number of points with the SRM chemical compositions would be unchanged in a different sample, and the same chemistry is used for all shifts from each of the three heats; hence there would be no change in chemistry input to the model. Second, the sample of SRM data is less than $5 \%$ of the calibration data, so since they are a small subset and in reasonable agreement with the model, sampling the SRM data differently cannot have much effect. Finally, the SRM sample only affects the CRP term, since the materials are all high $\mathrm{Cu}$, and the SRM heats have their own CRP coefficient specifically so that they cannot bias other parts of the model. 


\subsubsection{Calibration and Validation Datasets}

After the Chauvenet outliers, unusual irradiation points, and SRMs were removed, the database was partitioned into calibration and validation sets. The split was made on all remaining data, including PWR and BWR, with both high $\mathrm{Cu}(\mathrm{Cu}>0.072$ wt \%) and low $\mathrm{Cu}(\mathrm{Cu} \leq 0.072 \mathrm{wt} \%)$. The validation set is nominally $10 \%$ of the data, selected randomly by assigning uniform random numbers between 0 and 1 , then selecting the data with random numbers in the range 0.45 to 0.55 (the middle tenth). The choice of the middle tenth was arbitrary; any other arbitrarily-selected tenth should work as well because the random numbers are approximately uniformly distributed. The data not selected in the process became the 90\% calibration set. The SRM samples defined in Table 3.1 were then added back into the appropriate calibration and validation sets.

As shown in Table 3.2, the splits in the PWR datasets have nearly the desired 90\%:10\% proportions, as does the split in the BWR high-Cu dataset. The BWR low-Cu set is really too small for the random sampling to give the desired proportions. (There should be two or three points in the BWR low-Cu validation set; random sampling produced only one.) However, even if the ideal $10 \%$ fraction were selected, the BWR low-Cu validation set would be too small.

Table 3.2. Distribution of data by dataset (including SRM samples)

\begin{tabular}{lccc}
\hline \multirow{2}{*}{ Dataset $^{a}$} & $\begin{array}{c}\text { Total shifts } \\
\text { available }\end{array}$ & \multicolumn{2}{c}{ Sample size (\%) } \\
\cline { 3 - 4 } PWR, high Cu & 485 & Calibration set & Validation set \\
PWR, low Cu & 219 & $140(91 \%)$ & $45(9 \%)$ \\
BWR, high Cu & 124 & $196(89 \%)$ & $23(11 \%)$ \\
BWR, low Cu & 27 & $113(91 \%)$ & $11(9 \%)$ \\
Total & 855 & $26(96 \%)$ & $1(4 \%)$ \\
\hline
\end{tabular}

${ }^{a} \mathrm{High} \mathrm{Cu}:>0.072$ wt $\%$; low $\mathrm{Cu}: \leq 0.072$ wt $\%$.

A different random partitioning of the data into calibration and validation sets would be expected to make little or no difference in the results. The reason is that all the data, both calibration and validation sets, are reasonably consistent with the model as shown in Table 4.2 and in Figs. 4.8, 4.10, and 4.11. The greatest possible change in the calibration dataset would come from replacing $1 / 9$ of the calibration points with the $10 \%$ validation set (any other change would reuse more than $8 / 9$ of the calibration set). There is no significant difference between the calibration and validation sets as measured by the residuals relative to the model, (see Table 4.2) so replacing $1 / 9$ of the calibration set by the validation data should have little or no effect on the model.

\subsubsection{Range of Data by Independent Variable}

The independent variables used in the TTS model, together with the range and mean value of each variable, are shown in Table 3.3. An additional independent variable not listed in Table 3.3 is product form, which can take on the values forging, plate (in CE manufactured vessels or other vessels), weld (Linde 80, Linde 1092, or other), and standard reference material (SRM). Note that three exposure variables, fluence, time-averaged flux, and effective full power exposure time, are mathematically related such that any two of the three can be considered independent variables for modeling; the two that are used in the model given in Chap. 4 are fluence and flux.

The statistics in Table 3.3 are based on 855 datapoints, including all calibration and validation data, both PWR and BWR. Only the SRM data that were included in either calibration or validation sets have been included; there are additional SRM data that were not in those sets. Adding in the rest of the SRM 
Table 3.3. Independent variables in the embrittlement shift model and their ranges and mean values over all calibration and validation data (855 datapoints)

\begin{tabular}{clcc}
\hline Variable & \multicolumn{1}{c}{ Description } & Range & Mean \\
\hline$C u$ & Copper content (wt \%) & $0.01-0.41$ & 0.136 \\
$M n$ & Manganese content $(\mathrm{wt} \%)$ & $0.58-1.96$ & 1.300 \\
$N i$ & Nickel content $(\mathrm{wt} \%)$ & $0.044-1.26$ & 0.565 \\
$P$ & Phosphorous content $(\mathrm{wt} \%)$ & $0.003-0.031$ & 0.0119 \\
$\phi t$ & Neutron fluence, $\mathrm{E}>1 \mathrm{MeV}\left(\mathrm{n} / \mathrm{cm}^{2}\right)$ & $9.26 \times 10^{15}-7.13 \times 10^{19}$ & $6.50 \times 10^{18}$ \\
$\phi$ & Neutron flux, E $>1 \mathrm{MeV}\left(\mathrm{n} / \mathrm{cm}^{2} / \mathrm{s}\right)$ & $1.81 \times 10^{8}-9.71 \times 10^{11}$ & $5.13 \times 10^{10}$ \\
$T_{i}$ & Irradiation temperature $\left({ }^{\circ} \mathrm{F}\right)$ & $522-570$ & 545 \\
\hline
\end{tabular}

data would change the count and perhaps slightly bias the mean chemistry but would not affect the ranges because all data on each SRM heat have the same heat-average chemistry.

Some additional information about the variables in Table 3.3 is needed. The chemical composition variables are intended to represent the best available estimate of actual measured composition at the location where the shift is being analyzed. This is consistent with the use of average measured composition on surveillance samples from each heat (to the extent available) to develop the calibration database.

The values of fluence and flux variables are intended to be estimates at the actual location where the shift is to be estimated, with the flux estimate averaged over the total effective full power operating time. This is consistent with the estimates for the surveillance specimens, for which dosimetry was based on the actual capsule location and the time averaging was done by dividing total accumulated fluence by the effective full power operating time to estimate time-averaged flux.

The irradiation temperature is also intended to be a time-averaged estimate for the metal at the specific location where the shift is to be estimated. The best available metal temperature estimate for the surveillance specimens was the temperature of the coolant near the surveillance capsule, but coolant temperature may not be the best estimate of metal temperature in other cases.

The range of data given in Table 3.3 is not by itself sufficient for estimating the limits of applicability of the model given in Chap. 4. The actual coverage of the data over the fitting variables and combinations of variables varies considerably, so it is necessary to review the actual distribution of data in TTSDatabase8-04.xls to determine the ranges of variables and variable combinations that are supported by a reasonable amount of data. For instance, forgings with $\mathrm{Cu}>0.16 \mathrm{wt} \%$ or plates with $\mathrm{Cu}>0.25 \mathrm{wt}$ $\%$ are simply not available in the database, and the upper limit of $\mathrm{Cu}=0.41 \mathrm{wt} \%$ in Table 3.3 applies only to welds. As another example, there are no low $\mathrm{Mn}(\mathrm{Mn}<0.93 \mathrm{wt} \%)$ materials in the database except A508 class 2 forgings, and the range of other chemistry variables in such forgings is limited $(0.67 \leq \mathrm{Ni} \leq 0.86 \mathrm{wt} \%, 0.01 \leq \mathrm{Cu} \leq 0.16$ wt $\%$, and $0.004 \leq \mathrm{P} \leq 0.02 \mathrm{wt} \%)$. Thus, application of the model to any materials with $\mathrm{Mn}<0.93$ and values of $\mathrm{Ni}, \mathrm{Cu}$, or $\mathrm{P}$ outside the ranges corresponding to A508 class 2 forgings would be an extrapolation beyond the available data.

As an example of limits on exposure variable combinations, Table 3.3 shows that there are both highfluence and low-fluence data, and high-flux and low-flux data, so one might assume that the full range of the fluence/flux space is reasonably covered by data. Unfortunately, this assumption is not true. In fact, there are no high-fluence data at low flux. The highest available fluence in the database decreases as flux decreases, so in all the data with $\phi<1 \times 10^{10} \mathrm{n} / \mathrm{cm}^{2} / \mathrm{s}$, the highest available fluence is $\phi \mathrm{t}=1.9 \times 10^{18}$ $\mathrm{n} / \mathrm{cm}^{2}$. The highest available fluence in all the data with $\phi<1 \times 10^{9} \mathrm{n} / \mathrm{cm}^{2} / \mathrm{s}$ is only $\phi \mathrm{t}=2.8 \times 10^{17} \mathrm{n} / \mathrm{cm}^{2}$. Thus, estimating the shift at any fluence greater than $1.9 \times 10^{18} \mathrm{n} / \mathrm{cm}^{2}$ for $\phi \cong 1 \times 10^{10} \mathrm{n} / \mathrm{cm}^{2} / \mathrm{s}$ (and similarly for lower flux levels) is an extrapolation beyond the available data. 


\subsubsection{Comparison with Prior Databases}

The dataset used in the final calibration of most constants in the current model is larger (at 775 points) than the datasets used in prior modeling efforts. Some specific comparisons include

- The July 2000 draft model [8] and ASTM E900-2 models [10] were calibrated on 736 points

- The shift calibration set used in NUREG/CR-6551 [5] contained 609 points

- Regulatory Guide 1.99 Rev. 2 was based on 177 points.

In addition to the calibration data, there are additional data in the current TTS database that were not used for calibration but can be used to provide evidence of predictive capability of the TTS model. An additional 80 datapoints were randomly selected before the model development and reserved for validation purposes (see Table 3.2). An additional 81 randomly selected SRM shifts were excluded from the calibration and validation sets in the modeling effort discussed in this report, as shown in Table 3.1. These data are also available for comparison with the model, providing additional evidence of predictive capability. By comparison, all available non-outlier data were used in the earlier modeling efforts, leaving no independent surveillance data for validation and comparison.

Counting both the calibration points and the additional data available for validation and comparison, the calibration dataset for the current model is actually a sample of data from a $27 \%$ larger database (200 more usable points) than the one used in July 2000. The total TTS database is more than 5 times the size of the database used to develop Regulatory Guide $1.99 \mathrm{Rev}$. 2. More important than the gross increase in size, the additional points helped fill in areas of the database that were notably sparse in the earlier modeling efforts, including low copper, high fluence and long exposure time, and low flux data from BWR surveillance capsules. The imbalance in the NUREG/CR-6551 and July 2000 databases caused by a large number of points from just three SRM heats has also been addressed. Thus, the current calibration dataset, although only $5 \%$ larger than the one used for calibration in July 2000, is much better balanced.

\subsection{References}

1. Stallmann, F. W., J. A. Wang, F. B. K. Kam, and B. J. Taylor, "PR-EDB: Power Reactor Embrittlement Data Base, Version 2," NUREG/CR-4816 (ORNL/TM-10328/R2), U.S. Nuclear Regulatory Commission, Washington, D.C., 1994.

2. Tilly, L., and T. Hardin, BWRVIP-87: BWR Vessel and Internals Project Testing and Evaluation of BWR Supplemental Surveillance Program Capsules D, G, and H, EPRI Report TR-1000890, 2000.

3. Burgos, B., K. Hour, C. Potze, and K. Watkins, BWRVIP-111: BWR Vessel and Internals Project Testing and Evaluation of BWR Supplemental Surveillance Program Capsules E, F, and I, EPRI Report TR-1003553, 2002.

4. Odette, G. R., T. Yamamoto, and D. Klingensmith, The Effect of Dose Rate on Irradiation Hardening of RPV Steels: A Comprehensive Single Variable Database and Model Based Analysis, Letter Report UCSB-NRC-03/1, U.S. Nuclear Regulatory Commission, Washington, D.C., 2003.

5. Eason, E. D., J. E. Wright, and G. R. Odette, Improved Embrittlement Correlations for Reactor Pressure Vessel Steels, NUREG/CR-6551, U.S. Nuclear Regulatory Commission, Washington, D.C., 1998.

6. McConnell, P., W. L. Server, W. Oldfield, and F. M. Oldfield, Irradiated Nuclear Pressure Vessel Steel Data Base, EPRI NP-2428, Electric Power Research Institute, Palo Alto, Calif., June 1982.

7. ASTM, "Standard Test Method for Plane-Strain Fracture Toughness of Metallic Materials, E399-90, "Annual Book of ASTM Standards, Vol. 03.01ASTM International, 2005. 
8. Kirk, M., C. Santos, E. Eason, J. Wright, and G. R. Odette, "Updated Embrittlement Trend Curve for Reactor Pressure Vessel Steels," in Transactions of the $17^{\text {th }}$ International Conference on Structural Mechanics in Reactor Technology (SMiRT 17), Prague, Czech Republic, August 17-22, 2003, 2003.

9. Server, W., C. English, D. Naiman, and S. Rosinski, Charpy Embrittlement Correlations-Status of Combined Mechanistic and Statistical Bases for U.S. RPV Steels (MRP-45), EPRI Report 1000705, 2001.

10. ASTM, "Standard Guide of Predicting Neutron Radiation Damage to Reactor Vessel Materials, E706 (IIF), E900-02, “Annual Book of ASTM Standards, ASTM International, 2002. 


\section{Transition Temperature Shift (TTS) Model}

The primary objective of this chapter is to present the revised embrittlement shift model and to demonstrate that it is a good fit to the calibration data and to other data not used for calibration. The main steps that produced the model, summarized in Sects. 4.1 and 4.2, should be viewed as just the latest steps in an iterative process of data collection and review, use of physical insight, empirical model calibration, and post-calibration analysis that has now gone through four major iterations, dating back to and including the NUREG/CR-6551modeling effort [1]. The emphasis in this chapter on the final baseline model and its quality of fit on relevant sets of data is deliberate, since intermediate models and preliminary significance tests on subsets of the relevant data, though useful during the modeling process, do not fairly characterize the final model and can be misleading.

In addition to information presented in this chapter, the model is further justified by information in Chap. 5 on the shape and statistical significance of individual variable effects on relevant datasets, and by comparisons with an independent set of data showing similar trends, given in Chap. 6. The model presented in this chapter is considered the baseline model, and a slightly simplified model is presented in Sect. 7.3 that reflects the comparisons in Chap. 6 and subsequent sensitivity studies.

\subsection{TTS Analysis Methodology}

The following modeling guidelines were generally followed in developing the TTS model:

1. use results of mechanistic studies to guide the overall model and help identify variable effects that should be looked for in the TTS data;

2. use only the U.S. power reactor surveillance data for choosing appropriate mathematical fitting forms and calibrating the adjustable parameters;

3. fit each part of the model to the most relevant data, considering numerical trade-off issues as well as physical mechanism issues; and

4. make sure the model provides a reasonable fit in each of the key datasets, including high and low $\mathrm{Cu}$; high and low flux; and forging, plate, and weld.

The surveillance database has a very uneven distribution of data, signal-to-noise issues, and variable confounding, which limit what can be done under guideline 2. The imposed square root dependence of fluence in the MF term is an example where guideline 2 was relaxed in the present effort, and further relaxation of this guideline may be appropriate in the future to better reflect well-established results of mechanistic studies. Also, guidelines 3 and 4 are effectively constraints on the fit that imply that the sum of squares might be lower and that the calibrated constants might be somewhat different if they were simultaneously fitted to the entire database. Guidelines 3 and 4 were imposed because of lessons learned in past modeling iterations and because use of a single function to describe the entire database is not justified when different physical mechanisms are known to be active in different subsets of data (e.g., following from guideline 1, MF and CRP in low- and high- $\mathrm{Cu}$ subsets).

The revised model presented in this report is based on multiple radiation damage features identified in mechanistic studies, as discussed in Chaps. 1 and 2. The best-understood damage features are MFs and copper-rich precipitates CRPs, which are represented by separate terms in the model. Other damage features have been identified, involving $\mathrm{Ni}, \mathrm{Mn}, \mathrm{Si}$ and $\mathrm{P}$, which occur in materials both with and without $\mathrm{Cu}$ (see Sects. 2.1, 2.3.2, 2.4.1 and [2,3]). The MFs are important in materials with low $\mathrm{Cu}$ while both the $\mathrm{MF}$ and precipitation mechanisms are important in higher- $\mathrm{Cu}$ materials. In this report, the two terms are referred to as the "MF term" and the "CRP term," but it should be noted that in an empirically calibrated model, the model terms will reflect all the physical embrittlement mechanisms that are active in the data and not necessarily according to the labels used to refer to the model terms. Thus, associating one term 
with MFs and the other with precipitation mechanisms is a useful concept that is physically motivated, but it does not restrict the features each empirically calibrated model term may reflect.

The sum of the MF and CRP model terms estimates the total Charpy TTS at $30 \mathrm{ft}-\mathrm{lb}(41 \mathrm{~J})$. The U.S. surveillance TTS data are traditionally reported in degrees Fahrenheit, the same units used in the embrittlement model in Eq. (4-1). TTS may be converted by ${ }^{\circ} \mathrm{C}=\left({ }^{\circ} \mathrm{F}-32\right) / 1.8$.

$$
T T S=M F \text { term }+ \text { CRP term }
$$

The modeling guidelines specify that each part of the model should be calibrated on the most relevant subset of data. First, the MF term was initially developed on the low-Cu data, where, based on insights from guideline 1, it is the only term that applies. Then the MF term was held fixed while developing the CRP term. This approach provides temporary independence of the data domains for calibrating MF and CRP terms, preventing the fitting constants for fluence, temperature, chemistry, and product form variables that are present in both MF and CRP terms from trading off numerically during the nonlinear least squares iteration. Second, all available low-flux data, at both low and high $\mathrm{Cu}$, were used to calibrate the two effective fluence fitting constants, which apply at all $\mathrm{Cu}$ levels. A similar rationale led to use of both low- and high-Cu data for calibrating the $\mathrm{P} * \mathrm{Mn}$ term, which also applies at all $\mathrm{Cu}$ levels. Because low-flux data are scarce, all available low-flux data were used rather than setting aside a validation sample. Third, SRM plates were modeled as a separate product form so that the large number of measured TTS values for the three SRM plates could not unduly bias the model. By using a separate coefficient, the SRM plates, which are not part of any operating reactor vessel, cannot affect the calibration of coefficients for plates that are used in operating vessels. Finally, the PWR dataset ${ }^{1}$ is almost five times the size of the BWR dataset and so would dominate any model calibrated to the combined PWR and BWR data, so most of the preliminary model development was conducted on the PWR data; then the differences between the resulting PWR model and the BWR data were analyzed. This approach avoids the possibility that BWR trends could be masked by the much larger amount of PWR data, and it avoids confounding $\mathrm{T}_{\mathrm{i}}$ and flux, which both have relatively low values in the typical BWR data. ${ }^{2}$ The PWR data have a substantial range in $T_{i}$ and are not as much affected by flux, adequately separating these effects.

\subsubsection{Summary of Steps Taken to Develop a Draft Model}

The first step in the data analysis was to confirm the earlier definition of the border between low-Cu and high- $\mathrm{Cu}$ data for separating the MF and CRP mechanisms. The threshold value of $0.072 \mathrm{wt} \%$ for high- $\mathrm{Cu}$ behavior had been calibrated in earlier modeling efforts on smaller databases, so the purpose was to check that value on the current database. Then the MF part of the model was calibrated to all the available low-Cu PWR data. Calibration and validation samples were not used in this preliminary MF calibration step because the low-Cu PWR dataset (219 points) is not considered large enough to set aside a statistically meaningful number of data values for later use in a model validation step. Next, the CRP term was developed on the $90 \%$ calibration sample of the high-Cu PWR data, holding the MF term fixed. Then the BWR data were compared to the resulting PWR model to determine what changes to the PWR model would be needed to predict BWR data. The only change that was necessary was to add a flux effect (effective fluence) in both terms of the model, which was then calibrated to all BWR data and $90 \%$ of the PWR data at medium and lower flux. Then the flux-related fitting constants were held fixed, and the rest

\footnotetext{
1"Database" refers to the complete set of surveillance data while "dataset," "subset," or just "set" refer to part of the database.

2“"Typical BWR" data are those with flux $<2 \times 10^{10} \mathrm{n} / \mathrm{cm}^{2} / \mathrm{s}$ and $\mathrm{T}<535^{\circ} \mathrm{F}$. There are other data from surveillance specimens irradiated inside the shroud in BWR plants, and in an unusual BWR plant (Big Rock Point), that have flux and/or temperatures comparable to and higher than typical PWR irradiations.
} 
of the fitting parameters in the CRP term were calibrated to a $90 \%$ sample of PWR and BWR high-Cu data. This produced a draft model.

The draft model was reviewed in some detail, and several issues with it were identified. The P effect in the draft model (initially applicable only to higher $\mathrm{Cu}$ material) was contrary to clear $\mathrm{P}$ effects in lowor no- $\mathrm{Cu}$ materials tested in single-variable IVAR experiments [as shown in Ref. [4,5], Sect. 6.4.2, and Fig. 6.12 (c)] and to observations of $\mathrm{P}$ effects in low-Cu material worldwide, as discussed in Sect. 2.4.1. A significant interaction of $\mathrm{P}$ and $\mathrm{Mn}$ subsequently identified in the low-Cu surveillance data is the likely cause of the initial lack of an observed $\mathrm{P}$ effect in that subset of data. Potential improvements were identified for the draft method of choosing $\mathrm{Cu}$ saturation limits. An improved form for modeling $\mathrm{P}$ precipitation was also introduced. During the draft model review, the need for flux effects modeled by effective fluence in both MF and CRP terms, which was originally noted in the surveillance data, was confirmed on the independent IVAR database. The flux effects in both low and higher $\mathrm{Cu}$ IVAR materials are discussed in Sect. 6.6, and the flux effects in both low and higher $\mathrm{Cu}$ surveillance data are discussed in Sect. 5.2. These observations led to a second iteration of model calibration which is described in Sect. 4.1.2.

\subsubsection{Summary of Steps Taken to Calibrate the Baseline TTS Model}

In the second modeling iteration, the MF part of the model was again calibrated initially to all the available low-Cu PWR data. A preliminary MF term consisting of only a single calibrated coefficient, the temperature term from the draft model, and square root of fluence, was used in an attempt to model the interaction of $\mathrm{P}$ and Mn explicitly on the low-Cu PWR data without confounding that effect with product form effects and the effects of flux in BWR data. (Confounding of Mn and product form arises mainly from the lower average $\mathrm{Mn}$ in forging materials, as shown in more detail in Chap. 5, where effects of individual variables on TTS are discussed.) Then the resulting $\mathrm{P} * \mathrm{Mn}$ term was held fixed while three coefficients were calibrated on the low-Cu PWR data to account for all other effects of chemistry and microstructure in the low-Cu forging, plate, and weld materials. Because of this procedure, the proper interpretation of the MF coefficients is that they reflect composition and other differences between product forms, given that an interaction effect of $\mathrm{P}$ and $\mathrm{Mn}$ is explicitly modeled. In particular, a significant relationship between $\mathrm{P}, \mathrm{Mn}$, and $\mathrm{Ni}$ can be found by regression analysis in the low-Cu data, so the $\mathrm{P}^{*} \mathrm{Mn}$ term and coefficients in the MF term implicitly contain an effect of $\mathrm{Ni}$ as well as product form and Mn. Efforts to explicitly model the known Ni effect in low-Cu data [shown in Sect. 6.4 and Fig. 6.12 (a)] were unsuccessful, probably because of confounding.

The MF term, including the $\mathrm{P}$ and Mn fitting constants, was then held fixed while updating the coefficients and constants of the CRP term on a 90\% sample of the high-Cu PWR and BWR data. The other $10 \%$ sample of high-Cu data had been selected by random sampling as a validation set and were not used in this step. For this preliminary CRP update, the effective fluence fitting constants were held fixed at the values that had been calibrated in the draft model, to be updated in the next step for compatibility with the updated MF and CRP terms. Exploratory CRP term modeling was conducted in two forms, both with a single CRP coefficient, used to attempt to calibrate the Mn effect explicitly, and with separate CRP coefficients for forgings, plates in Combustion Engineering (CE) and non-CE manufactured vessels (which are significantly different in their shifts), welds, and SRMs. It was found that an explicit effect of Mn could not be calibrated in the CRP term, given the fact than the P*Mn effect and different coefficients for forgings, plates and welds were already included in the MF term. As in the MF term, regression analysis found that $\mathrm{Mn}$ is strongly correlated with and can be estimated from $\mathrm{Cu}$ and $\mathrm{Ni}$ in the high-Cu data, complicating the modeling of $\mathrm{Mn}$ in the surveillance data. The $\mathrm{Mn}$ effects on higher $\mathrm{Cu}$ materials are discussed in more detail in Sects. 5.1, 6.4, and 6.7.

The next step was to update the preliminary effective fluence (flux) fitting constants for compatibility with the updated MF and CRP terms. This was done in conjunction with an update to the P 
and Mn coefficients in the MF term. It was by then clear that the effective fluence and $\mathrm{P} * \mathrm{Mn}$ terms would be the only terms used for modeling those effects in both low- and high-Cu data, and thus they should be calibrated to both low- and high-Cu data. The dataset used for calibrating the four constants included all available low-flux data $\left(<5 \times 10^{10} \mathrm{n} / \mathrm{cm}^{2} / \mathrm{s}\right)$, all low-Cu data $(\leq 0.072 \mathrm{wt} \%)$, and $90 \%$ of the high-Cu PWR and BWR data. Better balance in the dataset is achieved by using all data in the subsets where data are relatively scarce (low flux and low $\mathrm{Cu}$ ) and a random sample of the data at high $\mathrm{Cu}$ and higher flux, which are more plentiful. The updated flux-related and $\mathrm{P} * \mathrm{Mn}$ fitting constants (four total) were held fixed for the remaining modeling.

The MF fitting constants (other than the four effective fluence and $\mathrm{P}^{*} \mathrm{Mn}$ constants) were then updated on the $90 \%$ calibration set of low-Cu data (BWR and PWR). This produced the final MF term coefficients and temperature slope. The final MF term and effective fluence constants were held fixed while the final constants for the CRP term were updated using the $90 \%$ sample of high-Cu BWR and PWR data as the fitting set. Thus, except for the four effective fluence and $\mathrm{P} * \mathrm{Mn}$ fitting constants, all fitting constants can be validated on the $10 \%$ samples of low- and high-Cu data reserved for that purpose. There is also partial validation of the two $\mathrm{P}^{*} \mathrm{Mn}$ fitting constants on the $10 \%$ random sample of high- $\mathrm{Cu}$ data. There is no validation set for the flux-related constants, as the low-flux data are so scarce that all available data were used to calibrate them. However, the comparison with flux effects in the IVAR data in Sect. 6.6 indicates that the physically based extrapolation of IVAR data to the BWR flux range is reasonably consistent with the flux-sensitive part of the TTS model. (see Fig. 6.20). The last remaining capsules (A, B, C) from the BWR Supplemental Surveillance Program (SSP) may provide additional lowflux surveillance data for validation when available [6].

The result of the fitting process described above is that the 2 flux-related constants in the model are based on all low flux data (and most of the high flux data), the 2 constants in the $\mathrm{P}^{*} \mathrm{Mn}$ part of the MF term are based on all available low- $\mathrm{Cu}$ data (and most of the high-Cu data) and the 22 other fitting constants are calibrated on a 90\% sample of BWR \& PWR data. The CRP fitting constants are based on a fixed MF model calibrated mainly to low-Cu data, which means that the $\mathrm{P}$ and $\mathrm{T}_{\mathrm{i}}$ terms and the product form coefficients in the CRP part of the model should be interpreted as the additional effect of those variables in high-Cu material, relative to the effects present in low-Cu material. This fitting approach provides both a good fit to both the low $\mathrm{Cu}$ data and to the higher $\mathrm{Cu}$ data, as is shown in Sect. 4.3.

\subsection{TTS Model}

Throughout this section, selected results are presented in the order originally developed, following the steps summarized above. Most preliminary models have been omitted to avoid confusion with the final model. The statistical justification for the final model form given in this section is presented in Sect. 4.3 , in the form of non-significant residual trends in all variables using the model, and in Chap. 5, in the form of significance analyses of the key variable effects.

\subsubsection{Threshold for High-Cu Behavior}

This subsection reports a preliminary study performed to see if the previously calibrated value of $\mathrm{Cu}$ threshold is reasonable for the enlarged dataset. This check is needed because the first step in the modeling effort is to split the data into low- and high- $\mathrm{Cu}$ sets based on a $\mathrm{Cu}$ threshold value, then calibrate an MF model on the low $\mathrm{Cu}$ data. Thus, the threshold value of $\mathrm{Cu}$ is needed to define "low $\mathrm{Cu}$ " before doing any calibration on the TTS data. The temperature dependence from the Jones \& Williams model [7], based on a completely separate set of data, is used to help confirm the threshold.

Some lack of precision in determining the threshold copper level for separating low-Cu and high-Cu behavior is unavoidable. For example, there is no practical difference between splitting the surveillance data at 0.07 and $0.072 \mathrm{wt} \% \mathrm{Cu}$, as there is only one datapoint in that range. The datapoint happens to be 
in the BWR subset, so that splitting on either 0.07 or $0.072 \mathrm{wt} \% \mathrm{Cu}$ produces identical results on the PWR subset. Thus, the uncertainty in the threshold is at least $0.002 \mathrm{wt} \%$, and, based on the method of determining it below, the actual uncertainty is probably at least $\pm 0.005 \mathrm{wt} \%$.

To determine the best value of copper for the split, the simple low-Cu model of Jones and Williams [7] (which was not used in the original calibration of the 0.072 value in [1]) was used to approximately account for the effects of temperature and fluence. The Jones and Williams model is usually presented in terms of Celsius temperature and dose measured by dpa. After conversion to Fahrenheit temperature and fluence in $\mathrm{n} / \mathrm{cm}^{2}$, it takes the form

$$
\text { Preliminary } M F \text { term }=2.766 \times 10^{-8}\left(1-0.001302 * T_{\mathrm{i}}\right) \sqrt{ } \phi t
$$

where the coefficient $2.766 \times 10^{-8}$ is fitted to the 214 PWR points in the Version 7-04 database with $\mathrm{Cu} \leq$ $0.072 \mathrm{wt} \%$. The temperature coefficient was not fitted; it was merely converted from the Jones and Williams value.

To determine whether $0.072 \mathrm{wt} \% \mathrm{Cu}$ is a reasonable splitting value, the available PWR data were split by $\mathrm{Cu}$ into categories in the range 0.02 to $0.10 \mathrm{wt} \% \mathrm{Cu}$. The lowest categories had an increment of 0.01 in copper, and categories above $0.05 \mathrm{wt} \% \mathrm{Cu}$ (where the threshold was expected to be) had an increment of 0.005 . For each category, the standard deviation and average value of the residuals relative to Eq. (4-2) were evaluated, and the average copper for each category was calculated. The coefficient $a_{1}=$ $2.766 \times 10^{-8}$ in Eq. (4-2) was also refitted to the data in each $\mathrm{Cu}$ category, holding the temperature coefficient and fluence exponent fixed.

Plotting the results, one can identify the approximate transition from MF to MF + CRP behavior by abrupt changes in three different measures: the best fit coefficient $a_{1}$, the standard deviation of residuals about Eq. (4-2), and the average residual relative to Eq. (4-2). Up to about $0.07 \mathrm{wt} \% \mathrm{Cu}$ in the PWR data, the results on all three measures, although scattered, do not show a consistent trend with $\mathrm{Cu}$. For $\mathrm{Cu}$ categories above $0.07 \mathrm{wt} \%$, the best fit coefficient increases, the average residual becomes increasingly negative, and the standard deviation increases, all indications that the CRP term must be added to reasonably fit the data. The results are shown in Figs. 4.1 through 4.3. The conclusion from this study is that $0.072 \mathrm{wt} \% \mathrm{Cu}$ is a reasonably good threshold for high copper behavior in the updated surveillance database. The same value was used in the NUREG/CR-6551 modeling effort [1] and all model revisions since then. An independent analysis of $\mathrm{Cu}$ threshold $\left(\mathrm{Cu}_{\mathrm{min}}\right)$ in the IVAR database found values in the range 0.06 to $0.08 \mathrm{wt} \%$, which are consistent with this estimate [see Sect. 6.4.1 and Fig. 6.8 (d)]. 


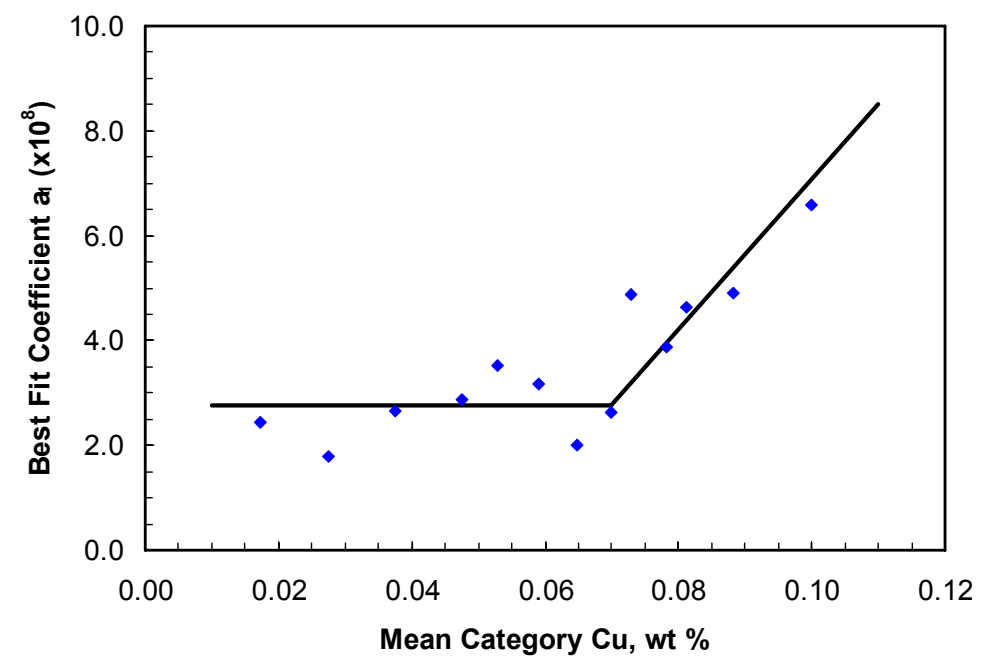

Fig. 4.1. Apparent location of $\mathrm{Cu}$ threshold based on coefficient in Eq. (4-2) fitted to each Cu category.

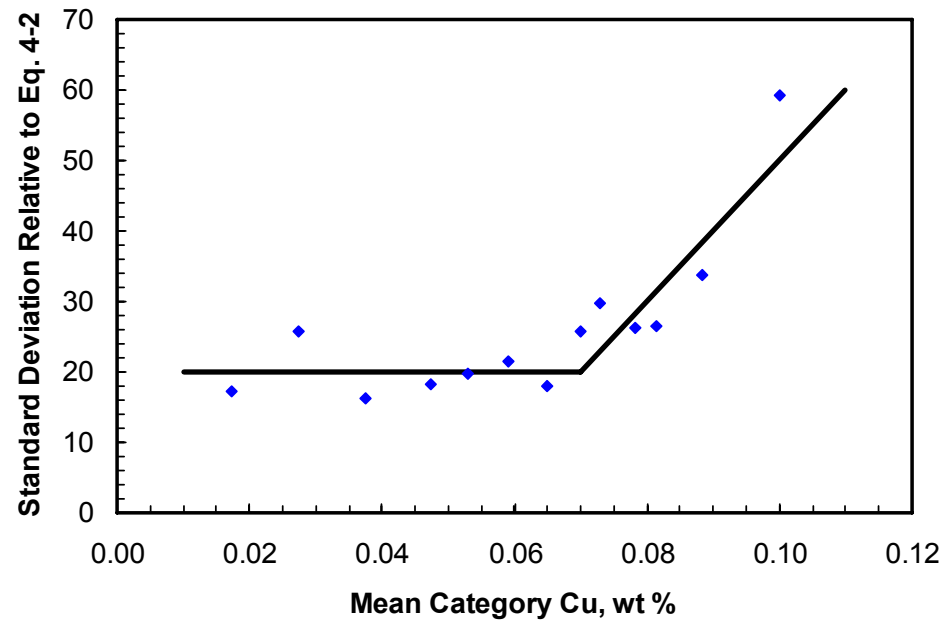

Fig. 4.2. Apparent location of $\mathrm{Cu}$ threshold based on standard deviation of residuals relative to Eq. (4-2) for each Cu category. 


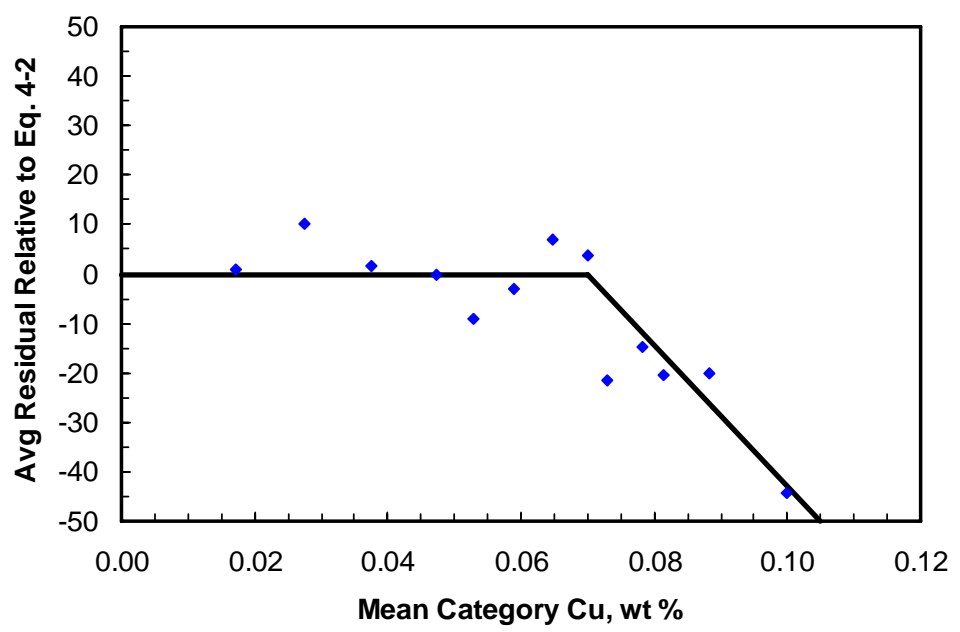

Fig. 4.3. Apparent location of $\mathrm{Cu}$ threshold based on average residual relative to Eq. (4-2) for each $\mathrm{Cu}$ category.

\subsubsection{Matrix Feature (MF) Term}

Having established that $\mathrm{Cu}=0.072$ wt \% is a reasonable value for separating low-Cu behavior (which can be modeled by just an MF term) from high-Cu behavior (which requires both MF and CRP terms), the next task is to calibrate an appropriate MF term for the low-Cu data. While fitting the Jones and Williams model in the preliminary work, it was apparent that the linear temperature term in that model is numerically more stable than the exponential (Arrhenius) form that had been previously used $[1,8]$. The ability to fit over the limited range of irradiation temperatures in surveillance data $\left(522-562^{\circ} \mathrm{F}\right.$ for PWRs) is comparable. Considering the comparable fitting capability, numerical stability advantages, and common use of the linear form in the radiation damage field (e.g., Ref [7] and Sect. 2.3.4), a decision was made to use the linear temperature term for the revised low-Cu model.

A decision was also made to hold the fluence exponent at the theoretical value of 0.5 for MF, rather than fit it to the data as in prior modeling efforts. This decision follows current common practice in the radiation damage field (see Sect. 2.4.2), and it was supported by preliminary models that gave fitted exponents slightly greater than 0.5 on the expanded low-Cu dataset. The fitted exponent in the July 2000 modeling effort [8] was slightly less than 0.5 , so the current and prior results of fitting the exponent could be viewed as clustering about 0.5 , depending on the database. A final advantage to fixing the MF term exponent at 0.5 is that it then cannot trade off numerically with the fluence function in the CRP term.

As a preliminary study, an MF term containing a single calibrated coefficient, the temperature term from the draft model, and the square root of fluence was calibrated to the Version 8-04 PWR low-Cu fitting set (219 points). The low-Cu residuals relative to this model [defined in Eq. (1-1)] show apparent trends with $\mathrm{P}, \mathrm{Mn}$, and $\mathrm{P}^{*} \mathrm{Mn}$. The slope of the low-Cu residuals is statistically significant for $\mathrm{Mn}$ and $\mathrm{P}^{*} \mathrm{Mn}$ but not for $\mathrm{P}$. However, the slope of the residuals with $\mathrm{P}$ is significant on the broader set of data affected by the final $\mathrm{P}^{*} \mathrm{Mn}$ interaction term (both low and high $\mathrm{Cu}$ ), as discussed in Sect. 5.1.4. Moreover, the $\mathrm{P}$ effect in the MF term is in a direction consistent with the $\mathrm{P}$ effects previously calibrated in NUREG/CR-6551 [1] and Draft 2000 models [8], as shown in Fig. 4.4, such that increased P appears to produce increased shift in low-Cu steels. The slope of the residuals throughout this section is always opposite in sign to the slope of the effect that is missing from the model. Thus, the negative slope to the residuals in Fig. 4.4 can be cured by adding to the model a term with a positive slope with P. 


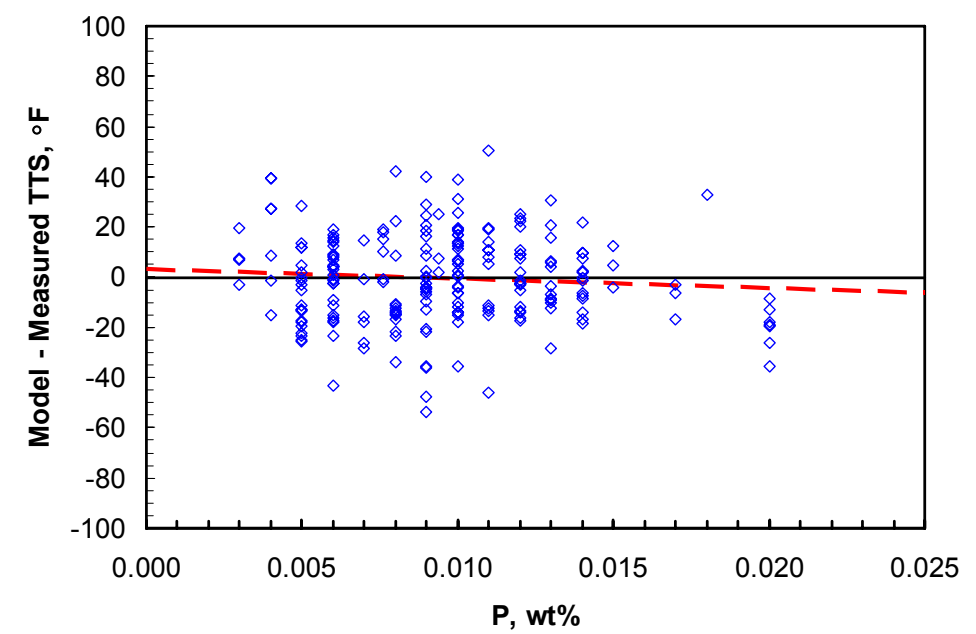

Fig. 4.4. Residuals in low-Cu PWR data relative to single coefficient model, plotted against $\mathbf{P}$. Dashed trend is not statistically significant.

The observation that the slope of the $\mathrm{P}$ residuals is not statistically significant in the preliminary models of the low-Cu surveillance database is contrary to studies worldwide that have found significant effects of $\mathrm{P}$ in low-Cu data [5,7], NUREG/CR-6551 and the previous Draft 2000 models [1,8], and controlled single-variable studies of the effect of $\mathrm{P}$ on no-Cu steel shown in Sect. 6.4 and Fig. 6.12c, which clearly show an effect of $\mathrm{P}$. The reason for the lack of statistical significance for the $\mathrm{P}$ effect in low-Cu PWR surveillance data appears to be the interaction of $\mathrm{P}$ with $\mathrm{Mn}$ that is observed in the current surveillance database. The residual effect of $\mathrm{P}$ is significant in high-Mn, low-Cu steels, as shown in Fig. 4.5, but not in low-Mn, low-Cu steels, as shown in Fig. 4.6. When those two sets are combined, the trend with $\mathrm{P}$ is not significant over the low-Cu PWR data currently available (all two sided tests on slope of fitted residual trend, $\mathrm{p}<0.05$ for significance). The high-Mn plot is for $\mathrm{Mn}>1.35 \mathrm{wt} \%$, while the low-Mn plot is for $\mathrm{Mn} \leq 1.35 \mathrm{wt} \%$, with $\mathrm{Ni}$ in the typical range $(\mathrm{Ni}>0.5 \mathrm{wt} \%)$ in both plots. The value of $1.35 \mathrm{wt} \%$ for $\mathrm{Mn}$ is approximately mid-range for the allowable content in the specification for RPV plates of SA 533 grade B class 1 steel, and is also approximately mid-range in the Mn values for a number of PWR data subsets evaluated in the analysis. Thus, the choice of $1.35 \mathrm{wt} \%$ is arbitrary but reasonable as a split for low- and high-Mn groups in these preliminary analyses. Moreover, the final calibrated term for $\mathrm{Mn}$ in the model is not dependent on the preliminary splits used for the various data subsets because it was calibrated to a broader dataset (see Sect. 5.1.5). The no-Cu IVAR materials showing a strong effect of $\mathrm{P}$ in controlled experiments had reasonably high $\mathrm{Mn}=1.6 \mathrm{wt} \%$, so these observations are reasonably consistent.

In addition to the residual trends with chemistry, there are statistically significant differences in average residual among the different product forms. This was determined by t-tests on the low-Cu residuals relative to a preliminary single-coefficient MF term model (without a $\mathrm{P} * \mathrm{Mn}$ term). Specifically, there is a statistically significant difference in average residuals for forging and plate and for forging and weld based on two sided t-tests and $\mathrm{p}<0.05$. Plate and weld average residuals are also significantly different on the broader dataset to which the MF coefficients apply (both low and high Cu materials), as discussed in Sect. 5.1. Thus, three separate MF coefficients were used for forging, plate, and weld. 


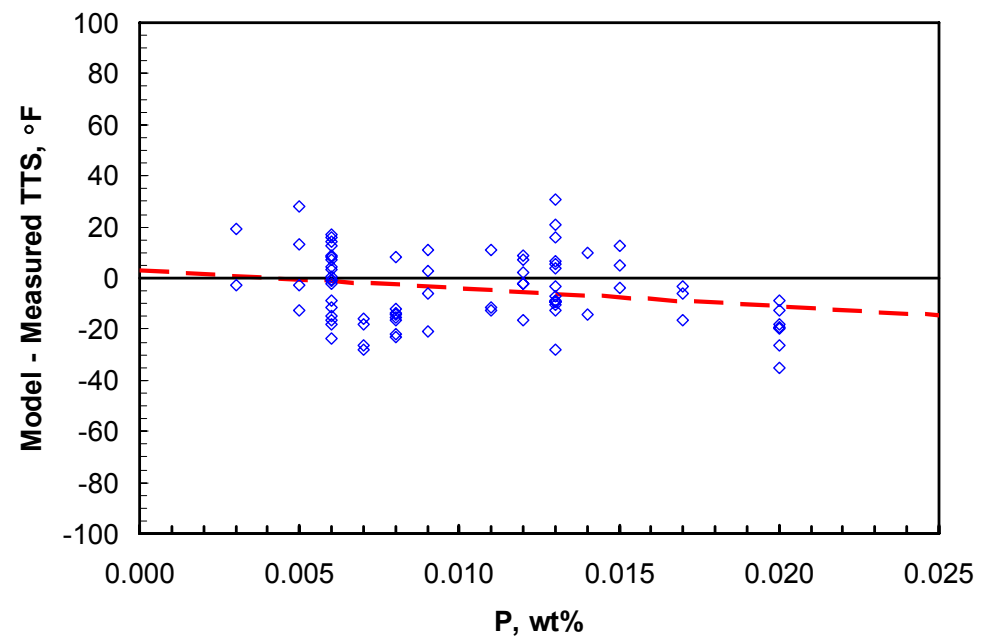

Fig. 4.5. Residuals in low-Cu PWR data with Mn $>1.35$ wt $\%$ relative to single coefficient model, plotted against $P$. Dashed trend is statistically significant.

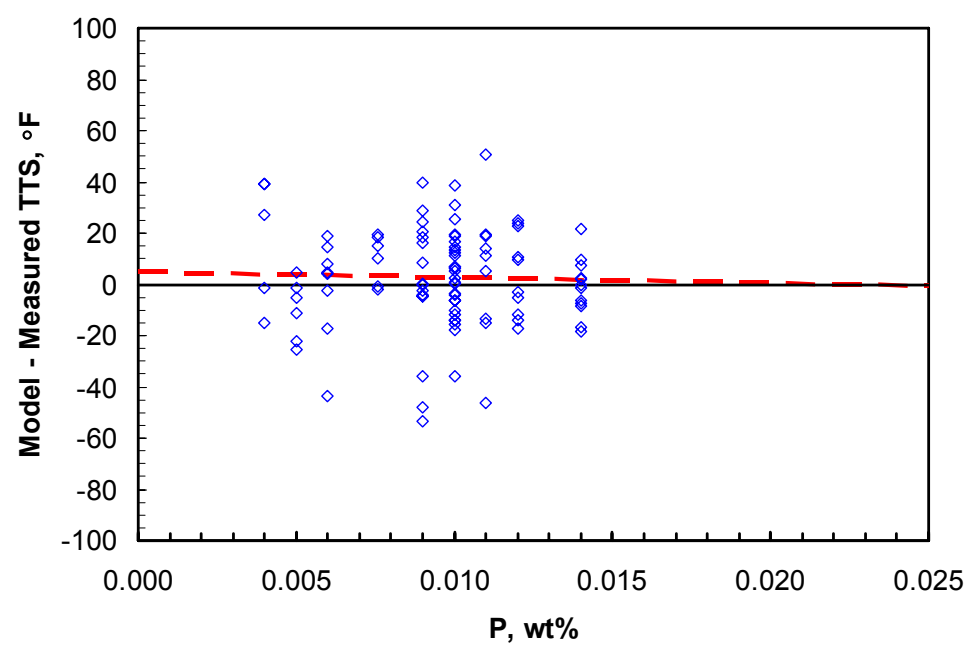

Fig. 4.6. Residuals in low-Cu PWR data with $\mathrm{Mn} \leq 1.35$ wt $\%$ relative to single coefficient model, plotted against $P$. Dashed trend is not statistically significant. 
The MF term was modified from the form shown in Eq. (4-2) to include a $\mathrm{P} * \mathrm{Mn}$ term and three separate product form coefficients for forging, plate, and weld. These enhancements eliminated the significant residual trends with $\mathrm{Mn}$ and $\mathrm{P} * \mathrm{Mn}$, flattened further the trend with $\mathrm{P}$, and eliminated the significant differences in average residual between the three product form groups. The final MF term, calibrated to a $90 \%$ sample of PWR and BWR low-Cu data in the analysis process described above, is

$$
\text { MF term }=A\left(1-0.001718 T_{i}\right)\left(1+6.13 P M n^{2.47}\right) \sqrt{\phi t_{e}}
$$

Where

$$
A=\left\{\begin{array}{cc}
1.140 \times 10^{-7} & \text { for forgings } \\
1.561 \times 10^{-7} & \text { for plates } \\
1.417 \times 10^{-7} & \text { for welds }
\end{array}\right\}
$$

Both MF and CRP terms use an effective fluence, calculated from fluence $\phi t$ and flux $\phi$ as

$$
\phi t_{e}=\left\{\begin{array}{c}
\phi t \quad \text { for } \phi \geq 4.39 \times 10^{10} \\
\phi t\left(\frac{4.39 \times 10^{10}}{\phi}\right)^{0.259} \text { for } \phi<4.39 \times 10^{10}
\end{array}\right\}
$$

The effective fluence in Eq. (4-4) is greater than actual fluence for flux below $4.39 \times 10^{10} \mathrm{n} / \mathrm{cm}^{2} / \mathrm{s}$, while above that breakpoint value, the fluence and effective fluence are the same (see Sect. 5.2.1 for discussion of effective fluence). The breakpoint, $4.39 \times 10^{10}$, and the exponent, 0.259 , were fitted simultaneously to all available low-flux data $\left(<5 \times 10^{10} \mathrm{n} / \mathrm{cm}^{2} / \mathrm{s}\right)$, all low-Cu data $(<0.072 \mathrm{wt} \%)$, and $90 \%$ of the high-Cu PWR and BWR data, as described in Sect. 4.1.2. Moreover, the fitted breakpoint, $4.39 \times 10^{10}$, remained relatively stable in preliminary and final calibration. The breakpoint approach was the result of preliminary analysis that showed only a slight residual trend with flux, in the expected direction but not statistically significant, in the PWR calibration data (which are mainly in the range $3 \times$ $10^{10} \leq \phi \leq 2 \times 10^{11} \mathrm{n} / \mathrm{cm}^{2} / \mathrm{s}$ ). This may have been due to the narrow flux range and other limitations of the PWR data, as there is strong evidence of flux effects in the PWR range in IVAR data, as discussed in more detail in Sect. 6.6.

Figure 4.7 shows typical curves based on Eq. (4-3) using the effective fluence form in Eq. (4-4) at assumed flux values of $1 \times 10^{9}$ and $1 \times 10^{10}$ and for any flux greater than $4.39 \times 10^{10} \mathrm{n} / \mathrm{cm}^{2} / \mathrm{s}$. The shifts estimated by the MF term at $1 \times 10^{9}$ and $1 \times 10^{10} \mathrm{n} / \mathrm{cm}^{2} / \mathrm{s}$, respectively, are factors of 1.63 and 1.21 times the shift at $4.39 \times 10^{10} \mathrm{n} / \mathrm{cm}^{2} / \mathrm{s}$ or higher flux. The statistical justification for including the effective fluence term in the MF term is given in Sect. 5.2, and additional supporting evidence is given in Sect. 6.6.2.

The MF term model given by Eq. (4-3) is a good fit to the low-Cu data, as shown by the plot of model TTS vs measured TTS in Fig. 4.8. In that figure, a perfect fit with no scatter would put all data along the 1:1 diagonal line. In any real case, there is scatter, but Fig. 4.8 shows the scatter is evenly distributed around the 1:1 line with no consistent deviation above or below. Both PWR and BWR points are shown, with open symbols for the calibration data and filled symbols for validation data. The good fit is confirmed by analyzing the residuals relative to Eq. (4-3), using all available low-Cu data (calibration and validation subsets, PWR and BWR, a total of 246 points). The residuals do not show statistically significant trends in fluence, flux, $\mathrm{T}_{\mathrm{i}}$, time, chemistry variables, product form, or the chemical interactions $\mathrm{P} * \mathrm{Mn}, \mathrm{P} * \mathrm{Ni}$, or $\mathrm{Mn} * \mathrm{Ni}$. Further details on the quality of fit and comparison of calibration and validation data are given in Sect. 4.3. 


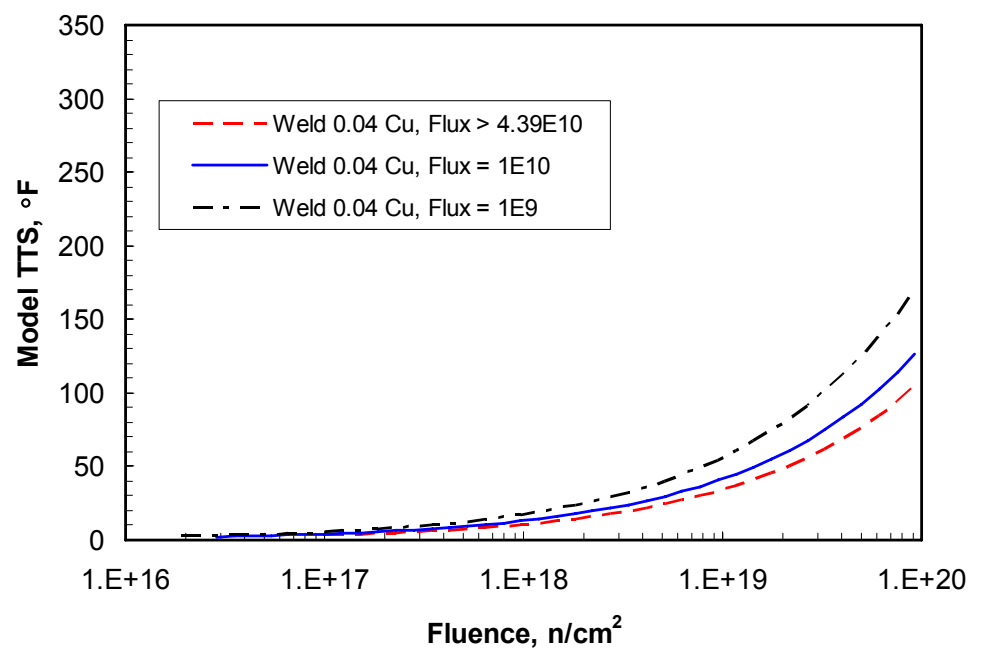

Fig. 4.7. Effect of effective fluence on MF term at various flux values.

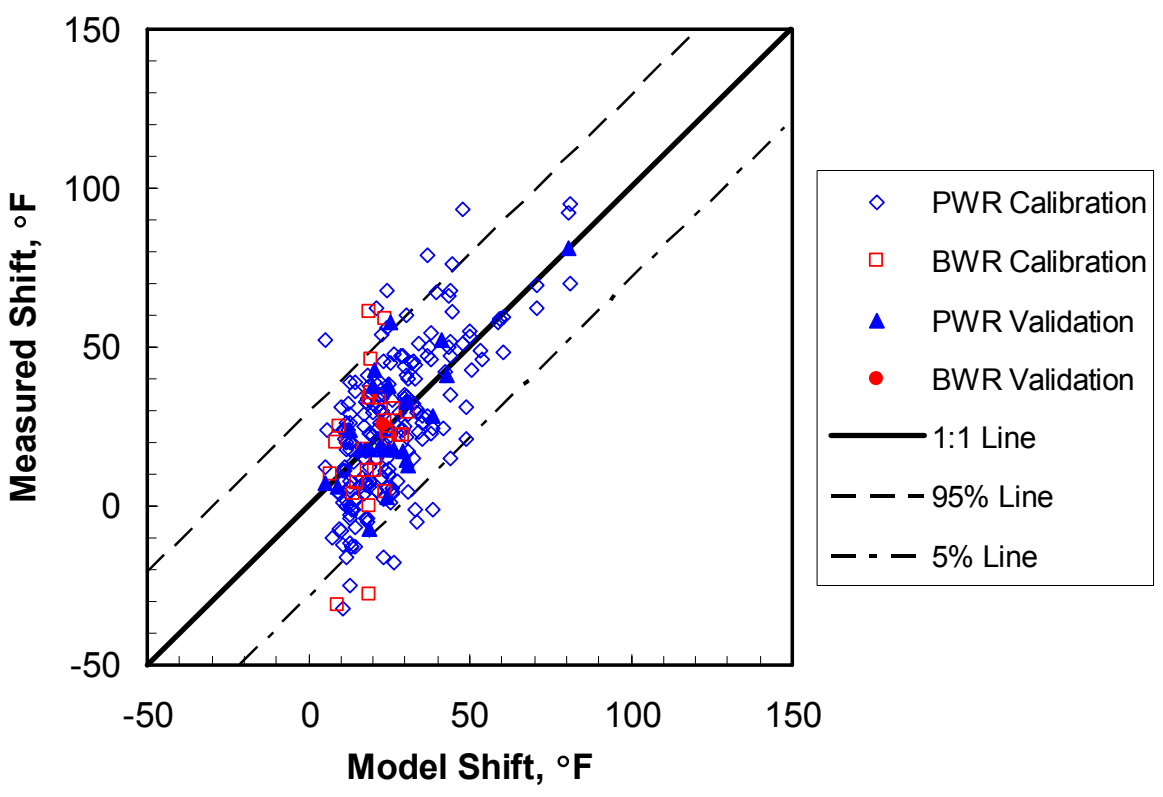

Fig. 4.8. Model shift vs measured shift, Eq. (4-3), all data with $\mathrm{Cu} \leq \mathbf{0 . 0 7 2}$ wt \%.

The $T_{i}$ term in Eq. (4-3a) has a steeper slope in the low-Cu surveillance data [-0.001718 in Eq. (4-3)] than in the Jones and Williams model [-0.001302 in Eq. (4-2)]. The different slopes may reflect differences in the materials used for calibration, different average values of fluence and other variables, or the fact that the temperature range of the surveillance data is smaller. The possibility of partial confounding of flux and temperature effects when fitting both effects simultaneously was ruled out by separately calibrating the temperature slope in narrow ranges of flux. The average of these subset values was the same as the overall calibration, and there was no obvious trend with flux, proving no apparent confounding of those variables. 
The effective fluence form shown in Eq. 4-4 is used in both MF and CRP terms; it is presented here because it is used in Eq. 4-3a. The effective fluence form was originally developed to cause the high $\mathrm{Cu}$ BWR data to agree with the preliminary model calibrated to high $\mathrm{Cu}$ PWR data. During preliminary modeling, using effective fluence in both the MF and CRP terms produced better fits to the high-Cu, lowflux data than using the effective fluence form in the CRP term only, as well as improving the fit to the low-Cu, low-flux data. Based on this empirical observation, the draft model from the first round of modeling had effective fluence in both terms. Significance tests on the draft model showed that disabling the flux effect by using fluence instead of effective fluence, in either the MF or CRP terms separately or both terms together, would produce significant mean residual errors on the low-flux BWR data with fluence $>10^{17} \mathrm{n} / \mathrm{cm}^{2}$. This provided a preliminary statistical justification for using effective fluence in both terms in the model, which was later confirmed on the baseline model as reported in Sect. 5.2.

There are only 27 low flux $\left(\phi<2 \times 10^{10} \mathrm{n} / \mathrm{cm}^{2} / \mathrm{s}\right)$ data points, all from exposure in BWRs, in the low$\mathrm{Cu}$ dataset. Four of those points are at such low fluence $\left(<2 \times 10^{17} \mathrm{n} / \mathrm{cm}^{2}\right)$ that very small shifts would be expected with or without a flux effect (see Fig. 4.7). Thus, the ability to statistically identify significant fitting trends or residual effects with respect to flux using just the low-Cu dataset is quite limited - there are not enough data, and the shifts in low-Cu data at such low fluence values are generally small. Using effective fluence in the MF term is justified on the broader dataset to which the MF term applies (both low and higher $\mathrm{Cu}$ materials) where the effective fluence term is significant, as shown in Sect. 5.2.

The flux effect in low $\mathrm{Cu}$ data was first identified in the surveillance data, but additional analysis of the low- $\mathrm{Cu}$ and no-Cu IVAR data confirmed that there is a statistically significant effect of flux in that dataset under controlled experimental conditions. The evidence in IVAR for a flux effect in no-Cu steels is shown in Sect. 6.6.2, providing independent support for a flux effect in the MF term. The IVAR data also show substantial effects of flux in the high-Cu materials, as has been known for some time (see Sect. 6.6.1 and [4,9]). The TTS and IVAR flux effects are in different flux ranges, reflecting the different flux ranges in those databases, but the physically based extrapolation of IVAR data to the BWR flux range is reasonably consistent with the flux-sensitive part of the TTS model (see Fig. 6.20).

\subsubsection{Copper-Rich Precipitate (CRP) Term}

As discussed in Sect. 2.2.3, the CRP term models the shift due to precipitation of $\mathrm{Cu}, \mathrm{P}$, and other elements, which is negligible at low fluence, rises rapidly over a higher range of fluence and saturates at high fluence. The appearance of the shift vs fluence curve is a plateau as shown in Fig. 4.9, with amplitude that depends on temperature, material chemistry, and product form, but not on fluence (above the saturation value) or on flux. The fluence values at which the CRP-related shift makes the transition to full amplitude depend on chemistry and flux (see Sects. 2.3.1, 2.3.2, and 2.3.3). High $\mathrm{Cu}$, low Ni, or low flux causes the transition to move toward lower fluence, while high $\mathrm{Ni}$, low $\mathrm{Cu}$, and higher flux cause the transition to move toward higher fluence. The saturating behavior and the effect of the variables is well known, shown with independent controlled experiments in IVAR data [4] and in British data [10], as discussed in Sect. 2.3.3. The precipitation behavior can be reasonably modeled by a tanh function, in the same form as Eq. (2-1) with $\mathrm{LSE}=0$ and $\mathrm{a}_{3}=1 / 2$, as is shown in Fig. 4.9, or by Avrami functions [4,11] which are similar in appearance to the tanh function. The alternative tanh form used in [12] to model the $\mathrm{Cu}$ effect was also tried, but it gave a substantially higher standard error (Se) than that used here. 


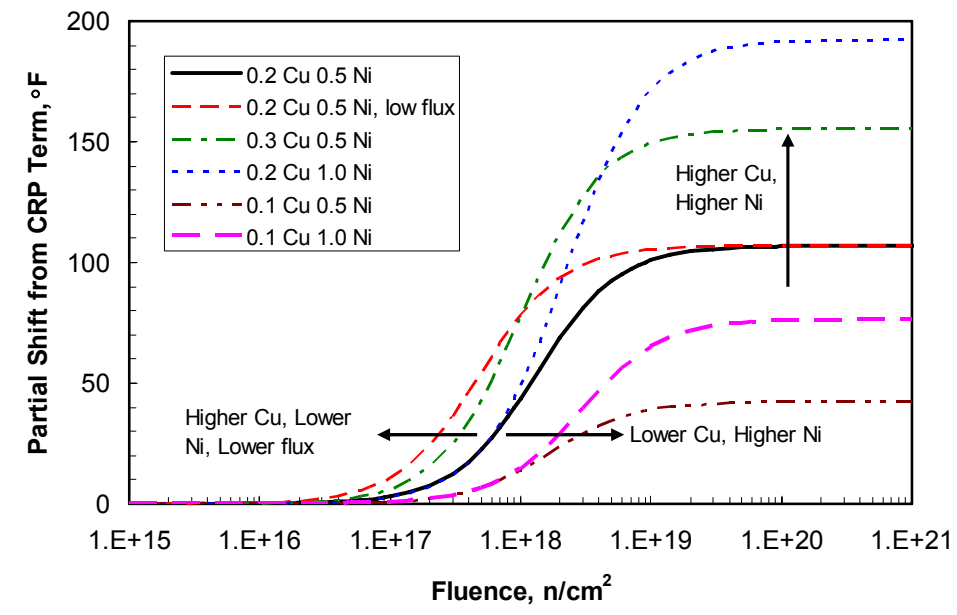

Fig. 4.9. Schematic of CRP term showing effect of key variables on changes in plateau height and location. Low flux is $10^{9} \mathrm{n} / \mathrm{cm}^{2} / \mathrm{s}$; all others are $10^{11} \mathrm{n} / \mathrm{cm}^{2} / \mathrm{s}$.

Following the steps summarized in Sect. 4.1, preliminary CRP term models were developed based on high-Cu PWR calibration data, which were then extended to adequately model the BWR data by simply incorporating an effective fluence (flux) model in both MF and CRP terms. Then both MF and CRP terms were recalibrated in a second round of modeling that addressed several issues in the preliminary models. The final result of this process is the following CRP term:

$$
\text { CRP term }=B\left(1+3.77 N i^{1.191}\right)\left(\frac{T_{i}}{543}\right)^{1.10} f\left(C u_{e}, P\right) g\left(C u_{e}, N i, \phi t_{e}\right)
$$

Where

$$
B=\left\{\begin{array}{c}
102.3 \text { for forgings } \\
102.5 \text { for plates in non - CE mfg. vessels } \\
135.2 \text { for plates in CE mfg. vessels } \\
155.0 \text { for welds } \\
128.2 \text { for SRM plates }
\end{array}\right\}
$$

It is clear in Eq. (4-5b) that calculations involving forgings and non-CE plates could use the same compromise coefficient of 102.4 with negligible $(\sim 0.1 \%)$ error. As in other recent model development efforts $[8,13]$, the shifts for plates in CE-manufactured vessels are statistically significantly larger than in non-CE manufactured vessels, an empirical observation for which an accepted physical explanation is not yet available. There is also a special SRM coefficient, used for matching results with the surveillance data on standard reference materials but not used for calculations related to materials in actual vessels (see Sects. 5.1.5 and 5.1.6 for detailed discussions of product form effects, 5.1.2 for Ni effect, and 5.2.2 for Ti dependence). 
The copper and phosphorus precipitation term in the model, $f\left(C u_{\mathrm{e}}, P\right)$, is built up from several functions. First, the effective $\mathrm{Cu}$ in solution, $\mathrm{Cu}$, which is the amount of $\mathrm{Cu}$ available for precipitation, is zero below a threshold value of 0.072 and is also limited by a maximum saturation value that reflects the amount of $\mathrm{Cu}$ available for precipitation. The $\mathrm{Cu}_{\max }$ value appears to depend on $\mathrm{Ni}$ concentration, heat treatment details, and possibly other factors (see Sects. 2.3 and 4.2.1). Hence, an effective $\mathrm{Cu}$ is defined as

$$
C u_{e}=\left\{\begin{array}{c}
0 \text { for } C u \leq 0.072 w t \% \\
\min \left[C u, \operatorname{Max}\left(C u_{e}\right)\right] \text { for } C u>0.072 w t \%
\end{array}\right\}
$$

where the upper limits

$$
\operatorname{Max}\left(\mathrm{Cu}_{e}\right)=\left\{\begin{array}{c}
0.243 \text { for typical }(\mathrm{Ni}>0.5 w t \%) \text { Linde } 80 \text { welds } \\
0.301 \text { for Linde } 1092 \text { welds } \\
0.370 \text { for all other materials }
\end{array}\right\}
$$

The $\mathrm{Cu}$ saturation limits, $\operatorname{Max}\left(\mathrm{Cu}_{\mathrm{e}}\right)$, can only be calibrated for materials with $\mathrm{Cu}$ above the limits. Only the materials that have very high $\mathrm{Cu}(\mathrm{Cu}>0.243 \mathrm{wt} \%$ for the lowest limit in the current database) can affect the calibrated saturation limits because materials with $\mathrm{Cu}$ below the limits show only increasing shifts with higher $\mathrm{Cu}$, with no limit behavior at all. The Linde 80 weld group is the only subset that has enough high- $\mathrm{Cu}$ data, with high enough $\mathrm{Cu}$ levels, to have confidence in the calibrated limits. This situation is discussed in detail in Sects. 5.1.3 and 7.2.

None of the forging materials in the surveillance database and only one of the plates have nominal $\mathrm{Cu}$ values above $0.243 \mathrm{wt} \%$ (and that plate is just above the limit at $0.25 \mathrm{wt} \%$ ), so the upper limits on $\mathrm{Cu}$ available for precipitation cannot be determined from the TTS database. Base metals should use the full $\mathrm{Cu}$ level, at least up to $\mathrm{Cu}=0.25 \mathrm{wt} \%$. This point is reiterated in Sect. 7.2.3, where the issue is further discussed under "Treatment of base metals."

The two large weld groups that have at least six welds with $\mathrm{Cu}>0.243 \mathrm{wt} \%$ (Linde 80 and Linde 1092) have individually calibrated $\mathrm{Cu}$ limits. It must be noted that the weld flux is only used as a means of grouping welds - there is no implication that weld flux directly affects the $\mathrm{Cu}$ limits. Most of the Linde 80 weld group have $0.52 \leq \mathrm{Ni} \leq 0.72 \mathrm{wt} \%$, although one Linde 80 weld has much lower $\mathrm{Ni}(\sim 0.1 \mathrm{wt} \%)$. The "typical Linde 80" group is defined as those welds with nominal $\mathrm{Ni}>0.5$ to distinguish the usual Linde 80 welds from the unusual low-Ni weld. The Linde 1092 weld group is generally in a higher $\mathrm{Ni}$ range than Linde 80 welds range, but some Linde 1092 welds have Ni values as low as $0.6 \mathrm{wt} \%$. The "all other" welds are generally at lower $\mathrm{Ni}(\leq 0.5 \mathrm{wt} \%)$. An attempt was made to consider Ni in calibrating the $\mathrm{Cu}_{\max }$ limits, but it was unsuccessful, as discussed in Sects. 5.1.3 and 7.2.

The effective $\mathrm{Cu}$ and $\mathrm{P}$ concentrations are simply combined on the basis that the observed precipitates generally include both $\mathrm{Cu}$ and $\mathrm{P}$ (see Sects. 2.3.2 and 2.4.1; see also [14,15]). Williams has noted that the mechanistic role of $\mathrm{P}$ appears to be similar to that of $\mathrm{Cu}$ in the precipitates, so that the sum of $\mathrm{Cu}$ and some multiple of $\mathrm{P}$ should be considered as the effective $\mathrm{Cu}$ available for precipitation ${ }^{3}$. The precipitation model includes a threshold concentration of both $\mathrm{Cu}(0.072)$ and $\mathrm{P}(0.008)$, below which precipitation is assumed to be negligible. The $\mathrm{Cu}$ threshold was calibrated in earlier studies and confirmed as reported above, while the $\mathrm{P}$ threshold $(0.008)$ is a current calibrated (fitted) value determined along with the other CRP fitting constants using the high- $\mathrm{Cu}$ calibration set and least squares. Consequently, $\mathrm{P}-0.008$ was linearly combined with the effective $\mathrm{Cu}-0.072$ as in Eq. (4-5e).

\footnotetext{
${ }^{3}$ Williams, T. J., email to E. D. Eason, 6/18/2004
} 
The chemistry term of the shift model in Regulatory Guide 1.99 Rev. 1 contained a linear combination of $(\mathrm{Cu}-0.08)$ and $(\mathrm{P}-0.008)$ terms, somewhat similar to the $\mathrm{Cu}$ and $\mathrm{P}$ terms in Eq. (4-5e).

$$
f\left(C u_{e}, P\right)=\left\{\begin{array}{c}
0 \text { for } C u \leq 0.072 \\
{\left[C u_{e}-0.072\right]^{0.668} \text { for } C u>0.072 \text { and } P \leq 0.008} \\
{\left[C u_{e}-0.072+1.359(P-0.008)\right]^{0.668} \text { for } C u>0.072 \text { and } P>0.008}
\end{array}\right\}
$$

The remaining CRP term is the saturating fluence function, which depends on $\mathrm{Cu}$ and $\mathrm{Ni}$ as well as flux and fluence.

$$
g\left(C u_{e}, N i, \phi t_{e}\right)=\frac{1}{2}+\frac{1}{2} \tanh \left[\frac{\log _{10}\left(\phi t_{e}\right)+1.139 C u_{e}-0.448 N i-18.120}{0.629}\right]
$$

The effective fluence from Eq. (4-4) and the effective $\mathrm{Cu}$ [limited by the values in Eqs. (4-5c) and (4-5d)] are used in the saturating term, not fluence and bulk $\mathrm{Cu}$ values.

The $\mathrm{Cu}$ and $\mathrm{Ni}$ effects inside the tanh function, which move the location of the transition laterally as shown on Fig. (4-9), are known physical effects as discussed in Sect. 2.3.3. These effects were previously explored as part of the NUREG/CR-6651 modeling effort, with similar results for the Ni term (see [1], p. 87). The database at the time was inadequate for calibrating the $\mathrm{Cu}$ effect, and numerical trade-offs were noted between $\mathrm{Cu}$ and $\mathrm{Ni}$ terms inside and outside of the tanh function, so including those terms was deferred. With the current database there was no difficulty calibrating the effects, and the $\mathrm{Cu}$ and Ni terms outside the tanh function did not change much as $\mathrm{Cu}$ and $\mathrm{Ni}$ terms inside the tanh function were calibrated. However, some change was noted, so the $\mathrm{Cu}$ and $\mathrm{Ni}$ fitting constants that control amplitude and those that control location of the transition are not completely numerically independent.

The model of high- $\mathrm{Cu}$ behavior $(\mathrm{Cu}>0.072 \mathrm{wt} \%)$ is given by Eqs. (4-1), (4-3), (4-4), and (4-5). It is a good fit, as shown by comparing model shifts and actual shifts for all PWR high-Cu data in Fig. 4.10 and for all BWR high-Cu data in Fig. 4.11. In the BWR plot, two symbols (small square or circle, larger diamond) are plotted for some points. These are the atypical BWR data, which were irradiated at unusual locations for a BWR (denoted BWb in database) and in an unusual BWR (Big Rock Point, denoted BWa in database) such that the flux levels are more typical of PWR than BWR exposure. Indeed, some of the flux levels in this atypical BWR set are higher than in most PWR surveillance capsules.

As detailed in Sect. 4.3, a further indication of the good fit is the fact that there are no significant residual trends in the high-Cu data with fluence, flux, $\mathrm{T}_{\mathrm{i}}$, exposure time, chemistry variables, and interactions $\mathrm{P} * \mathrm{Mn}, \mathrm{P} * \mathrm{Ni}, \mathrm{Cu}_{\mathrm{e}}{ }^{*} \mathrm{Ni}, \mathrm{Cu}_{\mathrm{e}}{ }^{*} \mathrm{Mn}$, and $\mathrm{Mn} * \mathrm{Ni}$. There is also no significant difference in average residual with product form variables or between high-flux $\left(>4.39 \times 10^{10} \mathrm{n} / \mathrm{cm}^{2} / \mathrm{s}\right)$ and low-flux $(\leq 4.39 \times$ $10^{10} \mathrm{n} / \mathrm{cm}^{2} / \mathrm{s}$ ) subsets. The high flux and low flux comparison is for plate and weld, since only one of the $\mathrm{BWR}$ low flux observations is high-Cu forging material. 


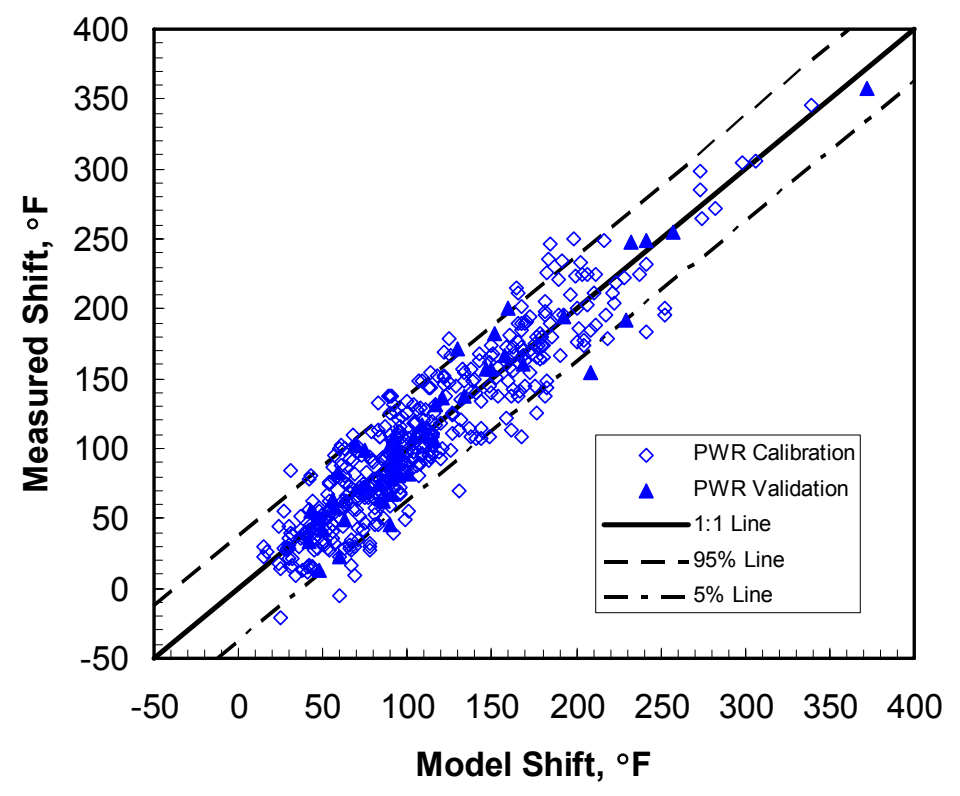

Fig. 4.10. Model shift vs measured shift, all PWR calibration and validation data with $\mathrm{Cu}>0.072 \mathrm{wt} \%$.

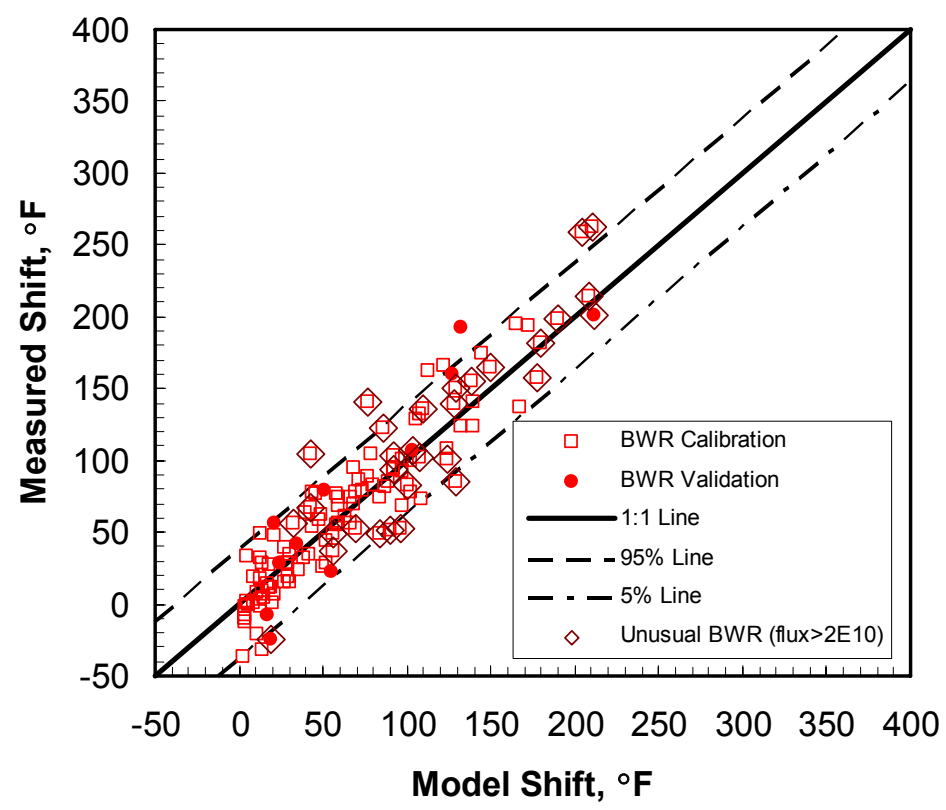

Fig. 4.11. Model shift vs measured shift, all BWR calibration and validation data with $\mathrm{Cu}>0.072 \mathrm{wt} \%$.

\subsection{Quality of Fit of Revised TTS Model}

This section presents several measures of goodness of fit of the revised TTS model and demonstrates predictive capability on surveillance data not used for fitting. Additional details on the TTS model trends, including analysis of their statistical significance on the available surveillance data, are given in Chap. 5. 


\subsubsection{Model TTS vs Measured TTS Plots}

There are several ways to assess the quality of fit of the multivariable TTS model given above. The first, and perhaps clearest overall assessment, is given in the model shift vs measured shift plots, Fig. 4.8 for low-Cu steels and Figs. 4.10 and 4.11 for high-Cu PWR and BWR data, respectively. Separate plots are given for those three subsets, both to avoid an overly dense cloud of points that cannot be interpreted easily and also to demonstrate the good fit in these important subsets of the surveillance database. A good model should be a reasonable fit to each of these major subsets (among others). The fact that the cloud of points on each of these plots is reasonably centered on the 1:1 line over the available range of measured shifts shows a reasonable fit. The plots also show that the fit is comparable on the calibration data used for model development (open symbols) and the validation sample used to show predictive capability (filled symbols).

\subsubsection{Statistical Measures}

Another typical indicator of the quality of fit is the standard error $\left(\mathrm{S}_{\mathrm{e}}\right)$, which is the standard deviation of residuals about the model, adjusted for the number of fitted constants in the model. Values of overall $S_{e}$ are given for the earlier models in [1,8], for example. However, with the expansion of the database in 2003-2004, it became apparent that $\mathrm{S}_{\mathrm{e}}$ is considerably smaller in the low-Cu data than in the high- $\mathrm{Cu}$ data. Thus, the overall $\mathrm{S}_{\mathrm{e}}$ depends on the relative amount of low- $\mathrm{Cu}$ and high-Cu data in the calibration set and hence has no validity as an indicator of quality of fit across different databases. Simply including less high- $\mathrm{Cu}$ data or increasing the amount of low-Cu data would have the same effect as improving the fit, in all cases causing the value of the overall $S_{e}$ to decrease. A similar result would be obtained by changing the proportion of forging and plate material (smaller $\mathrm{S}_{\mathrm{e}}$ ) relative to weld material (larger $S_{e}$ ). Because comparisons to the $S_{e}$ values found for the earlier models or possible future models on different databases would not be valid, the overall $\mathrm{S}_{\mathrm{e}}$ is not given for the revised model presented in this report.

A more valid measure than overall $\mathrm{S}_{\mathrm{e}}$ is the standard deviation $\left(\mathrm{S}_{\mathrm{d}}\right)$ of residuals about the model in the various subsets that have significantly different $S_{d}$, as given in Table 4.1. Standard deviation is used rather than standard error because many of the fitting constants in the model are fitted overall, not to the particular subsets shown in Table 4.1, and also because the $S_{d}$ values are based on both calibration data used for fitting and validation data that were not used in fitting. The traditional standard error concept is not well suited to these complexities. The SRM data have not been included in the plate $S_{d}$ values in Table 4.1, so that the tabulated values would be representative of plates in actual pressure vessels. The values of $S_{d}$ in Table 4.1 should be considered when setting margins or analyzing uncertainties in applications.

In Table 4.1, the standard deviation of high-Cu welds is significantly greater than the standard deviation for high- $\mathrm{Cu}$ plates and forgings. The standard deviation of low-Cu welds is significantly greater than the standard deviation for low-Cu plates (but not low-Cu forgings). The standard deviation of forging and plate are not significantly different from each other in either high or low-Cu material. The standard deviation in the high-Cu subset is significantly greater than the corresponding value for

Table 4.1 Standard deviation $\left(S_{d}\right)$ of residuals about the embrittlement shift model in various subsets, all PWR and BWR calibration and validation data except SRM All entries are TTS values measured in ${ }^{\circ} \mathrm{F}$

\begin{tabular}{|c|c|c|}
\hline \multirow{2}{*}{$\begin{array}{l}\text { Product } \\
\text { Form }\end{array}$} & \multicolumn{2}{|c|}{$\mathrm{S}_{\mathrm{d}}$ (points) } \\
\hline & $\mathrm{Cu} \leq 0.072 \mathrm{wt} \%$ & $\mathrm{Cu}>0.072 \mathrm{wt} \%$ \\
\hline Forging & $17.5(75)$ & $19.8(61)$ \\
\hline Plate & $15.0(78)$ & 20.9 (309) \\
\hline Weld & $18.6(93)$ & $26.3(213)$ \\
\hline
\end{tabular}
the same product form in the low-Cu subset for both plate and weld. All of these significance tests used the F-test on variance with significance if $\mathrm{p}<0.05$. 


\subsubsection{Residual Analysis}

Another way to assess the quality of fit is by analyzing residual plots for variables that are in the model and variables that are not. Residual plots show the difference between the model estimate and the actual shift, plotted against variables of interest. A good multivariable fit shows residuals scattered about the zero residual line with no apparent trend when plotted against the variables that are in the model and those that are not. Interactions of key variables can also be analyzed, and a fit that adequately reflects interaction nonlinearities will show no significant trend in the interactions. If the slope of the residual trend were significantly different from zero for a variable in the model, it would indicate that the model does not accurately reflect the first-order (linear) effect of that variable. Similarly, a nonlinear pattern in the residuals for a variable in the model would indicate a model inaccuracy of a nonlinear form. Finally, if the slope of the residual trend were significantly different from zero for a variable that is not in the model, it would indicate that the variable should be added to the model for a better fit.

The result of the residual analysis on the TTS model presented in this report is that there are no linear residual trends with slopes that are significantly different from zero, in either high- or low-Cu data, for all of the variables in the model and for all others that were analyzed. The statistical tests were two-sided, looking for a significant difference from zero slope in either direction. The residual plots are presented in Appendix F as Figs. F.1 through F.13 for low-Cu material and Figs. F.14 through F.28 for high-Cu material. The dashed line on each residual plot is the linear trend of the residuals, fitted to the residuals by least squares. Many of the trend lines are barely distinguishable from the zero residual line over the range of data. Moreover, there is no clear evidence of nonlinear trends in the residual plots for the modeling variables. Because the low- $\mathrm{Cu}$ residual indicates the quality of fit of the MF term separate from the CRP term, while both MF and CRP terms are reflected in the high-Cu residuals, it is useful to look at both measures of the fit. When the plots are interpreted, it is important to recall that the residual for these plots is defined as model TTS - measured TTS, so the residual and model slopes are reversed in sign. That is, a negative slope on these residual plots can be eliminated by making the slope of that variable more positive in the model.

The residual plots in Figs. F.1 through F.28 also show both calibration and validation points. Overall, there is no obvious difference between the residual trends in calibration and validation data. A statistical comparison of the fit to calibration and validation sets is presented in Sect. 4.3.4.

Additional residual analysis was performed by comparing the mean residuals of selected sets using Student- $t$ tests. No significant differences in mean residual were found for different product forms, in either low-Cu or high- $\mathrm{Cu}$ material. If there had been significant differences, they would suggest calibration issues with the MF or CRP coefficients. The residuals for the product form groups that were analyzed are plotted in Figs. F.10 for low-Cu and F.23 for high-Cu data. None of the mean residuals for these product form groups were significantly different from zero. An additional comparison found no significant difference in mean residual in high-flux $\left(\phi>4.39 \times 10^{10}\right)$ vs low-flux $\left(\phi \leq 4.39 \times 10^{10} \mathrm{n} / \mathrm{cm}^{2} / \mathrm{s}\right)$ categories in either low- $\mathrm{Cu}$ or high- $\mathrm{Cu}$ material. The low- $\mathrm{Cu}$ flux comparison was conducted on all product forms combined because the amount of low-flux, low-Cu data is relatively small and all three product forms are represented in both high- and low-flux categories. That is not the case in the high-Cu data, so high-Cu comparisons were made on plate and weld separately, and not for forging material (there are not enough forging data with low-flux and high- $\mathrm{Cu}$ ). As in the slope tests for continuous variables discussed above, the statistical tests were two-sided, looking for a significant difference between group means and from zero mean residual at $\mathrm{p}<0.05$.

\subsubsection{Calibration vs Validation Datasets and Predictive Capability}

The purpose of validation is to determine whether the model has predictive capability for data that were not used for calibrating it. In the present modeling effort, the validation is partial because there are insufficient data in the low-flux regime to be able to reserve a statistically meaningful sample of low-flux 
data for validation. However, all of the model except the two flux-related fitting constants can be validated on a significant sample of data that were randomly selected prior to the modeling effort and that were not used in fitting. Future quantitative validation of the flux effect on surveillance data may be possible when data become available from the last remaining capsules of the BWR SSP [6]. An overall validation of many effects in the TTS model, including the effects of flux in both MF and CRP terms, is provided by comparison with the IVAR data in Chap. 6 .

The validation set consists of a $10 \%$ random sample of data that was not used in developing most of the model or in the final calibration of most of the fitting constants. The parts of the model that did use points from the $10 \%$ sample during development and final calibration are the two constants in the effective fluence submodel and the two constants in the $\mathrm{P}^{*} \mathrm{Mn}$ submodel. Those four fitting constants were calibrated to the broadest possible dataset intended to reflect all low and high-Cu data, at both low and high flux. Because two of those subsets, low flux and low $\mathrm{Cu}$, are much smaller than the high-Cu subset of the surveillance data, all available data at medium to low flux $\left(\phi<5 \times 10^{10} \mathrm{n} / \mathrm{cm}^{2} / \mathrm{s}\right)$ and all available data at low-Cu were used to better balance the $90 \%$ sample of high-Cu data for this purpose.

The two flux-related constants are not validated on the surveillance data because the flux effect is mainly evident in the range $\phi<4.39 \times 10^{10} \mathrm{n} / \mathrm{cm}^{2} / \mathrm{s}$, and all available data in that range were needed and used for flux effect calibration. But the $\mathrm{P}^{*} \mathrm{Mn}$ term applies to all data, and the two constants in that term are validated by the 40 high- $\mathrm{Cu}$ validation points with $\phi>5 \times 10^{10} \mathrm{n} / \mathrm{cm}^{2} / \mathrm{s}$ which were not used to develop or calibrate the two constants. Additional confidence in the $\mathrm{P}^{*} \mathrm{Mn}$ term comes from theory and independent data that show strong P, Mn, and P-Mn interaction effects in low-Cu data (see Sects. 2.4.1 and 6.7.1). Additional confidence also comes from the fact that similar values for the $\mathrm{P}^{*} \mathrm{Mn}$ constants were calibrated during development on the PWR low-Cu data (219 points) and in the final calibration of those constants on the broadest possible set of high and low- $\mathrm{Cu}$, high and low flux points (830 points).

Some comparisons of calibration and validation data have been discussed previously (see Figs. 4.8, 4.10, and 4.11), and they also appear on the model vs actual shift plots and the residual plots in Appendix F. Qualitative comparisons of that sort show reasonable agreement of calibration and validation data. A more quantitative method of validation is to compare mean residual and $S_{d}$ of residuals between key calibration and validation subsets.

Table 4.2 shows reasonable agreement of the mean and standard deviation of residuals between highand low-flux, high- and low-Cu calibration and validation subsets. None of the differences between mean residuals and only one of the differences between $S_{d}$ in corresponding calibration and validation subsets is statistically significant, as indicated by the superscript a in Table 4-2 (two-sided t test on means, F test on $\mathrm{S}_{\mathrm{d}}$, significant if $\left.\mathrm{p}<0.05\right)$. The one difference in $\mathrm{S}_{\mathrm{d}}$ that is significant involves only two points in the validation set, a clearly inadequate sample for comparing $S_{d}$ values. So the statistics on validation sets generally confirm the calibrated model and the estimated $S_{d}$ values. Also, none of the mean residuals in Table 4.2 is significantly different from zero, confirming the overall quality of fit on these 8 subsets. The one validation mean that might appear different from zero, -7.9 in the high-Cu, low flux group, is for a small sample (15 points) and is not significantly different from zero based on a $t$ test (which accounts for the greater uncertainty in small samples). 
Table 4.2. Comparison of calibration and validation subsets by mean and standard deviation $\left(S_{d}\right)$ of residuals

The number of points is shown for subsets smaller than 40 points

\begin{tabular}{lcccc}
\hline \multirow{2}{*}{ Subset } & \multicolumn{2}{c}{ Mean (points) } & \multicolumn{2}{c}{$\mathrm{S}_{\mathrm{d}}$ (points) } \\
\cline { 2 - 5 } & Calibration & Validation & Calibration & Validation \\
\hline $\begin{array}{l}\mathrm{Cu} \leq 0.072, \\
\phi>4.39 \times 10^{10}\end{array}$ & 0.0 & $0.9^{a}(22)$ & 17.0 & $14.6^{a}(22)$ \\
$\begin{array}{l}\mathrm{Cu} \leq 0.072, \\
\phi \leq 4.39 \times 10^{10}\end{array}$ & 1.7 & $-2.1^{a}(2)$ & 19.5 & $0.2(2)$ \\
$\mathrm{Cu}>0.072$, & & & & \\
$\phi>4.39 \times 10^{10}$ & 0.5 & $1.4^{a}$ & 23.1 & $22.7^{a}$ \\
$\mathrm{Cu}>0.072$, & & $-7.9^{a}(15)$ & 21.2 & $26.7^{a}(15)$ \\
$\phi \leq 4.39 \times 10^{10}$ & 0.6 &
\end{tabular}

${ }^{a}$ The difference from the calibration value is not statistically significant.

\subsubsection{Comparison with SRM Data}

Although not set up as a statistical validation study, the SRM data provide additional insight on the predictive capability of the model, because about two-thirds of the SRM datapoints were not used in any way for calibrating any part of the model. The three SRM heats have their own CRP coefficients, so any partial validation from the SRM data is not necessarily representative of all plates or of the forgings and welds. The comparison of measured and model estimates of shift for the SRM data in Fig. 4.12 shows that the model is a reasonable representation of embrittlement behavior for all the data on the three SRM heats, with almost all SRM data falling within the $5 \%$ and $95 \%$ bounds (which were based on $S_{d}$ for high$\mathrm{Cu}$ plates).

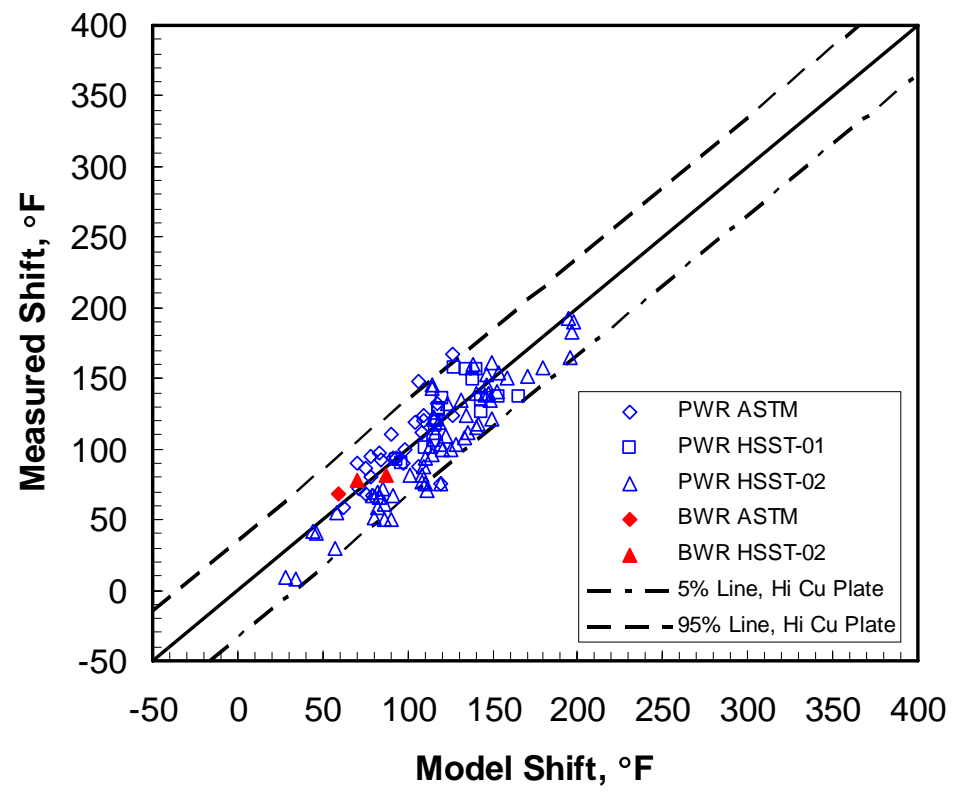

Fig. 4.12. Model shift vs measured shift for all SRM data. 
Looking closer at Fig. 4.12, it appears that some of the points from Plate HSST-02 (triangle symbols) are reasonably distributed about the 1:1 line and others are offset below the 1:1 line (over-predicted) by an amount that is similar over a range of fluence (or shift). This evidence provides some support for the concept expressed at an ASTM E10.02 meeting in January 2004 that the average value of unirradiated $\mathrm{T}_{30}$ used for all shifts with this heat may not be representative of the specific piece of material sent to some of the plants because of inhomogeneity in the plate. Due to the SRM sampling, which limited the impact of this heat on the model, and the separate coefficient for SRM materials, which isolated all SRM materials from the coefficients fitted to other materials, the effect of this uncertainty on the predictions for vessel materials is considered to be negligible.

\subsubsection{Numerical Convergence Checks}

The calibration of the model relies on nonlinear least squares. As with any nonlinear solution algorithm, convergence is not guaranteed, and the solution to a nonlinear problem may not be unique. These uncertainties were addressed by fitting all the key intermediate and final models more than once, using different initial estimates of the fitting parameters and two completely different least squares solution algorithms. The solutions agreed to several digits, converging from different initial estimates and using different algorithms. This information provides a check on model implementation and inputs (which are quite different for the two solution algorithms) and reasonable confidence in the convergence of the results.

\subsubsection{Comparison with Prior Models}

The present model is similar in overall format to the NUREG/CR-6551 and July 2000 draft models $[1,8]$, although differing in details. It includes all the effects except one from the prior models, although sometimes in different form. The linear MF temperature term is somewhat simpler than the Arrhenius term previously used. The $\mathrm{P}$ term in the MF part of the earlier models has been replaced by a $\mathrm{P}^{*} \mathrm{Mn}$ interaction in the MF part and a P precipitation term in the CRP part. The explicit use of Mn is new, but as in previous models, $\mathrm{Mn}$ is implicitly present in the form of lower coefficients for forgings, which have generally lower Mn compared to plates and welds.

The flux-time effect inside the tanh function in the July 2000 model has been replaced by a stronger flux effect in both MF and CRP terms, using the effective fluence form, which is a better fit to the larger set of low-flux data now available and is supported by mechanistic understanding and studies of an independent database (see Sects. 2.3.1, 2.4.2, and 6.6). The previously identified long-time bias, represented by a constant offset in the July 2000 model, is not evident in the data now available (see Figs. C.4 and C.17), so it is omitted from the present model. It appears that roughly doubling the amount of long time data and using a separate SRM coefficient caused that bias to disappear. The location of the transition to CRP plateau behavior now varies with $\mathrm{Cu}, \mathrm{Ni}$, and flux; it varied only with flux or time in the earlier models. These well-established effects are evident in the IVAR data [4,5], had been previously considered in the NUREG/CR-6551 fitting effort [1], and have been observed and calibrated in other databases [12].

\subsection{References}

1. Eason, E. D., J. E. Wright, and G. R. Odette, Improved Embrittlement Correlations for Reactor Pressure Vessel Steels, NUREG/CR-6551, U.S. Nuclear Regulatory Commission, Washington D.C., 1998.

2. Odette, G. R., "Radiation Induced Microstructural Evolution in Reactor Pressure Vessel Steels," pp. 137-48 in Microstructure of Irradiated Materials, MRS Symposium Proceedings, Volume 373, Pittsburgh, 1995. 
3. Odette, G. R., and G. E. Lucas, "Embrittlement of Nuclear Reactor Pressure Vessels," J. of Metals 53 (7), 18-22, 2001.

4. Odette, G. R., T. Yamamoto, and D. Klingensmith, The Effect of Dose Rate on Irradiation Hardening of RPV Steels: A Comprehensive Single Variable Database and Model Based Analysis, Letter Report UCSB-NRC-03/1, U.S. Nuclear Regulatory Commission, Washington, D.C., 2003.

5. Odette, G. R., G. E. Lucas, R. D. Klingensmith et al., The Effects of Composition and Heat Treatment on Hardening and Embrittlement of Reactor Pressure Vessel Steels, NUREG/ CR-6778, U.S. Nuclear Regulatory Commission, Washington, D.C., 2003.

6. Chap. 3 (p. 3-3 regarding status of SSP Capsules A, B, C) in BWR Vessel and Internals Project Updated BWR Integrated Surveillance Program (ISP) Implementation Plan, BWRVIP-86-A, EPRI Report 1003346, October 2002.

7. Jones, R. B., and T. J. Williams, "The Dependence of Radiation Hardening and Embrittlement on Irradiation Temperature," pp. 569-90 in Effects of Radiation on Materials: $17^{\text {th }}$ International Symposium, ASTM STP 1270, Ed. D. S. Gelles et al., American Society for Testing and Materials, 1996.

8. Kirk, M., C. Santos, E. Eason, J. Wright, and G. R. Odette, "Updated Embrittlement Trend Curve for Reactor Pressure Vessel Steels," in Transactions of the $17^{\text {th }}$ International Conference on Structural Mechanics in Reactor Technology (SMiRT 17), Prague, Czech Republic, August 17-22, 2003 (2003).

9. EPRI, Workshop on Dose Rate Effects in Reactor Pressure Vessel Materials Held November 12-14, 2001, Olympic Valley Calif., Electric Power Research Institute Conference Proceedings (CD), EPRI 1006981 (2002).

10. Williams, T. J., D. Ellis, C. A. English, and J. Hyde, "A Model of Irradiation Damage in High Nickel Submerged Arc Welds," Int. Jour. of Pressure Vessels and Piping, 79 649-60, 2002.

11. Odette, G. R., T. Yamamoto, D. Klingensmith, and H. Kishimoto, The Effect of Flux and Irradiation Temperature on Hardening in RPV Steels, Letter Report UCSB-NRC-03/2, 2003.

12. Williams, T. J., and D. Ellis, "A Mechanistically Based Model of Irradiation Damage in Low Alloy Steel Submerged Arc Welds," pp. 8-27 in Effects of Radiation on Materials: 20 ${ }^{\text {th }}$ International Symposium, ASTM STP 1405, ed. S. T. Rosinski et al. ASTM, 2001.

13. Server, W., C. English, D. Naiman, and S. Rosinski, Charpy Embrittlement Correlations - Status of Combined Mechanistic and Statistical Bases for U.S. RPV Steels (MRP-45), EPRI Report 1000705, 2001.

14. Miller, M. K., P. Pareige, and M. G. Burke, "Understanding Pressure Vessel Steels: An Atom Probe Perspective," Materials Characterization 44, 235-54, 2000.

15. Sokolov, M. A., R. K. Nanstad, and M. K. Miller, "Fracture Toughness and Atom Probe Characterization of a Highly Embrittled RPV Weld," Effects of Radiation on Materials: $21^{\text {st }}$ International Symposium, ASTM STP 1447, Ed. M. L. Grossbeck, T. R. Allen, R. G. Lott, and A. S. Kumar, ASTM International, West Conshohocken, PA, 2004. 


\section{Effects of Individual Variables on TTS}

In this chapter, individual variable effects in the TTS model given by Eqs. (4-1), (4-3), (4-4), and (4-5) are discussed in more detail. Plots are presented that show the trends in TTS as a function of variables that are in the TTS model, in most cases for various values and combinations of other variables. These are the same variable effects on which the residual plots in Appendix F are based. The fact that the residuals are reasonably distributed about the zero residual lines implies that the measured data are reasonably distributed about the TTS model trends shown in this chapter.

The second objective of this chapter is to show that the individual variable effects in the TTS model are statistically significant on the applicable surveillance data. The level of probability (p) used in this report for concluding that an effect is significant is $\mathrm{p}<0.05$ unless otherwise stated. This is a typical significance level for engineering, where it is sometimes referred to as the $95 \%$ significance level. That level of significance means that the chance the observed effect or difference could arise from random variation is estimated to be less than $5 \%$. Statistical significance is one form of evidence supporting the TTS model, and most effects in the model are also supported by physical understanding and other independent data, as indicated below by cross-references to the relevant parts in Chaps. 2 and 6 .

\subsection{Material Variables}

\subsubsection{Copper}

Copper affects embrittlement in several ways. The matrix features in materials with $\mathrm{Cu} \leq 0.072 \mathrm{wt} \%$ show a simple square root dependence on fluence (as shown in Fig. 4.7) without much effect of $\mathrm{Cu}$. Materials with higher $\mathrm{Cu}$ have the same matrix features as low-Cu materials plus a saturating precipitation effect that depends on $\mathrm{Cu}$, shown by the CRP term in Fig. 5.1. The CRP plateau in Fig. 5.1 both increases in amplitude and shifts to lower fluence as the $\mathrm{Cu}$ level increases. The lateral shift is somewhat easier to see by focusing on the point on the transition that is halfway to full amplitude, marked by dots on Fig. 5.1. The transition occurs over a similar range in fluence at all $\mathrm{Cu}$ levels, which has the effect of increasing the slope of the transition as the $\mathrm{Cu}$ level increases. The sum of the square root dependence in Fig. 4.7 and the saturating plateau in Fig 5.1 is a rather "bumpy" curve for total shift in higher $\mathrm{Cu}$ materials, as shown in Fig 5.2.

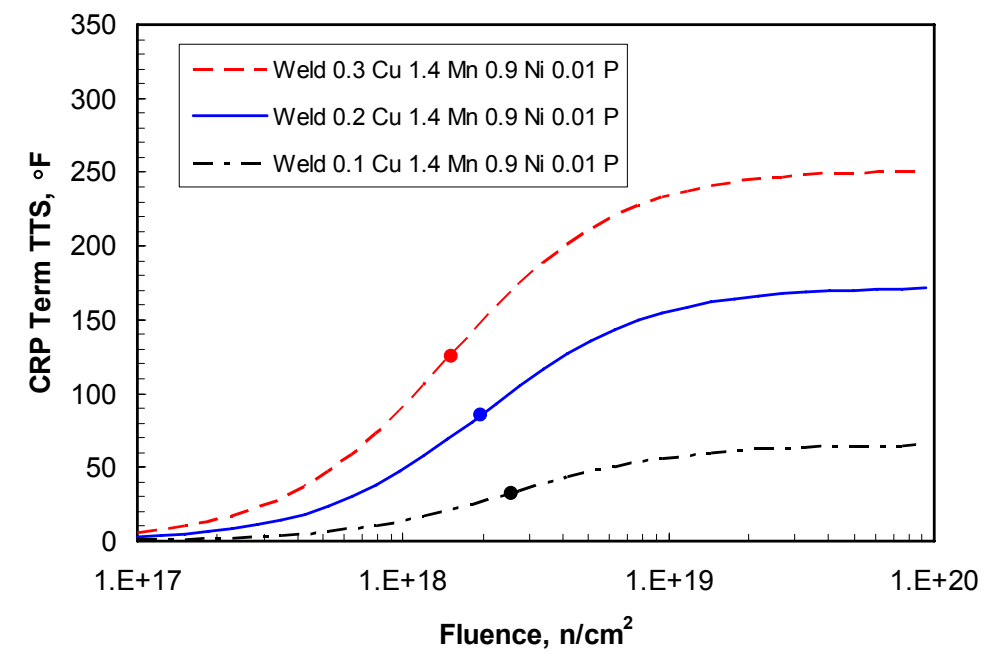

Fig. 5.1. Effect of $\mathrm{Cu}$ on CRP term amplitude and transition fluence. Dots indicate half amplitude location. 


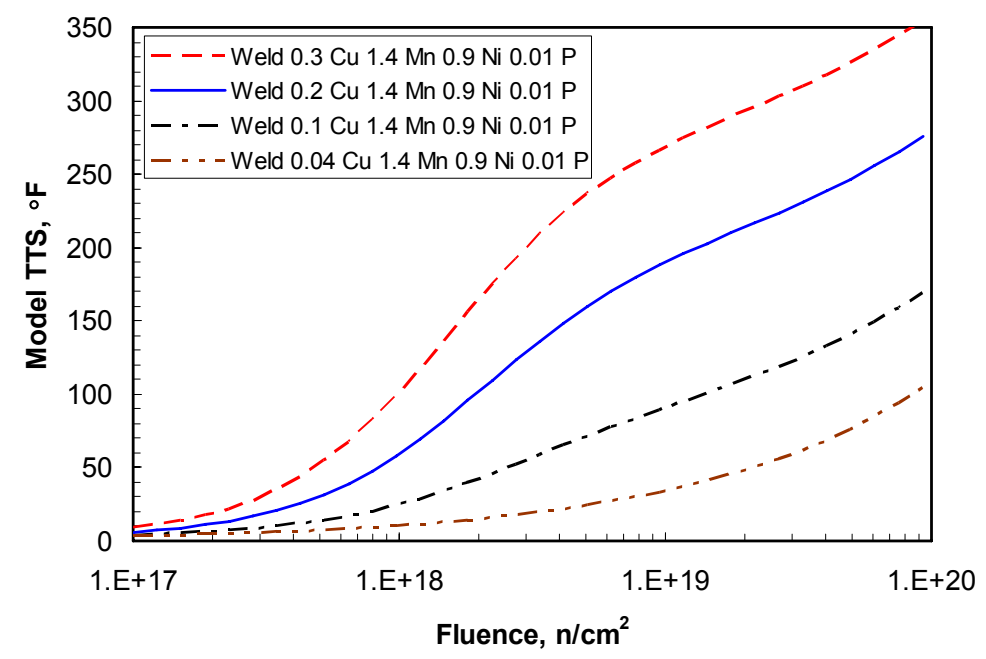

Fig. 5.2. Effect of $\mathrm{Cu}$ and fluence on TTS Model (MF term + CRP term).

The same effect shown in Figs. 5.1 and 5.2 can be plotted against $\mathrm{Cu}$, as shown for average Ni in Fig. 5.3. There is also an interaction of $\mathrm{Cu}$ and $\mathrm{Ni}$, such that the effect of $\mathrm{Cu}$ is enhanced at high Ni. The synergistic effect of $\mathrm{Cu}$ and $\mathrm{Ni}$ occurs with all product forms but is somewhat more complicated for high$\mathrm{Cu}$ welds because different $\mathrm{Cu}$ saturation levels apply in the three weld groups, which also have different ranges of $\mathrm{Ni}$, as shown in Fig. 5.4. The saturation levels of $\mathrm{Cu}$ are discussed in Sect. 5.1.3. Because of the strong $\mathrm{Cu}-\mathrm{Ni}$ interaction, it is important to have no significant residual trend relative to the interaction variable $\mathrm{Cu}_{\mathrm{e}}{ }^{*} \mathrm{Ni}$, as is the case with the TTS model (see Fig. C.27). Note in both Figs. 5.3 and 5.4 the initial flat segment of the curve corresponds to low-Cu material, where the shift does not vary much with $\mathrm{Cu}$, and the final flat segment at high $\mathrm{Cu}$ reflects the limit of $\mathrm{Cu}$ available for precipitation, $\mathrm{Cu}_{\max }$.

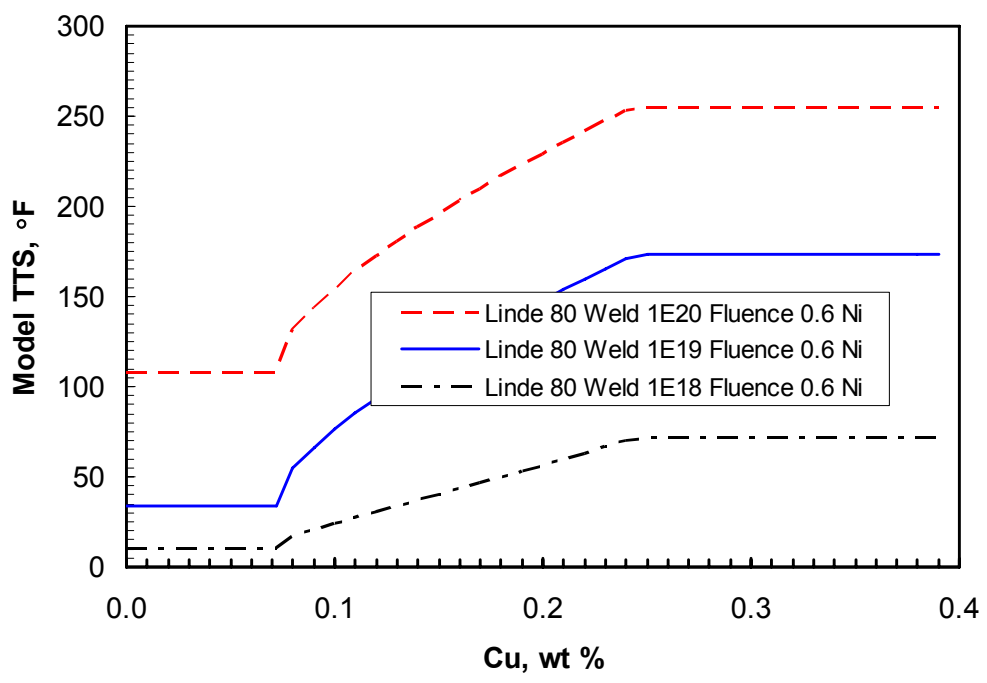

Fig. 5.3. Effect of $\mathrm{Cu}$ on TTS for average Ni Linde 80 welds at three fluence values. 


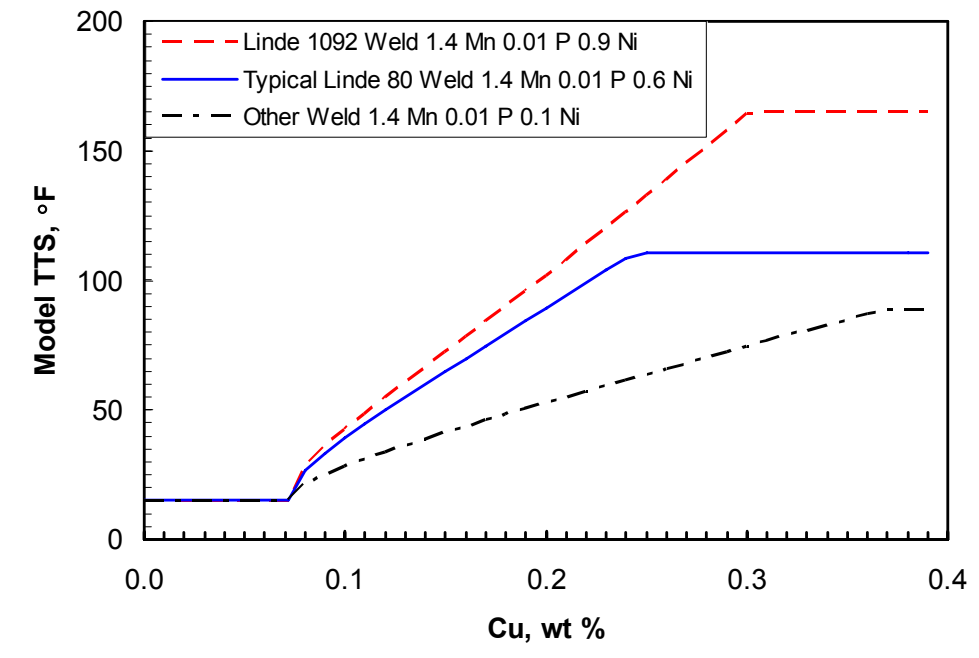

Fig. 5.4. Effect of $\mathrm{Cu}$ on TTS for three weld groups and $2 \times 10^{18} \mathrm{n} / \mathrm{cm}^{2}$ fluence.

The effect of $\mathrm{Cu}$ on the amplitude of the CRP term can be easily shown to be statistically significant by disabling it, using the average $\mathrm{Cu}$ value $(0.1729 \mathrm{wt} \%)$ in the $\mathrm{Cu}$ term for all the high-Cu calibration and validation data. The residual slope that results from disabling the term is significant on the high-Cu calibration and validation data. A one-sided test is appropriate because the direction of the residual slope from disabling the effect is known in advance. Similarly, the $\mathrm{Cu}$ effect on the CRP term plateau location can be shown to be significant by using the average $\mathrm{Cu}$ value in the tanh term while continuing to use the actual $\mathrm{Cu}$ values in the amplitude of the CRP term. As both effects of $\mathrm{Cu}$ are separately significant, the combined effect of taking $\mathrm{Cu}$ entirely out of the TTS model is also significant.

\subsubsection{Nickel}

There are effects of $\mathrm{Ni}$ on CRP amplitude and plateau location, as there are for $\mathrm{Cu}$. There is an overall enhancement of shift by increasing $\mathrm{Ni}$ for any particular $\mathrm{Cu}$, as shown in Fig. 5.5. The CRP plateau in Fig. 5.5 both increases in amplitude and shifts to higher fluence as the Ni level increases. The lateral shift due to increasing $\mathrm{Ni}$ is in the opposite direction to that caused by increasing $\mathrm{Cu}$, as can be seen by focusing on the point on the transition that is halfway to full amplitude, marked by dots on both Figs. 5.1 and 5.5. Because of the lateral shift, the fluence effect curves at various Ni converge essentially to a single curve at low fluence, as shown in Fig. 5.5 for the CRP term and in Fig. 5.6 for total shift (MF term + CRP term).

The convergence to essentially a single curve at low fluence corresponds to very small effects of $\mathrm{Ni}$ at low fluence and much larger effects at higher fluence, as shown for medium $\mathrm{Cu}$ levels in Fig. 5.7. Another implication is that the fluence at which the precipitation effect begins to be important varies more strongly with $\mathrm{Cu}$ than with $\mathrm{Ni}$ (compare the range in the fluence at TTS $=10^{\circ} \mathrm{F}$ on Figs. 5.1 and 5.5). For the higher $\mathrm{Cu}$ levels in welds, where the $\mathrm{Cu}$ saturation limits apply, there are different $\mathrm{Ni}$ curves within weld groups based on the different maximum $\mathrm{Cu}$ levels, as shown by the dashed curves in Fig. 5.8. Note that the curve segments for maximum $\mathrm{Cu}$ in Fig. 5.8 are upper bounds on TTS under the conditions used for plotting, and many materials within those weld categories will have lower $\mathrm{Cu}$ than the maximum and, hence, smaller shifts. 


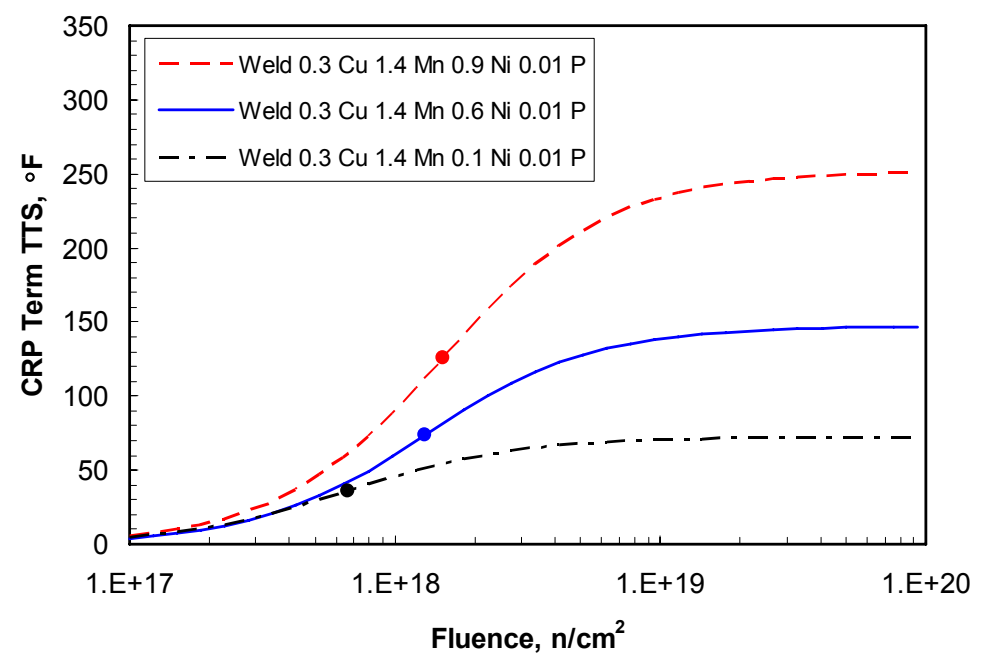

Fig. 5.5. Effect of $\mathrm{Ni}$ on $\mathrm{CRP}$ term amplitude and transition fluence. Dots indicate half amplitude location.

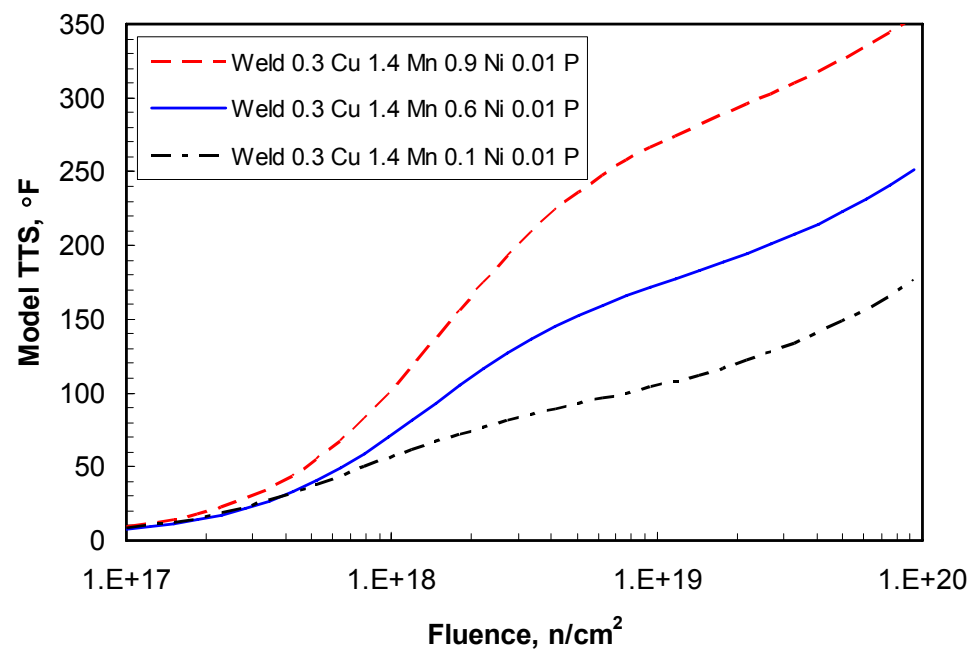

Fig. 5.6. Effect of Ni and fluence on TTS (MF term + CRP term).

Both the effect of Ni on CRP amplitude and the effect of Ni on plateau location are statistically significant, as can be shown by temporarily disabling the effects by using the average Ni in each of those places (separately), then testing the significance of the resulting residual trend slope. The procedure is exactly as described above for $\mathrm{Cu}$, and the average $\mathrm{Ni}$ value on the high- $\mathrm{Cu}$ calibration and validation data is $0.5444 \mathrm{wt} \%$. 


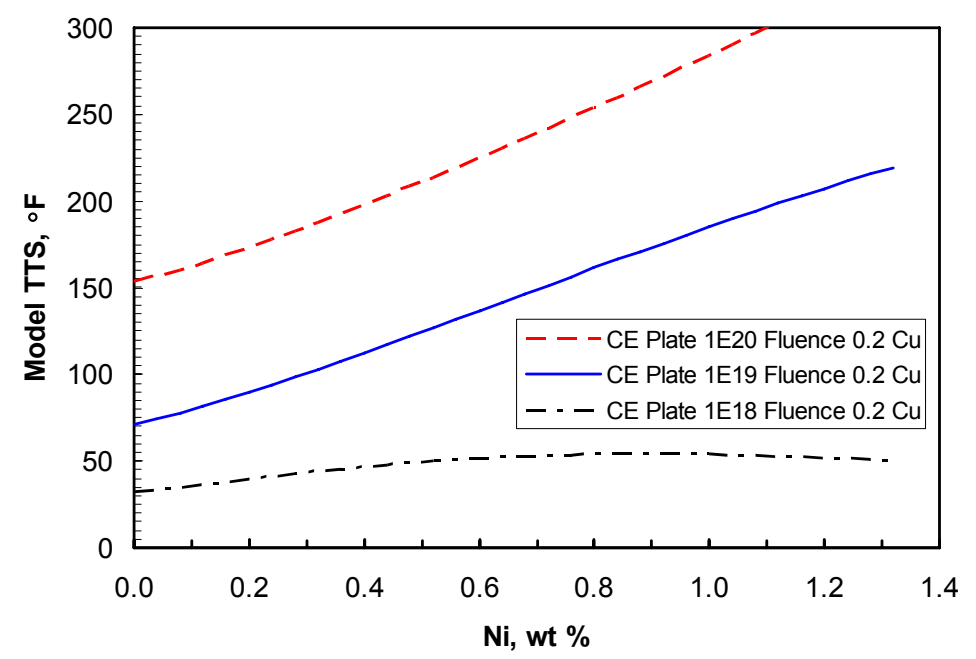

Fig. 5.7. Effect of $\mathrm{Ni}$ on TTS at typical $\mathrm{Cu}$ and three fluence values.

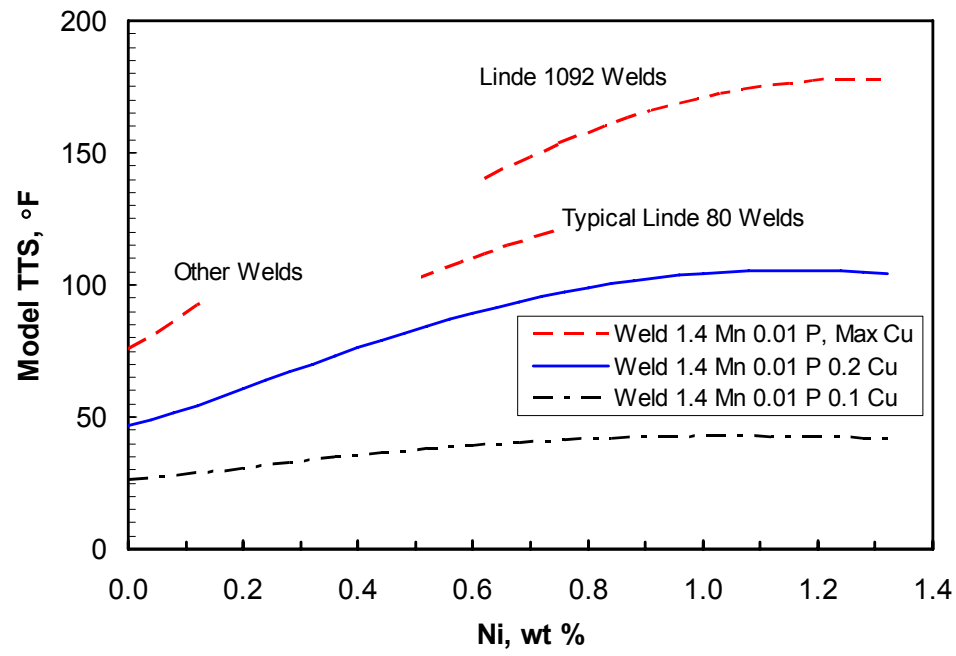

Fig. 5.8. Effect of $\mathrm{Ni}$ on TTS at various $\mathrm{Cu}$ levels and $2 \times$ $10^{18} \mathrm{n} / \mathrm{cm}^{2}$ fluence. The curves at $\mathrm{Cu}_{\max }$ for each weld group are plotted over representative $\mathrm{Ni}$ ranges.

\subsubsection{Maximum Cu Limits, $\mathrm{Cu}_{\max }$}

Calibration of the limits on the maximum copper available for precipitation present several challenges due to limited data, including insufficient data to calibrate limits applicable to base metals. None of the forging materials in the surveillance database has nominal $\mathrm{Cu}$ high enough to be usable for calibrating $\mathrm{Cu}_{\max }$; only one of the plates does, and that plate is just above the lowest calibrated limit for welds of $0.25 \mathrm{wt} \%$. The reason that lower- $\mathrm{Cu}$ materials are unusable for calibration is that the maximum $\mathrm{Cu}$ limit for a group of materials is determined solely by the materials with $\mathrm{Cu}$ values equal to or greater than the limit. The materials with lower $\mathrm{Cu}$ levels show only an increasing effect of $\mathrm{Cu}$, with no limit behavior at all. Because of this, maximum $\mathrm{Cu}$ limits can only be determined if there are several materials that have $\mathrm{Cu}$ levels at and above the calibrated limit. There are only two weld groups, identified by their weld flux (Linde 80 and Linde 1092), for which there are at least six different material chemistries with 
$\mathrm{Cu}>0.243 \mathrm{wt} \%$ (the value of the lowest calibrated limit). The limits for any other welds must be based on very sparse data and information from other research, since there are insufficient data in the surveillance database for calibration.

The information available at this time suggests that the maximum $\mathrm{Cu}$ available for precipitation depends on the Ni concentration, postweld heat treatment, and possibly other factors as discussed in Sect. 2.3.2 and [1]. Thus, it is appropriate to consider Ni content as a possible way to group materials in large enough groups for estimating $\mathrm{Cu}$ maxima, and it is also appropriate to consider the two large high-

$\mathrm{Cu}$ weld groups, within which individual welds have similar welding and heat treatment details. Weld flux is just a way of grouping welds that may have similar welding procedure and heat treatment, with no implication that the particular flux used for the welding directly affects the embrittlement of the steel. Thus, the two weld groups with sufficient high-Cu data to be separately considered, Linde 80 and Linde 1092, were examined in more detail, and Ni categories were also considered.

Three statistically justifiable categories are presented for the baseline model in Chap. 4: typical Linde 80 welds (the most common Linde 80 welds with nominal $\mathrm{Ni}>0.5$ ), Linde 1092 welds, and other welds. Low-Ni, medium-Ni, and high-Ni categories were also considered, but the current surveillance database is not adequate for calibrating Ni-based categories. A key difficulty with Ni categories in the surveillance database is that the Ni ranges of the particular Linde 80 and Linde 1092 datapoints that establish the $\mathrm{Cu}$ limits overlap substantially, yet those two weld categories have statistically significantly different limits. An additional difficulty with a Ni approach is that there are very few datapoints outside the medium-Ni range (nominally 0.5 to $0.75 \mathrm{wt} \% \mathrm{Ni}$ ) that have high enough $\mathrm{Cu}$ to be usable. Calibrating $\mathrm{Cu}_{\max }$ as a continuous function of $\mathrm{Ni}$ is not feasible at this time, either. The detailed discussion of these issues is given in Sect. 7.2, where a two-category version of the three $\mathrm{Cu}_{\max }$ categories in the baseline model is recommended for the simplified model presented in Sect. 7.3. All results in Chaps. 4, 5, and 6 were generated with the baseline model containing $3 \mathrm{Cu}_{\max }$ values.

The baseline $\mathrm{Cu}_{\max }$ effect is statistically significant, as can be shown by taking out the variation of $\mathrm{Cu}_{\max }$ by weld group and analyzing the residuals that result from this change. The simplest way to take out the effect is to calibrate a model with a single best-fit value of $\mathrm{Cu}_{\max }(0.2646 \mathrm{wt} \% \mathrm{Cu})$ that is otherwise exactly like the baseline model. The average residual for the single-value $\mathrm{Cu}_{\max }$ model in the "other welds" category $\left(-11.8^{\circ} \mathrm{F}\right)$ is significantly below the average residual of typical Linde 80 welds $\left(8.7^{\circ} \mathrm{F}\right)$, which is significantly above the average residual of Linde 1092 welds $\left(-6.3^{\circ} \mathrm{F}\right)$. It should be noted that the "other welds" and Linde 1092 weld groups do not have significantly different average residuals in this analysis, so there is also statistical merit in a slightly simpler "typical Linde 80 " vs "all other" grouping, as discussed in Sect. 7.2.

Only two welds establish the calibrated $\mathrm{Cu}_{\max }$ value for the baseline "other welds" category. One is an atypical Linde 80 weld in Crystal River 3 with very low Ni $(0.1 \mathrm{wt} \%)$ and high $\mathrm{Cu}(0.41 \mathrm{wt} \%)$. The other is a Rotterdamse weld from Sequoyah 1 with $\mathrm{Ni}=0.125 \mathrm{wt} \%$ and $\mathrm{Cu}=0.37 \mathrm{wt} \%$. Since it is effectively based on just two dissimilar welds, the limit on $\mathrm{Cu}$ for the baseline "other welds" should be considered highly uncertain. Although there are many Linde 1092 welds in the database, the $\mathrm{Cu}_{\max }$ value for the baseline Linde 1092 weld category is also not very well established, as there are only three Linde 1092 welds at and above the calibrated $\mathrm{Cu}_{\max }$ level $(0.301 \mathrm{wt} \% \mathrm{Cu})$. The only weld group that has a wellestablished $\mathrm{Cu}_{\max }$ value is the typical Linde 80 weld group, which is calibrated on at least 21 Linde 80 welds that have $\mathrm{Cu}$ levels at and above the calibrated $\mathrm{Cu}_{\max }$ value.

\subsubsection{Phosphorus}

The $\mathrm{P}$ effect in low-Cu surveillance materials ranges from almost no effect for low-Mn materials like ASTM A508 class 2 forgings to a clear linear effect of $\mathrm{P}$ for higher-Mn materials. This is a consequence of the $\mathrm{P}-\mathrm{Mn}$ interaction, as shown in Fig. 5.9. The $\mathrm{P}$ effect in high-Cu material is the sum of the effect given by the MF term and an increase in the amplitude of the CRP plateau as $\mathrm{P}$ increases. 


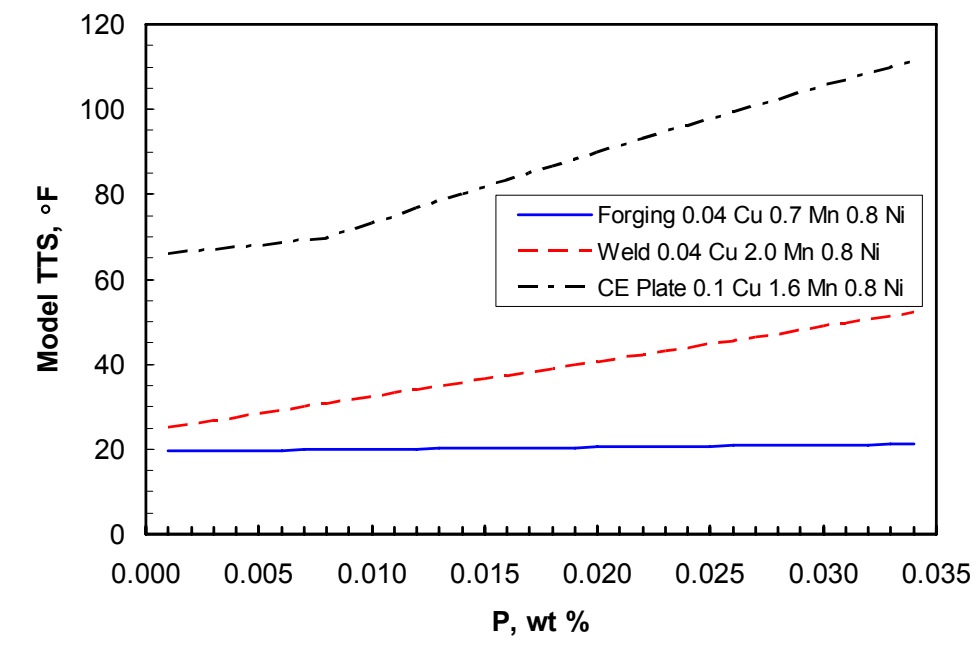

Fig. 5.9. Effect of $P$ on TTS at high- and low-Cu and $6.5 \times 10^{18} \mathrm{n} / \mathrm{cm}^{2}$ fluence.

The increase in CRP term plateau amplitude due to increased $\mathrm{P}$ is similar in form but smaller in magnitude compared to the effects of $\mathrm{Cu}$ and $\mathrm{Ni}$ on plateau amplitude as described previously. In fact, based on Eq. (4-5e), increasing P from the threshold level of $0.008 \mathrm{wt} \%$ to the maximum value observed in the surveillance data, $0.031 \mathrm{wt} \%$, will increase the CRP plateau amplitude the same amount as an increase in $\mathrm{Cu}$ from 0.072 to $0.103 \mathrm{wt} \%$. P does not produce a lateral shift in the location of the plateau on the fluence plot, as do $\mathrm{Cu}$ and Ni. There will be an additional increase in TTS from the $\mathrm{P}^{*} \mathrm{Mn}^{2.47}$ term in the MF term, depending on the material Mn. Thus, there are two slopes on the curve of TTS vs. P for high- $\mathrm{Cu}$ material, a flatter slope coming from the MF term (at a slope that depends on $\mathrm{Mn}$ ), which extends from $\mathrm{P}=0$ to the threshold value $\mathrm{P}=0.008 \mathrm{wt} \%$, then a steeper slope at higher $\mathrm{P}$, where both the MF and CRP terms contribute some effect. This is shown by the CE plate curve in Fig. 5.9.

The incorporation of phosphorus in the CRP term during model development was partly justified during model development by the fact that doing so eliminates the otherwise statistically significant residual trend with phosphorus in the high-Cu data. Similarly, incorporating phosphorus in the MF term was partly justified by the fact that doing so addresses the otherwise statistically significant residual trend with $\mathrm{P} * \mathrm{Mn}$ and the significant trend with $\mathrm{P}$ in the low-copper data at above-average Mn (Figs. 4.5 and 4.6 depict these residual effects graphically). In both cases additional justification came from theory and results of independent research on other databases, as discussed in Sects. 2.3.2 and 2.4.1. The other data show $\mathrm{P}$ effects in both low- $\mathrm{Cu}$ and high-Cu data, as shown in Sects. 6.4 and 6.7. Hardening features that are enriched in $\mathrm{P}$ are also observed in the radiation-damaged microstructure (see Sect. 2.4.1 and [2]). The way in which the empirical terms were incorporated in the model is also supported by the fact that the model produces no significant residual trends with phosphorus or its interactions with other key chemistry variables $\left(\mathrm{P}^{*} \mathrm{Ni}\right.$ and $\left.\mathrm{P}^{*} \mathrm{Mn}\right)$, both in low- and high-Cu surveillance data, as discussed in Sect. 4.3, and shown in the figures in Appendix F.

After the model was completed, the significance of the P effects in both terms of the model was evaluated. If the final $\mathrm{P}^{*} \mathrm{Mn}^{2.47}$ effect in the MF term is disabled by setting $\mathrm{P}$ and $\mathrm{Mn}$ to their average values (0.0119 and 1.30 respectively over all calibration and validation data) for the MF term but not the CRP term, the slopes of the resulting three residual trends for $\mathrm{P}, \mathrm{Mn}$, and $\mathrm{P}^{*} \mathrm{Mn}$ (over all calibration and validation data) are all statistically significant. The same result is obtained if the value of $\mathrm{P} * \mathrm{Mn}^{2.47}$ is set equal to its average value 0.02463 instead of setting $\mathrm{P}$ and $\mathrm{Mn}$ to their separate averages, while leaving $\mathrm{P}$ at its actual value in the CRP term. The significance tests are one-sided in this case because the directions 
of the residual slopes from disabling the $\mathrm{P}$ term are known in advance. If the $\mathrm{P}$ effect in the CRP term is disabled by using the average value of $\mathrm{P}$, but the $\mathrm{P}^{*} \mathrm{Mn}^{2.47}$ term in the MF term is still active, using actual $\mathrm{P}$ and $\mathrm{Mn}$, the slope of the residuals is also statistically significant. Disabling both $\mathrm{P}^{*} \mathrm{Mn}^{2.47}$ in the MF term and the $\mathrm{P}$ effect in the CRP term also produces significant residual slope errors. This is evidence that both $\mathrm{P}$ terms, individually and together, are statistically significant in the final model.

\subsubsection{Manganese, Product Form}

The effect of $\mathrm{Mn}$ is partly explicit, via the $\mathrm{P}^{*} \mathrm{Mn}^{2.47}$ part of the MF term, and partly implicit in the MF and CRP term coefficients and correlations with other composition variables. As noted in the discussion of $\mathrm{P}$ in Sect. 5.1.4, the explicit effect of $\mathrm{Mn}$ can be shown to be statistically significant by putting the average values of $\mathrm{P}$ and $\mathrm{Mn}$ in the MF term and analyzing the residual slopes relative to Mn and $\mathrm{P} * \mathrm{Mn}$ over all affected data. But an additional effect of $\mathrm{Mn}$ (and probably other variables) is included in the MF and CRP term coefficients. This is shown in Fig. 5.10, which plots the effect of Mn for selected product forms in high- $\mathrm{Cu}$ and low-Cu materials. The explicit effect of $\mathrm{Mn}$, coming from the $\mathrm{MF}$ term, is shown by the curves identified in the legend, each of which is plotted over the corresponding range of $\mathrm{Mn}$ in the surveillance database for high- $\mathrm{Cu}$ or low- $\mathrm{Cu}$ materials and for the particular product form group. The apparent implicit effect of Mn (and probably other variables) in the MF and CRP term coefficients is suggested by the arrows, which approximately pass through the mid-range Mn values for each product form group (see Sect. 5.1.6 for additional product form information). Note that the apparent implicit effect of $\mathrm{Mn}$ is at least as large as the explicit effect in low-Cu material and clearly larger than the explicit effect in high-Cu material for the examples shown on Fig. 5.10.

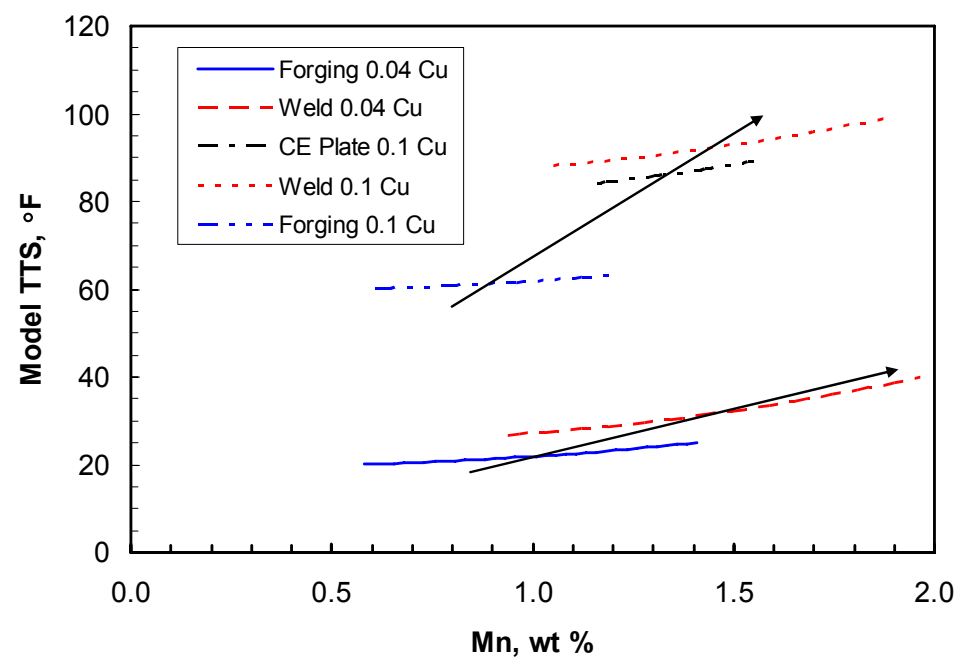

Fig. 5.10. Effect of Mn on TTS at high- and low-Cu and $6.5 \times 10^{18} \mathrm{n} / \mathrm{cm}^{2}$ fluence, $0.8 \mathrm{wt} \% \mathrm{Ni}$ and $0.02 \mathrm{wt} \% \mathrm{P}$. Curves represent the explicit Mn effect from the MF term, arrows suggest the apparent effect of average Mn implicit in the MF and CRP term coefficients.

As noted in Sect. 4.1, attempts were made to capture as much of the Mn effect as possible explicitly, by initially calibrating Mn effects with single MF and CRP coefficients. The fact that this effort did not take out more of the apparent $\mathrm{Mn}$ trend in the coefficients may indicate confounding of variables and suggests that other variables in addition to Mn may contribute to the apparent $\mathrm{Mn}$ trend in the product form groups. 
Most of the apparent $\mathrm{Mn}$ effect for typical high-Cu surveillance materials is in the TTS model coefficients, not the explicit $\mathrm{P}^{*} \mathrm{Mn}^{2.47}$ term, as shown in Fig. 5.10. Thus, although the coefficients are identified in Eqs. (4-3b) and (4-5b) by product form labels, they also may be surrogates for average composition. For this reason, comparisons of the TTS model with plates or welds from other databases, which may have substantially lower Mn than typical surveillance materials, should use the most appropriate coefficient for the average composition, as is done for IVAR SMMS materials with $\mathrm{Mn}=0$ and 0.8 in Fig. 6.8(c). The justification is the fact that the forgings have considerably lower average Mn than plates or welds in the surveillance database. The coefficients may also include unmodeled effects of $\mathrm{Ni}$, particularly affecting the MF coefficients, as noted in Sect. 5.1.6.

The hypothesis that the "product form" effect in previous TTS models could be a manganese effect (at least in part) was supported during the present analysis by the finding that either a manganese term or coefficients that vary by product form (or both) could reduce the residual difference between forging and other material groups. Introducing a $\mathrm{P}-\mathrm{Mn}$ interaction effect in the MF term helped to account for the otherwise significant residual trend with $\mathrm{Mn}$ in the low-Cu surveillance data, but there is still a significant difference in the coefficients for forgings and other materials, where the main composition difference is Mn.

\subsubsection{Additional Comments on Composition and Product Form}

The surveillance database contains numerical dependencies and confounding among the composition and product form variables used for fitting, of which the Mn issues discussed above are but one example. Independent variation of composition variables is possible, and some composition variables can and do vary reasonably independently within and between product forms (e.g., wide variations in $\mathrm{Cu}$ in some welds, depending on the weld wire copper coating or lack of copper coating, $\sim 10 \times$ variation in $\mathrm{P}$ across the database, $\sim 2 \times$ variation in Mn between A 508 class 2 and class 3 forgings, $\sim 6 \times$ variation in $\mathrm{Ni}$ between low $\mathrm{Ni}$ and typical Linde 80 welds). Composition variables have been intentionally varied independently over moderate ranges in RPV-like research materials, as in IVAR (see Sect. 6.1), which allows separation of variables in those studies. But the composition values in the surveillance materials do tend to occur in "clumps" and limited ranges corresponding to typical steelmaking practice for some materials, as shown in Appendix G.

The effect of typical steelmaking practices is to reduce the independence of composition and "product form" variables. Mn can be roughly estimated from $\mathrm{Ni}$ in both low-Cu and high-Cu data, using a statistically significant linear regression model. Mn can also be roughly estimated from $\mathrm{Cu}$ content in the high- $\mathrm{Cu}$ data by a statistically significant regression, and from forging vs plate and weld. Additional evidence includes the fact that the various product form groups do have different average values of $\mathrm{Ni}$ (see Appendix G). The average values of $\mathrm{Cu}$ also vary considerably by product form, but the average values of $\mathrm{P}$ do not. Other composition, microstructural, and mechanical property (e.g., unirradiated yield strength, upper shelf energy) variables may also be incorporated in what is referred to as the "product form effect." These numerical dependencies that exist in the surveillance database must be accepted and worked around in calibrating the TTS model, as it is not feasible now to include different steels in the surveillance capsules to help separate the effects of composition variables. The result is that the explicit $\mathrm{Mn}$ effect in the MF term may be partly confounded with a $\mathrm{Ni}$ effect, and the $\mathrm{Ni}$ and $\mathrm{Cu}$ effects in the CRP term may be partly confounded with a Mn effect, and all composition effects may be partially confounded with the product form effect.

As long as the TTS model is applied to estimate shifts in steels that are the same or similar to the steels represented in the surveillance database, the dependencies among the composition variables and coefficients cause no problems and the model should be directly applicable. The design of surveillance programs is intended to ensure that the surveillance materials are the same as, or as similar as possible to, the limiting materials in plants. However, the correlations among theoretically independent variables in 
the TTS model should be considered when trying to reconcile results with other databases that have more independent variables, such as IVAR. In particular, the inability to calibrate an explicit Ni effect in the MF term on surveillance data, despite IVAR results [Figs. 6.10, 6.11, and 6.12(a)] suggesting there should be one, may be caused by the partial confounding of $\mathrm{Ni}$ with $\mathrm{Mn}$ and product form in the surveillance data. Similarly, $\mathrm{Mn}$ is known to affect high-Cu shifts [Figs. 6.7 and 6.8(c)], so the inability to calibrate an explicit Mn effect in the CRP term may be related to partial confounding of Mn with Ni, $\mathrm{Cu}$, and product form as well as to the explicit Mn effect contribution from the MF term.

Fortunately, over the range of compositions that are present in both the surveillance and IVAR databases, the agreement with the TTS model is generally good, as shown in Sects. 6.3 and 6.4. This suggests that the numerical dependencies in the surveillance data discussed above have not prevented the calibration of a reasonably robust model. The TTS model provides both a reasonable representation of the surveillance data and a reasonable approximation of the main independent composition trends indicated by IVAR results, as shown and discussed in Chap. 6.

The product form effects are statistically significant, as can be demonstrated by taking out the variation in coefficients. If a single coefficient is used in the MF term, all differences in mean residual between forging, plate, and weld are statistically significant over the data affected by that change (all calibration and validation data). If a single coefficient is calibrated in the CRP term, all product forms (including $\mathrm{CE}$ vs non-CE plates discussed in more detail in the following paragraphs) have significantly different mean residuals over the relevant data (high- $\mathrm{Cu}$ calibration and validation). These are all onesided comparisons because the previously calibrated effect was intentionally disabled by using a single coefficient. Clearly the product form effect, including both microstructural variations and all related implicit composition effects, is a significant contribution in the model.

As first identified in the July 2000 modeling effort [3], there is a statistically significant difference between plates in CE-manufactured vessels and plates in other vessels, with the CE plates having greater shifts. The significant difference was independently noted by Professor Naiman in [4]. This difference was thought possibly to be caused by the grouping of SRMs with other plates (which was done in 2000). The current analysis proves otherwise because the difference between CE and non-CE plates is still significant in the present TTS model, where SRMs have their own coefficient in the CRP term to ensure that they could not affect the $\mathrm{CE}$ or non-CE plate coefficients.

The physical cause of the "CE plate effect" has not yet been identified, but the empirical evidence of it is strong. The coefficients in Eq. (4-5b) indicate that for the same composition and exposure, the CRP contribution to shift is about a third larger for plates in CE manufactured vessels, producing a difference in mean shift that is significant $(\mathrm{p}<0.0001)$ on a substantial amount of data $(181 \mathrm{CE}$ points, 128 non-CE points). Possible causes include any of the physical differences that could be associated with different manufacturers, including vessel fabrication and heat treatment practices, any material differences that may not be fully accounted for in the composition terms in the model, differences in surveillance programs including testing, capsule placement, and exposure variable estimates, and possibly differences in plant operation that may be associated with the manufacturer of the vessel. Some have questioned the statistical association of the plate differences with different manufacturers on the basis that any effects associated with the vessel manufacturer should also show up in weld data. However, the Linde 1092 and Linde 80 weld groups have significantly different $\mathrm{Cu}$ saturation limits in Eq. (4-5d), and they are in fact associated with $\mathrm{CE}$ and non-CE vessel manufacturers, respectively, with the highest-Cu members of the $\mathrm{CE}$ weld group having greater shifts because of the higher $\mathrm{Cu}_{\max }$ limit. Thus, broadly consistent $\mathrm{CE}$ vs non-CE shift differences are observed in both plates and welds. Differences in Ni content may contribute to this weld difference, although the substantial overlap in Ni content of the specific Linde 80 and Linde 1092 welds that have high enough $\mathrm{Cu}$ to establish the significance of $\mathrm{Cu}_{\max }$ limits suggests that other factors may also be relevant (see Sect. 7.2.2). At this point, the difference in shift of CE and non-CE plates is adopted and accepted as a purely empirical part of the model. 


\subsection{Exposure Variables}

\subsubsection{Fluence, Effective Fluence, and Flux}

The effect of fluence on radiation damage is well known and has been shown in several previously discussed plots, including Fig. 4.7 for the MF term, Figs. 4.9, 5.1, 5.5 for the CRP term, and Figs. 5.2 and 5.6 for both terms combined. The effective fluence approach adjusts the fluence effect to account for greater embrittlement damage at the same fluence under lower flux conditions. The effect of a lower flux in the effective fluence approach is numerically equivalent to the effect of a higher fluence, hence the name.

For the MF term, lower flux in the effective fluence form has the same effect as multiplying the MF shift by a factor greater than 1 . The magnitude of the fluence multiplier can be determined by introducing Eq. (4-4) into Eq. (4-3) and working through the algebra. For instance, the effective fluence for a flux value of $1 \times 10^{9} \mathrm{n} / \mathrm{cm}^{2} / \mathrm{s}$ is a factor of 2.67 times the nominal fluence. The multiplier on shifts estimated by the MF term at a flux of $1 \times 10^{9}$ is a factor of $1.63(=\sqrt{2} .67)$ times the shift at $4.39 \times 10^{10} \mathrm{n} / \mathrm{cm}^{2} / \mathrm{s}$ or higher flux. The multiplier on MF shift arising from a particular lower value of flux is the same at any fluence, for any temperature, composition or product form. The effect of flux on the fluence plot is shown in Fig. 4.7.

For the CRP term, lower flux causes the CRP plateau to be reached at a lower nominal fluence, but it does not increase the amplitude of shift at high fluence after reaching the CRP plateau. The effect of flux on CRP plateau location at various flux levels is shown in Fig. 5.11. Lower flux shifts the plateau to lower fluence without changing its amplitude. Combined with the MF term effect, the total shift is as shown in Fig. 5.12.

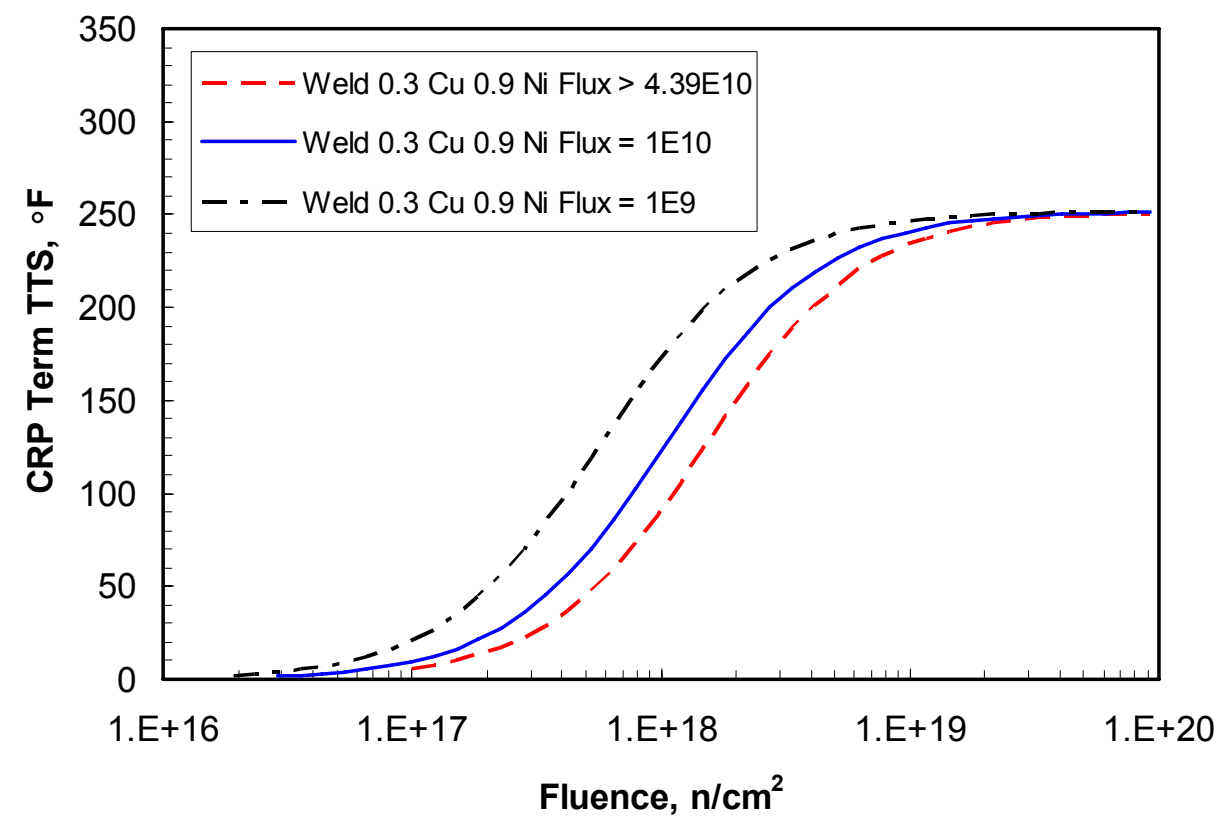

Fig. 5.11. Effect of flux on TTS in CRP term. 


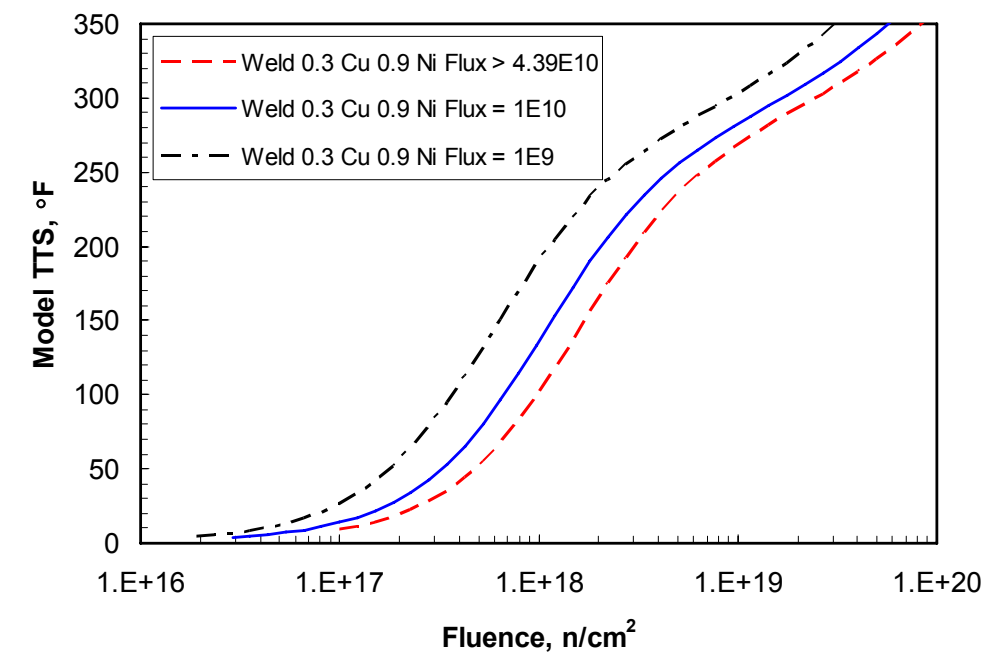

Fig. 5.12. Effect of flux on total TTS (MF term + CRP term) for $\phi \mathrm{t}<8 \times 10^{16} \mathrm{n} / \mathrm{cm}^{2}$.

In the surveillance data, the apparent effect of flux above $4.39 \times 10^{10} \mathrm{n} / \mathrm{cm}^{2} / \mathrm{s}$ has a flatter slope compared to the effect below that value. This can be seen in the flat residuals on Figs. F.2 and F.15, which would show a residual slope above $4.39 \times 10^{10} \mathrm{n} / \mathrm{cm}^{2} / \mathrm{s}$ if there were a strong effect of flux in that range. The observation of a much flatter, nonsignificant slope in PWR data motivated the piecewise form of effective fluence used in the TTS model. The break point is a calibrated value from the fit to both lowand high-flux data, and a completely flat function above the break point was assumed for the calibration, based on the observed nonsignificant effect in the PWR surveillance data. Note that the lack of a strong flux effect in the PWR data may be caused by limitations of the surveillance data, as discussed in Sect. 6.6.1. The piecewise form differs somewhat from a simple power law form, although they can give roughly similar results over the range in flux found in surveillance data $\left(\phi>4 \times 10^{8} \mathrm{n} / \mathrm{cm}^{2} / \mathrm{s}\right)$ as shown in Fig. 5.13.

Both curves in Fig. 5.13 are approximations of the fitted recombination model applied to individual materials in Sect. 6.6. The main difference is that the exponents for the curves in Fig. 5.13, though different from each other, are both constant values that would apply to many materials, while in the fitted recombination model the exponent is fitted to individual materials and is (theoretically) a function of flux, $\mathrm{Cu}$ diffusion rates, and other variables. Additional discussion of the flux effect, including the similarities and likely reasons for the differences between surveillance and IVAR results, is provided in Sect. 6.6. Figure 6.20 is a key result, showing that the extension of the flux dependent trend in the TTS model to higher flux levels is in reasonable agreement with the IVAR results, and the physically based extrapolation of the IVAR recombination model to the BWR flux range is in reasonable agreement with the TTS model.

It is easy to check the significance of effective fluence in each term of the TTS model separately and the combined effect in both terms. For the MF term, the relevant data includes both low-Cu and high- $\mathrm{Cu}$ data with sufficiently high fluence $\left(\phi t>8 \times 10^{16} \mathrm{n} / \mathrm{cm}^{2}\right)$ to exhibit measurable effects. The significance test is done by simply using fluence instead of effective fluence in the MF term, while continuing to use effective fluence in the CRP term. The significance test asks whether the slope of the residuals is significantly greater than zero on all data with $\phi<4.39 \times 10^{10} \mathrm{n} / \mathrm{cm}^{2} / \mathrm{s}$ and $\phi \mathrm{t}>8 \times 10^{16} \mathrm{n} / \mathrm{cm}^{2}$. The first condition arises because only the data with $\phi<4.39 \times 10^{10} \mathrm{n} / \mathrm{cm}^{2} / \mathrm{s}$ show a substantial flux effect, while the second condition is based on the fact that measured shift is essentially zero below $\phi \mathrm{t}=8 \times 10^{16} \mathrm{n} / \mathrm{cm}^{2}$, even at low flux, as shown in Fig. 5.14. Thus, data that do not meet both conditions are not useful for 


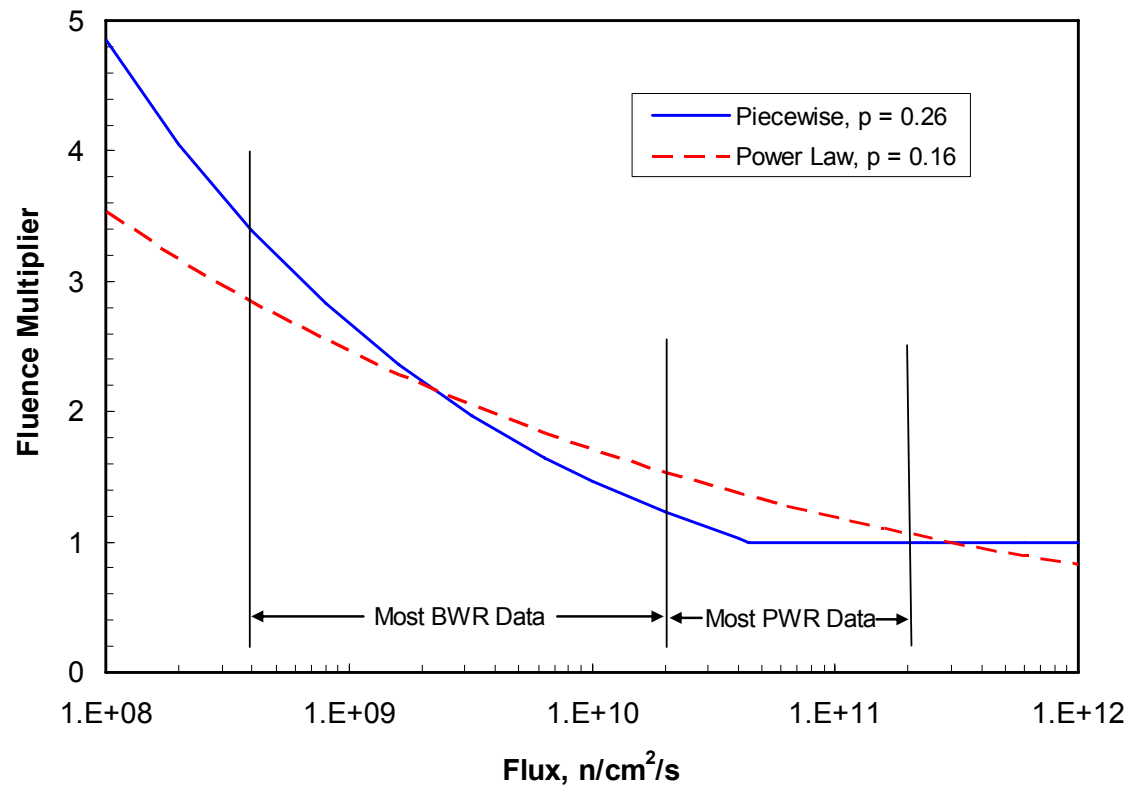

Fig. 5.13. Schematic of effective fluence in the TTS model (solid curve) and a power law form with $p=0.16$ (dashed curve).

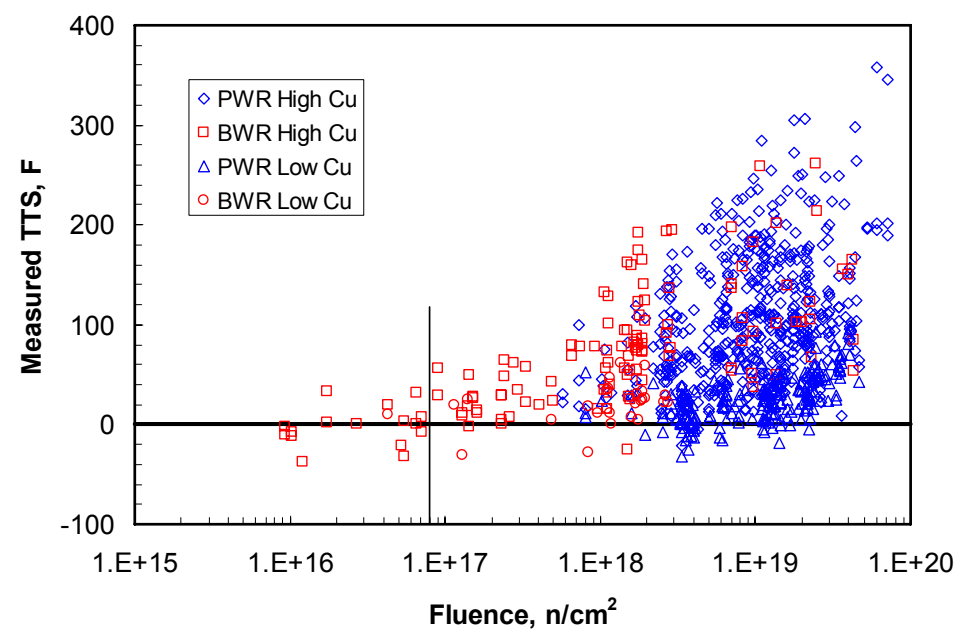

Fig. 5.14. Measured shift is essentially zero (avg. $\left.=-1.3^{\circ} \mathrm{F}\right)$ for $\phi \mathrm{t}<8 \times 10^{16} \mathrm{n} / \mathrm{cm}^{2}$.

determining whether fluence or effective fluence should be used. The significance test is one-sided because the direction of the residual slope resulting from taking out the flux effect is known in advance. The slope of the residuals is statistically significant, confirming the significance of using effective flux in the MF term. Without flux in the MF term, there is also a statistically significant mean residual, $-7.5^{\circ} \mathrm{F}$, in the 103 BWR datapoints with $\phi<2 \times 10^{10} \mathrm{n} / \mathrm{cm}^{2} / \mathrm{s}$ and $\phi \mathrm{t}>8 \times 10^{16} \mathrm{n} / \mathrm{cm}^{2}$, again showing the need for using effective fluence in the MF term.

Similarly, one can test for the significance of the flux effect on high $\mathrm{Cu}$ data, using effective fluence in the MF term and nominal fluence in the CRP term, or just use fluence (no flux effect) in both terms for 
all data. Those analyses show that flux, modeled as effective fluence, is also significant in the CRP term and in both terms used together. The residuals for the latter case, again meeting the two tests $\phi<4.39 \times$ $10^{10} \mathrm{n} / \mathrm{cm}^{2} / \mathrm{s}$ and $\phi \mathrm{t}>8 \times 10^{16} \mathrm{n} / \mathrm{cm}^{2}$, are shown in Fig. 5.15, where there is no doubt that the use of fluence rather than effective fluence leads to large negative residuals at low flux and a significant unmodeled trend with flux. Without a flux effect in either term, the average residual in the 103 BWR datapoints with $\phi<2 \times 10^{10} \mathrm{n} / \mathrm{cm}^{2} / \mathrm{s}$ and $\phi \mathrm{t}>8 \times 10^{16} \mathrm{n} / \mathrm{cm}^{2}$ is $-15.9^{\circ} \mathrm{F}$, which is significantly less than zero. A large cloud of higher-flux $\left(\phi>4.39 \times 10^{10} \mathrm{n} / \mathrm{cm}^{2} / \mathrm{s}\right)$ data has been omitted from Fig. 5.15. Because the effective fluence term in Eq. (4-4) has no effect on those data, the residuals are the same with or without effective fluence. Therefore, Figs. C.2 and C.15 show the residuals from $\phi>4.39 \times 10^{10} \mathrm{n} / \mathrm{cm}^{2} / \mathrm{s}$ points for both fluence and effective fluence.

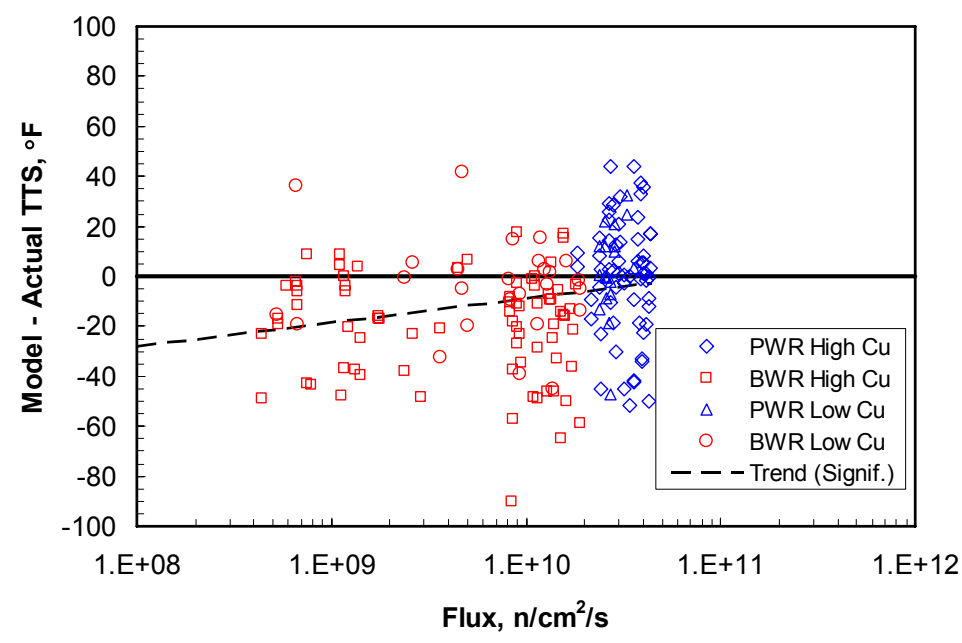

Fig. 5.15. Significant residual trend using fluence instead of effective fluence, $\phi<4.39 \times 10^{10} \mathrm{n} / \mathrm{cm}^{2} / \mathrm{s}$ and $\phi t>8 \times 10^{16}$ $\mathrm{n} / \mathrm{cm}^{2}$.

The flux effect, implemented as effective fluence in both MF and CRP terms, is also supported by significant trends with flux in the independent IVAR database. As in the surveillance data, the effects of flux are apparent in both the no-Cu steels (see Sect. 6.6.2) and the Cu-bearing steels (see Sect. 6.6.1) that are in the IVAR database.

Although the calibration data range included a few flux observations as low as $\phi=1.8 \times 10^{8} \mathrm{n} / \mathrm{cm}^{2} / \mathrm{s}$, as shown in Table 3.3, the flux values in the surveillance database below about 4 to $6 \times 10^{8} \mathrm{n} / \mathrm{cm}^{2} / \mathrm{s}$ correspond to fluence below about $8 \times 10^{16} \mathrm{n} / \mathrm{cm}^{2}$, where the observed shifts are near zero with or without the effective fluence term, as shown in Fig. 5.14. Thus, the useful flux range in the calibration data is $\phi \geq 4 \times 10^{8} \mathrm{n} / \mathrm{cm}^{2} / \mathrm{s}$ (the range of the data shown on Fig. 5.15), and applications to lower flux should be considered extrapolations beyond the currently available and usable data. Moreover, as noted in Chap. 3, the highest fluence that is available in surveillance data decreases as flux decreases, such that there are no data in the surveillance database with $\phi<2 \times 10^{10} \mathrm{n} / \mathrm{cm}^{2} / \mathrm{s}$ and $\phi \mathrm{t}>5.1 \times 10^{18} \mathrm{n} / \mathrm{cm}^{2}$. Applications at $\phi<2$ $\times 10^{10} \mathrm{n} / \mathrm{cm}^{2} / \mathrm{s}$ and fluences higher than $5.1 \times 10^{18} \mathrm{n} / \mathrm{cm}^{2}$ (and, similarly, above lower fluence values at lower flux levels) are also extrapolations beyond the currently available data. 


\subsubsection{Irradiation Temperature}

The TTS model presented in Chap. 4 requires values of $T_{i}$, the average temperature of the metal during irradiation. The surveillance database uses the time-averaged coolant temperature in the vicinity of the surveillance capsule as the best available estimate of Charpy specimen metal temperature during irradiation. This choice was one of necessity - the only other direct information on surveillance specimen temperature during irradiation is thermal monitor (melt wire) results, which only give an approximation of the maximum temperature reached over a period of time, not the average. In applications of the model, one should use the best available estimate of time-averaged metal temperature at the specific location in the vessel wall being analyzed, which will not be coolant temperature in many applications (e.g., at the tip of an assumed buried flaw or at any other location in the vessel wall).

The temperature effect in the MF term is in the same form as that used by Jones and Williams [5], but the calibrated coefficient on temperature is somewhat larger than in their studies. The temperature coefficient in the MF term for surveillance data is also larger than the value found in IVAR, though the overall trends with temperature in the most similar steels are similar (see Sect. 6.5). The fact that the temperature effect in the TTS model adequately fits the surveillance data is supported by the zero residual slopes in Figs. C.3 and C.16.

The temperature effect is shown for low- $\mathrm{Cu}$ and high- $\mathrm{Cu}$ cases on Fig. 5.16. The magnitude of the effect in this example is about 0.88 degrees increased shift per degree temperature decrease for the low$\mathrm{Cu}$ conditions (MF term only) and 0.65 degrees increased shift per degree temperature decrease for the high- $\mathrm{Cu}$ conditions shown on Fig. 5.16 (using both terms). These "degree shift per degree temperature" estimates depend on the conditions assumed when estimating them and would be different under other conditions (e.g., other product forms, different chemistry, different fluence).

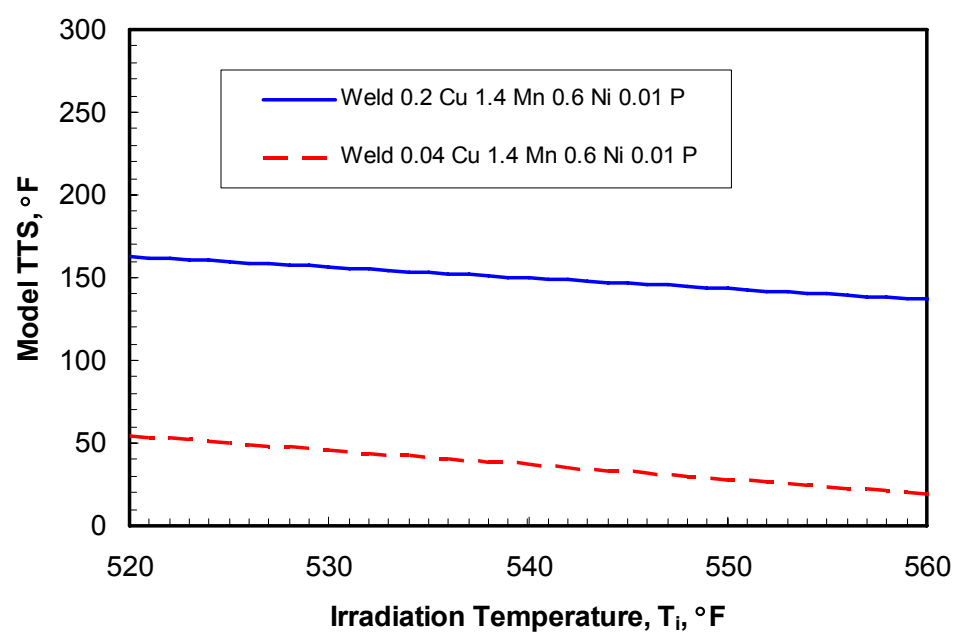

Fig. 5.16. Effect of estimated irradiation temperature.

The $T_{i}$ effect is statistically significant in the MF term, as can be demonstrated by noting the significant slope to the residuals that results from disabling it (setting $T_{i}$ equal to the average value over all calibration and validation data).

The $T_{i}$ effect in the CRP term is just a small empirical correction factor, partially counteracting the MF $T_{i}$ effect to approximate the somewhat flatter overall temperature effect observed in high-Cu surveillance data compared to low $\mathrm{Cu}$ surveillance data. The $\mathrm{T}_{\mathrm{i}}$ correction in the CRP term has little effect on estimated shift, amounting to $-4.3 \%,+5.5 \%$ of the value of the CRP term (and hence a smaller percentage of total TTS) over the full range of temperatures in the database. The form of the correction 
term was designed to facilitate removal without recalibration by setting it equal to 1 for all $\mathrm{T}_{\mathrm{i}}$, because CRP $\mathrm{T}_{\mathrm{i}}$ terms were found to be small in prior calibration efforts.

A linear residual analysis finds that the baseline $T_{i}$ term is not statistically significant, as shown by setting the CRP $\mathrm{T}_{\mathrm{i}}$ term equal to 1 and checking the significance of the slope of the line fitted to residuals over all the high- $\mathrm{Cu}$ data (both calibration and validation). However, additional analysis of the data that are most affected by the CRP $T_{i}$ term (the points at least $\pm 15^{\circ} \mathrm{F}$ from average $T_{i}$ ), again with no $T_{i}$ term in the CRP part, indicates a significant difference in low- and high- $T_{i}$ residuals, suggesting a nonlinear trend in the same direction as the baseline $T_{i}$ term and a significant average residual at low temperature. The direction of the apparent temperature trend in the surveillance data is a slightly flatter slope in the high-Cu data than in low-Cu data, as shown in Fig. 5-16, while the IVAR data from controlled experiments show the opposite trend-a steeper slope in high-Cu data than in low-Cu data, as shown in Figs. 6.13 and 6.14. The questionable significance and contrary direction of the trend led to additional analysis reported in Sect. 7.1. The CRP $T_{i}$ term is included in the baseline model used for the results in Chaps. 4, 5, and 6 but has been dropped from the simplified model presented in Sect. 7.3 based on that analysis.

It should be noted that the way the temperature effect is implemented in the TTS model, with a strong temperature term in the MF part and a small correction in the CRP term (or no CRP $\mathrm{T}_{\mathrm{i}}$ term), works because (a) the temperature trend in low $\mathrm{Cu}$ surveillance materials is relatively strong and (b) the CRP term contribution to total shift is roughly comparable to the MF term contribution to total shift in the surveillance data. If some plant applications have much larger CRP contributions relative to the MF contributions than in the surveillance data, and if those applications are at temperatures far from the average in the surveillance database, the TTS model may underestimate the temperature effect.

\subsection{References}

1. Odette, G. R., and G. E. Lucas, "Embrittlement of Nuclear Reactor Pressure Vessels," J. of Metals 53(7), 18-22, 2001.

2. Miller, M. K., P. Pareige, and M. G. Burke, "Understanding Pressure Vessel Steels: An Atom Probe Perspective," Materials Characterization 44, 235-54, 2000.

3. Kirk, M., C. Santos, E. D. Eason, J. E. Wright, and G. R. Odette, "Updated Embrittlement Trend Curve for Reactor Pressure Vessel Steels," in Proc. Structural Mechanics in Reactor Technology (SMiRT) Conference, 2003.

4. Server, W., C. English, D. Naiman, and S. Rosinski, Charpy Embrittlement Correlations—Status of Combined Mechanistic and Statistical Bases for U.S. RPV Steels (MRP-45), EPRI Report 1000705, 2001.

5. Jones, R. B., and T. J. Williams, "The Dependence of Radiation Hardening and Embrittlement on Irradiation Temperature," pp. 569-90 in Effects of Radiation on Materials: $17^{\text {th }}$ International Symposium, ASTM STP 1270, Ed. D. S. Gelles et. al., American Society for Testing and Materials, 1996. 


\section{Comparison of the IVAR Database with the Calibrated TTS Model Predictions}

The purpose of this chapter is to compare the trends in the Irradiation Variable (IVAR) database with the TTS model described in Chap. 4. The direct comparison was performed after the modeling effort in Chaps. 3 to 5 was completed, though preliminary IVAR results on some of the variable trends were available earlier.

\subsection{The IVAR Program and Database}

The main objective of the IVAR program was to develop an irradiation hardening and microstructure database that provides a high-accuracy and high-resolution map of the individual and combined effects of key RPV steel embrittlement variables, including: irradiation temperature $\left(\mathrm{T}_{\mathrm{i}}\right)$, flux $(\phi)$, fluence $(\phi t)$, alloy composition $(\mathrm{Cu}, \mathrm{Ni}, \mathrm{Mn}, \mathrm{P}, \mathrm{C}, \mathrm{N}, \mathrm{Mo}, \mathrm{Sn} / \mathrm{As} / \mathrm{Sb}, \mathrm{B})$, heat treatment and product form. Other IVAR objectives were to study embrittlement mechanisms, to explore phenomena like late-blooming Mn-Ni rich phases, and to develop a better understanding of deformation and fracture micromechanics in irradiated RPV steels. The main focus of this chapter is to compare the IVAR yield stress change $\left(\Delta \sigma_{\mathrm{y}}\right)$ database to the predictions of the TTS model fitted to the surveillance data described in Chap. 4.

The IVAR database includes stress-strain curves determined by testing sub-sized tensile specimens and associated information on CRPs and MFs derived from a variety of microanalytical characterization techniques, with special emphasis on the small-angle neutron scattering (SANS) and resistivity-Seebeck coefficient (RSC) methods [1,2]. The irradiations were carried out in three flux $(\phi)$ regimes and three irradiation temperatures $\left(\mathrm{T}_{\mathrm{i}}\right)$ in the IVAR facility at the University of Michigan Ford Research Reactor [3]. The IVAR facility was designed by UCSB and ORNL and was operated by ORNL and University of Michigan staff for a total of 27,650 reactor hours. The high- and low-flux irradiation assemblies and the specimen subcapsule configuration are shown in Fig. 6.1. The nominal flux levels were $\approx 8 \times 10^{10}$ (low), $\approx 3 \times 10^{11}$ (medium) and $\approx 8 \times 10^{11}$ (high) $\mathrm{n} / \mathrm{cm}^{2}-\mathrm{s}, \mathrm{E}>1 \mathrm{MeV}$. The irradiation temperatures were 270, 290 and $310^{\circ} \mathrm{C}$. The irradiations at different fluxes were carried out over overlapping fluence ranges from $\approx 0.006$ to $3.6 \times 10^{19} \mathrm{n} / \mathrm{cm}^{2}$.

The specimens were contained in a total of 80 individual capsules, which occupied one of the 54 IVAR locations for various periods of time. Capsules were inserted and removed during scheduled reactor shutdown periods by using a transfer cask to move the entire irradiation assembly to a nearby hot cell. When not occupied by an actual capsule, a dummy block was inserted in the location to provide a stable neutronic environment. Extensive 3-D neutronics calculations were carried out by ORNL to provide a flux map of the entire IVAR facility [4]. The map was validated and calibrated by multiple activation reaction measurements. The flux map was found to be consistent with individual capsule $\mathrm{Ni}$ and $\mathrm{Fe}$ dosimetry wire measurements, made as part of the IVAR program, to within $\approx \pm 7 \%$. The specimens in a capsule were assigned a fluence corresponding to the full power irradiation time and the nominal flux at the center of each capsule. The capsule temperatures were continuously monitored by 49 thermocouples, that also provided feedback control for achieving the prescribed conditions, which remained extremely stable and close to the nominal set points. Coupled with the thermocouple monitors, extensive heat transfer calculations showed that the specimen temperatures were within $\pm 5^{\circ} \mathrm{C}$ of the nominal values [5].

The IVAR alloy matrix included 41 split melt model steels (SMMS) with systematic single and combined variations in the alloy $\mathrm{Cu}, \mathrm{Mn}, \mathrm{Ni}$, and $\mathrm{P}$ and other compositional variables. The balance of elements was nominally the same and was selected to match typical A533B RPV plate steels. The SMMS were melted in final (approximately) 10-kg batches. One set of alloys with controlled composition variations, from Laval University, was hot-rolled to 27-mm plate (LV alloys) and the other set, produced at Sheffield University and acquired in collaboration with AEA Technology, was hot-rolled to 18-mm plate (CM alloys). The baseline heat treatment for the LV SMMS was as follows: austenitize at $900^{\circ} \mathrm{C}$ for $1 \mathrm{~h}$, air cool, temper at $664^{\circ} \mathrm{C}$ for $4 \mathrm{~h}$, air cool, stress relieve at $600^{\circ} \mathrm{C}$ for $40 \mathrm{~h}$, and air cool. The baseline heat treatment for the CM SMMS was as follows: austenitize at $900^{\circ} \mathrm{C}$ for $30 \mathrm{~min}$, salt quench to $450^{\circ} \mathrm{C}$ 


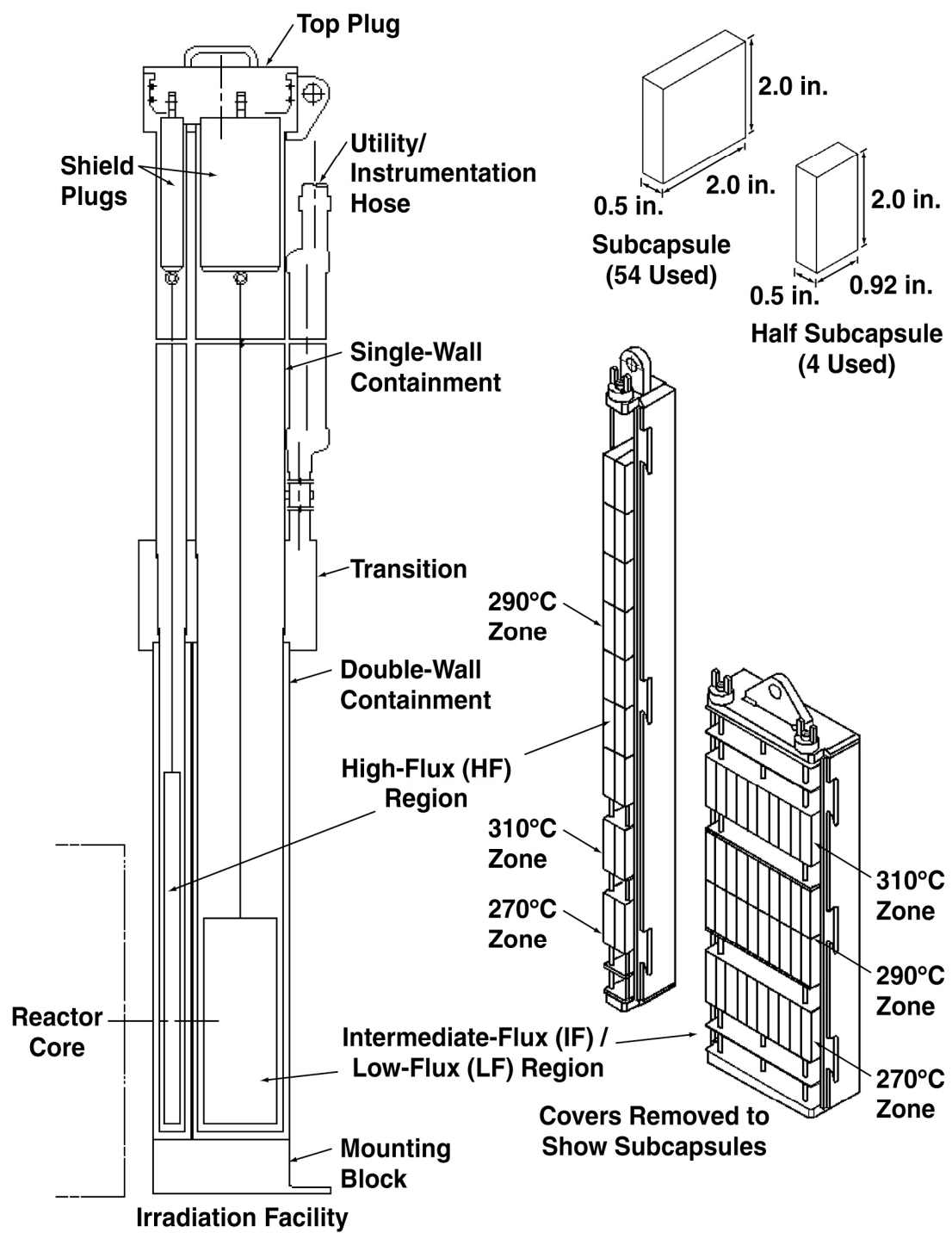

Fig. 6.1. The IVAR irradiation facility and specimen capsules.

and hold for $10 \mathrm{~min}$, temper at $660^{\circ} \mathrm{C}$ for $4 \mathrm{~h}$, air cool, stress relieve at $607^{\circ} \mathrm{C}$ for $24 \mathrm{~h}$, cool at $8^{\circ} \mathrm{C} / \mathrm{h}$ to $300^{\circ} \mathrm{C}$, and air cool. Additionally, a set of $9 \mathrm{CM}$ SMMSs were heat treated to 15 combinations of stress relief times and temperatures and then irradiated at high flux and at $290^{\circ} \mathrm{C}$ to $0.85 \times 10^{19} \mathrm{n} / \mathrm{cm}^{2}$ [6]. The SMMS experiments were complemented by irradiations of 14 commercial or program steels, including 10 welds, 3 plates, and 1 forging. Further perspective on the objectives and character of the SMMS is given in the following paragraphs.

A subset of the IVAR data is compared with the TTS model predictions in the remainder of this chapter. The IVAR data used in this report are summarized in Appendix B. The compositions and heat treatments of the alloys are shown in Table 6.1; the irradiation conditions are summarized in Table 6.2. The alloys include the following; their nominal compositions are noted.

- Twelve $\mathrm{Cu}$-bearing SMMSs: $\mathrm{Cu} \approx 0.11$ to $0.43 \mathrm{wt} \%, \mathrm{Ni} \approx 0.18$ to $1.7 \mathrm{wt} \%, \mathrm{Mn} \approx 0.0$ to $1.69 \mathrm{wt} \%$, and $\mathrm{P} \approx 0.002$ to $0.008 \mathrm{wt} \%$

- Nine nominally $\mathrm{Cu}$-free SMMSs plus one SMMS with $\mathrm{Cu}=0.05: \mathrm{Cu} \approx 0.01$ to 0.05 wt \%, $\mathrm{Ni}=0$ to $1.68 \mathrm{wt} \%, \mathrm{Mn}=0.01$ to $1.67 \mathrm{wt} \%$, and $\mathrm{P}=0.003$ to $0.035 \mathrm{wt} \%$ 
Table 6.1. Alloy compositions and heat treatments

Composition (wt \%)

\begin{tabular}{|c|c|c|c|c|c|c|c|c|c|c|}
\hline Alloy & $\mathrm{Cu} \%$ & $\mathrm{Ni} \%$ & $\mathrm{Mn} \%$ & $\mathrm{Cr} \%$ & Мо\% & $\mathrm{P} \%$ & $\mathrm{C} \%$ & $\mathrm{Si} \%$ & HT $\#^{a}$ & Prod. Form \\
\hline CM1 & 0.01 & 0.01 & 1.67 & 0.04 & 0.56 & 0.003 & 0.13 & 0.15 & 1 & $\mathrm{SMMS}^{b}$ \\
\hline CM3 & 0.02 & 0.85 & 1.60 & 0.00 & 0.49 & 0.006 & 0.13 & 0.16 & 1 & SMMS \\
\hline CM4 & 0.02 & 0.86 & 1.53 & 0.05 & 0.55 & 0.031 & 0.16 & 0.16 & 1 & SMMS \\
\hline CM5 & 0.02 & 0.86 & 1.61 & 0.04 & 0.53 & 0.035 & 0.15 & 0.16 & 1 & SMMS \\
\hline CM6 & 0.02 & 1.68 & 1.50 & 0.05 & 0.54 & 0.007 & 0.15 & 0.17 & 1 & SMMS \\
\hline CM8 & 0.01 & 0.86 & 0.01 & 0.04 & 0.55 & 0.004 & 0.13 & 0.14 & 1 & SMMS \\
\hline CM9 & 0.01 & 0.86 & 0.85 & 0.04 & 0.55 & 0.003 & 0.15 & 0.15 & 1 & SMMS \\
\hline CM10 & 0.02 & 0.88 & 1.66 & 0.05 & 0.53 & 0.008 & 0.16 & 0.17 & 1 & SMMS \\
\hline CM13 & 0.11 & 0.83 & 1.61 & 0.00 & 0.51 & 0.004 & 0.15 & 0.16 & 1 & SMMS \\
\hline CM14 & 0.11 & 0.83 & 1.62 & 0.00 & 0.52 & 0.040 & 0.16 & 0.17 & 1 & SMMS \\
\hline CM19 & 0.42 & 0.85 & 1.63 & 0.01 & 0.51 & 0.005 & 0.16 & 0.16 & 1 & SMMS \\
\hline CM21 & 0.42 & 0.84 & 0.01 & 0.02 & 0.58 & 0.002 & 0.14 & 0.14 & 1 & SMMS \\
\hline $\mathrm{CM} 22$ & 0.42 & 0.84 & 0.84 & 0.02 & 0.56 & 0.002 & 0.14 & 0.14 & 1 & SMMS \\
\hline CM31 & 0.01 & 0.86 & 1.65 & 0.05 & 0.51 & 0.006 & 0.16 & 0.17 & 1 & SMMS \\
\hline LB & 0.40 & 0.18 & 1.35 & 0.06 & 0.53 & 0.005 & 0.16 & 0.22 & 2 & SMMS \\
\hline LC & 0.41 & 0.86 & 1.44 & 0.06 & 0.55 & 0.005 & 0.14 & 0.23 & 2 & SMMS \\
\hline LD & 0.38 & 1.25 & 1.38 & 0.07 & 0.53 & 0.005 & 0.19 & 0.23 & 2 & SMMS \\
\hline LG & 0.01 & 0.74 & 1.37 & 0.05 & 0.55 & 0.005 & 0.16 & 0.22 & 2 & SMMS \\
\hline LH & 0.11 & 0.74 & 1.39 & 0.05 & 0.55 & 0.005 & 0.16 & 0.24 & 2 & SMMS \\
\hline LI & 0.20 & 0.74 & 1.37 & 0.05 & 0.55 & 0.005 & 0.16 & 0.24 & 2 & SMMS \\
\hline MD & 0.27 & 0.57 & 1.61 & 0.10 & 0.41 & 0.017 & 0.08 & 0.62 & 3 & Weld \\
\hline $62 \mathrm{~W}$ & 0.23 & 0.60 & 1.61 & 0.12 & 0.39 & 0.020 & 0.08 & 0.59 & 4 & Weld \\
\hline $63 \mathrm{~W}$ & 0.30 & 0.69 & 1.65 & 0.10 & 0.43 & 0.016 & 0.10 & 0.63 & 5 & Weld \\
\hline $65 \mathrm{~W}$ & 0.22 & 0.60 & 1.45 & 0.09 & 0.39 & 0.015 & 0.08 & 0.48 & 6 & Weld \\
\hline BWA & 0.21 & 0.63 & 1.69 & 0.14 & 0.40 & 0.014 & 0.08 & 0.45 & 7 & Weld \\
\hline BWC & 0.08 & 0.62 & 1.30 & 0.08 & 0.31 & 0.009 & 0.08 & 0.37 & 8 & Weld \\
\hline WP & 0.04 & 1.65 & 1.43 & 0.05 & 0.39 & 0.011 & 0.06 & 0.50 & 9 & Weld \\
\hline A 302 & 0.14 & 0.20 & 1.20 & 0.24 & 0.60 & 0.015 & 0.21 & 0.28 & 10 & Plate \\
\hline JRQ & 0.14 & 0.82 & 1.40 & 0.12 & 0.50 & 0.019 & 0.18 & 0.25 & 11 & Plate \\
\hline OV1 & $<0.02$ & $<0.02$ & 1.60 & $<0.02$ & $<0.02$ & $<0.002$ & $<0.02$ & $<0.02$ & 12 & Model alloy \\
\hline OV12 & $<0.02$ & $<0.02$ & $<0.02$ & $<0.02$ & $<0.02$ & $<0.002$ & $<0.02$ & $<0.02$ & 12 & Model alloy \\
\hline OV9 & $<0.02$ & $<0.02$ & $<0.02$ & $<0.02$ & $<0.02$ & 0.025 & $<0.02$ & $<0.02$ & 12 & Model alloy \\
\hline OV10 & $<0.02$ & $<0.02$ & 1.60 & $<0.02$ & $<0.02$ & 0.025 & $<0.02$ & $<0.02$ & 12 & Model alloy \\
\hline
\end{tabular}

${ }^{a}$ Heat Treatments:

1. Heat treatment for $\mathrm{CM}$ alloys: austenitize $900^{\circ} \mathrm{C} 30 \mathrm{~min} /$ salt quench $450^{\circ} \mathrm{C} 10 \mathrm{~min} /$ temper $660^{\circ} \mathrm{C} 4 \mathrm{~h}$ air cool $/$ stress relief $607^{\circ} \mathrm{C} 24 \mathrm{~h}$ followed by slow cool $@ 8^{*} \mathrm{C} / \mathrm{h}$ to $300^{\circ} \mathrm{C}$ air cool.

2. Heat treatment for Laval alloys: austenitize $900^{\circ} \mathrm{C} 1 \mathrm{~h} /$ air cool/temper $664^{\circ} \mathrm{C} 4 \mathrm{~h}$ air cool/stress relief $600^{\circ} \mathrm{C} 40 \mathrm{~h}$ to $300^{\circ} \mathrm{C} /$ air cool.

3. PWHT $607^{\circ} \mathrm{C}, 22.5$.

4. Submerged Arc Weld (SAW )(stress relieved (SR) 8 cycles of $6 \mathrm{~h}$ at $593-621^{\circ} \mathrm{C}$.

5. SAW, SR $48 \mathrm{~h}$ at $593-621^{\circ} \mathrm{C}$.

6. SAW, SR $80 \mathrm{~h}$ at $593-621^{\circ} \mathrm{C}$.

7. PWHT, $607^{\circ} \mathrm{C}$ for $15 \mathrm{~h}$, furnace cool.

8. PWHT, $607^{\circ} \mathrm{C}$ for $13.5 \mathrm{~h}$, furnace cool.

9. Austenitized $920^{\circ} \mathrm{C}$, water quench; tempered $600^{\circ} \mathrm{C}, 42 \mathrm{~h}, 650^{\circ} \mathrm{C}, 6 \mathrm{~h}$; slow cooled.

10. Normalized and tempered; $1700^{\circ} \mathrm{F} 6.5 \mathrm{~h} /$ warer quench $/ 1625^{\circ} \mathrm{F} 6.5 \mathrm{~h} /$ warer quench $/ 1200^{\circ} \mathrm{F} 6.5 \mathrm{~h} /$ warer quench $/ 125^{\circ} \mathrm{F} 6.5 \mathrm{~h} /$ furnace cool.

11. Normalized@ $900^{\circ} \mathrm{C}$, quenched @ $880^{\circ} \mathrm{C}$, tempered at $665^{\circ} \mathrm{C}$ for $12 \mathrm{~h}, \mathrm{SR}$ at $620^{\circ} \mathrm{C} 40 \mathrm{~h}$.

12. Normalize @ $775^{\circ} \mathrm{C}, 17 \mathrm{~h}$; forced helium quench.

${ }^{b}$ SMMS are Split Melt Model Steels 
Table 6.2. Irradiation conditions

\begin{tabular}{|c|c|c|c|}
\hline Capsule ID & $\mathrm{T}_{\mathrm{i}}\left({ }^{\circ} \mathrm{C}\right)$ & $\phi\left(\mathrm{n} / \mathrm{cm}^{2}-\mathrm{s}\right)$ & $\phi t\left(\mathrm{n} / \mathrm{cm}^{2}\right)$ \\
\hline A5 & 290 & $5.1 \times 10^{11}$ & $4.0 \times 10^{17}$ \\
\hline $\mathrm{T} 1$ & 290 & $7.8 \times 10^{11}$ & $7.0 \times 10^{17}$ \\
\hline $\mathrm{T} 2$ & 290 & $7.8 \times 10^{11}$ & $1.8 \times 10^{18}$ \\
\hline $\mathrm{T} 3$ & 290 & $7.8 \times 10^{11}$ & $3.4 \times 10^{18}$ \\
\hline $\mathrm{T} 4$ & 290 & $9.7 \times 10^{11}$ & $7.5 \times 10^{18}$ \\
\hline SR1 & 290 & $9.7 \times 10^{11}$ & $8.5 \times 10^{18}$ \\
\hline Piggyback $^{a}$ & 290 & $7.7 \times 10^{11}$ & $1.0 \times 10^{19}$ \\
\hline $\mathrm{T} 5$ & 290 & $7.8 \times 10^{11}$ & $1.4 \times 10^{19}$ \\
\hline T6 & 290 & $9.7 \times 10^{11}$ & $3.3 \times 10^{19}$ \\
\hline $\mathrm{T} 7$ & 270 & $9.2 \times 10^{11}$ & $3.8 \times 10^{18}$ \\
\hline $\mathrm{T} 8$ & 270 & $9.2 \times 10^{11}$ & $1.5 \times 10^{19}$ \\
\hline T9 & 310 & $9.8 \times 10^{11}$ & $4.0 \times 10^{18}$ \\
\hline $\mathrm{T} 10$ & 310 & $9.8 \times 10^{11}$ & $1.5 \times 10^{19}$ \\
\hline $\mathrm{T} 11$ & 290 & $2.6 \times 10^{11}$ & $4.0 \times 10^{17}$ \\
\hline $\mathrm{T} 12$ & 290 & $3.2 \times 10^{11}$ & $1.0 \times 10^{18}$ \\
\hline $\mathrm{T} 13$ & 290 & $3.1 \times 10^{11}$ & $2.4 \times 10^{18}$ \\
\hline $\mathrm{T} 14$ & 290 & $3.2 \times 10^{11}$ & $4.8 \times 10^{18}$ \\
\hline $\mathrm{T} 15$ & 290 & $2.6 \times 10^{11}$ & $8.5 \times 10^{18}$ \\
\hline T16 & 290 & $3.0 \times 10^{11}$ & $1.6 \times 10^{19}$ \\
\hline $\mathrm{T} 17$ & 270 & $2.5 \times 10^{11}$ & $4.3 \times 10^{18}$ \\
\hline $\mathrm{T} 18$ & 270 & $3.6 \times 10^{11}$ & $1.7 \times 10^{19}$ \\
\hline T19 & 310 & $2.3 \times 10^{11}$ & $4.0 \times 10^{18}$ \\
\hline $\mathrm{T} 20$ & 310 & $3.4 \times 10^{11}$ & $1.6 \times 10^{19}$ \\
\hline A1 & 290 & $7.0 \times 10^{10}$ & $6.0 \times 10^{16}$ \\
\hline $\mathrm{A} 2$ & 290 & $7.0 \times 10^{10}$ & $1.0 \times 10^{17}$ \\
\hline A3 & 290 & $7.0 \times 10^{10}$ & $2.3 \times 10^{17}$ \\
\hline A4 & 290 & $7.0 \times 10^{10}$ & $3.2 \times 10^{17}$ \\
\hline $\mathrm{T} 21$ & 290 & $1.0 \times 10^{11}$ & $3.0 \times 10^{17}$ \\
\hline $\mathrm{T} 22$ & 290 & $1.0 \times 10^{11}$ & $1.1 \times 10^{18}$ \\
\hline $\mathrm{T} 23$ & 290 & $8.0 \times 10^{10}$ & $2.4 \times 10^{18}$ \\
\hline $\mathrm{T} 24$ & 290 & $8.0 \times 10^{10}$ & $4.0 \times 10^{18}$ \\
\hline
\end{tabular}

${ }^{a}$ Piggyback irradiations were not part of the IVAR program. These ORNL irradiations were carried out in space-compatible subcapsules prepared by UCSB in the mouths of large CT specimens in the Tenth HSSI Irradiation Series capsules.

- Five commercial welds: $\mathrm{Cu} \approx 0.21$ to $0.30 \mathrm{wt} \%, \mathrm{Ni} \approx 0.6$ to $0.69 \mathrm{wt} \%, \mathrm{Mn} \approx 1.45$ to $1.69 \mathrm{wt} . \%$, and $\mathrm{P} \approx 0.014$ to $0.020 \mathrm{wt} \%$

- Two low-copper commercial welds: $\mathrm{Cu} \approx 0.04$ to $0.06 \mathrm{wt} \%, \mathrm{Ni} \approx 0.6$ to $1.65 \mathrm{wt} \%, \mathrm{Mn} \approx 1.3$ to 1.43 wt $\%$, and $\mathrm{P} \approx 0.009$ to 0.011 wt \%

- Two program plates: $\mathrm{Cu} \approx 0.14 \mathrm{wt} \%, \mathrm{Ni} \approx 0.2$ to $0.82 \mathrm{wt} \%, \mathrm{Mn} \approx 1.2$ to $1.4 \mathrm{wt} \%$, and $\mathrm{P} \approx 0.015$ to $0.019 \mathrm{wt} \%$

- Four simple ferritic model alloys: $\mathrm{Fe}, \mathrm{Fe}+1.6$ wt. $\% \mathrm{Mn}, \mathrm{Fe}+0.025$ wt \% P, and Fe +1.6 wt \% Mn + 0.025 wt $\% \mathrm{P}$

Tests on flat tensile specimens with $9 \times 2 \times 0.5 \mathrm{~mm}$ gauge section were conducted on a computercontrolled, semiautomated tensile instrument designed and constructed by UCSB on an Instron 1100 tabletop load frame. Cartridges of 29 specimens were loaded and tested in sequence. Each cartridge included two unirradiated reference steels with precisely known yield and ultimate stresses to provide a continuous system calibration. Except in a few cases, a minimum of two tensile tests was carried out for 
each alloy-irradiation condition. Replicate tensile tests showed an average standard deviation in $\Delta \sigma_{\mathrm{y}}$ to be $\approx \pm 15 \mathrm{MPa}$.

Some additional perspective on the objectives and character of SMMSs is useful. The use of SMMS was specifically intended to provide precise and controlled variations in key compositional variables and variable combinations. The SMMS baseline composition, fabrication route, and heat treatment schedule were selected to produce microstructures and properties that closely match those found in "typical" RPV plates. The baseline heat treatment generally produced prior austenite grain sizes of $\approx 50 \mu \mathrm{m}$ and plate microstructures ranging from tempered bainite to mixed tempered bainite-ferrite. The corresponding unirradiated $\sigma_{\mathrm{y}}$ ranged from 400 to $525 \mathrm{MPa}$, again very similar to the range of strength levels of typical A533B-type RPV steels. Thus, the SMMS are similar to their commercial counterparts, except that (1) they are generally likely to exhibit less melt-to-melt variability than is characteristic of the wide range of heavy- section RPV plates, and (2) the variation of key elements is intentionally wider than in typical commercial steelmaking practice in order to identify variable trends and interactions. Thus, the matrix includes some chemistry combinations that do not generally occur in commercial plates, such as higherthan-typical $\mathrm{Ni}$ and $\mathrm{Cu}$ and lower-than-typical $\mathrm{P}$ and $\mathrm{Mn}$ and high $\mathrm{P}$ with low $\mathrm{Cu}$.

Given their wide range of compositions, there may be some differences in the microstructures of the SMMSs compared to what might characterize the corresponding range and average of plates in the TTS database. For example, some SMMS microstructures may differ somewhat from the average for the plates in the TTS database in particular details, such as dislocation densities. Thus, the SMMSs can be thought of as a unique product form or as a small set of product forms that differ from those found in the TTS database. The TTS database itself has three product form coefficients for plate as well as additional product form coefficients for welds and forgings, so calibrating different coefficients for different product forms is a normal feature of the TTS model.

Further details on the IVAR facility and materials are provided in Ref. [6].

\subsection{Comparison of TTS Model Predictions and IVAR Data Trends-The TTS to $\Delta \boldsymbol{\sigma}_{\mathrm{y}}$ Conversion}

The objective is to compare predictions of the TTS model developed in Chap. 4 to various subsets of the IVAR database. To make this comparison, it is necessary to convert the TTS model predictions to $\Delta \sigma_{\mathrm{y}}$ by using a relation,

$$
\Delta \sigma_{\mathrm{y}}=\mathrm{TTS} / \mathrm{C}_{\mathrm{c}}
$$

As discussed in Chap. 2, the conversion factor $\mathrm{C}_{\mathrm{c}}$ depends on a number of variables, including the unirradiated Charpy properties and the $\Delta \sigma_{\mathrm{y}}$ itself. Eq. (6-1) can be expressed as

$$
\mathrm{C}_{\mathrm{c}}=\mathrm{C}_{0}+\mathrm{C}_{1} \mathrm{TTS}+\mathrm{C}_{2} \mathrm{TTS}^{2} \ldots
$$

Here the units are ${ }^{\circ} \mathrm{C} / \mathrm{MPa}, \mathrm{MPa},{ }^{\circ} \mathrm{C}$ for $\mathrm{C}_{\mathrm{c}}, \Delta \sigma_{\mathrm{y}}$ and TTS, respectively.

Previous studies have shown that the $\mathrm{C}_{\mathrm{c}}$ for welds is typically somewhat larger than for plates [6]. For welds the expression was based on slightly modifying a fit to $\Delta \sigma_{\mathrm{y}}-$ TTS data in Ref. [6] with data reported by English from the PSF experiment [7], yielding

$$
\mathrm{C}_{\mathrm{cw}}=0.55+1.2 \times 10^{-3} \mathrm{TTS}-1.33 \times 10^{-6} \mathrm{TTS}^{2} \ldots
$$

This expression is also approximately equivalent to the model-based $\mathrm{C}_{\mathrm{c}}$ described in Chap. 2, as well as and the formulation reported in NUREG/CR-6778 [6]. For plates, Eq. (6-2) was least-square fit to a compilation of ç - TTS data in Ref. [8], yielding

$$
\mathrm{C}_{\mathrm{cp}}=0.45+1.945 \times 10^{-3} \mathrm{TTS}-5.496 \times 10^{-6} \mathrm{TTS}^{2}+8.473 \times 10^{-9} \mathrm{TTS}^{3}
$$


The corresponding $\Delta \sigma_{\mathrm{y}}$-TTS relations are shown in Fig. 6.2. While Eqs. (6-3) and (6-4) provide reasonable conversions of TTS to $\Delta \sigma_{\mathrm{y}}$, the actual values for any particular alloy, and the effective average for the TTS database, may vary from these "generic" estimates, which adds an element of uncertainty to any quantitative TTS model IVAR $\Delta \sigma_{\mathrm{y}}$ comparisons. Based on the fits of available data, estimated conversion uncertainties for the $\Delta \sigma_{\mathrm{y}}$ derived from the TTS model predictions are on average $\oplus \pm 10 \%$ up to $20 \%$ in some individual cases.

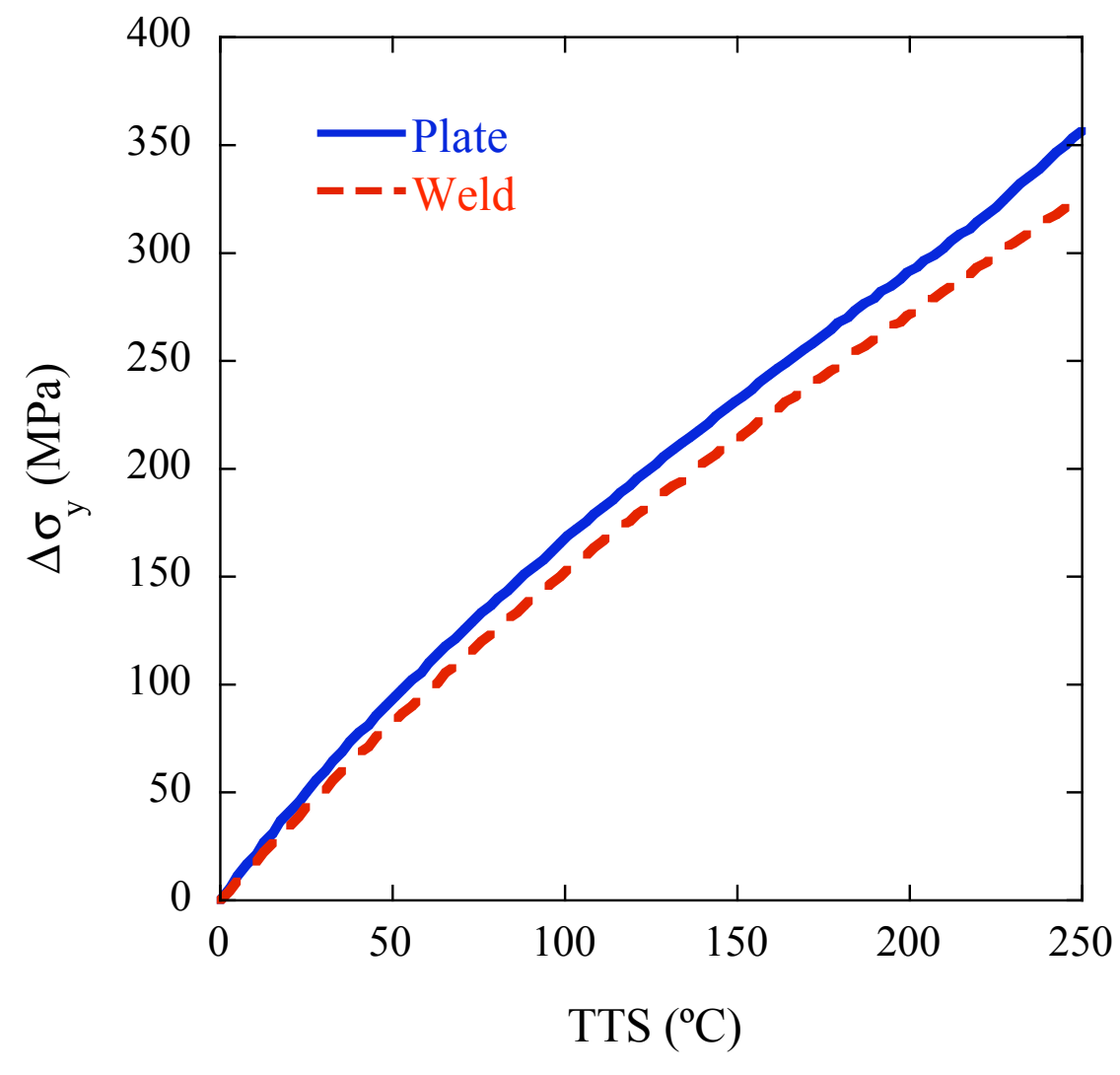

Fig. 6.2. The relations used to convert TTS predictions to $\Delta \sigma_{\mathrm{y}}$ data for welds and plates.

\subsection{The Fluence and Irradiation Temperature Dependence of $\Delta \sigma_{\mathrm{y}}$ in Welds and Plates}

Given the TTS to $\Delta \sigma_{\mathrm{y}}$ conversion uncertainties noted above, coupled with possible product form differences, especially for the IVAR SMMS case, modest differences between the TTS model predictions and the IVAR $\Delta \sigma_{\mathrm{y}}$ are not unexpected. The welds and plates discussed in this section are among the IVAR alloys that are the most similar to the steels in the TTS database. Figures $6.3 \mathrm{a}-\mathrm{f}$ show the IVAR data and the corresponding Chap. 4 TTS model predictions converted to $\Delta \sigma_{\mathrm{y}}$ curves plotted against square root fluence for six submerged-arc welds. The irradiations were carried out at $290^{\circ} \mathrm{C}$ at three dose rates, representing the low (squares $\approx 8 \times 10^{10} \mathrm{n} / \mathrm{cm}^{2}$ ), intermediate (circles $\approx 3 \times 10^{11} \mathrm{n} / \mathrm{cm}^{2}$ ), and high (diamonds $\approx 8 \times 10^{11} \mathrm{n} / \mathrm{cm}^{2}$ ) IVAR flux regimes, respectively. Five of these welds (MW, 62W, 63W, $65 \mathrm{~W}$, and BWA) contain more than $0.2 \mathrm{wt} \% \mathrm{Cu}$, while one weld (BWC) contains only $\approx 0.06 \mathrm{wt} \% \mathrm{Cu}$, which is below the $0.072 \mathrm{wt} \%$ threshold for CRP contributions to TTS. Figures $6.3 \mathrm{~g}-\mathrm{h}$ show similar plots for the A302B and JRQ plates with intermediate $\mathrm{Cu}(\approx 0.14 \mathrm{wt} \%$ in both cases $)$ using the CE-plate 

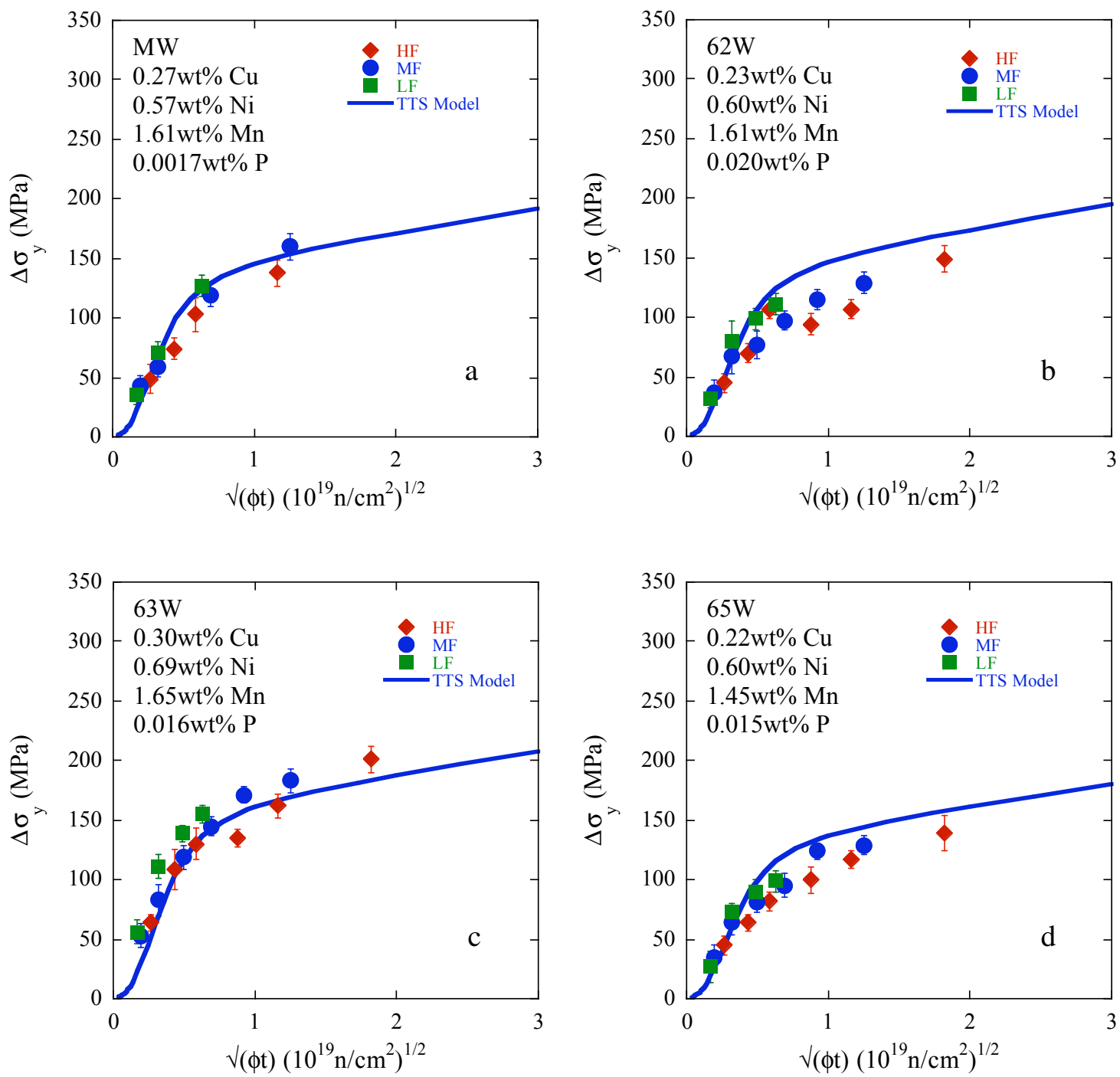

Fig. 6.3 a-d. The TTS model predictions and the IVAR $\Delta \sigma_{\mathrm{y}}$ data plotted against the square root of fluence for four commercial Cu-bearing welds.

coefficients in the TTS model. In the case of the $\mathrm{Cu}$ bearing welds, the TTS model is in generally good agreement with IVAR weld $\Delta \sigma_{\mathrm{y}}$ data, especially at low flux and fluence. However, the TTS model tends to slightly over predict the IVAR $\Delta \sigma_{\mathrm{y}}$ data at higher flux and fluence in 3 out of the 5 cases $(62 \mathrm{~W}, 65 \mathrm{~W}$, BWA). These differences are generally within the expected TTS model and IVAR $\Delta \sigma_{y}$ data uncertainties. The TTS model prediction is also in good agreement with the IVAR $\Delta \sigma_{\mathrm{y}}$ for the low $\mathrm{Cu}$ weld as well as for the plate $\Delta \sigma_{\mathrm{y}}$ data at low and intermediate flux.

The TTS model does not predict the systematic dose-rate effects observed in comparing HF, MF, and LF subsets in Fig. 6.3, or those found in the SMMS comparison that are discussed below. (Note: due to the expanded fluence scale the dose rate effects do not appear to be large in these figures. The effects of dose rate are more clearly shown in Sect. 6.6 [see Figs. 6.15 to 6.18].) The TTS model does include a dose rate effect, but it applies to the effect of flux noted when comparing typical BWR with PWR surveillance data. The typical BWR flux range is well below the range of the IVAR flux levels, which 

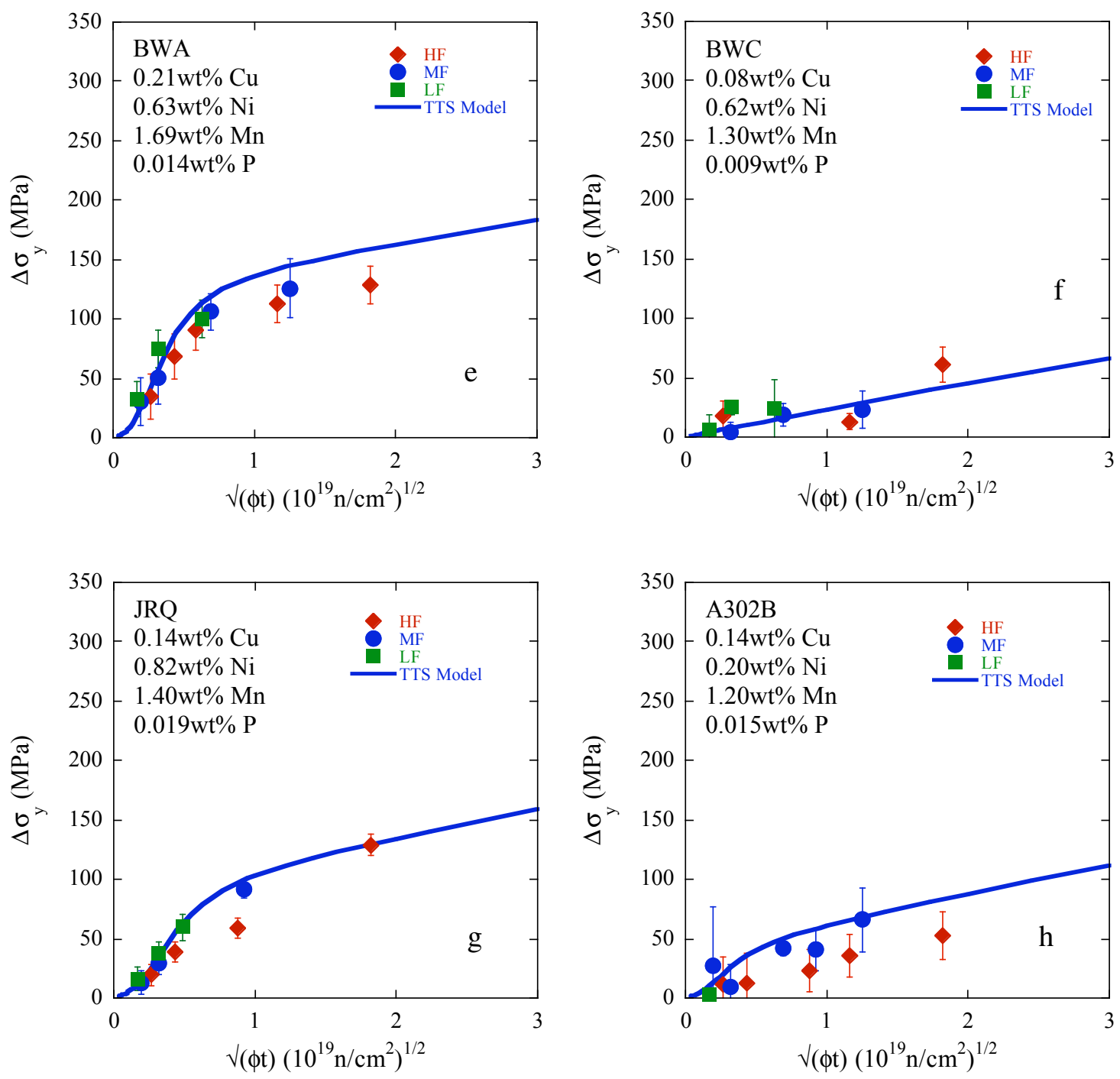

Fig. $6.3 \mathrm{e}-\mathrm{h}$. The TTS model predictions and the IVAR $\Delta \sigma_{\mathrm{y}}$ data plotted against the square root of fluence for a Cu-bearing, and low-Cu weld and two commercial plates.

overlap the PWR range. This different range of flux effects captured in the TTS model vs the flux effects present in the IVAR data affects many of the comparisons throughout this chapter, as discussed in some detail in Sect. 6.6.

Figure 6.4 compares the TTS model predictions to the available $\Delta \sigma_{\mathrm{y}}$ data for the same welds and plates at $\approx 0.43$ and $\approx 1.6 \times 10^{19} \mathrm{n} / \mathrm{cm}^{2}$, for medium flux IVAR irradiations at 270,290 and $310^{\circ} \mathrm{C}$. MW and JRQ IVAR data are not available at higher and lower irradiation temperatures. The TTS model lines are plotted only over the range of $T_{i}$ in the surveillance database. The irradiation temperature dependence predicted by the TTS model is somewhat stronger than for the low-Cu IVAR $\Delta \sigma_{\mathrm{y}}$ data and is, on average, slightly weaker than the IVAR data for $\mathrm{Cu}$ bearing welds. However, the overall agreement is again generally good and within the expected TTS model and IVAR data uncertainties. 

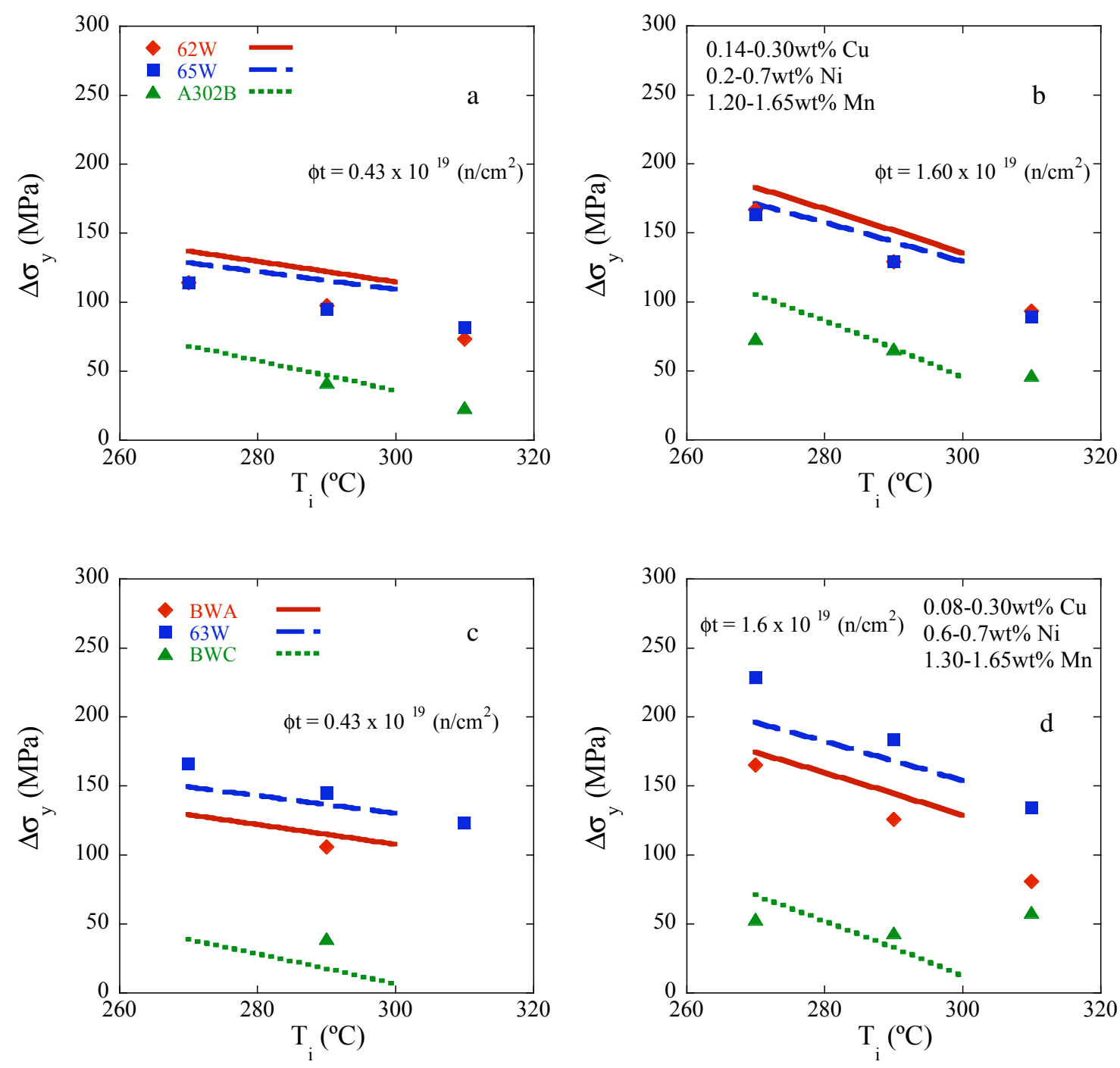

Fig. 6.4. The TTS model predictions and the IVAR $\Delta \sigma_{\mathrm{y}}$ data at two fluences for intermediate flux irradiations of five commercial welds and a plate at 270,290 , and $310^{\circ} \mathrm{C}$.

\subsection{The Fluence Dependence of $\Delta \sigma_{y}$ in the SMMS Irradiated at $290^{\circ} \mathrm{C}$}

\subsubsection{Cu-Bearing SMMS Irradiated at $290^{\circ} \mathrm{C}$}

For comparisons with SMMS, the $\mathrm{Cu}_{\max }$ categories and coefficients used in the TTS model must be determined in part by expert judgment. The SMMS with $\mathrm{Ni} \geq 0.75 \mathrm{wt} \%$ are assigned $\mathrm{Cu}_{\max }=0.30 \mathrm{wt} \%$, the value for the higher $\mathrm{Ni}$ weld category (Linde 1092) in the TTS database. Lower Ni SMMS $(\mathrm{Ni}<0.75$ wt \%) are assigned $\mathrm{Cu}_{\max }=0.24 \mathrm{wt} \%$, the value for the medium $\mathrm{Ni}$ weld group (typical Linde 80 ) in the TTS data.

The nominal baseline composition for the SMMS described in the following paragraphs and elsewhere in this chapter are as follows: medium $0.8 \mathrm{wt} \% \mathrm{Ni}$; higher $1.4(\mathrm{LV})$ to $1.6(\mathrm{CM}) \mathrm{wt} \% \mathrm{Mn}$; and low $0.005 \mathrm{wt} \% \mathrm{P}$. The results are described in terms of composition variations with respect to these 
baseline values. Figure 6.5 compares TTS model $\Delta \sigma_{\mathrm{y}}$ vs square root fluence curves to the IVAR data for the three baseline medium-Ni SMMS compositions with varying $\mathrm{Cu} \approx 0.1,0.2$ and $0.4 \mathrm{wt} \% \mathrm{Cu}$ (bulk)
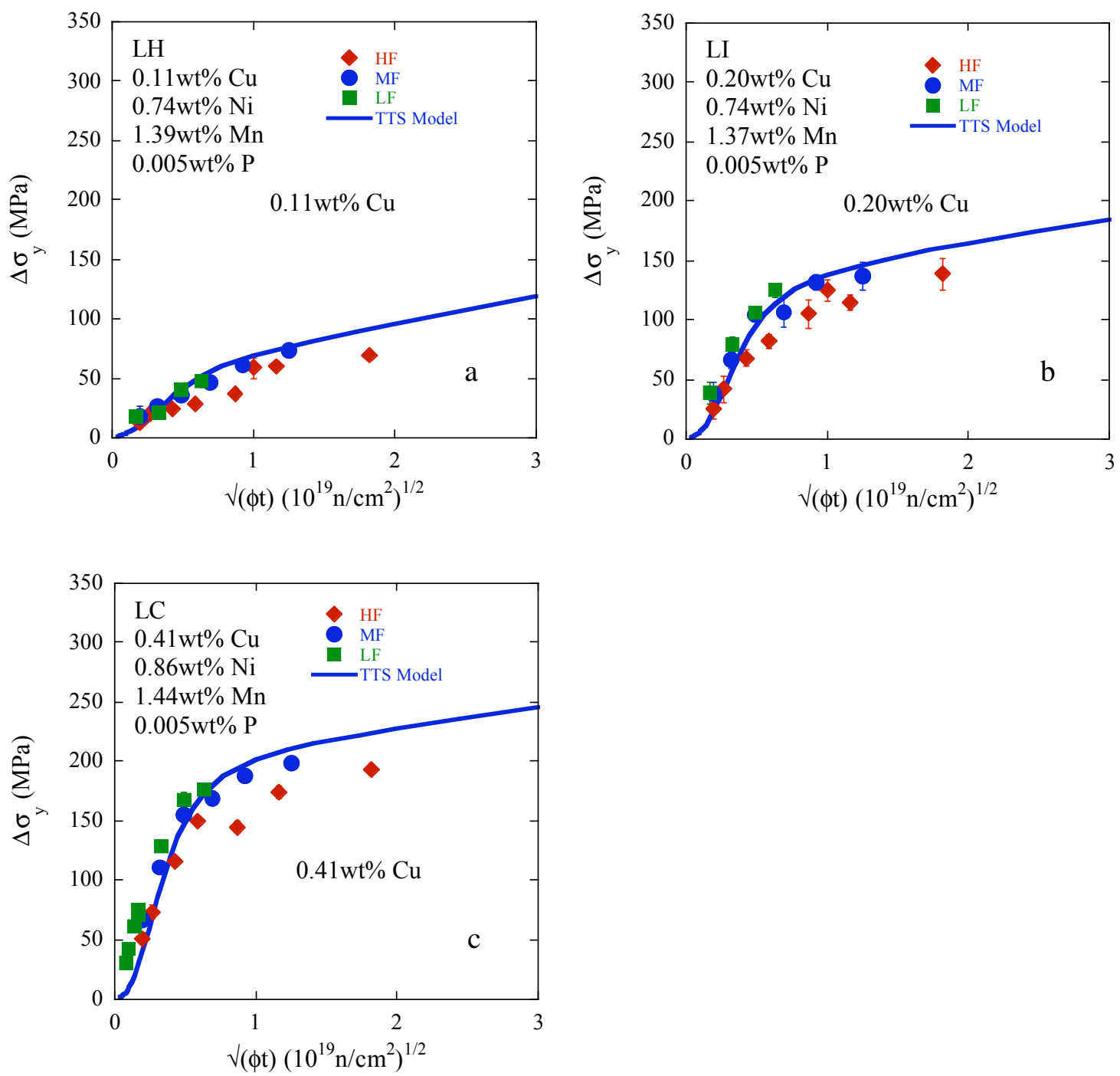

Fig. $6.5 \mathrm{a}-\mathrm{c}$. The TTS model predictions and the IVAR $\Delta \sigma_{\mathrm{y}}$ data plotted against the square root of fluence for three 0.8 wt \% Ni SMMS with 0.1 (a), 0.2 (b), and 0.4 (c) wt \% Cu.

irradiated at $290^{\circ} \mathrm{C}$ at low, medium, and high IVAR flux levels. The solid line is the TTS model prediction, with the set of plate product form coefficients that provide the best agreement. As noted above, the $\mathrm{Cu}_{\max }$ was $0.30 \mathrm{wt} \% \mathrm{Cu}$ for $\mathrm{Ni} \geq 0.75 \mathrm{wt} \%$ and $0.24 \mathrm{wt} \% \mathrm{Cu}$ for $\mathrm{Ni}<0.75 \mathrm{wt} \%$. Figure 6.6 shows similar plots for a high $\approx 0.4 \mathrm{wt} \% \mathrm{Cu}$ (bulk) SMMS composition with varying $\mathrm{Ni} \approx 0.2,0.8$, and $1.3 \mathrm{wt} \%$. Note the $0.4 \% \mathrm{Cu}$ and $1.3 \mathrm{wt} \% \mathrm{Ni}$ SMMS is outside the range of all surveillance plates, although there are welds with similar compositions. Figure 6.7 shows the corresponding plots for a high $\approx 0.4$ wt $\% \mathrm{Cu}$ (bulk), medium-Ni SMMS baseline composition with varying $\mathrm{Mn} \approx 0,0.8$ and $1.6 \mathrm{wt} \%$. The coefficients for forgings were used for the 0 and $0.8 \mathrm{wt} \% \mathrm{Mn}$ SMMS because a substantial part of the Mn effect in the TTS model is implicit in the differences between forging and other coefficients. With the exception of the $0.0 \mathrm{wt} \% \mathrm{Mn}$ alloy, whose composition is far outside the TTS database, the overall agreement is good. 

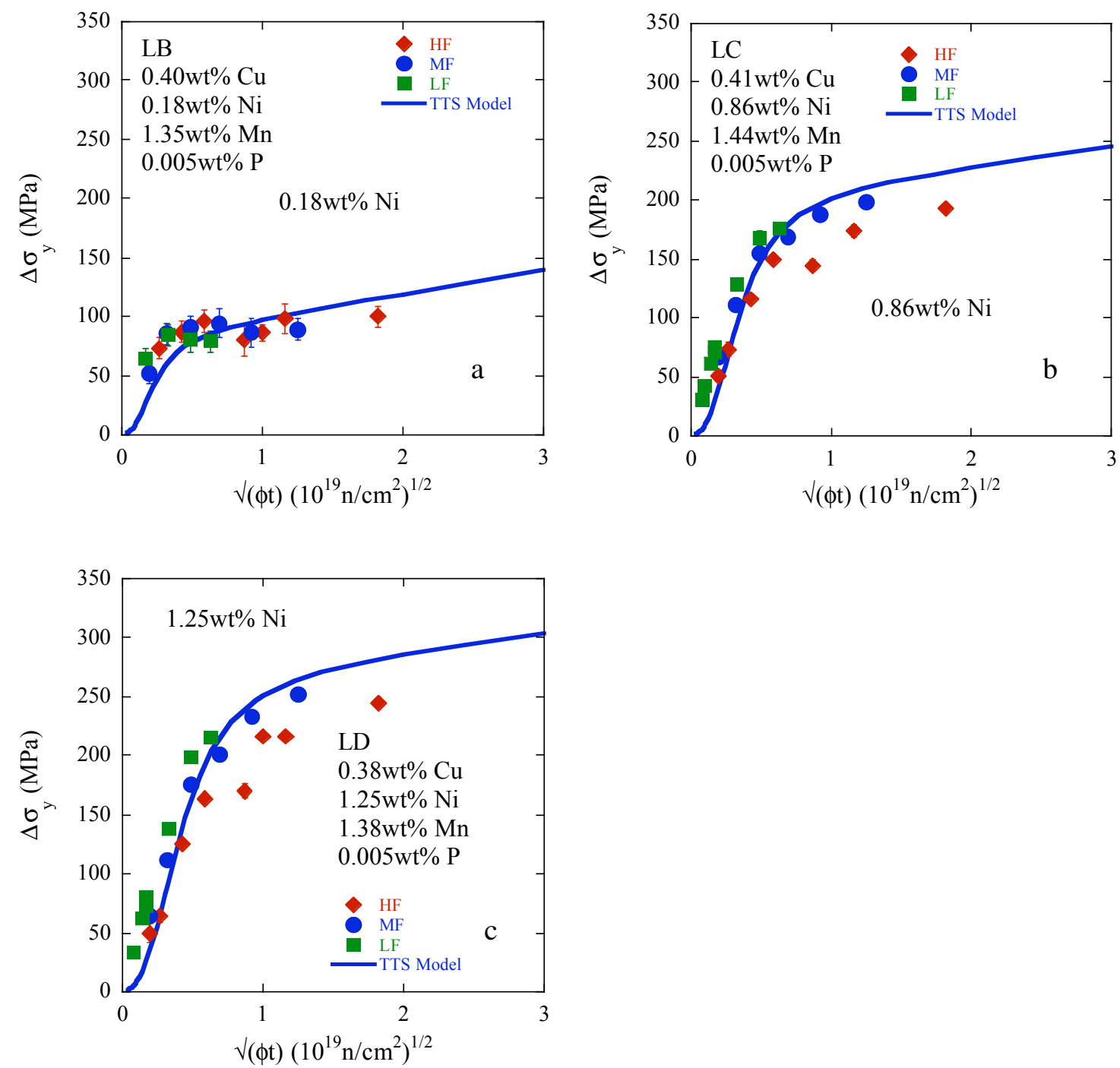

Fig. 6.6 a-c. The TTS model predictions and the IVAR $\Delta \sigma_{\mathrm{y}}$ data plotted against the square root of fluence for three $0.4 \mathrm{wt} \% \mathrm{Cu}$ SMMS with 0.2 (a) 0.4 (b) and 1.25 (c) wt \% Ni.

Figure 6.8 a cross plots the $\mathrm{Cu}$ dependence predicted by the TTS model for CE-Plate coefficient $(\mathrm{B}=135.2)$ and $\mathrm{Cu}_{\max }=0.30 \mathrm{wt} \%$, and the IVAR $\Delta \sigma_{\mathrm{y}}$ data for SMMS baseline composition at 0.24 and $1.6 \times 10^{19} \mathrm{n} / \mathrm{cm}^{2}$ for $290^{\circ} \mathrm{C}$ irradiations at the intermediate IVAR flux. The lines are the TTS model predictions covering the range of compositions in the surveillance plate database. A similar cross plot for $\mathrm{Ni}$ variations is shown in Fig. $6.8 \mathrm{~b}$ for the SMMS with $0.4 \mathrm{wt} \% \mathrm{Cu}, 1.4 \mathrm{wt} \% \mathrm{Mn}$, and $0.005 \mathrm{wt} \% \mathrm{P}$. In this case curves are given for both $\mathrm{Cu}_{\max }=0.24$ (dashed) and $0.30 \mathrm{wt} \%$ (solid). Figure $6.8 \mathrm{c}$ shows the corresponding $\mathrm{Mn}$ cross plot for the SMMS medium-Ni baseline composition, assuming $\mathrm{Cu}_{\max }=0.30 \mathrm{wt}$ $\%$. The effect of Mn shown in Fig. 6.8c is based on using the CE-Plate coefficient (solid lines) for higher $\mathrm{Mn}$ and forging coefficient $(\mathrm{B}=102.3$, dashed lines) for lower Mn. The arrows in Fig. 6.8(a-c) show the average $\mathrm{Cu}, \mathrm{Ni}$, and $\mathrm{Mn}$ contents of the steels in the TTS plate database. Overall, the TTS model 
predictions of the effects of $\mathrm{Ni}, \mathrm{Cu}$ and $\mathrm{Mn}$ (as reflected in the forging coefficients), are in reasonably good agreement with the IVAR $\Delta \sigma_{\mathrm{y}}$ data trends.
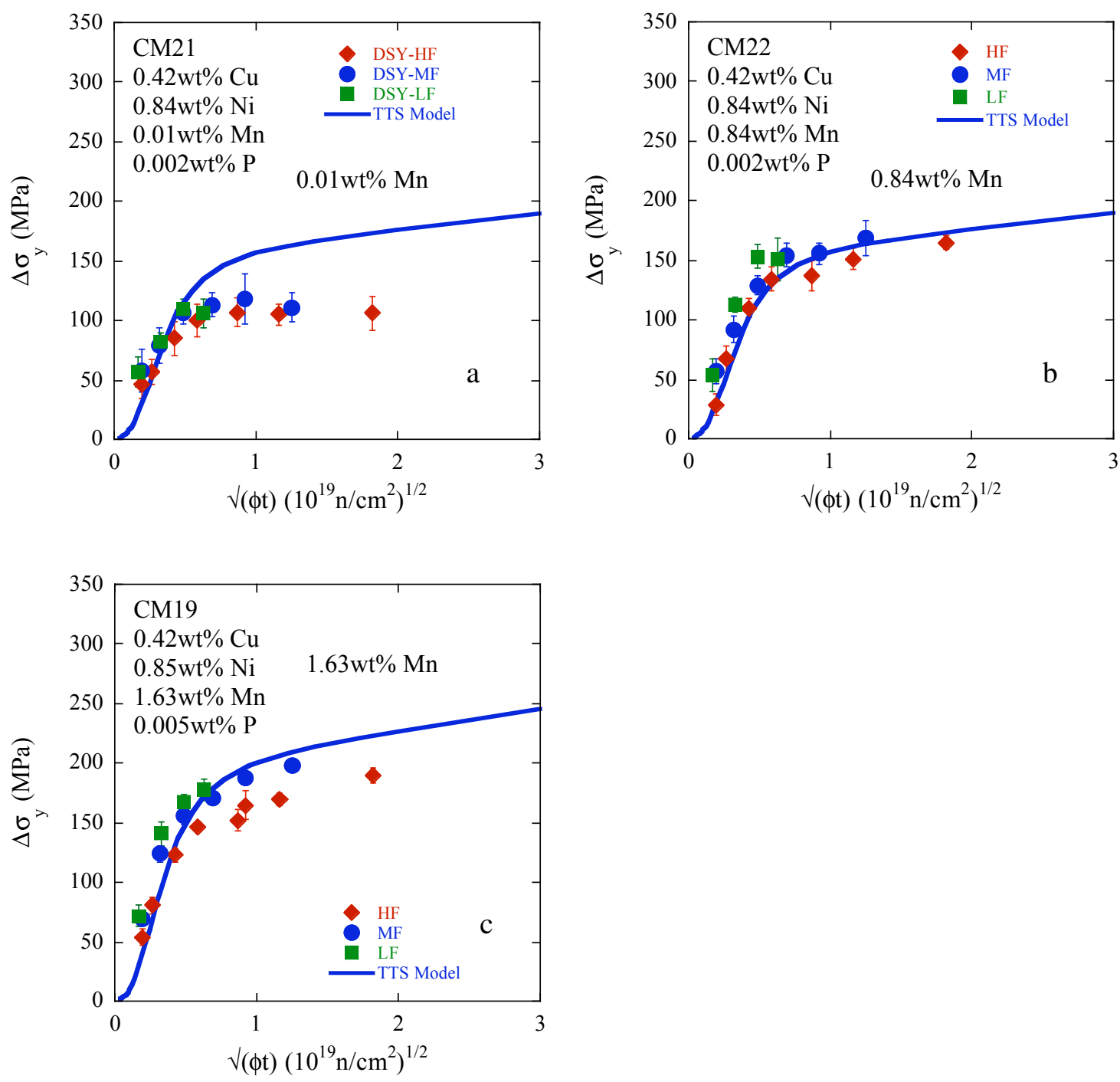

Fig. 6.7 a-c. The TTS model predictions and the IVAR $\Delta \sigma_{\mathrm{y}}$ data plotted against the square root of fluence for three $0.4 \mathrm{wt} \% \mathrm{Cu}$ SMMS with 0.0 (a), 0.8 (b), and 1.6 (c) wt \% Mn.

Figure 6.8 also supports the TTS fit of a $\mathrm{Cu}_{\max }$ between $\approx 0.24$ and 0.3 wt. \%. Figure $6.8 \mathrm{~d}$ shows that the IVAR data are also consistent with a minimum $\mathrm{Cu}$ for $\mathrm{CRP}$ formation, $\mathrm{Cu}_{\min }$. Here, the CRP hardening of LV SMMS baseline composition is estimated by subtracting the $\Delta \sigma_{\mathrm{y}}$ for the $\mathrm{Cu}$ free alloy (LG), from the corresponding $\Delta \sigma_{\mathrm{y}}$ for alloys with 0.1 (LH), 0.2 (LI) and 0.4 (LC) wt. \% Cu alloys, and plotting the result against the square root of $\mathrm{Cu}$. These data are for irradiations to 0.48 and $1.56 \times 10^{19}$ $\mathrm{n} / \mathrm{cm}^{2}$ at intermediate flux and $290^{\circ} \mathrm{C}$. For the SMMS with $0.4 \mathrm{wt} \% \mathrm{Cu}$ (bulk) two values of $\mathrm{Cu}_{\max }=0.24$ and $0.30 \mathrm{wt} \%$ are shown. The $\Delta \sigma_{\mathrm{y}}$ scales with the square root of the precipitate volume fraction, hence, the dissolved $\mathrm{Cu}$ content. Thus the intercepts of the least-square lines with the with $\Delta \sigma_{\mathrm{y}}=0$ axis provide estimates of the $\mathrm{Cu}_{\min }$, of 0.073 and 0.063 (rounded) wt \% Cu for the lower and higher fluences, respectively. Note: the values of $\sqrt{ } \mathrm{Cu}=0.25$ and 0.5 correspond to $\mathrm{Cu}$ contents of 0.0625 and $0.25 \mathrm{wt} \%$, 
respectively. As expected, the $\mathrm{Cu}_{\min }$ threshold decreases slightly with increasing fluence, and is in good agreement with the value in the TTS model of $0.072 \mathrm{wt} \% \mathrm{Cu}$.
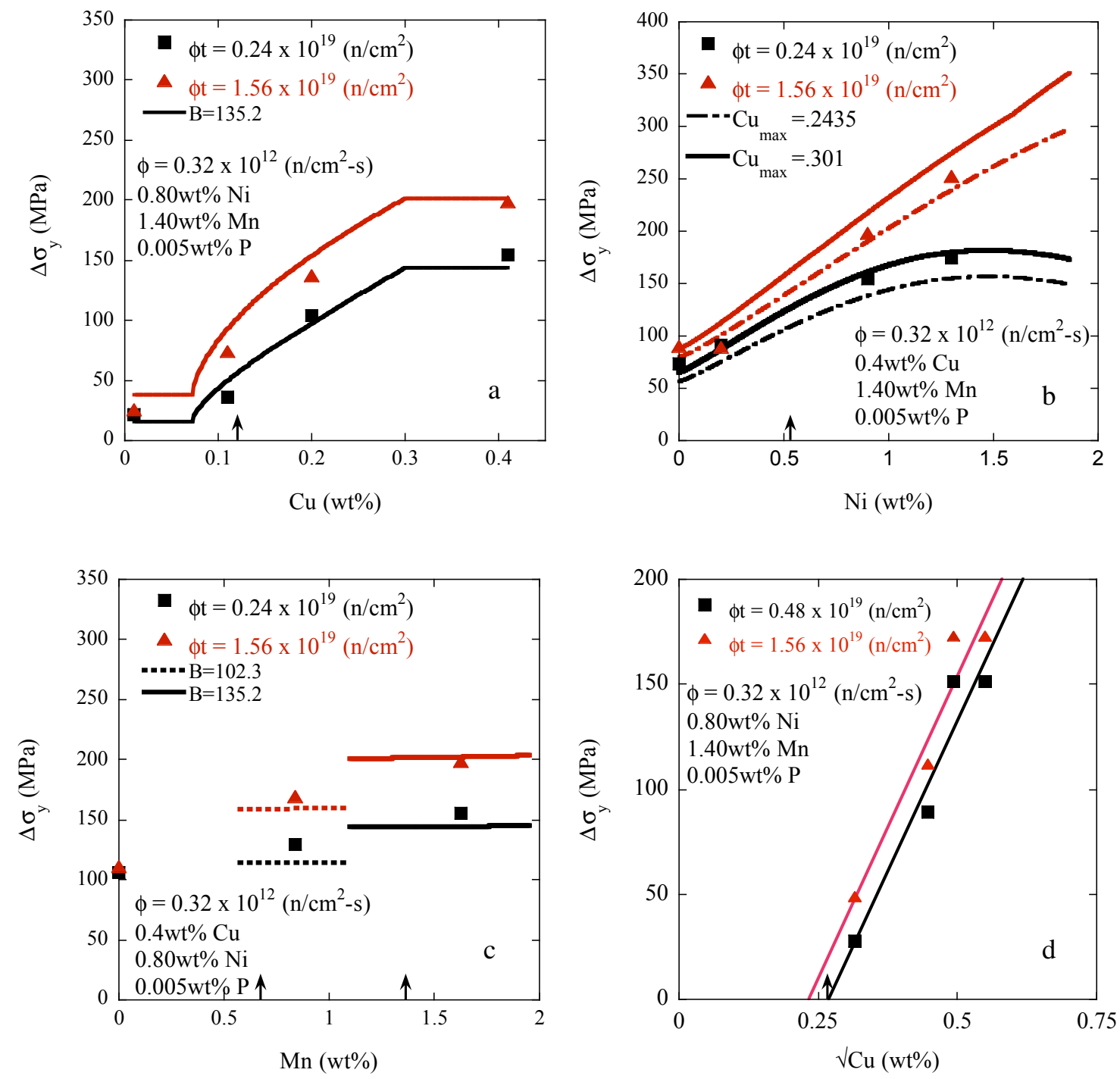

Fig. 6.8 a-d. The solid lines in a-c are TTS model predictions for a nominal CE plate product form coefficients $(B=135)$ and the IVAR $\Delta \sigma_{y}$ data at two fluences plotted against $\mathrm{Cu}(\mathrm{a}), \mathrm{Ni}$ (b), and $\mathrm{Mn}$ (c). The vertical arrows show the average compositions of these elements in the TTS plate database. The horizontal dotted lines in Fig. $6.8 \mathrm{c}$ are for the coefficients and average Mn composition of forgings in the TTS database. Fig. $6.8 \mathrm{~d}$ plots the estimated CRP hardening versus the square root of the dissolved $\mathrm{Cu}$ content to estimate $\mathrm{Cu}_{\min }$.

In summary, there is generally good agreement between the TTS model predictions and the IVAR $\Delta \sigma_{\mathrm{y}}$ data. However, there is a systematic effect of flux observed in the IVAR $\Delta \sigma_{\mathrm{y}}$ data shown in Figs. 6.3, 6.5, 6.6 and 6.7, that is not captured by the TTS model. The TTS model does predict flux dependence of $\Delta \sigma_{\mathrm{y}}$ but only below the range of the IVAR data. The probable reasons that the TTS model does not fully capture the effect of flux in the IVAR (and also the PWR surveillance) regimes are discussed in Sect. 6.6. 


\subsubsection{Low-Cu SMMS Irradiated at $290^{\circ} \mathrm{C}$}

Figure 6.9 compares the nominal TTS model $\Delta \sigma_{\mathrm{y}}$ curves plotted versus the square root of fluence (solid lines) to IVAR data for four nominally $\mathrm{Cu}$-free SMMS with similar compositions $(\approx 0.8 \mathrm{wt} \% \mathrm{Ni}, \approx$ 1.3 to $1.7 \mathrm{wt} \% \mathrm{Mn}$ and $\approx 0.005 \mathrm{wt} \% \mathrm{P}$ ) for $290^{\circ} \mathrm{C}$ irradiations at low, medium, and high flux. The slight curvature of these predicted lines is due to the nonlinear TTS-to- $\Delta \sigma_{\mathrm{y}}$ conversion. The dashed lines are least squares fits to the IVAR $\Delta \sigma_{\mathrm{y}}$ data, using the simple form, $\Delta \sigma_{\mathrm{y}}=\mathrm{CF} \sqrt{ }(\phi t)$, where $\mathrm{CF}$ is a fitted chemistry factor. The short dashed line in Fig. $6.9 \mathrm{~b}$ is the fit to the data leaving out the seemingly anomalous high-flux, high-fluence data point. Note there is a general trend for the measured $\Delta \sigma_{\mathrm{y}}$ for this irradiation condition to fall below the TTS model predictions. We believe this is due in part to a flux effect discussed in Sect. 6.6.2. Overall, the TTS MF model systematically over predicts the CF, and thus the $\Delta \sigma_{\mathrm{y}}$, for $\mathrm{Cu}$-free IVAR data by a factor of about 1.33 . However, the overall absolute average deviation $\approx 6.4 \mathrm{MPa}$ is not large.
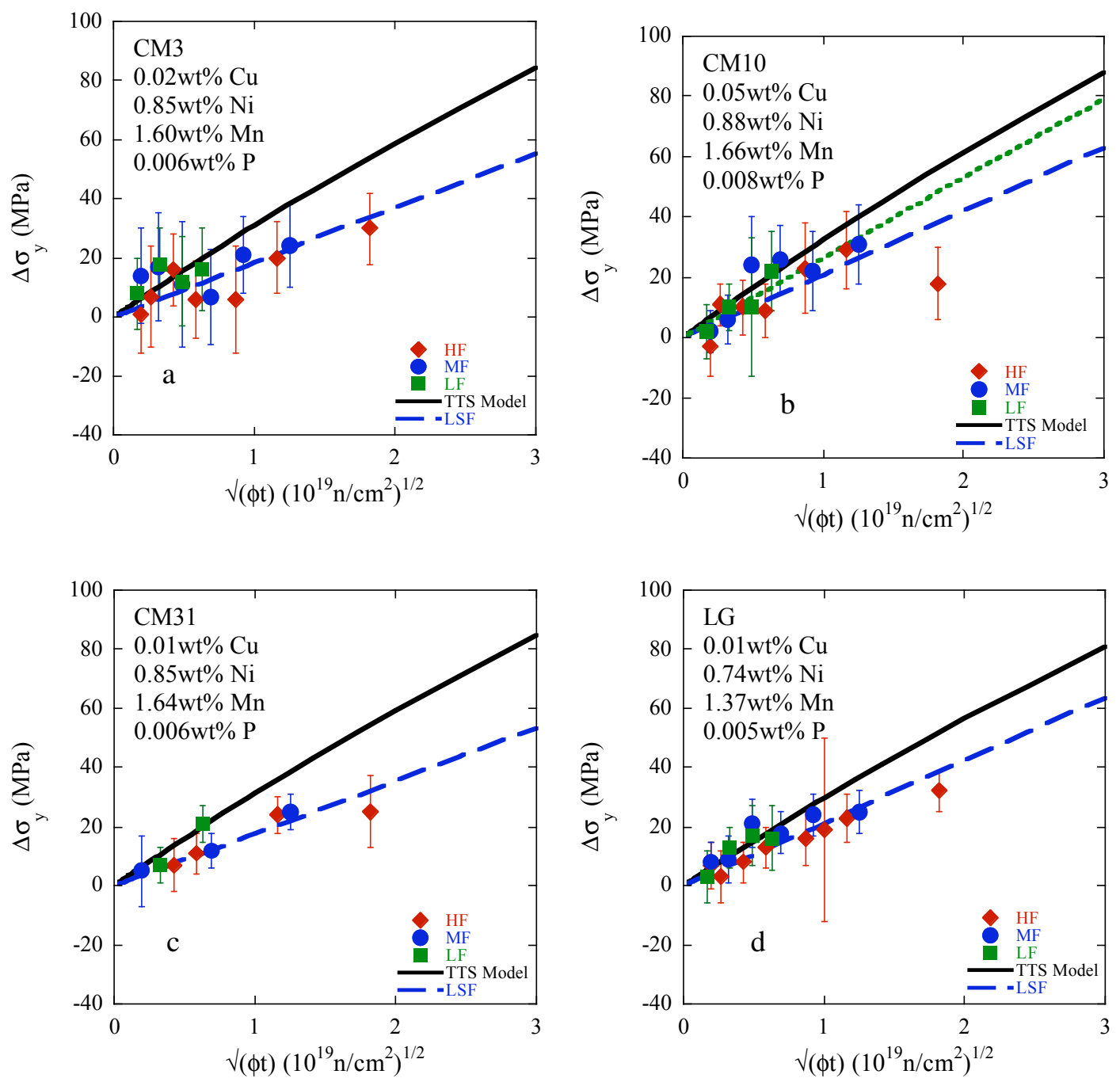

Fig. 6.9 a-d. The TTS model predictions (solid lines) and best fit chemistry factor (dashed lines) for the IVAR $\Delta \sigma_{\mathrm{y}}$ data plotted against the square root of fluence for Cufree SMMS. The dotted line in Fig. $6.9 \mathrm{~b}$ is the best fit ignoring the highest fluence data point. The TTS models over predict the $\Delta \sigma_{y}$ in the lower sensitivity Cu-free SMMS. 
Figures $6.10 \mathrm{a}$ and $\mathrm{b}$ show that the TTS model greatly over predicts and slightly under predicts the $\Delta \sigma_{\mathrm{y}}$ in $\mathrm{Cu}$-free $\mathrm{SMMS}$ with $\approx 1.6 \mathrm{wt} \% \mathrm{Mn}$ and $\approx 0.0$ and $\approx 1.68 \mathrm{wt} \% \mathrm{Ni}$, respectively. These very low-and high-Ni contents are outside the TTS database composition limits. Figures 6.10c and d show that the TTS model greatly and somewhat over predicts the IVAR $\Delta \sigma_{\mathrm{y}}$ for $\mathrm{Cu}$-free SMMS with $\approx 0.8 \mathrm{wt} \% \mathrm{Ni}$ for $\approx 0.0$ and $0.8 \mathrm{wt} \% \mathrm{Mn}$, respectively. In this case the forging MF coefficient, that is appropriate for alloys with lower Mn contents, was used in the TTS model. Again, the SMMS with no Mn is well outside the TTS database composition limits.
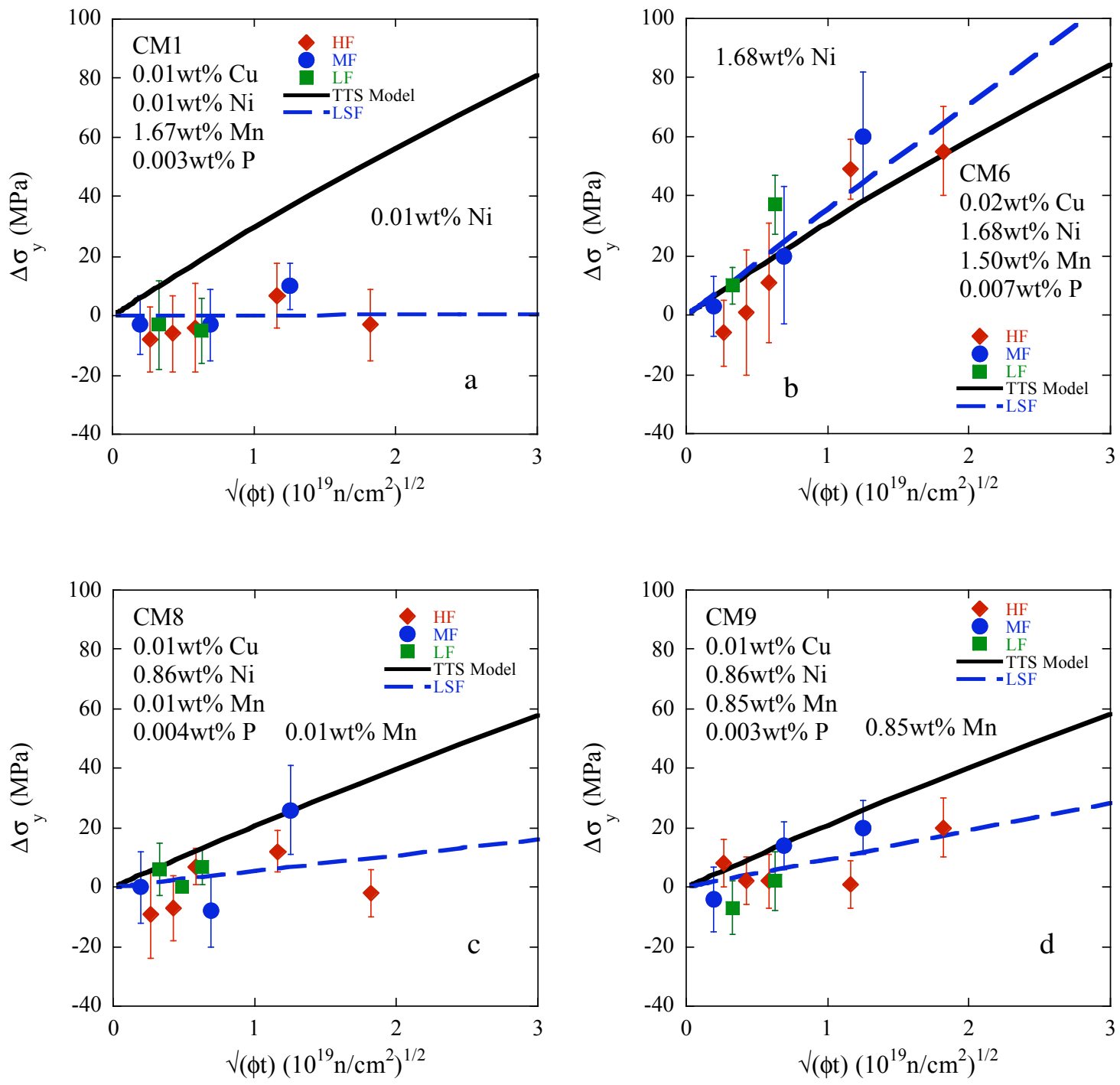

Fig. 6.10 a-d. The TTS model predictions (solid lines) and best fit chemistry factor (dashed lines) for the IVAR $\Delta \sigma_{y}$ data plotted against the square root of fluence for (a) and (b) 0.0 and 1.6 wt \% Ni Cu-free SMMS and (c) and (d) 0.0.and $1.6 \mathrm{wt} \% \mathrm{Mn}$. The TTS model over predicts $\Delta \sigma_{y}$ in the three low-sensitivity steels and slightly under-predicts the $\Delta \sigma_{y}$ for the high Ni SMMS. 
Figures $6.11 \mathrm{a}$ and $\mathrm{b}$ show that the TTS model slightly to significantly under predicts $\sigma_{\mathrm{y}}$ in $\approx 0.8 \mathrm{wt} \%$ $\mathrm{Ni}, \approx 1.6 \mathrm{wt} \% \mathrm{Mn}, \mathrm{Cu}$ free SMMS with $\approx 0.031$ and $0.035 \mathrm{wt} \% \mathrm{P}$. The underprediction is more severe relative to the dotted line in Fig. $6.11 \mathrm{~b}$, which is the fit without the high flux, high fluence data point. These P compositions are slightly outside the composition limits of the TTS plate database. Figure 6.11c shows that the TTS model also significantly under predicts $\Delta \sigma_{\mathrm{y}}$ for a very high $\mathrm{Ni} \approx 1.65 \mathrm{wt} \%$ weld with $\approx 0.04 \mathrm{wt} \% \mathrm{Cu}, 1.43 \mathrm{wt} \% \mathrm{Mn}$, and $0.011 \mathrm{wt} \% \mathrm{P}$. Since the Ni content in this case is far beyond the TTS database composition limits, these differences are not a specific matter of concern. However, these results reaffirm the importance of Ni in MF hardening that was found in the SMMS data.
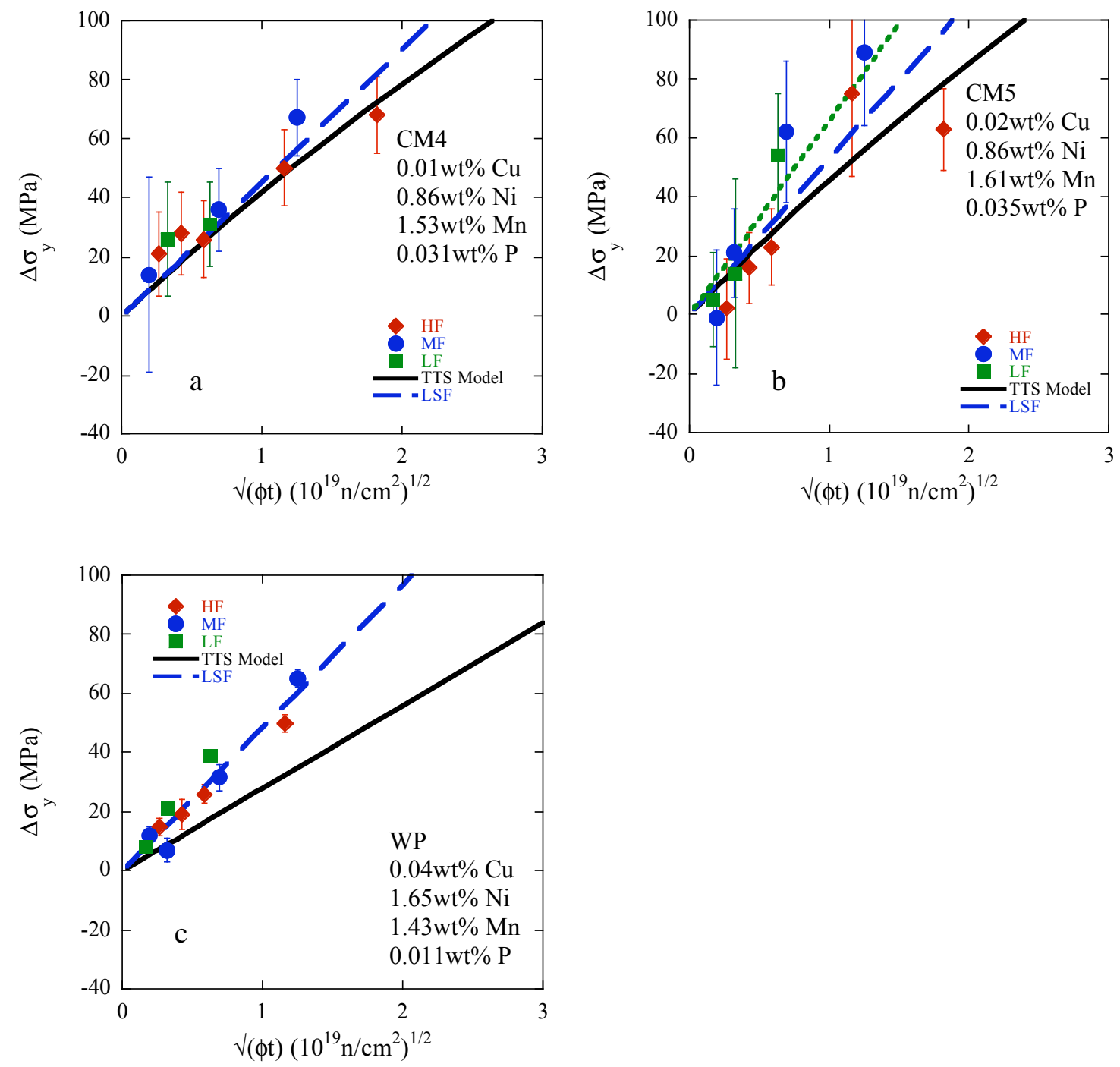

Fig. 6.11 a-c. The TTS model predictions (solid lines) and best fit chemistry factor (dashed lines) for the IVAR $\Delta \sigma_{y}$ data plotted against the square root of fluence: (a) and (b) for Cu-free SMMS with 0.031 (a) and $0.35 \mathrm{wt} \% \mathrm{P}$ (b); and a high Ni (1.6 wt \% Ni) low Cu weld (c). The dotted line in Figs. $6.11 \mathrm{~b}$ is the best fit ignoring the highest fluence data point. The TTS model under-predicts $\Delta \sigma_{y}$ in these sensitive steels. 
Figure 6.12 summarizes Figs. 6.9, 6.10, and 6.11 with cross-plots the fitted CF slopes of the IVAR data against $\mathrm{Ni}$ (Fig. 6.12a), Mn (Fig. 6.12b), P (Fig. 6.12c) and $\mathrm{Cu}$ (Fig. 6.12d). The open diamonds are for the $\mathrm{CF}$ fits after dropping the high flux and high fluence data point. The solid lines are the corresponding TTS model predictions. The arrows show the average composition of the plates (and where pertinent, forgings) in the surveillance database. The strong effect of Ni in Fig. 6.12a in the IVAR $\Delta \sigma_{\mathrm{y}}$ data is not reflected in the TTS MF model predictions. The effect of Mn shown in the IVAR $\Delta \sigma_{\mathrm{y}}$ data in Fig. 6.12b is primarily reflected in the TTS MF model coefficient differences between plate (higher Mn)
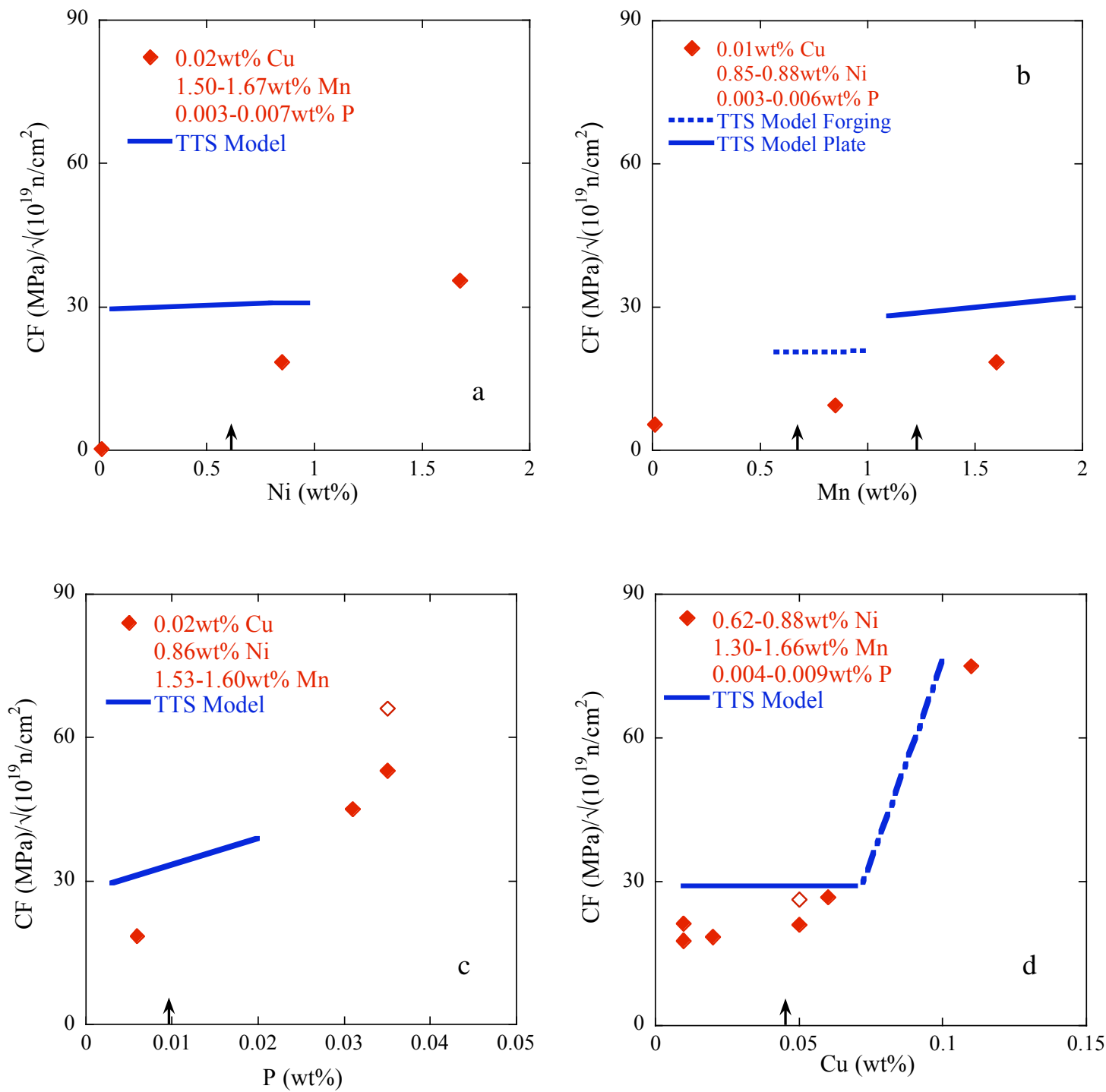

Fig. 6.12 a-d. The solid lines are TTS model predictions at $290^{\circ} \mathrm{C}$ for IVAR SMMS compositions, including one low-Cu weld, several Cu-free SMMS and a $0.1 \mathrm{wt} \% \mathrm{Cu}$ SMMS. The filled diamond symbols are the corresponding CF fits to the IVAR $\sigma_{y}$ data plotted against $\mathrm{Ni} \mathrm{(a),} \mathrm{Mn} \mathrm{(b),} \mathrm{P} \mathrm{(c),} \mathrm{and} \mathrm{Cu}$ (d). The vertical arrows show the average compositions of these elements in the TTS plate database. The horizontal dotted line in Fig. $6.12 \mathrm{~b}$ is for the TTS forging model. The open symbols are the IVAR data fits ignoring the highest fluence data points. The dashed dotted line in Fig. 12d includes the TTS model CRP contribution to hardening for $>0.072 \mathrm{wt} \% \mathrm{Cu}$. 
and forgings (lower Mn). The effect of $\mathrm{P}$ shown in Fig. 6.12c is stronger in the IVAR $\Delta \sigma_{\mathrm{y}}$ data than predicted by the TTS model. The IVAR $\Delta \sigma_{\mathrm{y}}$ data in Fig. $6.12 \mathrm{~d}$ shows there is a weak effect of Cu below the threshold for CRP formation that is not reflected in the TTS model. Note, the $0.1 \mathrm{wt} \% \mathrm{Cu}$ data is included to show the rapid increase of $\Delta \sigma_{\mathrm{y}}$ above the threshold for CRP formation of $0.072 \mathrm{wt} \%$.

Indeed, the effects of $\mathrm{Cu}$ that are not treated in the TTS MF model, as well as the corresponding weaker effect of $\mathrm{P}$, at least partially explain the apparent systematic TTS model over prediction of the low $\mathrm{Cu}$ IVAR $\Delta \sigma_{\mathrm{y}}$ data. This is due to the fact that the IVAR SMMS are generally cleaner than the low $\mathrm{Cu}$ alloys in the TTS database, which contain on average about $0.05 \mathrm{wt} \% \mathrm{Cu}$ and $0.011 \mathrm{wt} \% \mathrm{P}$. However, the generally higher $\mathrm{Ni}$ in the IVAR $\Delta \sigma_{\mathrm{y}}$ has the opposite effect in making the IVAR steels slightly more sensitive to irradiation hardening. The CF values fitted to the IVAR data are also generally biased to lower values by the high flux IVAR $\Delta \sigma_{\mathrm{y}}$ data. The analysis in Sect. 6.6.2 shows that high flux leads to systematically lower $\Delta \sigma_{y}$, even in low $\mathrm{Cu}$ steels. If the influence of the composition differences and flux effects are accounted for, the TTS MF model predictions and the IVAR $\Delta \sigma_{\mathrm{y}}$ data are in much better agreement. Details of this analysis will be presented in a future report.

Finally, we note that the TTS MF model also includes flux dependence, but only below the range of the IVAR data. Although the absolute flux dependent differences in the $\Delta \sigma_{\mathrm{y}}$ are much smaller than for the Cu-bearing alloys, as noted previously and discussed in Sect. 6.6.2, a systematic effect of flux is also observed in the low $\mathrm{Cu}$ and $\mathrm{Cu}$ free IVAR $\Delta \sigma_{\mathrm{y}}$ data. The reasons that the TTS MF model does not capture the effect of flux in the IVAR and PWR surveillance regimes are discussed in Sects. 6.6.1 and 6.6.2.

\subsection{The Irradiation Temperature Dependence of $\Delta \sigma_{\mathrm{y}}$ in the SMMS}

Figures 6.13 and 6.14 show intermediate flux IVAR $\Delta \sigma_{y}$ data plotted against the irradiation temperature $\left(\mathrm{T}_{\mathrm{i}}\right)$ for several of the same SMMS shown in Sect. 6.4 at fluences of $\approx 0.48$ and $1.6 \times 10^{19}$ $\mathrm{n} / \mathrm{cm}^{2}$, along with the corresponding TTS model curves using the coefficients for the CE plates. As shown in Fig. 6.13, the TTS model predicts stronger irradiation temperature dependence than is observed in the IVAR data for the four $\mathrm{Cu}$-free SMMS. In contrast, Fig. 6.14 shows that the opposite is the case in the more sensitive $\mathrm{Cu}$-bearing SMMS. The absolute irradiation temperature dependence (slope) of IVAR $\Delta \sigma_{\mathrm{y}}$ data increases with the alloy $\mathrm{Cu}, \mathrm{Ni}$, and $\mathrm{Mn}$ contents, as well as with fluence.
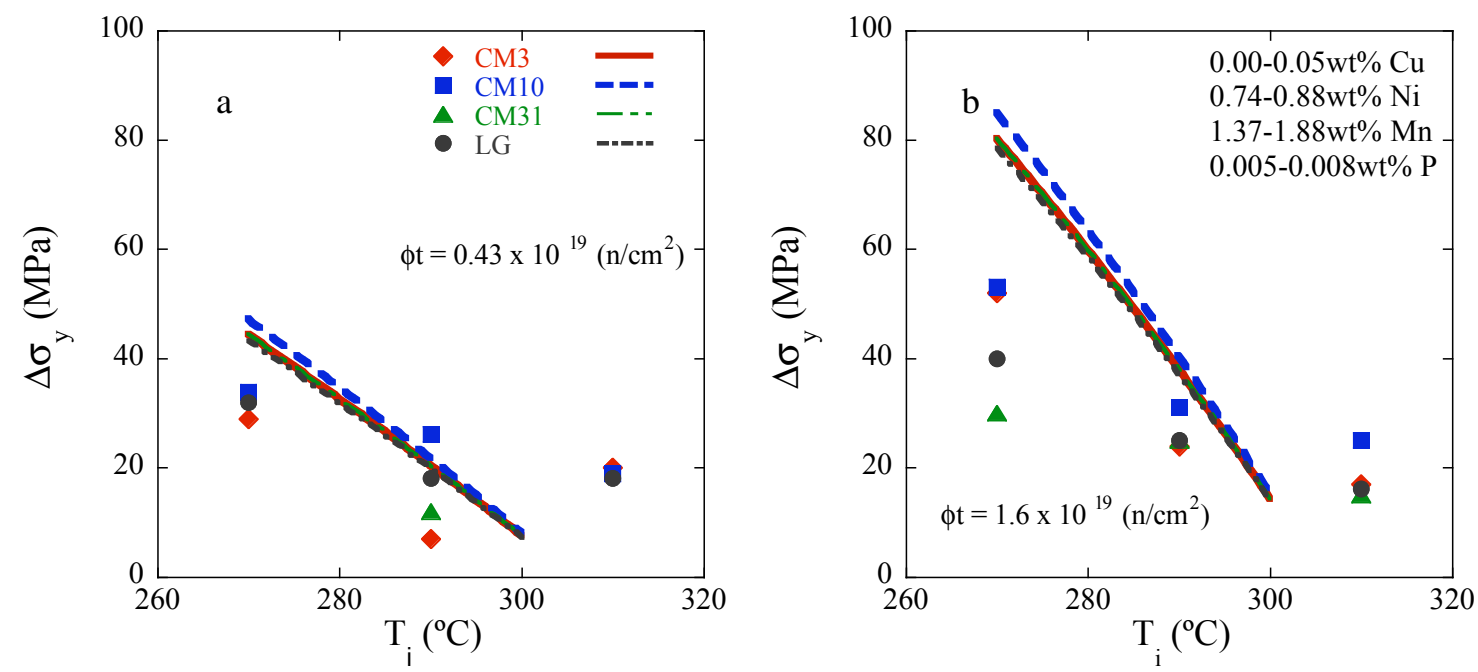

Fig. 6.13 a-b. TTS model predictions and the $\Delta \sigma_{\mathrm{y}}$ data for four Cu-free SMMS irradiated in IVAR at 270,390 , and $310^{\circ} \mathrm{C}$ at intermediate flux to two fluences. The TTS model predicts a stronger irradiation temperature dependence of $\Delta \sigma_{y}$ than observed in the IVAR SMMS. 

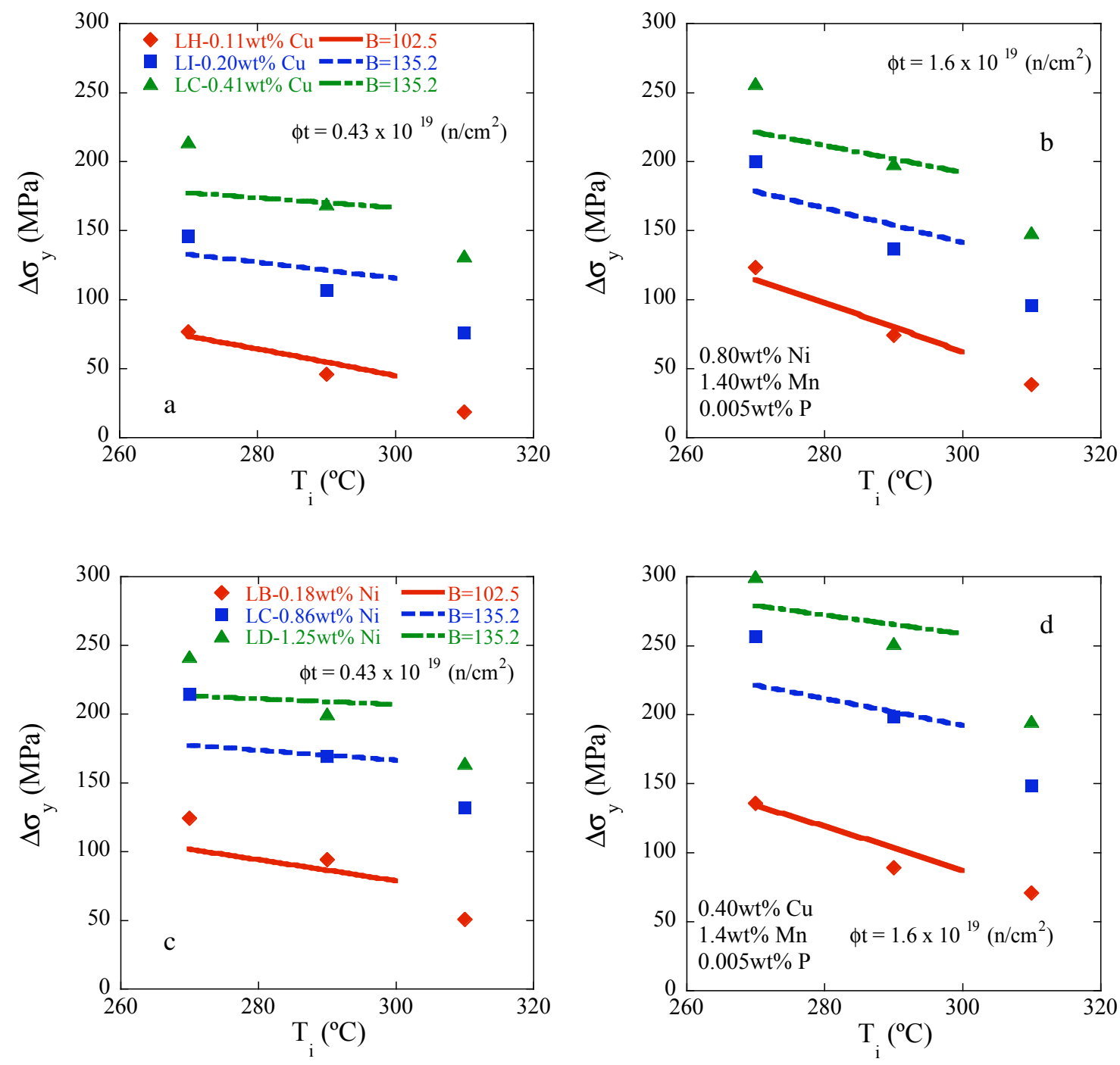

Fig. 6.14 a-d. TTS model predictions and the $\Delta \sigma_{y}$ data for intermediate flux IVAR irradiations to two fluences at 270,290 , and $310^{\circ} \mathrm{C}$ : (a) and (b) SMMS with $0.8 \mathrm{wt} \% \mathrm{Ni}$, 1.4 wt $\% \mathrm{Mn}$ and $0.1,0.2$, and 0.4 wt \% $\mathrm{Cu}$; (c) and (d) SMMS with $0.4 \mathrm{wt} \% \mathrm{Cu}, 1.4 \mathrm{wt} \%$ Mn and $0.2,0.8$ and 1.25 wt $\% \mathrm{Ni}$. The TTS model generally predicts weaker irradiation temperature dependence of $\sigma_{y}$ than observed in the Cu-bearing IVAR SMMS.

The reasons for the decrease in CRP contribution to hardening with increasing irradiation temperature were discussed in Chap. 2. In contrast, the TTS model predicts a weak increase in the CRP contribution to $\Delta \sigma_{\mathrm{y}}$ with increasing irradiation temperature. Possible reasons for this difference, and a recommended simplification which reduces the discrepancy, are discussed in Chap. 7. However, as shown in Fig. 6.4, the irradiation temperature dependence of the $\Delta \sigma_{\mathrm{y}}$ data for the lower-sensitivity IVAR welds and plates, which are most like the steels in the surveillance database, are more consistent with the TTS model predictions, compared to the $\Delta \sigma_{\mathrm{y}}$ vs $\mathrm{T}_{\mathrm{i}}$ trends in the higher-sensitivity IVAR SMMS shown here.

In summary, the IVAR database shows that both MF and CRP hardening contributions decrease with increasing irradiation temperature. This observation is consistent with the TTS model MF term but is not consistent with the TTS model CRP term presented in Chap. 4, that shows a weak, but opposite, trend. 

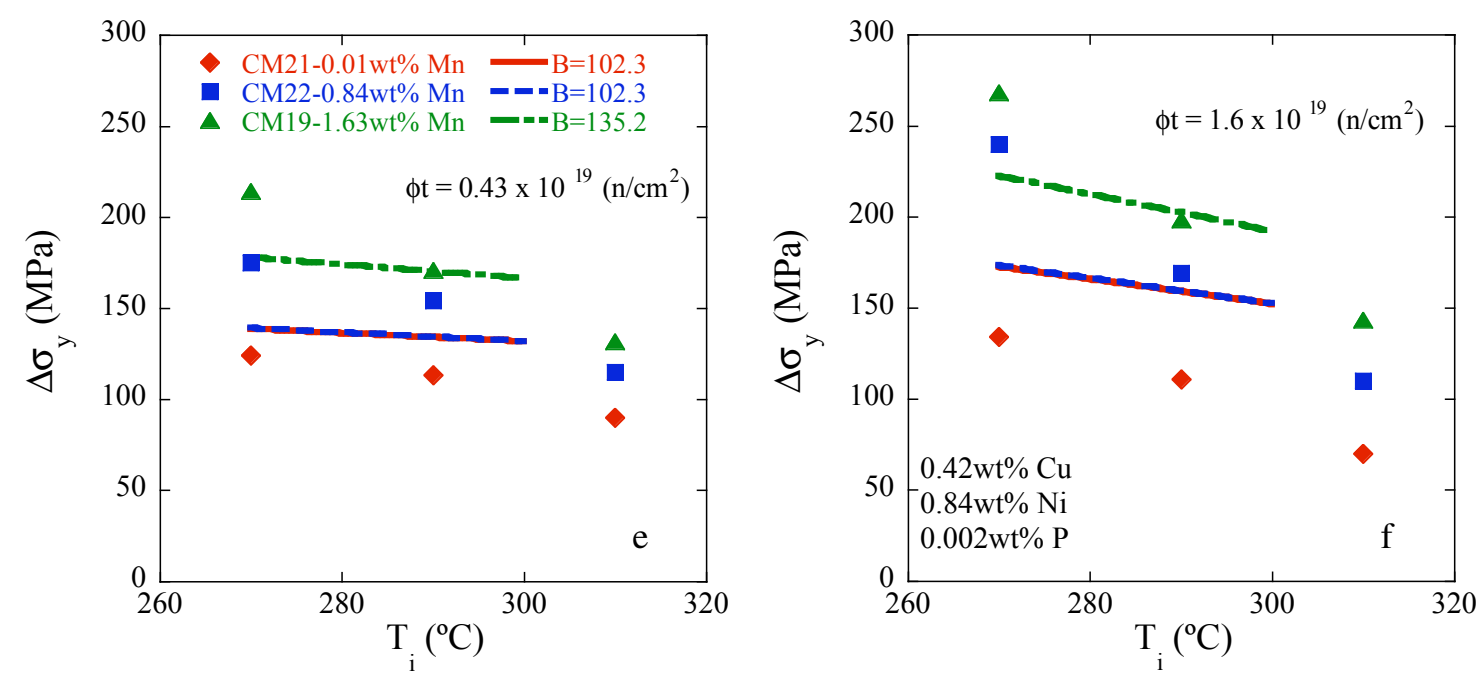

Fig. 6.14 e-f. The TTS model predictions and the $\Delta \sigma_{\mathrm{y}}$ data for intermediate flux IVAR irradiations to two fluences at 270,290 , and $310^{\circ} \mathrm{C}$ for SMMS with $0.4 \mathrm{wt} \% \mathrm{Cu} ; 0.8 \mathrm{wt} \%$ $\mathrm{Ni}$; and $\mathbf{0 . 0}, \mathbf{0 . 8}$, and $1.6 \mathrm{wt} \% \mathrm{Mn}$. The TTS model generally predicts weaker irradiation temperature dependence than observed in the IVAR results.

However, the CRP contribution in the TTS model is so small that the overall trend (MF plus CRP terms) in high $\mathrm{Cu}$ TTS model predictions follows the expected trend of decreasing $\Delta \sigma_{\mathrm{y}}$ with increasing irradiation temperature. The absolute irradiation temperature dependence predicted by the TTS model is stronger than observed in the low $\mathrm{Cu}$ SMMS and weaker than observed in Cu-bearing IVAR SMMS. However, the agreement between the IVAR data and the TTS model is better for the welds and plates that are most similar to surveillance steels.

\subsection{Flux Effects}

\subsubsection{Flux Effects on Hardening in Cu-Bearing Steels}

The systematic flux effect on CRP hardening observed in the IVAR database has been described in detail elsewhere [9] and will be only summarized briefly here. Examples of such flux effects were shown in Figs. 6.3, 6.5, 6.6, and 6.7. All these data show that the pre-plateau CRP hardening regime is shifted to lower fluence with decreasing flux. Note, while these effects may look small on an expanded $\Delta \sigma_{\mathrm{y}}$ vs $\log$ fluence scale, the average difference between the $\Delta \sigma_{\mathrm{y}}$ in the pre-plateau region for the highest and lowest flux measure in IVAR is $\approx 40 \%$.

As discussed in Chap. 2, flux effects in the IVAR data are believed to be primarily due to recombination enhanced by solute vacancy trapping, which reduces the efficiency of radiation enhanced diffusion (RED) of solutes. The net $\mathrm{Cu}$ diffusion per unit fluence, given by $\mathrm{D} * \mathrm{t} / \phi \mathrm{t}$, is smaller at higher flux. Thus, the hardening curves are shifted to higher fluence with increasing flux. The flux effect can be modeled using an effective fluence, $\phi \mathrm{t}_{\mathrm{e}}$, as

$$
\phi \mathrm{t}_{\mathrm{e}}=\phi \mathrm{t}\left(\phi_{\mathrm{r}} / \phi\right)^{\mathrm{p}}
$$

The $\phi_{\mathrm{r}}$ is an arbitrary reference flux that was taken as the intermediate IVAR flux of $3 \times 10^{11} \mathrm{n} / \mathrm{cm}^{2}-\mathrm{s}$ [9]. Since $\mathrm{p}$ is the slope of the $\log \left[\mathrm{D}^{*} \mathrm{t} / \phi \mathrm{t}\right](\phi)$ curve between $\log [\phi]$ and $\log [\phi \mathrm{r}]$ in principle the flux depends on the irradiation temperature, and the alloy composition and microstructure [9]. For example, recombination, hence $\mathrm{p}$, increases with higher alloy $\mathrm{Mn}$ and $\mathrm{Ni}$ contents because these solutes are vacancy 
traps. Also, $\mathrm{p}$ decreases with increasing irradiation temperature because the corresponding vacancy detrapping rates increase. The value of $p$ also depends on the choice of the reference flux, $\phi_{\mathrm{r}}$ (see Fig. 2.16), decreases from a limiting, high flux value of 0.5 in the recombination-dominated regime, and may approach 0 if the thermal diffusion coefficient of $\mathrm{Cu}, \mathrm{D}_{\mathrm{Cu}}$, is very small. However if, as expected, the lowtemperature $\mathrm{D}_{\mathrm{Cu}}$, is larger than estimates based on extrapolations of tracer diffusion data from high temperature, as indicated by thermal precipitation kinetics data in the range of 290 to $350^{\circ} \mathrm{C}$, $\mathrm{p}$ goes through a minimum and may reach values greater than 0.5 at low flux; in the limiting case where $\mathrm{D}^{*} \approx$ $\mathrm{D}_{\mathrm{Cu}}, \mathrm{p}$ may approach 1 at very low flux. These limiting values of $\mathrm{p}$ occur only if the actual and reference flux are in the same mechanism regime, in which $\mathrm{p}$ is independent of flux. More generally $\mathrm{p}$ is lower than the recombination limit of 0.5 and higher than the minimum flux independent value of 0 . To reiterate, the flux effect completely resides in $\mathrm{D}^{*}$; there is not flux effect if $\mathrm{D}^{*}$ is independent of flux.

Figures 6.15 to 6.18 show the same $\mathrm{Cu}$-bearing IVAR steel data presented in Figs. 6.3, 6.5, 6.6, and 6.7. The figures on the far left-hand side of Figs. 6.15 to 6.18 are for a solute vacancy trap enhanced recombination model for the CRP contribution to $\Delta \sigma_{\mathrm{y}}$ fitted to the individual alloy data sets and plotted on a common effective fluence, $\phi t_{\mathrm{e}}$, scale. In this case the MF term was modeled as $\Delta \sigma_{\mathrm{y}}=\mathrm{CF} \sqrt{ }\left(\phi \mathrm{t}_{\mathrm{e}}\right)$ where the $\mathrm{CF}$ is the fitted value for the individual alloy that has the same nominal composition, except for being $\mathrm{Cu}$ free, as the corresponding $\mathrm{Cu}$ bearing steel. In the cases where $\mathrm{Cu}$ free alloy data were not directly available, a simple linear correlation model, accounting for $\mathrm{Cu}, \mathrm{Ni}, \mathrm{Mn}$ and $\mathrm{P}$ effects in the $\mathrm{MF}$ for the $\mathrm{Cu}$ bearing alloy, was used to estimate the MF CF. As expected, the fitted recombination model (FRM) greatly reduces the flux dependence of $\Delta \sigma_{\mathrm{y}}$ in $\mathrm{Cu}$ bearing steels.

The systematic effect of flux is more clearly shown in the corresponding measured minus predicted $\Delta \sigma_{\mathrm{y}}$ vs flux residuals shown in Figs. 6.15 to 6.18. The figures in the middle show that the residuals for the FRM are generally well centered and, as expected, do not depend strongly on flux. In contrast, the figures on the far right hand side of Figs. 6.15 to 6.18 show that the residuals for the TTS model has a strong flux dependence, in some cases tending to slightly under predict the $\Delta \sigma_{\mathrm{y}}$ data at the low IVAR flux (squares), while over predicting the $\Delta \sigma_{\mathrm{y}}$ at the high IVAR flux (diamonds). In a number of cases, there is an overall bias in the average residual for the TTS model.

The TTS model also includes a flux effect on CRP and MF hardening, but with some key differences with respect to the IVAR data. Although the flux ranges of IVAR and surveillance data overlap, the calibrated TTS model has a flux effect only below $4.4 \times 10^{10} \mathrm{n} / \mathrm{cm}^{2}-\mathrm{s}$, which is lower than the flux range of the IVAR data. Note, above this threshold, a residual trend with flux can be detected in some surveillance datasets that is in the same direction as shown by the IVAR data. However, the magnitude of the effect in the TTS model residuals is not sufficiently large relative to the scatter in the data to be statistically significant. Possible reasons for this difference between the effects of dose rate in the TTS model and IVAR $\Delta \sigma_{\mathrm{y}}$ are discussed in Sect. 6.6.1.

The TTS model also assumes a fitted constant effective fluence scaling exponent, $\mathrm{p}=0.26$. Figure 6.19 plots $\mathrm{p}$ vs flux the recombination model shown previously in Fig. 2.16 , taking $\phi_{\mathrm{r}}=4.4 \times 10^{10}$ $\mathrm{n} / \mathrm{cm}^{2}$-s, using the same parameters that were described in Chap. 2. Curves are shown for trap concentrations, $\mathrm{X}_{\mathrm{t}}$, of 0.03 and 0.01 , effectively spanning the range of typical RPV steels with various Ni, $\mathrm{Mn} . \mathrm{Cu}, \ldots$ solute contents. Figs $6.19 \mathrm{a}$ and $6.19 \mathrm{~b}$ are for nominally high and low values of $\mathrm{D}_{\mathrm{Cu}}$, respectively. The constant $\mathrm{p}=0.26$ from the TTS model shown as the horizontal dashed line is in reasonable agreement with the physically based recombination model predictions in the flux range from $10^{9}$ and $10^{11} \mathrm{n} / \mathrm{cm}^{2}$-s: the average values of the predicted $\mathrm{p}$ are 0.29 and 0.20 for $\mathrm{X}_{\mathrm{t}}=0.03$ and 0.01 , respectively, for the low $\mathrm{D}_{\mathrm{Cu}}$ case; the corresponding values for the high $\mathrm{D}_{\mathrm{Cu}}$ case are 0.33 and 0.24. Overall, the FRM with the high $\mathrm{D}_{\mathrm{Cu}}$ is most consistent with the TTS model.

The fitted FRM can also be used to physically extrapolate the IVAR data to lower fluxes. The flux effect can be represented by a fluence-multiplier, $\mathrm{M}_{\phi}(\phi)=\phi \mathrm{t}_{\mathrm{e}} / \phi \mathrm{t}$, that is normalized to unity at a specified flux. Figure 6.20 plots $M_{\phi}$ curves normalized at $\phi_{\mathrm{r}}=8 \times 10^{10} \mathrm{n} / \mathrm{cm}^{2}$-s, in the borderline region of over- 

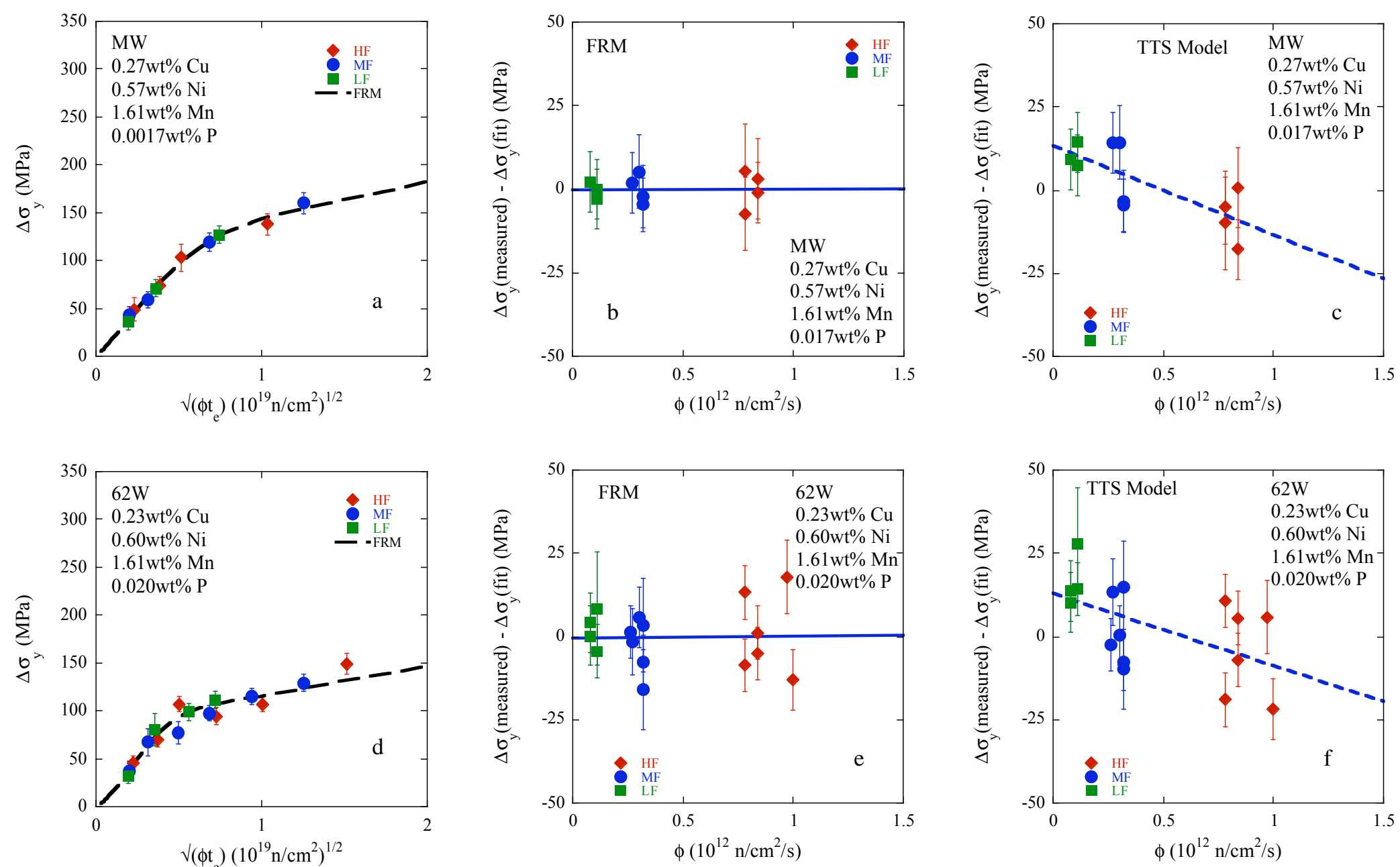

Fig. 6.15 a-f. (a, d) The fitted recombination model (FRM) predictions and the $290^{\circ} \mathrm{C}$ IVAR $\triangle \sigma_{\mathrm{y}}$ data for two Cu-bearing commercial welds on an effective fluence, $\phi t_{e}$, scale; (b, e) the corresponding measured minus predicted residuals for the FRM that are well centered and approximately independent of flux; $(c, f)$ the corresponding residuals for the TTS model, showing a systematic effect of flux that is not accounted for in the TTS model developed in Chap. 3. 

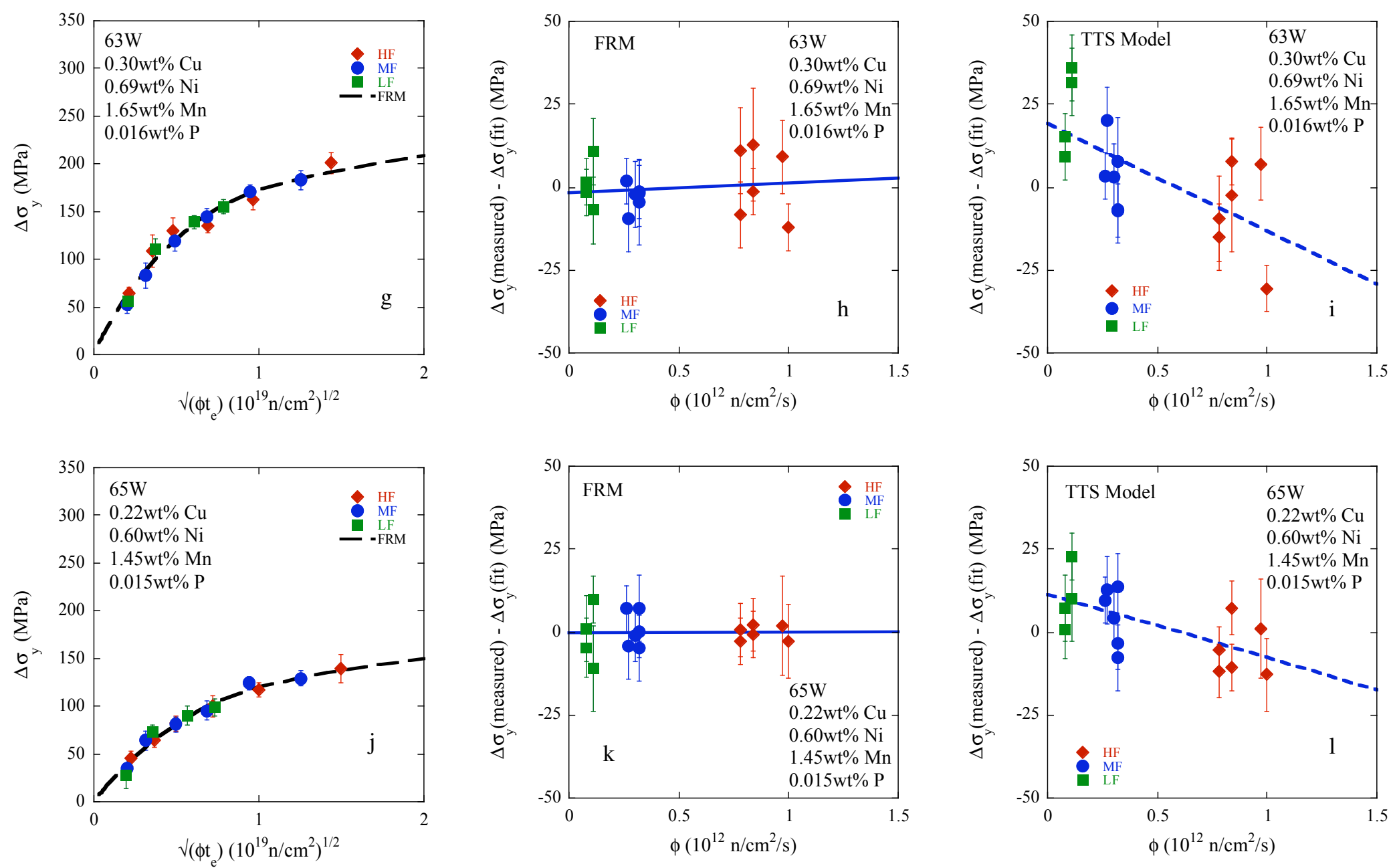

Fig. $6.15 \mathrm{~g}-\mathrm{I}$. (g, j) The fitted recombination model (FRM) predictions and the $290^{\circ} \mathrm{C}$ IVAR $\Delta \sigma_{\mathrm{y}}$ data for two Cu-bearing commercial welds on an effective fluence, $\phi t_{e}$, scale; $(h, k)$ the corresponding measured minus predicted residuals for the FRM that are well centered and approximately independent of flux; (i, l) the corresponding residuals for the TTS model, showing a systematic effect of flux that is not accounted for in the TTS model developed in Chap. 3. 

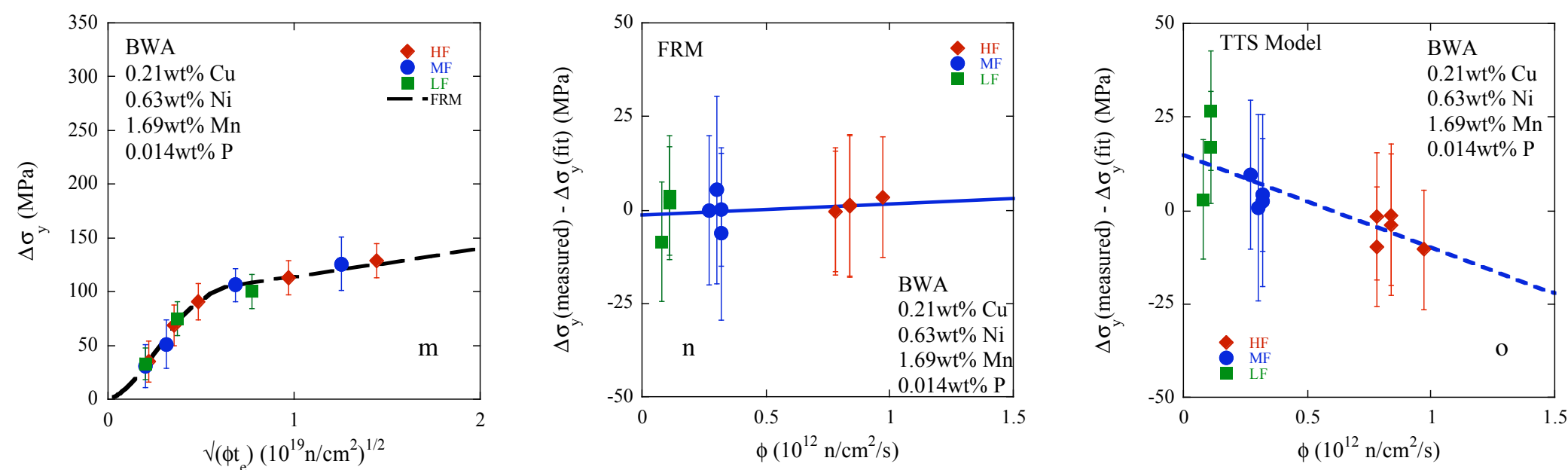

Fig. $6.15 \mathrm{~m}-0 .(\mathrm{m})$ The fitted recombination model (FRM) predictions and the $290^{\circ} \mathrm{C}$ IVAR $\Delta \sigma_{y}$ data for a Cu-bearing commercial welds on an effective fluence, $\phi t_{e}$, scale; $(n)$ the corresponding measured minus predicted residuals for the FRM that are well centered and approximately independent of flux; (o) the corresponding residuals for the TTS model, showing a systematic effect of flux that is not accounted for in the TTS model developed in Chap. 3. 


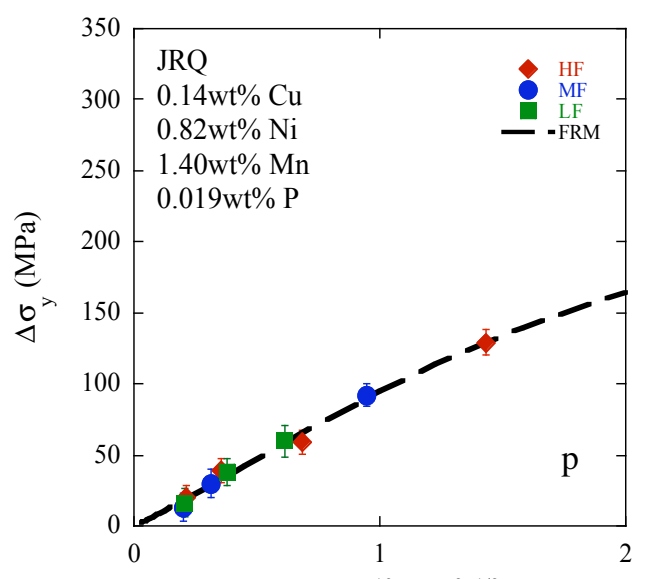

离 $\sqrt{(\phi \mathrm{t}})\left(10^{19} \mathrm{n} / \mathrm{cm}^{2}\right)^{1 / 2}$
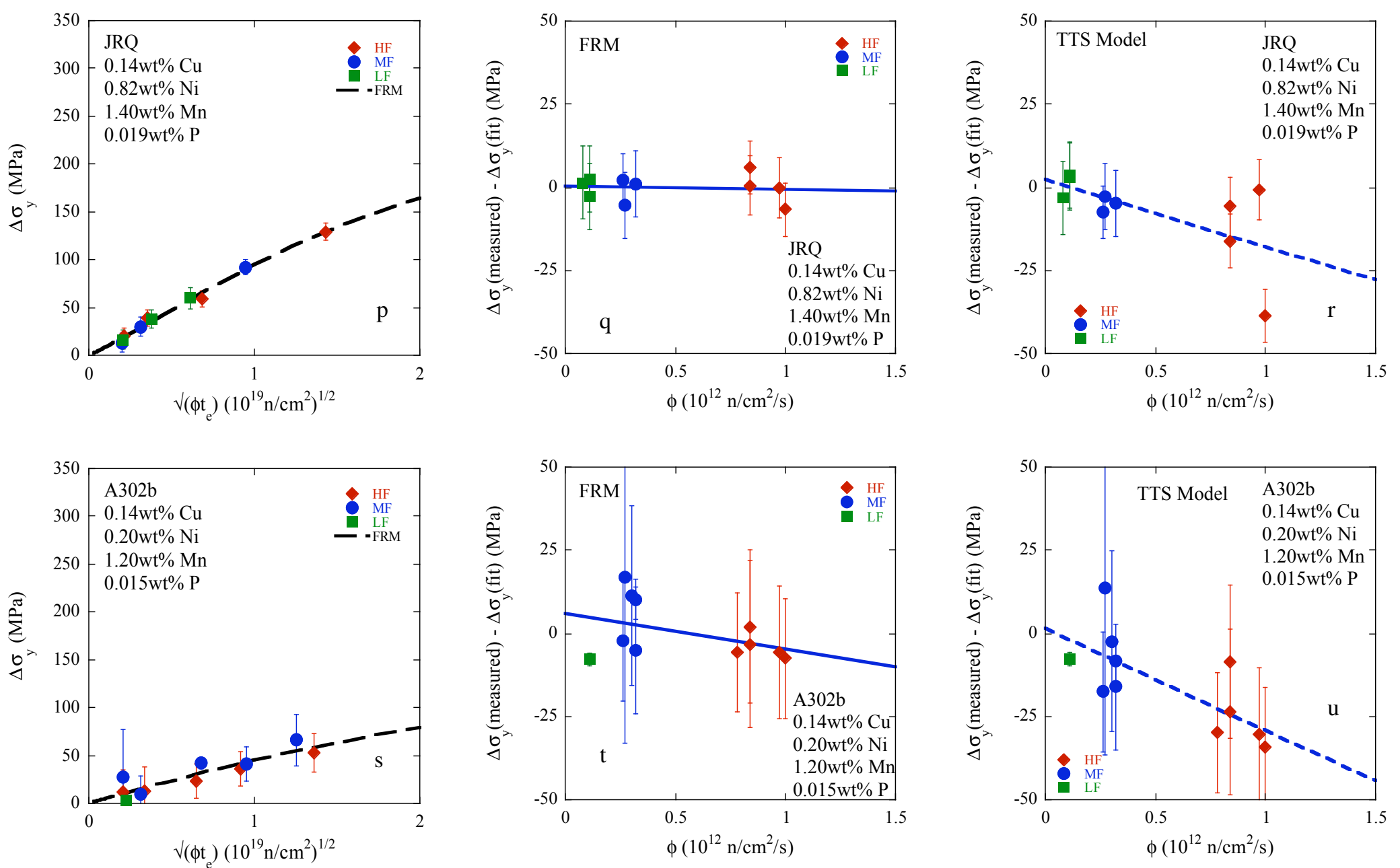

Fig. 6.15 p-u. (p, s) The fitted recombination model (FRM) predictions and the $290^{\circ} \mathrm{C}$ IVAR $\triangle \sigma_{y}$ data for two Cu-bearing commercial plates on an effective fluence, $\phi t_{e}$, scale; ( $\left.q, t\right)$ the corresponding measured minus predicted residuals for the FRM that are well centered and approximately independent of flux; $(r, u)$ the corresponding residuals for the TTS model, showing a systematic effect of flux that is not accounted for in the TTS model developed in Chap. 3. 

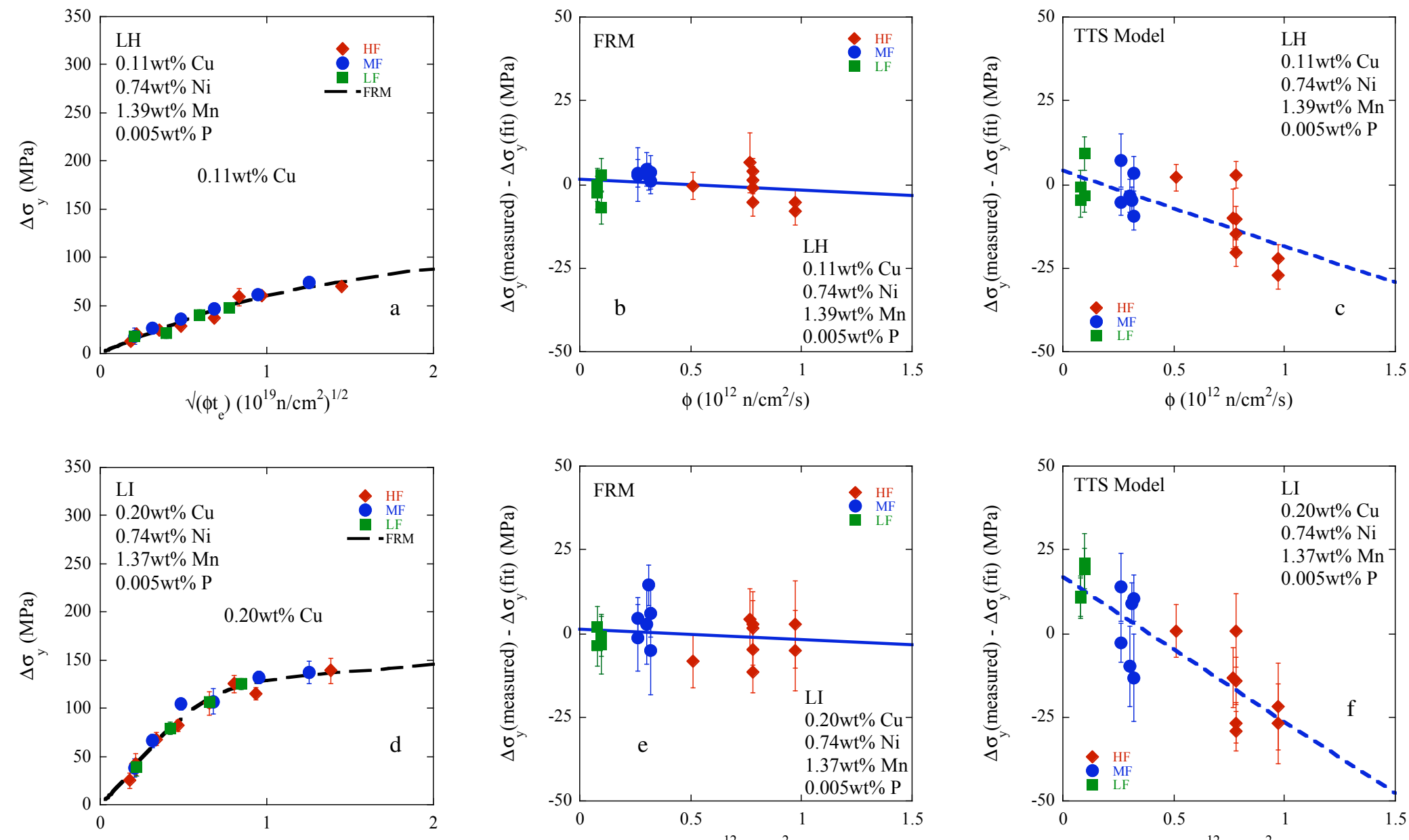

$\left.V_{\left(\phi \mathrm{e}_{\mathrm{e}}\right.}\right)\left(10^{19} \mathrm{n} / \mathrm{cm}^{2}\right)^{1 / 2}$
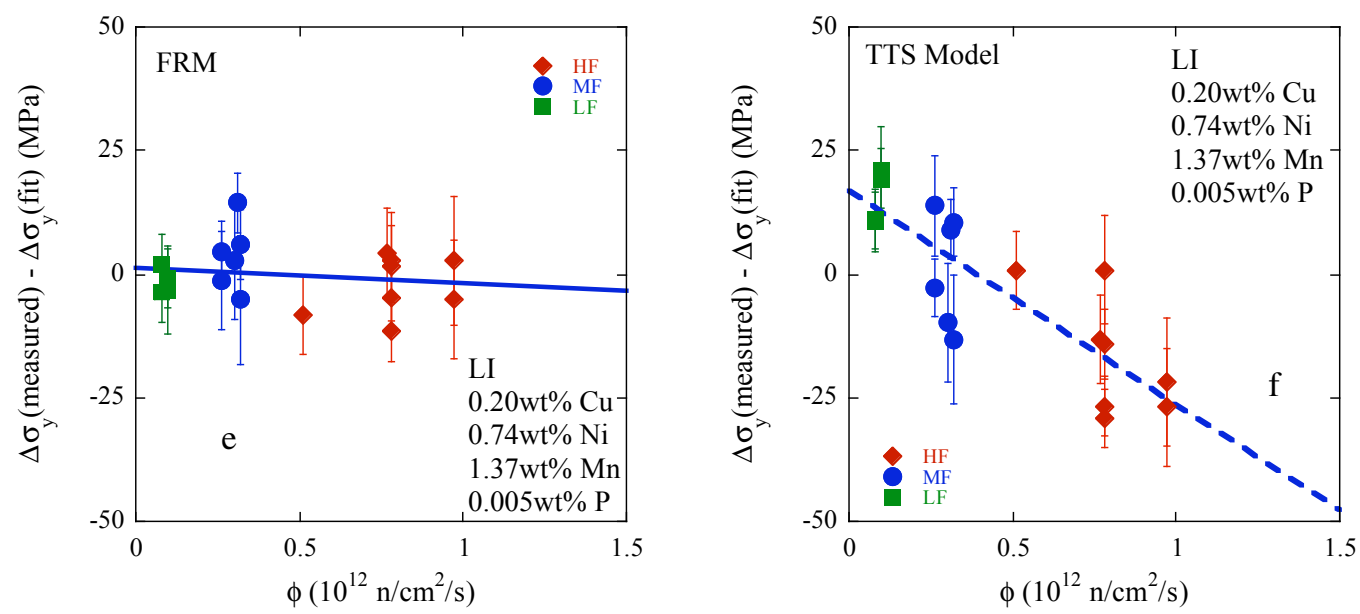

Fig. 6.16 a-f. (a, d) The fitted recombination model (FRM) predictions and the $290^{\circ} \mathrm{C}$ IVAR $\triangle \sigma_{\mathrm{y}}$ data for two SMMS with 0.8 wt $\% \mathrm{Ni}, 1.4 \mathrm{wt} \% \mathrm{Mn}$ and 0.1 and $0.2 \mathrm{wt} \% \mathrm{Cu}$ on an effective fluence, $\mathrm{t}_{\mathrm{e}}$, scale; (b, e) the corresponding measured minus predicted residuals for the FRM that are well centered and approximately independent of flux; (c, $f$ ) the corresponding residuals for the TTS model, showing a systematic effect of flux that is not accounted for in the TTS model developed in Chap. 3. 

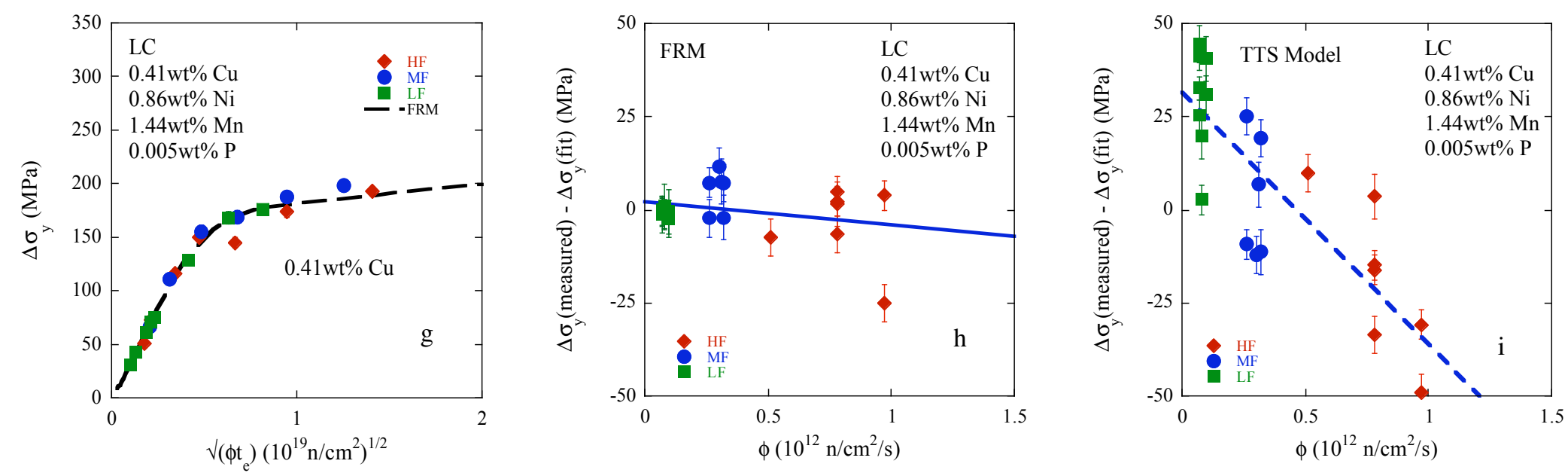

Fig. $6.16 \mathrm{~g}$-i. (g) The fitted recombination model (FRM) predictions and the $290^{\circ} \mathrm{C}$ IVAR $\triangle \sigma_{\mathrm{y}}$ data for a SMMS with 0.8 wt $\%$ $\mathrm{Ni}, 1.4 \mathrm{wt} \% \mathrm{Mn}$ and $0.4 \mathrm{wt} \% \mathrm{Cu}$ on an effective fluence, $\phi \mathrm{t}_{\mathrm{e}}$, scale; $(\mathrm{h})$ the corresponding measured minus predicted residuals for the FRM that are well centered and approximately independent of flux; (i) the corresponding residuals for the TTS model, showing a systematic effect of flux that is not accounted for in the TTS model developed in Chap. 3. 

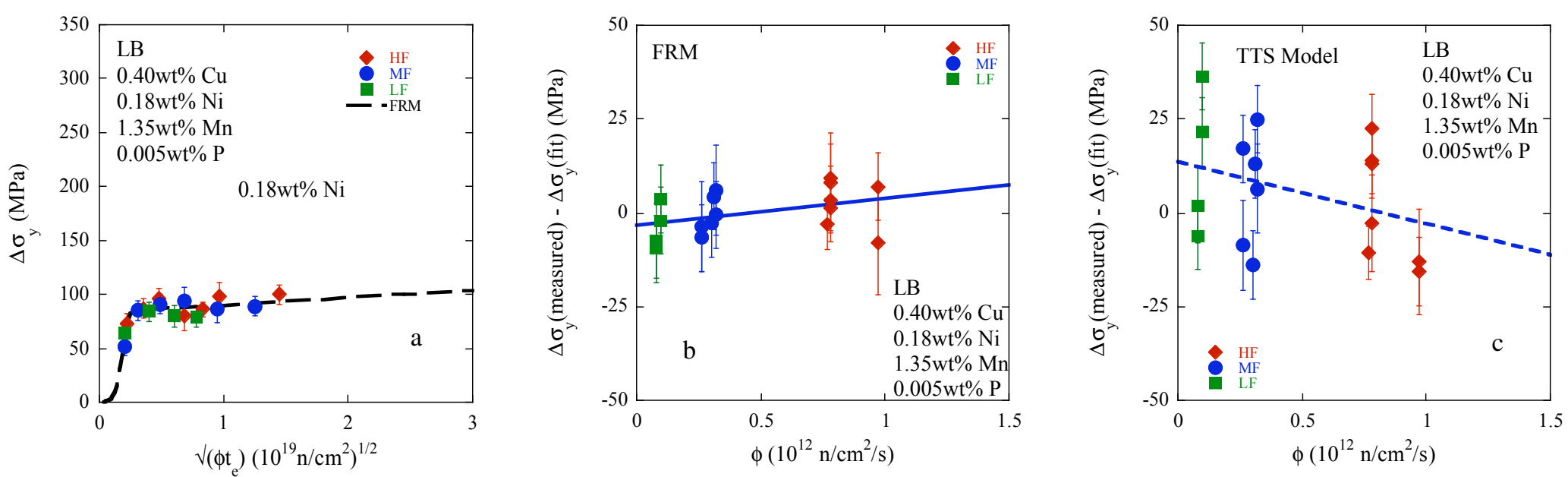

ì
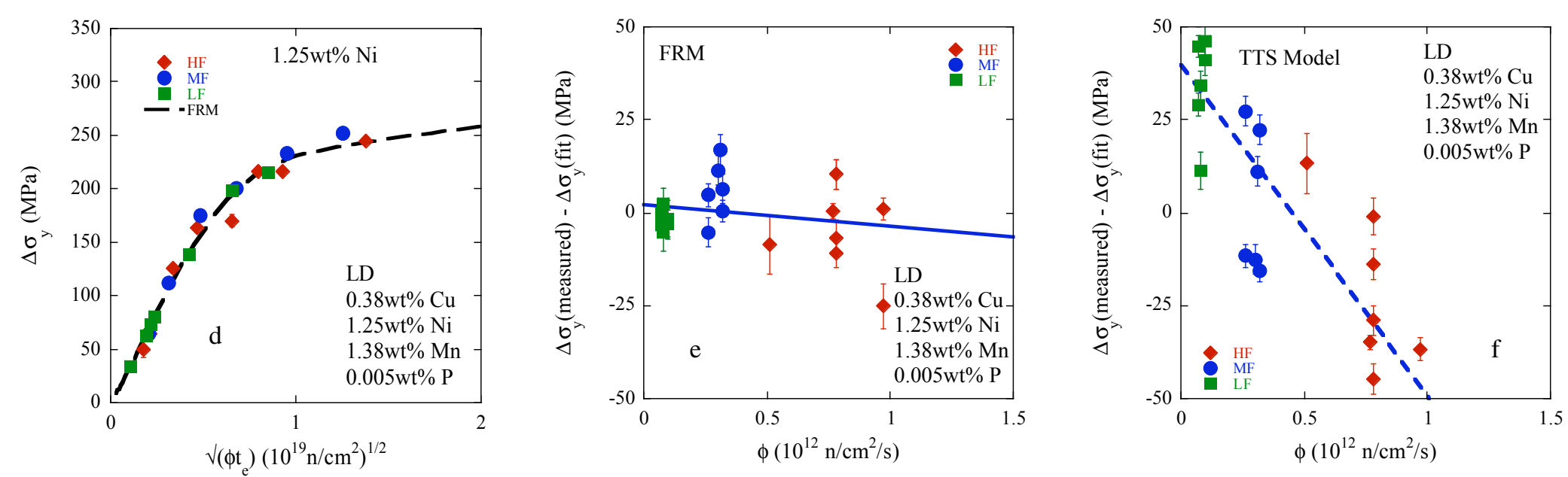

Fig. 6.17 a-f. (a, d) The fitted recombination model (FRM) predictions and the $290^{\circ} \mathrm{C}$ IVAR $\triangle \sigma_{y}$ data for two SMMS with 0.4 wt $\% \mathrm{Cu}, 1.4 \mathrm{wt} \% \mathrm{Mn}$, and 0.2 and $1.25 \mathrm{wt} \% \mathrm{Ni}$ on an effective fluence, $\phi \mathrm{t}_{\mathrm{e}}$, scale; $(\mathrm{b}, \mathrm{e})$ the corresponding measured minus predicted residuals for the FRM that are well centered and approximately independent of flux; (c, f) the corresponding residuals for the TTS model, showing a systematic effect of flux that is not accounted for in the TTS model developed in Chap. 3. 

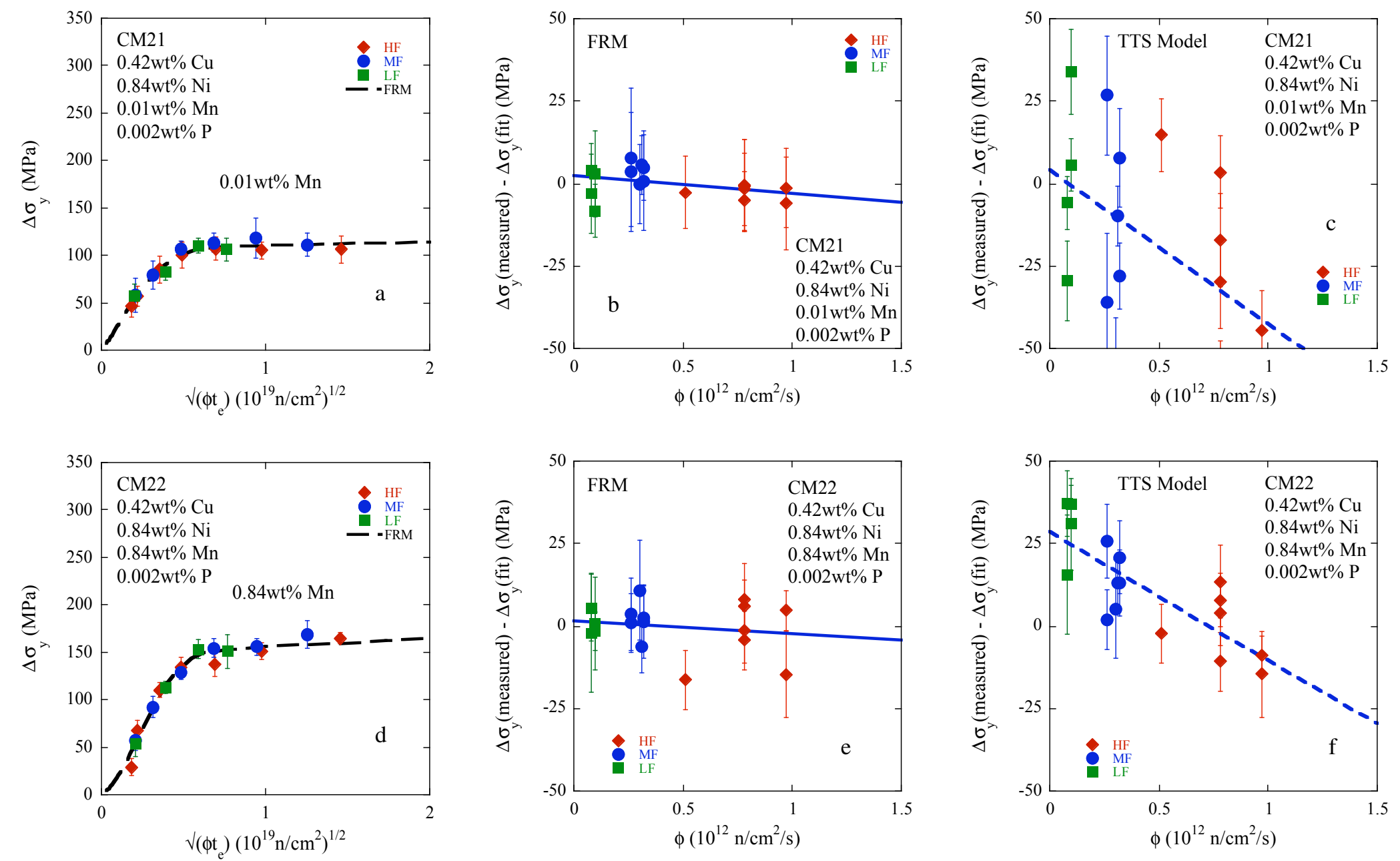

Fig. 6.18 a-f. (a, d) The fitted recombination model (FRM) predictions and the $290^{\circ} \mathrm{C}$ IVAR $\Delta \sigma_{\mathrm{y}}$ data for two SMMS with 0.4 wt $\% \mathrm{Cu}, 0.8 \mathrm{wt} \% \mathrm{Ni}$, and 0.0 and $0.8 \mathrm{wt} \% \mathrm{Mn}$ on an effective fluence, $\phi \mathrm{t}_{\mathrm{e}}$, scale; (b, e) the corresponding measured minus predicted residuals for the FRM that are well centered and approximately independent of flux; (c, $f$ ) the corresponding residuals for the TTS model, showing a systematic effect of flux that is not accounted for in the TTS model developed in Chap. 3. 

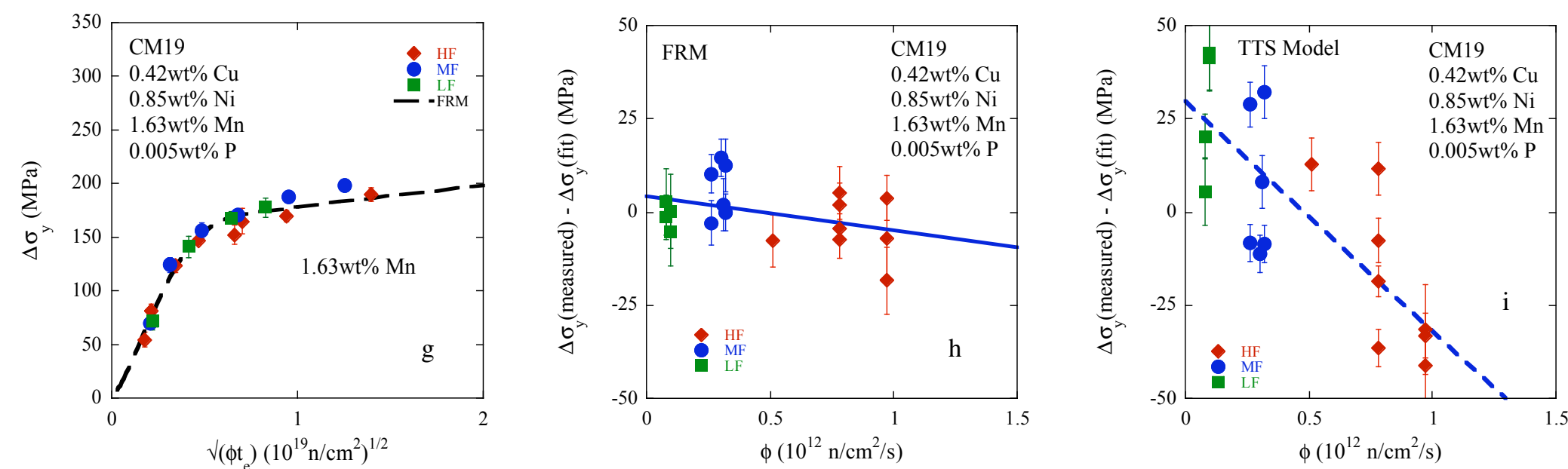

Fig. $6.18 \mathrm{~g}-\mathrm{i}$. (g) The fitted recombination model (FRM) predictions and the $290^{\circ} \mathrm{C}$ IVAR $\Delta \sigma_{\mathrm{y}}$ data for two SMMS with 0.4 wt $\% \mathrm{Cu}, 0.8 \mathrm{wt} \% \mathrm{Ni}$, and $1.6 \mathrm{wt} \% \mathrm{Mn}$ on an effective fluence, $\phi \mathrm{t}_{\mathrm{e}}$, scale; $(\mathrm{h})$ the corresponding measured minus predicted residuals for the FRM that are well centered and approximately independent of flux; (i) the corresponding residuals for the TTS model, showing a systematic effect of flux. that is not accounted for in the TTS model developed in Chap. 3. 

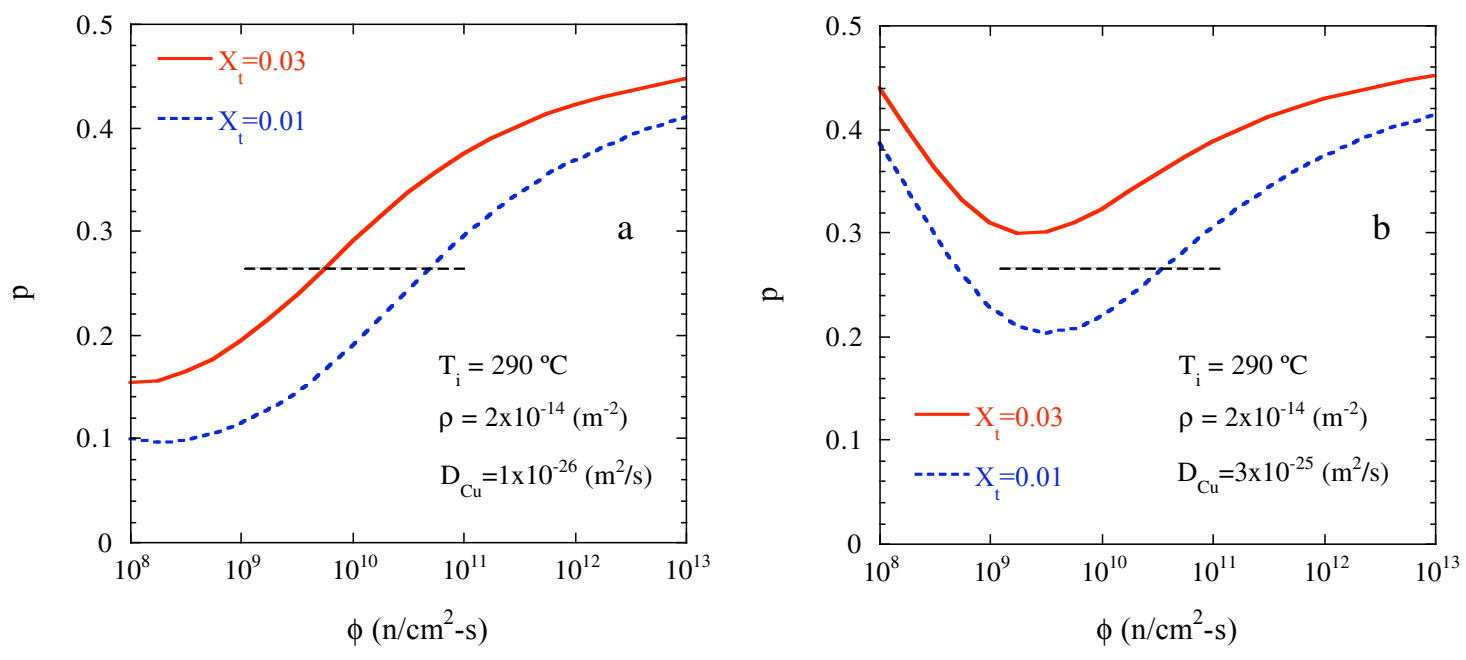

Fig. 6.19. The flux scaling $p$ values for a range of $X_{t}$ and low and high nominal $D_{c u}$. The horizontal dashed line represents the fitted value in the TTS model.
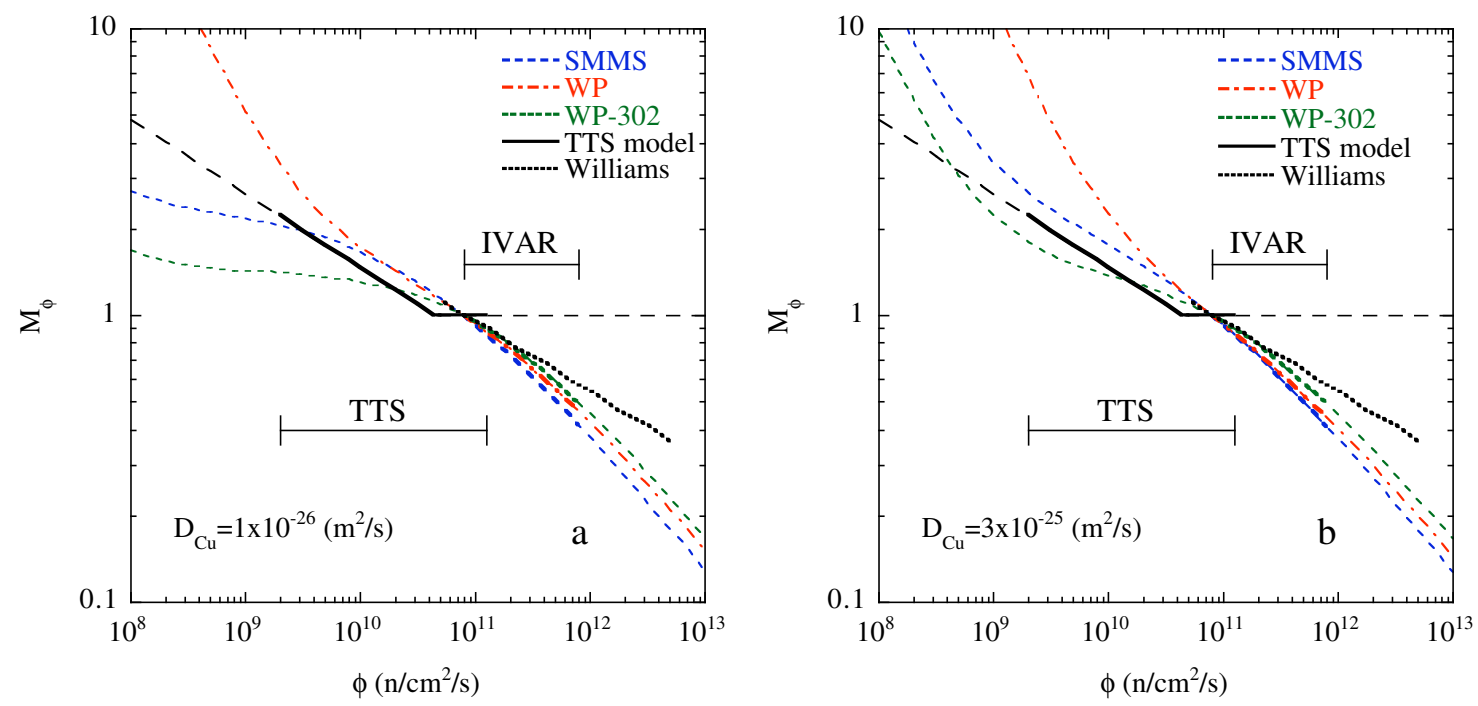

Fig. 6.20. The $M_{\phi}=\phi t_{\mathrm{e}} / \phi t$ effective fluence multiplying factor as a function of flux for the TTS model, the FRM for various subsets of IVAR data and the corresponding $M_{0}$ reported by Williams. The $M_{\phi}$ is normalized at $8 \times 10^{10} \mathrm{n} / \mathrm{cm}^{2}-\mathrm{s}$. The solid line is the TTS model plotted over the approximate range of TTS data: the dashed extensions indicate how the TTS model would extrapolate. Notably, the $\mathrm{M}_{\phi}$ curves are similar at a low flux $\left(2 \times 10^{9}\right.$ $\left.\mathrm{n} / \mathrm{cm}^{2}-\mathrm{s}\right)$. 
overlapping fluxes between the IVAR and TTS surveillance databases. Figures. 6.20a and 6.20b assume the nominal low and high estimates of $\mathrm{D}_{\mathrm{Cu}}$ (see Chap. 2). Separate averages are shown for the SMMS (solid line) and weld plus plate (long dashed line) alloy groupings. With one exception, the $\mathrm{FRM} \mathrm{M}_{\phi}(\phi)$ curves compare favorably with that for the TTS model, in spite of the latter's "hockey stick" shape. The exception is the high $D_{C u}$ FRM fitted to the weld and plate alloys. The $M_{\phi}$ in this case is significantly larger than for the TTS model at low flux. However, this is due to an anomalously large $\mathrm{M}_{\phi}$ in the A302B plate, hence, this curve is not considered to be very reliable. The corresponding curve that does not include the $\mathrm{A} 302 \mathrm{~B}$ data in the normalization is in better agreement with the TTS $\mathrm{M}_{\phi}$ curve. Figure 6.20 also shows that the $M_{\phi}$ function reported by Williams [10] is in good agreement with the IVAR results. Note, this figure also demonstrates the modest effect of flux predicted by the FRM in the region between $4.45 \times 10^{10}$ and $2 \times 10^{11} \mathrm{n} / \mathrm{cm}^{2}$-s, where a flux effect is not included in the TTS model because, for reasons discussed below, it did not appear to be significant in the surveillance data. Note, the effect of flux on $\phi t_{\mathrm{e}}$ is stronger than that of the corresponding $\Delta \sigma_{\mathrm{y}}$ which scales with dose to a power that is less

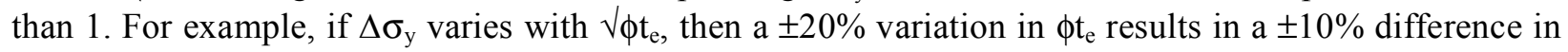
$\Delta \sigma_{\mathrm{y}}$.

The FRM $\Delta \sigma_{\mathrm{y}}$ hardening curves for the SMMS and welds tend to be shifted to slightly lower fluence compared to the TTS model predictions. The opposite is the case for the plates. This effect can be simply quantified in terms of the fluence at $50 \%$ of the maximum CRP hardening, $\phi \mathrm{t}_{0.5}$. Figure 6.21 shows the average $\phi \mathrm{t}_{0.5}$ for both models at $2 \times 10^{9}$ and $2 \times 10^{11} \mathrm{n} / \mathrm{cm}^{2}$-s for separate SMMS, weld and plate alloy groupings. As noted above, the FRM $\phi \mathrm{t}_{0.5}$ are lower for the SMMS and welds and higher for the plates compared with the TTS model.

The fact that the TTS model does not find a strong flux effect in the range of the IVAR experiment is not very surprising since:

- The PWR surveillance data and IVAR data have overlapping flux ranges, but the higher flux surveillance data are sparse. About $95 \%$ of the surveillance data have flux $<2 \times 10^{11} \mathrm{n} / \mathrm{cm}^{2}$-s, which is below the medium flux level $\left(3 \times 10^{11} \mathrm{n} / \mathrm{cm}^{2}-\mathrm{s}\right)$ in the IVAR irradiations. Thus, the TTS model calibration reflects mainly lower flux levels and only a small fraction of the IVAR flux range.

- It is inherently difficult to resolve an influence of any variable over the narrow range, in this case flux, especially since the effects are relatively small compared to the scatter in the TTS database.

- The TTS model is necessarily simplified and must compromise to provide average fits for $\mathrm{p}$ for a wide range of alloys and irradiation conditions, while the FRM model is physically based and is fitted to individual alloys, each with a large set of high quality data.

- Most of the high $\mathrm{Cu}$ TTS data in the IVAR flux range are at high fluence on, or approaching, the flux independent CRP hardening plateau, while the IVAR database explicitly explores a wide range of fluence, with many experimental observations for each alloy. Since the main effect of flux is to shift the fluence to reach rather than the magnitude of the CRP hardening plateau, it is not surprising that the TTS data do not show a flux effect.

- The Ni and Mn solute contents of the IVAR SMMS are, on average, somewhat higher than in the TTS database, thus enhancing recombination and flux effects.

In summary, as schematically illustrated in Fig. 6.22, it appears that a flux effect in the TTS surveillance database can be detected only over the wide range of fluxes corresponding to typical BWR and PWR surveillance capsule conditions. However, the more precise and well-controlled IVAR database shows a systematic flux effect that is remarkably consistent with simply extrapolating the flux dependence found in the TTS model to higher flux, in the region where the effect apparently flattens in fitting the surveillance database. There is no physical basis to expect an abrupt end to the flux dependent regime. Indeed, physical considerations suggest that the effect of flux should increase with increasing 


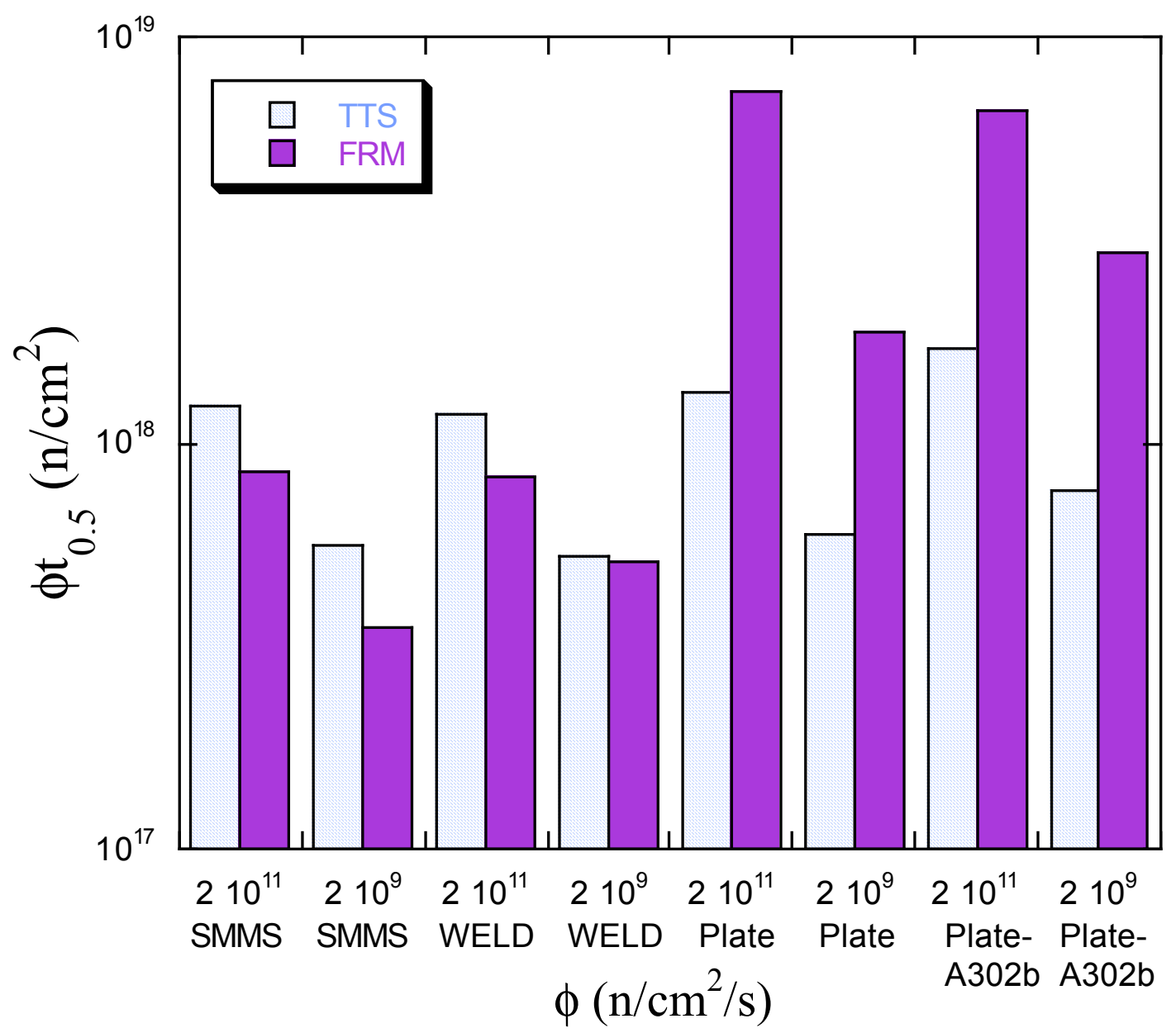

Fig. 6.21. The fluence at $50 \%$ of the maximum CRP hardening, $\phi t_{0.5}$, for the TTS and FRM models and various subsets of IVAR data ignoring the effect of the TTS to $\Delta \sigma_{y}$ conversion. The $\phi t_{0.5}$ is somewhat lower for the IVAR SMMS and commercial welds and higher for plates for the FRM vs TTS model.

dose rate. The use of a constant $\mathrm{p}$ approximation is supported by the analysis provided in this section. However, it must be recognized that the fitted $p$ in the TTS model is a compromise average that does not account for any of the relevant variables that affect $p$ such as the alloy composition, microstructure and irradiation temperature. The overall trends in the FRM and TTS models represented by a flux multiplier $\mathrm{M}_{\phi}(\phi)$ function are remarkably consistent over a wide range of flux, and are supported by independent results reported by Williams. Extrapolation of the TTS model to low flux, below the surveillance database, is clearly uncertain, since there are no relevant TTS data. However, it is notable that the TTS model extrapolation is bounded by the predictions of the FRM models that assume the high and low values for $\mathrm{D}_{\mathrm{Cu}}$. This suggests that from a mechanistic perspective, extrapolating the TTS model to low flux is justified by the current state of knowledge. 


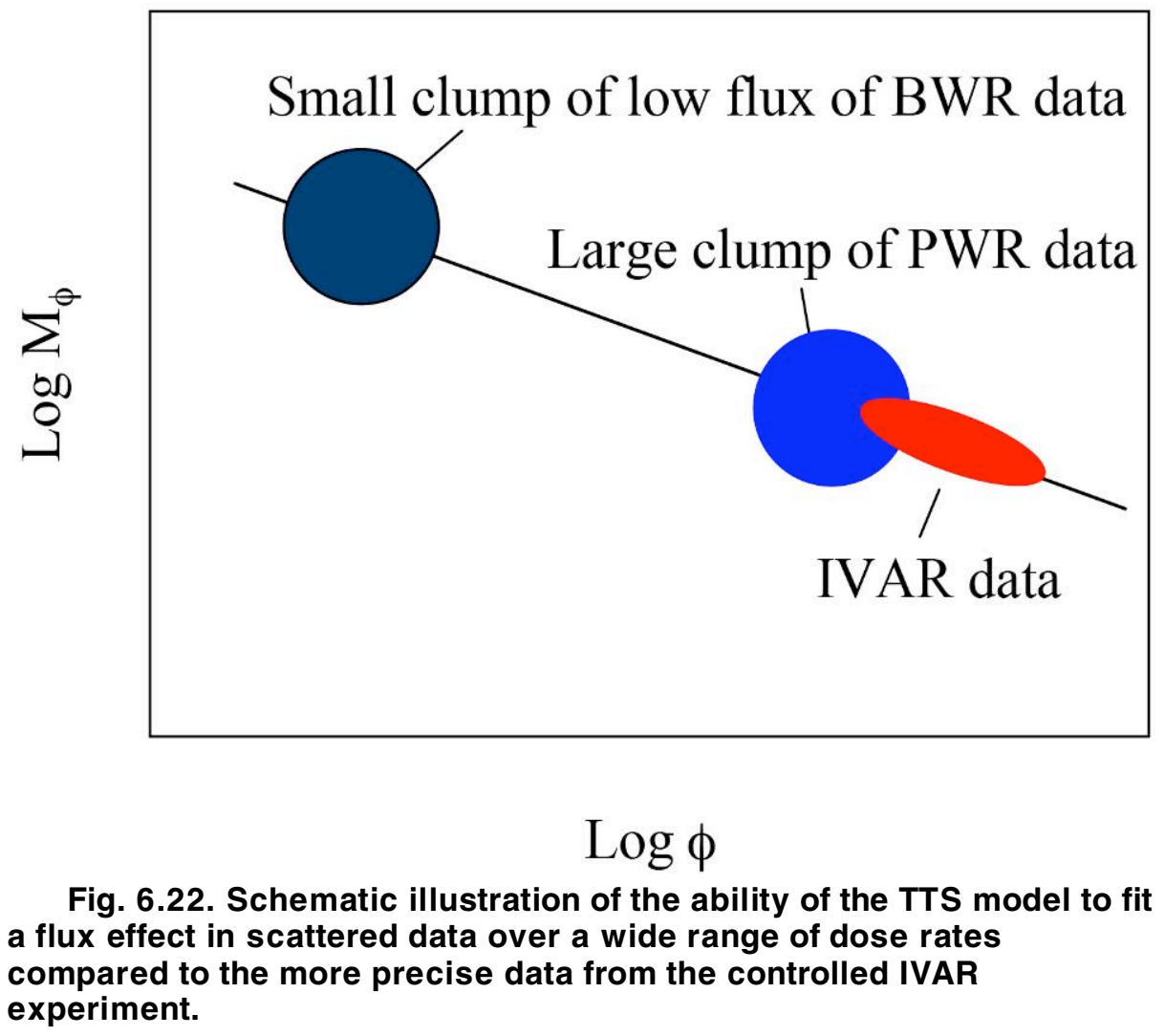

\subsubsection{Flux Effects on MF Hardening}

The conventional view is that the MF contribution to hardening does not depend on flux. However, as in the case of the CRP contribution, the MF hardening in the IVAR database on low $\mathrm{Cu}$ steels systematically increases with decreasing flux, as shown in Figs. 6.9 to 6.11. The effect of flux can also be accounted for by the effective fluence, $\phi t_{\mathrm{e}}$, by least square fitting the scaling exponent, $\mathrm{p}$, in Eq. (6-8) and CF parameters models for each alloy with the expression

$$
\Delta \sigma_{\mathrm{y}}=\mathrm{CF} \sqrt{ } \phi \mathrm{t}_{\mathrm{e}}=\mathrm{CF} \sqrt{ }\left[\phi \mathrm{t}\left(\phi_{\mathrm{r}} / \phi\right)^{\mathrm{p}}\right]
$$

Figures 6.23 to 6.25 show plots similar to those Figs. 6.15 to 6.18 , except that in this case they are for the low-Cu IVAR steels. The figures on the left are again the fits to the IVAR $\Delta \sigma_{\mathrm{y}}$ data plotted on an effective fluence, $\phi \mathrm{t}_{\mathrm{e}}$, scale. The figures in the middle are the measured minus predicted $\Delta \sigma_{\mathrm{y}}$ residuals plotted against flux for the effective fluence MF model in Eq. (6-9). The figures on the right are the corresponding residuals for the TTS model, which does not have a flux effect in the MF term in the flux range of the IVAR experiment, although it does at lower flux. As expected, the residuals for the fitted effective fluence MF model are generally well centered around 0, and show neither a significant average net bias nor any strong, systematic effect of flux. In contrast, the TTS model residuals show a systematic flux effect and are sometimes not well centered around 0 . The average fitted $\mathrm{p}$ is 0.42 for the data in Fig. 6.23, which represent steels most like those in the IVAR database. The corresponding $\mathrm{p}$ for fits to low and high sensitivity data in Figs 6.24 and 6.25 are 0.47 and 0.44 , respectively. The $\mathrm{p}$ for the single low $\mathrm{Cu}$ IVAR medium Ni weld is 0.125 . These $\mathrm{p}$ values are broadly consistent with the observed effect of flux on the CRP contribution to hardening. 

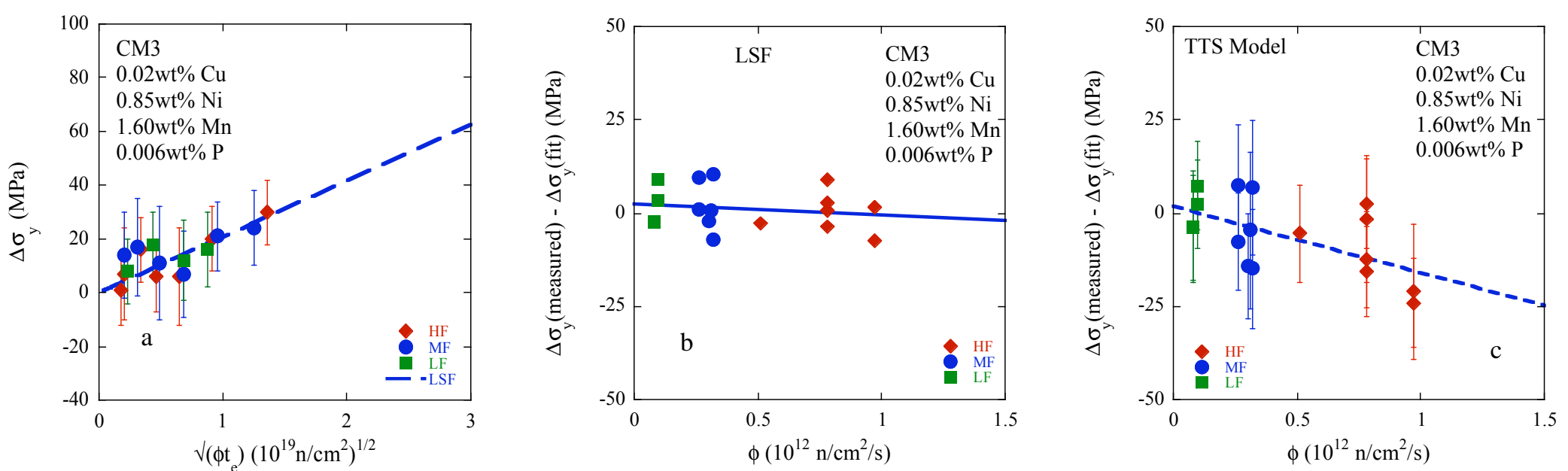

$\stackrel{i}{u}$
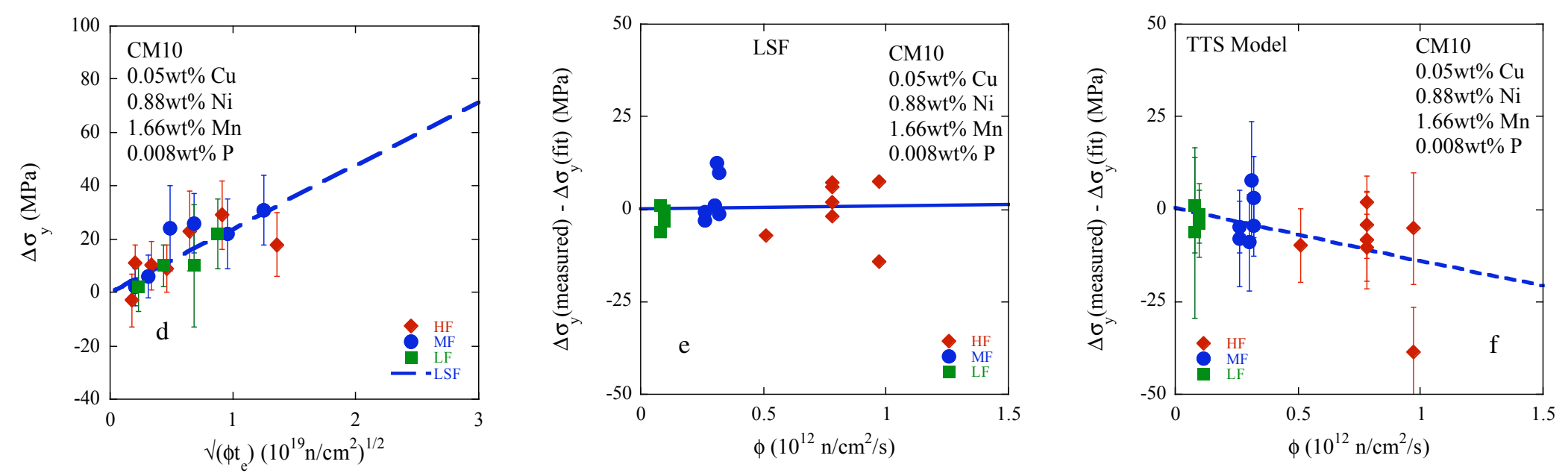

Fig. 6.23 a-f. (a, d) The fitted MF model for the $290^{\circ} \mathrm{C}$ IVAR $\Delta \sigma_{y}$ data on two Cu-free SMMS with $0.8 \mathrm{wt} \% \mathrm{Ni}, 1.6 \mathrm{wt} \% \mathrm{Mn}$, and $0.005 \mathrm{wt} \% \mathrm{P}$ on an effective fluence, $\phi \mathrm{t}_{\mathrm{e}}$, scale; $(\mathbf{b}, \mathbf{e})$ The corresponding measured minus predicted residuals for the $\mathrm{FRM}$ that are reasonably well-centered and approximately independent of flux; (c, f) The corresponding residuals for the TTS model, showing a systematic effect of flux. 

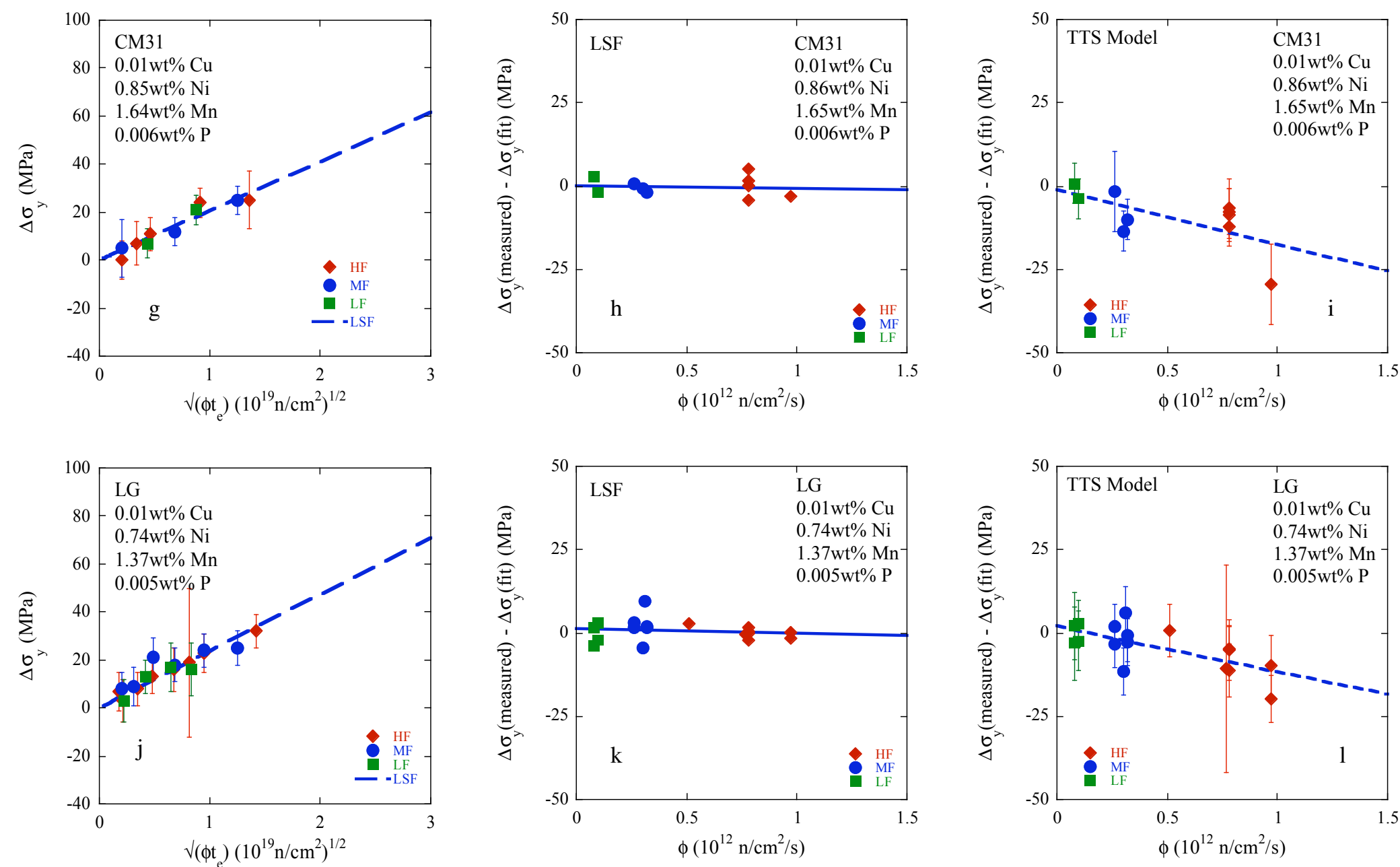

Fig. $6.23 \mathrm{~g}-\mathrm{I}$. (g, j) The fitted MF model for the $290^{\circ} \mathrm{C}$ IVAR $\Delta \sigma_{\mathrm{y}}$ data on two Cu-free SMMS with $0.8 \mathrm{wt} \% \mathrm{Ni}, 1.4$ to1.6 wt \% $\mathrm{Mn}$, and $\mathbf{0 . 0 0 5} \mathrm{wt} \% \mathrm{P}$ on an effective fluence, $\phi \mathrm{t}_{\mathrm{e}}$, scale; $(\mathbf{h}, \mathbf{k})$ The corresponding measured minus predicted residuals for the FRM that are reasonably well-centered and approximately independent of flux; (i, I) The corresponding residuals for the TTS model, showing a systematic effect of flux. 

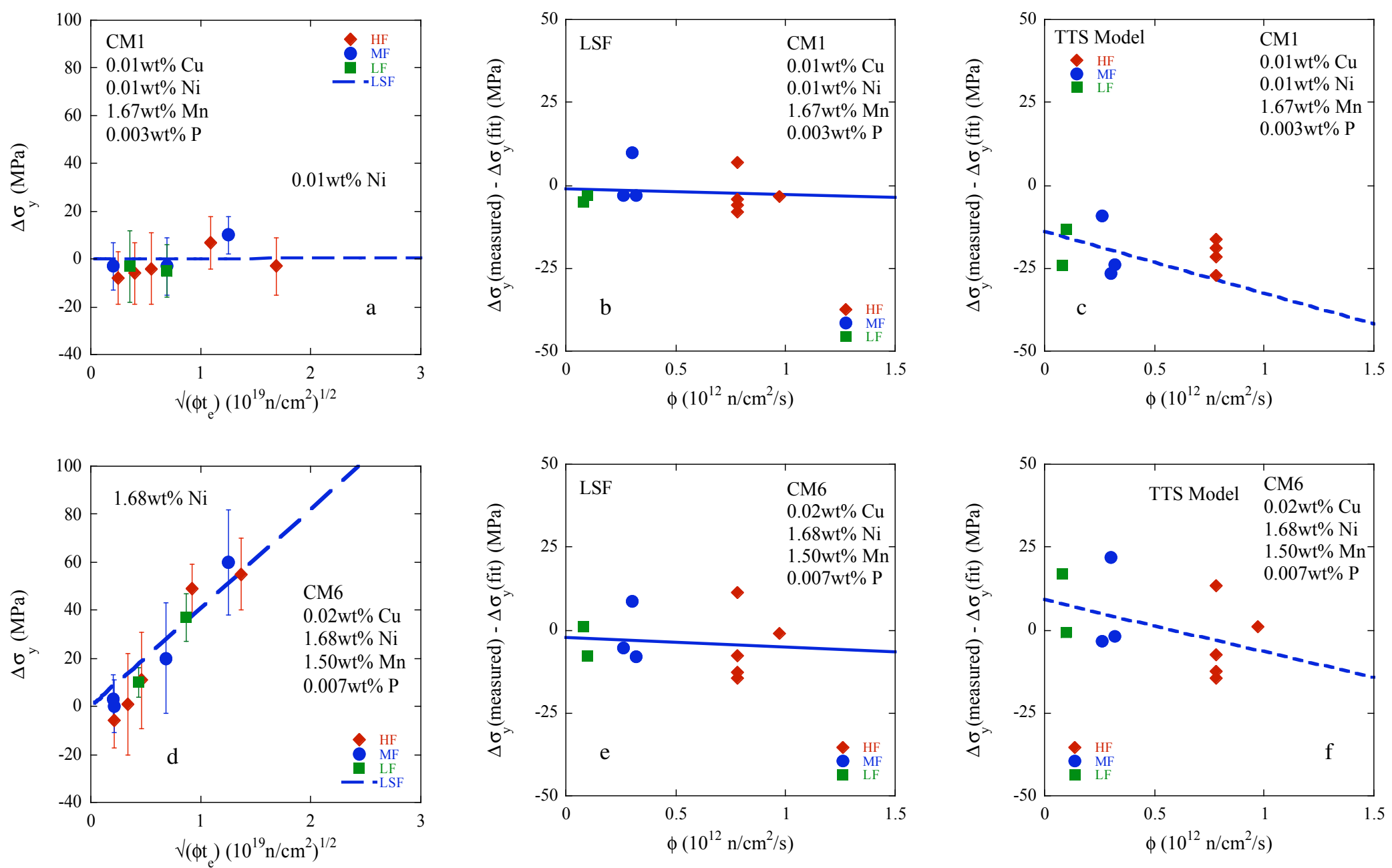

Fig. 6.24 a-f. (a, d) The fitted MF model for the $290^{\circ} \mathrm{C}$ IVAR $\Delta \sigma_{\mathrm{y}}$ data on two Cu-free SMMS with 0.8 and $1.6 \mathrm{wt} \% \mathrm{Ni}, 1.6 \mathrm{wt} \% \mathrm{Mn}$, and $0.005 \mathrm{wt} \% \mathrm{P}$ on an effective fluence, $\phi \mathbf{t}_{\mathrm{e}}$, scale; $(\mathbf{b}, \mathbf{e})$ The corresponding measured minus predicted residuals for the FRM that are reasonably well-centered and approximately independent of flux; (c, f) The corresponding residuals for the TTS model, showing a systematic effect of flux. 

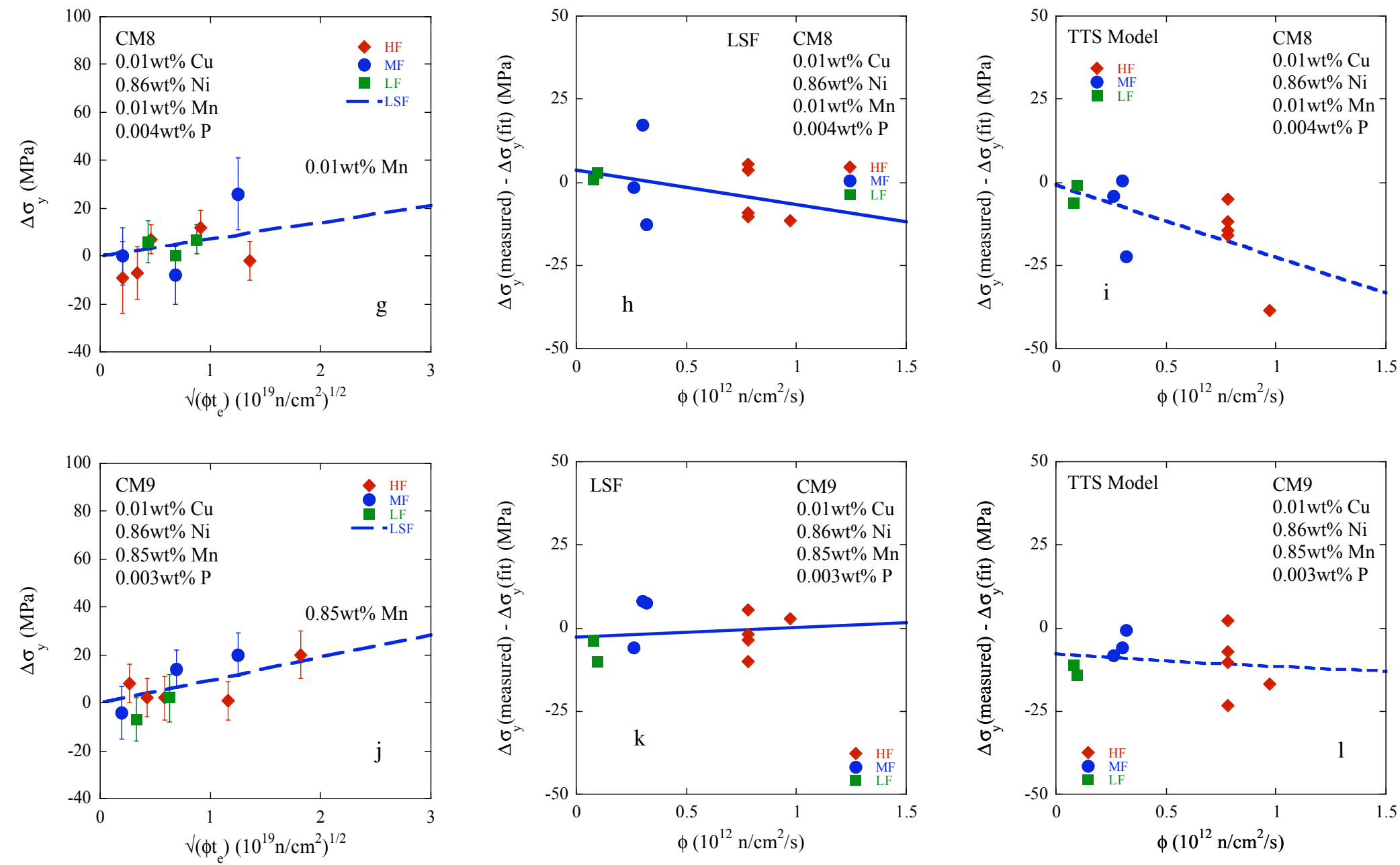

Fig. 6.24 g-l. (g, j) The fitted MF model for the $290^{\circ} \mathrm{C}$ IVAR $\Delta \sigma_{\mathrm{y}}$ data on two Cu-free SMMS with 0.8 wt $\% \mathrm{Ni}, 0.005 \mathrm{wt} \% \mathrm{P}$ and 0.0 and $0.8 \mathrm{wt} \% \mathrm{Mn}$ on an effective fluence, $\phi t_{\mathrm{e}}$, scale; $(h, k)$ The corresponding measured minus predicted residuals for the FRM that are reasonably well-centered and only moderately sensitive to flux in the $0.0 \mathrm{wt}, \% \mathrm{Mn}$ alloy; (i, l) The corresponding residuals for the TTS model, showing a systematic effect of flux. 

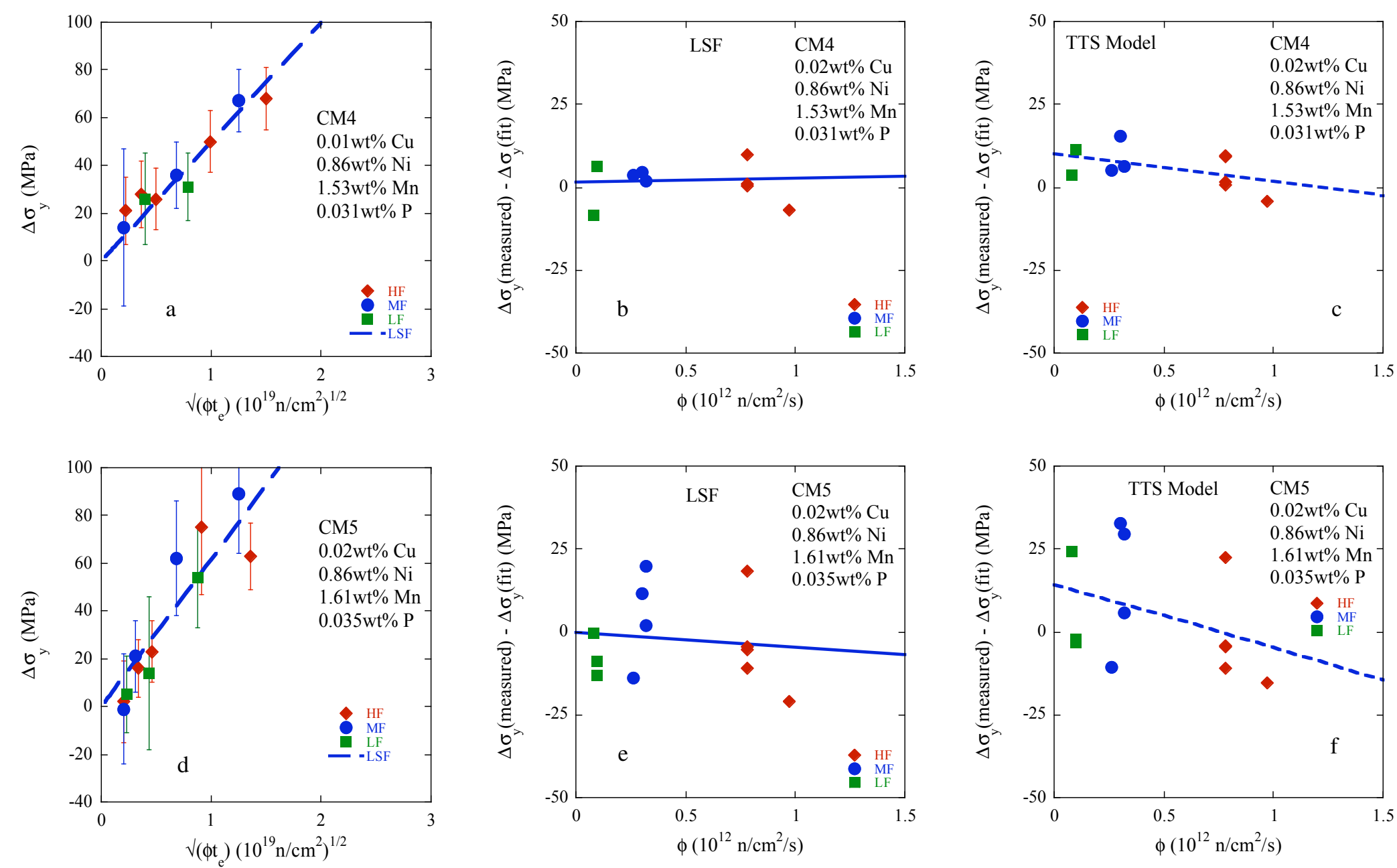

Fig. 6.25 a-f. (a, d) The fitted MF model for the $290^{\circ} \mathrm{C}$ IVAR $\Delta \sigma_{y}$ data for two Cu-free SMMS with 0.8 wt $\% \mathrm{Ni}, 1.6 \mathrm{wt} \% \mathrm{Mn}$, and 0.031 and $0.35 \mathrm{wt} \% \mathrm{P}$ on an effective fluence, $\phi \mathrm{t}_{\mathrm{e}}$, scale; $(\mathrm{b}, \mathbf{e})$. The corresponding measured minus predicted residuals for the FRM that are reasonably well centered and approximately independent of flux; (c, f) The corresponding residuals for the TTS model, showing a systematic effect of flux. 

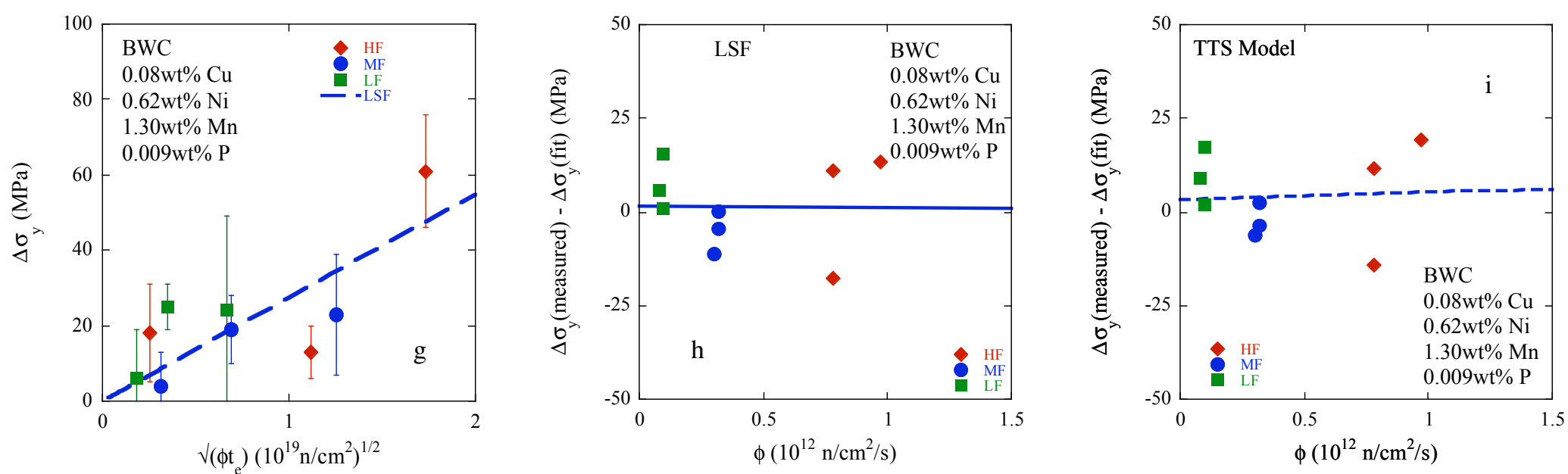

$\frac{1}{0}$
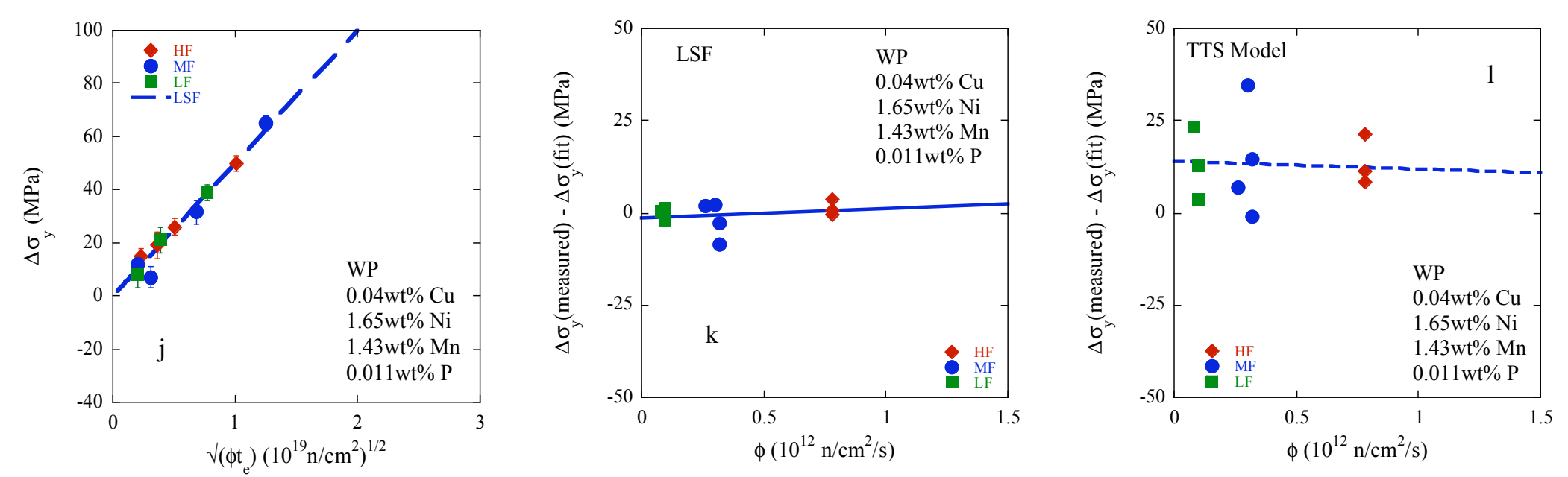

Fig. 6.25 g-l. (g, j) The fitted MF model for the $290^{\circ} \mathrm{C}$ IVAR $\Delta \sigma_{y}$ data for two low-Cu welds with $0.8-1.6$ wt $\%$ Ni on an effective fluence, $\phi t_{e}$, scale; $(h, k)$ The corresponding measured minus predicted residuals for the FRM that are reasonably wellcentered and approximately independent of flux; (i, I). The corresponding residuals for the TTS model, showing a systematic effect of flux for the high Ni weld. 
It is also clear that while the relative effect is fairly large, the absolute magnitude of the influence of flux on the MF hardening is small to modest because of the corresponding low values of $\Delta \sigma_{\mathrm{y}}$. Thus, given the minimal signal-to-noise ratio, a more detailed and quantitative analysis of flux effects on MF hardening has not yet been attempted. However, as discussed in Chap. 2, the effects are likely to be due to long-range solute diffusion. Thus, the physical arguments supporting a flux effect for the CRP contribution also apply to the MF as well.

In summary, weak but systematic flux effects are observed in low $\mathrm{Cu}$ IVAR steels. The IVAR results are broadly consistent with the use of a dose-rate-dependent effective fluence in the TTS MF model.

\subsection{Effects of Other Variables}

The TTS model includes a contribution to embrittlement from P in both the MF and CRP terms. The $\mathrm{P}$ effect in the MF term simply adds to the composition independent contributions and depends strongly on the alloy's Mn content. Since the IVAR SMMS with systematic variations on $\mathrm{P}$ all contained $\approx 1.6 \mathrm{wt}$ $\% \mathrm{Mn}$, the corresponding data cannot be used to directly address the specific issue of how $\mathrm{P}$ contributions in low $\mathrm{Cu}$ steels vary with $\mathrm{Mn}$. However, as shown in Fig. 6.26, IVAR irradiations of simple model alloys do support an Mn-P interaction. Fe alloyed with $0.0125 \mathrm{wt} \% \mathrm{~N}$ softens by $\Delta \sigma_{\mathrm{y}} \approx-31 \mathrm{MPa}$. This softening is probably due to some recovery of quench hardening vacancy-N clusters and, perhaps, annealing of a small amount of surface strain hardening introduced in preparing tensile specimens of this low strength material, as well as data scatter. Adding $0.025 \mathrm{wt} \% \mathrm{P}$ to Fe results in a $\Delta \sigma_{\mathrm{y}}=76 \mathrm{MPa}$, while the model alloy with $1.6 \mathrm{wt}$. $\% \mathrm{Mn}$ and $0.025 \mathrm{wt} \% \mathrm{P}$ hardens by a $\Delta \sigma_{\mathrm{y}}=151 \mathrm{MPa}$. The corresponding $\Delta \sigma_{\mathrm{y}}$ for the $\mathrm{Fe}-1.6 \mathrm{wt} \% \mathrm{Mn}$ model alloy is $46 \mathrm{MPa}$. Thus, there is clearly an additional contribution from a P-Mn interaction, beyond the individual contributions of $\mathrm{P}$ and $\mathrm{Mn}$.

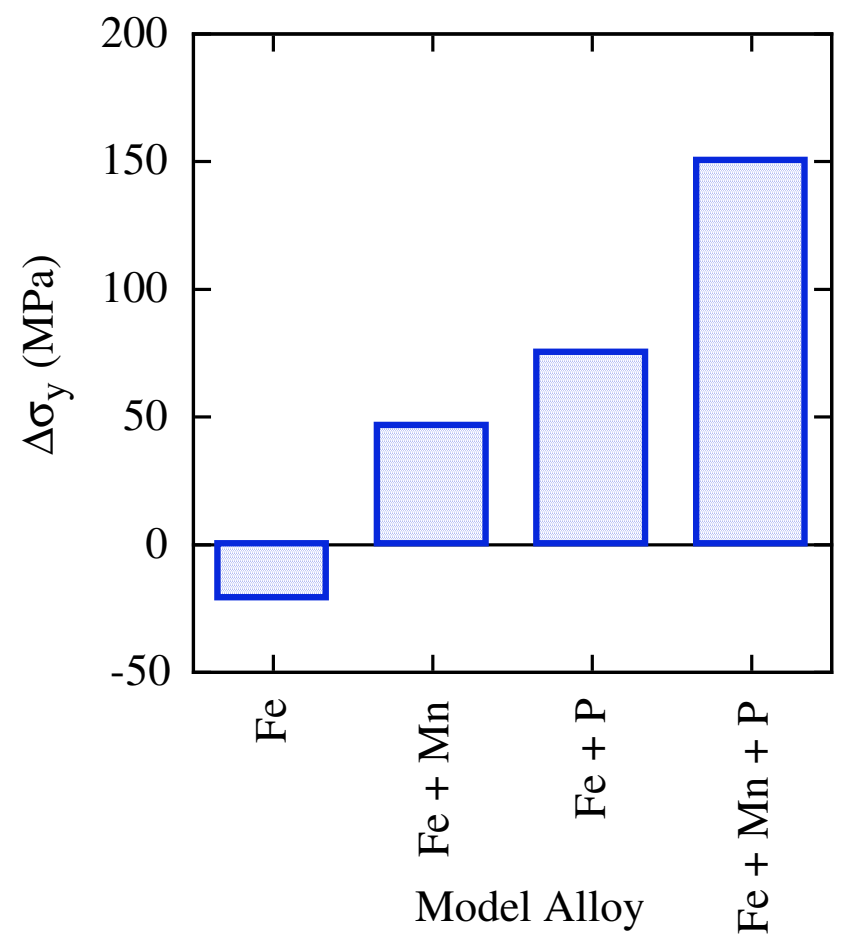

Fig. 6.26. The $\Delta \sigma_{y}$ for three simple model alloys irradiated in IVAR at high flux and $290^{\circ} \mathrm{C}$ to $1.77 \times$ $10^{19} \mathrm{n} / \mathrm{cm}^{2}$, showing a Mn-P interaction leading to higher hardening. 
The TTS model also includes a contribution of $\mathrm{P}$ to CRP embrittlement. In this case, $\mathrm{P}$ in excess of the empirically determined threshold of $0.008 \mathrm{wt} \%$, (note, the solubility of $\mathrm{P}$ is much lower than 0.008 wt \%) adds to the CRP hardening above the threshold of $0.072 \mathrm{wt} \% \mathrm{Cu}$. This TTS model contribution can be viewed as $\mathrm{P}$ adding to the hardening by CRPs, or, alternately, by forming separate phosphide precipitates whose flux, fluence, irradiation temperature, and $\mathrm{Ni}$ dependence approximately mirror those of the CRPs. However, as noted in Chap. 2, several earlier studies also showed that the effect of P decreases with increasing $\mathrm{Cu}[11,12]$.

As shown in Fig. 6.27, P effects, including P-Cu synergisms, are also observed in the IVAR database. Here the average $\sigma_{\mathrm{y}}$ at the four highest fluence data points for the high and intermediate flux IVAR irradiations at $290^{\circ} \mathrm{C}$ are plotted against $\mathrm{P}$ for both $\mathrm{Cu}$ free and $0.1 \mathrm{wt} \% \mathrm{Cu}$ SMMS. Least square fits yield $\mathrm{P}$ hardening chemistry factors, $\mathrm{CF}_{\mathrm{p}}$, which are the slopes of $\Delta \sigma_{\mathrm{y}}$ versus $\mathrm{P}$ fits in Fig. 6.27, of 1450 and $666 \mathrm{MPa} / \mathrm{wt} \% \mathrm{P}$ for $\mathrm{Cu}$ contents well below $(\approx 0.0 \mathrm{wt} \% \mathrm{Cu})$ and just above $(0.1 \mathrm{wt} \% \mathrm{Cu})$ the $\mathrm{CRP}$ threshold $(0.072$ wt. \% $\mathrm{Cu})$, respectively. The IVAR $\mathrm{CF}_{\mathrm{p}}$ are smaller than the equivalent values found by Jones [11], who also reported that $0.1 \mathrm{wt} \% \mathrm{Cu}$ reduced $\mathrm{CF}_{\mathrm{p}}$ by about $75 \%$, compared to about $55 \%$ in the subset of the Jones data shown in Fig. 6.27. Note, other subsets of the IVAR data also suggest a greater reduction in the $\mathrm{CF}_{\mathrm{p}}$ at $0.1 \mathrm{wt} \% \mathrm{Cu}$ than for the high fluence data shown in Fig. 6.27. Analyzing the IVAR results is somewhat complicated by the larger-than-average scatter in the unirradiated yield stress in the steels with higher P. However, the IVAR data, as well as all the test reactor results summarized in Chap. 2, are consistent with a significant effect of $\mathrm{P}$ in low-Cu steels that is reduced at higher $\mathrm{Cu}$.

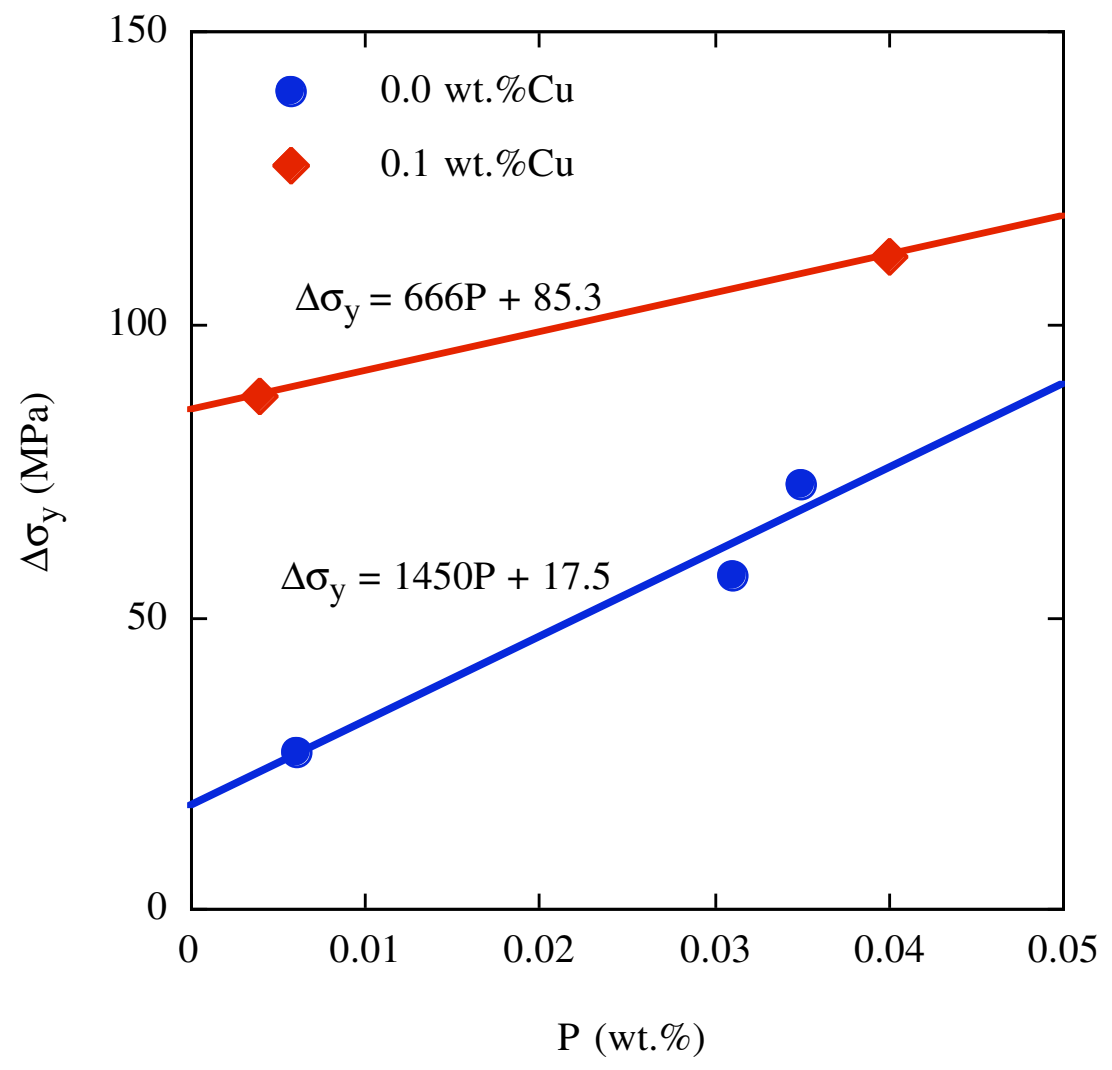

Fig. 6.27. Averaged high fluence $\sigma_{y}$ data for IVAR irradiations at intermediate and high flux at $290^{\circ} \mathrm{C}$ plotted as a function of $\mathbf{P}$ for SMMS with $0.8 \mathrm{wt} \% \mathrm{Ni}, 1.6 \mathrm{wt} \% \mathrm{Mn}$ and 0.0 and $0.1 \mathrm{wt} \% \mathrm{Cu}$. The data show a Cu-P synergism leading to decreased hardening due to $\mathrm{P}$ at higher $\mathrm{Cu}$, consistent with some previous observations. 
It is useful to compare the $\mathrm{P}$ chemistry factor $\left(\mathrm{CF}_{\mathrm{p}}\right)$ estimates from the IVAR data to those predicted by the TTS model. The $\mathrm{CF}_{\mathrm{p}}$ estimated from the TTS model depends on the flux, fluence, irradiation temperature, the alloy $\mathrm{Cu}$ and $\mathrm{Mn}$ contents, as well as the $\Delta \sigma_{\mathrm{y}} / \mathrm{TTS}$ conversion factor. In the case of low $\mathrm{Cu}$ steels, using nominal IVAR parameters of $1.6 \mathrm{wt} \% \mathrm{Mn}, \mathrm{T}_{\mathrm{i}}=290^{\circ} \mathrm{C}, \phi \mathrm{t}=1.5 \times 10^{19} \mathrm{n} / \mathrm{cm}^{2}$ and $\Delta \sigma_{\mathrm{y}} / \mathrm{TTS}=1.82 \mathrm{MPa} /{ }^{\circ} \mathrm{C}$, the TTS model estimate is $\mathrm{CF}_{\mathrm{p}} \approx 660 \mathrm{MPa} / \% \mathrm{P}$. This is lower than the value of $\mathrm{CF}_{\mathrm{p}}$ for the IVAR $\mathrm{Cu}$ free SMMS of $1450 \mathrm{MPa} / \mathrm{wt} \% \mathrm{P}$. For the $\approx 0.1 \mathrm{wt} \% \mathrm{Cu}, \approx 0.8 \mathrm{wt} \% \mathrm{Ni}$ and $\approx 1.6$ wt $\% \mathrm{Mn}$ alloy and a $\Delta \sigma_{\mathrm{y}} / \mathrm{TTS}=1.65 \mathrm{MPa} /{ }^{\circ} \mathrm{C}$, the TTS model estimate is $\mathrm{CF}_{\mathrm{p}} \approx 1820 \mathrm{MPa} / \mathrm{wt} \% \mathrm{P}$, which is higher than the corresponding IVAR $\mathrm{CF}_{\mathrm{p}}$ estimate of $666 \mathrm{MPa} / \mathrm{wt} \% \mathrm{P}$. Using the approximate average compositions of $\mathrm{Cu}$-bearing surveillance database plates of $\approx 0.14 \mathrm{wt} \% \mathrm{Cu}, \approx 0.6 \mathrm{wt} \% \mathrm{Ni}$ and $\approx$ 1.3 wt $\% \mathrm{Mn}$, the TTS model estimate is $\mathrm{CF}_{\mathrm{p}} \approx 1050 \mathrm{MPa} / \mathrm{wt} \% \mathrm{P}$. The TTS model estimate of $\mathrm{CF}_{\mathrm{p}}$ decreases further at higher $\mathrm{Cu}$; for example $0.25 \mathrm{wt} \% \mathrm{Cu}$, the TTS estimate is $\mathrm{CF}_{\mathrm{p}} \approx 880 \mathrm{MPa} / \mathrm{wt} \% \mathrm{P}$. These values compare reasonably with the estimated value $\mathrm{CF}_{\mathrm{p}} \approx 1060 \mathrm{MPa} / \mathrm{wt} \% \mathrm{P}$ found by averaging the slopes for the IVAR data shown in Fig. 6.27.

In summary, both the IVAR data and information in the literature support a significant contribution of $\mathrm{P}$ to $\Delta \sigma_{\mathrm{y}}$, especially in low $\mathrm{Cu}$ steels. Both theoretical considerations and IVAR model alloy data discussed in Chap. 2 are also consistent with a strong P-Mn interaction. Previous results in the literature, as well as the IVAR SMMS data, also indicate a significant P-Cu synergism, leading to a smaller P effect at higher $\mathrm{Cu}$ levels. The TTS model predicts that the effect of $\mathrm{P}$ is larger just above the CRP threshold of $0.072 \mathrm{wt} \% \mathrm{Cu}$. However the effect of P in the TTS model decreases with further increases in Cu beyond this threshold. Overall the effects of P predicted by the TTS model fall either somewhat on the low side (low $\mathrm{Cu}$ ) or within the expected range (for $>0.072 \mathrm{wt} \% \mathrm{Cu}$ ) found in other evaluations. The average contribution of $\mathrm{P}$ to hardening derived from the IVAR data and predicted by the TTS model are generally similar.

\subsection{Conclusions}

1. With the exception of the systematic effect of flux observed in the range of the IVAR database, the overall agreement between the TTS model predictions and the IVAR $\Delta \sigma_{\mathrm{y}}$ is reasonably good for the weld and plate alloys most like those in the TTS database.

2. The predictions of the TTS CRP model are also in remarkably good agreement with the IVAR $\Delta \sigma_{\mathrm{y}}$ data for Cu-bearing ( $\geq 0.072 \mathrm{wt} \% \mathrm{Cu}$ ) SMMS. However, the systematic dose rate effects observed in the SMMS $\Delta \sigma_{\mathrm{y}}$ data in the range of IVAR fluxes, are not explicitly captured by the TTS model. The effect of Mn observed in the IVAR database is reflected in product coefficients, rather than being treated directly.

3. While the absolute values are much smaller than the corresponding effects in the $\mathrm{Cu}$-bearing alloys, the systematic effect of flux observed in the range of the IVAR database for the low-Cu IVAR $\Delta \sigma_{y}$ data are also not captured in the TTS MF model.

4. As noted above, the IVAR database indicates a systematic flux effect in the range of $\approx 0.8$ to $8 \times 10^{11}$ $\mathrm{n} / \mathrm{cm}^{2}-\mathrm{s}$, which is not predicted by the TTS model, which finds a flux effect that begins below $4.4 \times 10^{10}$ $\mathrm{n} / \mathrm{cm}^{2}$-s. However, the predicted dose rate effects at lower fluxes are generally similar in both models. This observation suggests that detecting flux effects in the TTS surveillance database is difficult except over the widest range of dose rates. The IVAR results suggest that flux effects continue above $4.4 \times 10^{10}$ $\mathrm{n} / \mathrm{cm}^{2}$-s, where the fitted TTS model flattens. Use of a constant average $\mathrm{p}$ flux scaling approximation in the TTS model is generally supported by the analysis in this chapter as well as in Chap. 2. With the exception of the flux effect noted above, the predictions of the TTS MF model are in reasonably good agreement with the IVAR data, especially for alloys with compositions comparable to the preponderance of the low-Cu TTS plate database (like CM10 and BWC). However, a simple direct comparison shows that the TTS model tends to over predict the $\Delta \sigma_{\mathrm{y}}$ in $\mathrm{Cu}$-free medium-Ni, low-P SMMS. The differences are probably due to (a) the fact that the IVAR SMMS are, on average, cleaner than the alloys in the TTS 
surveillance database; and, (b) unaccounted for flux effects, that occur in the TTS database at fluxes below the IVAR range.

5. The TTS MF model does not reflect the significant effects of the wider range of Ni that are observed in the SMMS and $\Delta \sigma_{\mathrm{y}}$ data, or explicitly treat the effects of Mn. The effects of $\mathrm{P}$ are discussed below.

6. The IVAR database shows that both MF and CRP hardening contributions decrease with increasing irradiation temperature. The absolute irradiation temperature dependence predicted by the TTS model is generally stronger than observed in the low-Cu SMMS and weaker than observed in Cu-bearing SMMS. However, the agreement is reasonably good for the welds and plates that are most similar to steels in the TTS surveillance database. The IVAR data shows that the CRP contribution to $\Delta \sigma_{\mathrm{y}}$ decreases with increasing irradiation temperature, while the TTS model predicts a weak, but opposite effect.

7. The TTS model, IVAR data and information in the literature all support a significant contribution of $P$ to $\Delta \sigma_{\mathrm{y}}$. Both theoretical considerations and IVAR model alloy data are also consistent with a strong P-Mn interaction that is found in the TTS MF model. Previous results in the literature, as well as the IVAR SMMS data, also indicate a significant $\mathrm{P}-\mathrm{Cu}$ synergism, leading to a smaller $\mathrm{P}$ effect at higher $\mathrm{Cu}$ levels. In contrast, the TTS model predicts that the effect of $\mathrm{P}$ is larger above the CRP threshold of $0.072 \mathrm{wt} \%$ $\mathrm{Cu}$ and $0.008 \mathrm{wt} \% \mathrm{P}$. However the effect of $\mathrm{P}$ in the TTS model decreases with increasing $\mathrm{Cu}$ beyond this threshold. Overall the effects of P predicted by the TTS model fall either on the low side (low $\mathrm{Cu}$ ) or within the expected range (for $>0.072 \mathrm{wt} \% \mathrm{Cu}$ ) found in other evaluations.

\subsection{References}

1. Wirth, B. D., On the Character of Nano-Scale Features in Reactor Pressure Vessel Steels Under Neutron Irradiation, Ph.D. Thesis, the University of California, Santa Barbara (1998).

2. Odette, G. R. and Cowan, C., Use of Combined Electrical Resistivity and Seebeck Coefficient Measurements to Characterize Solute Redistribution Under Irradiation and Thermal Aging, Proceedings of the $10^{\text {th }}$ International Symposium on Environmental Degradation of Materials in Light Water Reactors, ed. G. S. Was et. al., NACE CD (2001).

3. Heatherly, D. W., Hurst, M. T., Thoms, K. R., Lucas, G. E., Odette, G. R. and Simpson, P. A., A New Versatile Materials Irradiation Facility, Trans $14^{\text {th }}$ Int Conf on Structural Mechanics in Reactor Technology 4G, SMIRT 341.

4. Remec, I., Baldwin, C. A. and Blakeman, E. D., Characterization of the Neutron Field in the HSSI/UCSB Irradiation Facility at the Ford Nuclear Reactor, NUREG/CR-6646 (U.S. Nuclear Regulatory Commission, Washington, DC, 1999).

5. Heatherly, D. W., K. R. Thoms, M. T. Hurst, and G. E. Giles, Heavy-Section Steel Irradiation Program's Reusable Irradiation Facilities, ORNL/TM-2002/77, Oak Ridge National Laboratory, Oak Ridge, Tennessee, 2004.

6. Odette, G. R., et al., The Effects of Composition and Heat Treatment on Hardening and Embrittlement of Reactor Pressure Vessel Steels, NUREG/CR-6778 (U.S. Nuclear Regulatory Commission, Washington, DC, 2003).

7. Odette, G. R., Lombrozo, P. M. and Wullaert, R. A., Relationship Between Irradiation Hardening and Embrittlement of Pressure Vessel Steels, pp. 840-62 in Effects of Radiation on Materials: 12th International Symposium, ASTM STP 870, ed. F.A. Garner et al. (American Society for Testing and Materials, West Conshohocken, Pa., 1985). 
8. English, C. A., Server, W. L. and Rosinski, S. T., Critical Review of Through-Wall Attenuation of Mechanical Properties in RPV Steels, pp. 221-243 Effects of Radiation on Materials: 21th International Symposium, ASTM STP 1447, ed. M. L Grossbeck et al. (American Society for Testing and Materials, West Conshohocken, Pa., 2004).

9. Odette, G., Yamamoto, R., T. and Klingensmith, R. D., On the Effect Of Dose Rate on Irradiation Hardening of RPV Steels, Philosophical Magazine 85, 779 (2005).

10. Williams, T. and Ellis, D., A mechanistically-Based Model of Irradiation Damage in Low Alloy Steel Submerged Arc Welds, pp. 8-27 in Effects of Radiation on Materials: 20th International Symposium, ASTM STP 1405, ed. S. T. Rosinski et al. (American Society for Testing and Materials, West Conshohocken, Pa., 2001).

11. Jones, R. B. and Buswell, J. T., The Interactive Roles of Phosphorus, Tin, and Copper in the Irradiation Embrittlement of PWR Pressure Vessel Steels, p. 111 in Environmental Degradation of Materials in Nuclear Power Systems-Water Reactors, Proceedings of the $3^{\text {rd }}$ International Symposium, ed. G.T. Theus et al. (Metallurgical Society, Warrendale, Pa., 1988).

12. Hawthorne, J. R., Exploratory Studies of Element Interactions and Composition Dependences in Radiation Sensitivity Development, NUREG/CR-4437 (U.S. Nuclear Regulatory Commission, Washington, DC, 1985). 



\section{Discussion and TTS Model Simplification}

The summary of current mechanistic understanding in Chap. 2 and the detailed comparison of the baseline model and IVAR data in Chap. 6 were completed after the development and analysis of the baseline model presented in Chaps. 3, 4, and 5. Two sensitivity studies were then conducted, which motivated minor changes to Eqs. (4-5a) and (4-5d) of the baseline model, as discussed in this chapter. These changes both simplify the baseline model and bring it into better agreement with the current mechanistic understanding and other data presented in Chaps. 2 and 6. The changes also address the two aspects of the baseline model that have the least statistical support from the surveillance database, the $T_{i}$ term and $\mathrm{Cu}_{\max }$ values in the CRP part of the model.

This chapter has two main purposes, to discuss the results of the sensitivity studies and to present a simplified model that implements the recommendations from those studies. All related information needed to properly apply the simplified model (e.g., the definitions of variables, units, revised standard deviation values for material groups) are also collected in Sect. 7.3 for ready reference.

\subsection{Discussion of the Irradiation Temperature Term in the CRP Part of the Baseline Model}

\subsubsection{Background}

During the development of the embrittlement shift model, it was assumed that there could be some effect of irradiation temperature, $\mathrm{T}_{\mathrm{i}}$, on both MF and CRP features based on prior theory and on observations discussed in Sect. 2.3.4. The effect of $T_{i}$ in the MF term has been studied by many authors; it is known to be a strong effect, and the accepted linear form presented by Jones and Williams [1] was used in the shift model. The best fitting form for the $T_{i}$ effect in the CRP part of the model has been studied much less, but a linear form appears to work, as given by Eq. (2-34).

Past experience with TTS models calibrated to the surveillance database suggested that the CRP $\mathrm{T}_{\mathrm{i}}$ term might turn out to be small relative to the stronger $T_{i}$ effect in the MF term, which applies to all materials. Consequently, the fitting form that was chosen for modeling the CRP $\mathrm{T}_{\mathrm{i}}$ effect was designed to allow removal of the term without recalibration. In particular, the effect was expressed as a multiplicative term in the CRP part that is a power law in the ratio of actual irradiation temperature, $T_{i}$, to the average irradiation temperature of the high-Cu calibration set $\left(T_{\text {avg }}=543.1^{\circ} \mathrm{F}\right)$; i.e.,

$$
\text { CRP } T_{i} \text { term }=\left[\frac{T_{i}}{T_{a v g}}\right]^{n}
$$

This term is capable of representing linear or nonlinear effects with either increasing or decreasing shifts as temperature increases, depending on the value and sign of the exponent. It should be considered an empirical "fix-up" term, adjusting the stronger $M F \mathrm{~T}_{\mathrm{i}}$ term (which was calibrated to low-Cu data) as needed to better fit the high-Cu surveillance data. No claim is made that the CRP $\mathrm{T}_{\mathrm{i}}$ term is an optimal fitting function for the surveillance data, but it is reasonably flexible and should be adequate for modeling a relatively small effect.

When the value of the CRP temperature term is unity, which will happen if the exponent, $n$, calibrates (or is set equal) to zero, the term has no effect on the calculated shift. For any exponent, if the $\mathrm{T}_{\mathrm{i}}$ term is removed from the model by setting the term equal to 1, the result would be an unbiased estimate overall, giving the same results as if the average irradiation temperature of the high-Cu calibration set were used for all CRP calculations at any $\mathrm{T}_{\mathrm{i}}$. 
The calibrated shift model confirmed the assumption that the CRP $\mathrm{T}_{\mathrm{i}}$ term might be a small contribution, as the calibrated exponent turned out to be 1.10. Thus, the CRP contribution from the $\mathrm{T}_{\mathrm{i}}$ term is only $-4.3 \%$ to $+5.5 \%$ of the CRP term value at average temperature, over the entire range of temperatures $\left(522-570^{\circ} \mathrm{F}\right)$ in the surveillance database. Since the CRP term is just part of the estimate of total shift, the contribution of the CRP $T_{i}$ term to total shift is always less than $\pm 5 \%$. Moreover, the direction of the calibrated CRP term is to increase the CRP contribution to shift as irradiation temperature increases, which is opposite to the direction of the MF term temperature effect, indicating that the high$\mathrm{Cu}$ surveillance data have a slightly flatter temperature trend than the low-Cu surveillance data, as shown in Fig. 5.16 (the difference in slope is hard to see). The effect of the CRP $\mathrm{T}_{\mathrm{i}}$ term in the shift model is typically much smaller than the MF $\mathrm{T}_{\mathrm{i}}$ term, so the slope of the overall irradiation temperature trend is in the same direction for both low- and high-Cu surveillance materials, tending to decrease the shift as $T_{i}$ increases.

The IVAR data and other data show a different trend, in which the higher $\mathrm{Cu}$ materials have a steeper temperature trend than the low-Cu materials, again with increasing irradiation temperature tending to decrease the hardening in both low- and high-Cu materials. Figures 6.14(a) and 6.14(b) show this trend, for example. Thus, the incremental CRP contribution from $T_{i}$ in controlled IVAR experiments is opposite in direction to the incremental CRP contribution from $T_{i}$ in surveillance materials. The overall temperature trend (combining both MF and CRP trends) is in the same direction and reasonably consistent between the IVAR data and surveillance data for materials with similar compositions, as shown in Fig. 6.4.

Because of the relatively small calibrated CRP $\mathrm{T}_{\mathrm{i}}$ effect and the questionable statistical significance (discussed in the next section), and because the effect of the calibrated CRP term is to flatten the overall temperature trend slope for high- $\mathrm{Cu}$ data rather than steepen it as shown in data from controlled experiments, a question was raised as to what the effect would be of removing the CRP $T_{i}$ term. That effect is analyzed in the next section as a sensitivity study, in which the only change from the baseline model was to remove the CRP $\mathrm{T}_{\mathrm{i}}$ term.

\subsubsection{Sensitivity Study on the CRP $T_{i}$ Term}

The irradiation temperature term from the CRP part of the TTS model was effectively removed from the model by temporarily setting its exponent $n=0$ in Eq. 7-1, which causes the value of the CRP temperature term to be unity for all temperatures. The effect of removing the temperature term was analyzed using all high- $\mathrm{Cu}$ calibration and validation data. This was done for direct comparison with the analysis done earlier for the baseline model in Chap. 4, which includes the temperature term in the CRP part. The analysis of the baseline model had shown a reasonably flat, nonsignificant residual trend with temperature in the high-Cu data, as shown in Fig. C.16.

After removing the temperature term from the CRP part of the model, there appears to be a small residual trend, as shown by the fitted linear residual trend line in Fig. 7.1. However, considering all high$\mathrm{Cu}$ calibration and validation data, the slope of this linear residual trend is not significantly less than zero. Deleting the CRP temperature term affects all high-Cu points to some extent, but the effect is predictably greatest at the high and low temperatures furthest from the average temperature. Thus, the planned approach for this sensitivity study was to examine the effect of removing the CRP temperature term on the residuals of points that are at least some $\Delta \mathrm{T}_{\mathrm{i}}$ interval above and below the average temperature. This approach can provide insight into whether removing the term would cause previously nonsignificant trends in variables other than $T_{i}$ to become significant. It also can determine whether there might be a trend in the $T_{i}$ residuals that is nonlinear. Such a trend would not necessarily be detected in the significance test on the slope of the CRP $T_{i}$ term because that test assumes a linear trend in the temperature residuals. 


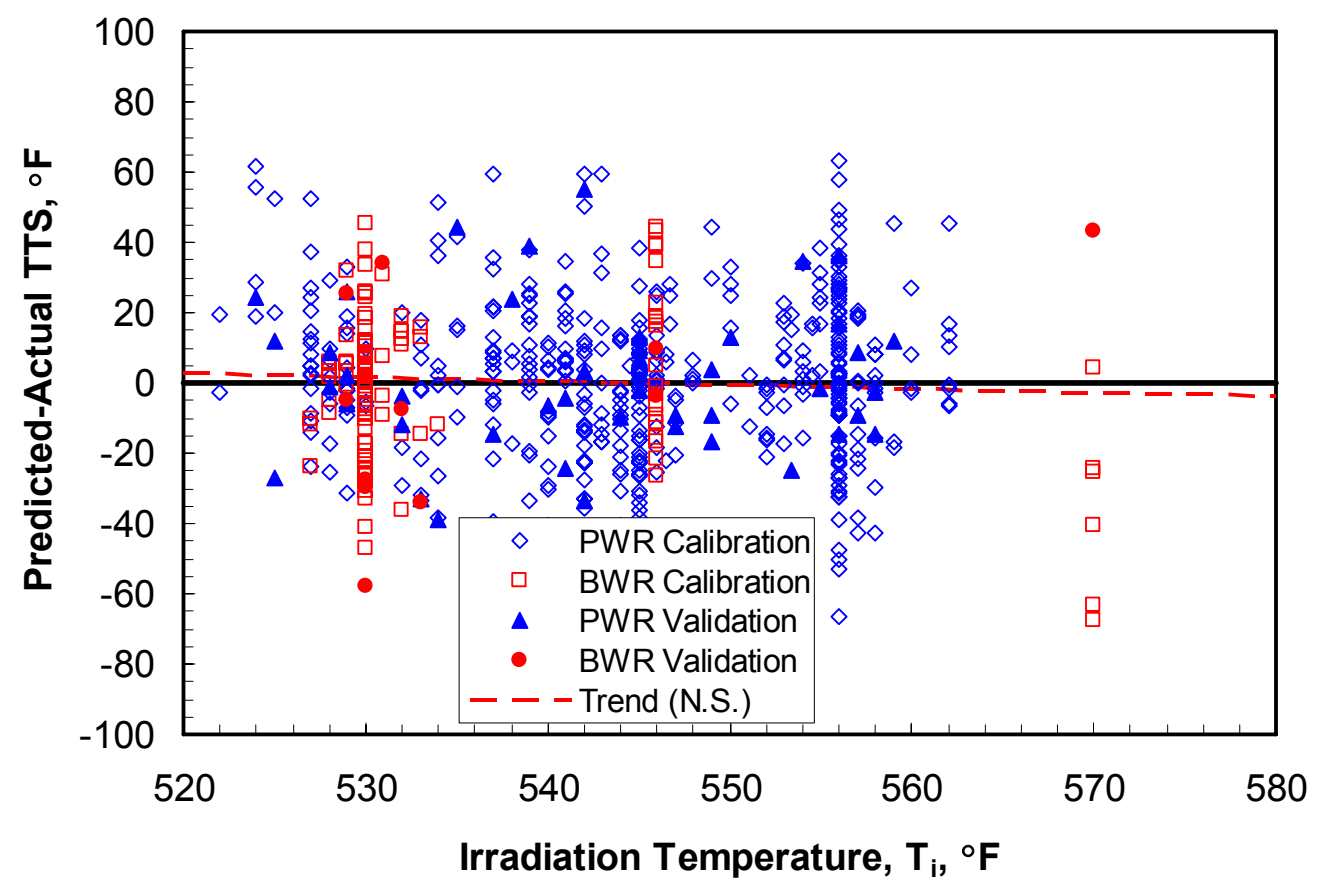

Fig. 7.1. Residual trend with temperature for high-Cu data, modified model without CRP $T_{i}$ term, showing apparent residual effect (slope is not significant).

The particular limiting values initially chosen for analysis, $\pm 10^{\circ}, \pm 15^{\circ}$, and $\pm 20^{\circ} \mathrm{F}$ from the average value of $T_{i}$ for high-Cu calibration data, were arbitrarily chosen to explore the competing issues of (a) being far enough from average temperature to show effects of deleting the term, if there are any, vs (b) having enough points to check significance using t-tests. If one does not go far enough out from the average temperature, a small effect should be expected to be nonsignificant, which is what is observed for the 312 points that are at least $\pm 10^{\circ} \mathrm{F}$ from the average temperature. If one goes too far from average temperature, there are not enough points for a credible significance analysis, which is what is observed for the nine points that are at least $\pm 20^{\circ} \mathrm{F}$ from the average temperature. The $\pm 15^{\circ} \mathrm{F}$ analysis is a reasonable compromise that spreads the high- and low-temperature sets adequately and retains 83 points in the analysis (about $14 \%$ of the high-Cu data).

The CRP $\mathrm{T}_{\mathrm{i}}$ term was removed, producing a modified model, and residuals relative to the modified model were calculated for each point. The results of $t$-tests for points at least $\pm 15^{\circ} \mathrm{F}$ from the average temperature are shown in Table 7.1. Two types of significance tests are summarized in Table 7.1. The first test determines whether the average residual at low temperatures is significantly greater than the average residual at high temperatures. If this difference in residuals at low and high temperatures is statistically significant, that is evidence that there may be a real $\mathrm{T}_{\mathrm{i}}$ trend in the high-Cu surveillance data, and as stated that trend would be in the same direction as the baseline CRP $\mathrm{T}_{i}$ term. The second test determines whether the average residual at low temperature is significantly greater than zero, and whether the average residual at high temperature is significantly less than zero. These tests determine if the average deviation from the model at low and high temperatures is large relative to the scatter. It is possible for a significant trend to exist when comparing the low- and high-temperature data, whether or not the average residuals are individually far enough from zero to be significant.

As shown in Table 7.1, the average residual of the low-temperature set is significantly greater than the average residual of the high temperature set. The significant difference is in the direction that suggests that the baseline CRP temperature trend may be real. That is, an effect that increases the incremental CRP 
contribution to shift as temperature increases would reduce both the under-prediction of high temperature points and the over-prediction of low-temperature points, thus counteracting the residual pattern that is observed after removing the CRP $\mathrm{T}_{\mathrm{i}}$ term. The average residuals at least $15^{\circ} \mathrm{F}$ above and below the average temperature are a factor of 2 different in absolute value (8.1 vs 3.7), an indication of nonlinearity. The fact that the low-temperature residual is significantly different from zero but the high temperature residual is not is another indication of nonlinearity. Thus, it appears that the residual trend from deleting the CRP $T_{i}$ term, which was not significant over the full range of $T_{i}$ when assumed to be linear, is statistically significant in the high and low-temperature data analyzed for Table 7.1. The significant difference in residuals and the significant average residual in the low-temperature data are visible in Fig. 7.1, where the points with $\mathrm{Ti} \leq 528^{\circ} \mathrm{F}$ do appear to be high relative to the zero residual line, and some points with $\mathrm{T}_{\mathrm{i}} \geq 558^{\circ} \mathrm{F}$ (especially at $\mathrm{T}_{\mathrm{i}}=570^{\circ} \mathrm{F}$ ) do appear to be low.

Table 7.1. Residual analysis of the points that are at least $15^{\circ} \mathrm{F}$ above and below average temperature, modified model without CRP $\mathrm{T}_{i}$ term

\begin{tabular}{|c|c|c|}
\hline & Data with $T_{i} \leq 528^{\circ} F$ & Data with $T_{i} \geq 558^{\circ} F$ \\
\hline Average residual & $8.1^{\circ} \mathrm{F}$ & $-3.7^{\circ} \mathrm{F}$ \\
\hline Sd of residuals & $20.9^{\circ} \mathrm{F}$ & $25.8^{\circ} \mathrm{F}$ \\
\hline Number of points & 48 & 35 \\
\hline $\begin{array}{l}\text { Significant difference in average } \\
\text { residual at high and low } T_{i}\end{array}$ & Yes, $8.1>-3.7$ & Yes, $8.1>-3.7$ \\
\hline Significant average residual? & Yes, average residual $8.1>0$ & No, average residual $-3.7 \sim 0$ \\
\hline
\end{tabular}

The fact that significant residual differences exist in the low- and high- $\mathrm{T}_{\mathrm{i}}$ data does not prove that a missing $T_{i}$ term is the cause. What it does suggest is that there are some apparently real differences in the average residuals of those subsets of data, which may be due to unmodeled temperature effects or other causes. The fact that the high- $\mathrm{Cu}$ data show a flatter $\mathrm{T}_{\mathrm{i}}$ trend than the low-Cu data in the collected surveillance data while the reverse is true in the controlled experiments is a strong indication that this particular residual trend may be due to causes other than an unmodeled $\mathrm{T}_{\mathrm{i}}$ trend.

The CRP $\mathrm{T}_{\mathrm{i}}$ term that is in the baseline model reduces the apparent temperature residual trend, but not by much, as can be shown using the same low- and high-temperature data and the same analysis as in Table 7.1. The absolute values of the average residuals at high and low temperature are each reduced by less than $2^{\circ} \mathrm{F}$ by incorporating the baseline $T_{i}$ term, which is not enough to be a significant reduction. The low-temperature average residual is still significantly greater than zero with or without the baseline $T_{i}$ term. Thus, by either the linear residual analysis or the analysis of low- and high-temperature subsets presented here, the baseline CRP $\mathrm{T}_{\mathrm{i}}$ term is not a significant improvement over having no CRP $\mathrm{T}_{\mathrm{i}}$ term at all.

\subsubsection{Detailed Review of Low- $T_{i}$ and High- $T_{i}$ Surveillance Data}

The points in the low- and high-temperature datasets from Table 7.1 were examined in some detail, looking for data-related anomalies that could explain the apparent $\mathrm{T}_{\mathrm{i}}$ trend in the surveillance data. In the high temperature subset, with $\mathrm{T}_{\mathrm{i}} \geq 558^{\circ} \mathrm{F}$, the points at $570^{\circ} \mathrm{F}$ appear to be unusual, as shown by Fig. 7.1. These points are all from the Big Rock Point reactor, an early 67 MW BWR demonstration plant that was not typical of later BWR designs and that has now been decommissioned. The Big Rock Point data are the main cause of the negative average residual at $T_{i} \geq 558^{\circ} \mathrm{F}$, as can be seen from the fact that the 7 Big Rock Point datapoints have an average residual of $-25^{\circ} \mathrm{F}$, while the average residual of the 28 other points with $\mathrm{T}_{\mathrm{i}} \geq 558^{\circ} \mathrm{F}$ is $1.6^{\circ} \mathrm{F}$, not significantly different from zero. Because of the small amount of Big Rock Point data and its relatively large scatter, the difference between the mean residuals of Big Rock Point and the other $\mathrm{T}_{\mathrm{i}} \geq 558^{\circ} \mathrm{F}$ data is not statistically significant in a t-test. No other plant, BWR or PWR, has 
estimated surveillance capsule temperature close to $570^{\circ} \mathrm{F}$, as is clear in Fig. 7.1. The Big Rock Point temperature is far above mean temperature, so those points have more influence on the slope of the $T_{i}$ term (more "leverage") than points nearer the mean temperature. Thus, there appear to be ample reasons, based on the unusual plant, the unusually large residual, and the $T_{i}$ value that is unusually higher than the mean temperature, to consider the data at $570^{\circ} \mathrm{F}$ to be possibly atypical and to consider the effect of excluding those seven points from $T_{i}$ term considerations. The underlying hypothesis that the Big Rock Point data may be different cannot be proven with the small sample of data, so this must be considered an exercise in exploring the implications of a hypothesis.

If the Big Rock Point data were excluded from consideration, the residual difference from low to high temperature groups with no CRP $\mathrm{T}_{\mathrm{i}}$ term would be reduced from $11.8=8.1-(-3.7)$ to $6.5=8.1-1.6$. The reduced difference $\left(6.5^{\circ} \mathrm{F}\right)$ between average residuals at low and high $\mathrm{T}_{\mathrm{i}}$ is not statistically significant. Thus, the exclusion of 7 datapoints from one atypical plant would change the statistical situation from an apparently real but nonlinear trend to no significant trend on either a linear or nonlinear (grouped data) basis.

There is no single plant in the low-temperature data group that has as much effect on significance as Big Rock Point does, but there is a possibly relevant pattern. The average residual in the $T_{i} \leq 528^{\circ} \mathrm{F}$ data is based on 9 BWR observations and 39 PWR observations. The significant positive average residual in the low-temperature group $\left(8.1^{\circ} \mathrm{F}\right.$ in Table 7.1$)$ is caused by the PWR data, as can be seen from the fact that the average BWR residual in the $\mathrm{T}_{\mathrm{i}} \leq 528^{\circ} \mathrm{F}$ range is $-4.7^{\circ} \mathrm{F}$, which is not significantly different from zero (on 9 points), while the average PWR residual in the $\mathrm{T}_{\mathrm{i}} \leq 528^{\circ} \mathrm{F}$ range is $+11.0^{\circ} \mathrm{F}$, which is significantly greater than zero. Note that $\mathrm{T}_{\mathrm{i}} \leq 528^{\circ} \mathrm{F}$ is an unusually low irradiation temperature range for PWRs, most of which have reported surveillance temperatures in the range $540-560^{\circ} \mathrm{F}$. Some of these unusually low PWR temperatures were reviewed by a working group of ASTM E10.02 subcommittee members in the 2004 data review, as indicated by comments in the appropriate cells of the database. But if some of these values are underestimates of the actual irradiation temperatures at the capsule locations, the shifts would be over-estimated, consistent with the positive average residual that is observed. This possibility of underestimated $T_{i}$ in the PWR data has not been resolved and would affect any future modeling activity.

\subsubsection{Conclusions and Recommendations from the CRP $\mathrm{T}_{\mathbf{i}}$ Term Sensitivity Study}

Removing the CRP temperature term from the TTS model contributes to an overall residual trend that is visible as shown in Fig. 7.1. Under a linear regression assumption, that residual trend is not significant, but analysis of the average residuals for the data furthest from $\left(\geq 15^{\circ} \mathrm{F}\right.$ above and below $)$ the average temperature show a significant difference, and for $\mathrm{T}_{\mathrm{i}} \leq 528^{\circ} \mathrm{F}$ the average residual is significantly greater than zero. The average residuals of the surveillance data at high and low $T_{i}$ are consistent with the direction of the trend in the baseline model, in which the high-Cu surveillance data show a flatter $\mathrm{T}_{\mathrm{i}}$ trend than the low-Cu data, and the significance results suggest this trend may be real, though the baseline CRP $\mathrm{T}_{\mathrm{i}}$ term does not adequately reflect the nonlinearity or strength of the trend. However, finding a significant difference between sets of data at low and high temperature does not prove that temperature causes the difference.

Data from controlled experiments show that high- $\mathrm{Cu}$ data have a steeper $\mathrm{T}_{\mathrm{i}}$ trend slope than low-Cu data, just opposite to the surveillance data. The IVAR temperature effect data are believed to be more reliable because they come from controlled experiments in which $T_{i}$ was deliberately varied for the same material and the irradiation temperatures were measured. By comparison, the different temperatures in the surveillance data also correspond to different heats and plants, possibly confounding the effects of multiple variables, and all of the surveillance $T_{i}$ values are estimates from coolant temperature. Thus, the IVAR results are the better indication of the actual $T_{i}$ trend, strongly suggesting that the surveillance data pattern may be due to other factors. 
Possible data issues have been identified that could explain the apparent trend in surveillance data, including unusual data from an unusual decommissioned plant (Big Rock Point) at the highest $T_{i}$ value in the database. Without the data from this one plant, the difference in average residuals at low and high $\mathrm{T}_{\mathrm{i}}$ in the surveillance data would become nonsignificant, though the average residual at low $T_{i}$ would still be significant. The positive average residual in the low- $T_{i}$ group is due to PWR data with unusually low $T_{i}$ values compared to typical PWR plants. Some of these temperature data have been recently reviewed, but additional checking may be warranted, since an underestimate of the actual $T_{i}$ values at the surveillance capsule in these PWR plants could explain the significant positive average residual. The possible contributions from these specific data issues must be regarded as unproven hypotheses.

A simplified model is recommended, in which the CRP $T_{i}$ term is removed from the baseline model by setting it to 1 for all $\mathrm{T}_{\mathrm{i}}$. The decision to present a simplified model is justified on the basis of the small, nonsignificant contribution of the baseline CRP $\mathrm{T}_{\mathrm{i}}$ term to shift estimates and the contrary direction of the baseline CRP $T_{i}$ term relative to controlled experiments. The fact that a statistically significant pattern exists in the surveillance data that suggests the need for a stronger, more nonlinear CRP $T_{i}$ term in the same direction as the baseline term was considered in forming this recommendation. That data pattern is not believed to be caused by a temperature effect, based on the contrary evidence from controlled experiments and the unusual nature of the particular datapoints responsible for the surveillance data pattern.

The baseline CRP $\mathrm{T}_{\mathrm{i}}$ term has little effect, so there is no motivation to revise all the results showing the baseline model in earlier chapters. Instead, the effects of the CRP $T_{i}$ term deletion and the recommended change to $\mathrm{Cu}_{\max }$ values discussed in Sect. 7.2 are analyzed together in Sect. 7.3, where a simplified model incorporating both changes is presented. Deleting the CRP $\mathrm{T}_{\mathrm{i}}$ term from the simplified model will slightly improve the agreement between the surveillance model and the IVAR data by slightly steepening the slopes of the TTS model plotted on Figs. 6.4, 6.14(a), and 6.14(b). The difference would be difficult to see, just as the change to exactly parallel lines (the result after deleting the CRP $\mathrm{T}_{\mathrm{i}}$ term) would be difficult to see in Fig. 5.16.

\subsection{Discussion of the Maximum $\mathrm{Cu}_{\mathrm{e}}$ Values for Material Groups}

\subsubsection{Background}

The baseline model includes calibrated maximum $\mathrm{Cu}$ values at which the $\mathrm{Cu}$ effect in the $\mathrm{CRP}$ term of the model saturates, referred to here as $\mathrm{Cu}_{\max }$ values. The values were calibrated originally in both $\mathrm{Ni}$ and weld flux categories, based on the understanding that the $\mathrm{Ni}$ level and heat treatment control the $\mathrm{Cu}$ saturation values (see Sect. 2.3.2) plus successful experience using weld flux categories in previous models. The $\mathrm{Cu}_{\max }$ values and categories were considered both based on Ni ranges and weld categories, with the latter approach being used in Eq. (4-5d) of the baseline model. The reasons why a Ni-based approach did not work on the surveillance data are discussed in Sects. 7.2.2 and 7.2.3.

The three categories in Eq. (4-5d) of the baseline model are typical Linde 80 welds (those with nominal $\mathrm{Ni}>0.5 \mathrm{wt} \%$ ), Linde 1092 welds, and all other materials. These categories apply to the CRP term, so they are only relevant to materials with higher $\mathrm{Cu}(\mathrm{Cu}>0.072 \mathrm{wt} \%)$. There are many materials in each of the three categories with $\mathrm{Cu}>0.072 \mathrm{wt} \%$, but most do not have high enough $\mathrm{Cu}$ to be affected by a saturation limit. The lowest calibrated saturation limit is 0.243 (typical Linde 80 category) so all materials with $\mathrm{Cu}<0.243 \mathrm{wt} \%$ are unaffected by any of the calibrated $\mathrm{Cu}$ limits because the limit is never reached. Materials with $\mathrm{Cu}$ levels below- $\mathrm{Cu}_{\max }$ show only an increasing shift as $\mathrm{Cu}$ increases, with no limit behavior at all. Thus, for calibrating $\mathrm{Cu}_{\max }$ values, only the data with $\mathrm{Cu}>0.243 \mathrm{wt} \%$ are potentially usable.

After calibrating the baseline model, it became apparent that more discussion is needed on the basis and description of the $\mathrm{Cu}_{\max }$ categories. The value of $\mathrm{Cu}_{\max }$ depends on $\mathrm{Ni}$, and for medium $\mathrm{Ni}$ materials 
$\mathrm{Cu}_{\max } \cong 0.25 \mathrm{wt} \%$ while for higher Ni materials, $\mathrm{Cu}_{\max }$ is higher, up to a solubility limit of about $0.3 \mathrm{wt} \%$ $\mathrm{Cu}$, as described in Sect. 2.3.2. The value of $\mathrm{Cu}_{\max }$ in the surveillance database for low-Ni materials is contrary to this expectation, with a calibrated value $\left(\mathrm{Cu}_{\max }=0.37 \mathrm{wt} \%\right)$ that exceeds both the solubility limit and the values of $\mathrm{Cu}_{\max }$ calibrated for the higher Ni materials. The Linde 1092 welds generally have higher $\mathrm{Ni}$ than the typical Linde 80 welds, so a higher calibrated value of $\mathrm{Cu}_{\max }$ is expected (and observed) for the Linde 1092 welds. But closer examination reveals that the higher calibrated value of $\mathrm{Cu}_{\max }$ is based on a small set of Linde 1092 welds with $\mathrm{Ni}$ values that substantially overlap the Ni range of the Linde 80 welds, thus casting doubt on $\mathrm{Ni}$ as the reason for the difference. It appears that the surveillance data are simply inadequate to confirm or calibrate the expected $\mathrm{Ni}$ effect on $\mathrm{Cu}_{\max }$, because of data limitations. The next section shows these limitations by a detailed examination of the surveillance data available for confirmation or calibration of the $\mathrm{Cu}_{\max }$ values.

\subsubsection{Analysis of Surveillance Data Usable for Calibrating the $\mathrm{Cu}_{\max }$ Values}

Table 7.2 shows the total number of $\mathrm{Cu}>0.072 \mathrm{wt} \%$ materials in each of the baseline $\mathrm{Cu}_{\max }$ categories and provides additional details on the materials with $\mathrm{Cu}>0.243$ that are potentially usable for calibrating the $\mathrm{Cu}_{\max }$ values. The baseline categories correspond roughly to the $\mathrm{Ni}$ ranges shown in parentheses, though all the Linde 1092 welds have been included in the high $\mathrm{Ni}$ category even though a few have medium Ni. The "all other" category only contains low-Ni materials in Table 7.2, so it will also be referred to as the "low-Ni" category in the following discussion.

Some of the difficulties in estimating $\mathrm{Cu}_{\max }$ limits from the surveillance data are readily apparent in Table 7.2. There are only 6 different weld chemistries available for low-Ni materials that have a high enough $\mathrm{Cu}$ level $(\mathrm{Cu}>0.243)$ to possibly help establish a $\mathrm{Cu}_{\max }$ value, despite the fact that there are 52 heats in the "all other" category with $\mathrm{Cu}>0.072$ and $\mathrm{Ni} \leq 0.5$. Only two of those 6 welds have $\mathrm{Cu}$ values at and above the calibrated limit of $\mathrm{Cu}_{\max }=0.37 \mathrm{wt} \%$, thus, the calibrated value for the category is effectively determined by only two welds from different vessel manufacturers, a clearly inadequate sample. Similarly, there are many Linde 1092 welds, spanning the Ni range from 0.6 to $1.26 \mathrm{wt} \%$, but only 6 different chemistries are available in the Linde 1092 welds with $\mathrm{Cu}>0.243 \mathrm{wt} \%$, and only 3 of those welds have $\mathrm{Cu}$ values at and above the calibrated limit of $\mathrm{Cu}_{\max }=0.301 \mathrm{wt} \%$. There are no high $\mathrm{Ni}$ $(\mathrm{Ni} \geq 0.75 \mathrm{wt} \%)$ materials other than Linde 1092 welds that have a high enough $\mathrm{Cu}$ value $(\mathrm{Cu}>0.243 \mathrm{wt}$ $\%$ ) to affect the value of $\mathrm{Cu}_{\max }$ for any category. Clearly, the samples available for calibrating two of the $\mathrm{Cu}_{\max }$ values are very small, both for the low-Ni data (two welds) and the Linde 1092 group (three welds).

Looking in more detail at Linde 1092 welds, despite the generally higher Ni level of most Linde 1092 welds $(0.6 \leq \mathrm{Ni} \leq 1.26 \mathrm{wt} \%)$ compared to typical Linde 80 welds $(0.52 \leq \mathrm{Ni} \leq 0.73 \mathrm{wt} \%)$, there are only 2 of the 6 Linde 1092 welds with $\mathrm{Cu}>0.243$ that have Ni values higher than the Linde 80 welds, and one of those has $\mathrm{Cu}=0.270$ so it does not affect the calibrated $\mathrm{Cu}_{\max }=0.301$ for Linde 1092 welds. Thus, there is almost total overlap in Ni range of the typical Linde 80 points and the Linde 1092 points that are actually relevant to the calibrated $\mathrm{Cu}_{\max }$. That overlap and the significant difference between Linde 80 and Linde 1092 calibrated $\mathrm{Cu}_{\max }$ values are the reasons why pure Ni categories were not used in the baseline model.

The quantity of usable data is much larger for typical Linde 80 welds than for any other category. "Typical Linde 80" implies the usual high-Cu, medium Ni Linde 80 welds, which nominally have Ni $>$ $0.5 \mathrm{wt} \%$, as opposed to a low-Ni Linde 80 weld in the database with $\mathrm{Ni}=0.1 \mathrm{wt} \%$ and a few Linde 80 welds with $\mathrm{Cu}<0.07 \mathrm{wt} \%$. There are 25 different heat chemistries in the medium Ni/typical Linde 80 category that are at and above the calibrated limit for that category $\left(\mathrm{Cu}_{\max }=0.243 \mathrm{wt} \%\right) .21$ of the 25 are identified specifically as Linde 80 welds and 3 more are B\&W welds that may be Linde 80 (the plant records should show whether they are or not, but requests to fill in this missing information during the 2004 ASTM review were not met, so the database used for analysis is missing the weld flux for those 3 welds). The only medium Ni heat that is clearly not Linde 80 is the plate heat with $\mathrm{Cu}=0.25 \mathrm{wt} \%$, 
which is not far enough above the calibrated $\mathrm{Cu}_{\max }=0.243 \mathrm{wt} \%$ to make any difference. Obviously, the $\mathrm{Cu}_{\max }$ value for the typical Linde 80 category is far better established than for any other category.

The low-Ni "all other" category is not so easy to identify with a particular weld group, because the two welds that establish the $\mathrm{Cu}_{\max }$ value are different (an unusual $\mathrm{B} \& \mathrm{~W}$ Linde 80 weld with $\mathrm{Ni}=0.1 \mathrm{wt} \%$ and high- $\mathrm{Cu}$ and a Rotterdamse weld with SMIT 89 flux and high $\mathrm{Cu}$ ). There are several Linde 0091 welds in the category, and two of them have $\mathrm{Cu}>0.243 \mathrm{wt} \%$ (the two unknown weld flux cases shown in Table 7.2 also may be Linde 0091), but the known and possible Linde 0091 welds did not affect the calibrated limit for the low-Ni category, so it should not be described as a Linde $0091 \mathrm{Cu}_{\max }$ value.

Table 7.2. Distribution of data for calibrating $\mathrm{Cu}$ saturation limits (calibration and validation data)

\begin{tabular}{|c|c|c|c|}
\hline & $\begin{array}{l}\text { All other materials } \\
(\mathrm{Ni} \leq 0.5 \mathrm{wt} \%)\end{array}$ & $\begin{array}{c}\text { Typical Linde } 80 \text { welds } \\
(0.5<\mathrm{Ni}<0.75 \text { wt } \% \text {, } \\
\text { excluding Linde } 1092 \\
\text { welds }) \\
\end{array}$ & $\begin{array}{l}\text { Linde } 1092 \text { welds } \\
(\mathrm{Ni} \geq 0.75 \text { wt \%) }\end{array}$ \\
\hline $\begin{array}{l}\text { Product forms with data } \\
\text { in the Category and } \\
\mathrm{Cu}>0.072\end{array}$ & P, SRM, W & F, P, SRM, W & $\mathrm{F}, \mathrm{P}, \mathrm{W}$ \\
\hline $\begin{array}{l}\text { Number of heats or } \\
\text { welds in the Category } \\
\text { with } \mathrm{Cu}>0.072\end{array}$ & 52 & 98 & 21 \\
\hline $\begin{array}{l}\text { Number of heats or } \\
\text { welds in the Category } \\
\text { with } \mathrm{Cu}>0.243 \mathrm{wt} \%\end{array}$ & 6 & 25 & 6 \\
\hline $\begin{array}{l}\text { Number of TTS } \\
\text { observations with } \\
\mathrm{Cu}>0.243 \text { wt } \%\end{array}$ & 19 & 65 & 20 \\
\hline $\begin{array}{l}\text { Description of heats or } \\
\text { welds in the Category } \\
\text { with } \mathrm{Cu}>0.243 \text { wt } \%\end{array}$ & $\begin{array}{l}1 \text { - Linde } 80 \text { weld } \\
2 \text { - Linde } 0091 \text { welds, } \\
\text { PWR vessels } \\
1 \text { - Rotterdamse SMIT } \\
89 \text { weld } \\
2 \text { - CE welds (unknown } \\
\text { weld flux, BWR } \\
\text { vessels) }\end{array}$ & $\begin{array}{l}1 \text { - CE plate } \\
21-\text { Typical Linde } 80 \\
\text { welds } \\
3-\text { B\&W welds } \\
\quad \text { (unknown weld flux, } \\
\text { may also be Linde } 80 \text { ) }\end{array}$ & $\begin{array}{l}6 \text { - Linde } 1092 \text { welds } \\
\text { (Note: only one of these six } \\
\text { Linde } 1092 \text { welds has } \mathrm{Ni} \geq \\
0.75 \text {, and there are no other } \\
\text { materials with } \mathrm{Ni} \geq 0.75 \text { and } \\
\mathrm{Cu}>0.243 \text { wt } \% \text { in the TTS } \\
\text { database) }\end{array}$ \\
\hline
\end{tabular}

\subsubsection{Specific Issues Regarding the $\mathrm{Cu}_{\max }$ Limits}

Using strict Ni categories to specify $\boldsymbol{C u}_{\max }$. Because of the overlap in the Ni ranges for Linde 80 and Linde 1092 welds, strictly imposing the Ni limits shown in Table 7.2, rather than using the weld group categories, would penalize the many Linde 80 welds by increasing their $\mathrm{Cu}_{\max }$ limit, just to accommodate a few medium Ni Linde 1092 welds. There would also be inadequate statistical basis for a high Ni value of $\mathrm{Cu}_{\max }$ if this were done. In fact there are only 2 materials in the entire surveillance database (both Linde 1092 welds) that have Ni levels above $0.73 \mathrm{wt} \%$ (the high end of the Linde 80 range) and high enough $\mathrm{Cu}(>0.243 \mathrm{wt} \%)$ to possibly contribute to a $\mathrm{Cu}_{\max }$ value. There is only one Linde 1092 weld that actually falls in the originally-defined high $\mathrm{Ni}$ range $(\mathrm{Ni} \geq 0.75 \mathrm{wt} \%)$ and has high enough $\mathrm{Cu}$. Thus, if the Linde 1092 welds were strictly divided by $\mathrm{Ni}$ level, most of the subset that affects $\mathrm{Cu}_{\max }$ would go into the medium $\mathrm{Ni}$ category (where they would increase the calibrated $\mathrm{Cu}_{\max }$ for all the Linde 80 welds) and the $\mathrm{Cu}_{\max }$ value for the high $\mathrm{Ni}$ category would be based on just 1 or 2 welds. It would then be highly uncertain, just as the $\mathrm{Cu}_{\max }$ value for the low-Ni "all other" category is.

Fitting $\mathbf{C} \boldsymbol{u}_{\max }$ as a continuous function of $\mathrm{Ni}$. If feasible, it would be preferable to have a continuous function of $\mathrm{Ni}$ for estimating $\mathrm{Cu}_{\max }$ rather than the discrete categories shown in Table 7.2. Unfortunately, a continuous function of $\mathrm{Ni}$ for predicting $\mathrm{Cu}_{\max }$ is not feasible because the currently-available 
surveillance data are inadequate for such an approach. The main problem is lack of data, which is evident in Table 7.2, where the lack of sufficiently high-Cu data at high and low-Ni is clear. Another problem is the pattern of the available data, which is evident when analyzing the residuals relative to a model that is otherwise like the baseline model but recalibrated for the best-fit single value of $\mathrm{Cu}_{\max }=0.2646 \mathrm{wt} \%$, as shown in Fig. 7.2. The points on Fig. 7.2 are the residuals relative to the single-value $\mathrm{Cu}_{\max }$ model for all the points in the surveillance database with $\mathrm{Cu}>0.243 \mathrm{wt} \%$, which are the only points that could possibly produce $\mathrm{Cu}_{\max }$ function that spans a range similar to the baseline $\mathrm{Cu}_{\max }$ values. A linear function would be the obvious choice of fitting function in Fig. 7.2, because there are really only two clumps of data that have high enough $\mathrm{Cu}$ to be usable for a saturation limit. Fitting anything other than a linear function to the two clumps of data would be debatable at best.

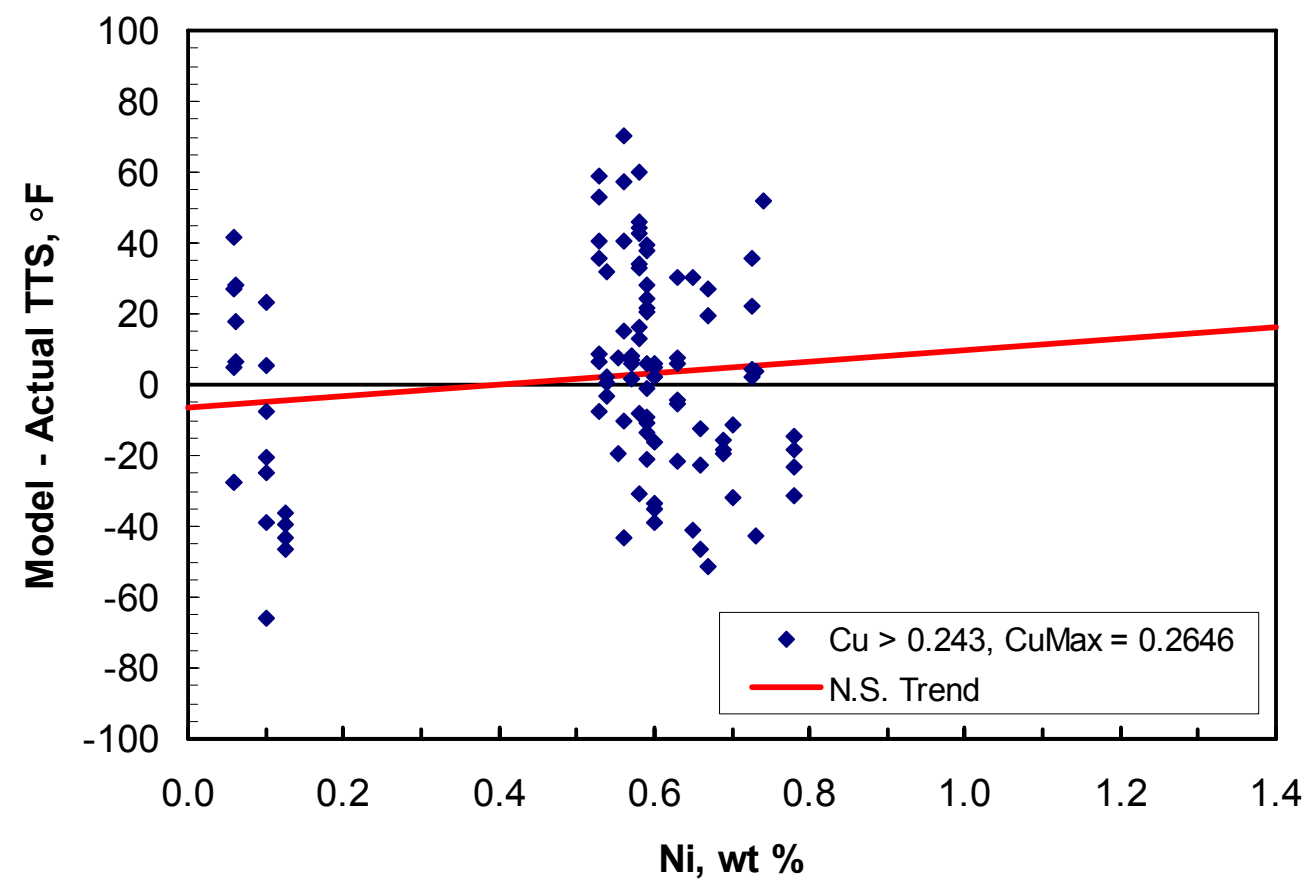

Fig. 7.2. Nonsignificant linear residual trend when a single calibrated $\mathrm{Cu}_{\max }$ value is used, all available data with $\mathrm{Cu}>0.243 \mathrm{wt} \%$.

In order to justify calibrating $\mathrm{Cu}_{\max }$ as a linear function of $\mathrm{Ni}$, there should be a significant linear trend in the residuals on Fig. 7.2. Instead, the slope of the apparent linear residual trend line is not statistically significant. The lack of significance does not prove that there is no effect of $\mathrm{Ni}$ on $\mathrm{Cu}_{\max }$, only that the effect, if any, is not large relative to the scatter in the surveillance data. For the residual definition used in Fig. 7.2, the positive apparent slope of the residual plot implies decreasing $\mathrm{Cu}_{\max }$ as $\mathrm{Ni}$ increases, opposite in sign relative to other results showing that higher $\mathrm{Ni}$ causes higher $\mathrm{Cu}_{\max }$ values (see Sect. 2.3.2). The results showing higher $\mathrm{Cu}_{\max }$ with higher $\mathrm{Ni}$ that are presented in Sect. 2.3.2 are based on controlled experiments and are considered more reliable than the surveillance residual trend. Thus, the facts that (a) the surveillance data show no significant effect of $\mathrm{Ni}$ on $\mathrm{Cu}_{\max }$ and (b) the slight apparent trend that is visible is contrary to the direction of the trend in controlled experiment data are both evidence that the surveillance data are inadequate to calibrate a continuous function $\mathrm{Cu}_{\max }(\mathrm{Ni})$. 
Significant differences in $\mathrm{Cu}>0.243$ wt \% residuals by weld group. Though the trend line shown in Fig. 7.2 does not indicate a significant trend of residuals with $\mathrm{Ni}$, significant differences are revealed when grouped by Linde 1092 welds, typical Linde 80 welds, and all other. The average residuals of the grouped data are statistically significantly different in t-tests. In particular, with a single $\mathrm{Cu}_{\max }$ value the average residual of the low-Ni "all other" data, $\left(-11.8^{\circ} \mathrm{F}\right)$, is significantly below the average residual of typical Linde 80 welds $\left(8.7^{\circ} \mathrm{F}\right)$, which is significantly above the average residual of Linde 1092 welds ($6.3^{\circ} \mathrm{F}$ ). Thus, there is a statistical justification for 3 grouped $\mathrm{Cu}_{\max }$ values but a lack of statistical justification for a linear function of $\mathrm{Ni}$ on the same set of surveillance data. It should be noted that the low-Ni and Linde 1092 groups do not have significantly different average residuals in the weld group analysis, so there is also statistical merit in a two-category "typical Linde 80" vs "all other" grouping, as discussed below.

Several possible reasons why the significant difference by weld group does not translate into a significant continuous trend with $\mathrm{Ni}$ have been identified. They include the known importance of postweld heat treatment details, the near-total overlap in Ni range of the high-Cu members of the Linde 1092 and Linde 80 weld groups, and the relative lack of surveillance data outside the medium Ni range. Additional possibilities include the fact that the overall difference in average residual of the two clumps of surveillance data is small relative to the scatter within each clump, making it difficult to justify any continuous function, and possible nonlinearity in the Ni effect that does not show up clearly in Fig. 7.2 because of the limited number of very high-Cu heats that are available. All the possibilities mentioned in this paragraph should be considered hypotheses, as the actual reasons are not yet established.

Uncertainty from medium $\mathrm{Ni} B \& W$ welds that may or may not be typical Linde 80 welds. Three of the medium $\mathrm{Ni}$ welds that were used for determining the value $\mathrm{Cu}_{\max }=0.243$ were not specifically identified as Linde 80 (weld flux is blank, vessel manufacturer is Babcock \& Wilcox), and they contribute 7 of the 65 points used in the $\mathrm{Cu}_{\max }$ calibration for that category. The $\mathrm{Cu}$ values for these welds are 0.26 , 0.31 , and 0.35 . There is also a $\mathrm{CE}$ plate at $\mathrm{Cu}=0.25 \mathrm{wt} \%$ that contributes another 7 points. The plate at $0.25 \mathrm{wt} \% \mathrm{Cu}$ and the weld at $0.26 \mathrm{wt} \% \mathrm{Cu}$ had little effect on the calibrated value of $\mathrm{Cu}_{\max }=0.243$ because the fitted $\mathrm{Cu}_{\max }$ value is close to the maximum actual $\mathrm{Cu}$ values for these materials (limiting $\mathrm{Cu}$ makes little difference when the unlimited value is nearly the same). Thus, there are really only 5 points from welds WDR302 and WQC102 that could have affected the calibrated value substantially. If those two welds are in fact Linde 80 welds, there is no issue and the calibrated value of $\mathrm{Cu}_{\max }=0.243$ is the best value to use. If not, there may be a slight inaccuracy in the calibrated value of $\mathrm{Cu}_{\max }$ for typical Linde 80 welds.

To bound the possible inaccuracy, all the data in the medium Ni category in Table 7.2 that are not specifically listed as Linde 80 welds were moved into the "Other" category, including the plate and the three $\mathrm{B} \& \mathrm{~W}$ welds that may be Linde 80 . The value of $\mathrm{Cu}_{\max }$ for the typical Linde 80 category was recalibrated, and it changed only slightly, from 0.243 to 0.247 . The third digit of the calibrated $\mathrm{Cu}_{\max }$ value is in any case somewhat uncertain and the measured composition is often only given to two digits, so whether the medium $\mathrm{Ni}$ welds with missing weld flux are in fact Linde 80 makes little difference.

Treatment of base metals. The proper value of $\mathrm{Cu}_{\max }$ for base metals is not adequately established by the surveillance data, because there is only one plate heat with high enough $\mathrm{Cu}$ to affect any of the $\mathrm{Cu}_{\max }$ values (and it is barely high enough, at $\mathrm{Cu}=0.25 \mathrm{wt} \%$ so it cannot substantially affect the calibrated $\mathrm{Cu}_{\max }$ limit). $\mathrm{Cu}$ levels in all other base metals do not exceed the $\mathrm{Cu}_{\max }$ limit calibrated for any of the groups, so no $\mathrm{Cu}$ limit behavior is observed. Thus, the base metals are assigned to the "all other" group, which means that their full $\mathrm{Cu}$ value is used without limit. This is an adequate approach up to $\mathrm{Cu} \cong 0.25$ wt $\%$,which includes all current surveillance base metals and most or all plant base metals.

Unusually high value of $\mathrm{Cu}_{\max }$ in the low-Ni category. The calibrated value of $\mathrm{Cu}_{\max }$ in the low-Ni or "all other" category is higher $(0.37 \mathrm{wt} \% \mathrm{Cu})$ than the expected value of about $0.3 \mathrm{wt} \%$ based on the solubility of $\mathrm{Cu}$ (see Sect. 2.3.2 and [2]). The low-Ni $\mathrm{Cu}_{\max }$ value is also higher than the calibrated value 
for the typical Linde 80 category, which has substantially higher $\mathrm{Ni}$, contrary to the evidence that increased $\mathrm{Ni}$ generally causes a higher $\mathrm{Cu}_{\max }$ limit. Moreover, the statistical case for the particular calibrated value in the low-Ni category is based on so few datapoints ( 8 points from 2 welds) that it is highly uncertain. Finally, the grouped statistical analysis described in the preceding subsection found no significant difference between residuals in the low-Ni and Linde 1092 data. Consequently, a sensitivity study was conducted to determine the effect of combining the low-Ni data into a larger "all other" category.

\subsubsection{Sensitivity Study on Combining the Low-Ni Group in a Larger "All Other” Grouping}

A sensitivity approach was taken, in which the only change from the baseline model was to combine the low-Ni data with one of the other groups, without recalibration. The low-Ni group has only 19 high$\mathrm{Cu}$ datapoints $(\mathrm{Cu}>0.243 \mathrm{wt} \%)$, and those are the only points that can be affected by the re-grouping changes being analyzed. Depending on the final $\mathrm{Cu}_{\max }$ value, not all of the 19 points will be affected. Thus, the approach used in the study was to apply the previously calibrated values of $\mathrm{Cu}_{\text {max }}$ for either the typical Linde 80 or Linde 1092 weld group to the low-Ni data and analyze the effects on the 19 points, without recalibration.

Combining the low-Ni and typical Linde 80 groups and using $\mathrm{Cu}_{\max }=0.243$ for all materials in those groups is not a reasonable choice. All 19 of the TTS points from the 6 welds with $\mathrm{Ni}<0.5$ and $\mathrm{Cu}>$ 0.243 are affected by this choice, and the average residual for those points is large $\left(-15.2^{\circ} \mathrm{F}\right)$, in the unconservative direction, and significantly less than zero. The average residual of the low-Ni group after combination is also significantly less than the average residual of the typical Linde 80 and Linde 1092 groups. Thus, this combination is not recommended.

Combining the low-Ni and Linde 1092 weld groups and using $\mathrm{Cu}_{\max }=0.301$ for all materials in those groups is a reasonable choice. The two $\mathrm{Cu}_{\max }$ values that would result in this case could be interpreted as values for typical Linde 80 welds $\left(\mathrm{Cu}_{\max }=0.243\right)$ and "all other" $\left(\mathrm{Cu}_{\max }=0.301\right)$. Only the 8 points from the 2 welds that currently establish the low-Ni $\mathrm{Cu}_{\max }$ value are affected by this choice, because all the other low-Ni welds have $\mathrm{Cu} \leq 0.279$ wt \%. Thus, the average residual for the 19 high-Cu points in the low-Ni group is $-7.1^{\circ} \mathrm{F}$ in this case, which is not significantly less than zero.

The standard deviation of residuals for all welds with $\mathrm{Cu}>0.072 \mathrm{wt} \%$ would increase slightly due to combining the Linde 1092 and "all other" categories in Table 7.2. The increase would be reduced if both the $\mathrm{Cu}_{\max }$ change and the deletion of the CRP $\mathrm{T}_{\mathrm{i}}$ term are implemented, as these two recommended changes partially offset in the weld category. The two-category approach with typical Linde 80 and "all other" has an advantage over the similar two-category approach in the ASTM E 900-2 model, because the only weld group that has a well-established $\mathrm{Cu}_{\max }$ value (typical Linde 80 ) would be the only weld group that would be specifically mentioned in the model. The value used for Linde 1092 welds would still be the best available estimate for that weld group, so the change from three to two values of $\mathrm{Cu}_{\max }$ would not affect the second-largest group of very high- $\mathrm{Cu}$ welds. The only other weld group that has 2 or more welds with $\mathrm{Cu}>0.243 \mathrm{wt} \%$ (Linde 0091) is also unaffected by the change, because the highest Cu level in such welds $(0.279 \mathrm{wt} \%)$ is below both the baseline three category "all other" limit and the revised twocategory "all other" limit.

It is better to use the calibrated Linde 1092 value $\left(\mathrm{Cu}_{\max }=0.301 \mathrm{wt} \%\right)$ for the two-category "all other" group rather than to recalibrate a new value for the combined group. This can be seen by analyzing the consequences of a recalibration. There are only 12 welds with $\mathrm{Cu}>0.243 \mathrm{wt} \%$ in the Linde 1092 and low-Ni categories combined. The shifts calculated for 7 of these 12 welds will not be affected by a recalibration, because their actual $\mathrm{Cu}$ values are below the $\mathrm{Cu}_{\max }$ values calibrated for both Linde 1092 and low-Ni categories, and hence below any recalibrated compromise value. If a single value were recalibrated for the remaining 5 welds in the combined category with $\mathrm{Cu}>0.243$ wt \%, it would be somewhat high for the 3 Linde 1092 welds and somewhat low for the 2 low-Ni welds. Thus, while the 
original Linde 1092 value would be a best estimate for 3 out of 5 welds and statistically acceptable on the other two welds, the recalibrated result would not be a best estimate for either the Linde 1092 or the low$\mathrm{Ni}$ subsets. More importantly, the particular $\mathrm{Cu}_{\max }$ value calibrated to the low-Ni welds is higher than expected based on $\mathrm{Cu}$ solubility, so the net effect of a recalibration would be to penalize the Linde 1092 welds by increasing their $\mathrm{Cu}_{\max }$ value to accommodate physically-questionable data.

In a two- $\mathrm{Cu}_{\max }$ approach, the model trends shown in Figs. 5.4 and 5.8 would be modified as shown for "other materials" in Fig. 7.3 and 7.4. The corresponding curve for typical Linde 80 welds would look similar to the $0.6 \mathrm{Ni}$ curve on Fig. 7.3, except the curve would be flat for any $\mathrm{Cu}>0.243$ instead of $\mathrm{Cu}>$ 0.301. The $0.3 \mathrm{Cu}$ curve on Fig. 7.4 would be plotted somewhat lower for the typical Linde 80 group, plotting at $\mathrm{Cu}_{\max }=0.243$ instead of 0.3 , and it would be plotted only over the relevant Ni range $0.52 \leq \mathrm{Ni}$ $\leq 0.73$.

The 8 points in the baseline "all other" group that are affected by the change from three to two $\mathrm{Cu}_{\max }$ categories are shown in Fig. 7.5, with small filled diamond symbols for the baseline model plotting location and large filled diamond symbols for the plotted location after incorporation in the revised "all other" category. The trend line has been updated, and it remains nonsignificant and almost indistinguishable from the baseline residual $\mathrm{Ni}$ trend (and almost indistinguishable from the horizontal "no-trend" line) after making the change. The same 8 points would move by the same amount on all other residual plots for materials with $\mathrm{Cu}>0.072$. None of the residual plots is affected by the change to such a degree that previously nonsignificant trends would become significant, as shown in Sect. 7.3.3.

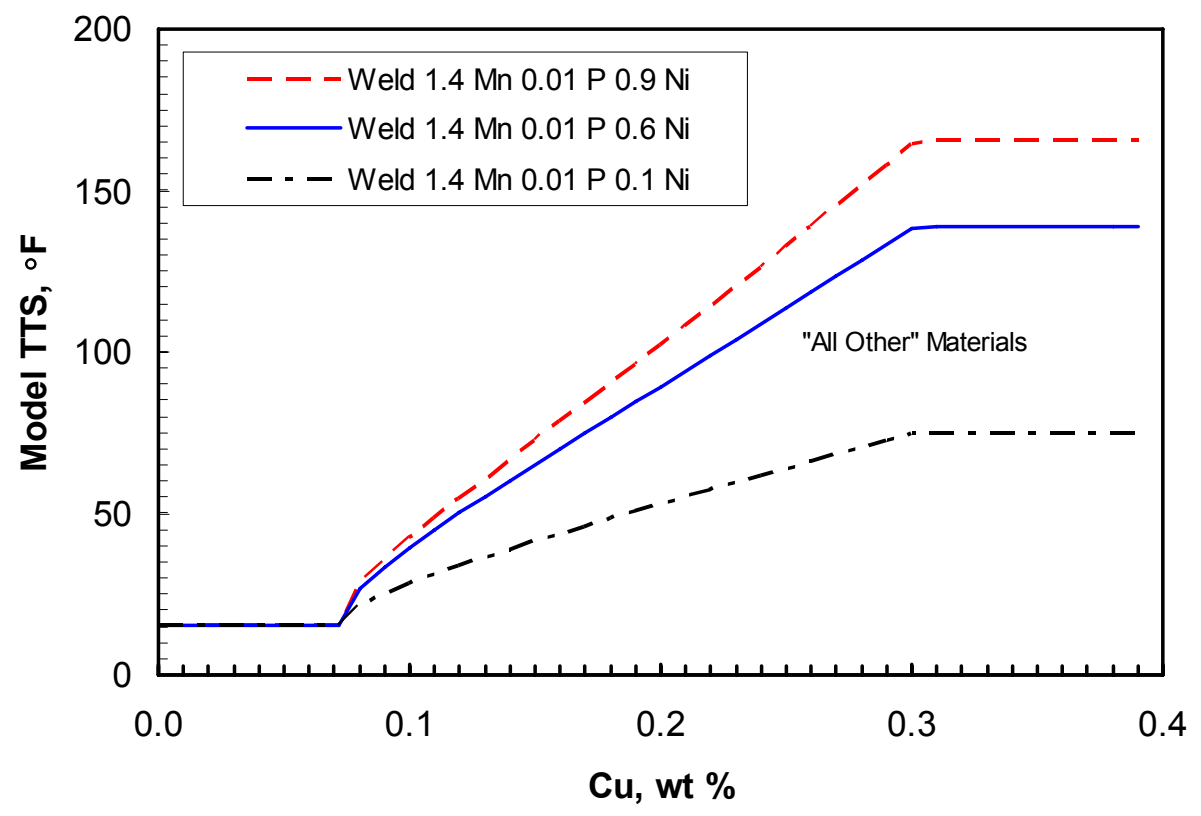

Fig. 7.3. Effect of Cu on TTS for various Ni levels, "all other" materials (similar to Fig. 5-4 but revised for two material group categories). 


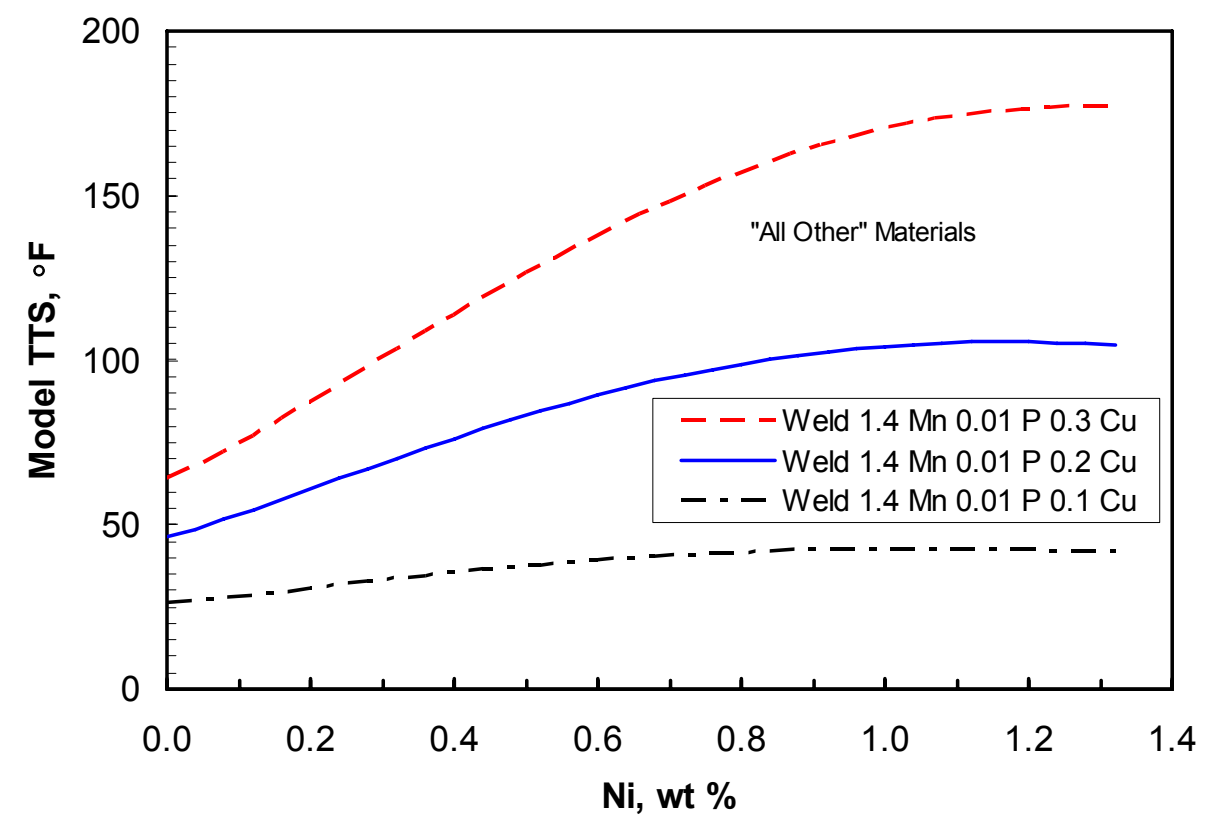

Fig. 7.4. Effect of Ni on TTS for various Cu levels, "All Other" group, and $2 \times 10^{18} \mathrm{n} / \mathrm{cm}^{2}$ fluence.

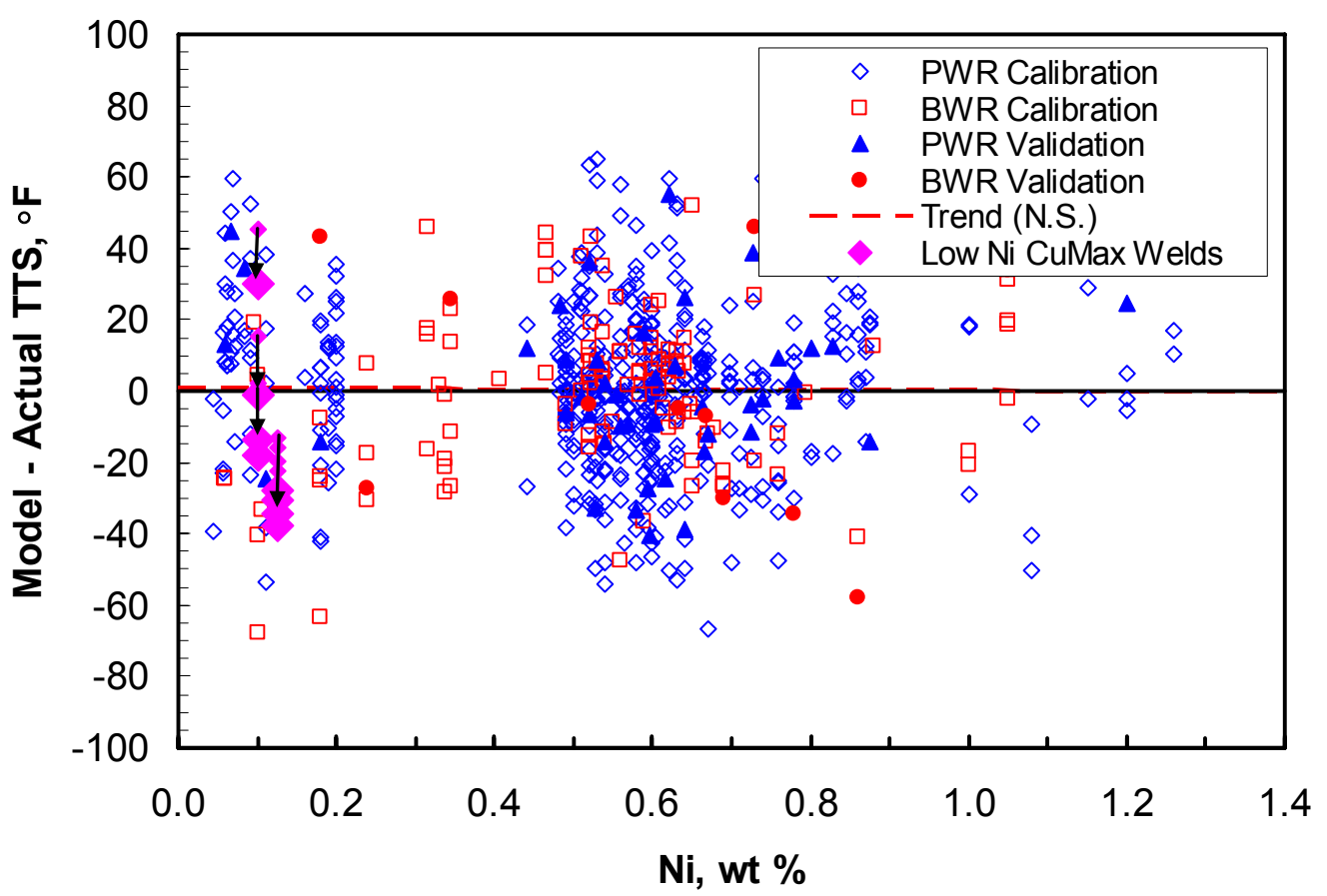

Fig. 7.5 Effect of using $\mathrm{Cu}_{\max }=\mathbf{0 . 3 0 1}$ on the low-Ni welds that determined the $\mathrm{Cu}_{\max }=0.37$ value for the "other weld" category in the baseline model. 


\subsubsection{Conclusions and Recommendations Regarding $\mathrm{Cu}_{\max }$}

Limitations of the surveillance data for establishing $\mathbf{C u}_{\max }$ values. The baseline values of $\mathrm{Cu}_{\max }$ for the Linde 1092 and "all other" categories in Eq. (4-5d) are not well established, being determined by only three and two welds respectively. The value of $\mathrm{Cu}_{\max }$ for the typical Linde 80 category is reasonably well established, calibrated to at least 21 different Linde 80 welds. The surveillance database is simply lacking in enough materials with sufficiently high $\mathrm{Cu}$ to reasonably establish the $\mathrm{Cu}_{\max }$ limits for materials other than typical Linde 80 welds.

The lack of sufficient surveillance data with high enough $\mathrm{Cu}$ and either low or high levels of $\mathrm{Ni}$ prevents the confirmation or calibration of the $\mathrm{Ni}$ effect on $\mathrm{Cu}_{\max }$ that has been identified in other data. The possibility of expressing $\mathrm{Cu}_{\max }$ as a continuous function of $\mathrm{Ni}$ has been evaluated, and it also is not feasible with the present database.

The surveillance database also does not contain enough plate or forging materials with high enough $\mathrm{Cu}$ levels to directly establish a $\mathrm{Cu}_{\max }$ limit for base metals. The assumption has been made that the full $\mathrm{Cu}$ level (which is $\leq 0.25 \mathrm{wt} \%$ ) should be used for all base materials.

Recommended change from three $\mathbf{C} u_{\max }$ values to two $\mathbf{C} \mathbf{u}_{\max }$ values. Based on the additional analysis in Sect. 7.2.4, the baseline "all other" category should be combined with the baseline Linde 1092 weld category in a new category labeled "all other materials". The typical Linde 80 category in the baseline model should be maintained. The $\mathrm{Cu}_{\max }$ value for the revised "all other" category would be $0.301 \mathrm{wt} \%$, which is equal to the calibrated value for the Linde 1092 welds. The value $\mathrm{Cu}_{\max }=0.301$ is somewhat low for the two low-Ni welds that determined $\mathrm{Cu}_{\max }$ in the baseline "all other" category, but not low enough to cause a statistically significant error in that category. No other materials are affected by the revised "all other" $\mathrm{Cu}_{\max }$ value, because they are all either included in the typical Linde 80 weld group or they have $\mathrm{Cu}$ levels below $0.301 \mathrm{wt} \%$. In particular, the four other very high-Cu welds from the baseline "all other" category all have $\mathrm{Cu} \leq 0.279 \mathrm{wt} \%$, so they are not affected by the revision.

\subsection{Simplified TTS Model}

In this section, a simplified model is presented incorporating the recommended changes of the previous two sections. The quality of fit and predictive capability of the simplified model are updated as well. All additional information needed to apply the model is included here for ready reference, even though some of this material was presented in earlier Chapters.

\subsubsection{Simplified Model Equations}

Implementing the recommendations in both Sects. 7.1.4 and 7.2.5 produces the following simplified model.

$$
\begin{gathered}
\text { TTS }=\text { MF term }+ \text { CRP term } \\
\text { MF term }=A\left(1-0.001718 T_{i}\right)\left(1+6.13 P M n^{2.47}\right) \sqrt{\phi t e} \\
A=\left\{\begin{array}{cc}
1.140 \times 10^{-7} & \text { for forgings } \\
1.561 \times 10^{-7} & \text { for plates } \\
1.417 \times 10^{-7} & \text { for welds }
\end{array}\right\} \\
\text { CRP term }=B\left(1+3.77 N i^{1.191}\right) f\left(C u_{e}, P\right) g\left(C u_{e}, N i, \phi t_{e}\right)
\end{gathered}
$$




$$
\begin{aligned}
& B=\left\{\begin{array}{c}
102.3 \text { for forgings } \\
102.5 \text { for plates in non }- \text { CE mfg. vessels } \\
135.2 \text { for plates in CE mfg. vessels } \\
155.0 \text { for welds } \\
128.2 \text { for SRM plates }
\end{array}\right\} \\
& C u_{e}=\left\{\begin{array}{c}
0 \quad \text { for } C u \leq 0.072 w t \% \\
\min \left[C u, \operatorname{Max}\left(C u_{e}\right)\right] \text { for } C u>0.072 w t \%
\end{array}\right\} \\
& \operatorname{Max}\left(C u_{e}\right)=\left\{\begin{array}{c}
0.243 \text { for typical }(\mathrm{Ni}>0.5) \text { Linde } 80 \text { welds } \\
0.301 \text { for all other materials }
\end{array}\right\} \\
& f\left(C u_{e}, P\right)=\left\{\begin{array}{c}
0 \text { for } C u \leq 0.072 \\
{\left[C u_{e}-0.072\right]^{0.668} \text { for } C u>0.072 \text { and } P \leq 0.008} \\
{\left[C u_{e}-0.072+1.359(P-0.008)\right]^{0.668} \text { for } C u>0.072 \text { and } P>0.008}
\end{array}\right\} \\
& g\left(C u_{e}, N i, \phi t_{e}\right)=\frac{1}{2}+\frac{1}{2} \tanh \left[\frac{\log _{10}\left(\phi t_{e}\right)+1.139 C u_{e}-0.448 N i-18.120}{0.629}\right]
\end{aligned}
$$

The effective fluence form in Eq. (7-5) applies to both the MF and CRP terms, Eqs. (7-3) and (7-4).

$$
\phi t_{e}=\left\{\begin{array}{c}
\phi t \quad \text { for } \phi \geq 4.39 \times 10^{10} \\
\phi t\left(\frac{4.39 \times 10^{10}}{\phi}\right)^{0.259} \text { for } \phi<4.39 \times 10^{10}
\end{array}\right\}
$$

\subsubsection{Variables, Units, Definitions, and Ranges of Applicability}

The units and descriptions of independent variables in Eqs. (7-2) through (7-5) are given in Table 7.3. The dependent variable, transition temperature shift (TTS), is estimated by Eq. (7-2) in degrees Fahrenheit. Both TTS and $\mathrm{T}_{\mathrm{i}}$ may be converted by the usual formula $\mathrm{T}\left({ }^{\circ} \mathrm{C}\right)=\left[\mathrm{T}\left({ }^{\circ} \mathrm{F}\right)-32\right] / 1.8$.

Table 7.3. Independent variables in the embrittlement shift model and their ranges and mean values over all calibration and validation data (855 datapoints)

\begin{tabular}{clcc}
\hline Variable & \multicolumn{1}{c}{ Description } & Range & Mean \\
\hline $\mathrm{Cu}$ & Copper content $(\mathrm{wt} \%)$ & $0.01-0.41$ & 0.136 \\
$\mathrm{Mn}$ & Manganese content $(\mathrm{wt} \%)$ & $0.58-1.96$ & 1.300 \\
$\mathrm{Ni}$ & Nickel content $(\mathrm{wt} \%)$ & $0.044-1.26$ & 0.565 \\
$P$ & Phosphorous content $(\mathrm{wt} \%)$ & $0.003-0.031$ & 0.0119 \\
$\phi t$ & Neutron fluence, $\mathrm{E}>1 \mathrm{MeV}\left(\mathrm{n} / \mathrm{cm}^{2}\right)$ & $9.26 \times 10^{15}-7.13 \times 10^{19}$ & $6.50 \times 10^{18}$ \\
$\phi$ & Neutron flux, $\mathrm{E}>1 \mathrm{MeV}\left(\mathrm{n} / \mathrm{cm}^{2} / \mathrm{s}\right)$ & $1.81 \times 10^{8}-9.71 \times 10^{11}$ & $5.13 \times 10^{10}$ \\
$T_{i}$ & Irradiation temperature $\left({ }^{\circ} \mathrm{F}\right)$ & $522-570$ & 545 \\
\hline
\end{tabular}


Two quantities are derived from the independent variables given in Table 7.3, the effective $\mathrm{Cu}$ in solution, $\mathrm{Cu}_{\mathrm{e}}$ as defined in Eq. (7-4c), and the effective fluence (or flux-adjusted fluence) $\phi t_{e}$ as defined by Eq. (7-5). An additional independent variable not listed in Table 7.3 is product form, which can take on the values for forging, plate (in CE manufactured vessels or other vessels), weld (typical Linde 80 or other), and SRM as defined in Eqs. (7-3b) and (7-4b).

Some additional information about the variables is needed for application of the model. The chemical composition variables are intended to represent the best available estimate of actual measured composition at the location where the shift is being analyzed. This is consistent with the use of average measured composition on surveillance samples from each heat (to the extent available) to develop the calibration database. Generic default chemical composition values could be considered if measurements or better estimates are unavailable. The average composition does vary significantly in high-Cu ( $>0.072$ wt \%) and low-Cu categories and by class of forging or plate material, weld group etc. so it is necessary to define any default values on statistically-relevant material groupings. A list of mean, standard deviation, minima, and maxima of composition variables for various material groups in the surveillance database is given in Appendix G.

The values of fluence and flux variables to be used in the model should be estimated at the actual location where the shift is to be estimated, with the flux estimate averaged over the relevant effective full power operating time. This is consistent with the estimates for the surveillance specimens, for which dosimetry was based on the actual capsule location and the time averaging was done by dividing total accumulated fluence by the effective full power operating time to estimate time-averaged flux. If an analysis is performed partway through the wall of the vessel, the average flux and fluence estimates should be made first at that specific location in the wall, using appropriate attenuation models, then those attenuated flux and fluence estimates should be used in the shift model. The location of greatest sensitivity to embrittlement will depend generally on both fluence and flux in the lower flux range.

The irradiation temperature used in the model should also be a time-averaged estimate for the metal at the specific location where the shift is to be estimated. The best available metal temperature estimate for the surveillance specimens was the temperature of the coolant near the surveillance capsule, but coolant temperature is not necessarily the best estimate of metal temperature in applications.

The overall range of data given in Table 7.3 is not by itself sufficient for estimating the limits of applicability of the model. The actual coverage of the data over the fitting variables and combinations of variables varies considerably, so it is necessary to review the actual distribution of data in TTSDatabase804.xls (Appendix C) to determine the ranges of variables and variable combinations that are supported by a reasonable amount of data. Some information on the ranges of chemical composition variables in the database is tabulated by product form in Appendix E, where, for instance, it is clear that forgings with $\mathrm{Cu}$ $>0.16 \mathrm{wt} \%$ or plates with $\mathrm{Cu}>0.25 \mathrm{wt} \%$ are simply not available in the database, and the upper limit of $\mathrm{Cu}=0.41 \mathrm{wt} \%$ in Table 7.3 applies only to welds. As another example, there are no low $\mathrm{Mn}(\mathrm{Mn}<0.93$ wt \%) materials in the database except A508 class 2 forgings, and the range of other chemistry variables in such forgings is limited $(0.67 \leq \mathrm{Ni} \leq 0.86 \mathrm{wt} \%, 0.01 \leq \mathrm{Cu} \leq 0.16 \mathrm{wt} \%$, and $0.004 \leq \mathrm{P} \leq 0.02 \mathrm{wt} \%)$. Thus, application of the model to any materials with $\mathrm{Mn}<0.93$ and values of $\mathrm{Ni}, \mathrm{Cu}$, or $\mathrm{P}$ outside the ranges corresponding to A508 class 2 forgings would be an extrapolation beyond the available data.

As an example of limits on exposure variable combinations, Table 7.3 shows that there are both highfluence and low-fluence data, and high-flux and low-flux data, so one might assume that the full range of the fluence/flux space is reasonably covered by data. Unfortunately, this assumption is not true. The highest available fluence in the database decreases as flux decreases, so in all the data with $\phi<1 \times 10^{10}$ $\mathrm{n} / \mathrm{cm}^{2} / \mathrm{s}$, the highest available fluence is $\phi \mathrm{t}=1.9 \times 10^{18} \mathrm{n} / \mathrm{cm}^{2}$. The highest available fluence in all the data with $\phi<1 \times 10^{9} \mathrm{n} / \mathrm{cm}^{2} / \mathrm{s}$ is only $\phi \mathrm{t}=2.8 \times 10^{17} \mathrm{n} / \mathrm{cm}^{2}$. Thus, estimating the shift at any fluence greater than $1.9 \times 10^{18} \mathrm{n} / \mathrm{cm}^{2}$ for $\phi \cong 1 \times 10^{10} \mathrm{n} / \mathrm{cm}^{2} / \mathrm{s}$ (and similarly for lower flux levels) is an extrapolation beyond the available data. 


\subsubsection{Goodness of Fit of the Simplified Model}

After deleting the CRP $\mathrm{T}_{\mathrm{i}}$ term and choosing two $\mathrm{Cu}_{\max }$ values instead of three, the quality of fit of the simplified model in Eqs. (7-2) through (7-5) is not significantly changed from the baseline model. Only the results for higher $\mathrm{Cu}$ materials $(\mathrm{Cu}>0.072 \mathrm{wt} \%)$ are affected by these simplifications, so that is the only subset of data discussed here; the results for lower $\mathrm{Cu}$ materials remain as given in Chap. 4. The model prediction vs actual shift plots for $\mathrm{Cu}>0.072 \mathrm{wt} \%$ data are similar to the baseline model, as shown by comparing Figs. 7.6 and 7.7 below with Figs. 4.10 and 4.11.

The table of standard deviations by product form is slightly changed from Table 4.1 which corresponds to the baseline model with the CRP $\mathrm{T}_{\mathrm{i}}$ term. After the simplifications, the high- $\mathrm{Cu}$ standard deviations change slightly, with the values decreasing slightly for forgings and increasing slightly for plates and welds, as highlighted in Table 7.4. The sum of squared residuals over the $\mathrm{Cu}>0.072$ wt \% calibration data increases about $1 \%$ with the simplifications. The mean residuals for the product forms listed

Table 7.4. Standard deviation $\left(S_{d}\right)$ of residuals about the simplified embrittlement shift model in various subsets, all PWR and BWR calibration and validation data except SRM All entries are TTS values measured in ${ }^{\circ} \mathrm{F}$

\begin{tabular}{|c|c|c|}
\hline \multirow{2}{*}{ Product Form } & \multicolumn{2}{|c|}{$\mathrm{S}_{\mathrm{d}}$ (points) } \\
\hline & $\mathrm{Cu} \leq 0.072 \mathrm{wt} \%$ & $\mathrm{Cu}>0.072 \mathrm{wt} \%$ \\
\hline Forging & $17.5(75)$ & $19.6(61)$ \\
\hline Plate & $15.0(78)$ & $21.2(309)$ \\
\hline Weld & $18.6(93)$ & $26.4(213)$ \\
\hline
\end{tabular}
in Table 7.4 are not significantly different from zero and pairs of means (forging vs plate, plate vs weld, forging vs weld) are not significantly different from each other.

None of the lines fitted to residual trends have a significant slope after the simplifications, both for variables in the model and for those variables not in the model that were checked. The plots showing this fact are given in Appendix $\mathrm{H}$. These can be compared directly to the corresponding plots for the baseline model (See Appendix F, Figs. F.14 through F.28). The differences are not easy to see, and both baseline and simplified models have reasonably flat residual plots that indicate a good fit.

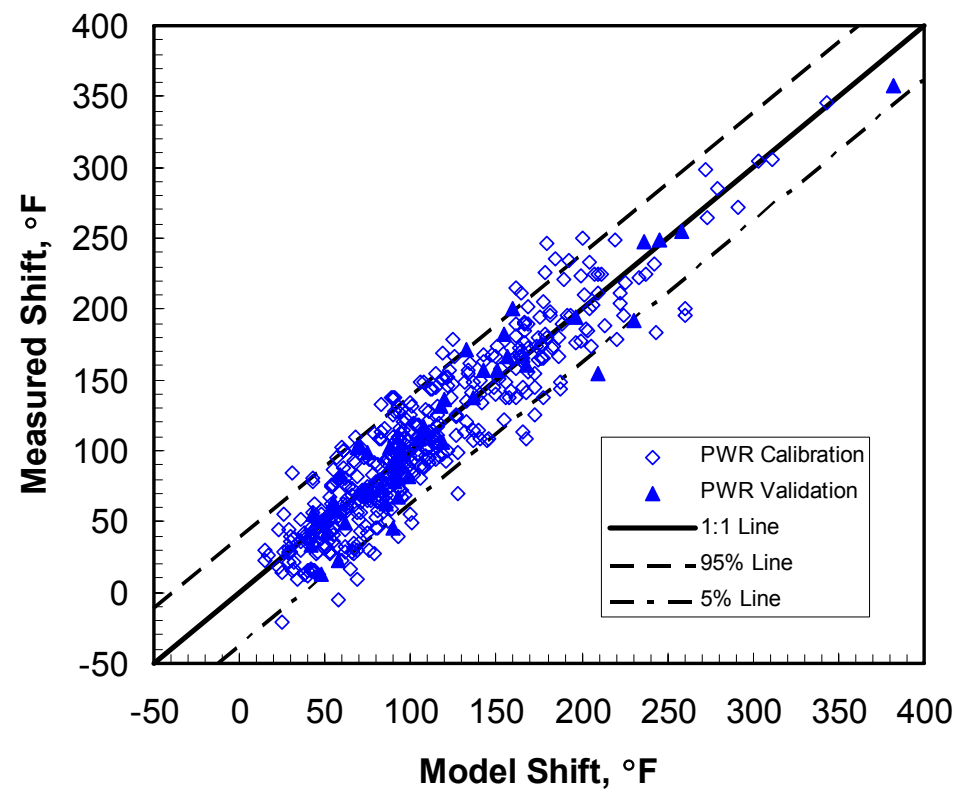

Fig. 7.6. Simplified Model shift (Eq. 7-2 through 7-5) vs measured shift, all PWR calibration and validation data with $\mathrm{Cu}>$ 0.072 wt \%. 


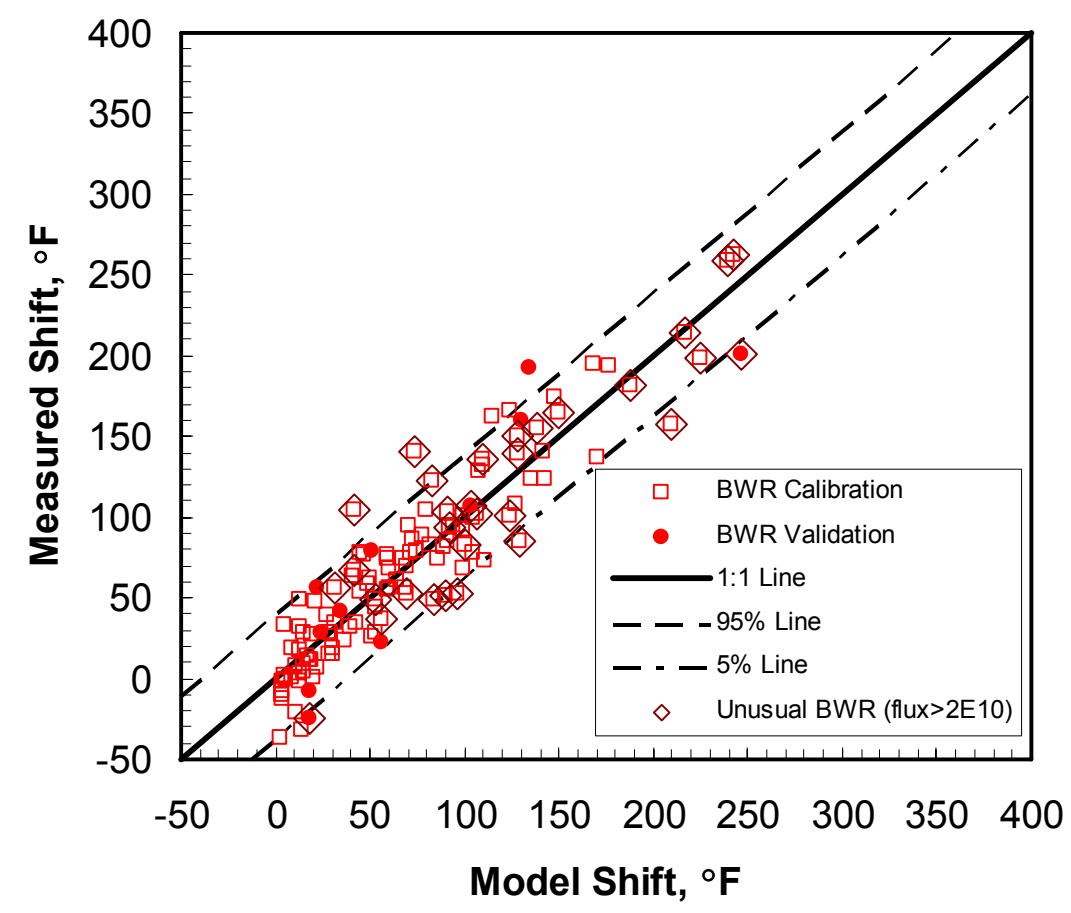

Fig. 7.7. Simplified Model shift (Eq. 7-2 through 7-5) vs measured shift, all BWR calibration and validation data with $\mathrm{Cu}>0.072 \mathrm{wt} \%$.

\subsubsection{Predictive Capability}

The simplified model retains the predictive capability of the baseline model on the high- $\mathrm{Cu}$ data, and the prediction of low-Cu data is not affected by the simplifications. Table 7.5 shows the statistical comparison of calibration and validation data for the simplified model, which can be compared to Table 4.2 for the baseline model. Though the highlighted numbers changed somewhat, the conclusion is the same - there is no significant difference between means or standard deviations of high- $\mathrm{Cu}(\mathrm{Cu}>$ $0.072 \mathrm{wt} \%$ ) calibration and validation data at either low or high flux. None of the mean residuals is significantly different from zero, either. Thus, the simplified model predicts data not used in fitting about as well as it fits the calibration data used to develop the model.
Table 7.5. Comparison of calibration and validation subsets by mean and standard deviation $\left(S_{d}\right)$ of residuals for the simplified TTS model

The number of points is shown for subsets smaller than 40 points

\begin{tabular}{lcccc}
\hline \multirow{2}{*}{ Subset } & \multicolumn{2}{c}{ Mean (points) } & \multicolumn{2}{c}{$\mathrm{S}_{\mathrm{d}}$ (points) } \\
\cline { 2 - 5 } & Calibration & Validation & Calibration & Validation \\
\hline $\begin{array}{l}\mathrm{Cu} \leq 0.072, \\
\phi>4.39 \times 10^{10}\end{array}$ & 0.0 & $0.9^{a}(22)$ & 17.0 & $14.6^{a}(22)$ \\
$\mathrm{Cu} \leq 0.072$, & & & & \\
$\phi \leq 4.39 \times 10^{10}$ & 1.7 & $-2.1^{a}(2)$ & 19.5 & $0.2(2)$ \\
$\mathrm{Cu}>0.072$, & & $2.9^{a}$ & 23.2 & $23.7^{a}$ \\
$\phi>4.39 \times 10^{10}$ & 0.4 & & & \\
$\mathrm{Cu}>0.072$, & 1.4 & $-7.5^{a}(15)$ & 21.3 & $26.1^{a}(15)$ \\
$\phi \leq 4.39 \times 10^{10}$ & & & &
\end{tabular}

${ }^{a}$ The difference from the calibration value is not statistically significant. 


\subsection{References}

1. Jones, R. B., and T. J. Williams, "The Dependence of Radiation Hardening and Embrittlement on Irradiation Temperature," pp. 569-90 in Effects of Radiation on Materials: 17th International Symposium, ASTM STP 1270, Ed. D. S. Gelles et al., American Society for Testing and Materials, 1996.

2. Odette, G. R., et al., The Effects of Composition and Heat Treatment on Hardening and Embrittlement of Reactor Pressure Vessel Steels, NUREG/CR-6778, U.S. Nuclear Regulatory Commission, Washington, D.C., 2003. 



\section{SUMMARY AND CONCLUSIONS}

The primary result of the present work is the physically motivated, empirically calibrated model of transition temperature shift (TTS) and the associated demonstration of the quality of fit and ability to predict data not used for fitting. This model is based on using separate terms for matrix features (MFs) and copper-rich precipitates (CRPs). It is a revision of earlier models of similar form, based on the larger surveillance database now available and on the continuing advances in mechanistic understanding. Following a detailed comparison in Chap. 6 of the baseline model given in Chap. 4 and the IVAR data, two sensitivity studies were conducted on the TTS data that resulted in a simplified model presented and discussed in Chap. 7. The following summary and conclusions are applicable to both models because the differences between the two models are slight and statistically nonsignificant.

The embrittlement mechanisms underlying the revised TTS model are reviewed in Chap. 2, noting practical simplifications of the set of complex, interacting processes controlling TTS. The TTS model reflects reasonably well-understood physical effects of composition, particularly the main effects and interactions of $\mathrm{Cu}, \mathrm{Ni}, \mathrm{P}$, and $\mathrm{Mn}$, and the exposure variables fluence, flux, and irradiation temperature. These variables cause changes in the magnitude and fluence dependence of the shift, especially the CRP contribution that is a saturating function of $\mathrm{Cu}, \mathrm{Ni}$, and flux. The model also includes empirical treatment of product form by using different coefficients, which reflect several physical variables, including heat treatment, preirradiation microstructure, and unmodeled effects of alloy composition, especially for $\mathrm{Mn}$ and for the likely effects of $\mathrm{Ni}$ on matrix features. The coefficients also reflect other factors not yet explained physically, such as the difference in shifts between plates in CE and non-CE-manufactured vessels.

The database used to calibrate the TTS model was updated in 2003-2004 to include an important set of 62 additional low-flux BWR shifts and an additional 140 PWR shifts. All available surveillance data, both old and new, were reviewed for completeness, discrepancies, and duplicates, and the database was improved in quality by addressing the issues identified in this review. Random sampling was used to ensure better balance of the standard reference material (SRM) data relative to the data from actual vessel materials and to reserve a $10 \%$ sample of data for validating the predictive capability of the model on data not used for fitting. The combined effect of the database expansion, review, and sampling effort was to produce a TTS calibration set that is larger than any dataset that was previously modeled, as well as being better balanced and of generally higher data quality.

The overall philosophy in calibrating the model was to fit the MF and CRP contributions to the most relevant data, which helps to avoid numerical tradeoffs between the various model terms. Thus, most of the MF constants are calibrated to low- $\mathrm{Cu}$ data $(\mathrm{Cu} \leq 0.072 \mathrm{wt} \%)$ since there is no CRP contribution in that case. The MF term, which applies at all $\mathrm{Cu}$ levels, is then held fixed while calibrating the CRP term, again to avoid trade-offs between variables that appear in both terms. A few constants were calibrated to the entire range of $\mathrm{Cu}$, either because of the scarcity of data (in the case of flux) or because the MF term is used for all data and there is no corresponding CRP term that can adjust for any differences in the lowand higher- $\mathrm{Cu}$ data (in the case of the P-Mn interaction term). Insights from prior research on embrittlement mechanisms are reflected in the model form and variables, and the fitting functions and calibrated constants are chosen to produce a reasonable empirical fit to the key TTS datasets, including high- and low- $\mathrm{Cu}$ material, PWR and BWR irradiations, and forging, plate, and weld product forms.

The revised model contains, sometimes in different form, all effects that were in the earlier NUREG/CR-6551 model [1], which was based on a substantially smaller database, plus additional effects that have been subsequently characterized. The main differences from earlier models are

- the use of a theoretical square root dependence on fluence and a linear temperature dependence in the MF term; 
- inclusion of an explicit P-Mn interaction in the MF term and a P precipitation term in the CRP term;

- separate CRP coefficients for plates in CE-manufactured vessels, plates in other vessels, and SRM plates (which are not part of any vessels);

- variation in the fluence at which the CRP term becomes important as a function of $\mathrm{Cu}, \mathrm{Ni}$, and flux; and

- incorporation of flux effects in both the MF and CRP terms by an effective fluence approach.

The goodness of fit of the model to the calibration and validation data is displayed in predicted vs measured plots, tabulated standard deviations within product form groups, and residual plots that separately consider the low-Cu data, where only the MF term applies, and the higher-Cu data, where both MF and CRP terms apply. Based on all of these measures, the fit to surveillance data is good, with no statistically significant residual trends found for any of the variables used for modeling or for other variables that were not.

The predictive capability of most aspects of the model is validated on a subset of surveillance data not used in model development, comparing means and standard deviations of residuals and predicted vs measured plots. The model also compares favorably with SRM data, most of which were not used in the calibration. The two fitting constants in the effective fluence part of the model are not validated by the sample of data set aside for validation because all of the relatively scarce low-flux data were needed for calibration. However, the effective fluence (flux effect) part of the model, as well as all other variable effects, are generally supported by comparisons with the IVAR data, which were also not used in calibrating the model.

The rather complex variable trends in the model are shown in a series of plots in Chap. 5, and each variable effect is discussed in some detail, including analysis of the statistical significance of the effect and discussion of variable confounding. All key terms in the TTS model are statistically significant for the relevant data, and most also have support from the physical understanding described in Chap. 2 and the separate set of data generated in the controlled IVAR experiments discussed in Chap. 6. The temperature correction in the CRP part of the baseline model presented in Chap. 4, a small adjustment that partially counteracts the temperature effect of the MF term to slightly improve the fit to the high-Cu surveillance data, is contrary to trends observed in controlled experiments; hence, this term is not included in the simplified model presented in Chap. 7.

The TTS model is compared in Chap. 6 to data from the controlled IVAR experiments, which are completely independent of the surveillance data used for calibrating the TTS model. The form of the TTS model is also reasonably independent of the IVAR data, although it was based in part on physical insights from the literature on embrittlement, which includes some previous results of IVAR experiments. However, specific fitting functions used in the TTS model, such as the saturating CRP function and effective fluence form, differ from the functions previously used to characterize IVAR data. Thus, the comparison with IVAR data in Chap. 6 can be considered an independent check on the TTS model.

Notably, the IVAR database is a very large collection of results from test reactor irradiations and postirradiation testing of materials selected to systematically study the effects of variables that influence radiation hardening and embrittlement. A quantitative comparison is made, using an empirical conversion between the TTS that characterizes the surveillance data and the yield strength increase that characterizes the IVAR data. Generally good agreement is shown between the TTS model and the IVAR results on welds and plates that are very similar to the surveillance materials. Good agreement is also found with IVAR results on the split-melt model steels over the range of compositions that encompass the surveillance database. Where some disagreements occur, they are generally associated with the wider variation of composition variables in the IVAR database and known limitations of the available surveillance data. The IVAR data generally provide more precise evaluations of the effects of individual 
variables and variable combinations under controlled conditions, while the TTS model reflects average effects of many variables over a wide range of alloys and irradiation conditions.

The main difference between the TTS and IVAR data trends is the range of flux over which significant dose rate effects are observed. This difference arises in part from the fact that the majority of observations in the IVAR database have higher flux than the 95th percentile flux in the TTS database, and there is a relatively narrow overlapping flux range in which both databases have substantial data. Physically based extrapolation of the flux effect identified in IVAR down to the lower flux levels of BWR surveillance data, based on an enhanced recombination mechanism, shows good agreement with the calibrated TTS flux effect, and numerical extrapolation of the flux-dependent part of the empirical TTS model to the higher flux levels in IVAR also shows agreement, suggesting that the two databases emphasize different parts of the same continuum of flux effects, as discussed in Sect. 6.6.1.

The key new insight in the TTS modeling effort, that flux effects are evident in both low-Cu (or no$\mathrm{Cu}$ ) and higher-Cu materials, is supported by the IVAR data, as discussed in Sect. 6.6.2. The flux effect in the MF term was originally incorporated in the TTS model because it is statistically significant in fitting the surveillance data. Since that empirical observation was made, a broadly similar MF flux effect was found in the low- $\mathrm{Cu}$ (no- $\mathrm{Cu}$ ) data from controlled experiments in the IVAR database, as described in Sect. 6.4.2. While the absolute flux effect on the MF term in the IVAR data is relatively small, it is systematic and can be rationalized based on mechanistic considerations discussed in Sect. 2.4.2. Thus, flux effects have been found to affect both MF and CRP terms in two independent databases and are included in the revised model.

In conclusion, the TTS model presented in this report is a good fit to the available surveillance data, with no significant residual trends in the model variables or in other variables that were considered, on both low- and higher-Cu subsets of the data. The slightly simplified version of the model given in Sect. 7.3 is recommended for applications. All major variable effects in the model are statistically significant, and most variable trends are also supported by physical understanding and quantitative agreement with trends in the independent IVAR database. The model has predictive capability for data not used in fitting, including a 10\% random sample of surveillance data reserved for validation, SRM data not used in fitting, and the independent IVAR database of test reactor irradiations.

\subsection{Reference}

1. Eason, E. D., J. E. Wright, and G. R. Odette, Improved Embrittlement Correlations for Reactor Pressure Vessel Steels, NUREG/CR-6551, U.S. Nuclear Regulatory Commission, Washington D.C., 1998. 



\section{Appendix A. Publications List}

Irradiation embrittlement of RPV beltline materials is currently evaluated using Regulatory Guide 1.99 Revision 2 (RG1.99/2), which presents methods for estimating the shift in Charpy transition temperature at $30 \mathrm{ft}-\mathrm{lb}$ (TTS) and the drop in Charpy upper shelf energy ( $\triangle \mathrm{USE}$ ). The purpose of the work reported here is to improve on the TTS correlation model in RG1.99/2 using the broader database now available and current understanding of embrittlement mechanisms.

This report is also a record of work performed in part at ORNL under the Heavy-Section Steel Irradiation (HSSI) Program, which is sponsored by the NRC Office of Nuclear Regulatory Research. A list of publications for the ORNL HSSI Program is given in Sect. A.1.

The HSSI Program includes both follow-on research and the direct continuation of work that was performed under the Heavy-Section Steel Technology (HSST) Program. Previous HSST reports related to irradiation effects in pressure vessel materials and those containing unirradiated properties of materials used in HSSI and HSST irradiation programs are listed in Sect. A.2.

\section{A.1 HSSI Program Reports}

1. F. M. Haggag, W. R. Corwin, and R. K. Nanstad, Martin Marietta Energy Systems, Inc., Oak Ridge Natl. Lab., Oak Ridge, Tenn., Irradiation Effects on Strength and Toughness of Three-Wire SeriesArc Stainless Steel Weld Overlay Cladding, USNRC Report NUREG/CR-5511 (ORNL/TM-11439), February 1990.

2. L. F. Miller, C. A. Baldwin, F. W. Stallman, and F. B. K. Kam, Martin Marietta Energy Systems, Inc., Oak Ridge Natl. Lab., Oak Ridge, Tenn., Neutron Exposure Parameters for the Metallurgical Test Specimens in the Sixth Heavy-Section Steel Irradiation Series, USNRC Report NUREG/CR-5409 (ORNL/TM-11267), March 1990.

3. S. K. Iskander, W. R. Corwin, and R. K. Nanstad, Martin Marietta Energy Systems, Inc., Oak Ridge Natl. Lab., Oak Ridge, Tenn., Results of Crack-Arrest Tests on Two Irradiated High-Copper Welds, USNRC Report NUREG/CR-5584 (ORNL/TM-11575), December 1990.

4. R. K. Nanstad and R. G. Berggren, Martin Marietta Energy Systems, Inc., Oak Ridge Natl. Lab., Oak Ridge, Tenn., Irradiation Effects on Charpy Impact and Tensile Properties of Low Upper-Shelf Welds, HSSI Series 2 and 3, USNRC Report NUREG/CR-5696 (ORNL/TM-11804), August 1991.

5. R. E. Stoller, Martin Marietta Energy Systems, Inc., Oak Ridge Natl. Lab., Oak Ridge, Tenn., Modeling the Influence of Irradiation Temperature and Displacement Rate on Radiation-Induced Hardening in Ferritic Steels, USNRC Report NUREG/CR5859 (ORNL/TM-12073), August 1992.

6. R. K. Nanstad, D. E. McCabe, and R. L. Swain, Martin Marietta Energy Systems, Inc., Oak Ridge Natl. Lab., Oak Ridge, Tenn., Chemical Composition RTNDT Determinations for Midland Weld WF-70, USNRC Report NUREG/CR-5914 (ORNL-6740), December 1992.

7. R. K. Nanstad, F. M. Haggag, D. E. McCabe, S. K. Iskander, K. O. Bowman, and B. H. Menke, Martin Marietta Energy Systems, Inc., Oak Ridge Natl. Lab., Oak Ridge, Tenn., Irradiation Effects on Fracture Toughness of Two High-Copper Submerged-Arc Welds, USNRC Report NUREG/CR5913 (ORNL/TM-12156/V1), October 1992.

8. S. K. Iskander, W. R. Corwin, and R. K. Nanstad, Martin Marietta Energy Systems, Inc., Oak Ridge Natl. Lab., Oak Ridge, Tenn., Crack-Arrest Tests on Two Irradiated High-Copper Welds, USNRC Report NUREG/CR-6139 (ORNL/TM-12513), March 1994. 
9. R. E. Stoller, Martin Marietta Energy Systems, Inc., Oak Ridge Natl. Lab., Oak Ridge, Tenn., $A$ Comparison of the Relative Importance of Copper Precipitates and Point Defects in Reactor Pressure Vessel Embrittlement, USNRC Report NUREG/CR-6231 (ORNL/TM-6811), December 1994.

10. D. E. McCabe, R. K. Nanstad, S. K. Iskander, and R. L. Swain, Martin Marietta Energy Systems, Inc., Oak Ridge Natl. Lab., Oak Ridge, Tenn., Unirradiated Material Properties of Midland Weld WF-70, USNRC Report NUREG/CR-6249 (ORNL/TM-12777), October 1994.

11. P. M. Rice and R. E. Stoller, Lockheed Martin Energy Systems, Oak Ridge Natl. Lab., Oak Ridge, Tenn., Microstructural Characterization of Selected AEA/UCSB Model FeCuMn Alloys, USNRC Report NUREG/CR-6332 (ORNL/TM-12980), June 1996.

12. J. H. Giovanola and J. E. Crocker, SRI International, Fracture Toughness Testing with Cracked Round Bars: Feasibility Study, USNRC Report NUREG/CR-6342 (ORNL/SUB/94-DHK60), April 2000 .

13. F. M. Haggag and R. K. Nanstad, Lockheed Martin Energy Systems, Oak Ridge Natl. Lab., Oak Ridge, Tenn., Effects of Thermal Aging and Neutron Irradiation on the Mechanical Properties of Three-Wire Stainless Steel Weld Overlay Cladding, USNRC Report NUREG/CR-6363 (ORNL/TM13047), May 1997.

14. M. A. Sokolov and D. J. Alexander, Lockheed Martin Energy Systems, Oak Ridge Natl. Lab., Oak Ridge, Tenn., An Improved Correlation Procedure for Subsize and Full-Size Charpy Impact Specimen Data, USNRC Report NUREG/CR-6379 (ORNL/TM-13088), March 1997.

15. S. K. Iskander and R. E. Stoller, Lockheed Martin Energy Research Corporation, Oak Ridge Natl. Lab., Oak Ridge, Tenn., Results of Charpy V-Notch Impact Testing of Structural Steel Specimens Irradiated at $\sim 30^{\circ} \mathrm{C}$ to $1 \times 10^{6}$ Neutrons $/ \mathrm{cm} 2$ in a Commercial Reactor Cavity, USNRC Report NUREG/CR-6399 (ORNL-6886), April 1997.

16. S. K. Iskander, P. P. Milella, and A. Pini, Lockheed Martin Energy Research Corporation, Oak Ridge Natl. Lab., Oak Ridge, Tenn., Results of Crack-Arrest Tests on Irradiated A 503 Class 3 Steel, USNRC Report NUREG/CR-6447 (ORNL-6894), February 1998.

17. P. Pareige, K. F. Russell, R. E. Stoller, and M. K. Miller, Lockheed Martin Energy Research Corporation, Oak Ridge Natl. Lab., Oak Ridge, Tenn., Influence of Long-Term Thermal Aging on the Microstructural Evolution of Nuclear Reactor Pressure Vessel Materials: An Atom Probe Study, USNRC Report NUREG/CR-6537(ORNL-13406), March 1998.

18. I. Remec, C. A. Baldwin, and F. B. K. Kam, Lockheed Martin Energy Research Corporation, Oak Ridge Natl. Lab., Oak Ridge, Tenn., Neutron Exposure Parameters for Capsule 10.05 in the HeavySection Steel Irradiation Program Tenth Irradiation Series, USNRC Report NUREG/CR-6600 (ORNL/TM-13548), October 1998.

19. I. Remec, C. A. Baldwin, and F. B. K. Kam, Lockheed Martin Energy Research Corporation, Oak Ridge Natl. Lab., Oak Ridge, Tenn., Neutron Exposure Parameters for the Dosimetry Capsule in the Heavy-Section Steel Irradiation Program Tenth Irradiation Series, USNRC Report NUREG/CR6601 (ORNL/TM-13549), October 1998.

20. D. E. McCabe, R. K. Nanstad, S. K. Iskander, D. W. Heatherly, and R. L. Swain, Lockheed Martin Energy Research Corporation, Oak Ridge Natl. Lab., Oak Ridge, Tenn., Evaluation of WF-70 Weld Metal from the Midland Unit 1 Reactor Vessel-Final Report, USNRC Report NUREG/CR-5736 (ORNL/TM-13748), November 2000.

21. Mikhail A. Sokolov and Randy K. Nanstad, Lockheed Martin Energy Research Corporation, Oak Ridge Natl. Lab., Oak Ridge, Tenn., Comparison of Irradiation-Induced Shifts of $K_{J c}$ and Charpy 
Impact Toughness for Reactor Pressure Vessel Steels, USNRC Report NUREG/CR-6609 (ORNL/TM-13755), November 2000.

22. S. K. Iskander, C. A. Baldwin, D. W. Heatherly, D. E. McCabe, I. Remec, and R. L. Swain, UTBattelle, LLC, Oak Ridge Nat'l. Lab., Oak Ridge, Tenn., Detailed Results of Testing Unirradiated and Irradiated Crack-Arrest Toughness Specimens from the Low-Upper-Shelf-Energy, High-Copper Weld, WF-70, USNRC Report NUREG/CR-6621 (ORNL/TM-13764), to be published (submitted July 2004).

23. D. J. Alexander, K. B. Alexander, M. K. Miller, and R. K. Nanstad, Lockheed Martin Energy Research Corporation, Oak Ridge Natl. Lab., Oak Ridge, Tenn., The Effect of Aging at $343^{\circ} \mathrm{C}$ on the Microstructure and Mechanical Properties of Type 308 Stainless Steel Welds, USNRC Report NUREG/CR-6628 (ORNL/TM-13767), November 2000.

24. M. K. Miller, P. Pariege, K. F. Russell, and R. E. Stoller, Lockheed Martin Energy Research Corporation, Oak Ridge Natl. Lab., Oak Ridge, Tenn., An Atom Probe Tomography Characterization of the Solute Distributions in a Neutron Irradiated and Annealed Pressure Vessel Steel, USNRC Report NUREG/CR-6629 (ORNL/TM-13768), November 2000.

25. I. Remec, C. A. Baldwin, and E. D. Blakeman, Lockheed Martin Energy Research Corporation, Oak Ridge Natl. Lab., Oak Ridge, Tenn., Characterization of the Neutron Field in the HSSI/UCSB Irradiation Facility at the Ford Nuclear Reactor, USNRC Report NUREG/CR-6646 (ORNL/TM1999/140), to be published (submitted September 1999).

26. P. M. Rice and R. E. Stoller, UT-Battelle, LLC, Oak Ridge Natl. Lab., Oak Ridge, Tenn., Hardening Behavior of Ferritic Alloys at High Doses and After Thermal Aging, USNRC Report NUREG/CR6643 (ORNL/TM-1999/297), to be published (submitted August 2000).

27. S. K. Iskander, J. T. Hutton, L. E. Creech, M. Suzuki, K. Onizawa, E. T. Manneschmidt, R. K. Nanstad, T. M. Rosseel, and P. S. Bishop, UT-Battelle, LLC, Oak Ridge Natl. Lab., Oak Ridge, Tenn., Attenuation of Charpy Impact Toughness Through the Thickness of a JPDR Pressure Vessel Weldment, USNRC Report NUREG/CR-6709 (ORNL/TM-2000-343), to be published (submitted January 2001).

28. K. Onizawa, E. van Walle, W. Pavinich, and R. K. Nanstad, UT-Battelle, LLC, Oak Ridge Natl. Lab., Oak Ridge, Tenn., Results and Analysis of The ASTM Round Robin On Reconstitution, USNRC Report NUREG/CR-6777, (ORNL/TM-2001/34) August 2002.

29. D. W. Heatherly, K. R. Thoms, M. T. Hurst, and G. E. Giles, UT-Battelle, LLC, Oak Ridge Natl. Lab., Oak Ridge, Tenn., Heavy-Section Steel Irradiation Program's Reusable Irradiation Facilities, USNRC Report NUREG/CR-6779, (ORNL/TM-2002/77) to be published (submitted February 2002).

30. R. K. Nanstad and D. E. McCabe, UT-Battelle, LLC, Oak Ridge Natl. Lab., Oak Ridge, Tenn., Preliminary Evaluation of Precracked Charpy Specimens for Determining Fracture Toughness of Reactor Pressure Vessel Weldment, New Coordinated Research Program, USNRC Report NUREG/CR-XXXX (ORNL/TM-2001/YYY), to be published (submitted July 2001).

31. M. A. Sokolov, R. K. Nanstad, I. Remec, C. A. Baldwin, and R. L. Swain, UT-Battelle, LLC, Oak Ridge Natl. Lab., Oak Ridge, Tenn., Fracture Toughness of an Irradiated, Highly Embrittled Reactor Pressure Vessel Weld, USNRC Report NUREG/CR-XXXX (ORNL/TM-2002/293), to be published (submitted December 2003).

32. M. K. Miller et al., UT-Battelle, LLC, Oak Ridge Natl. Lab., Oak Ridge, Tenn., APT Characterization of Highly Irradiated High-Nickel RPV Welds and Low-Upper-Shelf Midland Weld Materials, USNRC Report NUREG/CR-XXXX, ORNL/TM-2006/531), to be published. 
33. M. A. Sokolov et al., UT-Battelle, LLC, Oak Ridge Natl. Lab., Oak Ridge, Tenn., The Effects of Radiation on Fracture Toughness Curve Shape on a Low-Upper-Shelf Weld, USNRC Report NUREG/CR-XXXX, ORNL/TM-2006/532), to be published.

34. This report. E. D. Eason, M\&CS; R. K. Nanstad, UT-Battelle, LLC, Oak Ridge Natl. Lab., Oak Ridge, Tenn; G. R. Odette, UCSB; and T. Yamamato, A Physically Based Correlation of IrradiationInduced Transition Temperature Shifts for RPV Steels, USNRC Report NUREG/CR-XXXX, ORNL/TM-2006/530).

35. S. K. Iskander, R. K. Nanstad, C. A. Baldwin, D. W. Heatherly, M. K. Miller, and I. Remec, UTBattelle, LLC, Oak Ridge Natl. Lab., Oak Ridge, Tenn., Reirradiation Response of Irradiated and Thermally Treated Reactor Pressure Vessel Steels, USNRC Report NUREG/CR-XXXX, ORNL/TM2006/533), to be published.

\section{A.2 Reports Related to Irradiation Effects in Pressure Vessel Materials and to the Properties of Unirradiated Materials Used in the HSSI and HSST Programs}

C. E. Childress, Union Carbide Corp. Nuclear Div., Oak Ridge Natl. Lab., Oak Ridge, Tenn., Fabrication History of the First Two 12-in.-Thick A-533 Grade B, Class 1 Steel Plates of the Heavy-Section Steel Technology Program, ORNL-4313, February 1969.

T. R. Mager and F. O. Thomas, Westinghouse Electric Corporation, PWR Systems Division, Pittsburgh, Pa., Evaluation by Linear Elastic Fracture Mechanics of Radiation Damage to Pressure Vessel Steels, WCAP-7328 (Rev.), October 1969.

P. N. Randall, TRW Systems Group, Redondo Beach, Calif., Gross Strain Measure of Fracture Toughness of Steels, HSSTP-TR-3, Nov. 1, 1969.

L. W. Loechel, Martin Marietta Corporation, Denver, Colo., The Effect of Testing Variables on the Transition Temperature in Steel, MCR-69-189, Nov. 20, 1969.

W. O. Shabbits, W. H. Pryle, and E. T. Wessel, Westinghouse Electric Corporation, PWR Systems Division, Pittsburgh, Pa., Heavy-Section Fracture Toughness Properties of A533 Grade B Class 1 Steel Plate and Submerged Arc Weldment, WCAP-7414, December 1969.

C. E. Childress, Union Carbide Corp. Nuclear Div., Oak Ridge Natl. Lab., Oak Ridge, Tenn., Fabrication History of the Third and Fourth ASTM A-533 Steel Plates of the Heavy-Section Steel Technology Program, ORNL-4313-2, February 1970.

P. B. Crosley and E. J. Ripling, Materials Research Laboratory, Inc., Glenwood, Ill., Crack Arrest Fracture Toughness of A533 Grade B Class 1 Pressure Vessel Steel, HSSTP-TR-8, March 1970.

F. J. Loss, Naval Research Laboratory, Washington, D.C., Dynamic Tear Test Investigations of the Fracture Toughness of Thick-Section Steel, NRL-7056, May 14, 1970.

T. R. Mager, Westinghouse Electric Corporation, PWR Systems Division, Pittsburgh, Pa., PostIrradiation Testing of 2T Compact Tension Specimens, WCAP-7561, August 1970.

F. J. Witt and R. G. Berggren, Union Carbide Corp. Nuclear Div., Oak Ridge Natl. Lab., Oak Ridge, Tenn., Size Effects and Energy Disposition in Impact Specimen Testing of ASTM A533 Grade B Steel, ORNL/TM-3030, August 1970.

D. A. Canonico, Union Carbide Corp. Nuclear Div., Oak Ridge Natl. Lab., Oak Ridge, Tenn., Transition Temperature Considerations for Thick-Wall Nuclear Pressure Vessels, ORNL/TM-3114, October 1970. 
T. R. Mager, Westinghouse Electric Corporation, PWR Systems Division, Pittsburgh, Pa., Fracture Toughness Characterization Study of A533, Grade B, Class 1 Steel, WCAP-7578, October 1970.

W. O. Shabbits, Westinghouse Electric Corporation, PWR Systems Division, Pittsburgh, Pa., Dynamic Fracture Toughness Properties of Heavy-Section A533 Grade B Class 1 Steel Plate, WCAP-7623, December 1970.

C. E. Childress, Union Carbide Corp. Nuclear Div., Oak Ridge Natl. Lab., Oak Ridge, Tenn., Fabrication Procedures and Acceptance Data for ASTM A-533 Welds and a 10-in.-Thick ASTM A-543 Plate of the Heavy Section Steel Technology Program, ORNL-TM-4313-3, January 1971.

D. A. Canonico and R. G. Berggren, Union Carbide Corp. Nuclear Div., Oak Ridge Natl. Lab., Oak Ridge, Tenn., Tensile and Impact Properties of Thick-Section Plate and Weldments, ORNL/TM3211, January 1971.

C. W. Hunter and J. A. Williams, Hanford Eng. Dev. Lab., Richland, Wash., Fracture and Tensile Behavior of Neutron-Irradiated A533-B Pressure Vessel Steel, HEDL-TME-71-76, Feb. 6, 1971.

C. E. Childress, Union Carbide Corp. Nuclear Div., Oak Ridge Natl. Lab., Oak Ridge, Tenn., Manual for ASTM A533 Grade B Class 1 Steel (HSST Plate 03) Provided to the International Atomic Energy Agency, ORNL/TM-3193, March 1971.

P. N. Randall, TRW Systems Group, Redondo Beach, Calif., Gross Strain Crack Tolerance of A533-B Steel, HSSTP-TR-14, May 1, 1971.

C. L. Segaser, Union Carbide Corp. Nuclear Div., Oak Ridge Natl. Lab., Oak Ridge, Tenn., Feasibility Study, Irradiation of Heavy-Section Steel Specimens in the South Test Facility of the Oak Ridge Research Reactor, ORNL/TM-3234, May 1971.

H. T. Corten and R. H. Sailors, University of Illinois, Urbana, Ill., Relationship Between Material Fracture Toughness Using Fracture Mechanics and Transition Temperature Tests, T\&AM Report 346, Aug. 1, 1971.

L. A. James and J. A. Williams, Hanford Eng. Dev. Lab., Richland, Wash., Heavy Section Steel Technology Program Technical Report No. 21, The Effect of Temperature and Neutron Irradiation Upon the Fatigue-Crack Propagation Behavior of ASTM A533 Grade B, Class 1 Steel, HEDL-TME 72-132, September 1972.

P. B. Crosley and E. J. Ripling, Materials Research Laboratory, Inc., Glenwood, Ill., Crack Arrest in an Increasing K-Field, HSSTP-TR-27, January 1973.

W. J. Stelzman and R. G. Berggren, Union Carbide Corp. Nuclear Div., Oak Ridge Natl. Lab., Oak Ridge, Tenn., Radiation Strengthening and Embrittlement in Heavy-Section Steel Plates and Welds, ORNL-4871, June 1973.

J. M. Steichen and J. A. Williams, Hanford Eng. Dev. Lab., Richland, Wash., High Strain Rate Tensile Properties of Irradiated ASTM A533 Grade B Class 1 Pressure Vessel Steel, HEDL-TME 73-74, July 1973.

J. A. Williams, Hanford Eng. Dev. Lab., Richland, Wash., The Irradiation and Temperature Dependence of Tensile and Fracture Properties of ASTM A533, Grade B, Class 1 Steel Plate and Weldment, HEDL-TME 73-75, August 1973.

J. A. Williams, Hanford Eng. Dev. Lab., Richland, Wash., Some Comments Related to the Effect of Rate on the Fracture Toughness of Irradiated ASTM A553-B Steel Based on Yield Strength Behavior, HEDL-SA 797, December 1974. 
J. A. Williams, Hanford Eng. Dev. Lab., Richland, Wash., The Irradiated Fracture Toughness of ASTM A533, Grade B, Class 1 Steel Measured with a Four-Inch-Thick Compact Tension Specimen, HEDL-TME 75-10, January 1975.

J. G. Merkle, G. D. Whitman, and R. H. Bryan, Union Carbide Corp. Nuclear Div., Oak Ridge Natl. Lab., Oak Ridge, Tenn., An Evaluation of the HSST Program Intermediate Pressure Vessel Tests in Terms of Light-Water-Reactor Pressure Vessel Safety, ORNL/TM-5090, November 1975.

J. A. Davidson, L. J. Ceschini, R. P. Shogan, and G. V. Rao, Westinghouse Electric Corporation, Pittsburgh, Pa., The Irradiated Dynamic Fracture Toughness of ASTM A533, Grade B, Class 1 Steel Plate and Submerged Arc Weldment, WCAP-8775, October 1976.

J. A. Williams, Hanford Eng. Dev. Lab., Richland, Wash., Tensile Properties of Irradiated and Unirradiated Welds of A533 Steel Plate and A508 Forgings, NUREG/CR-1158 (ORNL/SUB79/50917/2), July 1979.

J. A. Williams, Hanford Eng. Dev. Lab., Richland, Wash., The Ductile Fracture Toughness of HeavySection Steel Plate, NUREG/CR-0859, September 1979.

K. W. Carlson and J. A. Williams, Hanford Eng. Dev. Lab., Richland, Wash., The Effect of Crack Length and Side Grooves on the Ductile Fracture Toughness Properties of ASTM A533 Steel, NUREG/CR1171 (ORNL/SUB-79/50917/3), October 1979.

G. A. Clarke, Westinghouse Electric Corp., Pittsburgh, Pa., An Evaluation of the Unloading Compliance Procedure for J-Integral Testing in the Hot Cell, Final Report, NUREG/CR-1070 (ORNL/SUB7394/1), October 1979.

P. B. Crosley and E. J. Ripling, Materials Research Laboratory, Inc., Glenwood, Ill., Development of a Standard Test for Measuring KIa with a Modified Compact Specimen, NUREG/CR-2294 (ORNL/SUB-81/7755/1), August 1981.

H. A. Domian, Babcock and Wilcox Company, Alliance, Ohio, Vessel V-8 Repair and Preparation of Low Upper-Shelf Weldment, NUREG/CR-2676 (ORNL/SUB/81-85813/1), June 1982.

R. D. Cheverton, S. K. Iskander, and D. G. Ball, Union Carbide Corp. Nuclear Div., Oak Ridge Natl. Lab., Oak Ridge, Tenn., PWR Pressure Vessel Integrity During Overcooling Accidents: A Parametric Analysis, NUREG/CR-2895 (ORNL/TM-7931), February 1983.

J. G. Merkle, Union Carbide Corp. Nuclear Div., Oak Ridge Natl. Lab., Oak Ridge, Tenn., An Examination of the Size Effects and Data Scatter Observed in Small Specimen Cleavage Fracture Toughness Testing, NUREG/CR-3672 (ORNL/TM-9088), April 1984.

W. R. Corwin, Martin Marietta Energy Systems, Inc., Oak Ridge Natl. Lab., Oak Ridge, Tenn., Assessment of Radiation Effects Relating to Reactor Pressure Vessel Cladding, NUREG/CR-3671 (ORNL-6047), July 1984.

W. R. Corwin, R. G. Berggren, and R. K. Nanstad, Martin Marietta Energy Systems, Inc., Oak Ridge Natl. Lab., Oak Ridge, Tenn., Charpy Toughness and Tensile Properties of a Neutron Irradiated Stainless Steel Submerged-Arc Weld Cladding Overlay, NUREG/CR-3927 (ORNL/TM-9709), September 1984.

J. J. McGowan, Martin Marietta Energy Systems, Inc., Oak Ridge Natl. Lab., Oak Ridge, Tenn., Tensile Properties of Irradiated Nuclear Grade Pressure Vessel Plate and Welds for the Fourth HSST Irradiation Series, NUREG/CR-3978 (ORNL/TM-9516), January 1985.

J. J. McGowan, Martin Marietta Energy Systems, Inc., Oak Ridge Natl. Lab., Oak Ridge, Tenn., Tensile Properties of Irradiated Nuclear Grade Pressure Vessel Welds for the Third HSST Irradiation Series, NUREG/CR-4086 (ORNL/TM-9477), March 1985. 
W. R. Corwin, G. C. Robinson, R. K. Nanstad, J. G. Merkle, R. G. Berggren, G. M. Goodwin, R. L. Swain, and T. D. Owings, Martin Marietta Energy Systems, Inc., Oak Ridge Natl. Lab., Oak Ridge, Tenn., Effects of Stainless Steel Weld Overlay Cladding on the Structural Integrity of Flawed Steel Plates in Bending, Series 1, NUREG/CR-4015 (ORNL/TM-9390), April 1985.

W. J. Stelzman, R. G. Berggren, and T. N. Jones, Martin Marietta Energy Systems, Inc., Oak Ridge Natl. Lab., Oak Ridge, Tenn., ORNL Characterization of Heavy-Section Steel Technology Program Plates 01, 02, and 03, NUREG/CR-4092 (ORNL/TM-9491), April 1985.

G. D. Whitman, Martin Marietta Energy Systems, Inc., Oak Ridge Natl. Lab., Oak Ridge, Tenn., Historical Summary of the Heavy-Section Steel Technology Program and Some Related Activities in Light-Water Reactor Pressure Vessel Safety Research, NUREG/CR-4489 (ORNL-6259), March 1986.

R. H. Bryan, B. R. Bass, S. E. Bolt, J. W. Bryson, J. G. Merkle, R. K. Nanstad, and G. C. Robinson, Martin Marietta Energy Systems, Inc., Oak Ridge Natl. Lab., Oak Ridge, Tenn., Test of 6-in.-Thick Pressure Vessels. Series 3: Intermediate Test Vessel V-8A - Tearing Behavior of Low Upper-Shelf Material, NUREG-CR-4760 (ORNL-6187), May 1987.

D. B. Barker, R. Chona, W. L. Fourney, and G. R. Irwin, University of Maryland, College Park, Md., A Report on the Round Robin Program Conducted to Evaluate the Proposed ASTM Standard Test Method for Determining the Plane Strain Crack Arrest Fracture Toughness, $\mathrm{K}_{\mathrm{Ia}}$, of Ferritic Materials, NUREG/CR-4966 (ORNL/SUB/79-7778/4), January 1988.

L. F. Miller, C. A. Baldwin, F. W. Stallman, and F. B. K. Kam, Martin Marietta Energy Systems, Inc., Oak Ridge Natl. Lab., Oak Ridge, Tenn., Neutron Exposure Parameters for the Metallurgical Test Specimens in the Fifth Heavy-Section Steel Technology Irradiation Series Capsules, NUREG/CR5019 (ORNL/TM-10582), March 1988.

J. J. McGowan, R. K. Nanstad, and K. R. Thoms, Martin Marietta Energy Systems, Inc., Oak Ridge Natl. Lab., Oak Ridge, Tenn., Characterization of Irradiated Current-Practice Welds and A533 Grade B Class 1 Plate for Nuclear Pressure Vessel Service, NUREG/CR-4880 (ORNL-6484/V1 and V2), July 1988.

R. D. Cheverton, W. E. Pennell, G. C. Robinson, and R. K. Nanstad, Martin Marietta Energy Systems, Inc., Oak Ridge Natl. Lab., Oak Ridge, Tenn., Impact of Radiation Embrittlement on Integrity of Pressure Vessel Supports for Two PWR Plants, NUREG/CR-5320 (ORNL/TM-10966), February 1989.

J. G. Merkle, Martin Marietta Energy Systems, Inc., Oak Ridge Natl. Lab., Oak Ridge, Tenn., An Overview of the Low-Upper-Shelf Toughness Safety Margin Issue, NUREG/CR-5552 (ORNL/TM11314), August 1990.

R. D. Cheverton, T. L. Dickson, J. G. Merkle, and R. K. Nanstad, Martin Marietta Energy Systems, Inc., Oak Ridge Natl. Lab., Oak Ridge, Tenn., Review of Reactor Pressure Vessel Evaluation Report for Yankee Rowe Nuclear Power Station (YAEC No. 1735), NUREG/CR-5799 (ORNL/TM-11982), March 1992. 



\section{Appendix B. Irradiation Variable (IVAR) Program Data Base}

Irradiation Variable (IVAR) program test reactor data, as described in Chap. 6, is collected in the IVAR Data Base (filename IVAR_database.doc).

The compact disc included in the pocket on the back cover of this report contains a copy of the IVAR Data Base, along with the Analysis Data Base (See Appendix C) and a pdf version of this report.

All three files are transmitted together if sent electronically. 



\section{Appendix C. Analysis Data Base}

The complete surveillance data base used for the modeling and analysis, as described in Chap. 3 of this report, is TTSDatabase8-04R1.xls, a file in Microsoft Excel format.

The compact disc included in the pocket on the back cover of this report contains a copy of TTSDatabase8-04R1.xls, along with the IVAR Data Base (See Appendix B) and a pdf version of this report.

All three files are transmitted together if sent electronically. 



\section{Appendix D. Explanations of the Specialized Terms and Parameters Used in Chapters 2 and 6}

\section{D.1 Terms related to radiation damage production, point defects and defect clusters}

Cascade: The small (several tens of $\mathrm{nm}$ ) region of the crystal lattice where the primary and secondary recoiling atoms slow down and initially produce a dense concentration of vacancies and self interstitial atom (SIA) and small clusters of these defects.

Cascade aging: The spatially correlated rearrangement of the cascade over the period of time from about $100 \mathrm{ps}$ up to a gs, involving additional recombination and clustering of vacancies, SIA and solutes. The residual SIA and SIA clusters quickly leave the cascade region. The vacancy clusters coarsen and form complexes with solutes. However, the vacancy clusters eventually dissolve leaving behind solute cluster remnants that can be very stable, and can even grow by the long-range diffusion of additional solutes. The solute clusters may be somewhat diffuse relative to well-formed precipitates. Vacancy cluster solute complexes and their solute remnants are believed to be one of the primary matrix features that contribute to hardening in both low $\mathrm{Cu}$ and $\mathrm{Cu}$-bearing steels.

Dislocation loop: A dislocation line that closes on itself to form a nearly circular loop; a prismatic loop is composed of edge dislocation segments.

Displacements-per-atom (dpa, nd): A measure of the neutron damage dose that represents the computed fraction of atoms that have been displaced from their atomic lattice positions. Also, in ASTM E 170: the mean number of times each atom of a solid is displaced from its lattice site during an exposure to displacing radiation, as calculated following standard procedures.

Fraction of vacancies that recombine and the fraction that escape recombination to reach sinks $\left(g_{r}\right.$ and $\left.g_{s}, n d\right)$ : The fraction of vacancies that recombine versus vacancies that escape recombination during long range diffusion and annihilate at sinks thus determining the steady-state vacancy concentration

Frenkel pair: The SIA and vacancy produced by an atomic displacement event.

Primary recoiling atom (PRA): The high-energy ionized atom that is created by interactions between neutrons and the atomic nucleus that slows down by collisions with other atoms and with electrons. The multiplying chain reaction atomic collisions continue until the n'th generation of collided atoms has insufficient energy to create additional displaced atoms.

Cascade recombination: The reaction between a vacancy and SIA in a displacement cascade that annihilates both defects, thus healing the crystal lattice. Cascade recombination rapidly annihilates about 60 to 70 percent of the vacancies SIA created by atomic displacements.

Self-interstitial atom (SIA): Two atoms sharing a single crystal lattice site

SIA clusters: Two-dimensional platelet shaped aggregates of SIA that are equivalent to a prismatic dislocation loop.

Vacancy: A crystal lattice site that is missing an atom.

Vacancy production cross-section $\left(\sigma_{v}\right.$, vacancies- $m^{2} /$ atom): The effective area per atom for a neutron to produce a vacancy that, when multiplied by neutron flux $\left(\phi, \mathrm{n} / \mathrm{m}^{2}-\mathrm{s}\right)$, gives the generation rate per lattice 
site of creating vacancies, $G_{v}$ (atom fraction of vacancies created/s). The rate of creating SIA, $G_{i}$, is the same as that for vacancies.

Vacancy cluster: Compact spherical or polyhedral three-dimensional aggregates of vacancies

Vacancy-solute cluster complex: A vacancy cluster with solutes segregated to and near the surface. These features form in aged cascades.

\section{D.2 Terms related to irradiation-induced changes in the yield stress, $\Delta \sigma_{y}(M P a)$, and transition temperature shifts, TTS $\left({ }^{\circ} \mathrm{C}\right)$.}

Critical stress for cleavage fracture $\left(\sigma^{*}, M P a\right)$ : The internal tensile stress near the tip of a notch or crack required to initiate cleavage fracture. In the case of Charpy tests, $\sigma^{*}$ has generally been found to be independent of temperature and irradiation.

Dislocations and dislocation density $\left(\rho, \mathrm{m}^{-2}\right)$ : Line defects that glide to propagate slip between atomic planes at a critical value of a resolved shear stress. Dislocations also serve as sinks for SIA and vacancies, especially at jogs which are short dislocation segments connecting longer dislocation segments that do not lie entirely in the same glide plane. The dislocation density, $\rho$, is the total length of dislocations per unit volume.

Dislocation Burger's vector (b $\mathrm{m}$ or $\mathrm{nm}, 0.248 \mathrm{~nm}$ ): The close packed spacing in the ferrite lattice that defines the unit distance of slip.

Elastic shear modulus of $\mathrm{Fe}(\mu, 80 \mathrm{GPa})$

Obstacle strength parameters $\alpha_{i}(n d)$ : The numerical coefficient $(\leq 1)$ that characterizes the strength of the i'th-type obstacle for retarding the glide of dislocations. Obstacles are classified as being weak (w), medium strength $(\mathrm{m})$ or strong $(\mathrm{o})$.

Orowon obstacle: A strong obstacle to dislocation glide, with $\alpha_{0}$ up to 1 , which is bypassed by a dislocation looping around, rather than shearing through, the feature. Fine scale $\mathrm{Mo}_{2} \mathrm{C}$ precipitates in low alloy steels are Orowon obstacles.

Parameters that characterize irradiation induced features $-f_{i}(\mathrm{nd}), N_{i}\left(/ \mathrm{m}^{3}\right), r_{i}(\mathrm{~m}$ or $\mathrm{nm})$ : The volume fraction, number density and radius of feature $i$ that retards dislocation glide and increases the critical resolved shear stress

Superposition and the superposition parameter $(S, n d)$ : The method used to combine the strengthening contributions from various obstacles to dislocation slip needed to predict the total $\sigma_{\mathrm{y}}$ and $\Delta \sigma_{\mathrm{y}}$. Superposition can be modeled by a superposition parameter, $S$, that depends on the strengths $\left(\alpha_{i}\right)$ of the different obstacles. The limiting superposition laws are linear sum (LS) and root sum of the squares (RSS) combinations of the individual contributions, $\sigma_{\mathrm{yi}}$. The $\mathrm{S}$ parameter can be used along with the medium strength $\left(\sigma_{y m}\right)$ and strong $\left(\sigma_{y o}\right)$ obstacle yield stress contributions to interpolate between LS and RSS laws to determine the net $\sigma_{\mathrm{y}}$ and $\Delta \sigma_{\mathrm{y}}$. The yield stress contribution from weak obstacles follows the LS law.

Taylor factor $(n d, T \approx 3)$ : The factor that relates the critical resolved shear stress to the uniaxial yield stress. 
Maximum elastic fracture temperature $T T_{10}$ and $T T S_{10}$ shifts $\left[T T S_{10}=T T_{10 i}\right.$ (irradiated) - $T T_{10 u}$ (unirradiated), $\left.{ }^{\circ} \mathrm{C}\right]$ : The highest temperature at which Charpy specimens undergo cleavage fracture in the linear elastic loading regime, prior to plastic yielding. This also marks the highest elastic load. At higher temperatures, if cleavage occurs, it is only after general plastic yielding. At lower temperatures the fracture is in the linear elastic regime, but occurs at a lower load compared to that at $\mathrm{TT}_{10}$. $\mathrm{The}^{\mathrm{TT}} \mathrm{T}_{10}$ condition typically occurs at about $10 \mathrm{~J}$ of absorbed energy. Irradiation hardening increases $\mathrm{TT}_{10}$, and $\mathrm{TTS}_{10}$ can be related to the temperature dependence of $\sigma_{\mathrm{y}}(\mathrm{T})$.

TTS due to the irradiation induced reduction in the Charpy upper shelf energy $\left(\operatorname{TTS}_{\text {use }},{ }^{\circ} \mathrm{C}\right)$ : Irradiation induced decreases in the Charpy upper shelf energy ( $\triangle U S E)$ lead to reductions in the slope of the energytemperature curve. This adds an increment of $\mathrm{TTS}_{\text {use }}$ to the $\mathrm{TTS}_{10}$ in the TTS at $41 \mathrm{~J}$. The $\Delta \mathrm{USE}$ can be correlated with $\Delta \sigma_{\mathrm{y}}$ and $\mathrm{TTS}_{\text {use }}$ from the observation that the lower-to-upper shelf transition occurs over an approximately constant temperature interval.

TTS to $\Delta \sigma_{y}$ ratio $\left(\mathrm{C}_{c},{ }^{\circ} \mathrm{C} / \mathrm{MPa}\right)$ : The overall coefficient relating TTS to $\Delta \sigma_{\mathrm{y}} \mathrm{C}_{\mathrm{c}}$ can be predicted by semiempirical models (Chap. 2) and represented by a polynomial with coefficients $\left(\mathrm{C}_{0}, \mathrm{C}_{1}, \mathrm{C}_{2}, ..\right)$ fitted to pairs of TTS- $\Delta \sigma_{\mathrm{y}}$ data (Chap. 6).

\section{D.3 Terms related to the concentrations and diffusion of solutes and defects and the annihilation of defects by sinks.}

Atomic volume of $\mathrm{Cu}$ and $\mathrm{Fe}$ atoms $\left(v_{c u}\right.$ and $\left.v_{f e}, \mathrm{~m}^{3}\right)$ : Volume occupied by a $\mathrm{Cu}$ or $\mathrm{Fe}$ atom $\approx 1.18 \times 10^{-29}$ and $\approx 1.15 \times 10^{-29} \mathrm{~m}^{3}$, respectively.

Chemical Cu diffusion coefficient ( $D_{c u}$ or $D_{C u}, \mathrm{~m}^{2} / \mathrm{s}$ ): The diffusion coefficient for $\mathrm{Cu}$ (and other solutes) that accounts for the effects of both finite concentrations of multiple alloying elements and the non-ideal solution behavior of the diffusing species. The effects of solutes on diffusion are due to their corresponding effect of solute $\mathrm{j}$ on vacancy concentrations and various atomic jump frequencies and can be approximately characterized by a factor $\left(1+\mathrm{X}_{\mathrm{j}} \mathrm{b}_{\mathrm{j}}\right)$ that multiplies the by the intrinsic diffusion coefficient. The effects of non-ideality are represented by the solution thermodynamic factor (TDF) that also multiplies the solute modified diffusion coefficient.

Weight fraction concentration of species $i,\left(C_{i}, w t \%\right)$ : The weight fraction of the species in $\%$.

Atomic fraction concentration of species $i,\left(X_{i}\right.$, atom fraction): The atomic fraction of the species. The species include solutes, vacancies, SIA and solute vacancy traps that can have units of the absolute atom fraction, $\%$, or atomic parts per million (appm).

Concentration of vacancies under irradiation $\left(X_{v}, n d\right)$ : The excess steady-state fractional concentration of vacancies that just balances vacancy production by displacements and their annihilation at sinks.

Effective fluence $\left(\phi t_{e}, n / \mathrm{m}^{2}\right)$ : The actual fluence adjusted for dose rate effects at a flux, $\phi$, different than a specified reference flux, $\phi_{r}$. The choice of $\phi_{\mathrm{r}}$ is arbitrary. However, once $\phi_{\mathrm{r}}$ is selected, the effective fluence at other fluxes can by determined by a scaling relation in the form $\phi t_{e}=\left(\phi_{\mathrm{r}} / \phi\right)^{\mathrm{p}}$ (see next definition for exponent $\mathrm{p}$ ).

Effective fluence scaling exponent ( $p, n d)$ : The exponent $(\mathrm{p} \leq 1)$ that scales the actual to effective fluence. The value of $p$ depends of the flux, reference flux, irradiation temperature and the alloy composition and microstructure. However, in fitting the TTS model to the surveillance database it is assumed that $\mathrm{p}$ is an average constant. 
Equilibrium concentration of vacancies $\left(X_{v e}, n d\right)$ : The concentration of vacancies in the absence of irradiation.

Intrinsic Cu tracer diffusion coefficient $\left(D_{c u}\right.$ or $\left.D_{C u}, \mathrm{~m}^{2} / \mathrm{s}\right)$ : The coefficient for the diffusion of $\mathrm{Cu}$ (and other solutes) at very low concentrations.

Molar volume of $\mathrm{Cu}\left(V_{c u}, \mathrm{~m}^{3} /\right.$ mole $)$ : Volume occupied by a $6.02 \times 10^{23}$ of $\mathrm{Cu}$ atoms $\approx 7.15 \times 10^{-6} \mathrm{~m}^{3} / \mathrm{mole}$.

Radiation enhanced $C u$ diffusion (RED) and the RED diffusion coefficient ( $\left.D^{*}, \mathrm{~m}^{2} / \mathrm{s}\right)$ : The enhancement of the diffusion rate of $\mathrm{Cu}$ (and other solutes) due to the excess concentration of vacancies under irradiation, that is characterized by a RED coefficient $\mathrm{D}^{*}$.

RED factor $\left(K, \mathrm{~m}^{4}\right)$ : The factor that when multiplied by flux gives the enhancement term for $\mathrm{Cu}$ (and other solute) diffusion under irradiation that adds to the thermal $\mathrm{Cu}$ coefficient of $\mathrm{Cu}, \mathrm{D}_{\mathrm{cu}}$.

Ratio of the Cu to self-diffusion coefficient $\left[D_{c u} / D_{s d}, n d\right]$ : The diffusion coefficients of solutes, such as $\mathrm{Cu}$, are generally not the same as those for the self-diffusion of matrix $(\mathrm{Fe})$ atoms. The corresponding ratio $\left[D_{c u} / D_{s d}\right]$ is much less temperature dependent than $D_{c u}$, and when multiplied by the irradiation enhanced self-diffusion coefficient, which can be estimated with some accuracy, provides a good means to model $\mathrm{D}^{*}$ at low temperatures.

Lattice recombination and recombination coefficient $(R, n d)$ : The reaction between a vacancy and SIA during long-range diffusion that annihilates both defects, thus healing the crystal lattice, where $\mathrm{R}$ is characterized by a recombination radius $\left(r_{r}, n m\right), \mathrm{D}_{\mathrm{v}}, \mathrm{D}_{\mathrm{i}}$ and the atomic volume of $\mathrm{Fe}, \mathrm{v}_{\mathrm{fe}}$.

Molar volume of $\mathrm{Cu}\left(V_{c u}, \mathrm{~m}^{3} / \mathrm{mole}\right)$ : Volume occupied by a $6.02 \times 10^{23}$ of $\mathrm{Cu}$ atoms.

Self-diffusion coefficient $\left(D_{s d}, \mathrm{~m}^{2} / \mathrm{s}\right)$ : The diffusion coefficient for an $\mathrm{Fe}$ atom in the ferrite matrix.

Vacancy and SIA sinks and sink strength $\left(S_{t}, m^{-2}\right)$ : A site where SIA and vacancy defects lose their individual identity (are annihilated). The total sink strength, $S_{t}$, is used in defect conservation equations. Dislocations are the primary defect sinks in RPV steels, and $S_{t}$ is often approximated as the total dislocation density, $\rho$.

Vacancy and SAI diffusion coefficients $\left(D_{v}\right.$ and $\left.D_{i}, \mathrm{~m}^{2} / \mathrm{s}\right)$ : The diffusion coefficients for thermally activated migration of vacancies and SIA that control the steady state concentration of these species that balances their production, recombination and annihilation rates. Within the framework of the rate theory models described in Chapter 2, for technical reasons it is not necessary to know $\mathrm{D}_{\mathrm{i}}$, which is much higher than $\mathrm{D}_{\mathrm{v}}$.

Solute vacancy trapping energy $\left(X_{t}\right.$ and $\left.H_{t}, k J / m o l e\right)$ : The trap concentration $\left(\mathrm{X}_{\mathrm{t}}\right)$ and binding energy $\left(\mathrm{H}_{\mathrm{t}}\right)$ between a vacancy and a solute atom trap, that adds to the activation energy for the jumps of vacancies between lattice sites.

Substitutional solute: A solute that replaces an Fe atom on a crystal lattice site.

Spherical cluster sink strength $\left(S_{s}, m^{-2}\right)$ : The sink strength for a dilute concentration of spherical sinks rate $\mathrm{S}_{\mathrm{s}} \approx 4 \pi \mathrm{r}_{\mathrm{s}} \mathrm{N}_{\mathrm{s}}$. Note features that act as sinks can also act as sources by emitting vacancies or solutes at a rate proportional to the sink strength 


\section{D.4 Terms related to irradiation induced features and their evolution under irradiation as well as fitting the IVAR $\Delta \sigma_{y}$ Data}

Activity $\left(a_{i j}, n d\right)$ and activity coefficient $\Gamma_{i j}(n d)$ of a element $i$ in phase $j$ : The activity, $\mathrm{a}_{\mathrm{ij}}$, of an element in solution is a measure of its effective thermodynamic concentration relative to its actual concentration as described by the activity coefficient $\Gamma_{\mathrm{ij}}=\mathrm{a}_{\mathrm{ij}} / \mathrm{X}_{\mathrm{ij}}$ that can be related to the solutes enthalpy of solution, $\mathrm{H}_{\mathrm{ij}}$.

Avrami transformation equation: A simple representation of the $\phi \mathrm{t}$ (or time) dependence of precipitate volume fraction $\left(f_{p}\right)$ and precipitate hardening $\Delta \sigma_{y p}$ that varies between 0 and $100 \%$ of the maximum values of $f_{p m}$ and $\Delta \sigma_{y p m}$. The parameters in Avrami model used to fit the IVAR $\Delta \sigma_{\mathrm{y}}$ data are $\Delta \sigma_{\mathrm{ypm}}, \phi \mathrm{t}_{\mathrm{e}}$ at $63 \%$ precipitation and maximum hardening, $\mathrm{p}$ that scales the effective fluence, $\phi \mathrm{t}_{\mathrm{e}}$ and $\beta$ that sets the $\phi \mathrm{t}_{\mathrm{e}}$ interval for the transformation.

Chemistry factor for matrix features $\left[C F, M P a / \sqrt{ }\left(10^{23} \mathrm{n} / \mathrm{m}^{2}\right)\right]$ : The composition dependent coefficient for matrix feature hardening that multiplies the square root of the fluence (or effective fluence) in fitting the low $\mathrm{Cu}$ IVAR data.

Coherency misfit strain parameter $(\delta \approx 0.03, n d)$ : The ratio of the difference between the lattice parameters for $\mathrm{Cu}$ and $\mathrm{Fe}$ divided by the lattice parameter of $\mathrm{Fe}$.

Cluster dynamics models are based on the rates of absorbing and emitting $C u$ by CRP clusters of size $n$ up to a maximum $n_{\max }[\beta(n)$ and $\alpha(n)$, number/cluster-s]: In the simplest cluster dynamics models of nucleation, growth and coarsening, CRPs (and MNPs) simultaneously dissolve by emitting $[\alpha(\mathrm{n})]$ and grow by absorbing $[\beta(\mathrm{n})]$ mobile $\mathrm{Cu}$ atoms $(\mathrm{n}=1)$, and other solute atoms, respectively.

$\mathrm{Cu}$ in equilibrium with $\mathrm{CRP}$ with a radius $r_{p}\left(X_{\text {cur }}, n d\right)$ : The solubility of $\mathrm{Cu}$ is higher for small precipitates due to the interface (Gibbs-Thompson effect) and misfit strain energies, as well as the bcc vs fcc crystal structure.

$\mathrm{Cu}$ rich precipitates (CRPS): Coherent bcc phases of $\mathrm{Cu}$ alloyed with varying amounts of $\mathrm{Mn}, \mathrm{Ni}, \mathrm{Si}$ and $\mathrm{P}$ with a fraction of $\mathrm{Cu}$ in the precipitate, $\mathrm{X}_{\text {cup }} \geq 0.5$.

Cu supersaturation $(S S, n d)$ : The ratio of the $\mathrm{Cu}$ in solution, $\mathrm{X}_{\mathrm{cu}}$, to the equilibrium solubility of $\mathrm{Cu}, \mathrm{X}_{\mathrm{cue}}$.

Effective fluence factor $\left(M_{\phi} n d\right)$ : The flux dependent factor that multiplies the actual fluence to determine the effective fluence, normalized to 1 at a reference flux. $M_{\phi}$ provides a convenient basis to compare different models of flux effects.

Enthalpy of solution of solute $i\left(H_{i}, \mathrm{~kJ} /\right.$ mole $)$ : The thermodynamic parameter that governs the activity and solubility limit of a solute.

Equilibrium solubility of $\mathrm{Cu}\left(X_{\text {cue }}, n d\right)$ : The solubility limit of $\mathrm{Cu}$ in equilibrium with nearly pure bcc $\mathrm{Cu}$ phase.

Fitted recombination model (FRM): The Avrami effective fluence model fit to IVAR $\Delta \sigma_{\mathrm{y}}$ data to optimize the parameters $\beta, \phi \mathrm{t}_{\mathrm{et}}, \Delta \sigma_{\mathrm{ypm}}$ and $\mathrm{p}$ for individual alloys.

Fluence at $50 \%$ of the maximum CRP hardening $\left(\phi t_{0.5}, 10^{23} \mathrm{n} / \mathrm{m}^{2}\right)$ : The fluence at $\Delta \sigma_{\mathrm{ypm}} / 2$. 
Irradiation temperature coefficient $\left(C_{T},{ }^{\circ} C^{-1}\right)$ : The temperature sensitivity coefficient in a function $\Delta \sigma_{\mathrm{y}}\left(\mathrm{T}_{\mathrm{i}}\right)=\Delta \sigma_{\mathrm{y}}(290)\left[1-\mathrm{C}_{\mathrm{T}}\left(\mathrm{T}_{\mathrm{i}}-290\right)\right]$.

Late blooming phases (LBPs): $\mathrm{Mn}, \mathrm{Ni}, \mathrm{Si}, \mathrm{P}$ phases (MNPs) that form in low or $\mathrm{Cu}$ free RPV steels that contain little or no $\mathrm{Cu}$. The LBP nucleation rate is low compared to those for CRPs and MNPs with significant contents of $\mathrm{Cu}$.

Manganese-nickel rich precipitates (MNPs): Coherent bcc phases of $\mathrm{Mn}, \mathrm{Ni}, \mathrm{Cu}$ and $\mathrm{P}$ with a fraction of $\mathrm{Cu}$ in the precipitate, $\mathrm{X}_{\text {cup }}<0.5$.

Matrix Features (MF): MF are defined as the obstacles to dislocation slip that form in both low $\mathrm{Cu}$ and $\mathrm{Cu}$-bearing steels. Possible MF include dislocation loops, dislocation solute atmospheres, phosphide precipitates (PP, separately defined) and especially vacancy cluster solute complexes and their solute cluster remnants.

Nucleation barrier: Below a critical size $\mathrm{Cu}$ clusters shrink faster than they grow, so CRPs (and other phases) must nucleate by statistical fluctuations. The rate of nucleation can be described by equations containing a pseudo activation energy barrier or directly simulated using cluster dynamics models.

Particle matrix interface energy $\left(\gamma_{p m}, \mathrm{~J} / \mathrm{m}^{2}\right)$ : The energy of the generally coherent interface separating CRPs and MNPs from the Fe matrix. The $\gamma_{\mathrm{pm}}$ depends on the composition of the precipitate.

Precipitate volume fraction and maximum volume fraction $\left(f_{p}\right.$ and $\left.f_{p m}, n d\right)$ : The volumetric fraction of precipitates up to a solute $(\mathrm{Cu})$ limited maximum.

Phosphide precipitates (PP): Solute atom-P precipitates such as $\mathrm{Mn}_{2} \mathrm{P}$ and $\mathrm{Mn}_{3} \mathrm{P}$.

\section{D.5 Terms related to experimental facilities and characterization methods}

Irradiation variable facility (IVAR): The facility at the University of Michigan Ford Research Reactor that was used to irradiate specimens that were tested to produce the IVAR database. The IVAR facility had three temperature zones in each of three flux regimes. The facility had 54 locations for subcapsules that were inserted and removed during reactor shutdown periods. Each subcapsule held up to several hundred specimens.

Resistivity-Seebeck coefficient characterization (RSC): Dissolved solutes contribute to the electrical resistivity and Seebeck coefficient of an alloy. The effects of an individual element, i, can be represented by individual coefficients $k_{i}$ and $\kappa_{i}$ for the resistivity and Seebeck coefficient, respectively. Clustering and precipitation removes solutes from solution and, thus, results in changes in the resistivity $(\Delta \rho)$ and Seebeck $(\Delta S)$ coefficient. The $k_{i}$ and $\kappa_{i}$ can be used along with the measured $\Delta \rho$ and $\Delta S$ to estimate the total amount of precipitation.

Model Alloys: Simple Fe-Mn-Ni-P-... ferritic alloys used to study embrittlement mechanisms.

Small angle neutron scattering characterization (SANS): Small microstructural features, like CRPs, produce scatter of a well-collimated cold neutron beam at small angles. The scattering intensity as a function of both the scattering angle and the angle from the direction of a strong imposed magnetic field is reduced to magnetic and nuclear scattering cross sections. These scattering cross sections are analyzed to provide CRP (and MNP) $r_{p}, N_{p}$ and $f_{p}$ parameters, as well as estimates of the CRP (and MNP) 
composition. MF can also be detected by SANS in some cases, but generally produce only weak scattering. Well defined PPs and LBP MNPs can also be characterized by SANS.

Split melt model steels (SMMS): Special small melt heats of low alloy steels with controlled variations in combinations of $\mathrm{Cu}, \mathrm{Ni}, \mathrm{Mn}, \mathrm{P}$ and other minor elements heat treated to produce mechanical properties and microstructures very similar to RPV plates. 



\section{Appendix E. Points Excluded from Fitting Sets}

Twelve points were removed from the database during the model development process, as listed by group below.

\section{E.1 Low Cu Chauvenet Outliers}

See the description of Chauvenet outlier analysis in the Introduction for information on this analysis technique.

- Heat FGIN02, plant Ginna, forging in capsule T (1 point),

- Heat WFA201, plant Farley 2, weld in capsules X and Z (2 points),

- Heat WCL101, plant Callaway 1, weld in capsule U (1 point)

The above four points were identified as Chauvenet outliers during preliminary low-Cu modeling and remained Chauvenet outliers relative to the revised model. FGIN02 has the largest recorded negative shift $\left(-35^{\circ} \mathrm{F}\right)$ in all the PWR data (the physical impossibility of such a large negative shift suggests a possibly incorrect value of unirradiated $\mathrm{T}_{30}$ ), and WFA201 has the lowest recorded Mo of all materials in the database (the Mo value was checked by a member of ASTM E10.02 and is believed to be correct).

\section{E.2 High-Cu Chauvenet Outliers}

- Heat PBR_01, plant Big Rock Point, plate in capsule 124 (1 point)

This is one of the atypical BWR points, with the highest flux, fluence, and temperature of all data in the database $\left(1.63 \times 10^{12} \mathrm{n} / \mathrm{cm}^{2} / \mathrm{s}\right.$ flux, $1.07 \times 10^{20} \mathrm{n} / \mathrm{cm}^{2}$ fluence, $\left.570^{\circ} \mathrm{F}\right)$. It was found to be a Chauvenet outlier in the preliminary amplitude study of high- $\mathrm{Cu}$, high-fluence $\left(>2 \times 10^{19} \mathrm{n} / \mathrm{cm}^{2}\right)$ data and was excluded from further consideration. It could also be excluded on the basis of its unusual irradiation, which is well beyond the flux and fluence values expected in applications, so it is listed below as well.

The four Chauvenet outliers identified in the NUREG/CR-6551 [1] and July 2000 [2] modeling efforts remained outliers relative to the revised model:

- Heat WCK101, plant Cook 1, weld in capsules U and T (2 points)

- Heat WSQ201, plant Sequoyah 2, weld in capsule U (1 point)

- Heat WTM201, plant Surry 2, weld in capsule W1 (1 point)

\section{E.3 Unusual Irradiations}

Three points in the high- $\mathrm{Cu}$ subset were excluded from the modeling sets because they were irradiated in two reactors at substantially different irradiation temperatures $\left(T_{i}\right)$ or in both a PWR and a BWR reactor at different flux values. Since the damage accumulation varies with $T_{i}$ and flux, representative values of $\mathrm{T}_{\mathrm{i}}$ and flux for these three points were considered more uncertain than usual:

- Heat PMON01, plants Monticello (BWR) and Prairie Island 1(PWR), plate in capsule W

- Heat WTP301, plants Turkey Point 3 and Davis Besse 1, weld in capsules V and A5

- Heat WZN101, plants Zion 1 and Davis Besse 1, weld in capsules Y and A5

Other points irradiated in two reactors were left in the modeling datasets if the reactors were of like kind and similar $\mathrm{T}_{\mathrm{i}}$. Where small $\mathrm{T}_{\mathrm{i}}$ variations occurred during irradiation, usually due to operating condition changes, a time-weighted average $\mathrm{T}_{\mathrm{i}}$ was used. 
As noted above, the following point can be considered both an unusual irradiation and a Chauvenet outlier, so it is listed in both places.

- Heat PBR_01, plant Big Rock Point, plate in capsule 124 (1 point)

This is one of the atypical BWR points, with the highest flux, fluence, and temperature of all data in the database $\left(1.63 \times 10^{12} \mathrm{n} / \mathrm{cm}^{2} / \mathrm{s}\right.$ flux, $1.07 \times 10^{20} \mathrm{n} / \mathrm{cm}^{2}$ fluence, $\left.570^{\circ} \mathrm{F}\right)$.

\section{E.4 References}

1. Eason, E. D., J. E. Wright, and G. R. Odette, Improved Embrittlement Correlations for Reactor Pressure Vessel Steels, NUREG/CR-6551, U.S. Nuclear Regulatory Commission, Washington D.C., 1998.

2. Kirk, M., C. Santos, E. D. Eason, J. E. Wright, and G. R. Odette, "Updated Embrittlement Trend Curve for Reactor Pressure Vessel Steels," Proc. Structural Mechanics in Reactor Technology (SMiRT) Conference, 2003, 2003. 


\section{Appendix F. Residual Plots for Low-Cu and High-Cu Datasets, Baseline Model}

The plots in Figs. F.1 through F.28 are based on the following definition of residual:

$$
\text { Residual }=\text { Model TTS }- \text { Measured TTS, }
$$

which implies that a negative residual is an underestimate by the model of the actual shift. The linear trend of the residuals fitted by least squares is shown by a dashed line on each plot. A residual plot for a variable in the model that shows no significant residual slope trend, denoted "N.S." in the legend, indicates that the model correctly captures the trend of the variable to first order. A similar result for a variable not included in the model implies that the variable would be unlikely to improve the fit significantly if it were added.

The residual plots for the low-Cu data, Figs. F.1 through F.13, are based on the matarix feature (MF) term model, Eqs. (4-3) and (4-4). The residual plots for the high-Cu data, Figs. F.14 through F.28, are based on the complete model, including both MF and copper-rich-precipitate (CRP) terms, i.e., Eqs. (4-1), (4-3), (4-4), and (4-5). 


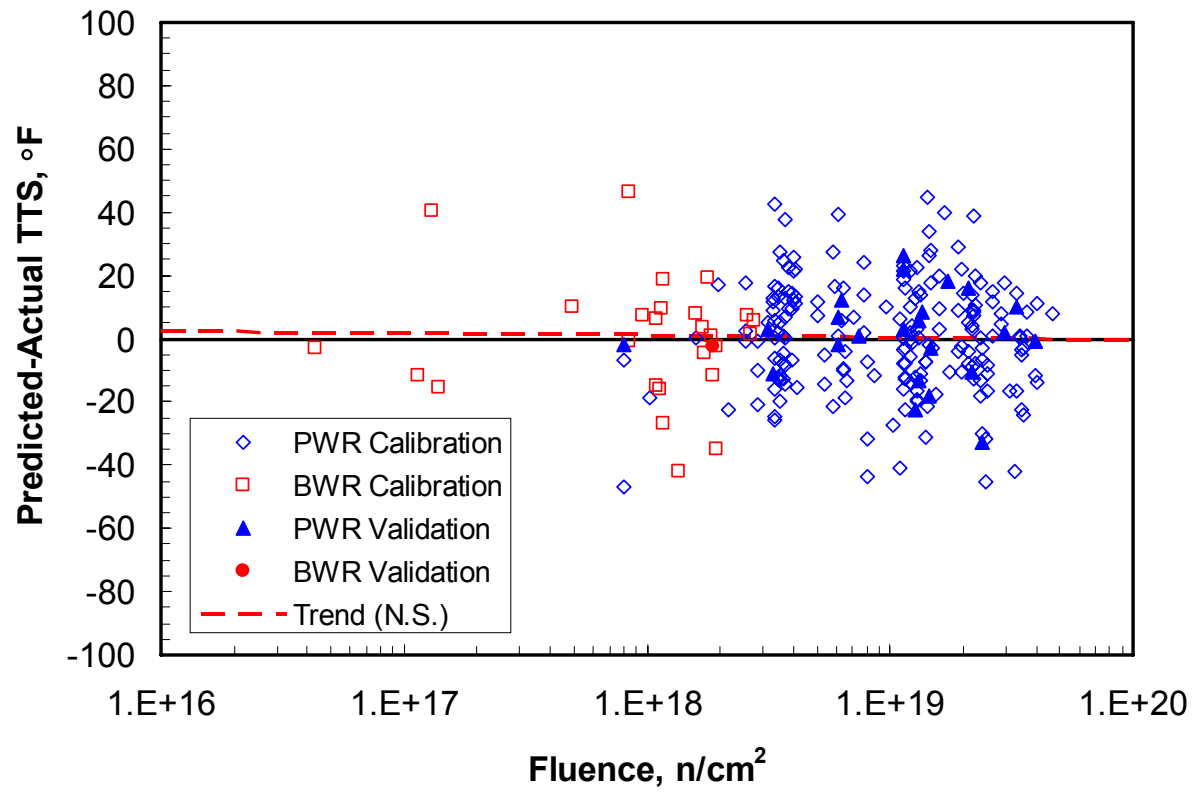

Fig. F.1. Residuals plotted against fluence, calibration and validation data with $\mathrm{Cu} \leq 0.072$ wt $\%$.

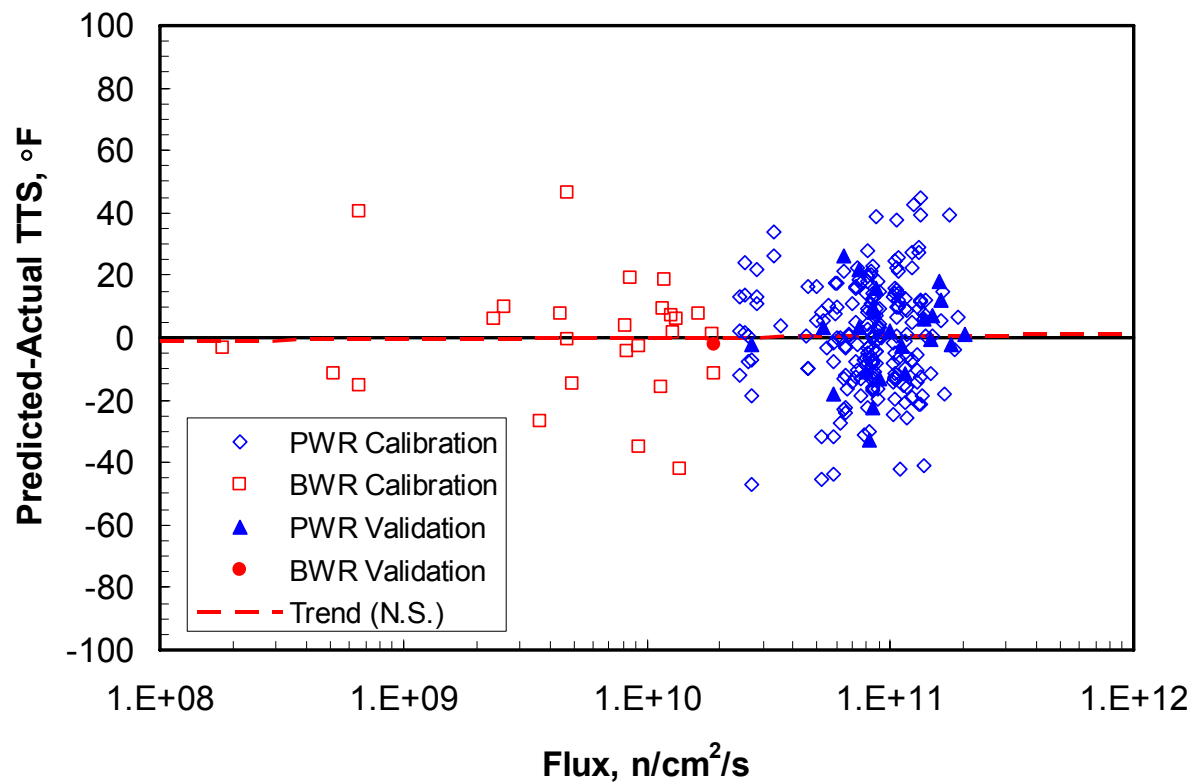

Fig. F.2. Residuals plotted against flux, calibration and validation data with $\mathrm{Cu} \leq 0.072$ wt \%. 


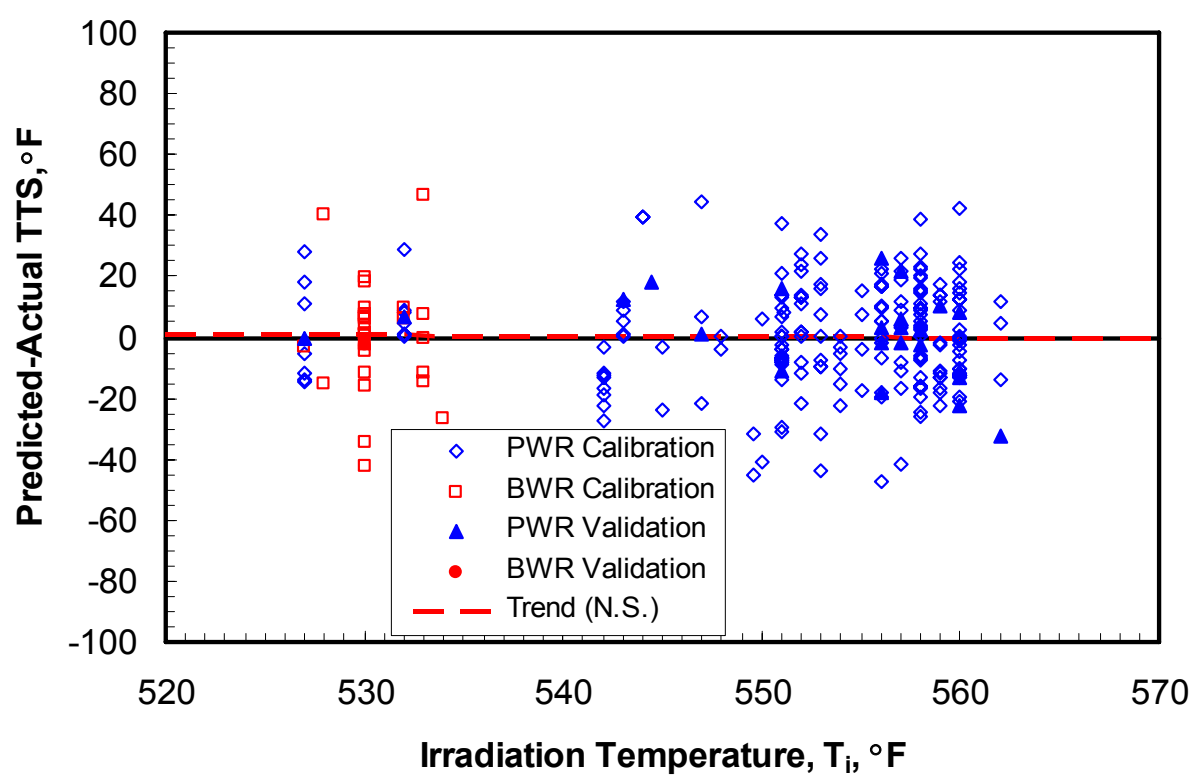

Fig. F.3. Residuals plotted against $T_{i}$, calibration and validation data with $\mathrm{Cu} \leq 0.072$ wt \%.

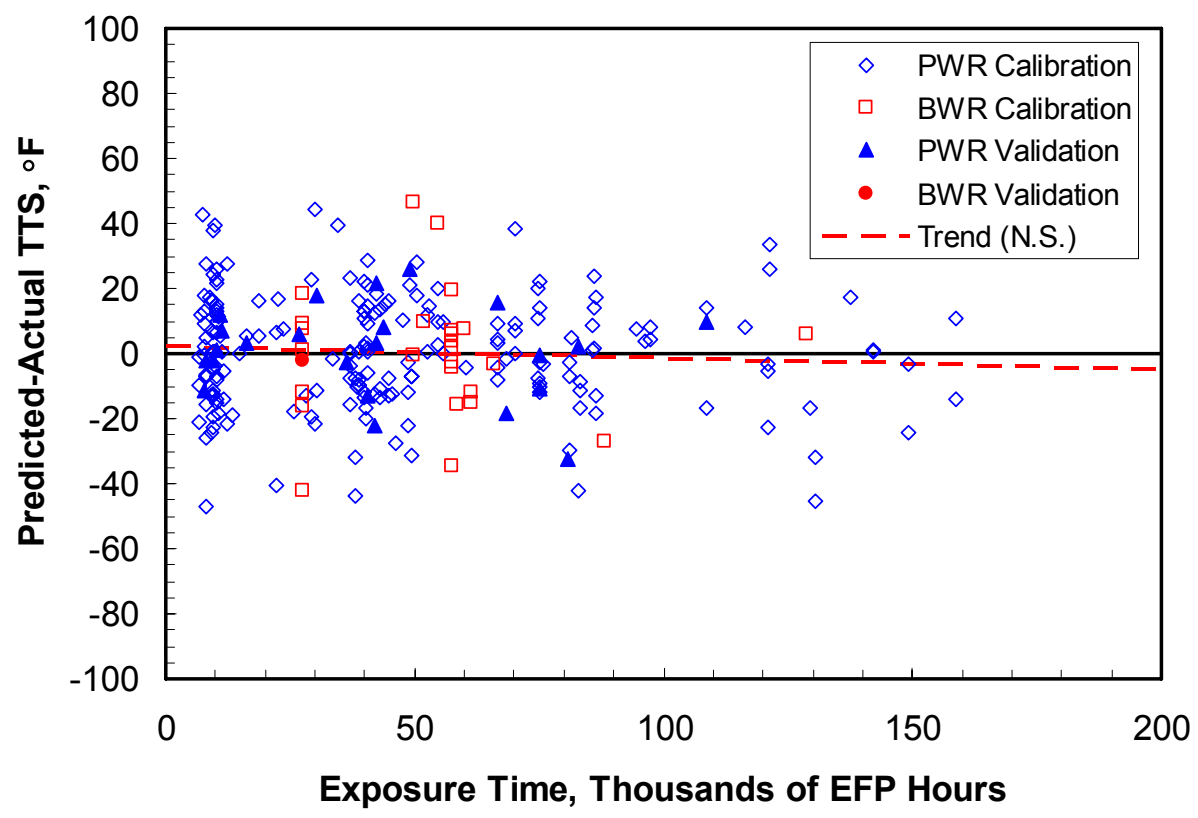

Fig. F.4. Residuals plotted against time, calibration and validation data with $\mathrm{Cu} \leq 0.072$ wt \%. 


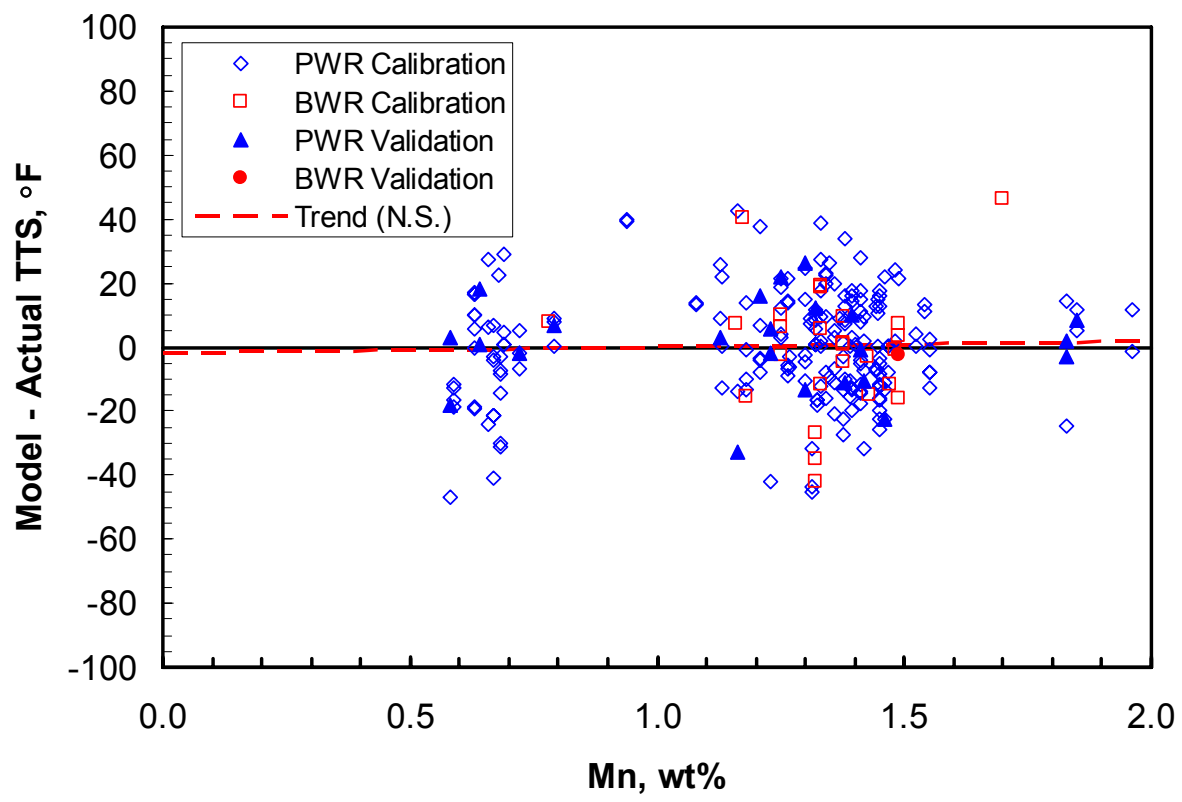

Fig. F.5. Residuals plotted against $\mathrm{Mn}$, calibration and validation data with $\mathrm{Cu} \leq 0.072$ wt \%.

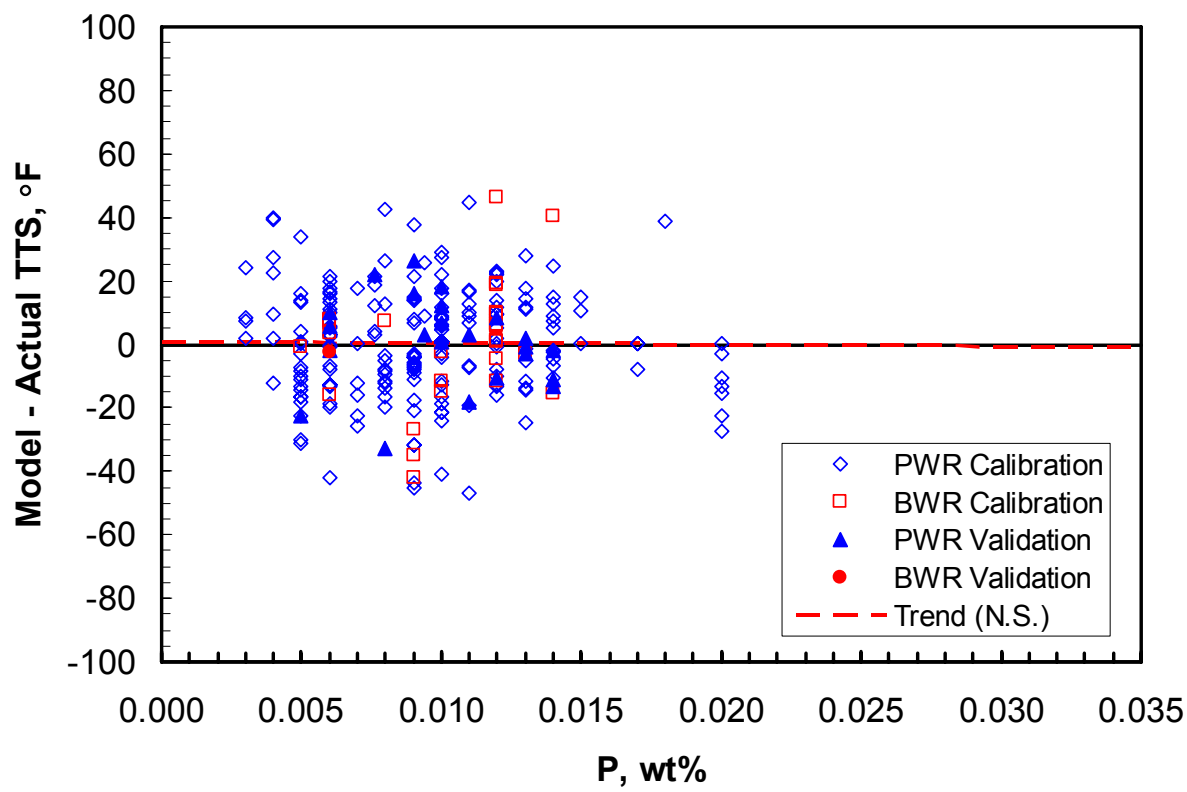

Fig. F.6. Residuals plotted against $P$, calibration and validation data with $\mathrm{Cu} \leq \mathbf{0 . 0 7 2}$ wt \%. 


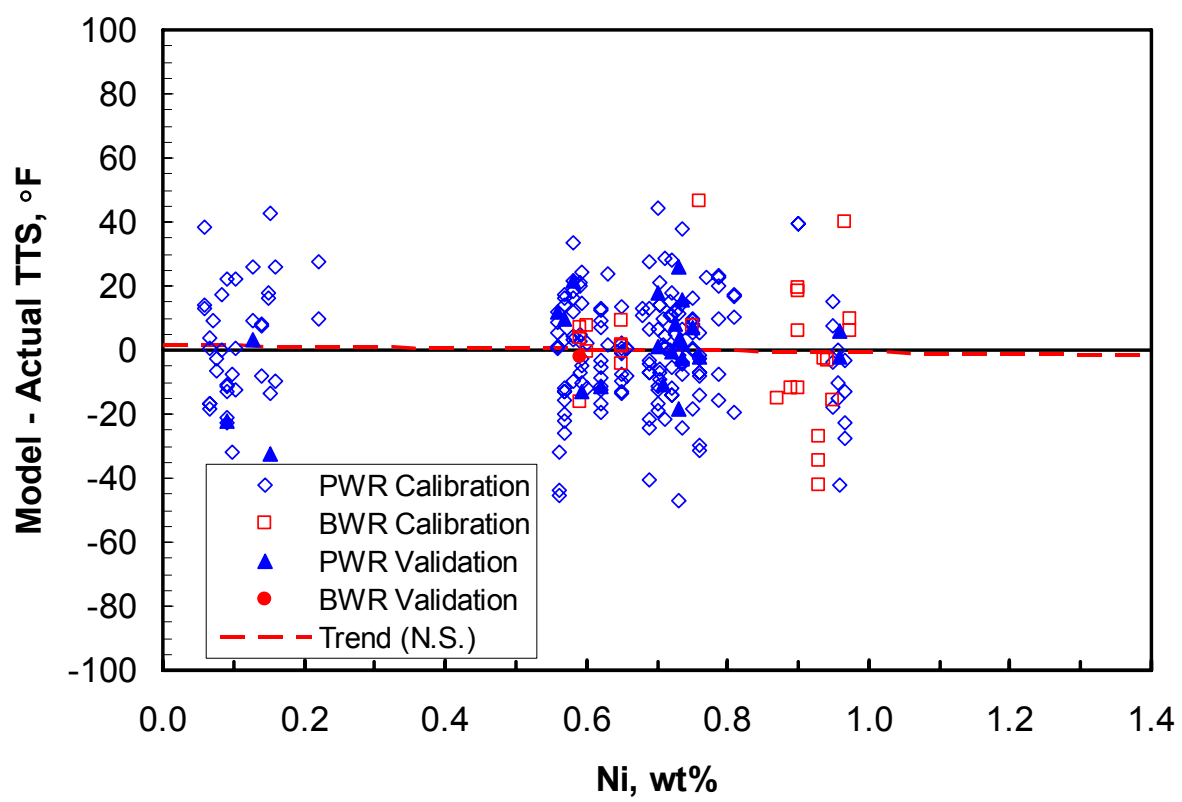

Fig. F.7. Residuals plotted against Ni, calibration and validation data with $\mathrm{Cu} \leq \mathbf{0 . 0 7 2}$ wt \%.

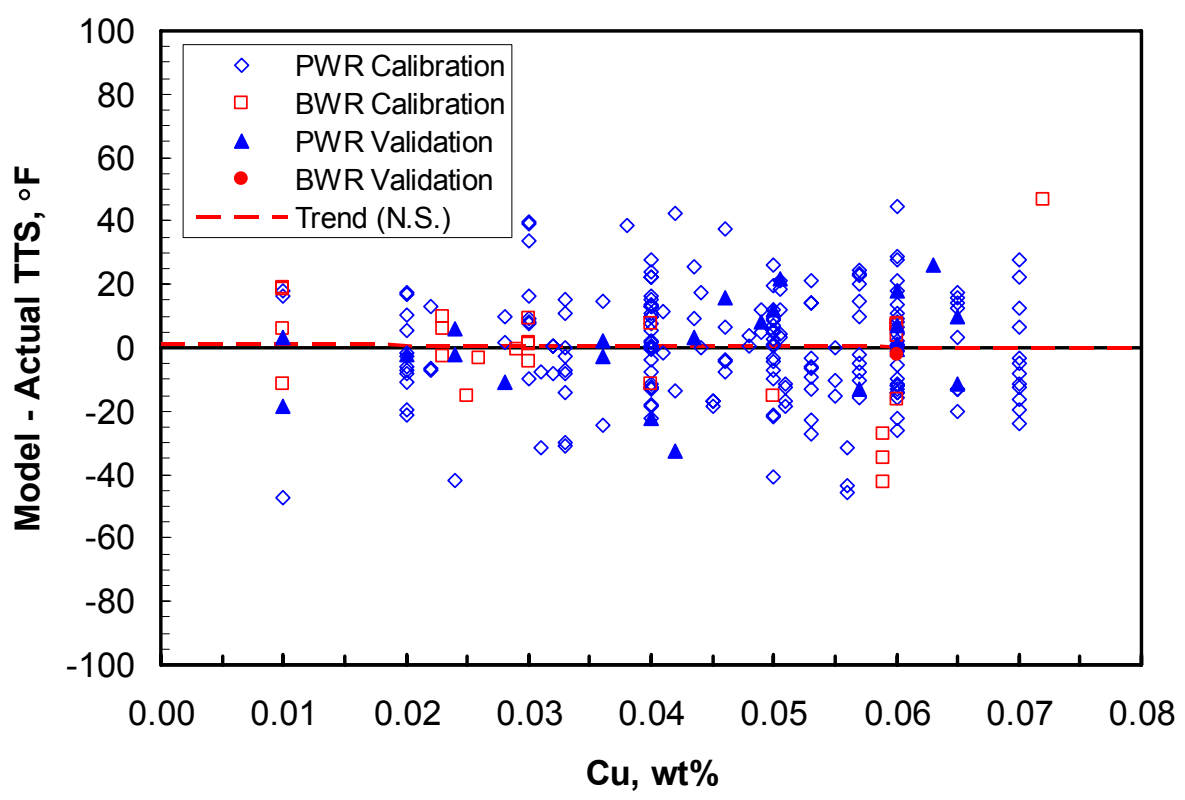

Fig. F.8. Residuals plotted against $\mathrm{Cu}$, calibration and validation data with $\mathrm{Cu} \leq 0.072$ wt \%. 


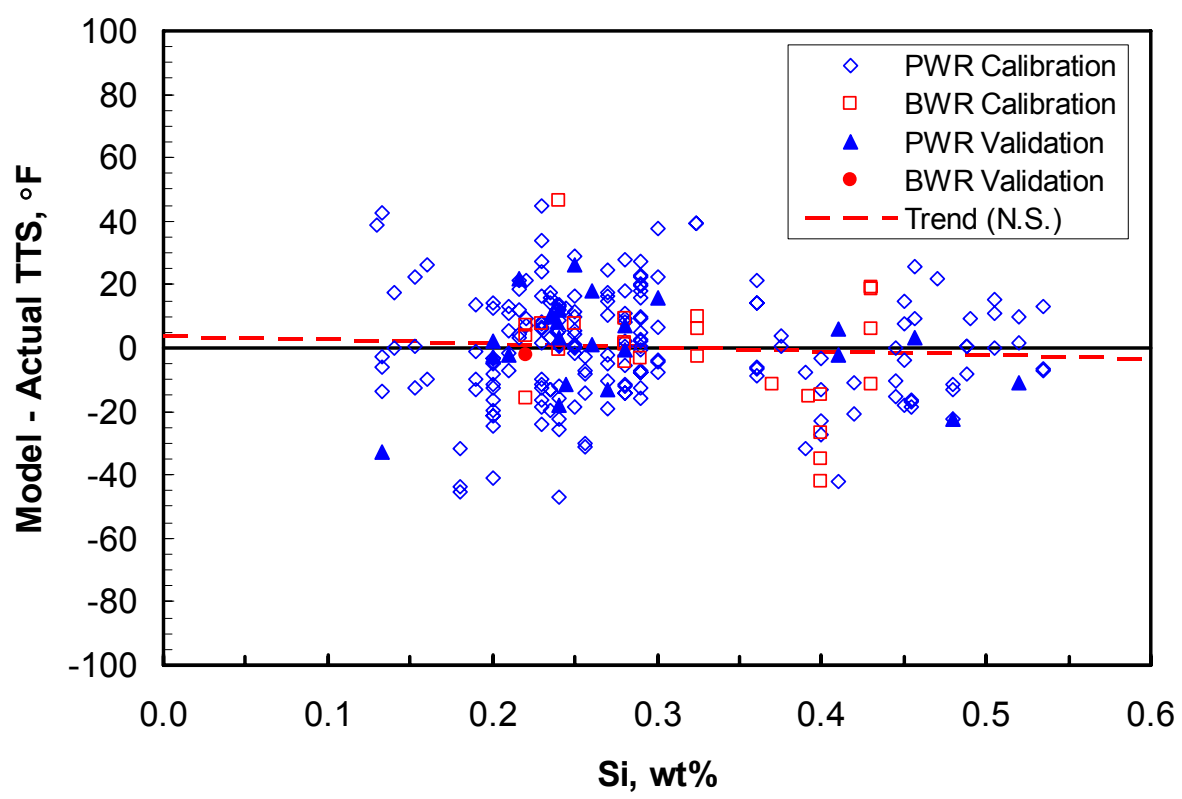

Fig. F.9. Residuals plotted against $\mathrm{Si}$, calibration and validation data with $\mathrm{Cu} \leq \mathbf{0 . 0 7 2}$ wt \%.

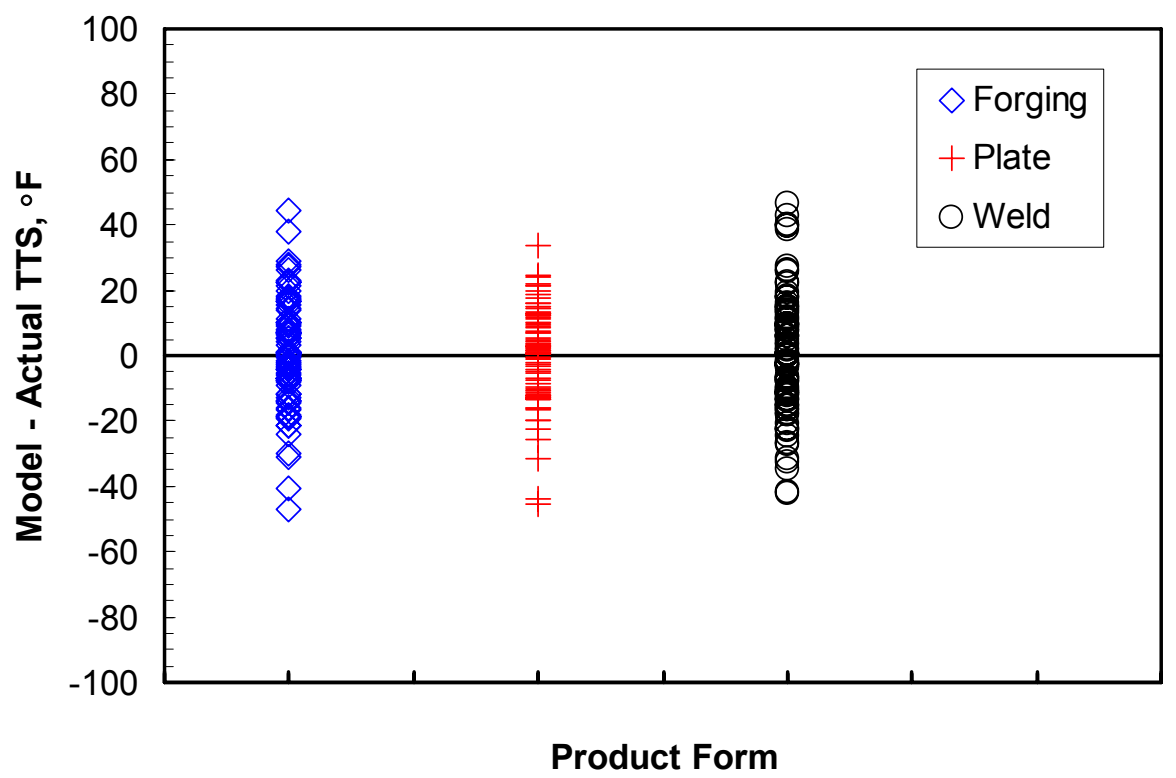

Fig. F.10. Residuals plotted against Product Form, calibration and validation data with $\mathrm{Cu} \leq \mathbf{0 . 0 7 2}$ wt \%. 


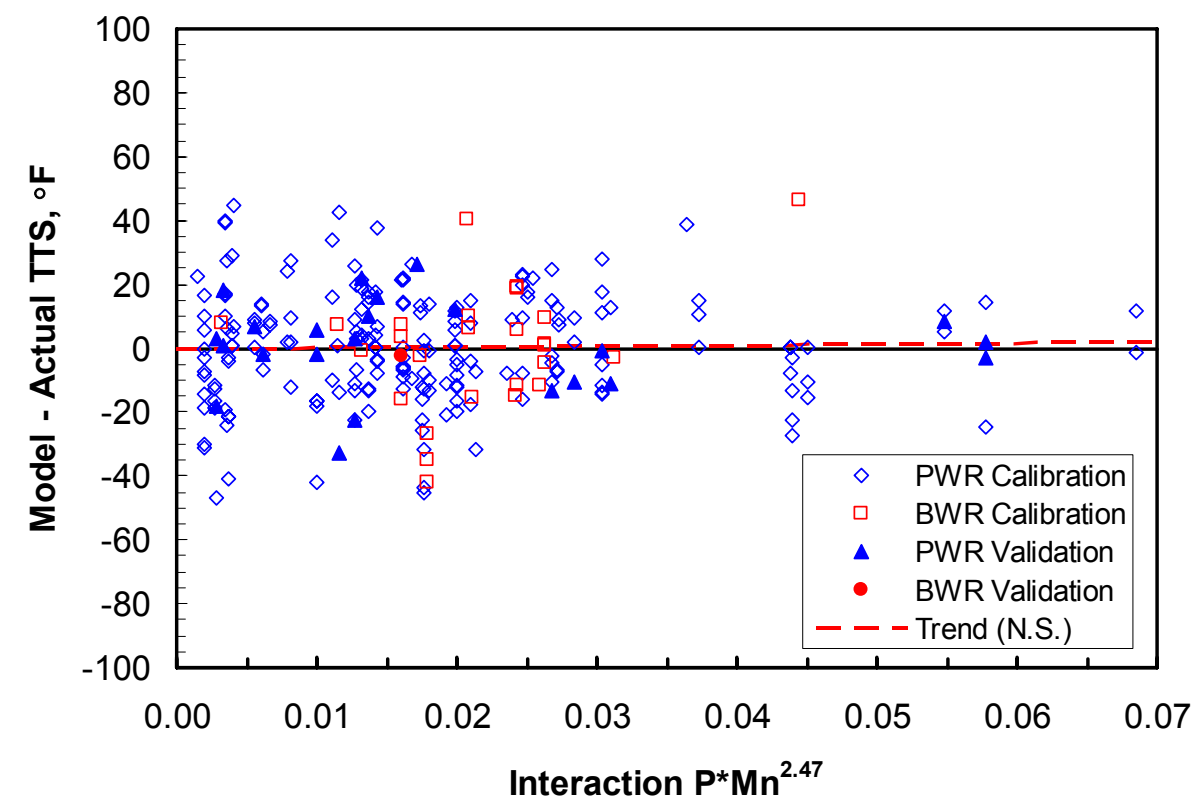

Fig. F.11. Residuals plotted against $P * M^{2.47}$, calibration and validation data with $\mathrm{Cu} \leq \mathbf{0 . 0 7 2} \mathrm{wt} \%$.

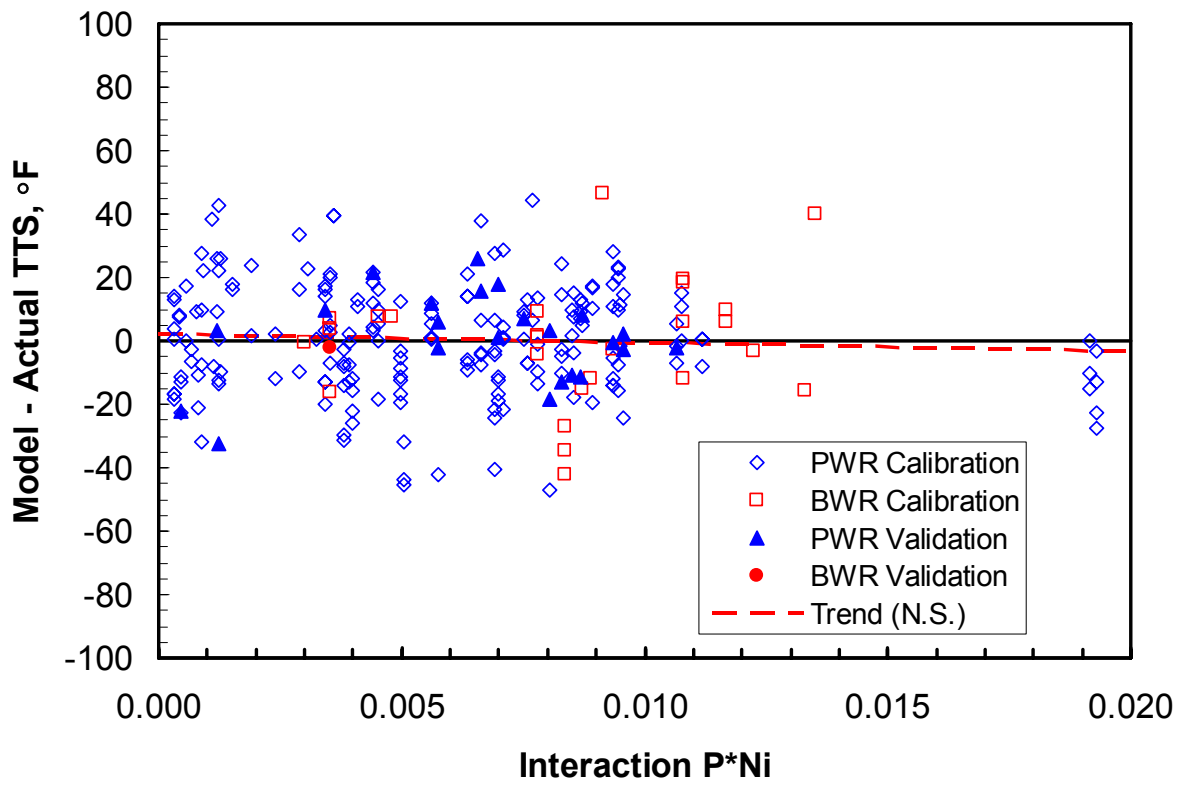

Fig. F.12. Residuals plotted against $\mathrm{P} * \mathrm{Ni}$, calibration and validation data with $\mathrm{Cu} \leq 0.072$ wt \%. 


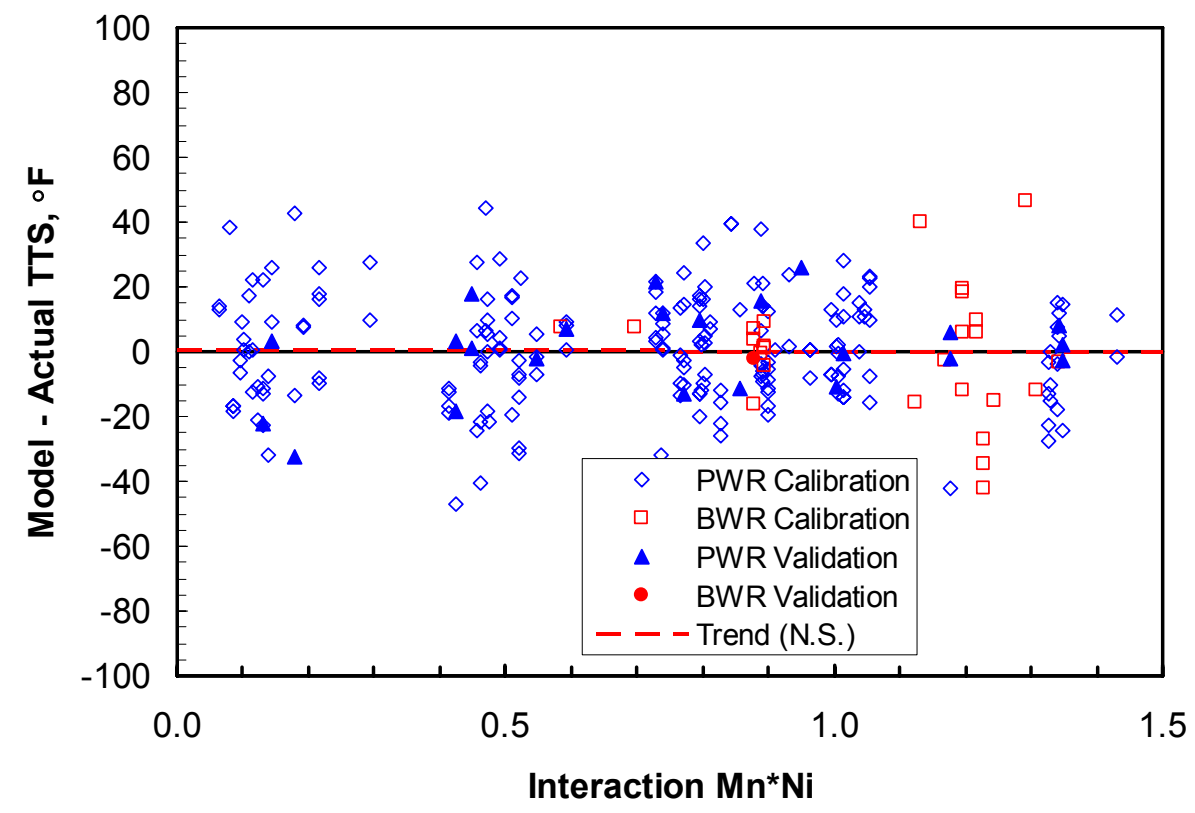

Fig. F.13. Residuals plotted against $\mathrm{Mn} * \mathrm{Ni}$, calibration and validation data with $\mathrm{Cu} \leq \mathbf{0 . 0 7 2}$ wt \%.

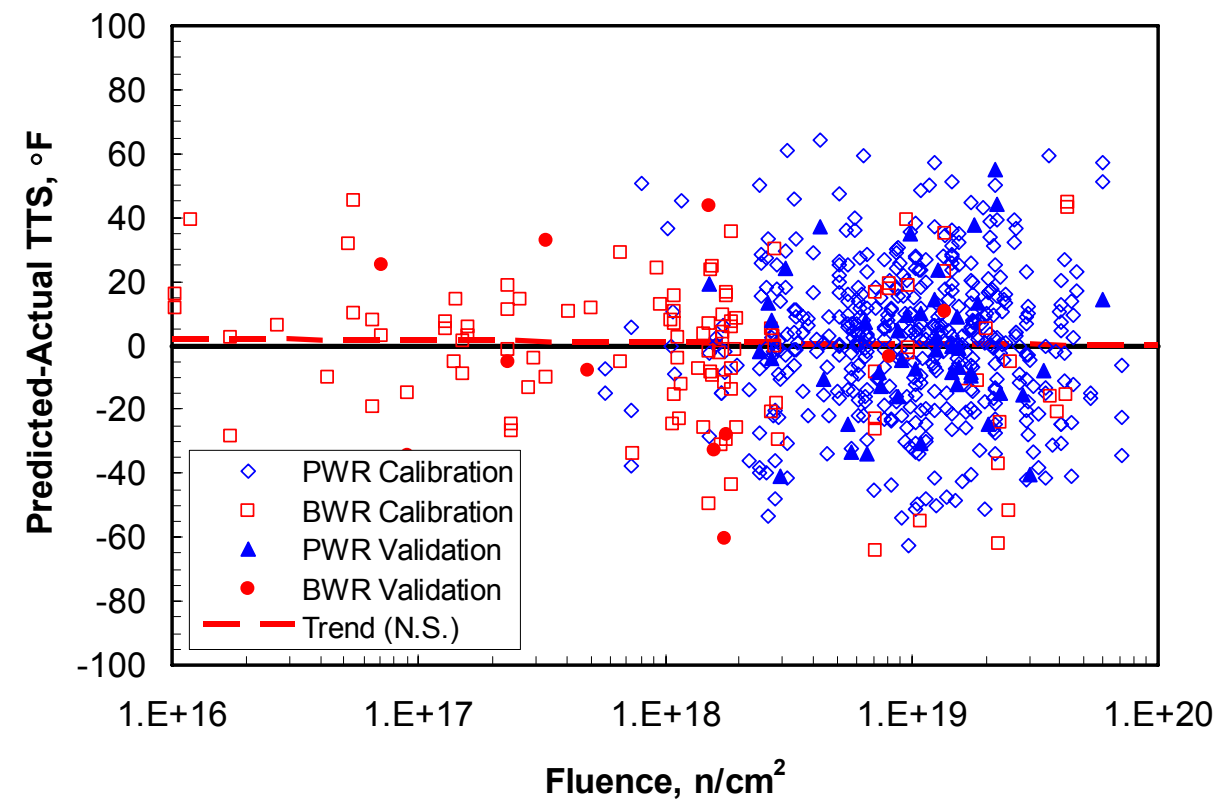

Fig. F.14. Residuals plotted against Fluence, calibration and validation data with $\mathrm{Cu}>0.072 \mathrm{wt} \%$. 


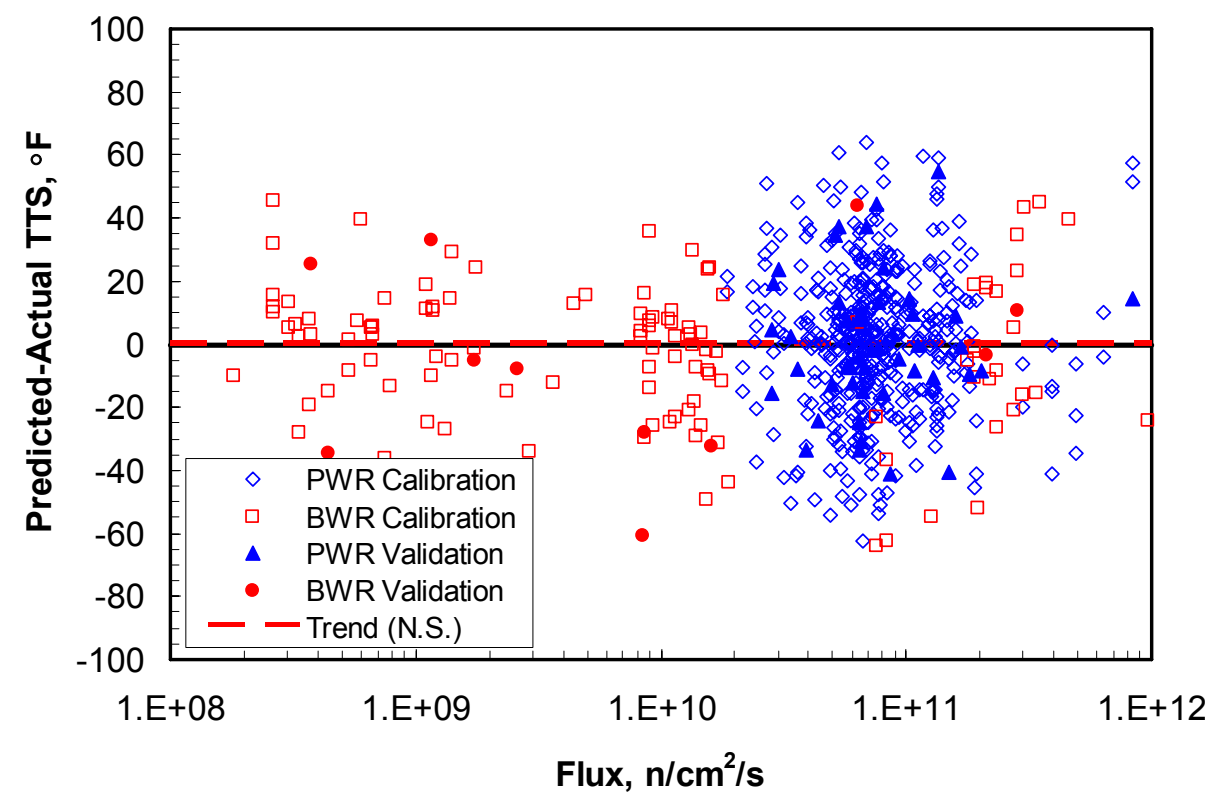

Fig. F.15. Residuals plotted against Flux, calibration and validation data with $\mathrm{Cu}>0.072$ wt \%.

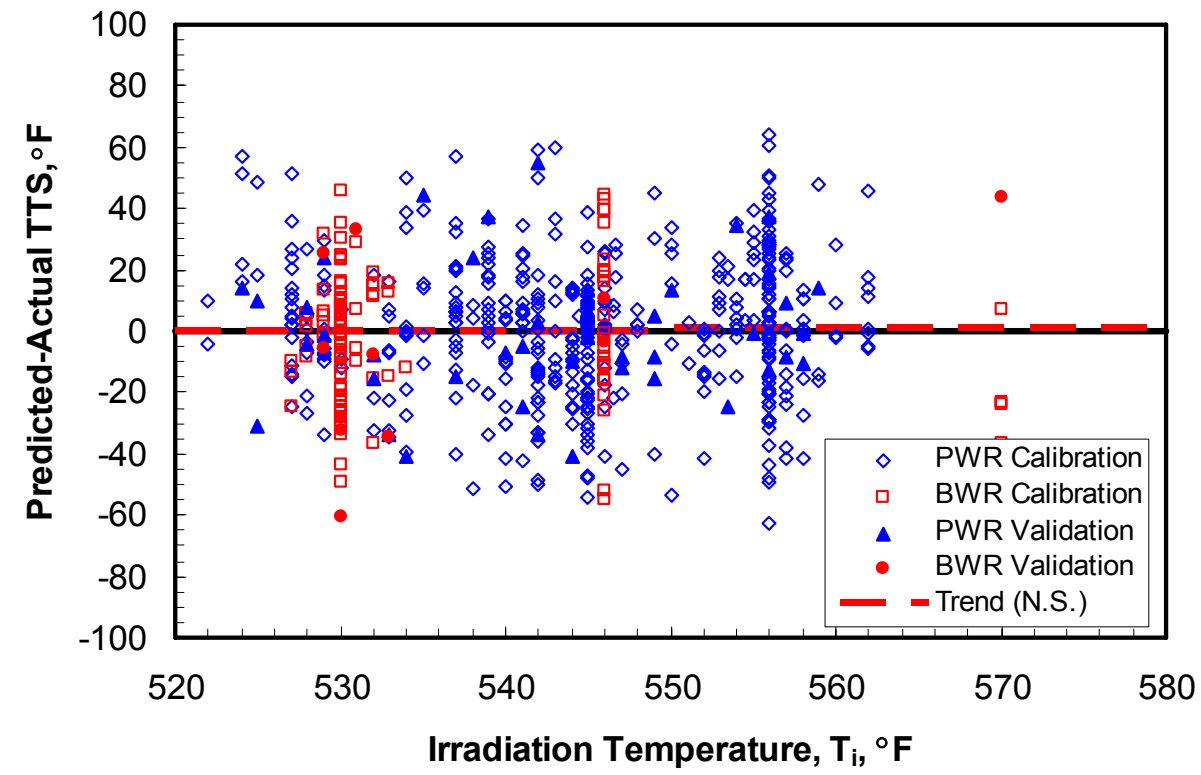

Fig. F.16. Residuals plotted against $T_{i}$, calibration and validation data with $\mathrm{Cu}>0.072$ wt \%. 


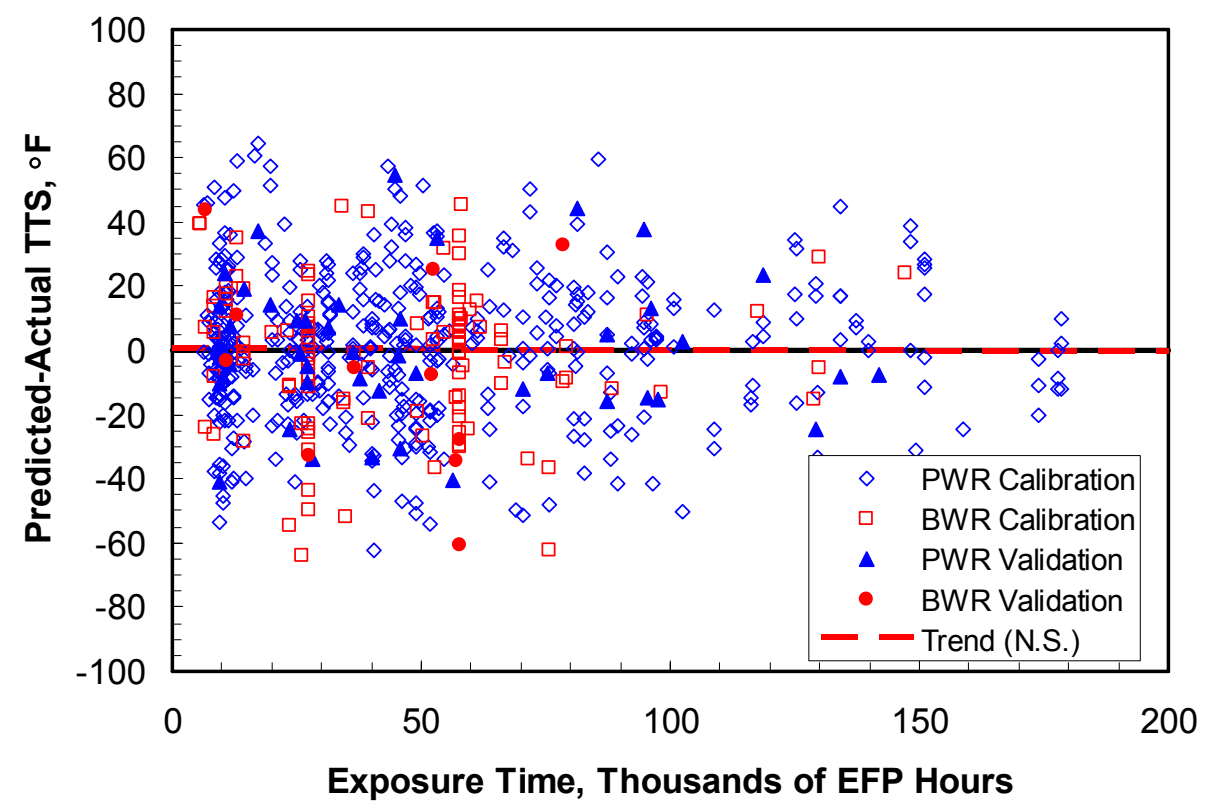

Fig. F.17. Residuals plotted against Time, calibration and validation data with $\mathrm{Cu}>0.072$ wt \%.

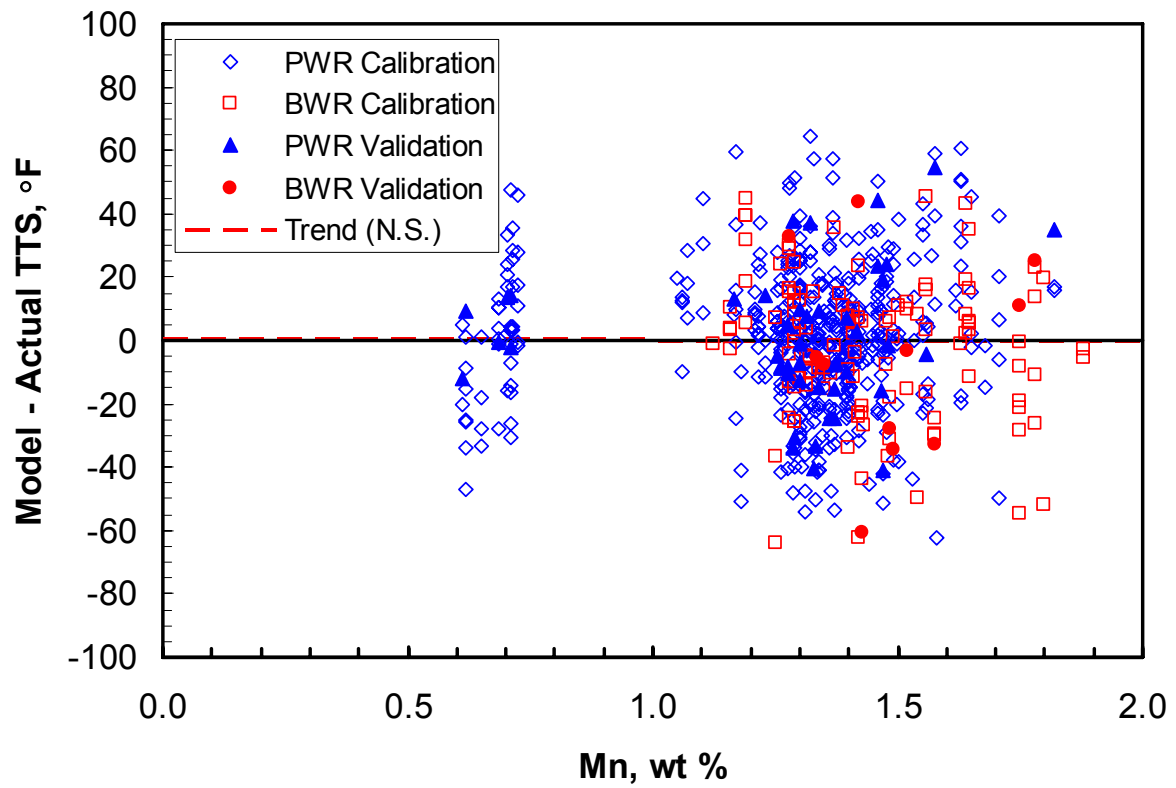

Fig. F.18. Residuals plotted against $M n$, calibration and validation data with $\mathrm{Cu}>0.072$ wt \%. 


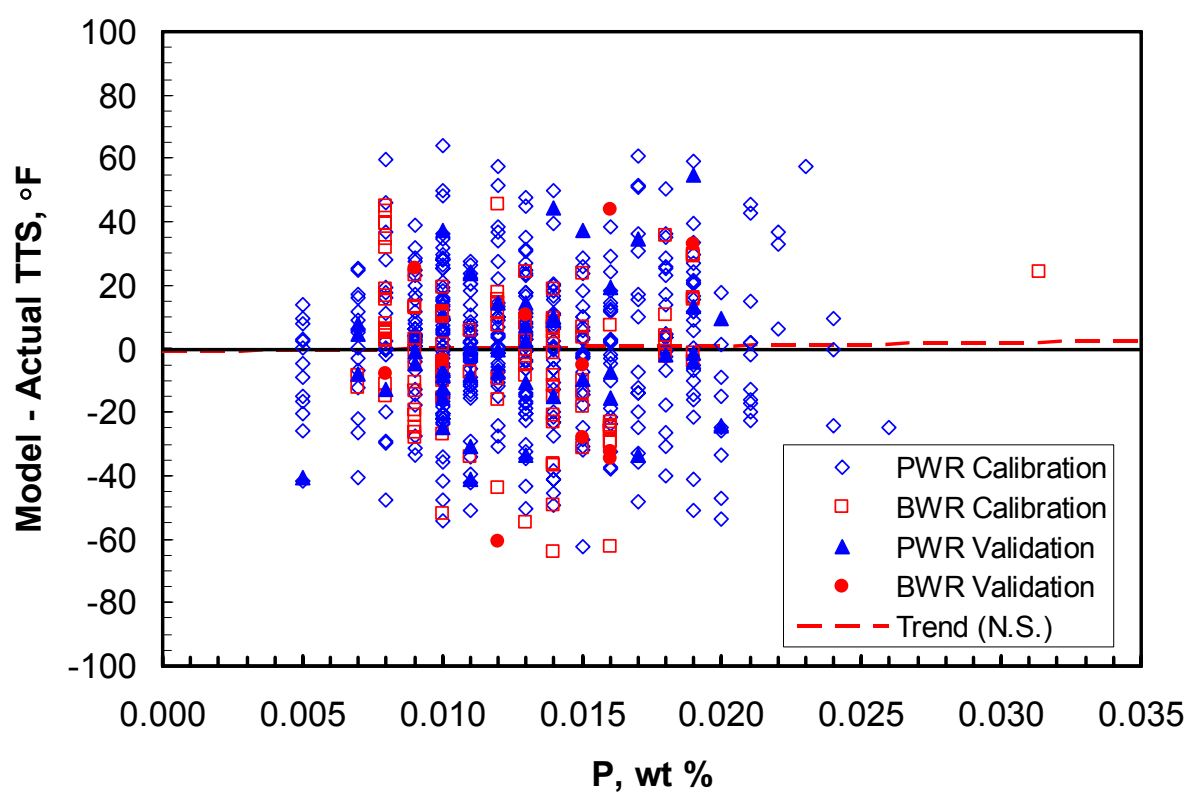

Fig. F.19. Residuals plotted against $P$, calibration and validation data with $\mathrm{Cu}>0.072$ wt \%.

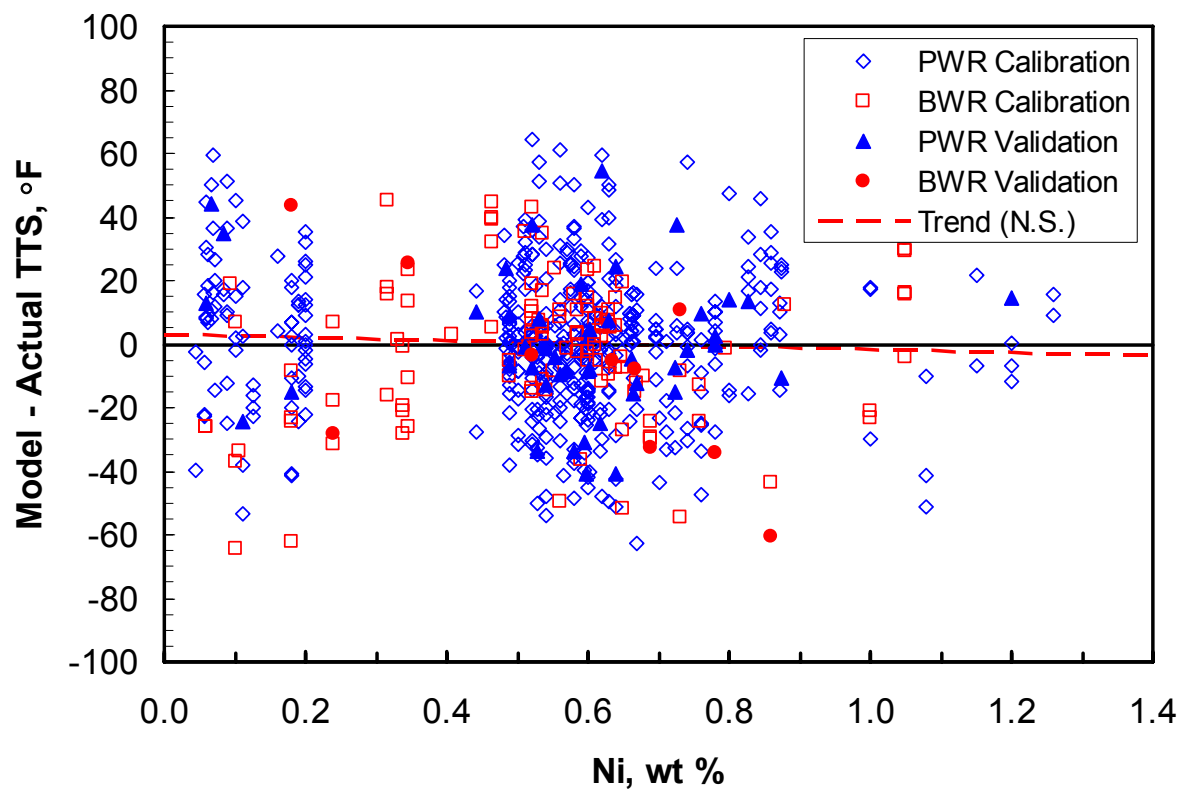

Fig. F.20. Residuals plotted against $\mathrm{Ni}$, calibration and validation data with $\mathrm{Cu}>0.072$ wt \%. 


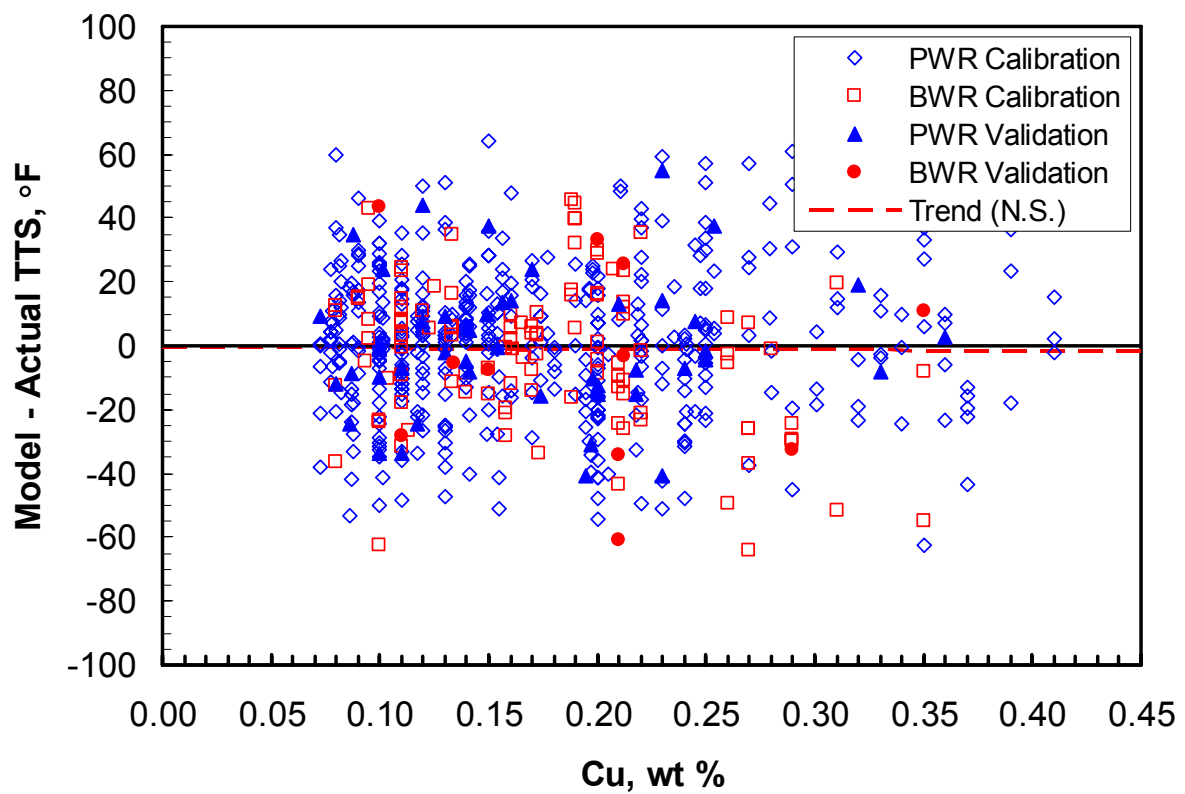

Fig. F.21. Residuals plotted against $\mathrm{Cu}$, calibration and validation data with $\mathrm{Cu}>0.072$ wt \%.

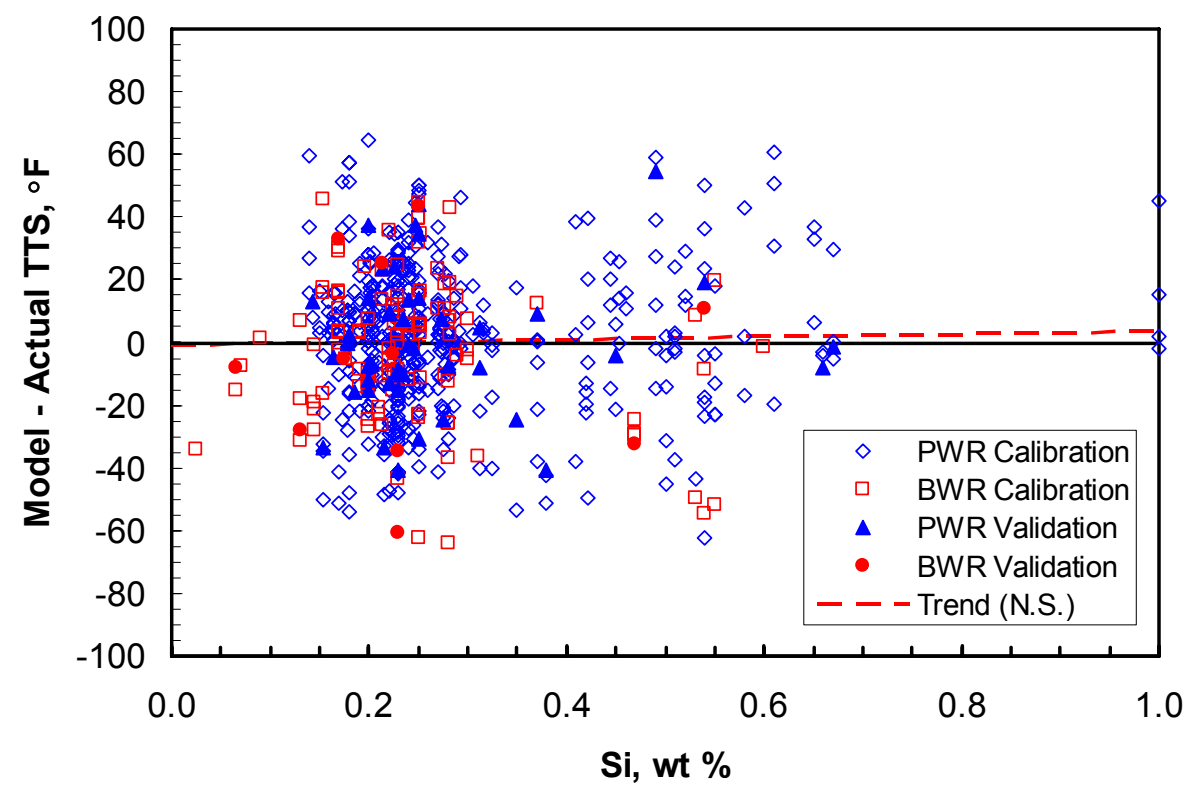

Fig. F.22. Residuals plotted against Si, calibration and validation data with $\mathrm{Cu}>0.072$ wt \%. 


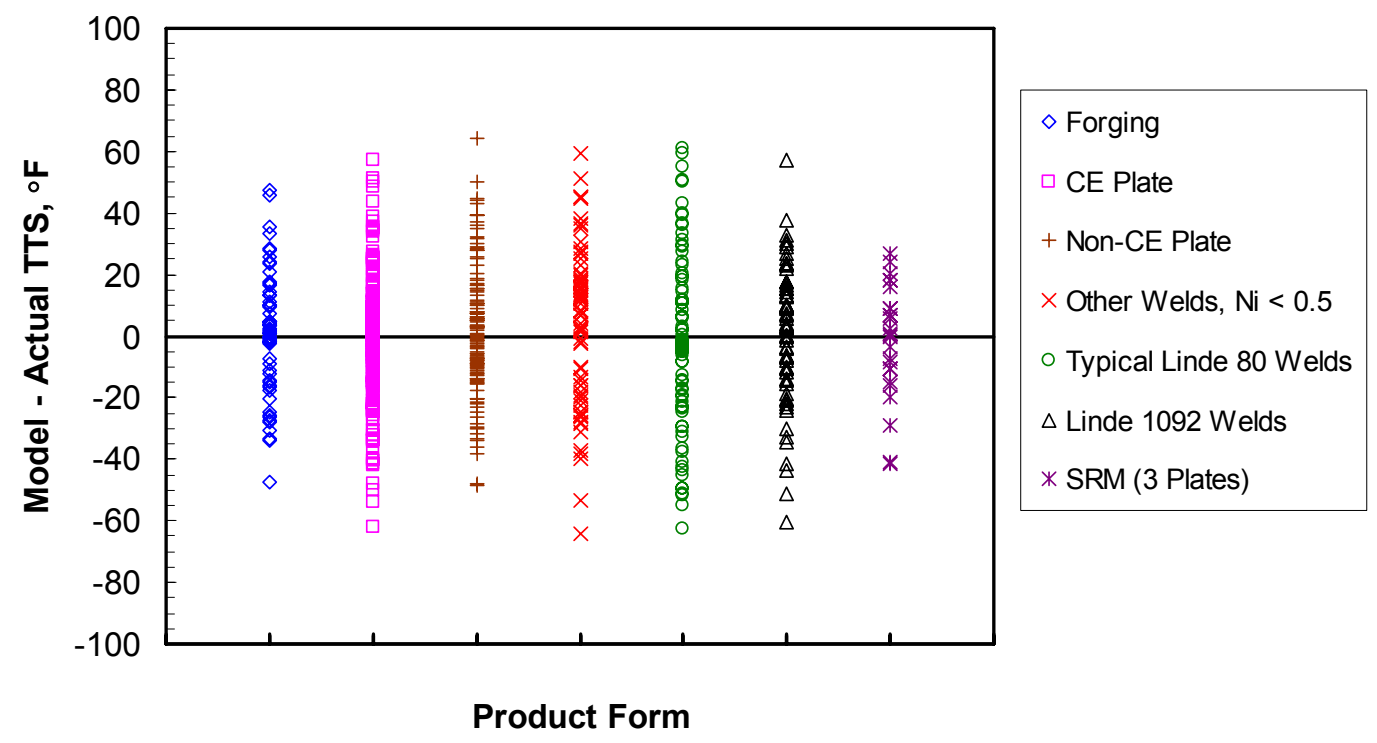

Fig. F.23. Residuals plotted against Product Form, calibration and validation data with $\mathrm{Cu}>0.072$ wt \%.

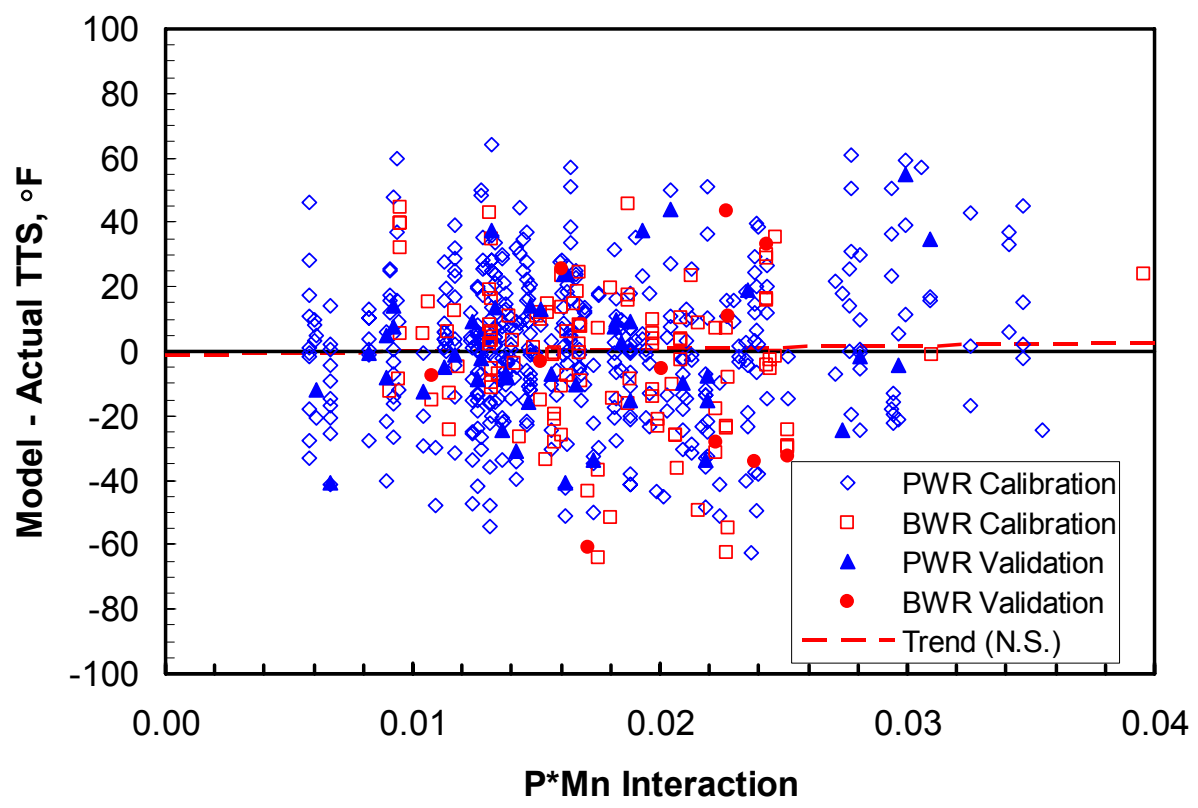

Fig. F.24. Residuals plotted against $P * M n$, calibration and validation data with $\mathrm{Cu}>0.072$ wt \%. 


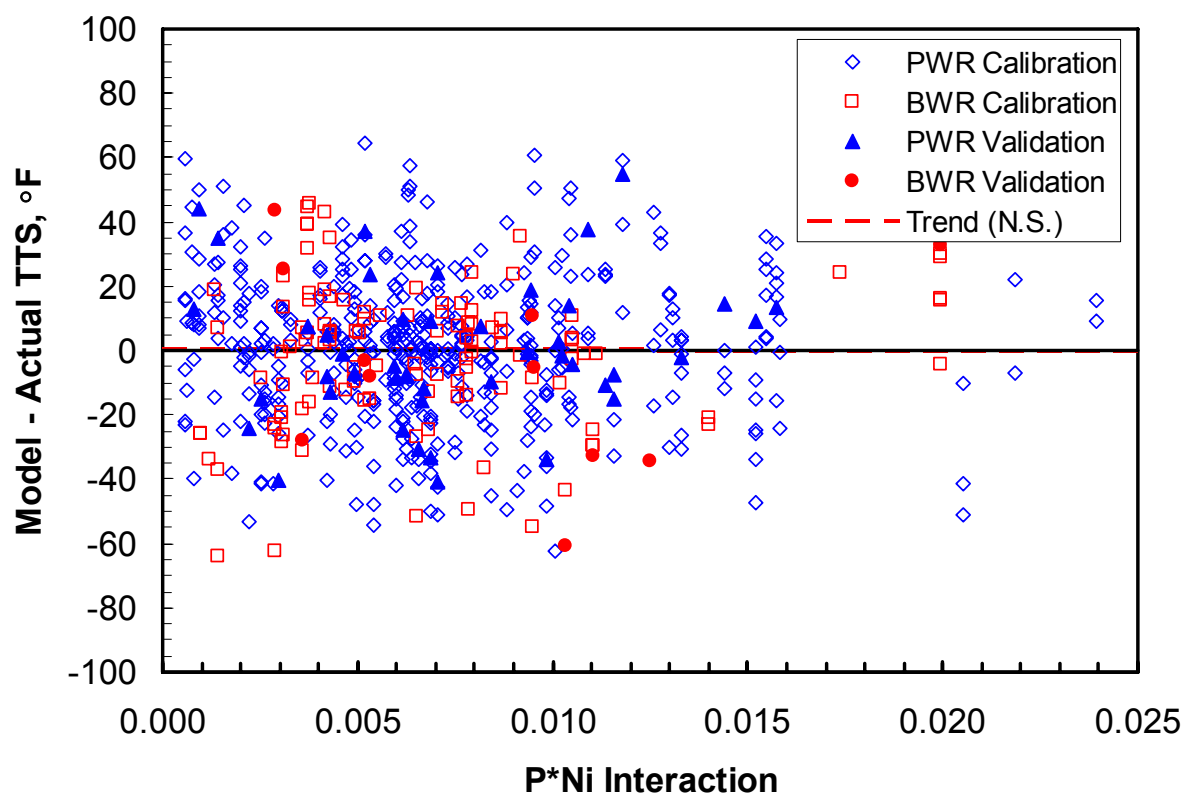

Fig. F.25. Residuals plotted against $P * N i$, calibration and validation data with $\mathrm{Cu}>0.072$ wt \%.

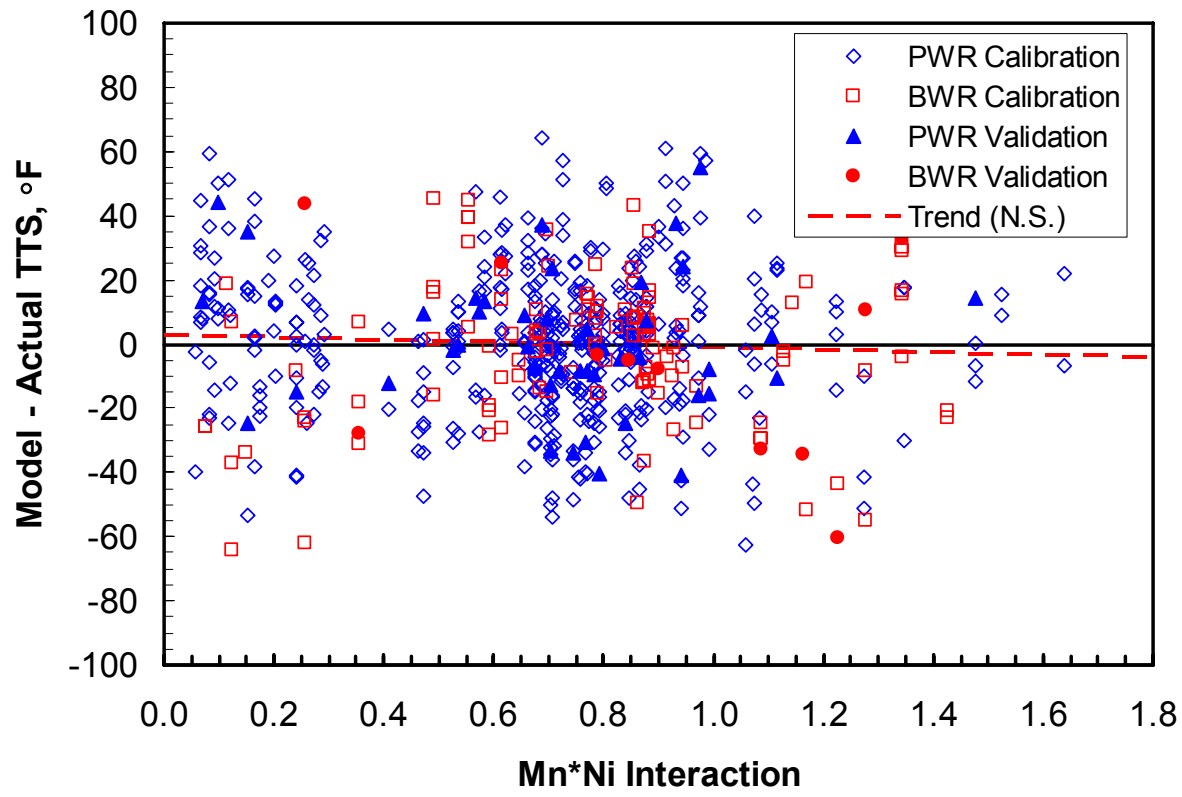

Fig. F.26. Residuals plotted against $\mathrm{Mn} * \mathrm{Ni}$, calibration and validation data with $\mathrm{Cu}>0.072$ wt \%. 


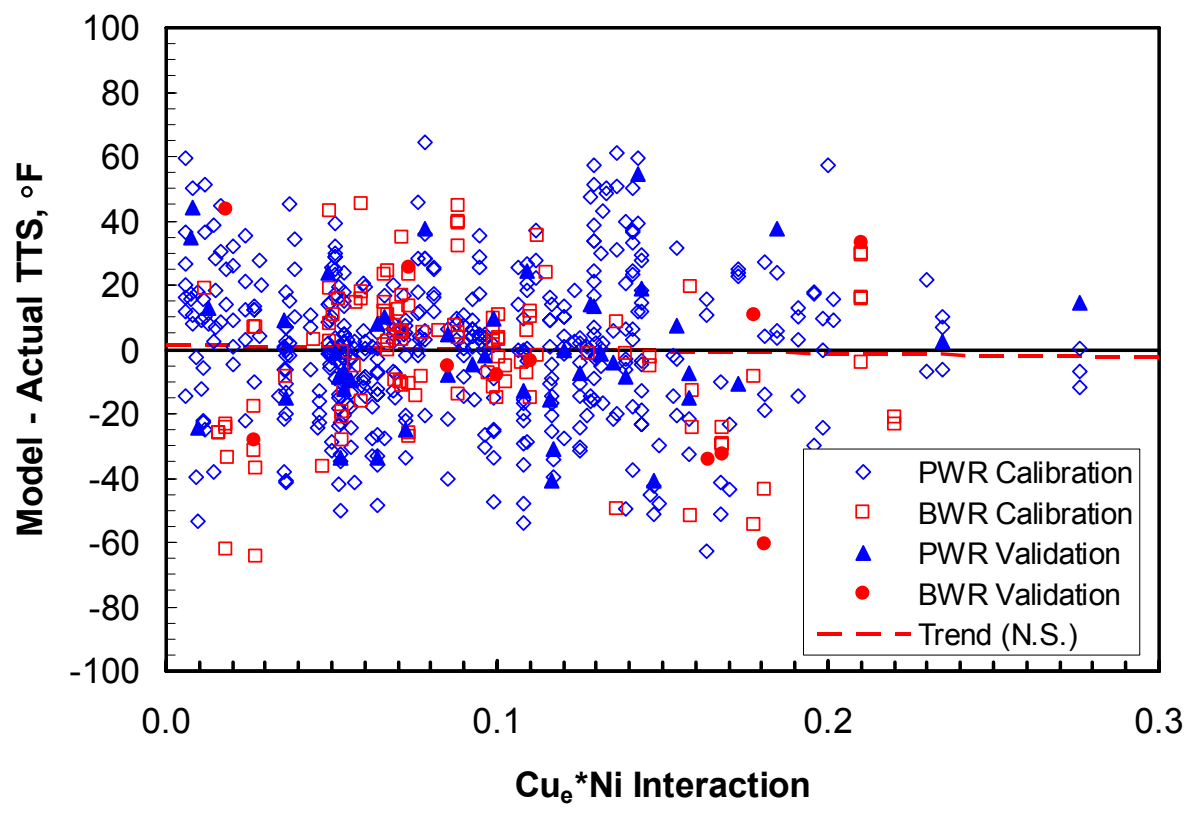

Fig. F.27. Residuals plotted against $\mathrm{Cu}_{\mathrm{e}}{ }^{* \mathrm{Ni}}$, calibration and validation data with $\mathrm{Cu}>0.072 \mathrm{wt} \%$.

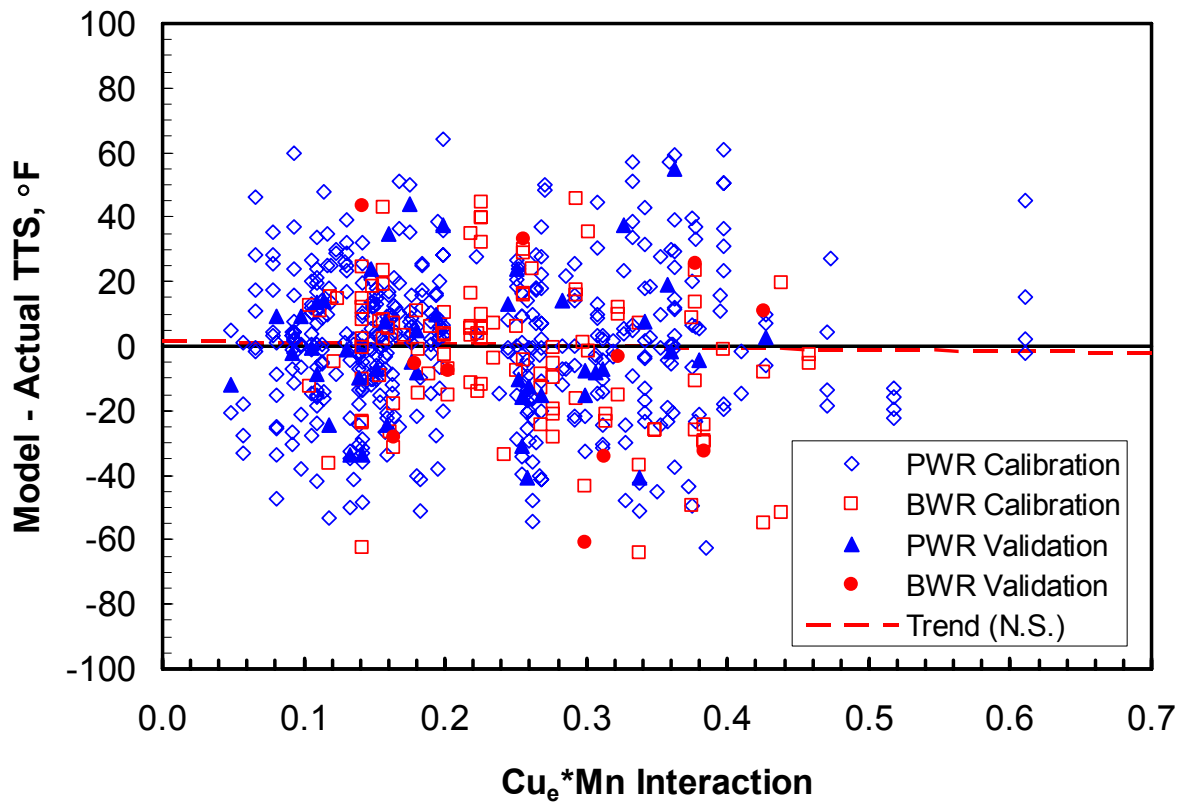

Fig. F.28. Residuals plotted against $\mathrm{Cu}_{\mathrm{e}}{ }^{*} \mathrm{Mn}$, calibration and validation data with $\mathrm{Cu}>0.072$ wt \%. 



\section{Appendix G. Means, Standard Deviations, and Range of Chemistry Variables by Material Group, Surveillance Data}

The statistics in the following tables were calculated for the referenced material groups in the surveillance database, giving each heat in each group equal weight regardless of the number of shift estimates available for that heat. The number of heats used in the statistics for each sample is given by $n$.

Table G.1. Low-Cu Materials

$\mathrm{Cu} \leq 0.072 \mathrm{wt} \%$ except as noted

\begin{tabular}{|c|c|c|c|c|}
\hline & $\mathrm{Mn}$ & $\mathrm{P}$ & $\mathrm{Ni}$ & $\mathrm{Cu}$ \\
\hline \multicolumn{5}{|c|}{ Forging A508 Class 2 (low $\mathrm{Cu}), \mathrm{n}=15$} \\
\hline Minimum & 0.5800 & 0.0040 & 0.6900 & 0.0100 \\
\hline Mean & 0.6646 & 0.0092 & 0.7321 & 0.0443 \\
\hline Std deviation & 0.0525 & 0.0027 & 0.0356 & 0.0175 \\
\hline Maximum & 0.7900 & 0.0140 & 0.8100 & 0.0700 \\
\hline \multicolumn{5}{|c|}{ Forging A508 Class $3(1$ heat has $\mathrm{Cu}>0.072$ wt $\%), n=6$} \\
\hline Minimum & 1.2070 & 0.0090 & 0.6970 & 0.0460 \\
\hline Mean & 1.2897 & 0.0105 & 0.7290 & 0.0593 \\
\hline Std deviation & 0.0776 & 0.0018 & 0.0321 & 0.0105 \\
\hline Maximum & 1.4100 & 0.0130 & 0.7870 & 0.0770 \\
\hline \multicolumn{5}{|c|}{ Plate A533B1 (low Cu), $\mathbf{n}=21$} \\
\hline Minimum & 1.1600 & 0.0030 & 0.5600 & 0.0290 \\
\hline Mean & 1.3759 & 0.0080 & 0.6079 & 0.0506 \\
\hline Std deviation & 0.1058 & 0.0034 & 0.0339 & 0.0124 \\
\hline Maximum & 1.5500 & 0.0140 & 0.6800 & 0.0700 \\
\hline \multicolumn{5}{|c|}{ Low-Cu welds $\mathrm{Ni} \leq 0.5$ (some Linde $0091 \&$ Linde 124$), \mathrm{n}=19$} \\
\hline Minimum & 1.0800 & 0.0030 & 0.0600 & 0.0100 \\
\hline Mean & 1.3372 & 0.0082 & 0.1074 & 0.0369 \\
\hline Std deviation & 0.1338 & 0.0035 & 0.0433 & 0.0136 \\
\hline Maximum & 1.5500 & 0.0180 & 0.2200 & 0.0700 \\
\hline \multicolumn{5}{|c|}{ Low-Cu welds $0.5<\mathrm{Ni}<0.75($ some Linde $80 \&$ LW320), $\mathrm{n}=7$} \\
\hline Minimum & 1.4170 & 0.0110 & 0.6570 & 0.0220 \\
\hline Mean & 1.6303 & 0.0133 & 0.7091 & 0.0344 \\
\hline Std deviation & 0.2375 & 0.0021 & 0.0276 & 0.0088 \\
\hline Maximum & 1.9600 & 0.0170 & 0.7360 & 0.0490 \\
\hline \multicolumn{5}{|c|}{ Low-Cu welds $\mathrm{Ni} \geq 0.75$ (most Linde 124,1 has $\mathrm{Cu}>0.072 \mathrm{wt} \%), \mathrm{n}=16$} \\
\hline Minimum & 0.9370 & 0.0040 & 0.7600 & 0.0100 \\
\hline Mean & 1.3164 & 0.0115 & 0.9203 & 0.0397 \\
\hline Std deviation & 0.1692 & 0.0043 & 0.0540 & 0.0199 \\
\hline Maximum & 1.7000 & 0.0200 & 0.9730 & 0.0800 \\
\hline
\end{tabular}


Table G.2. High-Cu Materials

$\mathrm{Cu}>0.072$ wt \%

\begin{tabular}{|c|c|c|c|c|}
\hline & $\mathrm{Mn}$ & $\mathrm{P}$ & $\mathrm{Ni}$ & $\mathrm{Cu}$ \\
\hline \multicolumn{5}{|c|}{ Forging A508 Class $2($ high $\mathrm{Cu}), \mathrm{n}=9$} \\
\hline Minimum & 0.6100 & 0.0080 & 0.6700 & 0.0800 \\
\hline Mean & 0.6810 & 0.0141 & 0.7770 & 0.1220 \\
\hline Std deviation & 0.0433 & 0.0047 & 0.0635 & 0.0313 \\
\hline Maximum & 0.7250 & 0.0200 & 0.8600 & 0.1600 \\
\hline \multicolumn{5}{|c|}{ Plate A302B and A302B1, $n=18$} \\
\hline Minimum & 1.2626 & 0.0070 & 0.0560 & 0.0900 \\
\hline Mean & 1.3878 & 0.0134 & 0.2524 & 0.1400 \\
\hline Std deviation & 0.0585 & 0.0060 & 0.1794 & 0.0468 \\
\hline Maximum & 1.4600 & 0.0314 & 0.6300 & 0.2400 \\
\hline \multicolumn{5}{|c|}{ Plate A302BM, $n=19$} \\
\hline Minimum & 1.1600 & 0.0070 & 0.4410 & 0.0950 \\
\hline Mean & 1.3669 & 0.0111 & 0.5407 & 0.1812 \\
\hline Std deviation & 0.1297 & 0.0033 & 0.0571 & 0.0449 \\
\hline Maximum & 1.6450 & 0.0180 & 0.6300 & 0.2500 \\
\hline \multicolumn{5}{|c|}{ Plate A533B1 (high Cu), $\mathrm{n}=52$} \\
\hline Minimum & 1.2100 & 0.0050 & 0.4810 & 0.0730 \\
\hline Mean & 1.3505 & 0.0108 & 0.5755 & 0.1303 \\
\hline Std deviation & 0.0686 & 0.0029 & 0.0616 & 0.0412 \\
\hline Maximum & 1.5000 & 0.0170 & 0.7580 & 0.2410 \\
\hline \multicolumn{5}{|c|}{ Other high $\mathrm{Cu}$ welds $(\mathrm{Ni}<0.5)$ (some Linde 0091, others), $\mathrm{n}=24$} \\
\hline & $\mathrm{Mn}$ & $\mathrm{P}$ & $\mathrm{Ni}$ & $\mathrm{Cu}$ \\
\hline Minimum & 1.0500 & 0.0080 & 0.0440 & 0.0800 \\
\hline Mean & 1.3685 & 0.0144 & 0.1560 & 0.1849 \\
\hline Std deviation & 0.2320 & 0.0040 & 0.1113 & 0.0883 \\
\hline Maximum & 1.8200 & 0.0210 & 0.4070 & 0.4100 \\
\hline \multicolumn{5}{|c|}{ Typical Linde 80 welds $(\mathrm{Ni}>0.5), \mathrm{n}=32$} \\
\hline Minimum & 1.2600 & 0.0090 & 0.5200 & 0.0800 \\
\hline Mean & 1.5475 & 0.0152 & 0.6073 & 0.2800 \\
\hline Std deviation & 0.1291 & 0.0031 & 0.0508 & 0.0624 \\
\hline Maximum & 1.8800 & 0.0220 & 0.7300 & 0.3900 \\
\hline \multicolumn{5}{|c|}{ Linde 1092 welds, $\mathbf{n}=\mathbf{2 0}$} \\
\hline Minimum & 1.1220 & 0.0120 & 0.6000 & 0.1550 \\
\hline Mean & 1.3316 & 0.0158 & 0.8689 & 0.2349 \\
\hline Std deviation & 0.1323 & 0.0036 & 0.2024 & 0.0610 \\
\hline Maximum & 1.5700 & 0.0240 & 1.2600 & 0.3600 \\
\hline
\end{tabular}




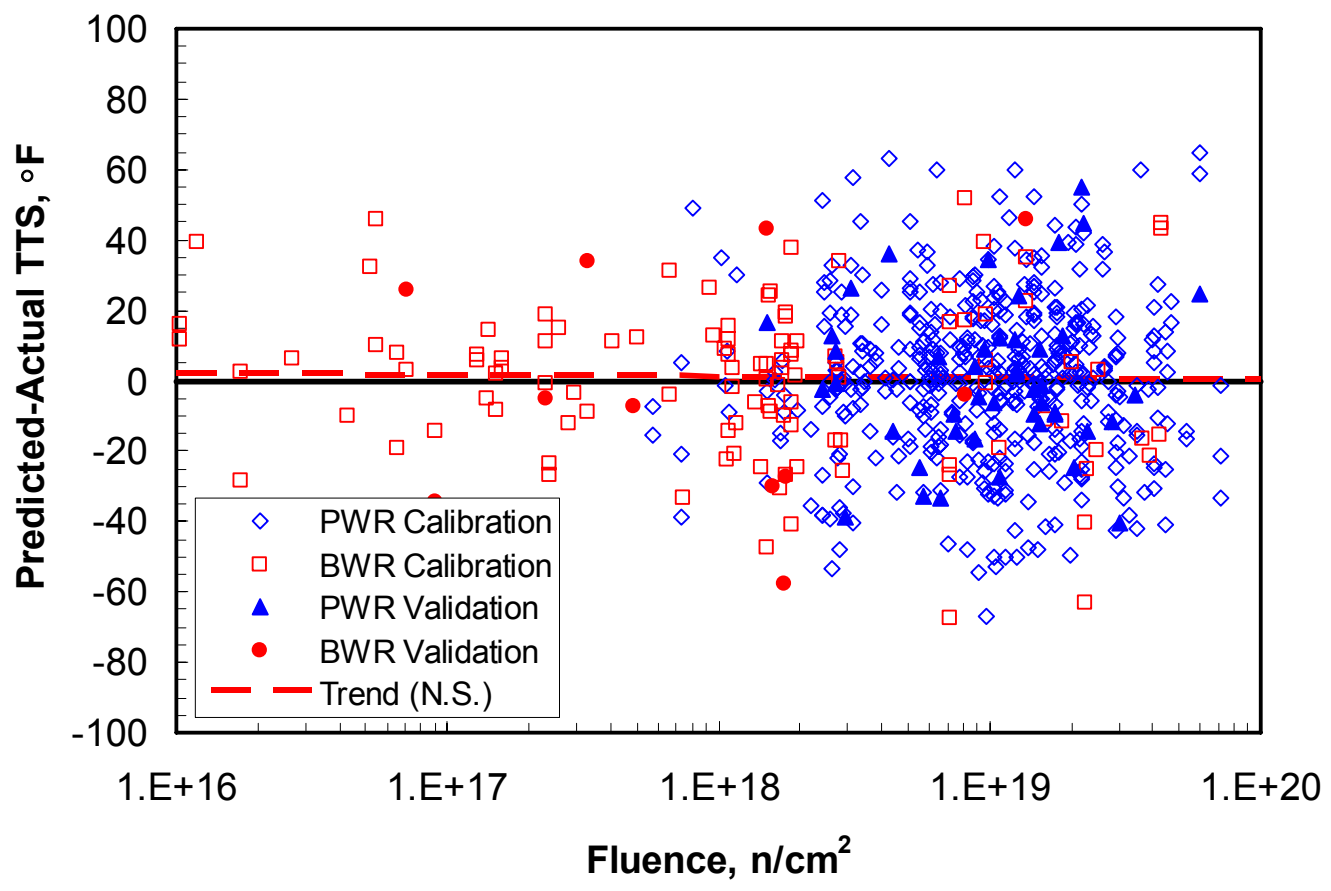

Fig. H.1. Residuals plotted against fluence, calibration and validation data with $\mathrm{Cu}>0.072$ wt \%.

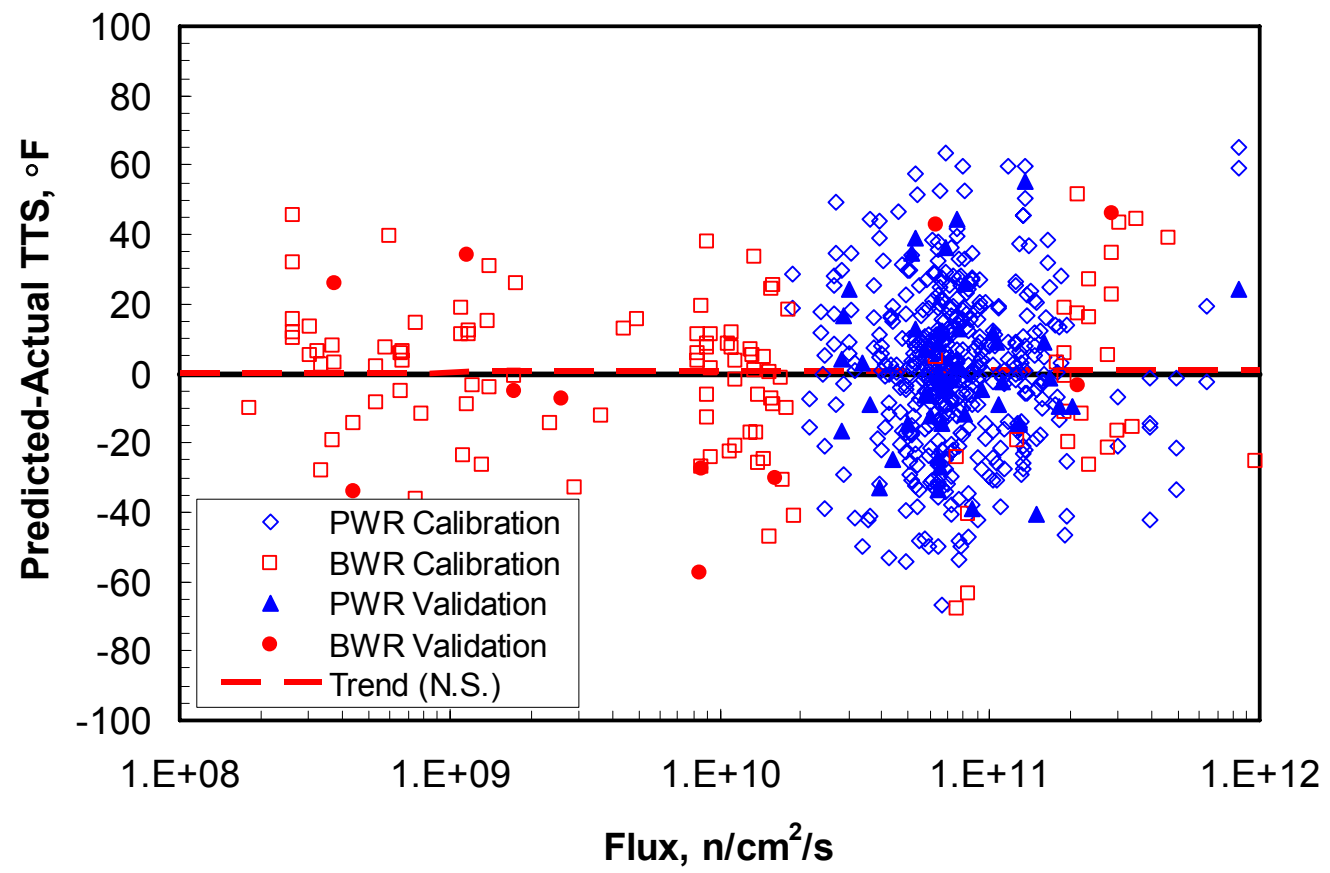

Fig. H.2. Residuals plotted against Flux, calibration and validation data with $\mathrm{Cu}$ $>0.072$ wt \%. 


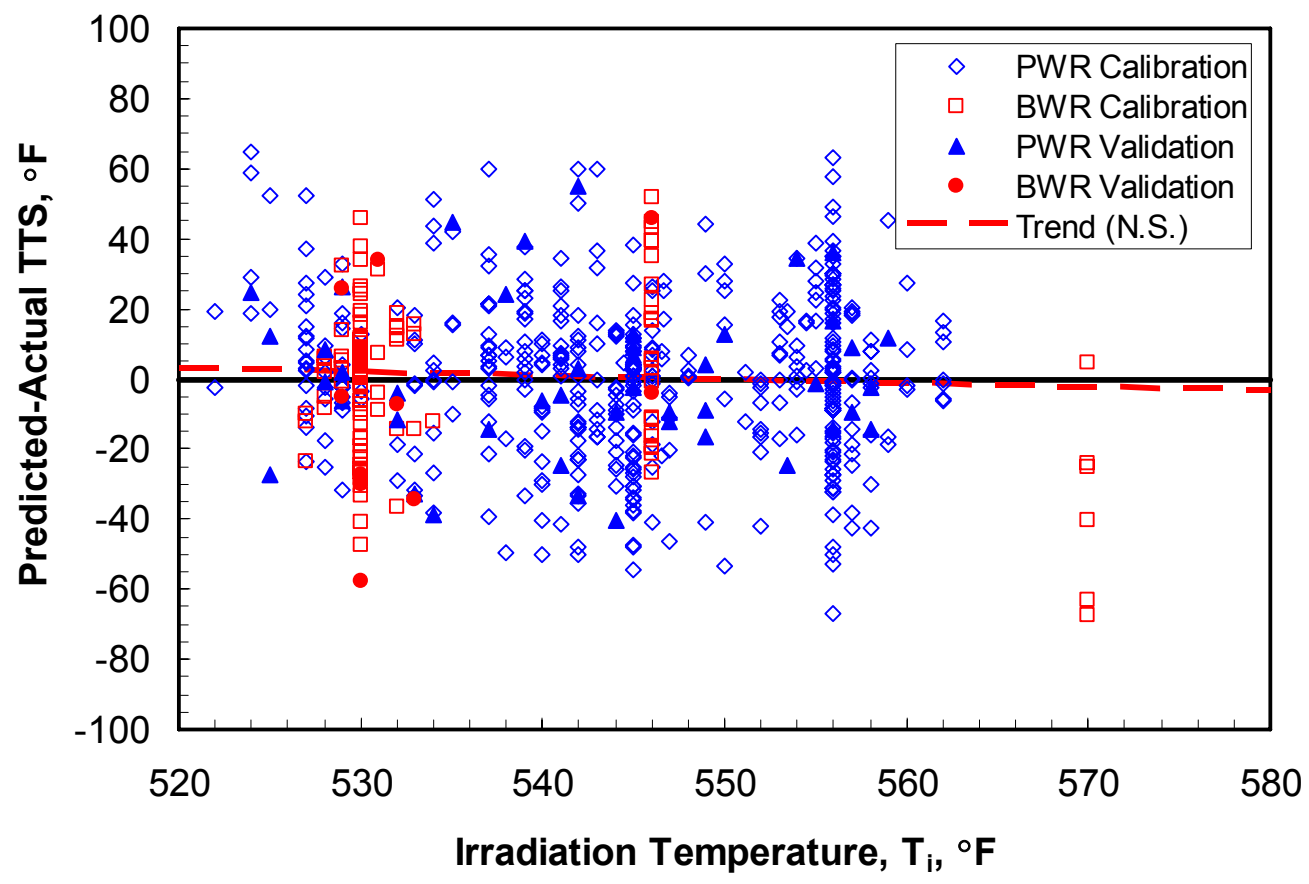

Fig. H.3. Residuals plotted against $T_{i}$, calibration and validation data with $\mathrm{Cu}>0.072$ wt \%.

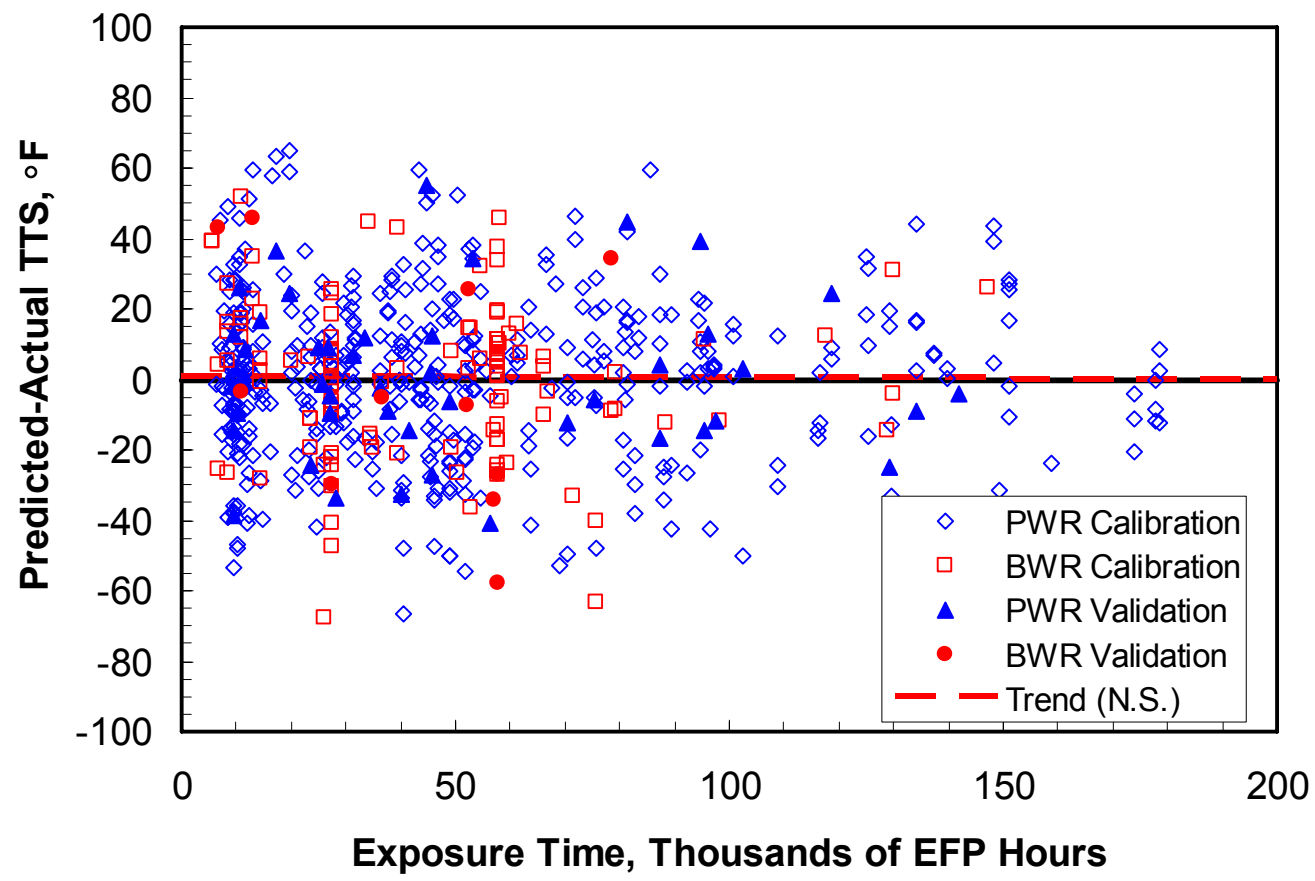

Fig. H.4. Residuals plotted against exposure time, calibration and validation data with $\mathrm{Cu}>0.072$ wt \%. 


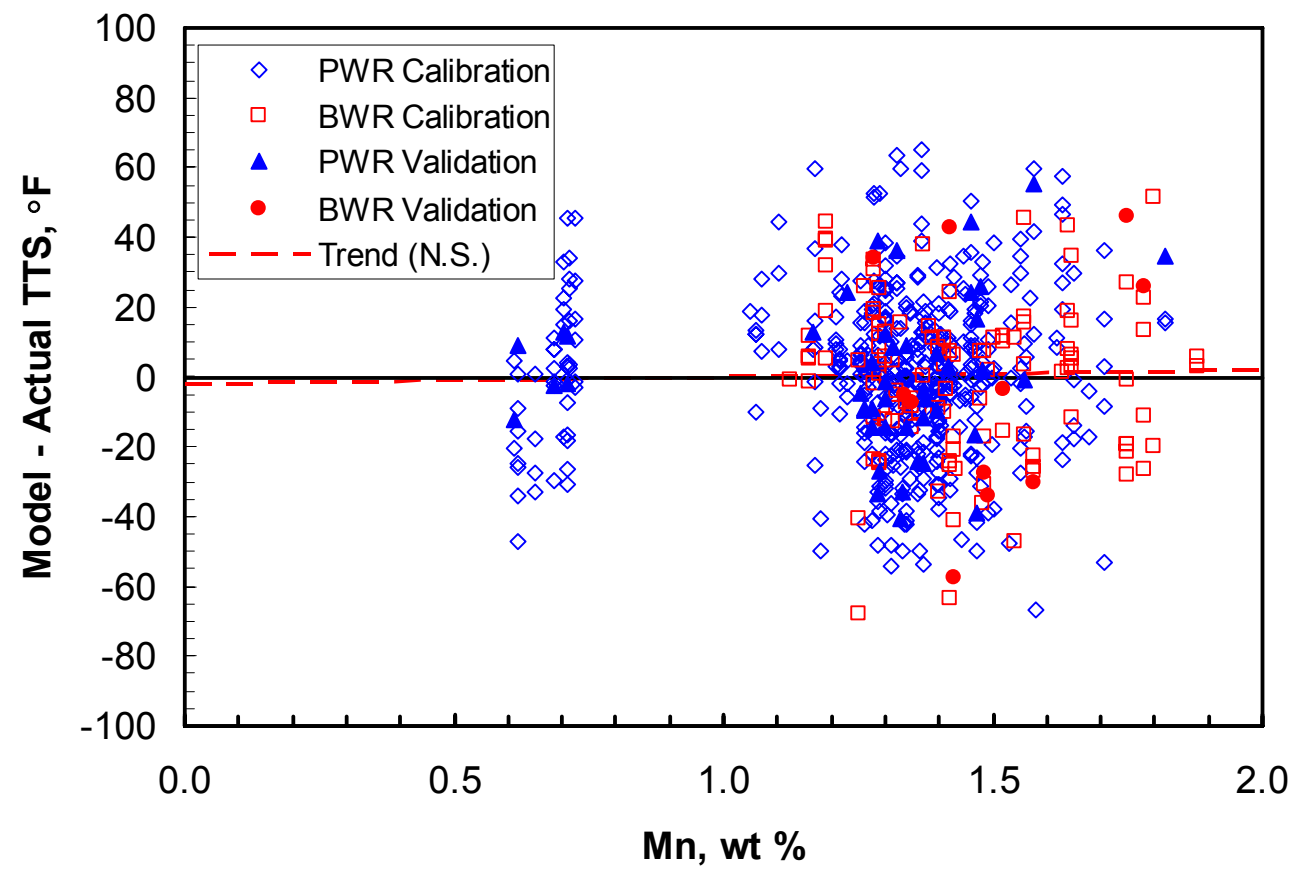

Fig. H.5. Residuals plotted against $\mathrm{Mn}$, calibration and validation data with $\mathrm{Cu}>0.072$ wt \%.

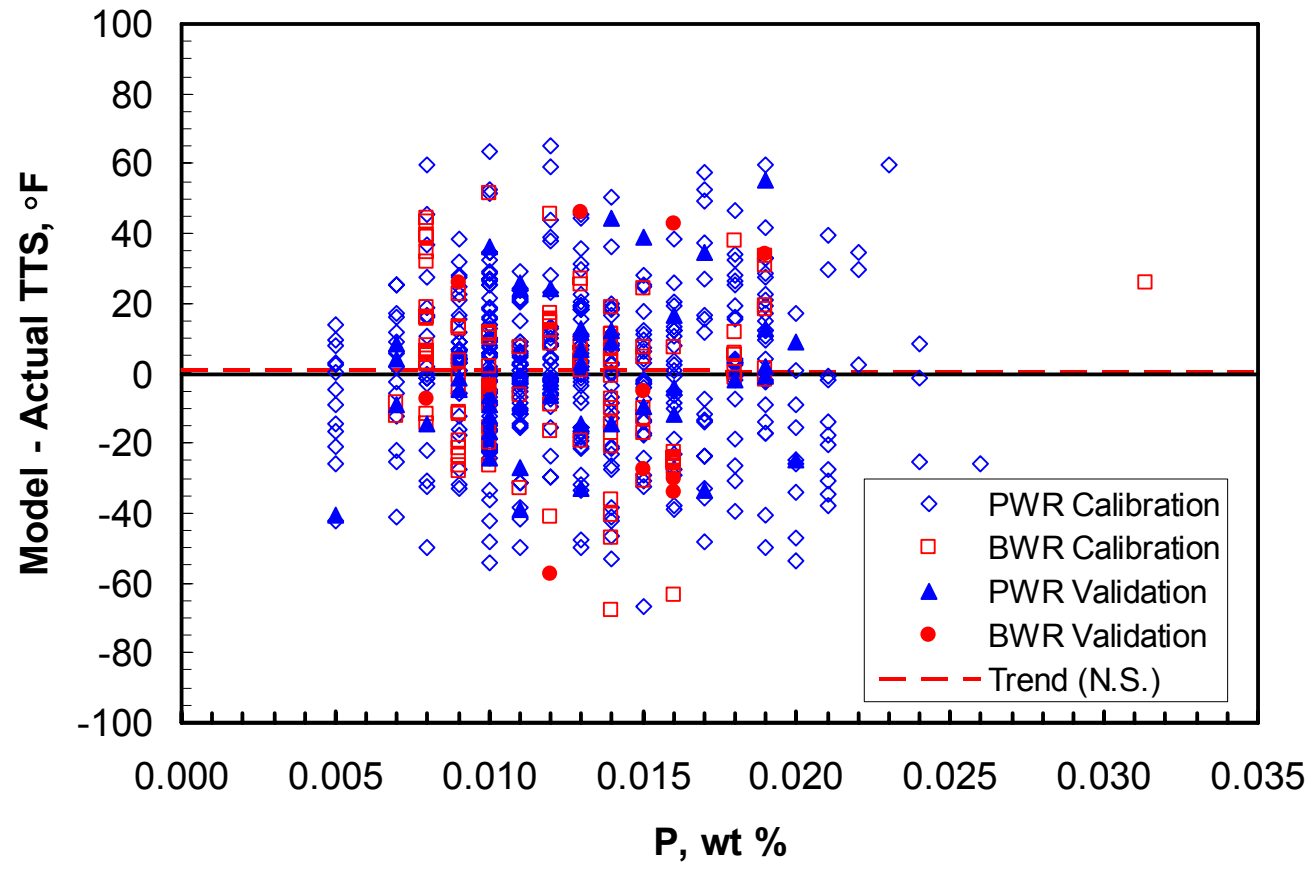

Fig. H.6. Residuals plotted against $P$, calibration and validation data with $\mathrm{Cu}>0.072$ wt \%. 


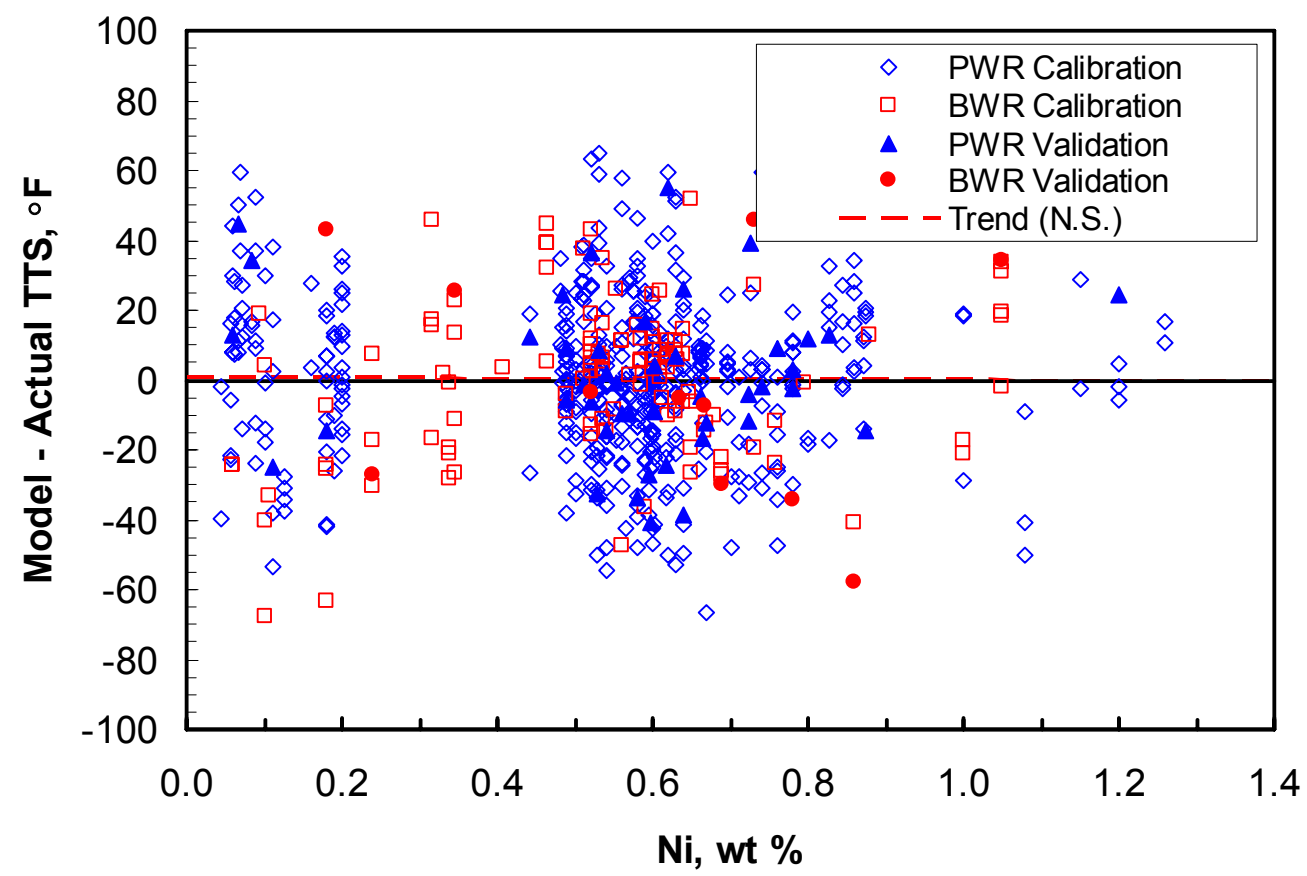

Fig. H.7. Residuals plotted against $\mathrm{Ni}$, calibration and validation data with $\mathrm{Cu}>0.072$ wt $\%$.

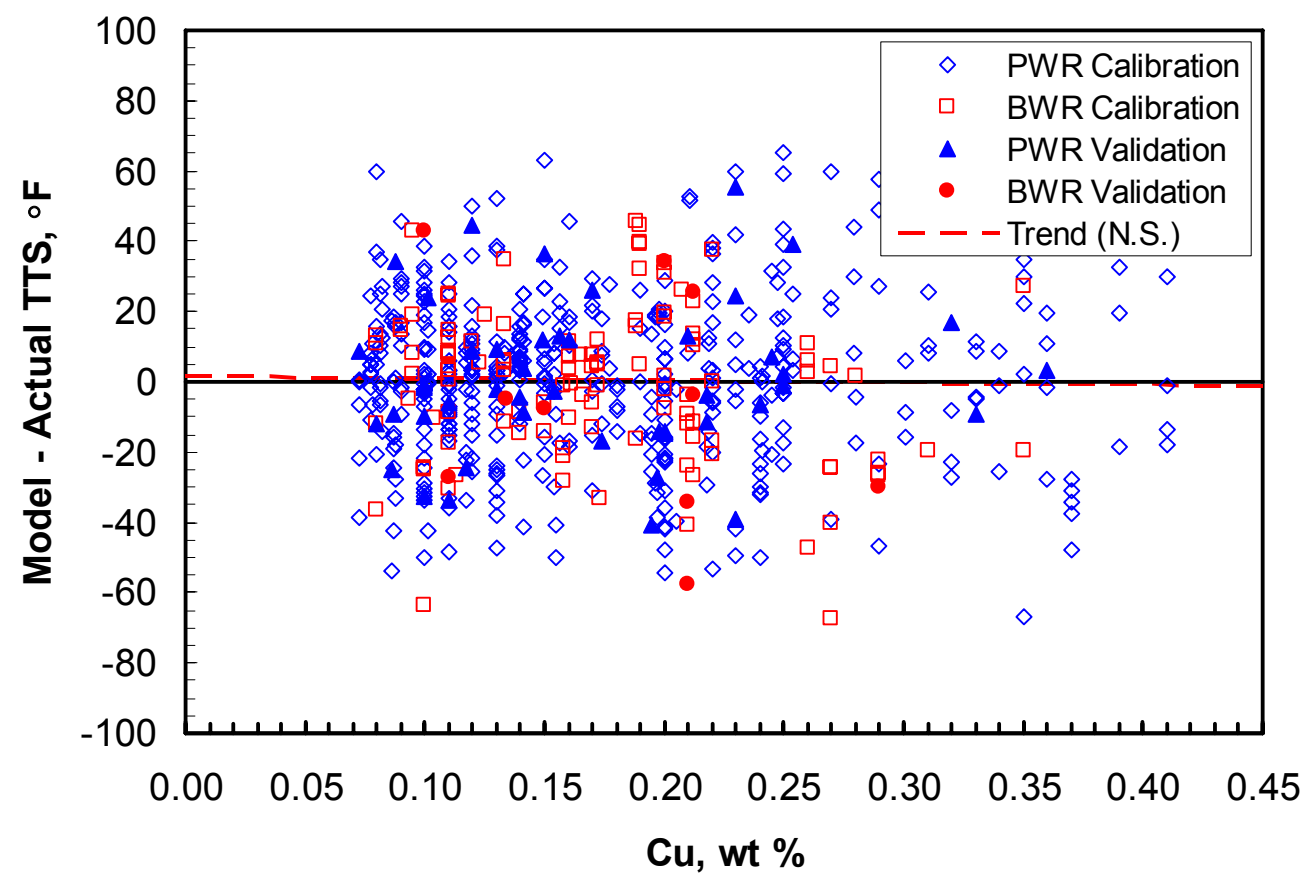

Fig. H.8. Residuals plotted against $\mathrm{Cu}$, calibration and validation data with $\mathrm{Cu}>0.072$ wt \%. 


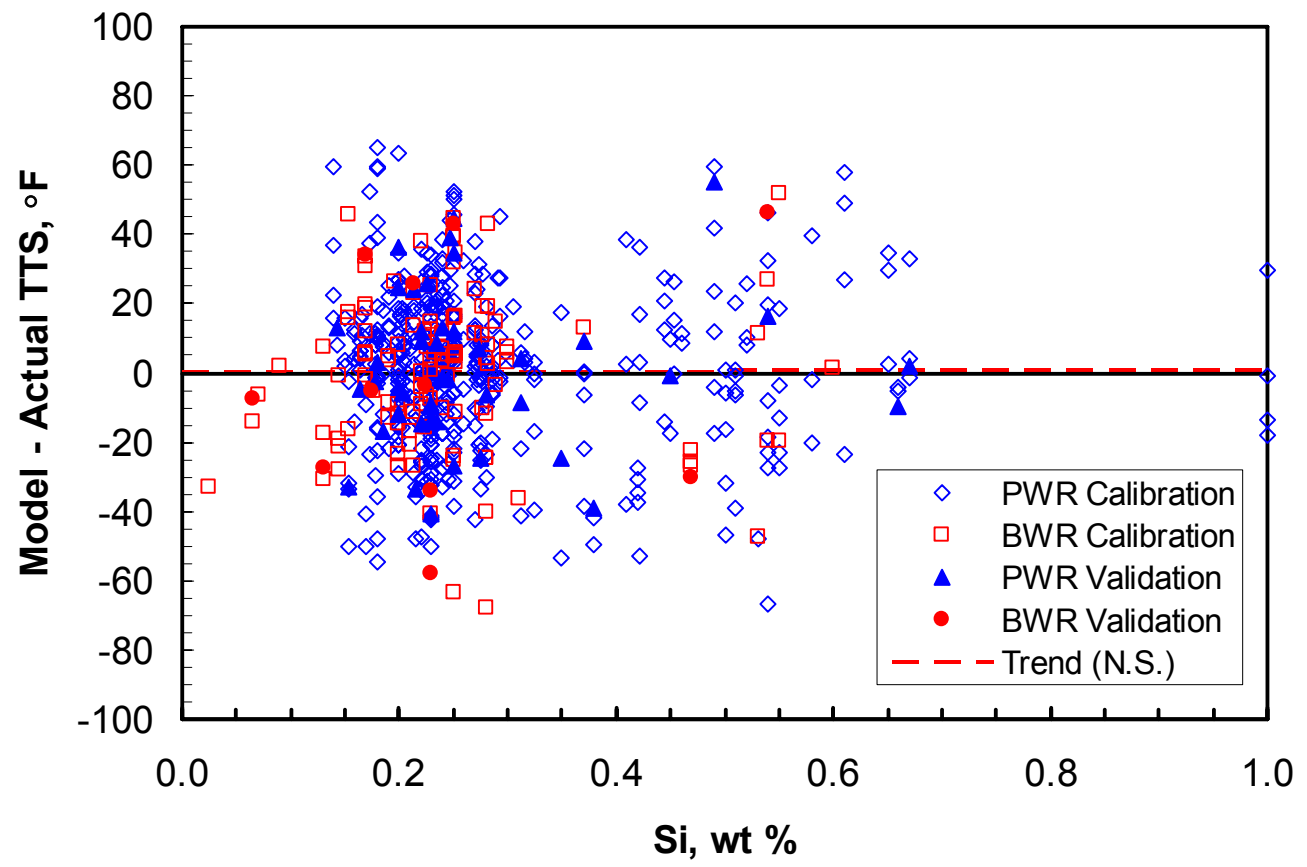

Fig. H.9. Residuals plotted against $\mathrm{Si}$, calibration and validation data with $\mathrm{Cu}>0.072$ wt $\%$.

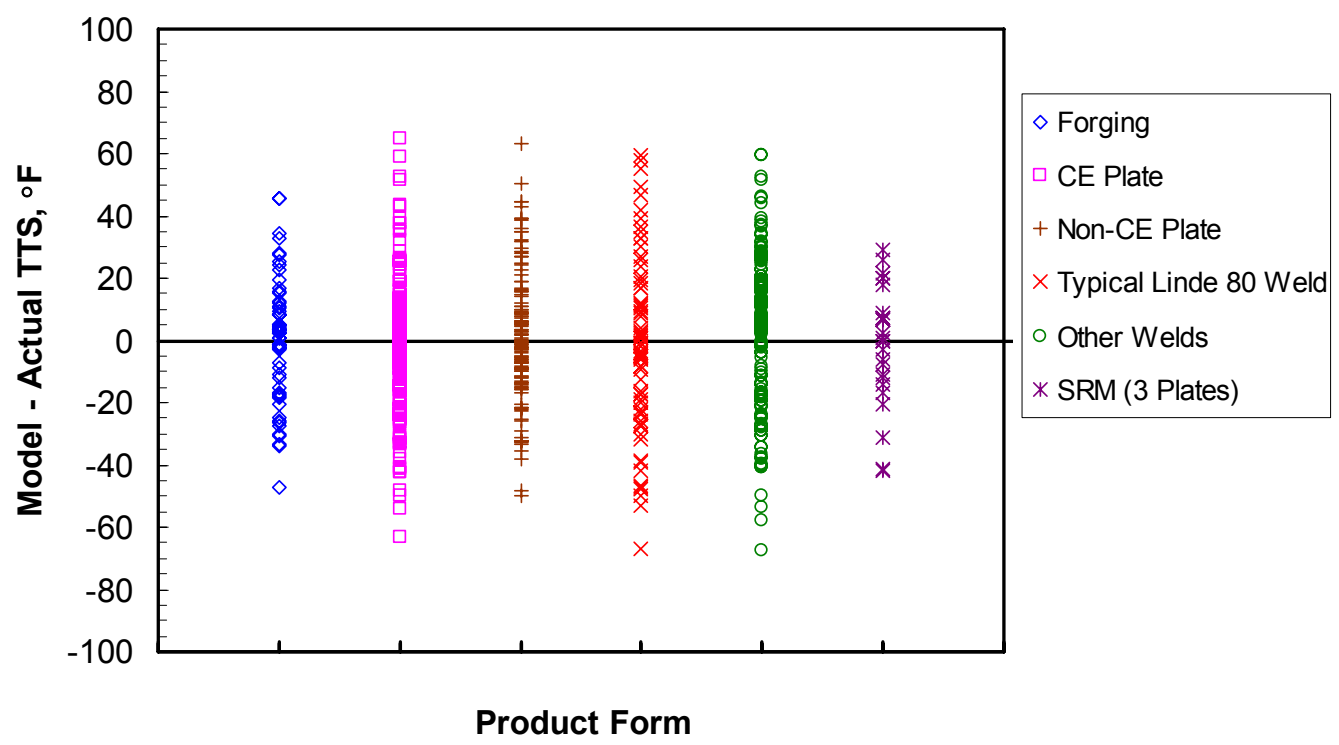

Fig. H.10. Residuals plotted against product form, calibration and validation data with $\mathrm{Cu}>0.072$ wt \%. 


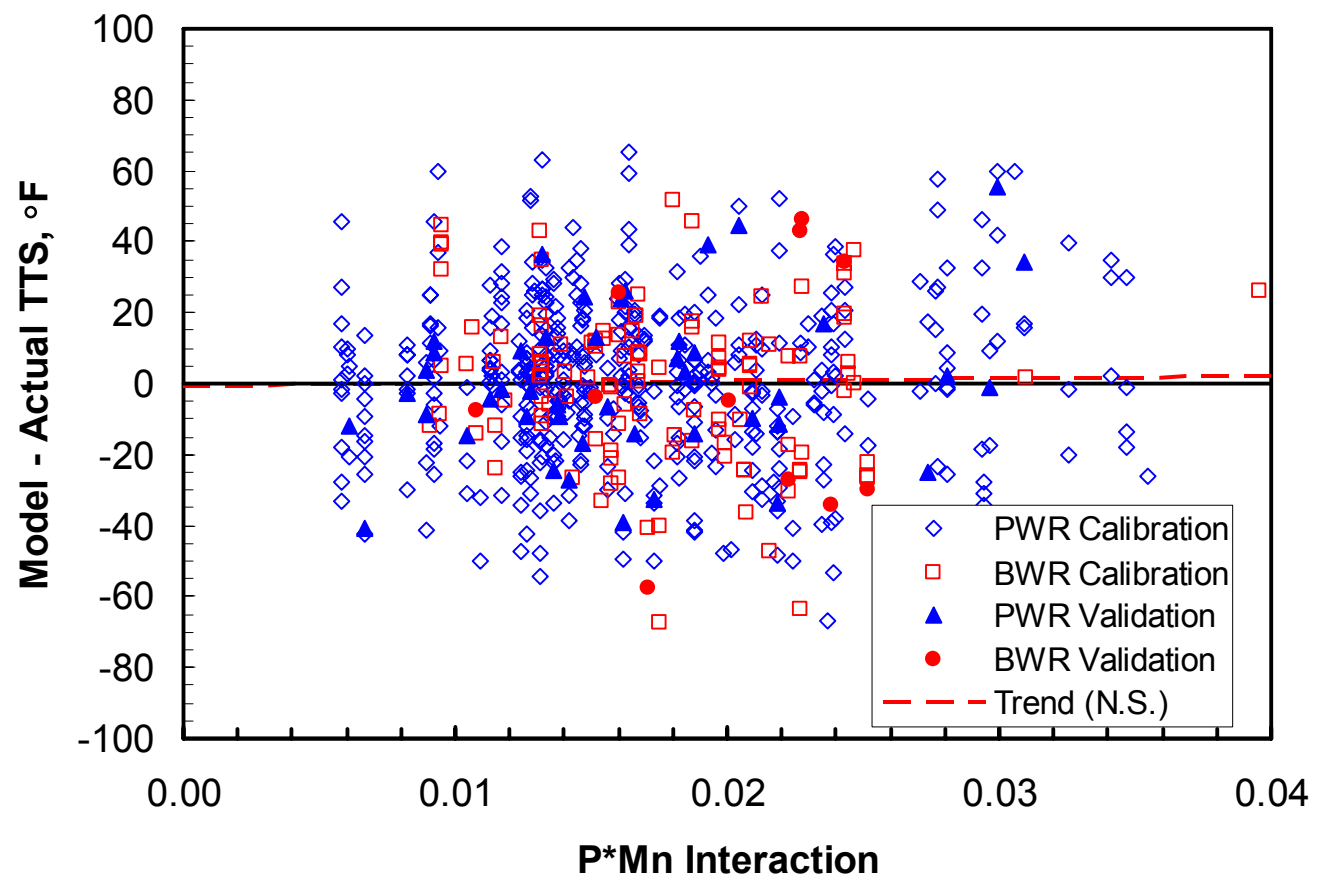

Fig. H.11. Residuals plotted against $P * M n$, calibration and validation data with $\mathrm{Cu}>0.072$ wt \%.

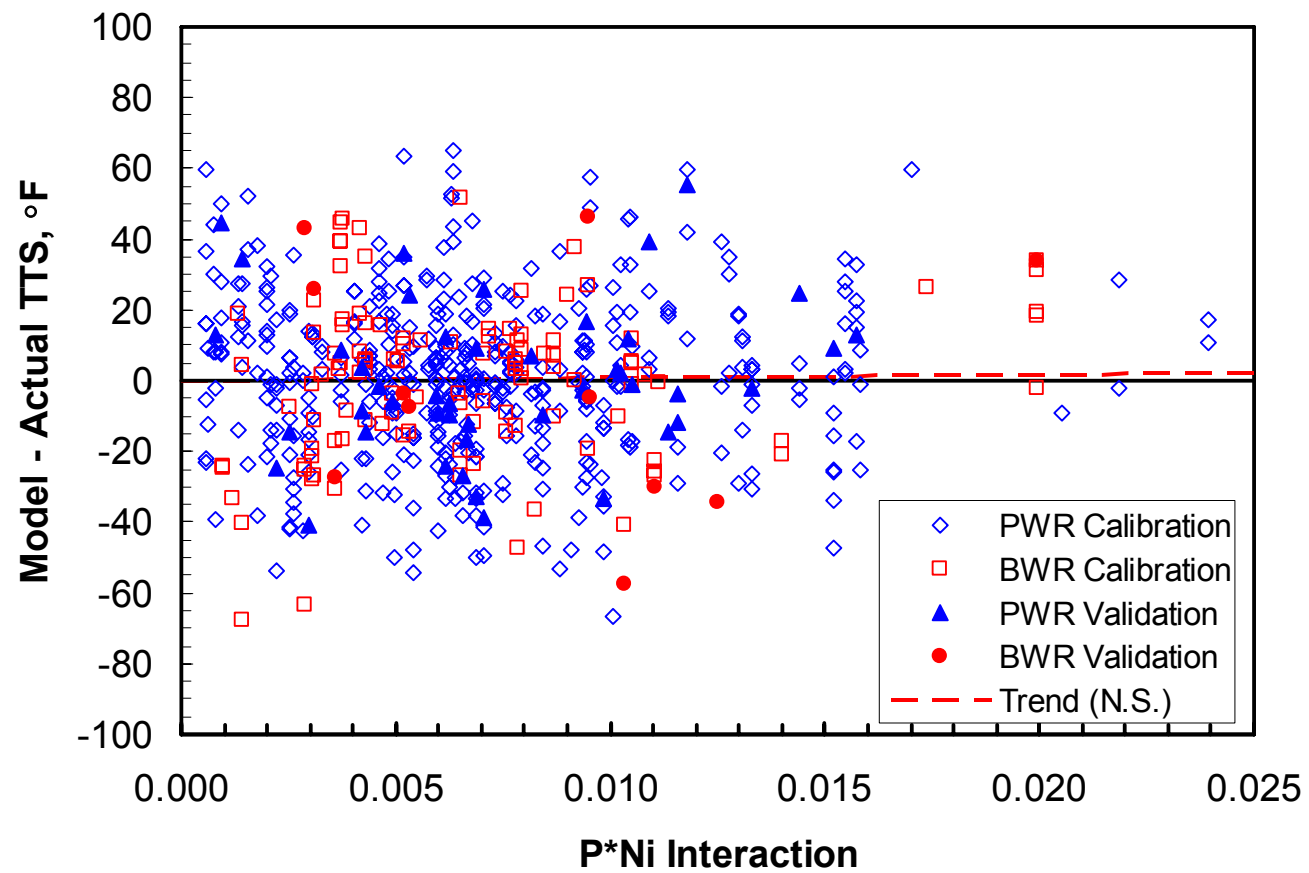

Fig. H.12. Residuals plotted against $\mathrm{P} * \mathrm{Ni}$, calibration and validation data with $\mathrm{Cu}>0.072$ wt \%. 


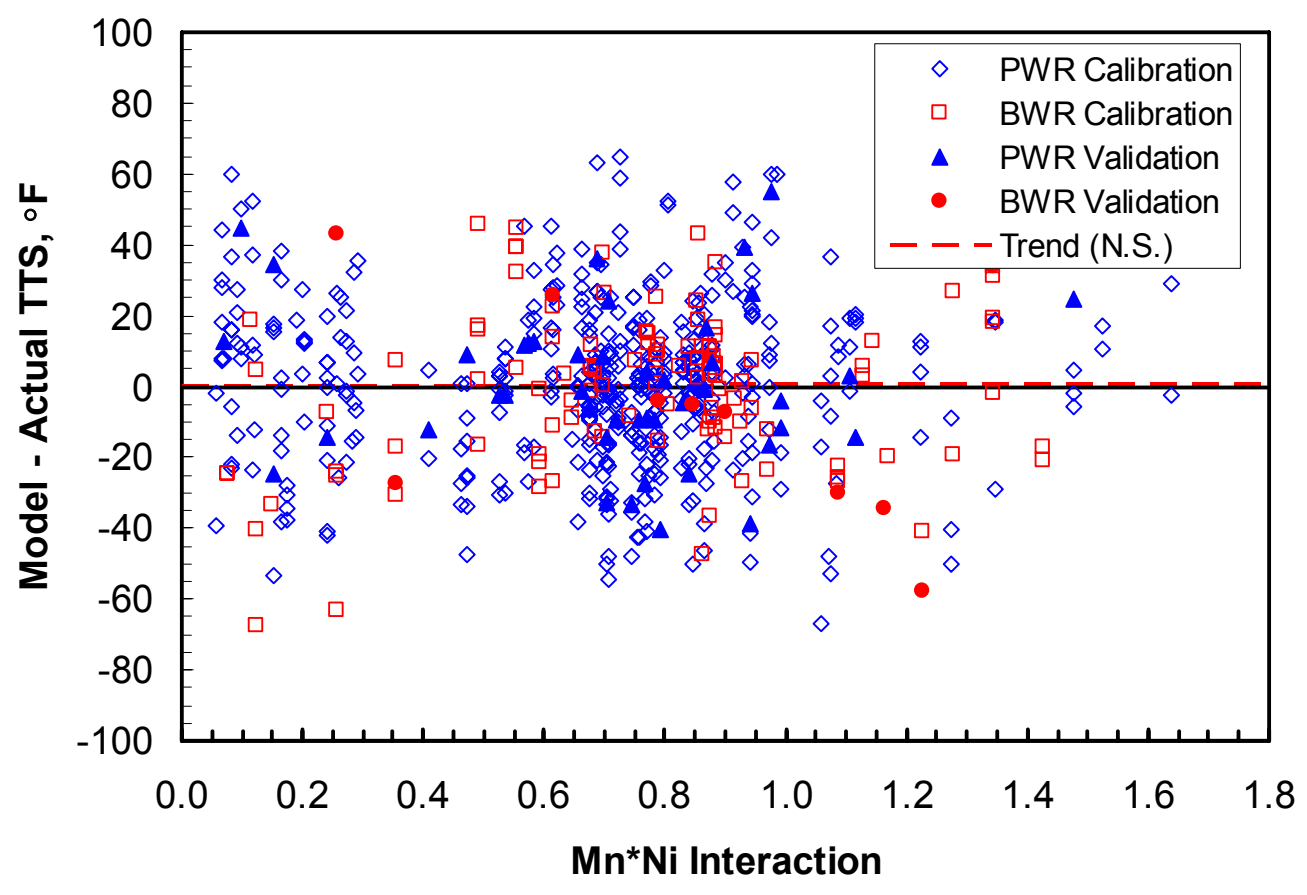

Fig. H.13. Residuals plotted against $\mathrm{Mn} * \mathrm{Ni}$, calibration and validation data with Cu $>0.072$ wt \%.

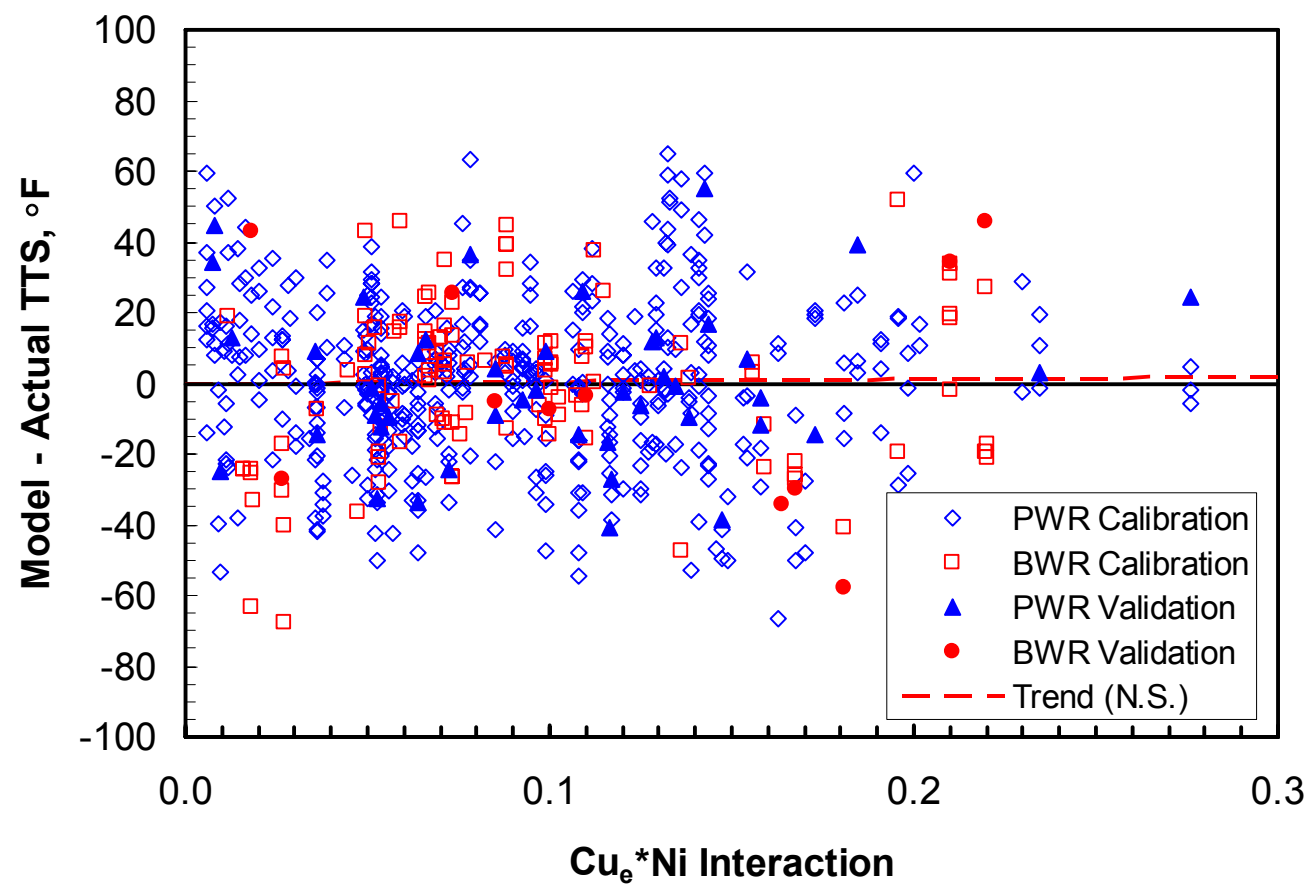

Fig. H.14. Residuals plotted against $\mathrm{Cu}_{\mathrm{e}}{ }^{* \mathrm{Ni}}$, calibration and validation data with $\mathrm{Cu}>0.072$ wt \%. 


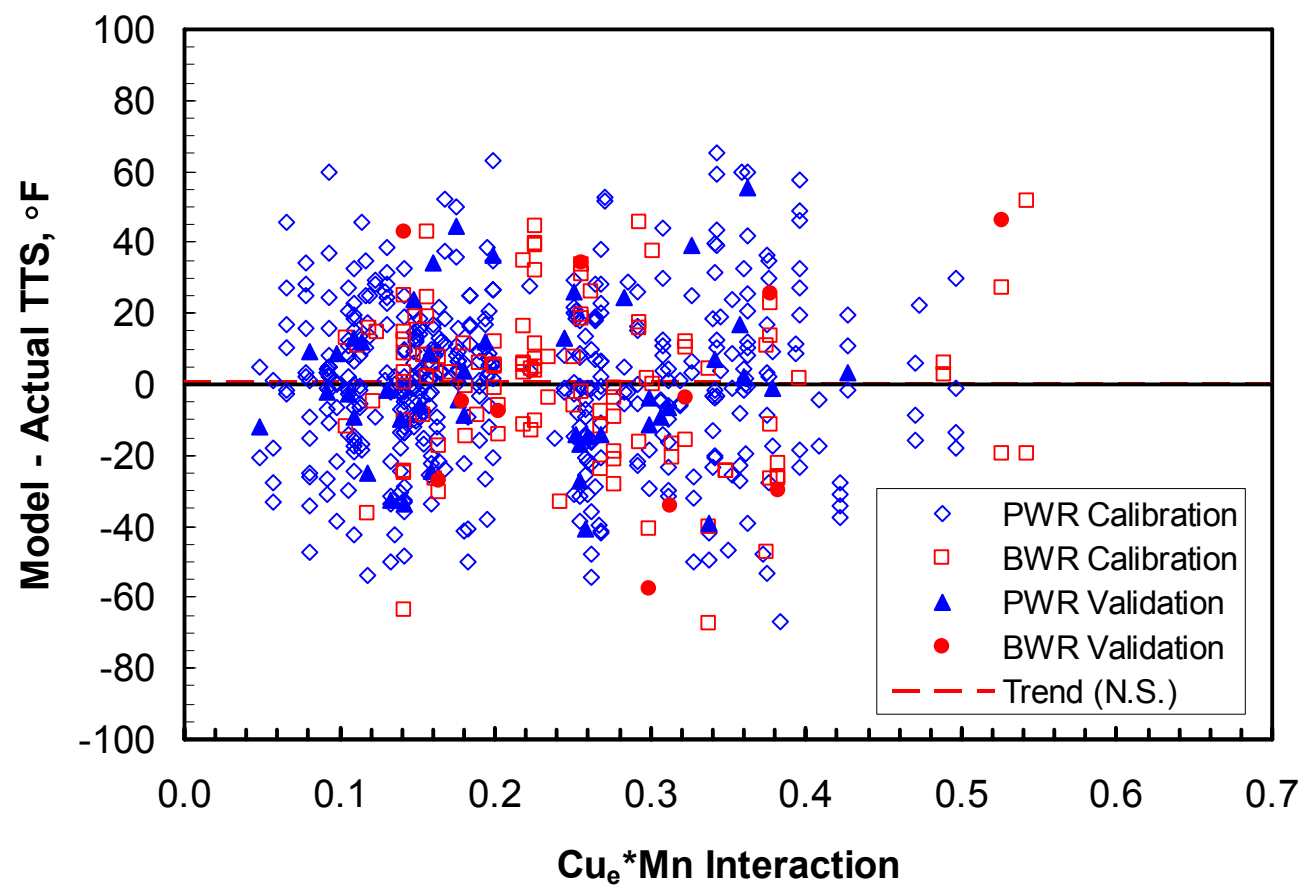

Fig. H.15. Residuals plotted against $\mathrm{Cu}_{\mathrm{e}} * \mathrm{Mn}$, calibration and validation data with $\mathrm{Cu}>0.072$ wt \%. 


\section{INTERNAL DISTRIBUTION}

1. B. R. Bass

2. W. R. Corwin

3. D. W. Heatherly

4. S. K. Iskander

5. L. K. Mansur

6. D. E. McCabe

7. J. G. Merkle

8. M. K. Miller

9-16. R. K. Nanstad

17. I. Remec

\author{
18-25. T. M. Rosseel \\ 26. J. J. Simpson \\ 27. M. A. Sokolov \\ 28. R. E. Stoller \\ 29. R. L Swain \\ 30. K. R. Thoms \\ 31. J. A. Wang \\ 32. S. J. Zinkle \\ 33. Office of Technical Information \\ and Classification
}

\section{EXTERNAL DISTRIBUTION}

34. AEA Technology Nuclear Science, Harwell, Didcot, Oxforshire OX1100QJ, United Kingdom

$$
\text { C. A. English }
$$

35. ATI, Inc., Suite 160, 210 Crow Canyon Place, San Ramon, CA 94583

$$
\text { W. L. Server }
$$

36-37. Areva, N. P., 25589 Fishers Hill Ct., Chantilly, VA 20152

$$
\begin{aligned}
& \text { B. Hall } \\
& \text { K. Yoon }
\end{aligned}
$$

38. Bettis Atomic Power Laboratory, Westinghouse Electric Corp., P. O. Box 79 West Mifflin, PA 15122

$$
\text { M. G. Burke }
$$

39. BWXT Services, Inc., 2016 Mount Athos Rd., Lynchburg, VA 24504

$$
\text { K. Hour }
$$

40-42. Central Institute for Nuclear Studies, CEN/SCK, Reactor Physics Department, Boeretang 200, B-2400 Mol. Belgium
M. Scibetta
E. Van Walle
E. Lucon 
43. DOE Oak Ridge Operation Office, P. O. Box 2001, Oak Ridge, TN 37831-6269

\section{S. R. Martin}

44-46. EPRI, 1300 West W.T. Harris Blvd., Charlotte, NC 28262
S. T. Rosinski
R. G. Carter
J. Spanner

47. Fraunhofer Institute für Werkstoffmechanik Wöhlerstr. 11-13 79108 Freiburg, Germany

W. Boehme

48. Health \& Safety Executive, Nuclear Safety Directorate, HM Nuclear Installation Inspectorate, St. Peter's House, Stanley Precinct, Bootle, Merseyside L203LZ, United Kingdom

$$
\text { R. D. Nicholson }
$$

49. Joint Research Center, Institute for Advanced Materials, P. O. Box 2 Petten, 1775 Petten, The Netherlands

L. Debarberis

50. Korea Atomic Energy Research Institute, Nuclear Material Technology Division, 150 Dukjin-dong, Yusong-ku, 305-353 Daejon, Korea

\section{Bong Sang Lee}

51-60. Modeling and Computer Services, LLC, 6560 Gunpark Dr., Suite B, Boulder, CO 80301

E. D. Eason

61. Nuclear Research Institute, Division of Nuclear Materials, 25068 REZ, Czech Republic M. Brumovsky

62-68. NRC, RES/DFERR, Washington, DC, 20555-0001
M. C. Cunningham
D. A. Jackson
M. T. EricksonKirk
S. M. Malik
E. Focht
C. E. Moyer
R. O. Hardies
R. L. Tregoning

69-72. NRC, NRR/DE, Washington, DC, 20555-0001
B. J. Elliot
C. J. Fairbanks
A. L Hiser
M. A. Mitchell 
73. NRC/ONR/DE, Washington, DC, 20555-0001

M. E. Mayfield

74. NRC, NMSS, SPPO
E. M. Hackett

75. NRC, ACRS, ITSB, Washington, DC, 20555-0001

C. G. Santos

76. Pavinich, W. A., 1113 Laurel Hill Road, Knoxville, TN 37923

77. Prometey, Central Research, Institute of Structural Materials, 193167 Sankt

Petersburg, Russia

$$
\text { V. A. Nikolaev }
$$

78. Rolls-Royce and Associates LTD., P. O. Box 31, Raynesway, Derby DE24 8BJ, United Kingdom

\section{T. J. Williams}

79. Russian Research Center-Kurchatov Institute, 1 Kurchatov Sq., Moscow 123182, Russia
A. A. Chernobaeva

80-81. Paul Scherrer Institute, CH-5233 VILLIGEN SPI, Switzerland

\section{J. Bertsch}

82. Siemens AG, KWU S514, Freyeslebenstrasse 1, D-8520 Erlangen, Germany

$$
\text { R. Langer }
$$

83-87. Staatliche Materialprufungsanstalt and Der Universitat Stuttgart, Pfaffenwaldring 32, 70569 Stuttgart, Germany
U. Eisele
J. Fohl
H. Huber
R. Gillot
E. Roos

88. Swiss Federal Nuclear Safety Inspectorate, CH 5232 Villigen-HSK, Switzerland
D. Kalkhof
P. Tipping

89. Texas A\&M University College Station, TX 77843-3123

R. Chona 
90. The Welding Institute, Abington Hall, Abington, Cambridge, CB1 6 AL, United Kingdom

C. S. Wiesner

91-92. Tokai Research Establishment, Japan Atomic Energy Research Institute, 2-4 Shirakatashirane, Tokai-mura, Naka-gun, Ibaraki 319-1195, Japan

\section{Suzuki \\ K. Onizawa}

93. Tokai University, Dept. of Nuclear Engineering, 1117, Kitakaname, Hiratsuka-shi, Kanagawa-Ken 259-12, Japan

Prof. Shiori Ishino

94-114. University of California, Department of Chemical and Nuclear Engineering, Ward Memorial Drive, Santa Barbara, CA 93106
G. E. Lucas
T. Yamamoto
G. R. Odette

115. U.S. Department of Energy, Office of Nuclear Science and Technology, 19901 Germantown Rd., Germantown, MD 20874-1290

R. M. Versluis

116. VTT Manufacturing Technology, P. O. Box 1704, FIN-02044 VTT, Finland M. Valo

117. Westinghouse, P. O. Box, 500, Mail Code 9483-1903, Windsor, CT 60695

$$
\text { S. T. Byrne }
$$

118. Westinghouse Electric Corporation, P. O. Box 335, Pittsburgh, PA 15230

$$
\text { W. Bamford }
$$

119. Westinghouse R\&D Center, 1310 Beulah Rd., Pittsburgh, PA 15325

$$
\text { R. G. Lott }
$$




\begin{tabular}{l}
$\begin{array}{l}\text { NRC FORM } 335 \\
(2-89)\end{array}$ \\
$\begin{array}{l}\text { NRCM 1102, } \\
3201,3202\end{array}$ \\
$\begin{array}{l}\text { BIBLIOGRACLEAR REGULATORY COMMISSION } \\
\text { (See instructions on the reverse) }\end{array}$ \\
\hline
\end{tabular}

1. REPORT NUMBER
(Assigned by NRC, Add Vol., Supp., Rev., and Addendum Numbers, if any.)

2. TITLE AND SUBTITLE

NUREG/CR -XXXX

ORNL/TM - 2006/530

A Physically Based Correlation of Irradiation-Induced Transition

Temperature Shifts for RPV Steels

3. DATE REPORT PUBLISHED

\begin{tabular}{l|l} 
MONTH & YEAR
\end{tabular}

4. FIN OR GRANT NUMBER

5. AUTHOR(S)

6. TYPE OF REPORT

E. D. Eason, M\&CS; G. R. Odette, UCSB; R. K. Nanstad, UT-Battelle, LLC, Oak Ridge Natl. Lab., Oak Ridge, Tenn; and T. Yamamato, UCSB

NUREG/CR

7. PERIOD COVERED (Inclusive Dates)

8. PERFORMING ORGANIZATION - NAME AND ADDRESS (If NRC, provide Division, Office or Region, U.S. Nuclear Regulatory Commission, and mailing address; if contractor, provide name and mailing address.)

Heavy-Section Steel Irradiation Program

Materials Science and Technology Division

Oak Ridge National Laboratory

Oak Ridge, TN 37831-6161

9. SPONSORING ORGANIZATION - NAME AND ADDRESS (If NRC, type "Same as above"; if contractor, provide NRC Division, Office or Region, U.S. Nuclear Regulatory Commission, and mailing address.

Component Integrity Branch

Division of Fuel, Engineering \& Radiological Research

Office of Nuclear Regulatory Research

US Nuclear Regulatory Commission

Washington DC 20555-0001

10. SUPPLEMENTARY NOTES

11. ABSTRACT (200 words or less)

Irradiation embrittlement of reactor pressure vessel beltline materials is currently evaluated using U.S. Nuclear Regulatory Commission Regulatory Guide 1.99 Revision 2 (RG1.99/2), which presents methods for estimating the shift in Charpy transition temperature at $30 \mathrm{ft}-\mathrm{lb}$ (TTS) and the drop in Charpy upper shelf energy ( $\triangle \mathrm{USE}$ ). The purpose of the work reported here is to improve on the TTS correlation in RG1.99/2 using the broader database now available and current understanding of embrittlement mechanisms.

The key areas expanded in the current database are low-flux, low-copper, and long-time, high-fluence exposures, previously sparse areas. The model incorporates both physically motivated features and empirical calibration to the U.S. power reactor surveillance data. It contains two terms, corresponding to the best-understood radiation damage features, matrix damage and copper-rich precipitates. The new model includes the variables copper, nickel, and fluence that are in RG1.99/2, but also includes irradiation temperature, neutron flux, phosphorus, and manganese.

The report includes a chapter summarizing embrittlement mechanisms and one comparing the Irradiation Variables (IVAR) database with the TTS model predictions. The key new insight in the TTS modeling effort, that flux effects are evident in both low (or no) copper and higher copper materials, is supported by the IVAR data.

12. KEY WORDS/DESCRIPTORS (List words or phrases that will assist researchers in locating the report.)

Transition temperature shift

Embrittlement mechanisms

Copper rich precipitates

Matrix features

Material variables

Flux effects

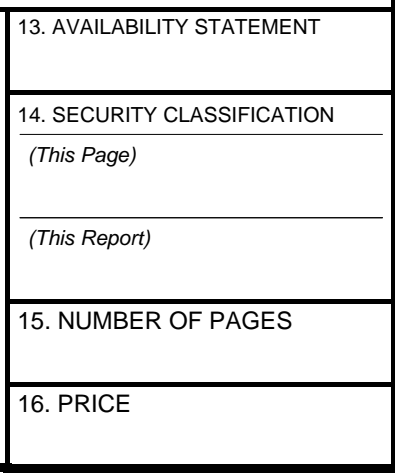


